\title{
2012 Service Academy Gender Relations Survey
}




\section{Service Academy Gender Relations Survey}

\section{Introduction}

The Department of Defense (DoD) continues to emphasize sexual assault and sexual harassment response and prevention at the Service Academies. Each of the Academies have implemented and expanded programs to educate students on these issues and to provide reporting and victim care procedures. Continuing evaluation of these programs is critical to reducing instances of sexual assault and sexual harassment as the Academies strive to provide a safe educational and leadership development environment for their students. This survey note discusses findings from the 2012 Service Academy Gender Relations Survey (2012 SAGR), a source of information for evaluating these programs and for assessing the gender-relations environment at the Academies.

The 2012 SAGR is the fifth in a series of surveys mandated by U.S. Code Title 10, as amended by Section 532 of the John Warner National Defense Authorization Act for Fiscal Year 2007 (10USC $\$ 4361)$. This survey assesses the incidence of sexual assault and sexual harassment and gender-related issues at the U.S. Military Academy (USMA), the U.S. Naval Academy (USNA), the U.S. Air Force Academy (USAFA), and the U.S. Coast Guard Academy (USCGA). ${ }^{1}$

This survey note and accompanying briefings (Appendices A- $\mathrm{D}^{2}$ ) provide information on the annual prevalence rates of sexual assault, sexual harassment and sexist behavior, and stalking-related behaviors; a discussion of students' perceptions of Academy culture with respect to sexual assault and sexual harassment; the availability and effectiveness of sexual assault and sexual harassment training; and perceptions of program effectiveness in reducing or preventing sexual assault and sexual harassment.

The 2012 SAGR was fielded in March and April 2012. Completed surveys were received from 5,425 eligible respondents (1,513 from USMA, 1,574 from USNA, 1,537 from USAFA, and 801 from USCGA). The overall weighted response rate was 73\% (79\% for USMA, 70\% for USNA, $68 \%$ for USAFA, and $80 \%$ for USCGA). ${ }^{3}$

This survey note provides results for USMA, USNA, USAFA, and USCGA by gender and class year. When 2012 SAGR questions are comparable to questions in the previous 2006, 2008, and 2010 surveys, an analysis of trends is also presented. When a result is annotated as higher or lower than another result, the reader should understand that to be a statistically significant difference at the .05 level of significance. A finding annotated as no change did not achieve a .05 level of difference.

\footnotetext{
${ }^{1}$ This survey was also administered to students at the U.S. Merchant Marine Academy (USMMA). The results for USMMA are reported separately.

${ }^{2}$ See Appendix A for USMA results, Appendix B for USNA results, Appendix C for USAFA results, and Appendix D for USCGA results.

${ }^{3}$ Further details on survey methodology can be found in the 2012 SAGR Statistical Methodology Report (DMDC 2012).
} 


\section{Overview}

The ability to calculate annual prevalence rates is a distinguishing feature of this survey. This report includes rates of unwanted sexual contact, unwanted gender-related behaviors, and stalking-related behaviors experienced during the Academic Program Year 2011-2012 (defined in the survey questions as June 2011 through the date of survey administration in March or April 2012).

Unwanted Sexual Contact. The 2012 SAGR survey includes a measure of unwanted sexual contact (i.e., sexual assault). Although this term does not appear in the Uniform Code of Military Justice (UCMJ), it is used as an umbrella term intended to include certain acts prohibited by the UCMJ. For the purposes of the 2012 SAGR survey, the term "unwanted sexual contact" means intentional sexual contact that was against a person's will or which occurred when the person did not or could not consent, and includes completed or attempted sexual intercourse, sodomy (oral or anal sex), penetration by an object, and the unwanted touching of genitalia and other sexually-related areas of the body. Students were asked questions related to personal experiences of unwanted sexual contact between June 2011 and the time they took the survey. Students who indicated they experienced unwanted sexual contact were then asked to provide details on their experience. The measure of unwanted sexual contact was developed for the 2006 survey. Trend comparisons on unwanted sexual contact are presented from surveys administered in 2006, 2008, 2010, and 2012. Also included for the first time in 2012 SAGR is a measure of unwanted sexual contact prior to entering the Academy.

Unwanted Gender-Related Behaviors. The 2012 SAGR includes measures of unwanted genderrelated behaviors (i.e., sexual harassment and sexist behavior) derived from the Sexual Experiences Questionnaires (Fitzgerald et al., 1988; Fitzgerald, Gelfand, \& Drasgow, 1995). To determine the extent of unwanted gender-related behaviors, students were provided a list of 12 sexual harassment behaviors and four sexist behaviors, and were asked to indicate how often they had experienced the behaviors since June 2011. The 12 sexual harassment behaviors comprise three components of sexual harassment—crude/offensive behavior (e.g., repeatedly told sexual stories or jokes that are offensive); unwanted sexual attention (e.g., unwanted attempts to establish a romantic sexual relationship despite efforts to discourage it); and sexual coercion (e.g., treated badly for refusing to have sex). In order to determine how to "count" the frequency of sexual harassment behaviors, a counting algorithm was used. To be included in the calculation of the sexual harassment rate, students must have experienced at least one behavior defined as sexual harassment and indicated they considered some or all of the behaviors to be sexual harassment. The measures of unwanted gender-related behaviors are consistent with the methodology used in 2006, 2008, and 2010 surveys. Trend comparisons on unwanted genderrelated behaviors are presented from surveys administered in 2006, 2008, 2010, and 2012.

Stalking-Related Behaviors. The 2012 SAGR included a measure of stalking that is designed to conform to the UCMJ definition of stalking as "a course of conduct directed at a specific person that would cause a reasonable person to fear death or bodily harm, including sexual assault." Trend comparisons on stalking-related behaviors are presented from surveys administered in 2006, 2008, 2010, and 2012. 


\section{U.S. Military Academy}

\section{Unwanted Sexual Contact.}

Overall, $10.7 \%$ of women and

$1.7 \%$ of men indicated they

experienced unwanted sexual

contact in 2012. The

percentage of women was

higher in 2012 than in 2010

and no change for men. Of the $10.7 \%$ of women who indicated experiencing unwanted sexual contact, $44 \%$ (19 percentage points higher than 2010) indicated they experienced unwanted sexual touching only. Twenty-four percent (15 percentage points lower than 2010) indicated the incident included attempted sex, with or without sexual touching; and 30\% (unchanged from 2010) indicated they experienced completed sex, with or without sexual touching and/or attempted sex. ${ }^{4}$

Unwanted Sexual Contact Details. Of the $10.7 \%$ of women who indicated experiencing unwanted sexual contact, nearly all (95\% - 3 percentage points lower than 2010) identified the offender as male, and most (82\% - 10 percentage points lower than 2010) indicated the offender was a fellow cadet. Forty-six percent indicated alcohol and/or drugs were involved and $11 \%$ indicated threats and physical force were used (both unchanged from 2010). Nineteen percent (unchanged from 2010) indicated they reported the incident to a military authority or organization. The main reasons those women chose to report the incident were: it was the right thing to do (82\%), to stop the offender from hurting others $(73 \%)$, to stop the offender from hurting them again $(65 \%)$, and to seek help dealing with an emotional incident $(65 \%)$. The main reasons women chose not to report the incident were: they thought it was not important enough to report (75\% - 19 percentage points higher than 2010), they did not want people gossiping about them (74\% - unchanged from 2010), and they did not want anyone to know (70\% - 8 percentage points higher than 2010).

Prior Unwanted Sexual Contact. Students were asked to indicate if they experienced any of the unwanted sexual contact behaviors prior to entering the Academy. The prior experience rate was $16.4 \%$ for women and $3.7 \%$ for men.

Unwanted Gender-Related Behaviors. Forty-nine percent of women and $8 \%$ of men indicated experiencing sexual harassment in 2012 (both unchanged from 2010). Eighty-two percent of women ( 2 percentage points lower than 2010) and $43 \%$ of men (unchanged from 2010) indicated experiencing crude/offensive behavior. Fifty-two percent of women (5 percentage points lower than 2010) and $12 \%$ of men (unchanged from 2010) indicated experiencing unwanted sexual attention. Seventeen percent of women (3 percentage points lower than 2010) and 4\% of men (unchanged from 2010) indicated experiencing sexual coercion. Ninety-one percent of women (3 percentage points lower than 2010) and $33 \%$ of men (unchanged from 2010) indicated experiencing sexist behavior.

Unwanted Gender-Related Behavior Details. Ninety-three percent of women and $51 \%$ of men indicated experiencing one or more of the unwanted gender-related behaviors (i.e., crude/offensive behavior, unwanted sexual attention, sexual coercion, sexist behavior) in 2012. Of those, the majority of women (85\% - 4 percentage points higher than 2010) and men (67\% - unchanged from 2010)

\footnotetext{
${ }^{4}$ Details of unwanted sexual contact for men are not reportable due to the small number of men who experienced unwanted sexual contact.
} 
identified the offender as a fellow cadet. Nine percent of women and $2 \%$ of men discussed the situation with a military authority or organization (both unchanged from 2010).

Stalking. Few women $(4.4 \%$ - 1.2 percentage points lower than 2010$)$ and men $(0.2 \%$ - unchanged from 2010) indicated that they experienced stalking-related behaviors that caused them fear of physical harm or sexual assault (the requirement to meet the legal definition of stalking).

\section{U.S. Naval Academy}

\section{Unwanted Sexual Contact.}

Overall, $15.1 \%$ of women and $2.6 \%$ of men indicated they experienced unwanted sexual contact in 2012. There were no changes in the percentages of women or men in 2012 from 2010. Of the $15.1 \%$ of women who indicated experiencing unwanted sexual contact, 29\% (10 percentage points lower than 2010) indicated they experienced unwanted sexual touching only. Twenty-two percent (unchanged from 2010) indicated the incident included attempted sex, with or without sexual touching; and 43\% (13 percentage points higher than 2010) indicated they experienced completed sex, with or without sexual touching and/or attempted sex. ${ }^{5}$

Unwanted Sexual Contact Details. Of the $15.1 \%$ of women who indicated experiencing unwanted sexual contact, $100 \%$ identified the offender as male (unchanged from 2010), and most (76\% unchanged from 2010) indicated the offender was a fellow midshipman. Sixty-five percent indicated alcohol and/or drugs were involved and 9\% indicated threats and physical force were used (both unchanged from 2010). Eleven percent (unchanged from 2010) indicated they reported the incident to a military authority or organization. The main reasons those women chose to report the incident were: to seek closure on the incident (83\%), to seek help dealing with an emotional incident (74\%), and it was the right thing to do $(72 \%)$. The main reasons women chose not to report the incident were: they took care of it themselves (77\% - 10 percentage points higher than 2010$)$, they did not want people gossiping about them (71\% - unchanged from 2010), and they did not want anyone to know (68\% - 7 percentage points higher than 2010).

Prior Unwanted Sexual Contact. Students were asked to indicate if they experienced any of the unwanted sexual contact behaviors prior to entering the Academy. The prior experience rate was $22.3 \%$ for women and $3.8 \%$ for men.

Unwanted Gender-Related Behaviors. Sixty-one percent of women (unchanged from 2010) and $10 \%$ of men (7 percentage points lower than 2010) indicated experiencing sexual harassment in 2012. Ninety percent of women and $51 \%$ of men indicated experiencing crude/offensive behavior (both unchanged from 2010). Sixty-three percent of women and $19 \%$ of men indicated experiencing unwanted sexual attention (both unchanged from 2010). Twenty-one percent of women and $5 \%$ of men indicated experiencing sexual coercion (both unchanged from 2010). Ninety-four percent of

\footnotetext{
${ }^{5}$ Details of unwanted sexual contact for men are not reportable due to the small number of men who experienced unwanted sexual contact.
} 
women (2 percentage points lower than 2010) and 46\% of men (unchanged from 2010) indicated experiencing sexist behavior.

Unwanted Gender-Related Behavior Details. Ninety-six percent of women and $61 \%$ of men indicated experiencing one or more of the unwanted gender-related behaviors (i.e., crude/offensive behavior, unwanted sexual attention, sexual coercion, sexist behavior) in 2012. Of those, the majority of women (81\% - unchanged from 2010) and men (70\% - 6 percentage points higher than 2010) identified the offender as a fellow midshipman. Six percent of women (3 percentage points lower than 2010 ) and $3 \%$ of men (unchanged from 2010) discussed the situation with a military authority or organization.

Stalking. Few women (5.8\% - unchanged from 2010) and men (1.0\% - unchanged from 2010) indicated that they experienced stalking-related behaviors that caused them fear of physical harm or sexual assault (the requirement to meet the legal definition of stalking).

\section{U.S. Air Force Academy}

\section{Unwanted Sexual Contact.}

Overall, $11.2 \%$ of women and $1.7 \%$ of men indicated they experienced unwanted sexual contact in 2012. There were no changes in the percentages of women or men in 2012 (unchanged from 2010) indicated they experienced unwanted sexual touching only. Twenty-two percent (11 percentage points lower than 2010) indicated the incident included attempted sex, with or without sexual touching; and 54\% (15 percentage points higher than 2010) indicated they experienced completed sex, with or without sexual touching and/or attempted sex. ${ }^{6}$

Unwanted Sexual Contact Details. Of the $11.2 \%$ of women who indicated experiencing unwanted sexual contact, nearly all (97\% - 3 percentage points lower than 2010) identified the offender as male, and most (85\% - unchanged from 2010) indicated the offender was a fellow cadet. Sixty percent (12 percentage points higher than 2010) indicated alcohol and/or drugs were involved and 10\% (unchanged from 2010) indicated threats and physical force were used. Fifteen percent (unchanged from 2010) indicated they reported the incident to a military authority or organization. The main reasons those women chose to report the incident were: to seek help dealing with an emotional incident $(82 \%)$, to stop the offender from hurting others $(74 \%)$, to seek justice $(64 \%)$, and it was the right thing to do (64\%). The main reasons women chose not to report the incident were: they took care of it themselves (66\% - unchanged from 2010), they did not want anyone to know (63\% unchanged from 2010), and they did not want people gossiping about them (62\% - unchanged from 2010).

\footnotetext{
${ }^{6}$ Details of unwanted sexual contact for men are not reportable due to the small number of men who experienced unwanted sexual contact.
} 
Prior Unwanted Sexual Contact. Students were asked to indicate if they experienced any of the unwanted sexual contact behaviors prior to entering the Academy. The prior experience rate was $22.9 \%$ for women and $4.6 \%$ for men.

Unwanted Gender-Related Behaviors. Forty-four percent of women (9 percentage points lower than 2010) and 11\% of men (unchanged from 2010) indicated experiencing sexual harassment in 2012. Seventy-seven percent of women (7 percentage points lower than 2010) and $43 \%$ of men (4 percentage points lower than 2010) indicated experiencing crude/offensive behavior. Fifty-two percent of women ( 5 percentage points lower than 2010) and 11\% of men (unchanged from 2010) indicated experiencing unwanted sexual attention. Seventeen percent of women (3 percentage points lower than 2010) and $3 \%$ of men (unchanged from 2010) indicated experiencing sexual coercion. Eighty-five percent of women (4 percentage points lower than 2010) and 37\% of men (unchanged from 2010) indicated experiencing sexist behavior.

Unwanted Gender-Related Behavior Details. Eighty-nine percent of women and $51 \%$ of men indicated experiencing one or more of the unwanted gender-related behaviors (i.e., crude/offensive behavior, unwanted sexual attention, sexual coercion, sexist behavior) in 2012. Of those, the majority of women (80\%) and men (72\%) identified the offender as a fellow cadet (both unchanged from 2010). Six percent of women and $0 \%$ of men discussed the situation with a military authority or organization (both unchanged from 2010).

Stalking. Few women (4.9\% - unchanged from 2010) and men (0.3\% - unchanged from 2010) indicated that they experienced stalking-related behaviors that caused them fear of physical harm or sexual assault (the requirement to meet the legal definition of stalking).

\section{U.S. Coast Guard Academy}

Unwanted Sexual Contact. Overall, $9.8 \%$ of women and $0.7 \%$ of men indicated they

experienced unwanted sexual contact in 2012. The percentage of women was higher in 2012 than in 2010 and the percentage of men was lower. Of the $9.8 \%$ of women who indicated experiencing unwanted sexual contact, $23 \%(12$

\begin{tabular}{|c|l|l|}
\hline \multicolumn{3}{|c|}{ Unwanted Sexual Contact } \\
\hline $\mathbf{2 0 0 8}$ & \multicolumn{1}{|c|}{$\mathbf{2 0 1 0}$} & $\mathbf{2 0 1 2}$ \\
\hline Women: $5.6 \%$ & Women: $7.8 \%$ & Women: $9.8 \%$ \\
Men: $2.4 \%$ & Men: $2.9 \%$ & Men: $0.7 \%$ \\
\hline
\end{tabular}

percentage points lower than 2010) indicated they experienced unwanted sexual touching only. Thirtynine percent (22 percentage points higher than 2010) indicated the incident included attempted sex, with or without sexual touching; and 39\% (unchanged from 2010) indicated they experienced completed sex, with or without sexual touching and/or attempted sex. ${ }^{7}$

Unwanted Sexual Contact Details. Of the $9.8 \%$ of women who indicated experiencing unwanted sexual contact, $100 \%$ identified the offender as male ( 7 percentage points higher than 2010), and most (76\% - unchanged from 2010) indicated the offender was a fellow cadet. Fifty-nine percent (unchanged from 2010) indicated alcohol and/or drugs were involved and $8 \%$ (8 percentage points higher than 2010) indicated threats and physical force were used. Seven percent (12 percentage points

\footnotetext{
${ }^{7}$ Details of unwanted sexual contact for men are not reportable due to the small number of men who experienced unwanted sexual contact.
} 
lower than 2010) indicated they reported the incident to a military authority or organization. The main reasons women chose not to report the incident were: they took care of it themselves $(86 \%-15$ percentage points higher than 2010), they did not think it was important enough to report (73\% unchanged from 2010), and they did not want people gossiping (69\% - unchanged from 2010).

Prior Unwanted Sexual Contact. Students were asked to indicate if they experienced any of the unwanted sexual contact behaviors prior to entering the Academy. The prior experience rate was $15.8 \%$ for women and $3.0 \%$ for men.

Unwanted Gender-Related Behaviors. Forty percent of women (unchanged from 2010) and 10\% of men (7 percentage points lower than 2010) indicated experiencing sexual harassment in 2012. Seventy-six percent of women (unchanged from 2010) and 46\% of men (13 percentage points lower than 2010) indicated experiencing crude/offensive behavior. Forty-two percent of women (6 percentage points higher than 2010) and $13 \%$ of men ( 7 percentage points lower than 2010) indicated experiencing unwanted sexual attention. Eleven percent of women (unchanged from 2010) and $4 \%$ of men ( 2 percentage points lower than 2010) indicated experiencing sexual coercion. Seventy-seven percent of women ( 3 percentage points lower than 2010) and 40\% of men (9 percentage points lower than 2010) indicated experiencing sexist behavior.

Unwanted Gender-Related Behavior Details. Eighty-four percent of women and 55\% of men indicated experiencing one or more of the unwanted gender-related behaviors (i.e., crude/offensive behavior, unwanted sexual attention, sexual coercion, sexist behavior) in 2012. Of those, the majority of women (80\% - unchanged from 2010) and men (59\% - 8 percentage points lower than 2010) identified the offender as a fellow cadet. Seven percent of women ( 3 percentage points higher than 2010 ) and $2 \%$ of men (unchanged from 2010) discussed the situation with a military authority or organization.

Stalking. Few women (3.3\% - unchanged from 2010) and men (0.4\% - unchanged from 2010) indicated that they experienced stalking-related behaviors that caused them fear of physical harm or sexual assault (the requirement to meet the legal definition of stalking).

\section{Survey Methodology}

Statistical Design. The total sample consisted of 7,259 students drawn from the student rosters provided to DMDC by USMA, USNA, USAFA, and USCGA. After excluding students who could not participate for various reasons (e.g., medical excuses, emergency leave, left the Academy, were foreign nationals, or were exchange students from another Academy), the final eligible sample was 7,258 students. Surveys were completed by 5,425 students $^{8}$ yielding an overall weighted response rate for eligible respondents of $73 \%$. The entire population of female students was selected for the survey. ${ }^{9}$ For male students at USMA, USNA, and USAFA, a single-stage, nonproportional stratified random sampling ${ }^{10}$ procedure was employed to ensure adequate sample sizes for the reporting categories. At

\footnotetext{
8“Completed" is defined as answering $50 \%$ or more of the questions asked of all participants, at least one sub-item in Q12as (unwanted gender-related behaviors), and a valid response to Q19 (unwanted sexual contact).

${ }^{9}$ Because of the relatively small number of female students at the Academies, all eligible female students are included in the sample to produce reliable results.

${ }^{10}$ In stratified random sampling, all members of a population are categorized into homogeneous groups. For example, members might be grouped by gender, class, and Academy (all male, senior USMA students in one group; all male, junior
} 
USCGA, all male students were selected for the survey. ${ }^{11}$ Data were weighted to reflect each Academy's population as of March 2012.

Survey Administration. Data were collected in March and April 2012. A team from DMDC administered the anonymous paper-and-pen survey in group sessions. Separate sessions were held for female and male students. After checking in, each student was handed a survey, ${ }^{12}$ an envelope, a pen, and an Academy-specific information sheet. The information sheet included details on where students could obtain help if they became upset or distressed while taking the survey or afterwards. Students were briefed on the purpose of the survey and the importance of participation, but completion of the survey itself was voluntary. Students could leave the session at the completion of the mandatory briefing if they did not wish to take the survey. Students returned completed or blank surveys (depending on whether they chose to participate) in sealed envelopes to survey staff as they exited the session.

Presentation of Results. Each finding in 2012 SAGR is presented in graphical or tabular form along with its margin of error. The margin of error represents the degree of certainty that the percentage or mean would fall within the interval in repeated samples of the population. For example, if 55\% of individuals selected an answer and the margin of error was \pm 3 , in repeated surveyed samples from the population the percentage of individuals selecting the same answer would be between $52 \%$ (55 minus 3 ) and $58 \%$ (55 plus 3 ) in $95 \%$ of the samples. Because the results of comparisons are based on a weighted, representative sample, the reader can infer that the results generalize to the population at each Academy, within the margin of error. The annotation "NR" used throughout the Appendices indicates that a specific result is not reportable due to low reliability.

Statistical Comparisons. Only statistically significant group comparisons are discussed in this survey note. Comparisons are generally made along a single dimension (e.g., class year) at a time. In this type of comparison, the responses for one group are compared to the weighted average of the responses of all other groups in that dimension. Thus within the current survey year, the percentage of each subgroup is compared to its respective "all other" group (i.e., the total population minus the group being assessed). For example, responses of senior women at USAFA are compared to the weighted average of the responses from junior, sophomore, and freshman USAFA women (e.g., women in all other classes at USAFA). When comparing results across survey years (e.g., 2012 compared to 2010), statistical tests for differences between means are used. All comparisons are made at the .05 level of significance.

USMA students in another; etc.). Students were chosen at random within each group. Weights were used so that counts represent the total population by aggregating the sample to the population.

${ }^{11}$ Because of the relatively small number of male students at USCGA, all eligible male students are included in the sample to produce reliable results.

${ }^{12}$ Survey booklets contained no printed identifying or tracking information. 


\section{References}

DMDC. (2012). 2012 Service Academy Gender Relations Survey: Statistical methodology report (Report No. 2012-032). Arlington, VA: Author.

Fitzgerald, L. F., Gelfand, M. J., \& Drasgow, F. (1995). Measuring sexual harassment: Theoretical and psychometric advances. Basic and Applied Social Psychology, 17, 425-445.

Fitzgerald, L. F., Shullman, S. L., Bally, N., Richards, M., Swecker, J, Gold, Y., Ormerod, M., \& Weitzman, L. (1988). The incidence and dimensions of sexual harassment in academia and the workplace. Journal of Vocational Behavior, 32, 152-175.

John Warner National Defense Authorization Act for Fiscal Year 2007, Pub. L. No. 109-364, H.R. 5122 (2006).

Prepared by: Paul Cook and Lindsay Rock

Survey Design, Analysis, \& Operations Branch

Human Resources Strategic Assessment Program, DMDC

For further information, see http://www.dmdc.osd.mil/surveys.

Additional copies of this report may be obtained from:

Defense Technical Information Center

ATTN: DTIC-BRR

8725 John J. Kingman Rd., Suite \#0944

Ft. Belvoir, VA 22060-6218

Or from:

http://www.dtic.mil/dtic/order.html

Ask for report by ADA 532279 


\section{Service Academy Gender Relations Survey}

Appendix A: U.S. Military Academy

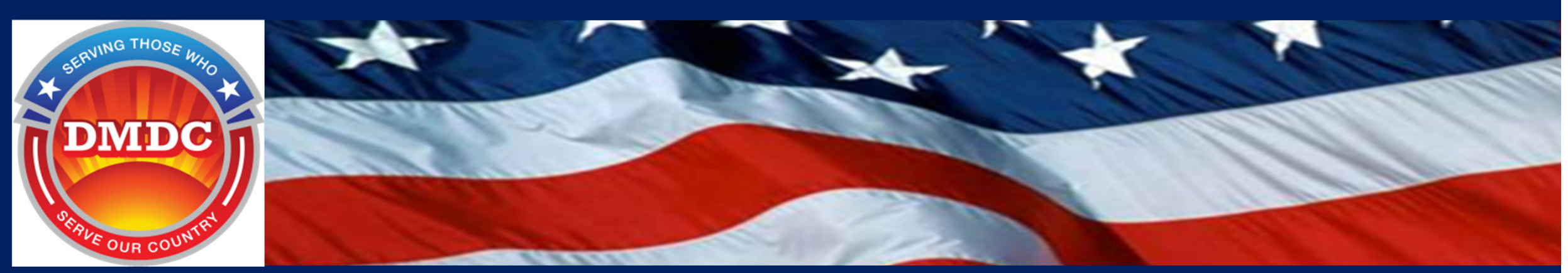




\section{Appendix A \\ U.S. Military Academy \\ Contents}

Slide

$\checkmark \quad$ Introduction ...................................................................................... 3

- Unwanted Sexual Contact ................................................................ 7

- Unwanted Gender-Related Behaviors ........................................... 49

• Stalking ........................................................................................... 75

• Training ............................................................................................. 83

๑ Student Perceptions .................................................................. 93 


\section{Introduction}

- Paper survey administered to cadets in March - April 2012

- 703 female and 1,162 male students surveyed, weighted response rate of $88 \%$ and $77 \%$, respectively

\section{- Appendix includes results from survey items related to:}

- Results of unwanted sexual contact experienced in the past academic year

- For women who had experienced unwanted sexual contact in the past academic year, details of the incident that had the greatest impact (characteristics of the offender, location, actions taken, reporting, etc.). Details for men are not reportable due to the small number of men who experienced unwanted sexual contact.

- Unwanted sexual contact experienced prior to entering the Academy

- Unwanted gender-related behaviors experienced in the past academic year (sexual harassment, crude and offensive behavior, unwanted sexual attention, sexual coercion, and sexist behavior)

- For students who experienced unwanted gender-related behavior in the past academic year, details of the incident that had the greatest impact (characteristics of the offender, reporting)

- Stalking experienced in the past academic year

- Training received in preventing and responding to sexual assault and sexual harassment

- Student perceptions about culture and climate at the Academy

- For each survey item, briefing includes the following:

- Graphic displays of overall results by gender, survey year, and class year

- Tables showing results for grouped items by reporting categories (e.g., gender, survey year, and class year) 


\section{Introduction to Briefing Slides}

\section{- Graphics showing results by reporting categories (gender, survey year, and class year)}

- Statistical tests are used to compare each subgroup to previous survey years and other class years

- For items with trends, 2012 survey year is compared to the other survey years $(2006,2008,2010)$

- For the current 2012 survey year, each class year (e.g., freshmen) is compared to the average of the other class years (sophomore, junior, senior)

- Results are presented on the bottom of the slide for survey year and class year comparisons that are statistically significant

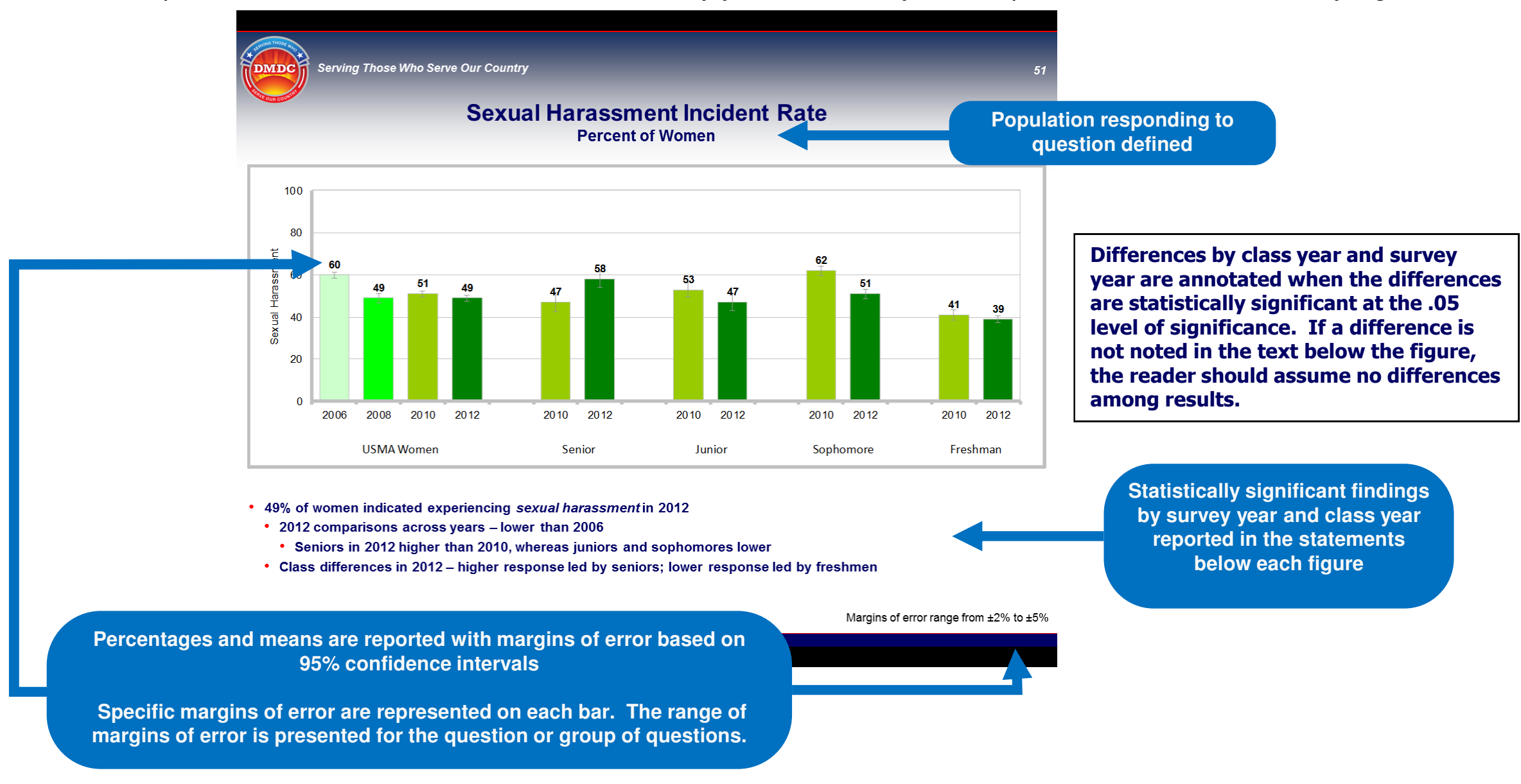




\section{Introduction to Briefing Slides}

\section{- Tables showing results for grouped items by reporting categories (gender, survey year,} and class year)

- Statistical tests are used to compare each subgroup to previous survey years and other class years

- For items with trends, 2012 survey year is compared to the other survey years $(2006,2008,2010)$

- For the current 2012 survey year, each class year (e.g., freshmen) is compared to the average of the other class years (sophomore, junior, senior)

- Results are presented within the table for survey year and class year comparisons that are statistically significant

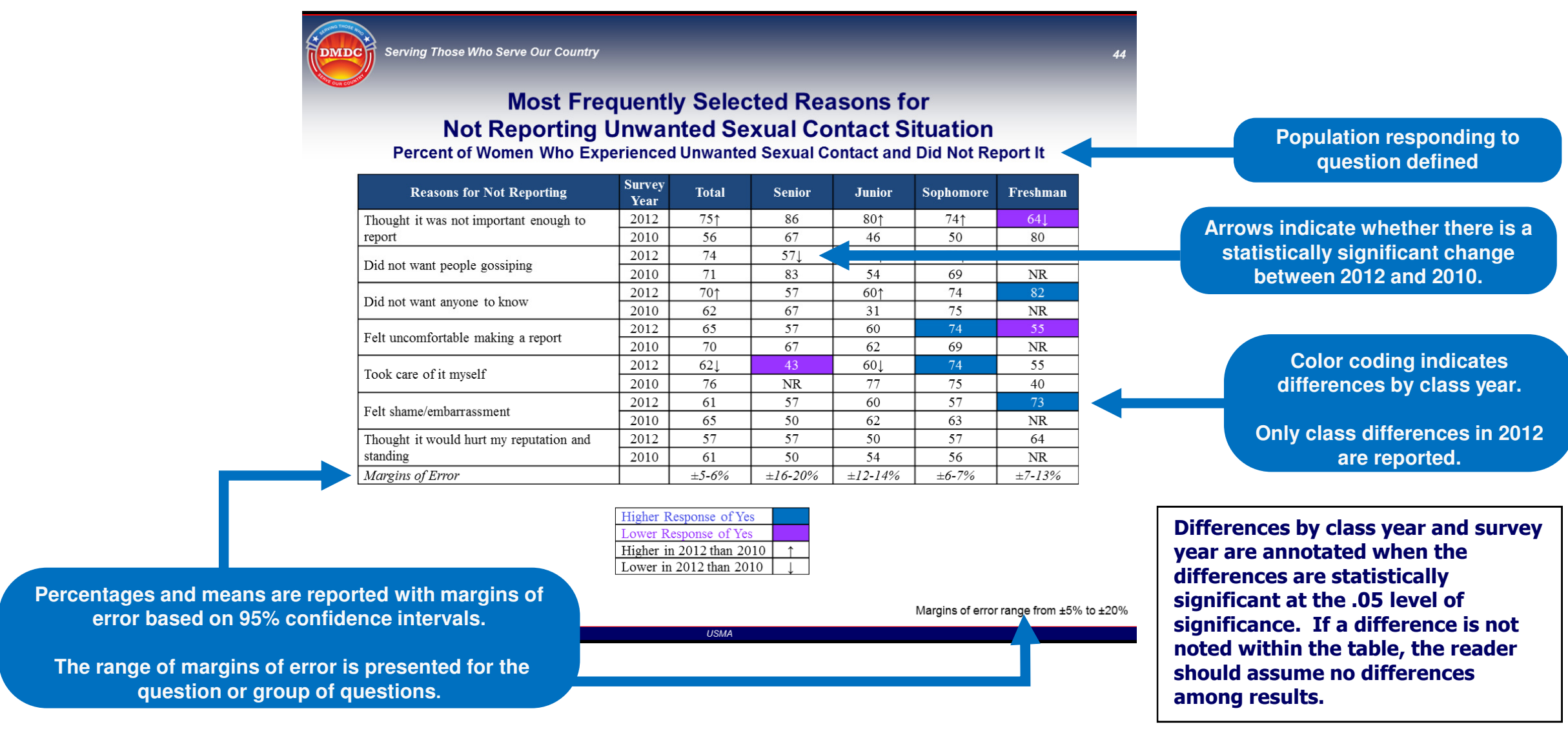




\section{Contents}

$\checkmark$ Unwanted Sexual Contact ............................................................. 7

- Most Serious Behaviors Experienced.......................................... 11

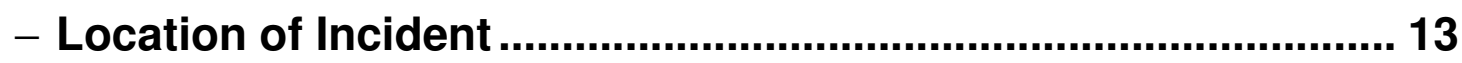

- Summer Experience......................................................................... 15

- Characteristics of Offender............................................................. 17

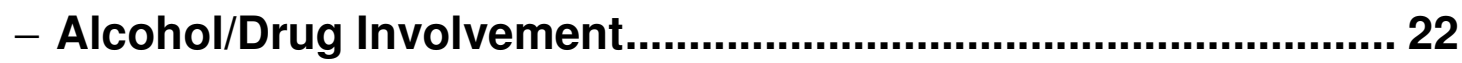

- Use of Threats/Force ................................................................ 27

- Experience of Sexual Harassment/Stalking ................................ 32

- Consequences of the Situation ..................................................... 25

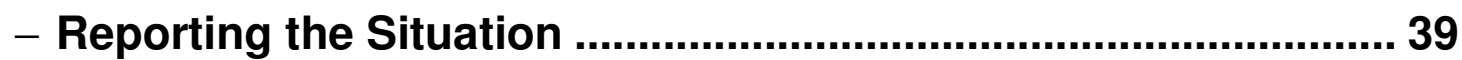

- Experience of Unwanted Sexual Contact Prior to Entering the Academy .............................................................................. 47

- Unwanted Gender-Related Behaviors ............................................. 49

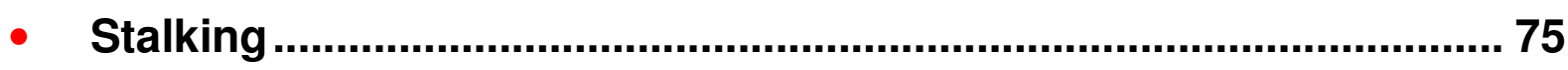

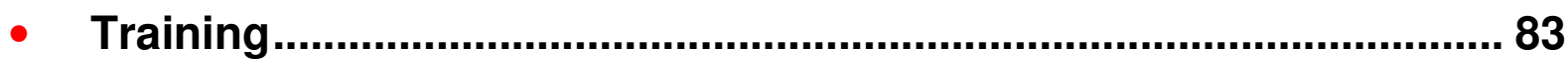

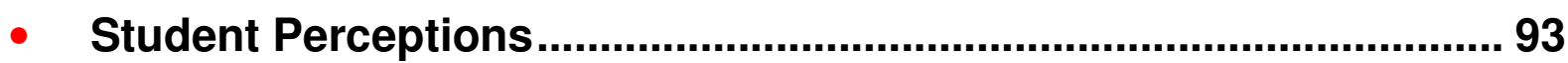




\section{Unwanted Sexual Contact Incident Rates}

\section{- Definition and measure of unwanted sexual contact:}

- The 2012 SAGR survey includes a measure of unwanted sexual contact (i.e., sexual assault). Although this term does not appear in the Uniform Code of Military Justice $(\mathrm{UCMJ})$, it is used to refer to a range of activities that is used as an umbrella term intended to include certain acts prohibited by the UCMJ.

- Unwanted sexual contact is measured in the 2012 SAGR survey by asking students to refer to experiences since June 2011 in which they experienced any of the following intentional sexual contacts that were against their will or which occurred when they did not or could not consent in which someone...

- Sexually touched them (e.g., intentional touching of genitalia, breasts, or buttocks) or made them sexually touch someone,

- Attempted to make them have sexual intercourse, but was not successful,

- Made them have sexual intercourse,

- Attempted to make them perform or receive oral sex, anal sex, or penetration by a finger or object, but was not successful, or

- Made them perform or receive oral sex, anal sex, or penetration by a finger or object.

- A student is counted in the unwanted sexual contact incident rate if he or she replied "yes" to any of the behaviors listed.

- The 2012 SAGR survey was administered in March and April 2012. The timeframe looking back to June 2011 is intended to include behaviors experienced in the majority of Academic Program Year 2011-2012 to be consistent with the timeframe for reporting incidents of sexual assault at the Academies. 


\section{Unwanted Sexual Contact Incident Rate Percent of Women}

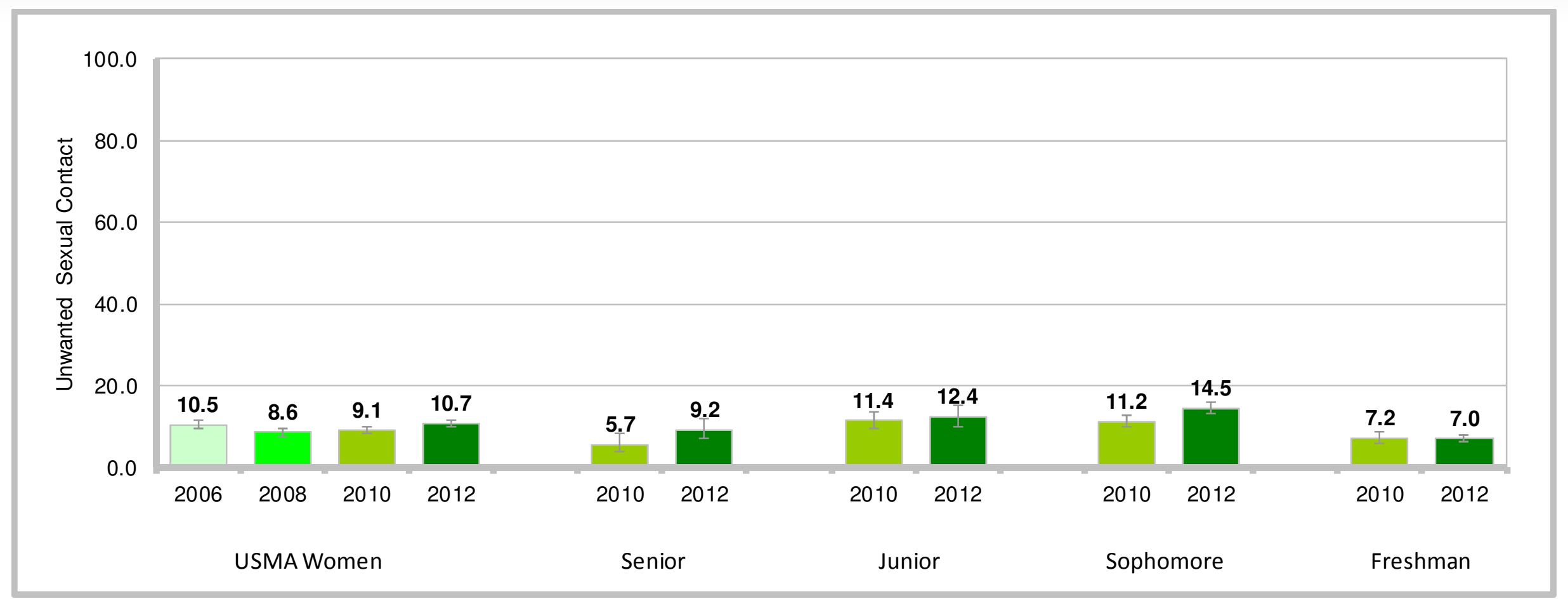

- $10.7 \%$ of women indicated experiencing unwanted sexual contact in 2012

- 2012 comparisons across years - higher than 2010, 2008

- Seniors and sophomores in 2012 higher than 2010

- Class differences in 2012 - higher response led by sophomores; lower response led by freshmen 


\section{Unwanted Sexual Contact Incident Rate}

Percent of Men

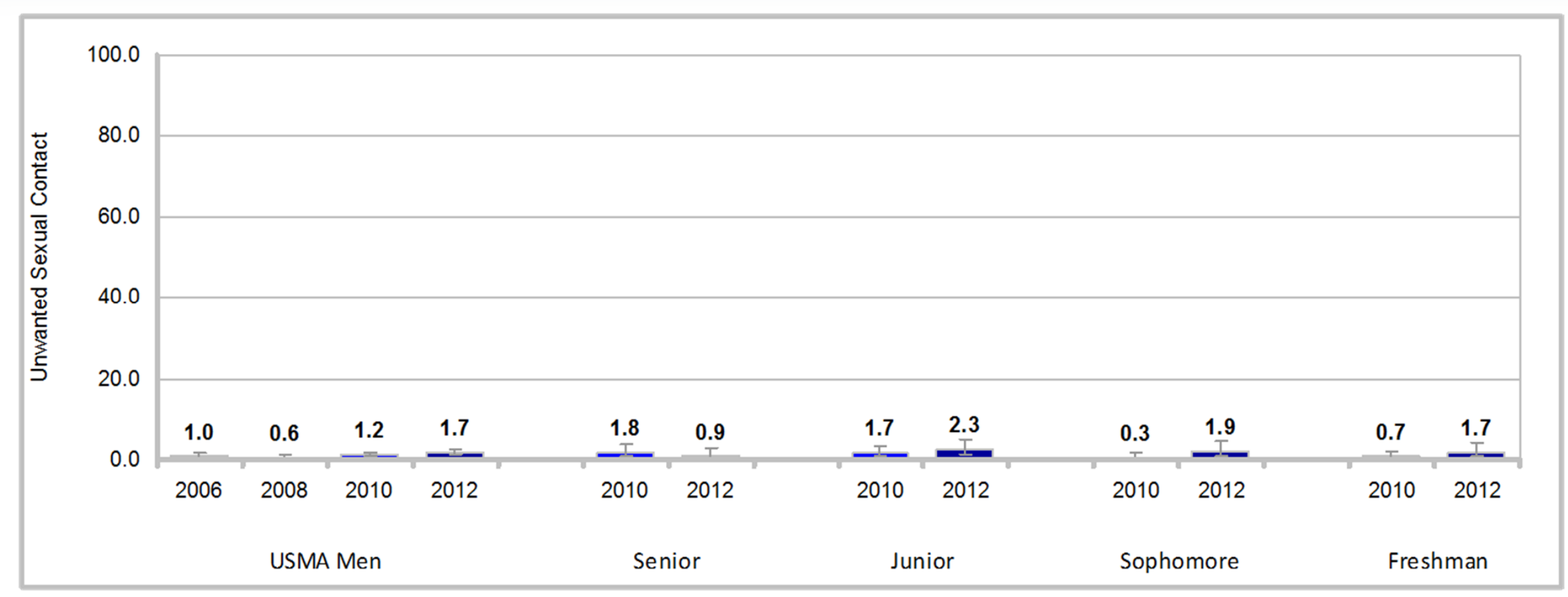

- $1.7 \%$ of men indicated experiencing unwanted sexual contact in 2012

- 2012 comparisons across years - higher than 2008

- Class differences in 2012 - no differences 


\section{Contents}

Slide

- Introduction ............................................................................................ 3

- Unwanted Sexual Contact ................................................................ 7

$\checkmark$ Most Serious Behaviors Experienced...................................... 11

- Location of Incident ........................................................................ 13

- Summer Experience................................................................... 15

- Characteristics of Offender......................................................... 17

- Alcohol/Drug Involvement........................................................... 22

- Use of Threats/Force ..................................................................... 27

- Experience of Sexual Harassment/Stalking ............................. 32

- Consequences of the Situation .................................................. 35

- Reporting the Situation ............................................................ 39

- Experience of Unwanted Sexual Contact Prior to Entering

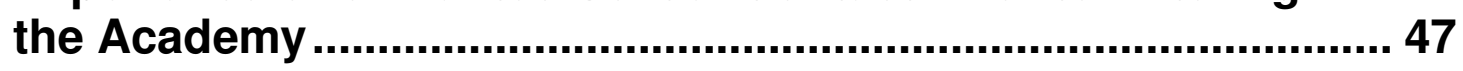

- Unwanted Gender-Related Behaviors ............................................. 49

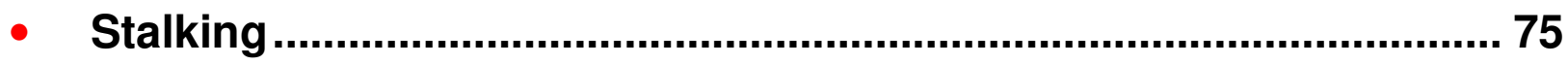

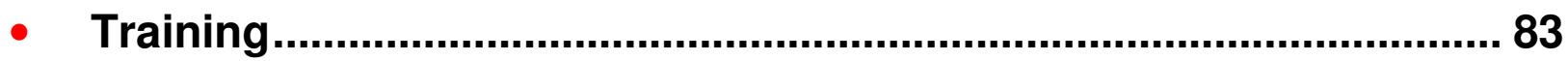

- Student Perceptions .................................................................... 93 


\section{Most Serious Unwanted Sexual Contact Behaviors Experienced} Percent of Women Who Experienced Unwanted Sexual Contact

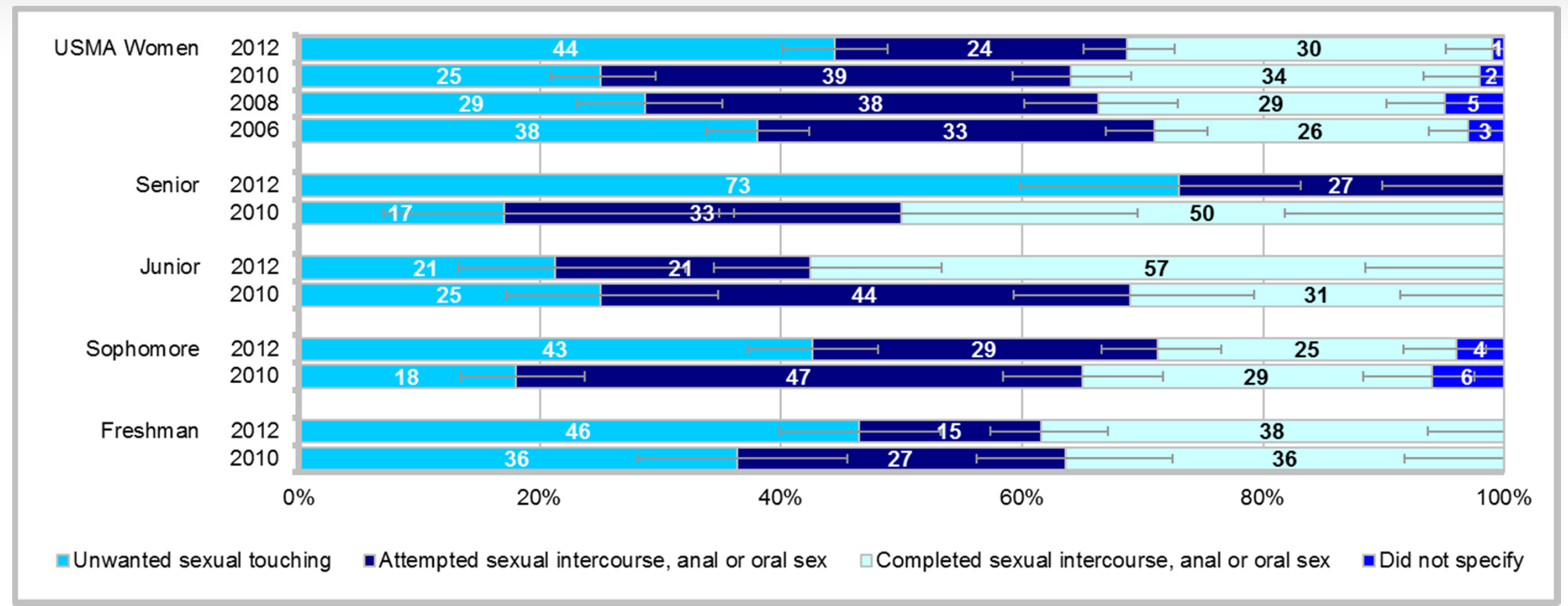

- Of the $10.7 \%$ of women who experienced USC, $44 \%$ indicated experiencing touching; $24 \%$ indicated experiencing attempted sex; $30 \%$ indicated experiencing completed sex; and $1 \%$ did not specify

- 2012 comparisons across years - touching higher than 2010, 2008; attempted sex lower than 2010, 2008, 2006; did not specify lower than 2008, 2006

- Seniors and sophomores indicating touching in 2012 higher than 2010; juniors, sophomores, and freshmen indicating attempted sex in 2012 lower than 2010; juniors indicating completed sex in 2012 higher than 2010

- Class differences in 2012 - higher response of touching led by seniors; higher response of completed sex led by juniors and freshmen; higher response of did not specify led by sophomores 


\section{Contents}

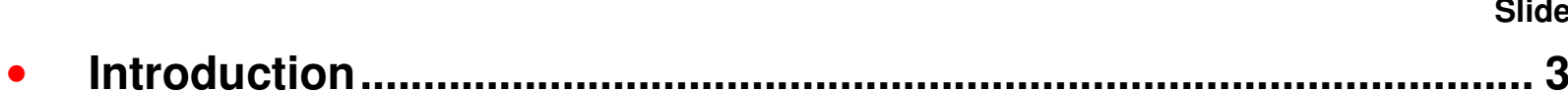

- Unwanted Sexual Contact ................................................................ 7

- Most Serious Behaviors Experienced........................................... 11

$\checkmark$ Location of Incident .......................................................................... 13

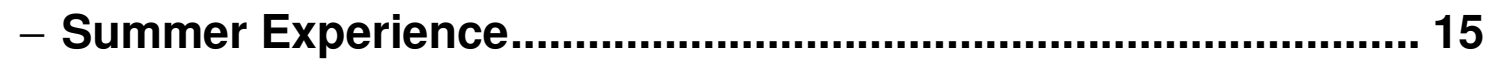

- Characteristics of Offender .......................................................... 17

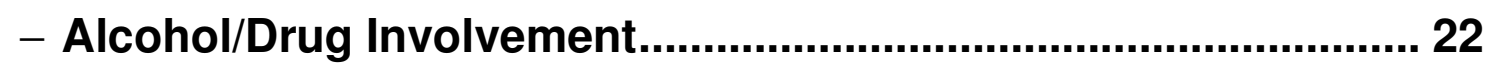

- Use of Threats/Force ................................................................. 27

- Experience of Sexual Harassment/Stalking ............................... 32

- Consequences of the Situation ................................................ 35

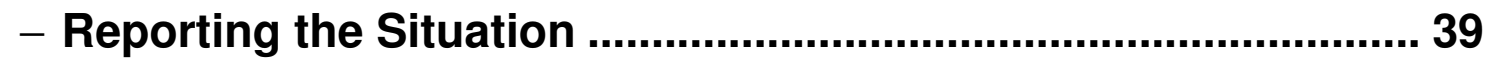

- Experience of Unwanted Sexual Contact Prior to Entering the Academy ..................................................................................... 47

- Unwanted Gender-Related Behaviors ............................................. 49

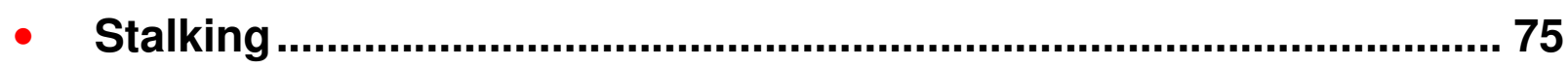

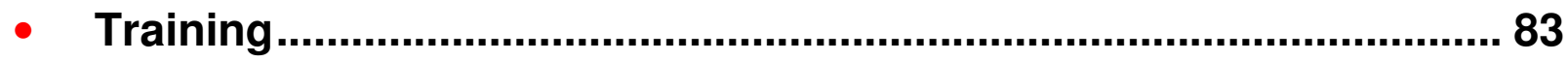

- Student Perceptions ................................................................................ 93 


\section{Location of Unwanted Sexual Contact Incident Percent of Women Who Experienced Unwanted Sexual Contact}

\begin{tabular}{|c|c|c|c|c|c|}
\hline Location of Incident & Total & Senior & Junior & Sophomore & Freshman \\
\hline On Academy grounds in dormitory/living area & 53 & 27 & 64 & 58 & 54 \\
\hline On Academy grounds not in dormitory/living area & 34 & 36 & 21 & 35 & 46 \\
\hline $\begin{array}{l}\text { Off Academy grounds at a social event (such as a } \\
\text { party) }\end{array}$ & 18 & 27 & 21 & 15 & 8 \\
\hline $\begin{array}{l}\text { Off Academy grounds at an Academy sponsored } \\
\text { event }\end{array}$ & 8 & 18 & NR & 8 & 8 \\
\hline Off Academy grounds other & 15 & NR & 43 & 12 & 0 \\
\hline Margins of Error & $\pm 3-5 \%$ & $\pm 13-14 \%$ & $\pm 11-12 \%$ & $\pm 4-6 \%$ & $\pm 0-7 \%$ \\
\hline
\end{tabular}

\begin{tabular}{|l|l|}
\hline Higher Response of Yes & \\
\hline Lower Response of Yes & \\
\hline
\end{tabular}

- New question in 2012; no trend data available 


\section{Contents}

Slide

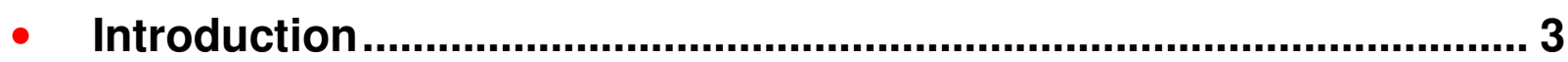

- Unwanted Sexual Contact ............................................................... 7

- Most Serious Behaviors Experienced....................................... 11

- Location of Incident ...................................................................... 13

$\checkmark$ Summer Experience.................................................................... 15

- Characteristics of Offender........................................................ 17

- Alcohol/Drug Involvement.......................................................... 22

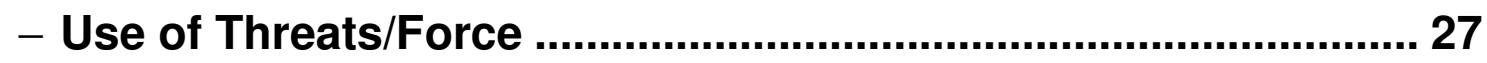

- Experience of Sexual Harassment/Stalking ............................... 32

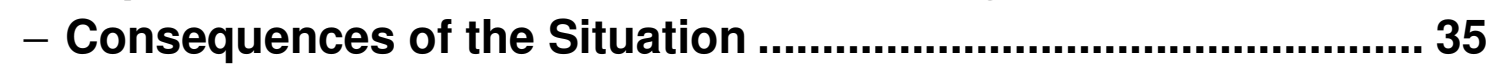

- Reporting the Situation .............................................................. 39

- Experience of Unwanted Sexual Contact Prior to Entering

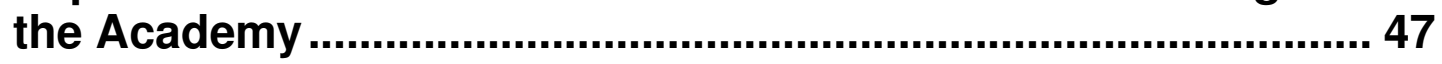

- Unwanted Gender-Related Behaviors ............................................... 49

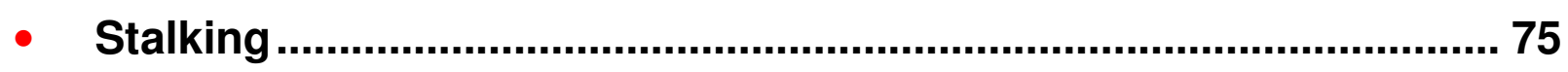

- Training ................................................................................................. 83

- Student Perceptions.................................................................... 93 


\section{Unwanted Sexual Contact Incident Occurred During Summer Experience/Training \\ Percent of Women Who Experienced Unwanted Sexual Contact}

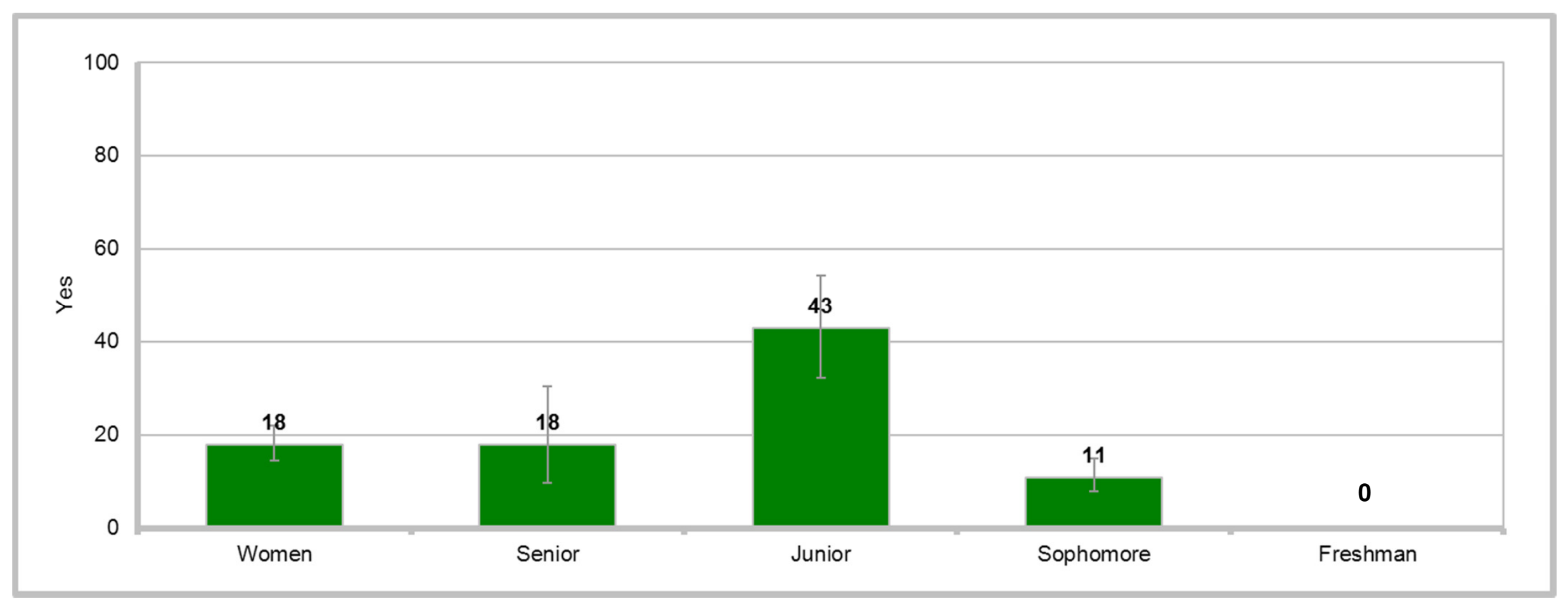

- Of the $10.7 \%$ of women who experienced USC, $18 \%$ indicated the offense occurred during summer experience/training

- 2012 comparisons across years - new question in 2012; no trend data available

- Class differences in 2012 - higher response led by juniors; lower response led by sophomores and freshmen 


\section{Contents}

Slide

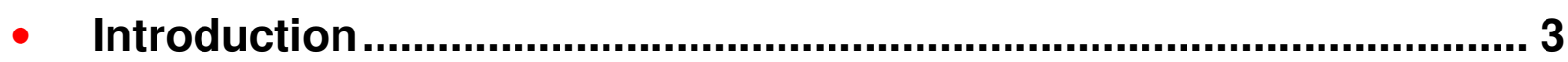

- Unwanted Sexual Contact ............................................................... 7

- Most Serious Behaviors Experienced....................................... 11

- Location of Incident ..................................................................... 13

- Summer Experience.................................................................. 15

$\checkmark$ Characteristics of Offender........................................................ 17

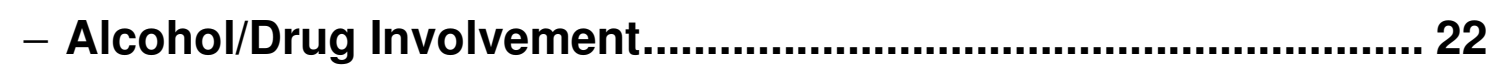

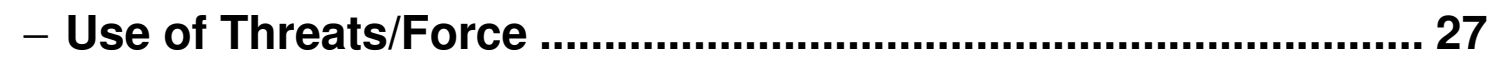

- Experience of Sexual Harassment/Stalking ............................... 32

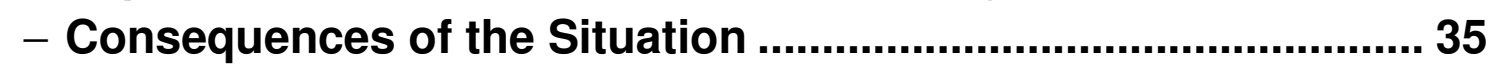

- Reporting the Situation ............................................................ 39

- Experience of Unwanted Sexual Contact Prior to Entering

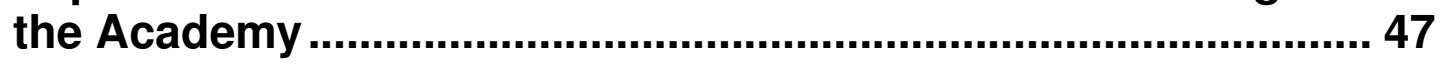

- Unwanted Gender-Related Behaviors ................................................ 49

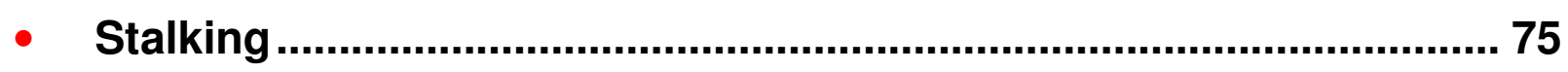

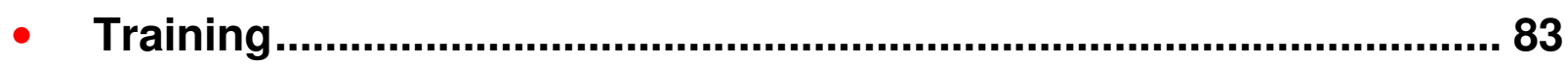

- Student Perceptions.................................................................... 93 


\section{Combinations of Offender Affiliations Percent of Women Who Experienced Unwanted Sexual Contact}

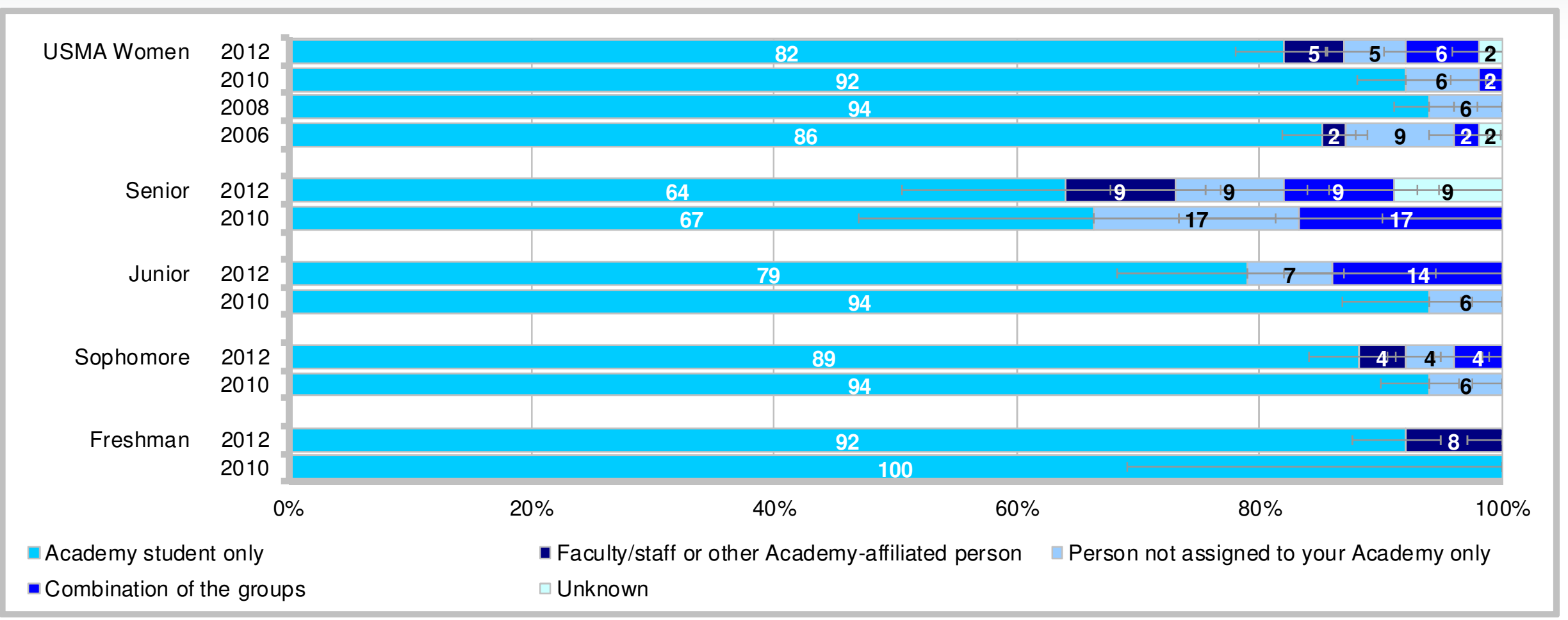

- Of the $10.7 \%$ of women who experienced USC, $82 \%$ indicated the offender was Academy student only; $5 \%$ indicated faculty/staff; $5 \%$ indicated person not assigned to the Academy; $6 \%$ indicated combination of the groups; and $2 \%$ indicated the offender was unknown

- 2012 comparisons across years - Academy student only lower than 2010, 2008; faculty/staff higher than 2010, 2008, 2006; person not assigned to the Academy lower than 2006; combination of the groups higher than 2010, 2008, 2006; unknown higher than 2010, 2008

- Juniors, sophomores, and freshmen indicating Academy student only in 2012 lower than 2010; seniors, sophomores, and freshmen indicating faculty/staff in 2012 higher than 2010; juniors and sophomores indicating combination of the groups in 2012 higher than 2010; seniors indicating unknown in 2012 higher than 2010

- Class differences in 2012 - Academy student only led by freshmen and sophomores 


\section{Gender of the Offender Percent of Women Who Experienced Unwanted Sexual Contact}

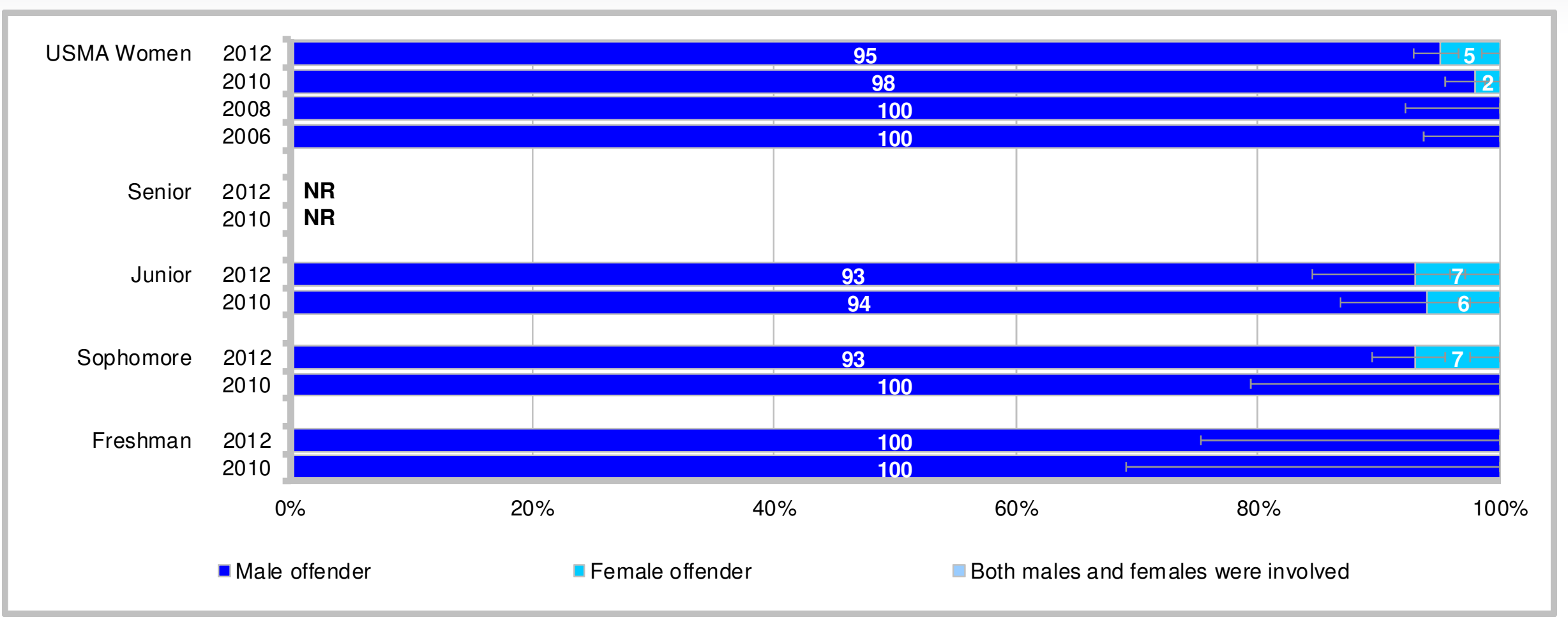

- Of the $10.7 \%$ of women who experienced USC, $95 \%$ indicated the offender was male; $5 \%$ indicated the offender was female - 2012 comparisons across years - male offender lower than 2010, 2008, 2006; female offender higher than 2010, 2008,2006 - Sophomores indicating male offender in 2012 lower than 2010; sophomores indicating female offender in 2012 higher than 2010

- Class differences in 2012 - male offender led by freshmen 


\section{Multiple Offenders Involved \\ Percent of Women Who Experienced Unwanted Sexual Contact}

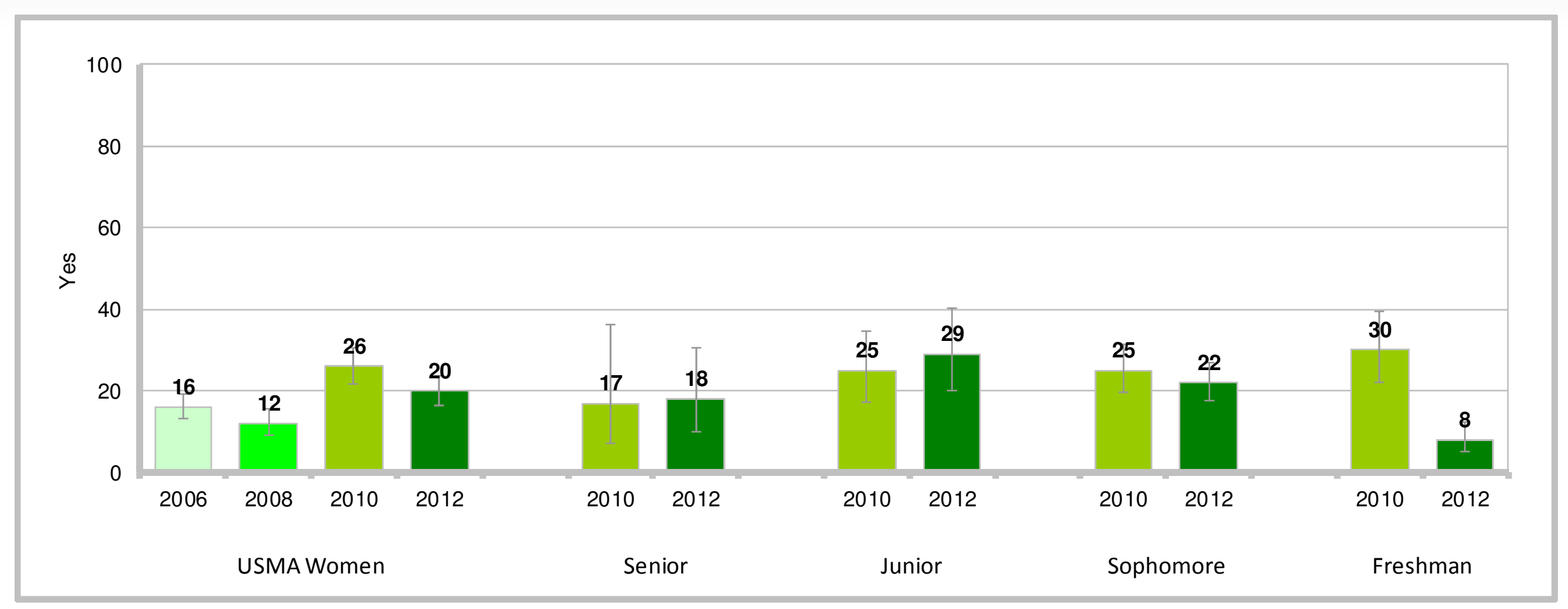

- Of the $10.7 \%$ of women who experienced USC, $20 \%$ indicated multiple offenders were involved

- 2012 comparisons across years - lower than 2010, higher than 2008

- Freshmen in 2012 lower than 2010

- Class differences in 2012 - lower response led by freshmen 


\section{Offender Was a "Creeper" (i.e., someone who is socially awkward)} Percent of Women Who Experienced Unwanted Sexual Contact Involving an Academy Student

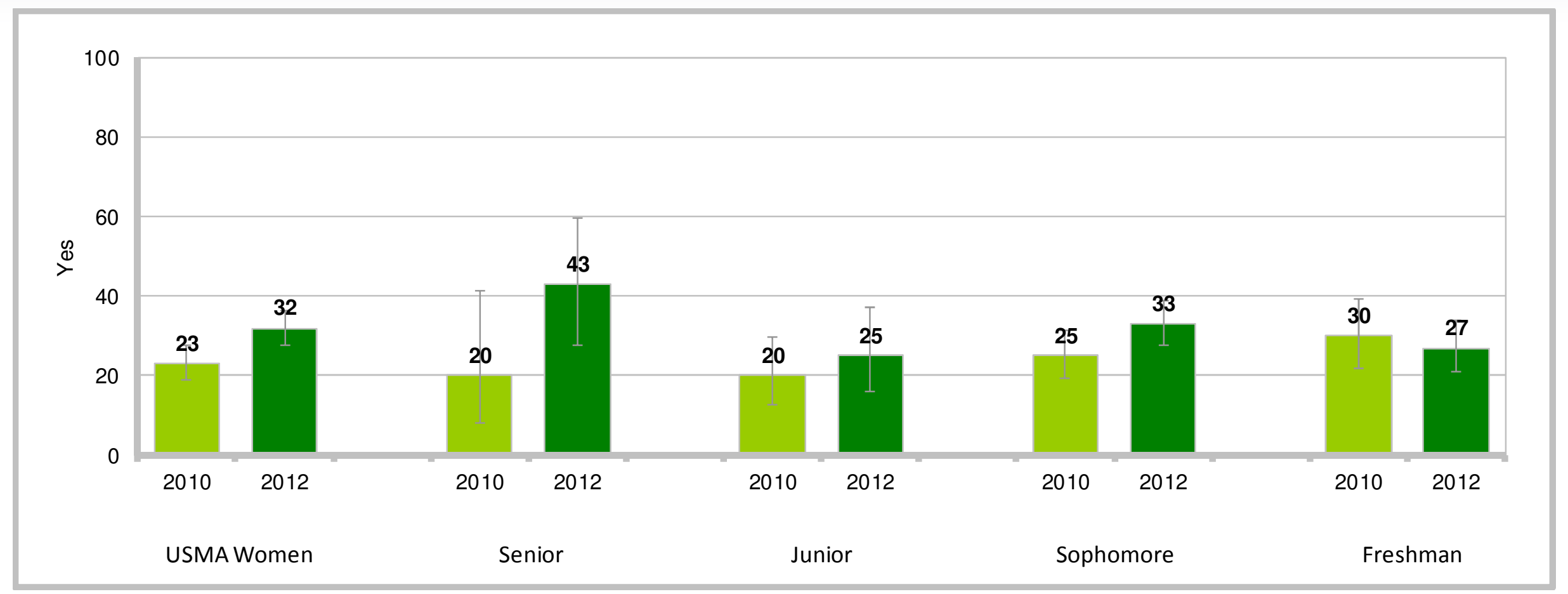

- Of the $10.7 \%$ of women who experienced USC, $32 \%$ indicated the offender was a "creeper"

- 2012 comparisons across years - higher than 2010

- Class differences in 2012 - no differences 


\section{Contents}

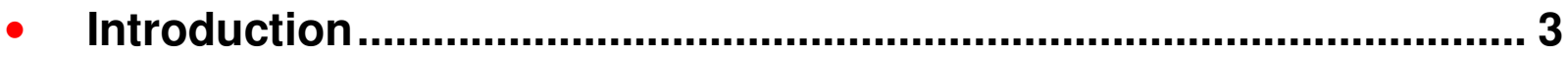

- Unwanted Sexual Contact ............................................................... 7

- Most Serious Behaviors Experienced....................................... 11

- Location of Incident ..................................................................... 13

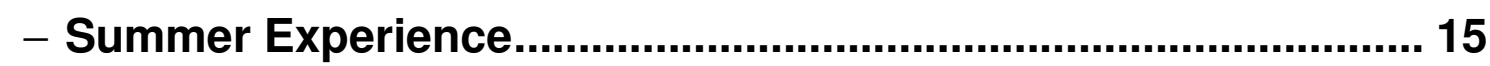

- Characteristics of Offender....................................................... 17

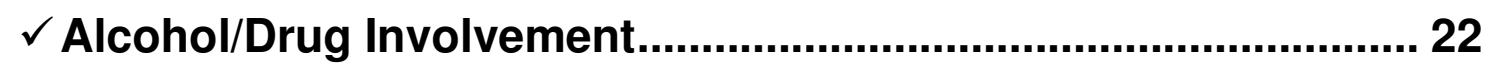

- Use of Threats/Force .............................................................. 27

- Experience of Sexual Harassment/Stalking ............................... 32

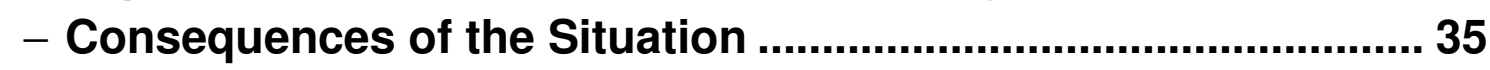

- Reporting the Situation ............................................................ 39

- Experience of Unwanted Sexual Contact Prior to Entering

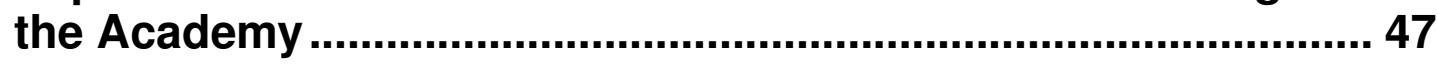

- Unwanted Gender-Related Behaviors ................................................ 49

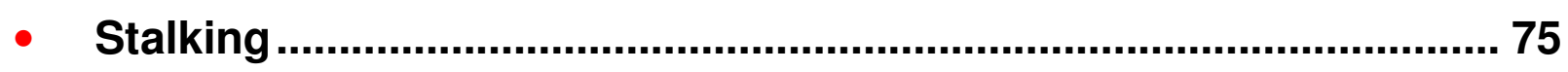

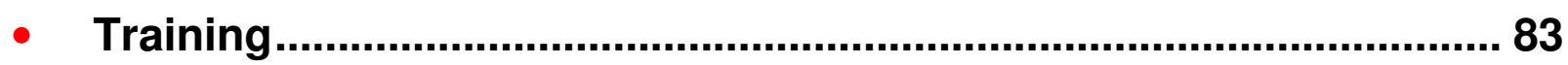

- Student Perceptions.................................................................... 93 


\section{Use of Alcohol and/or Drugs in the Incident Percent of Women Who Experienced Unwanted Sexual Contact}

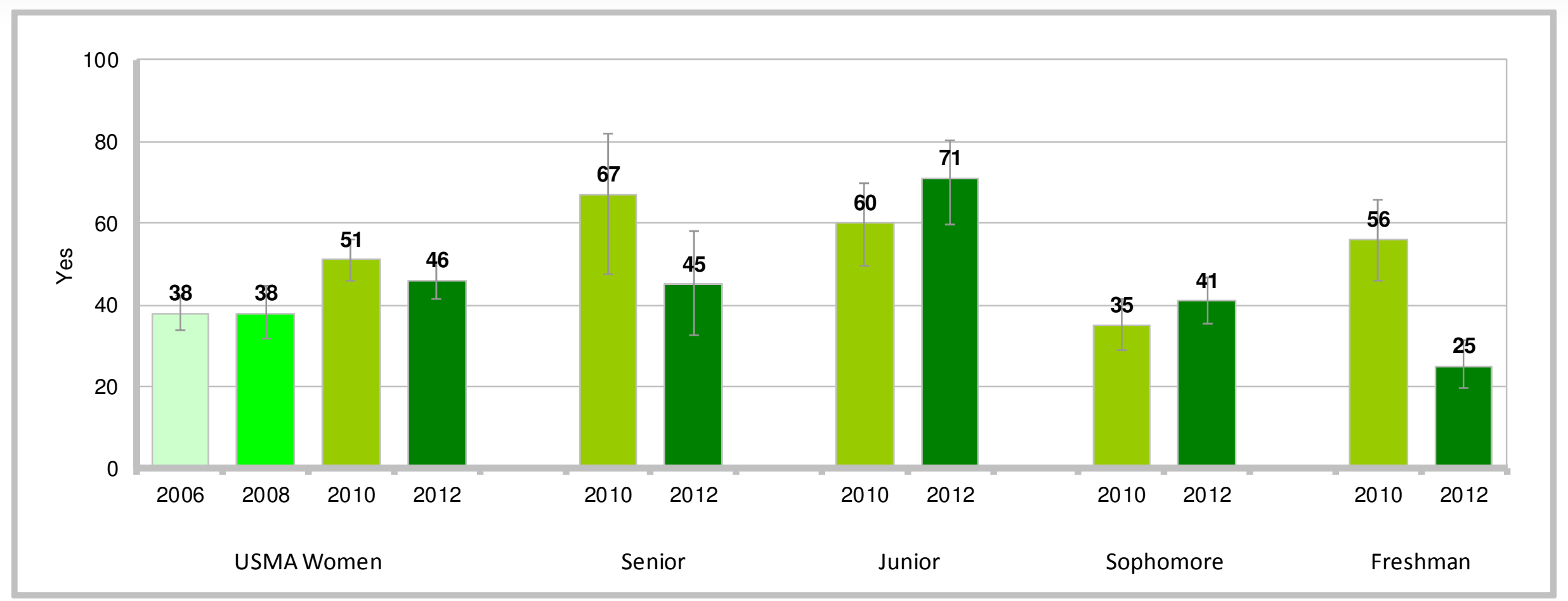

- Of the $10.7 \%$ of women who experienced USC, $46 \%$ indicated alcohol and/or drugs were involved

- 2012 comparisons across years - higher than 2008, 2006

- Freshmen in 2012 lower than 2010

- Class differences in 2012 - higher response led by juniors; lower response led by freshmen 


\section{Use of Alcohol Before the Incident Percent of Women Who Experienced Unwanted Sexual Contact}

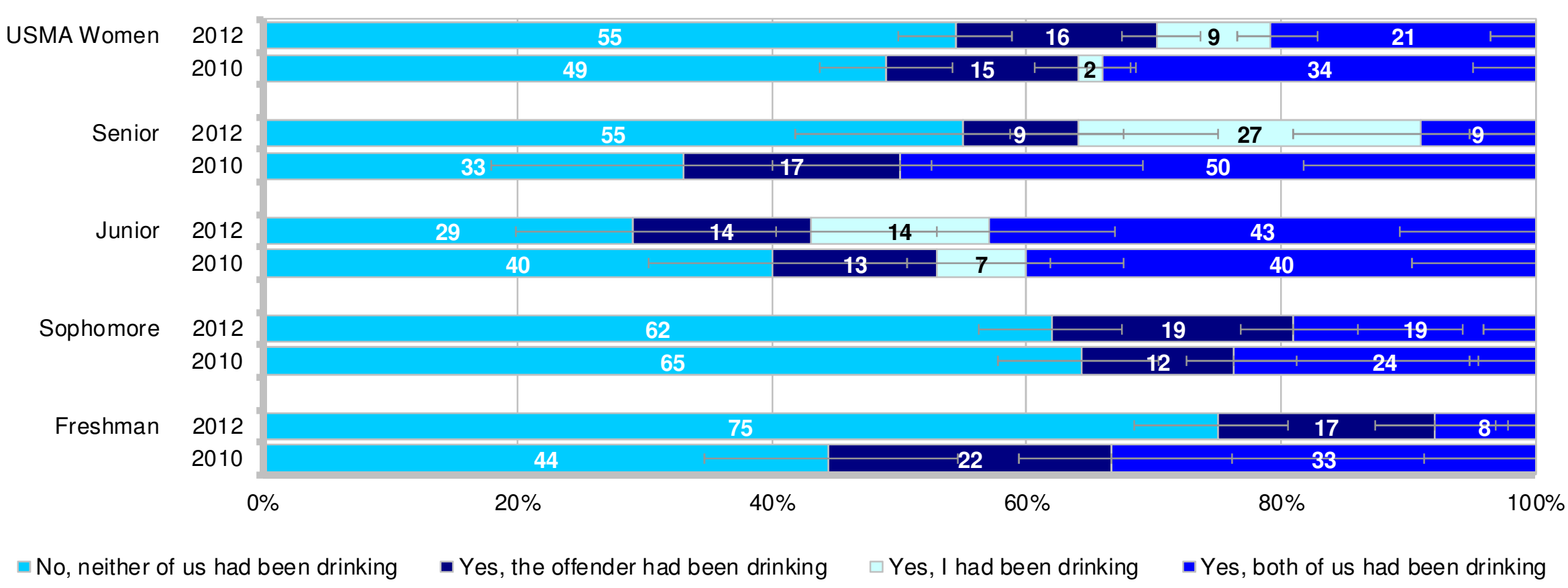

- Of the $10.7 \%$ of women who experienced USC, $55 \%$ indicated neither of them had been drinking; $16 \%$ indicated the offender had been drinking; $9 \%$ indicated they had been drinking; and $21 \%$ indicated both had been drinking

- 2012 comparisons across years - they had higher than 2010; both lower than 2010

- Freshmen indicating neither in 2012 higher than 2010; sophomores indicating the offender in 2012 higher than 2010; seniors and freshmen indicating both in 2012 lower than 2010

- Class differences in 2012 - neither led by freshmen and sophomores; they had led by seniors; both led by juniors 


\section{Use of Drugs Before the Incident Percent of Women Who Experienced Unwanted Sexual Contact}

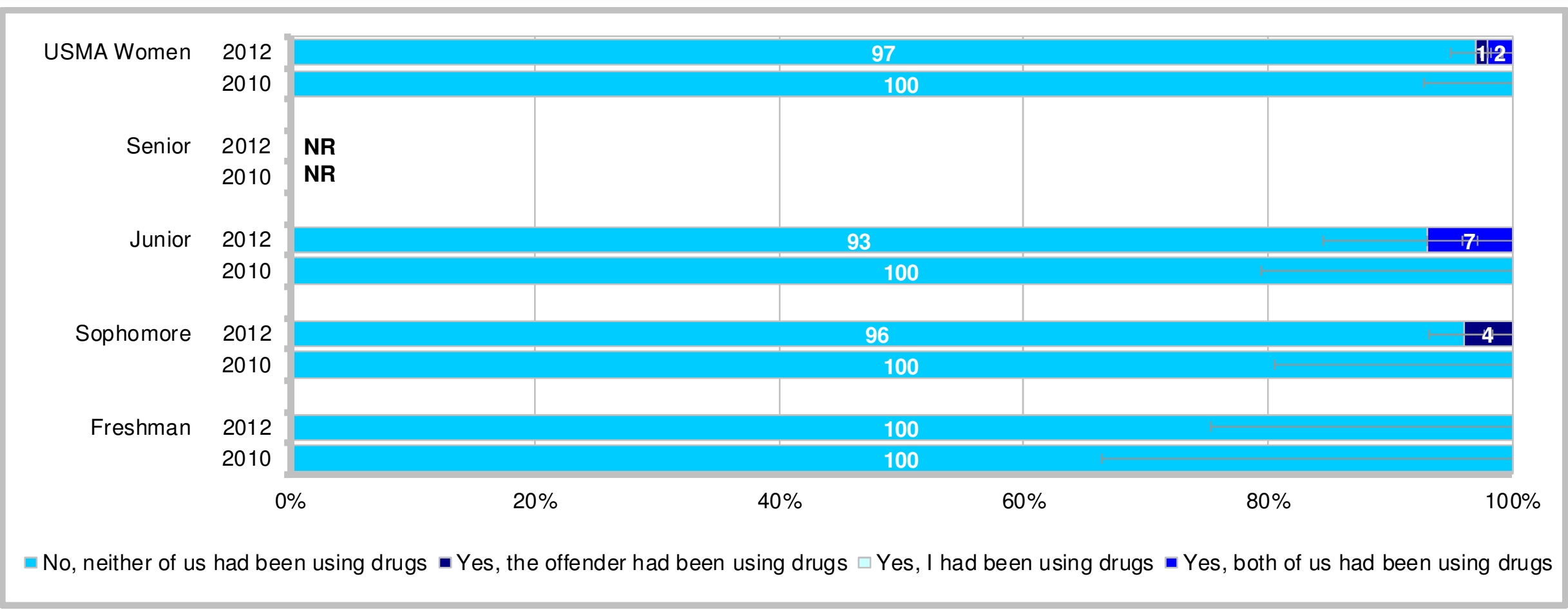

- Of the $10.7 \%$ of women who experienced USC, $97 \%$ indicated neither of them had been using drugs; $1 \%$ indicated the offender had been using drugs; and $2 \%$ indicated both had been using drugs

- 2012 comparisons across years - neither lower than 2010; both higher than 2010

- Juniors and sophomores indicating neither in 2012 lower than 2010; sophomores indicating the offender in 2012 higher than 2010; juniors indicating both in 2012 higher than 2010

- Class differences in 2012 - neither led by freshmen in 2012; the offender led by sophomores 


\section{Use of Knock Out Drugs Before the Incident Percent of Women Who Experienced Unwanted Sexual Contact}

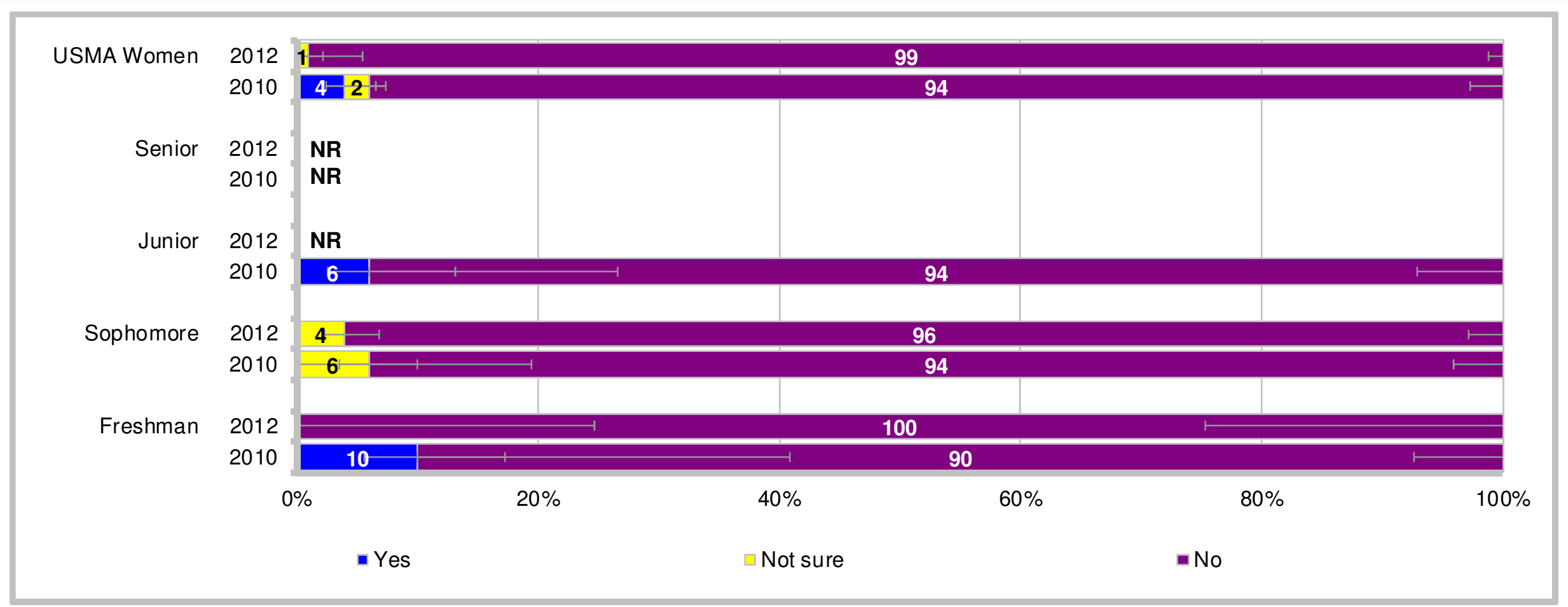

- Of the $10.7 \%$ of women who experienced USC, $99 \%$ indicated the offender did not use drugs to knock them out; $1 \%$ indicated they were not sure; $0 \%$ indicated the offender used drugs to knock them out

- 2012 comparisons across years - the offender did not use drugs to knock them out higher than 2010; the offender used drugs to knock them out lower than 2010

- Freshmen indicating the offender did not use drugs to knock them out in 2012 higher than 2010; freshmen indicating the offender used drugs to knock them out in 2012 lower than 2010

- Class differences in 2012 - not sure led by sophomores; did not use drugs to knock them out led by freshmen 


\section{Contents}

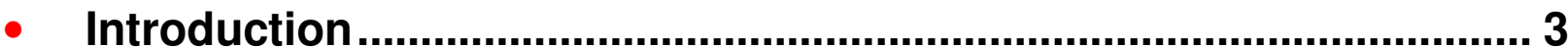

- Unwanted Sexual Contact ................................................................... 7

- Most Serious Behaviors Experienced.......................................... 11

- Location of Incident ....................................................................... 13

- Summer Experience..................................................................... 15

- Characteristics of Offender....................................................... 17

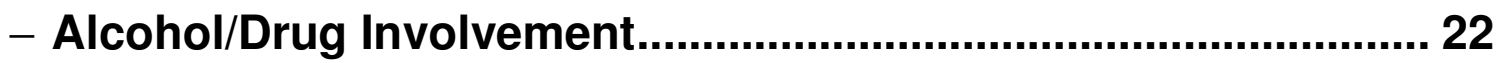

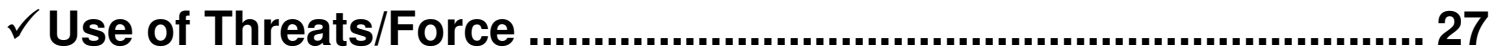

- Experience of Sexual Harassment/Stalking ............................... 32

- Consequences of the Situation ................................................ 35

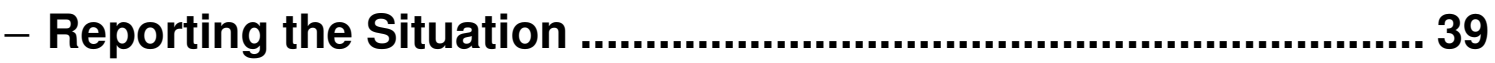

- Experience of Unwanted Sexual Contact Prior to Entering the Academy ..................................................................................... 47

- Unwanted Gender-Related Behaviors .............................................. 49

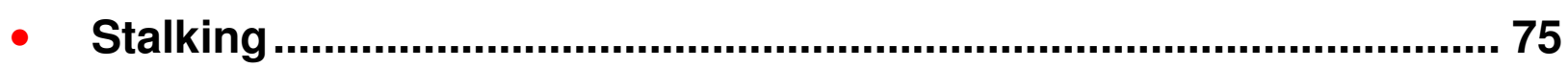

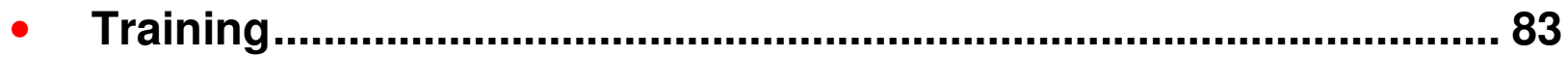

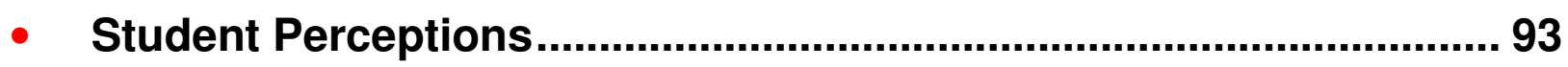




\section{Use of Threats and Physical Force in the Incident Percent of Women Who Experienced Unwanted Sexual Contact}

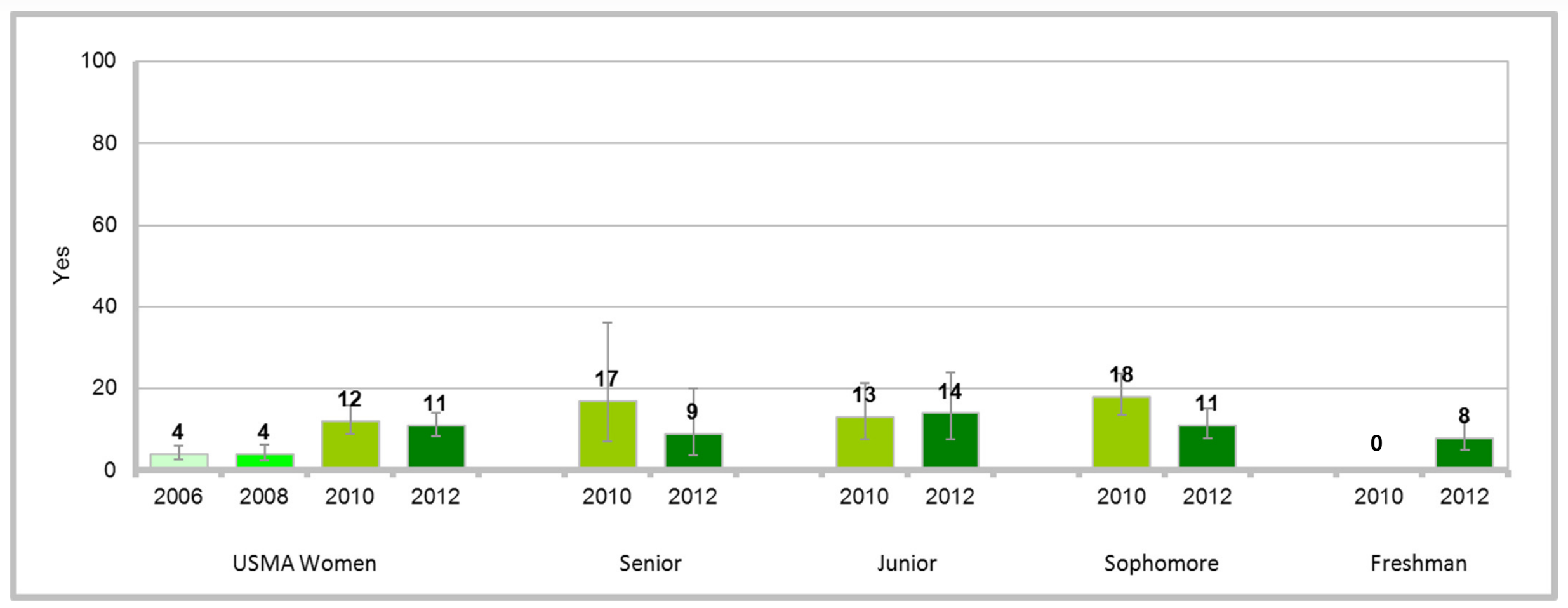

- Of the $10.7 \%$ of women who experienced USC, $11 \%$ indicated the situation involved threats and force

- 2012 comparisons across years - higher than 2008, 2006

- Sophomores in 2012 lower than 2010, whereas freshmen higher

- Class differences in 2012 - no differences 


\section{Offender Threatened To Ruin Your Reputation if You Did Not Consent \\ Percent of Women Who Experienced Unwanted Sexual Contact}

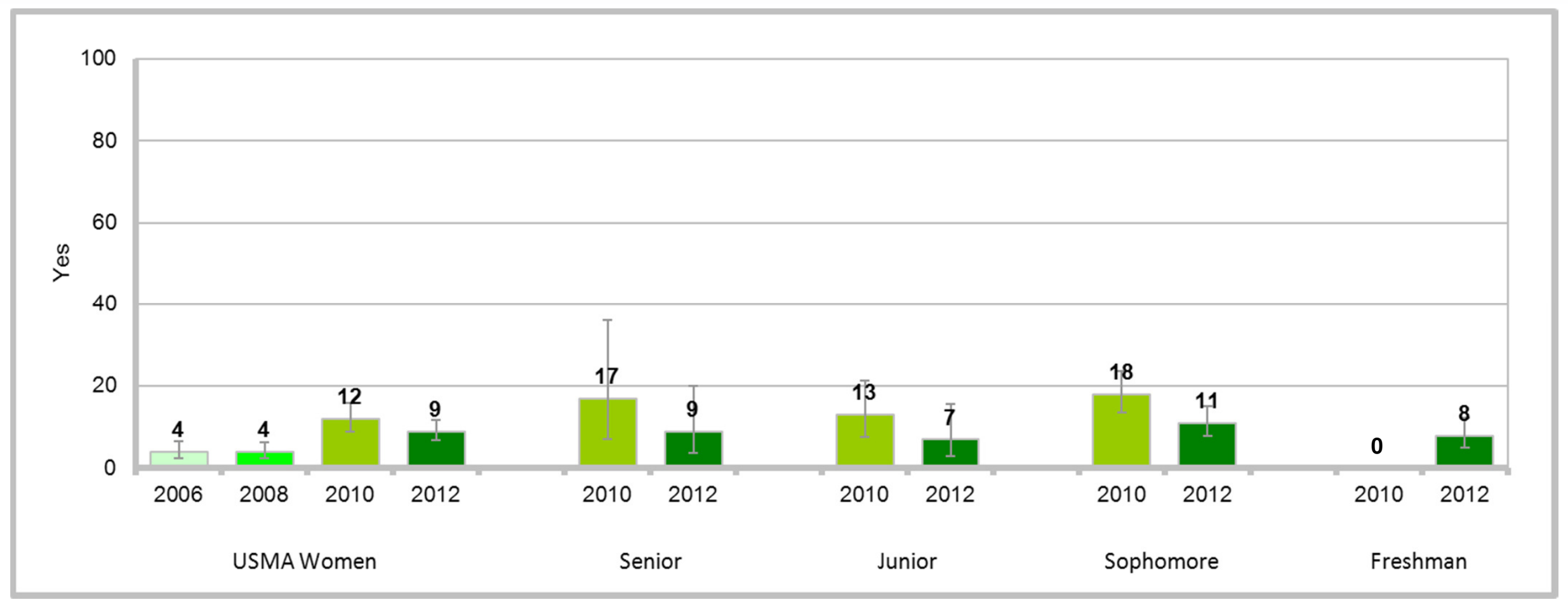

- Of the $10.7 \%$ of women who experienced USC, $9 \%$ indicated the offender threatened to ruin their reputation

- 2012 comparisons across years - higher than 2008, 2006

- Sophomores in 2012 lower than 2010, whereas freshmen higher

- Class differences in 2012 - no differences 


\section{Offender Threatened To Harm You if You Did Not Consent Percent of Women Who Experienced Unwanted Sexual Contact}

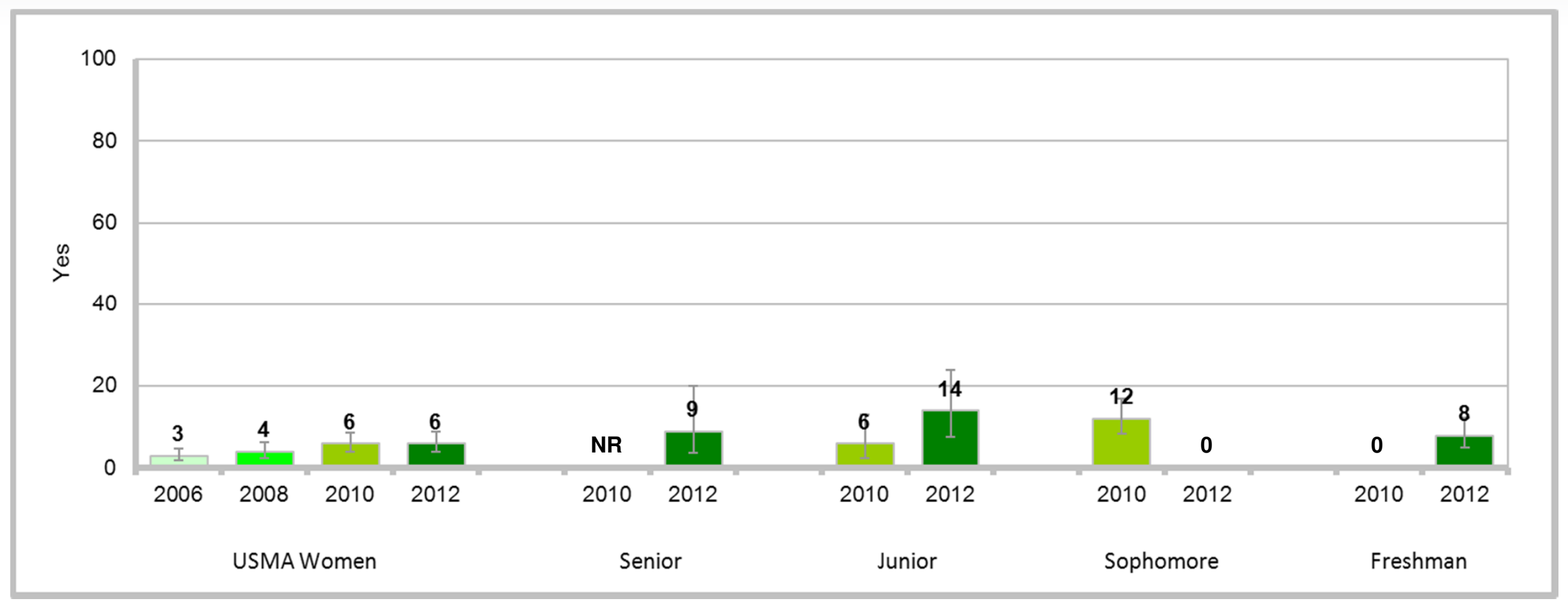

- Of the $10.7 \%$ of women who experienced USC, $6 \%$ indicated the offender threatened to harm them

- 2012 comparisons across years - higher than 2006

- Sophomores in 2012 lower than 2010, whereas freshmen higher

- Class differences in 2012 - lower response led by sophomores 


\section{Offender Used Some Degree of Physical Force}

Percent of Women Who Experienced Unwanted Sexual Contact

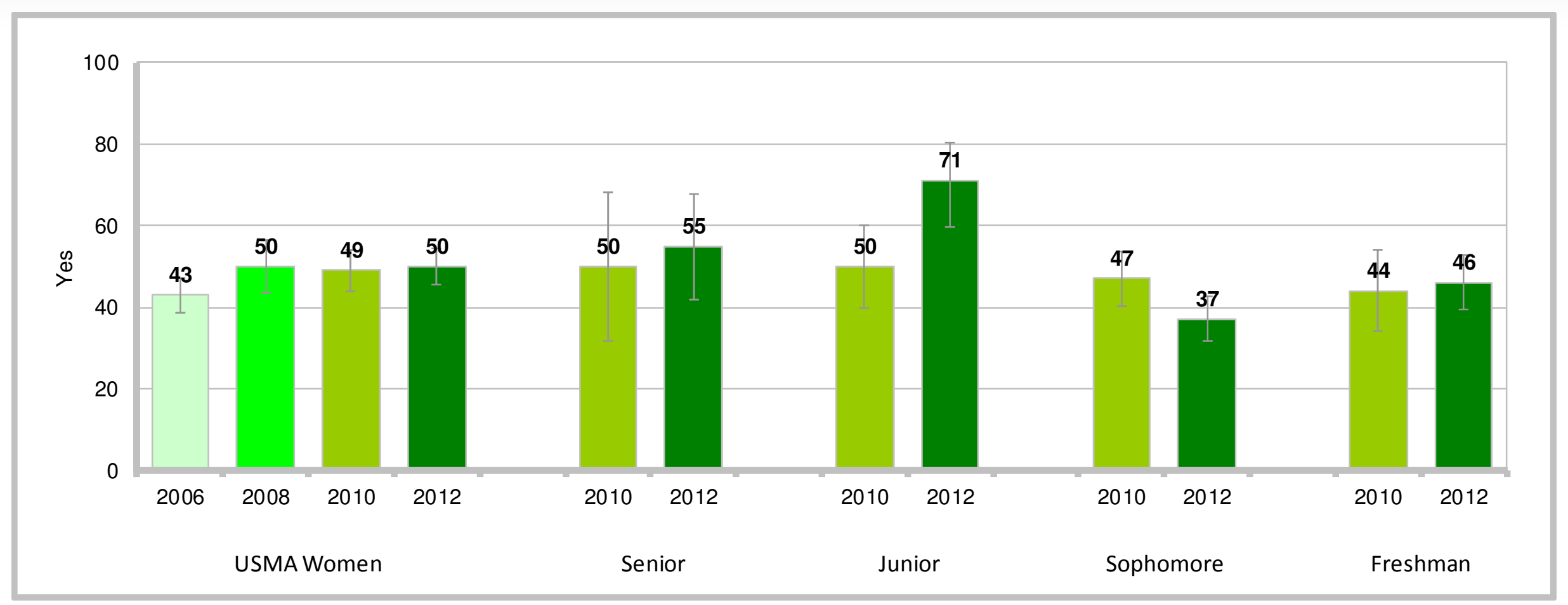

- Of the $10.7 \%$ of women who experienced USC, $50 \%$ indicated the offender threatened to use some degree of physical force

- 2012 comparisons across years - higher than 2006

- Juniors in 2012 higher than 2010, whereas sophomores lower

- Class differences in 2012 - higher response led by juniors; lower response led by sophomores 


\section{Contents}

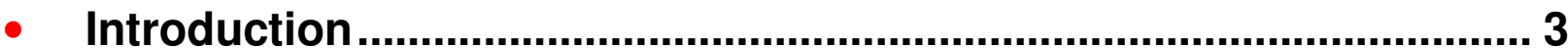

- Unwanted Sexual Contact ................................................................... 7

- Most Serious Behaviors Experienced........................................... 11

- Location of Incident ....................................................................... 13

- Summer Experience..................................................................... 15

- Characteristics of Offender....................................................... 17

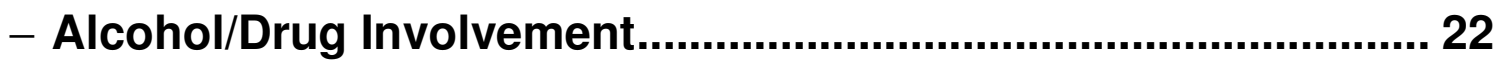

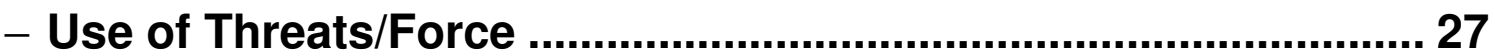

$\checkmark$ Experience of Sexual Harassment/Stalking ............................... 32

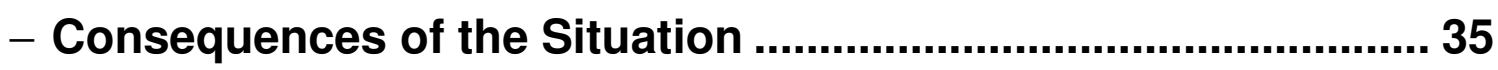

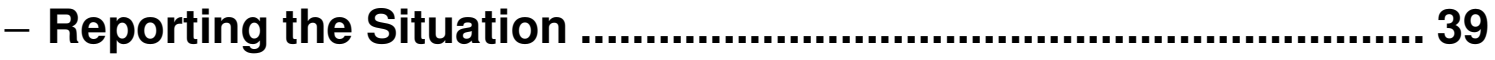

- Experience of Unwanted Sexual Contact Prior to Entering the Academy .................................................................................... 47

- Unwanted Gender-Related Behaviors .............................................. 49

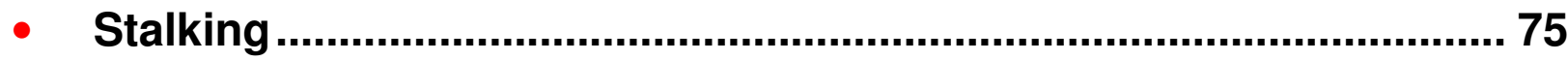

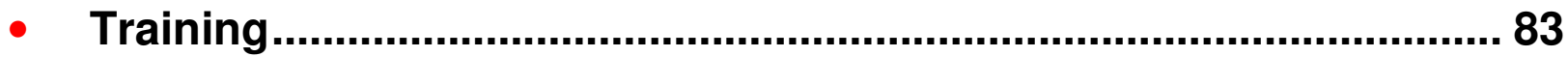

- Student Perceptions..................................................................... 93 


\section{Offender Sexually Harassed and/or Stalked You Before or After the Incident} Percent of Women Who Experienced Unwanted Sexual Contact

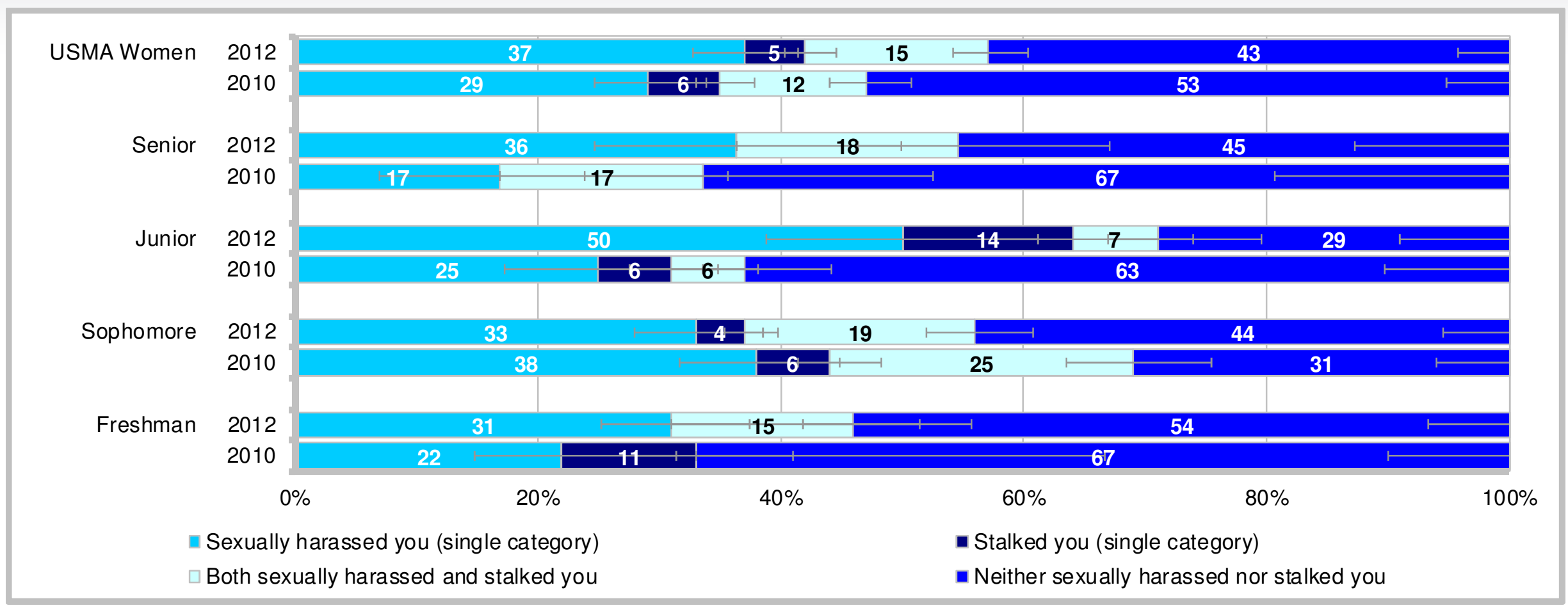

- Of the $10.7 \%$ of women who experienced USC, $37 \%$ indicated the offender sexually harassed them; $5 \%$ indicated the offender stalked them; $15 \%$ indicated the offender both sexually harassed and stalked them; and $43 \%$ indicated the offender neither sexually harassed nor stalked them

- 2012 comparisons across years - sexually harassed higher than 2010; neither lower than 2010

- Juniors indicating sexually harassed in 2012 higher than 2010; freshmen indicating stalked in 2012 lower than 2010; freshmen indicating both in 2012 higher than 2010; juniors and freshmen indicating neither in 2012 lower than 2010, whereas sophomores higher

- Class differences in 2012 - sexually harassed led by juniors; stalked led by juniors; neither led by freshmen 


\section{When Offender Sexually Harassed and/or Stalked You Percent of Women Who Experienced Unwanted Sexual Contact}

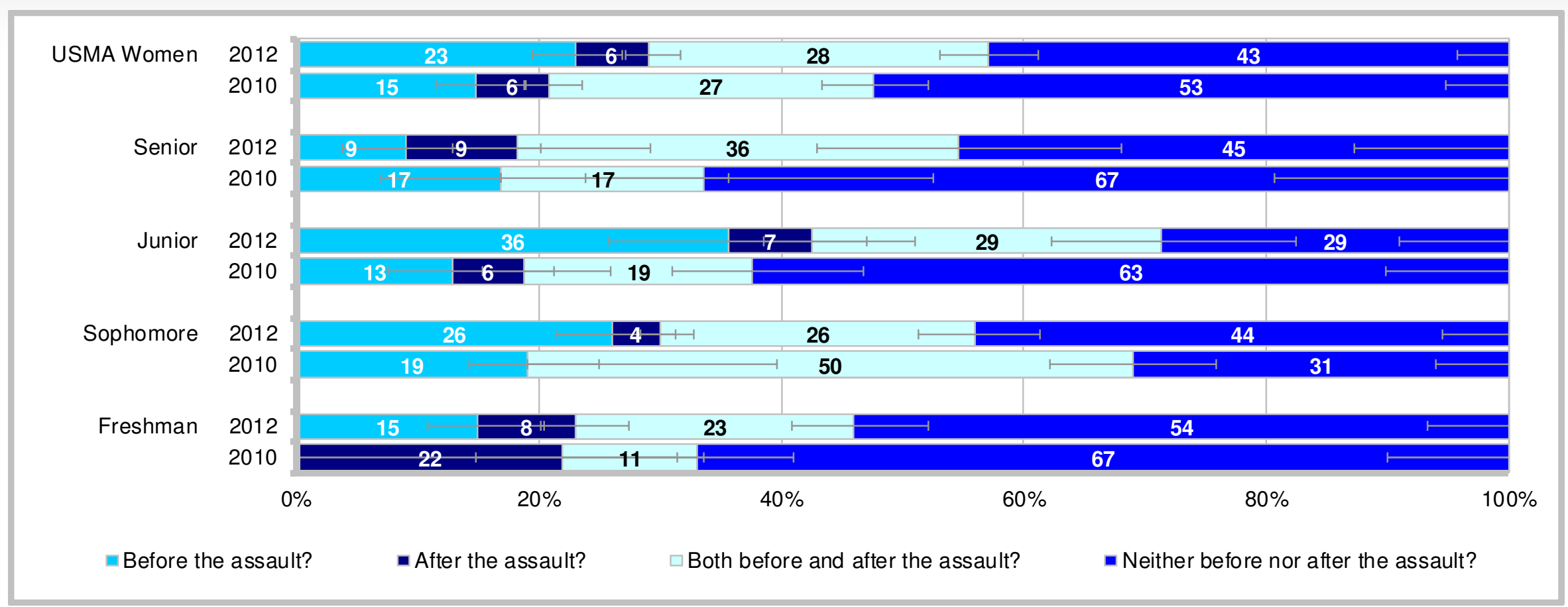

- Of the $10.7 \%$ of women who experienced USC, $23 \%$ indicated the offender sexually harassed or stalked them before the assault; $6 \%$ indicated the offender sexually harassed or stalked them after the assault; $28 \%$ indicated the offender sexually harassed or stalked them both before and after the assault; and $43 \%$ indicated neither before nor after the assault

- 2012 comparisons across years - before higher than 2010; neither lower than 2010

- Juniors and freshmen indicating before in 2012 higher than 2010; sophomores indicating after in 2012 higher than 2010, whereas freshmen lower; sophomores indicating both in 2012 lower than 2010, whereas freshmen higher; juniors and freshmen indicating neither in 2012 lower than 2010, whereas sophomores higher

- Class differences in 2012 - before led by juniors; neither led by freshmen 


\section{Contents}

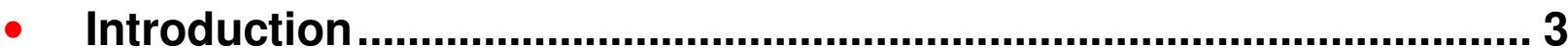

- Unwanted Sexual Contact ................................................................... 7

- Most Serious Behaviors Experienced............................................ 11

- Location of Incident ....................................................................... 13

- Summer Experience..................................................................... 15

- Characteristics of Offender....................................................... 17

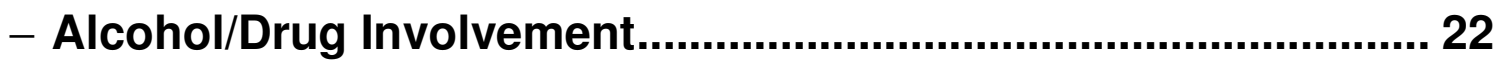

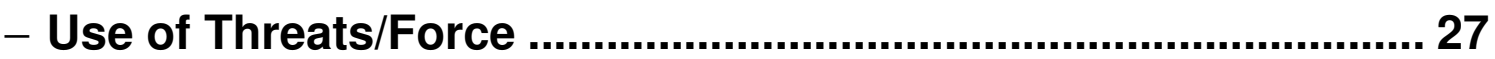

- Experience of Sexual Harassment/Stalking ............................... 32

$\checkmark$ Consequences of the Situation .................................................... 35

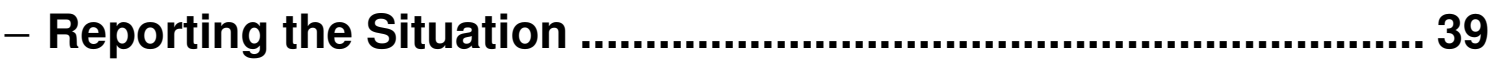

- Experience of Unwanted Sexual Contact Prior to Entering the Academy ..................................................................................... 47

- Unwanted Gender-Related Behaviors .............................................. 49

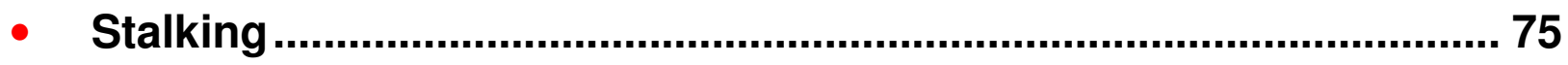

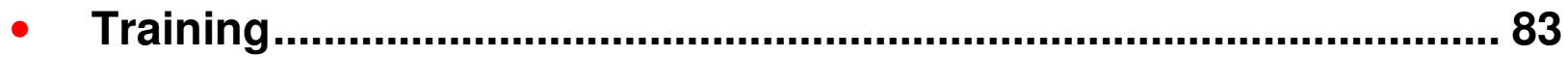

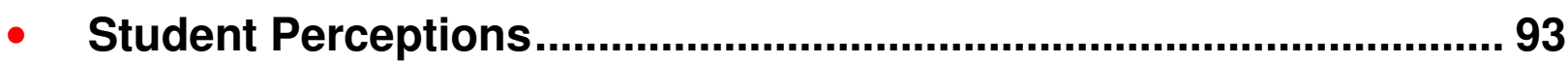




\section{Considered Requesting a Transfer to Another Company Percent of Women Who Experienced Unwanted Sexual Contact}

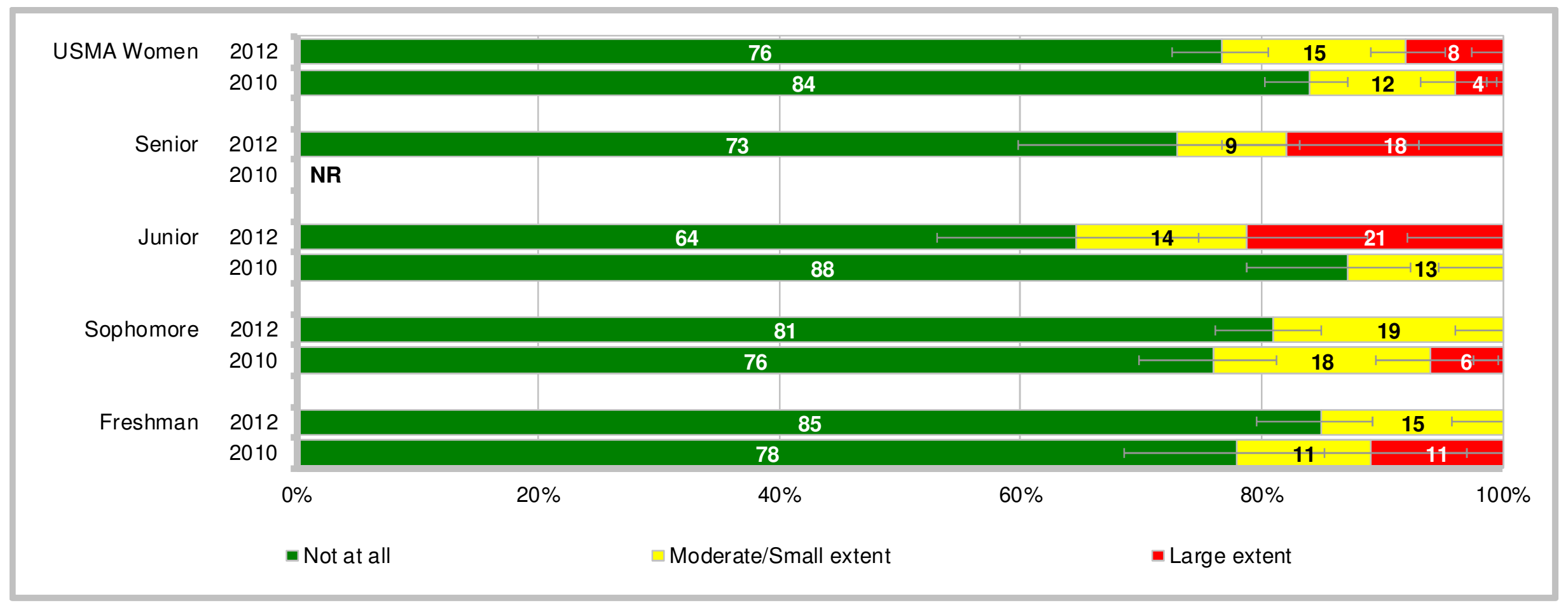

- Of the $10.7 \%$ of women who experienced USC, $23 \%$ indicated they considered a transfer to some extent; $76 \%$ indicated not at all

- 2012 comparisons across years - not at all lower than 2010

- Juniors indicating not at all in 2012 lower than 2010; juniors indicating large extent in 2012 higher than 2010, whereas sophomores and freshmen lower

- Class differences in 2012 - not at all led by freshmen; large extent led by juniors 


\section{Thought About Leaving Your Academy Percent of Women Who Experienced Unwanted Sexual Contact}

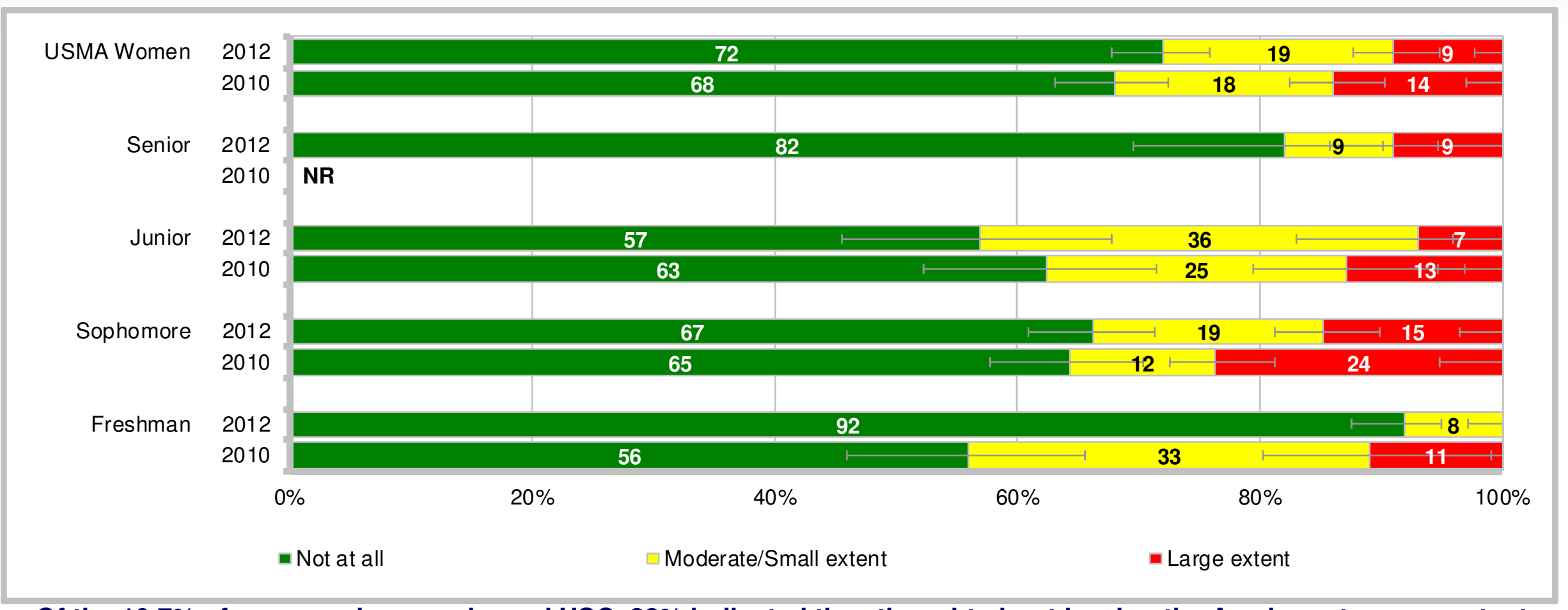

- Of the $10.7 \%$ of women who experienced USC, $28 \%$ indicated they thought about leaving the Academy to some extent; $72 \%$ indicated not at all

- 2012 comparisons across years - large extent lower than 2010

- Freshmen indicating not at all in 2012 higher than 2010; sophomores indicating moderate/small extent in 2012 higher than 2010, whereas freshmen lower; sophomores and freshmen indicating large extent in 2012 lower than 2010

- Class differences in 2012 - not at all led by freshmen; moderate/small extent led by juniors; large extent led by sophomores 


\section{Your Academic Performance Suffered Percent of Women Who Experienced Unwanted Sexual Contact}

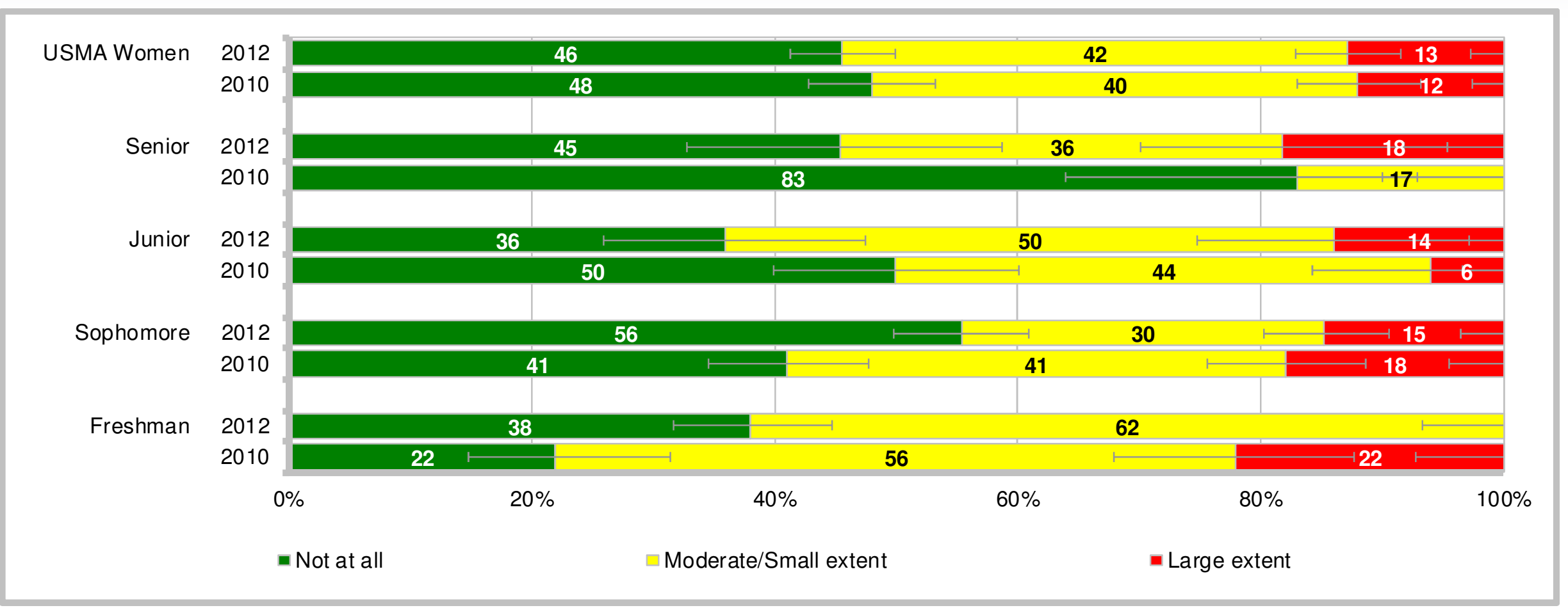

- Of the $10.7 \%$ of women who experienced USC, $55 \%$ indicated their academic performance suffered to some extent; $46 \%$ indicated not at all

- 2012 comparisons across years - no differences

- Seniors indicating not at all in 2012 lower than 2010, whereas sophomores and freshmen higher; sophomores indicating moderate/small extent in 2012 lower than 2010; freshmen indicating large extent in 2012 lower than 2010

- Class differences in 2012 - not at all led by sophomores 


\section{Contents}

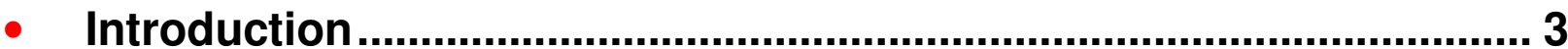

- Unwanted Sexual Contact ................................................................... 7

- Most Serious Behaviors Experienced.......................................... 11

- Location of Incident ....................................................................... 13

- Summer Experience..................................................................... 15

- Characteristics of Offender....................................................... 17

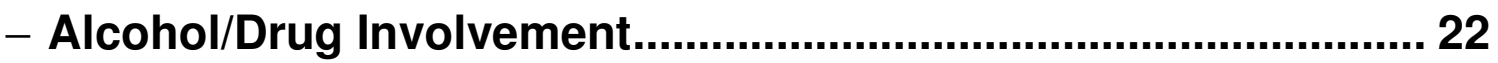

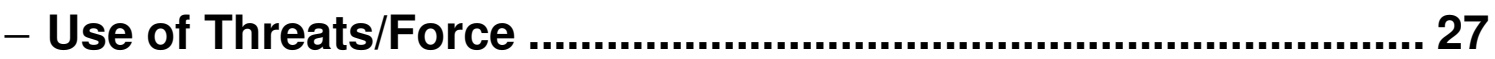

- Experience of Sexual Harassment/Stalking ............................... 32

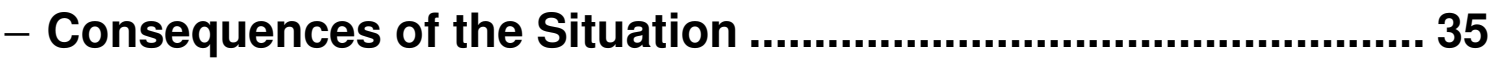

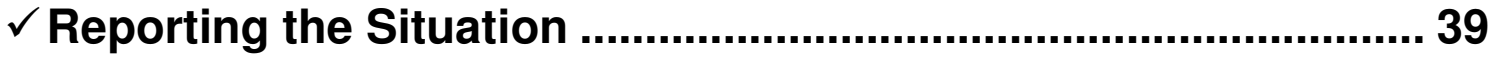

- Experience of Unwanted Sexual Contact Prior to Entering the Academy ..................................................................................... 47

- Unwanted Gender-Related Behaviors .............................................. 49

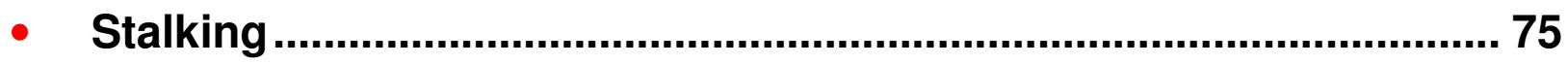

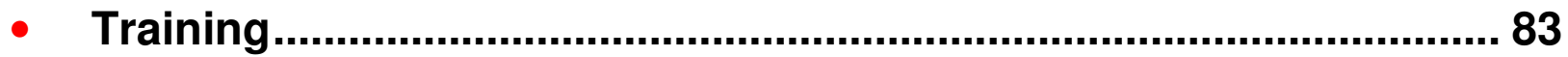

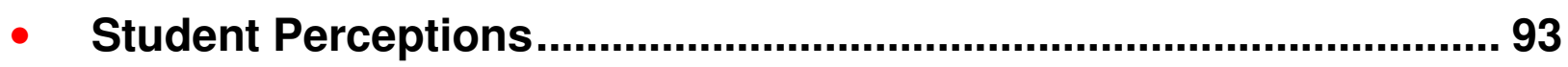




\section{Reported This Situation to a Military Authority or Organization Percent of Women Who Experienced Unwanted Sexual Contact}

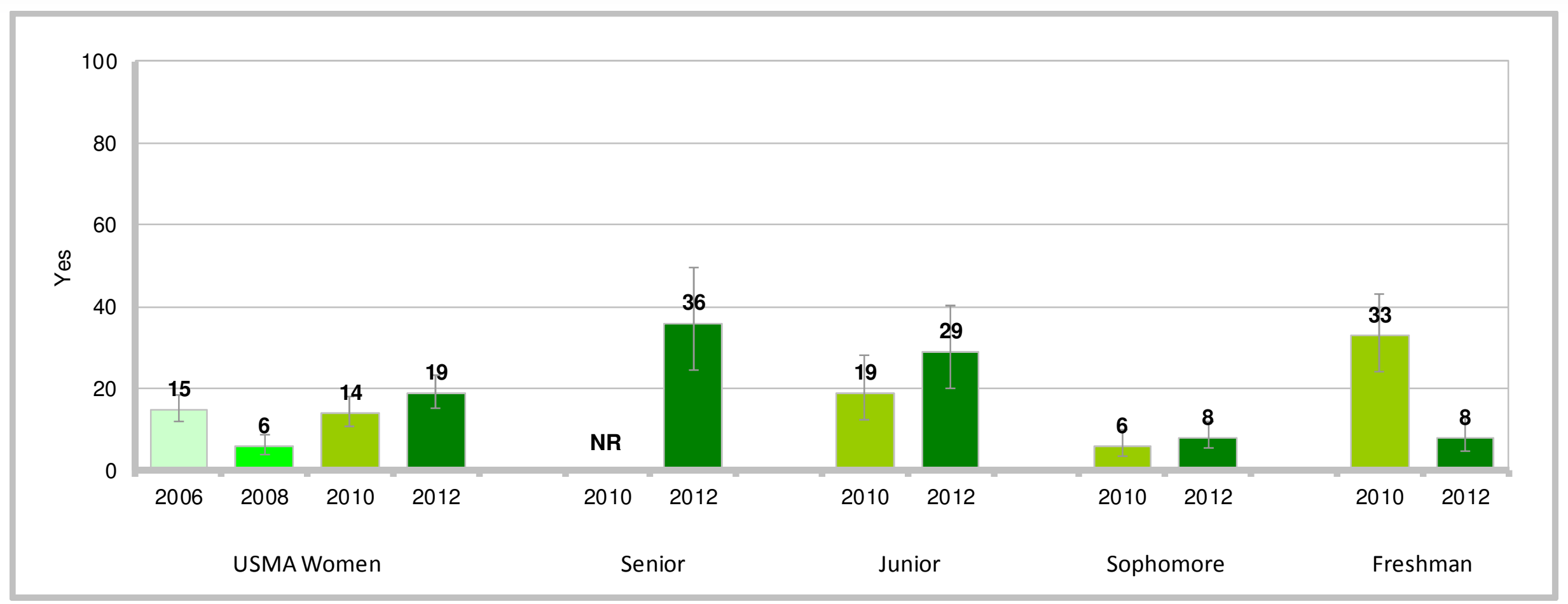

- Of the $10.7 \%$ of women who experienced USC, $19 \%$ indicated they reported the situation

- 2012 comparisons across years - higher than 2008

- Freshmen in 2012 lower than 2010

- Class differences in 2012 - higher response led by seniors; lower response led by sophomores and freshmen 


\section{Type of Report Made to a Military Authority}

Percent of Women Who Experienced Unwanted Sexual Contact and Reported It

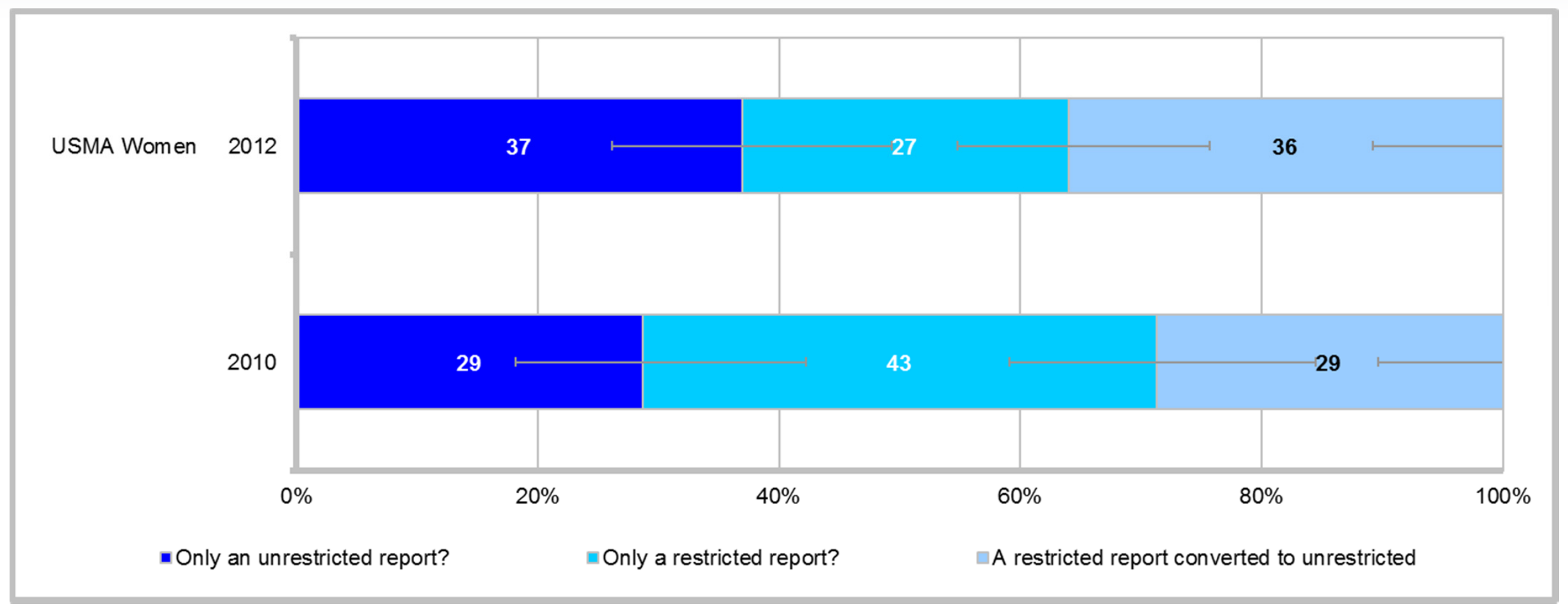

- Of the $19 \%$ of women who reported the situation, $37 \%$ indicated they made only an unrestricted report; $27 \%$ made only a restricted report; and $36 \%$ made a restricted report converted to unrestricted

- 2012 comparisons across years - no differences

- Results by class year not reportable 


\section{When Report Made to a Military Authority}

Percent of Women Who Experienced Unwanted Sexual Contact and Reported It

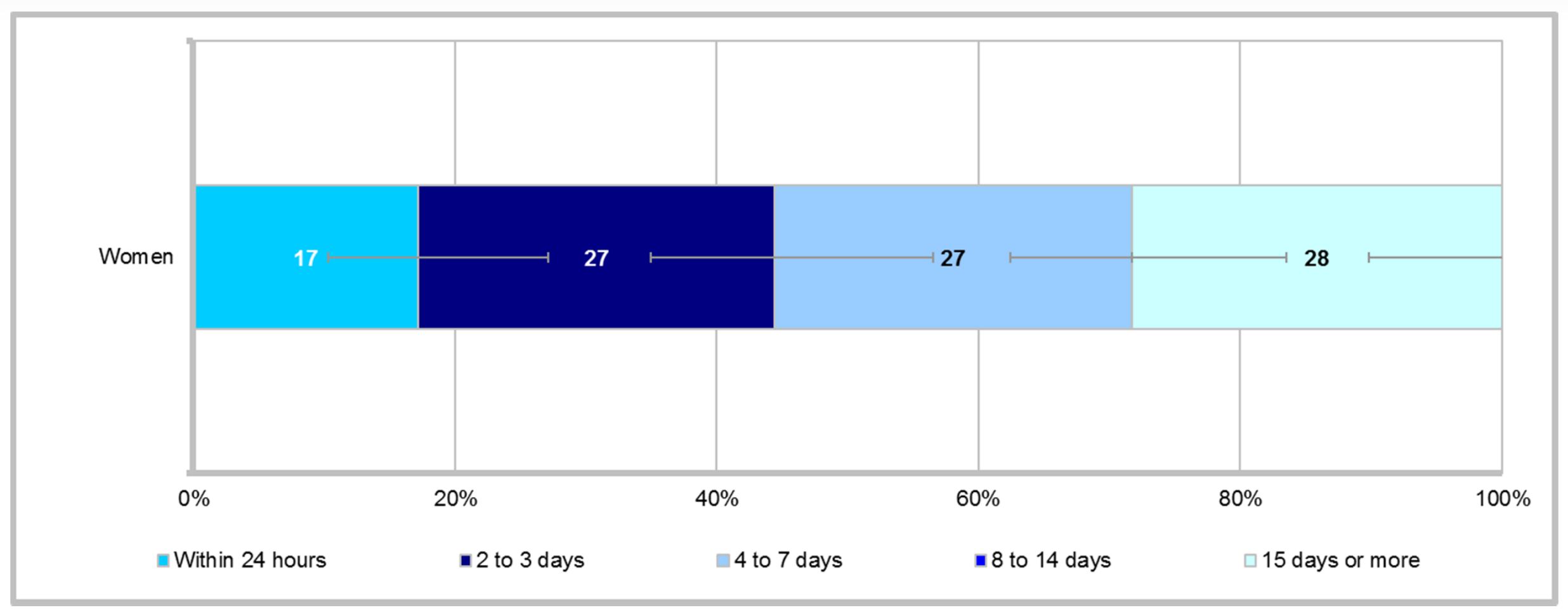

- Of the $19 \%$ of women who reported, $17 \%$ indicated they made their report within 24 hours; $27 \%$ within 2 to 3 days; $27 \%$ within 4 to 7 days; $0 \%$ within 8 to 14 days; and $28 \%$ after 15 days or more

- 2012 comparisons across years - new question in 2012; no trend data available

- Results by class year not reportable 


\section{Reason for Delay in Reporting the Situation}

Percent of Women Who Experienced Unwanted Sexual Contact and Waited at Least 24 Hours to Report It

\begin{tabular}{|l|c|}
\hline \multicolumn{1}{|c|}{ Reasons for Delay in Reporting } & Total \\
\hline $\begin{array}{l}\text { Wanted to seek advice first from a friend or } \\
\text { family member }\end{array}$ & 68 \\
\hline $\begin{array}{l}\text { Wanted to think about the situation before } \\
\text { deciding to report }\end{array}$ & 57 \\
\hline $\begin{array}{l}\text { Wanted to seek advice/counseling from a } \\
\text { professional before deciding to report }\end{array}$ & 45 \\
\hline Other* & 43 \\
\hline Margins of Error & $\pm 14-15$ \\
\hline
\end{tabular}

\footnotetext{
*Other reasons given for delay in reporting the situation included: did not originally plan on reporting; got up the courage to report it; had to wait because of time constraints; and was not able to pretend it didn't actually happen.
}

- New question in 2012; no trend data available

- Results by class year not reportable 


\section{Most Frequently Selected Reasons for Reporting Unwanted Sexual Contact Situation Percent of Women Who Experienced Unwanted Sexual Contact and Reported It}

\begin{tabular}{|l|c|}
\hline \multicolumn{1}{|c|}{ Reasons for Reporting } & Total \\
\hline It was the right thing to do & 82 \\
\hline Stop the offender from hurting others & 73 \\
\hline Stop the offender from hurting you again & 65 \\
\hline Seek help dealing with an emotional incident & 65 \\
\hline Margins of Error & \pm 13 \\
\hline
\end{tabular}

- New question in 2012; no trend data available

- Results by class year not reportable 


\section{Most Frequently Selected Reasons for} Not Reporting Unwanted Sexual Contact Situation Percent of Women Who Experienced Unwanted Sexual Contact and Did Not Report It

\begin{tabular}{|l|c|c|c|c|c|c|}
\hline \multicolumn{1}{|c}{ Reasons for Not Reporting } & $\begin{array}{l}\text { Survey } \\
\text { Year }\end{array}$ & Total & Senior & Junior & Sophomore & Freshman \\
\hline \multirow{2}{*}{$\begin{array}{l}\text { Thought it was not important enough to } \\
\text { report }\end{array}$} & 2012 & $75 \uparrow$ & 86 & $80 \uparrow$ & $74 \uparrow$ & $64 \downarrow$ \\
\cline { 2 - 7 } & 2010 & 56 & 67 & 46 & 50 & 80 \\
\hline \multirow{2}{*}{ Did not want people gossiping } & 2012 & 74 & $57 \downarrow$ & $80 \uparrow$ & $78 \uparrow$ & 73 \\
\cline { 2 - 7 } & 2010 & 71 & 83 & 54 & 69 & $\mathrm{NR}$ \\
\hline \multirow{2}{*}{ Did not want anyone to know } & 2012 & $70 \uparrow$ & 57 & $60 \uparrow$ & 74 & 82 \\
\cline { 2 - 7 } & 2010 & 62 & 67 & 31 & 75 & $\mathrm{NR}$ \\
\hline \multirow{2}{*}{ Felt uncomfortable making a report } & 2012 & 65 & 57 & 60 & 74 & 55 \\
\cline { 2 - 7 } & 2010 & 70 & 67 & 62 & 69 & $\mathrm{NR}$ \\
\hline \multirow{2}{*}{ Took care of it myself } & 2012 & $62 \downarrow$ & 43 & $60 \downarrow$ & 74 & 55 \\
\cline { 2 - 7 } & 2010 & 76 & $\mathrm{NR}$ & 77 & 75 & 40 \\
\hline \multirow{2}{*}{ Felt shame/embarrassment } & 2012 & 61 & 57 & 60 & 57 & 73 \\
\cline { 2 - 7 } & 2010 & 65 & 50 & 62 & 63 & $\mathrm{NR}$ \\
\hline $\begin{array}{l}\text { Thought it would hurt my reputation and } \\
\text { standing }\end{array}$ & 2012 & 57 & 57 & 50 & 57 & 64 \\
\hline & 2010 & 61 & 50 & 54 & 56 & $\mathrm{NR}$ \\
\hline Margins of Error & & $\pm 5-6 \%$ & $\pm 16-20 \%$ & $\pm 12-14 \%$ & $\pm 6-7 \%$ & $\pm 7-13 \%$ \\
\hline
\end{tabular}

Higher Response of Yes

Lower Response of Yes

Higher in 2012 than 2010

Lower in 2012 than 2010 


\section{Would Make the Same Decision About Reporting or Not Reporting if Could Do It Over}

Percent of Women Who Experienced Unwanted Sexual Contact and Reported or Did Not Report to a Military Authority

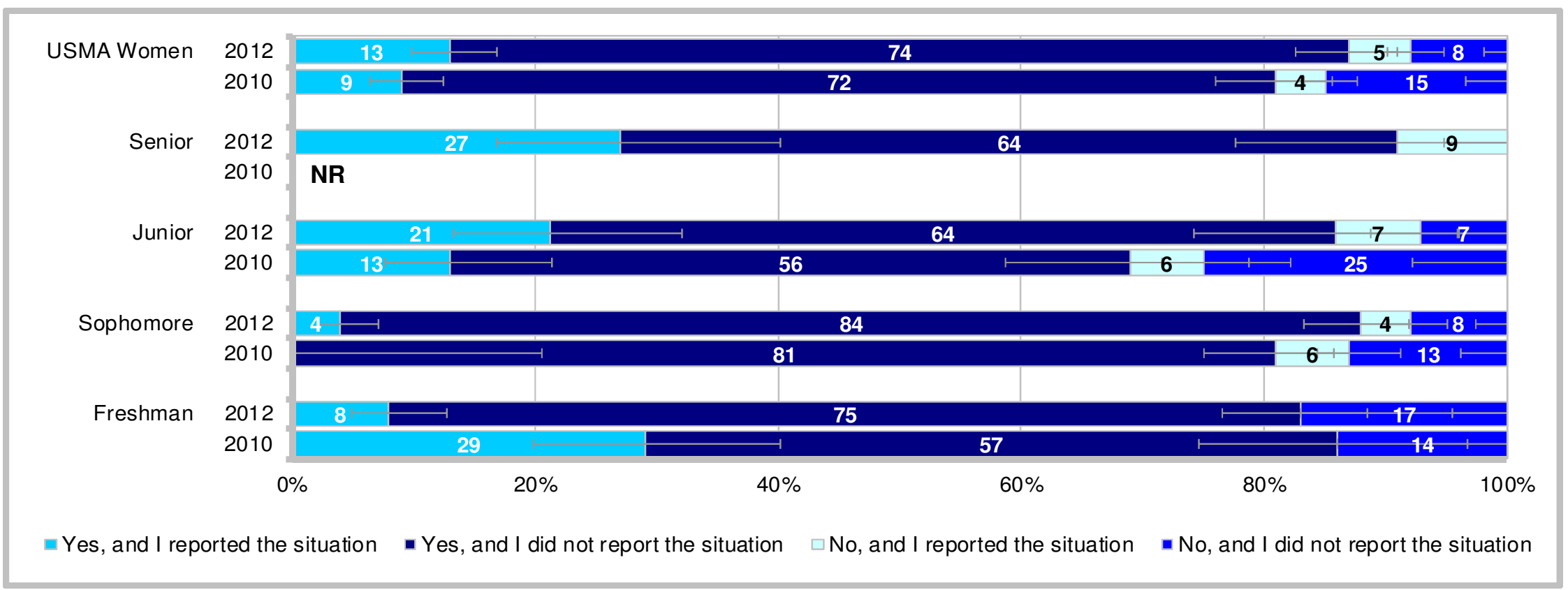

- Of the $10.7 \%$ of women who experienced USC, $13 \%$ indicated they reported the situation and would make the same decision again; $74 \%$ indicated they did not report the situation and would make the same decision again; $5 \%$ indicated they reported but would not make the same decision again; and $8 \%$ indicated they did not report the situation but would not make the same decision again

- 2012 comparisons across years - did not report but would not make the same decision lower than 2010

- Sophomores indicating reported and would make the same decision in 2012 higher than 2010, whereas freshmen lower; freshmen indicating did not report and would make the same decision in 2012 higher than 2010; juniors indicating did not report but would not make the same decision in 2012 lower than 2010

- Class differences in 2012 - reported and would make the same decision led by seniors; did not report and would make the same decision led by sophomores; and did not report but would not make the same decision led by freshmen 


\section{Contents}

Slide

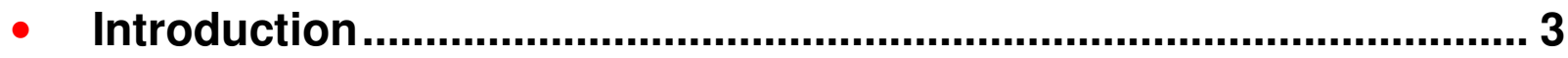

- Unwanted Sexual Contact ............................................................... 7

- Most Serious Behaviors Experienced....................................... 11

- Location of Incident ..................................................................... 13

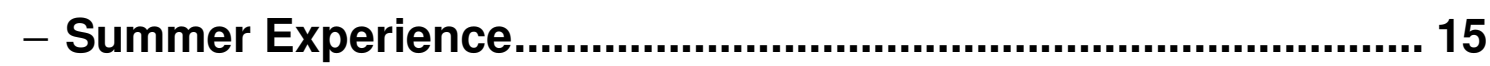

- Characteristics of Offender......................................................... 17

- Alcohol/Drug Involvement......................................................... 22

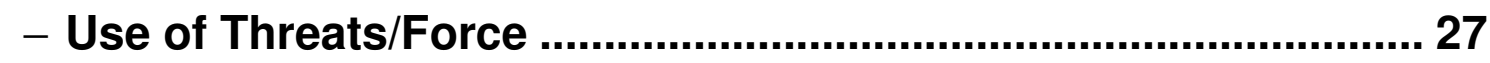

- Experience of Sexual Harassment/Stalking ............................. 32

- Consequences of the Situation .................................................. 35

- Reporting the Situation ............................................................ 39

$\checkmark$ Experience of Unwanted Sexual Contact Prior to Entering

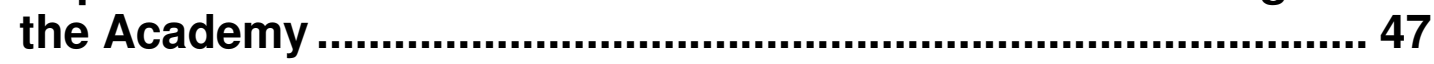

- Unwanted Gender-Related Behaviors ................................................ 49

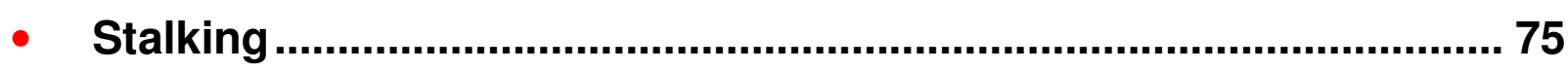

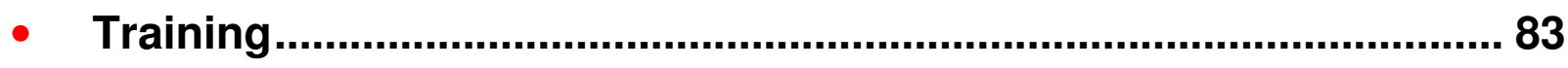

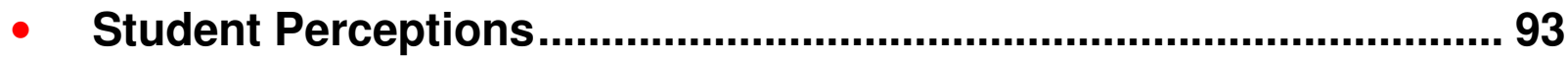




\section{Unwanted Sexual Contact Incident Rate Prior to Entering the Academy} Percent of All Students

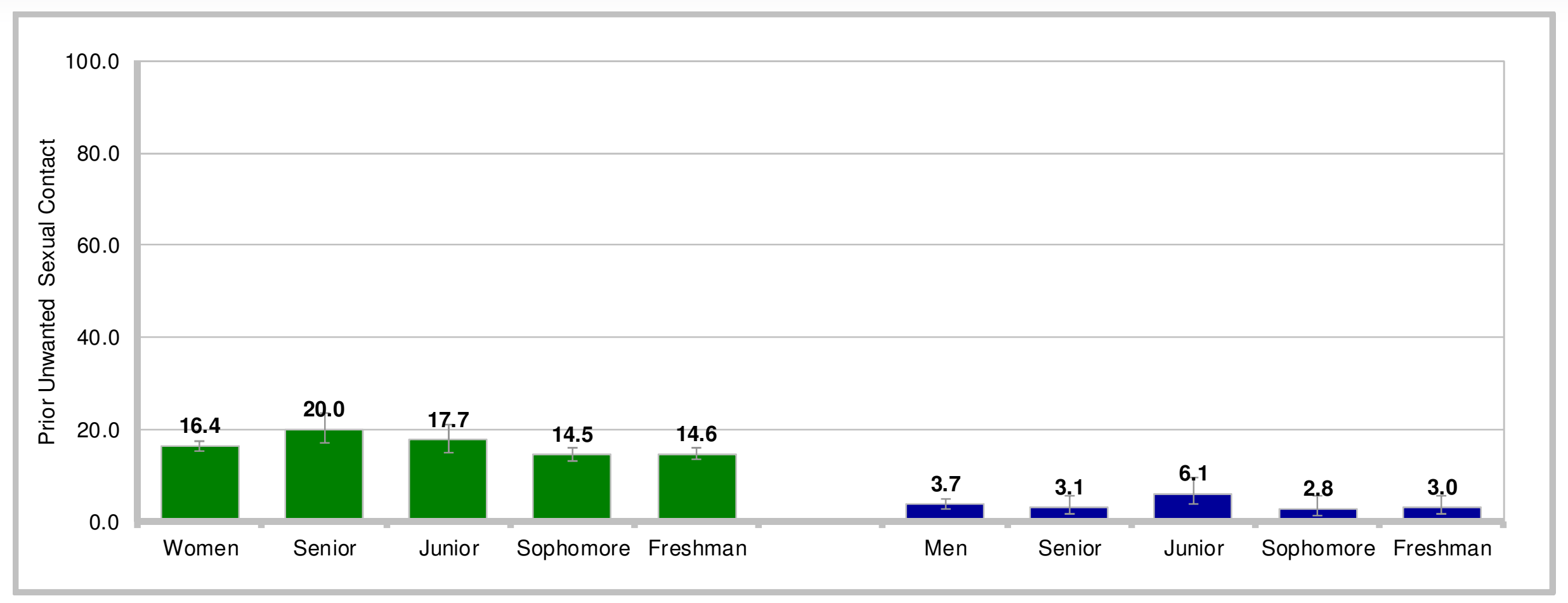

- $16.4 \%$ of women and $3.7 \%$ of men indicated experiencing unwanted sexual contact prior to entering the Academy

- 2012 comparisons across years - new question in 2012; no trend data available

- Class differences in 2012 - for women, higher response led by seniors; for men, no differences 


\section{Contents}

Slide

- Introduction ................................................................................ 3

- Unwanted Sexual Contact ........................................................... 7

$\checkmark \quad$ Unwanted Gender-Related Behaviors ........................................... 49

- Sexual Harassment ............................................................... 51

- Categories of Behaviors Associated with Sexual Harassment.................................................................................. 54

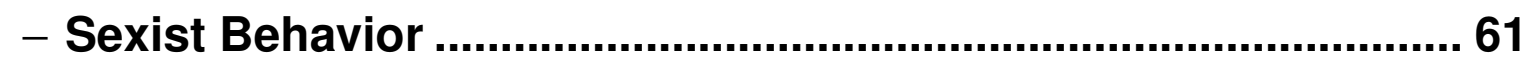

- Organizational Affiliation of Offender ..................................... 64

- Discussing/Reporting Experiences............................................6 67

- Response to Reporting.......................................................... 70

- Reasons for Not Reporting ................................................. 72

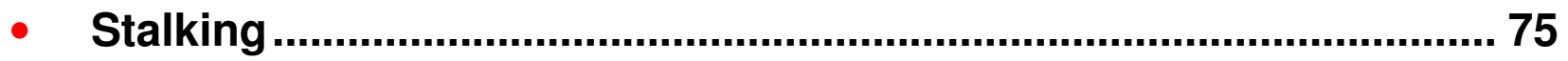

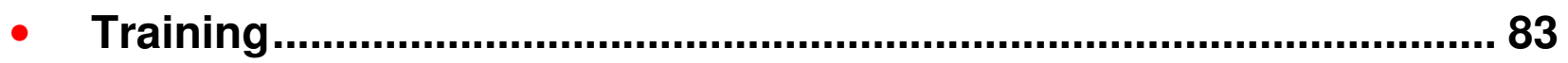

- Student Perceptions ...................................................................... 93 


\section{Unwanted Gender-Related Behaviors:}

\section{Sexual Harassment and Sexist Behavior Incident Rates}

\section{- Definition and measure of sexual harassment and sexist behavior:}

- DoD defines sexual harassment as "a form of sex discrimination that involves unwelcome sexual advances, requests for sexual favors, and other verbal or physical conduct of a sexual nature when:

- Submission to such conduct is made either explicitly or implicitly a term or condition of a person's job, pay, or career, or

- Submission to or rejection of such conduct by a person is used as a basis for career or employment decisions affecting that person, or

- Such conduct has the purpose or effect of unreasonably interfering with an individual's work performance or creates an intimidating, hostile, or offensive working environment" (Department of Defense, 1995).

- The core measure of unwanted gender-related behaviors on the 2012 SAGR consists of 17 items

- Sexual harassment measure has 12 items in a three-factor structure and a single "labeling" item

- Crude/Offensive Behavior - four items regarding offensive or embarrassing verbal/nonverbal behaviors of a sexual nature

- Unwanted Sexual Attention - four items regarding unwanted attempts to establish a sexual relationship

- Sexual Coercion - four items regarding classic quid pro quo instances of special treatment or favoritism conditioned on sexual cooperation

- One "labeling" item that asks if the student considers any of the selected behaviors to be sexual harassment

- Sexist behavior measure has four items that include verbal/nonverbal behaviors that convey insulting, offensive, and/or condescending attitudes based on gender of student

- Write-in comments analyzed separately

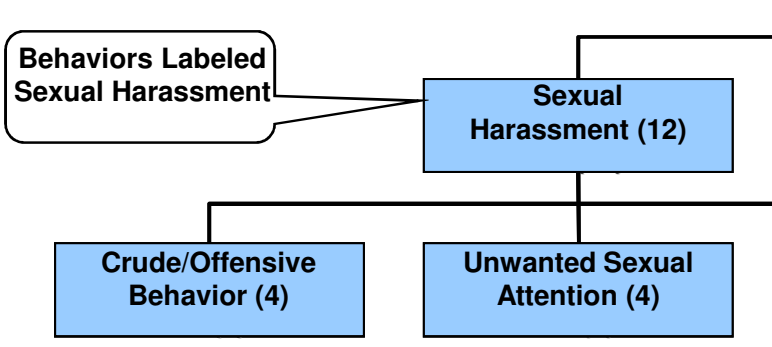

Write-in Comment (1)

Sexual Coercion (4) 


\section{Contents}

- Introduction 3

- Unwanted Sexual Contact ................................................................... 7

- Unwanted Gender-Related Behaviors .............................................. 49

$\checkmark$ Sexual Harassment ................................................................. 51

- Categories of Behaviors Associated with Sexual Harassment.................................................................................... 55

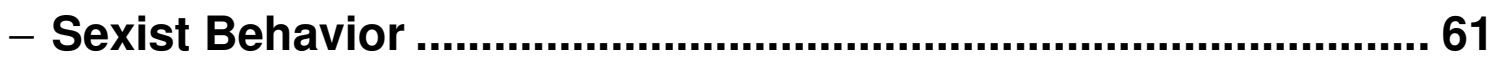

- Organizational Affiliation of Offender ........................................ 64

- Discussing/Reporting Experiences............................................. 67

- Response to Reporting.............................................................. 70

- Reasons for Not Reporting ............................................................. 72

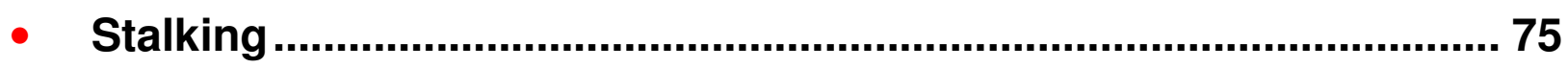

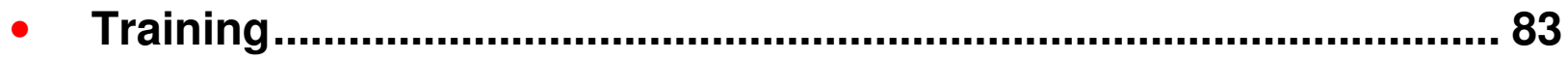

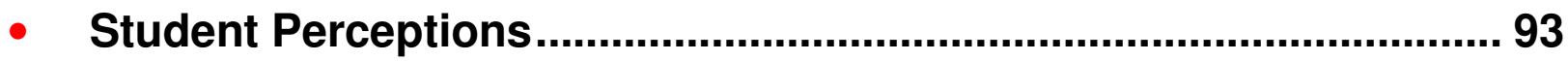




\section{Sexual Harassment Incident Rate Percent of Women}

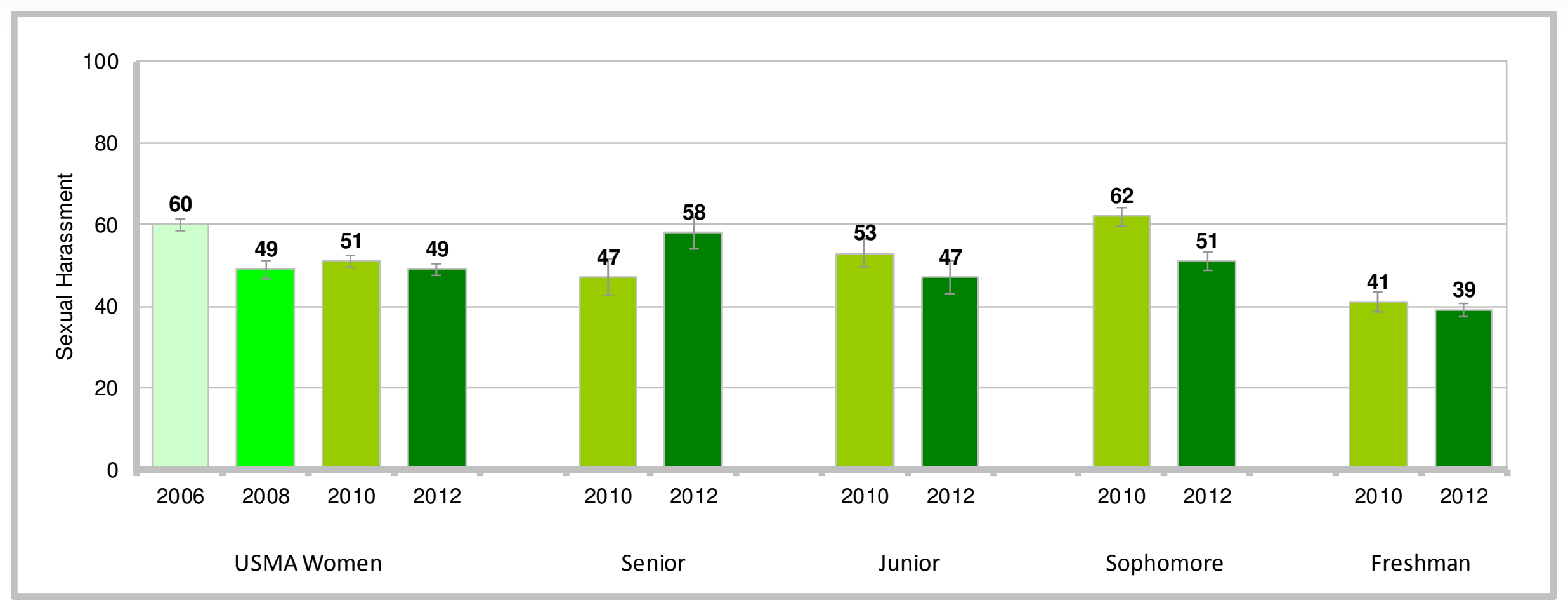

- $49 \%$ of women indicated experiencing sexual harassment in 2012

- 2012 comparisons across years - lower than 2006

- Seniors in 2012 higher than 2010, whereas juniors and sophomores lower

- Class differences in 2012 - higher response led by seniors; lower response led by freshmen 


\section{Sexual Harassment Incident Rate}

Percent of Men

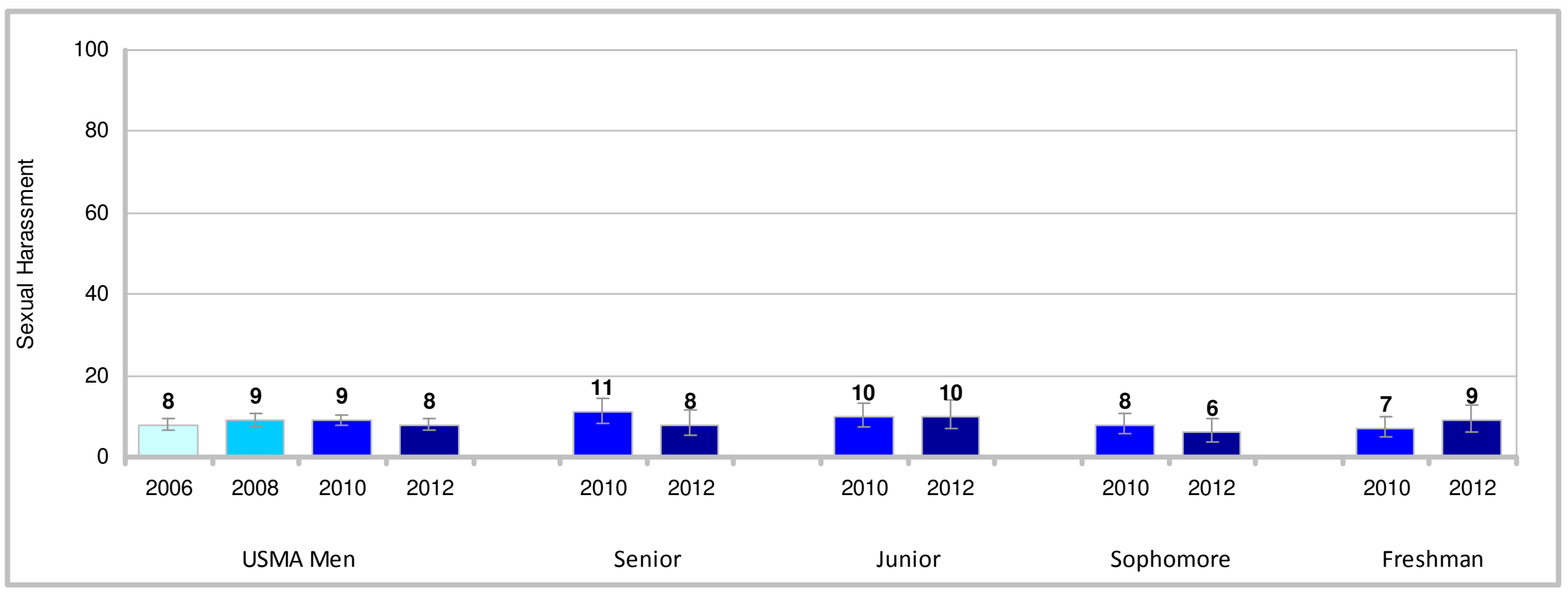

- 8\% of men indicated experiencing sexual harassment in 2012

- No differences by survey year or class year 


\section{Contents}

Slide

- Introduction ................................................................................. 3

- Unwanted Sexual Contact ........................................................... 7

- Unwanted Gender-Related Behaviors ......................................... 49

- Sexual Harassment .................................................................. 51

$\checkmark$ Categories of Behaviors Associated with Sexual Harassment.................................................................................. 54

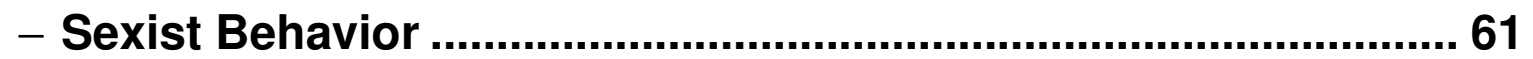

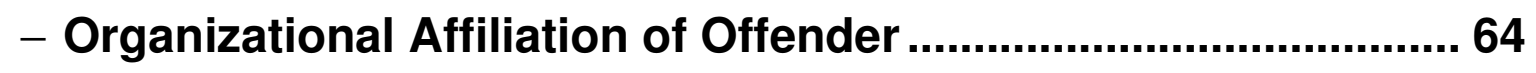

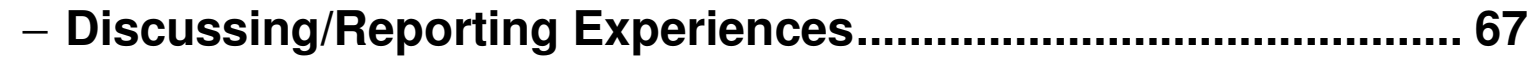

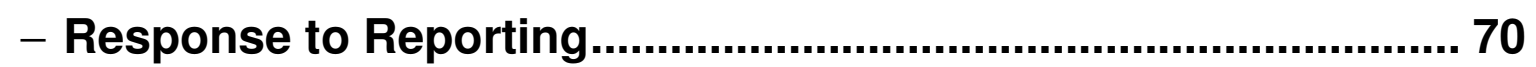

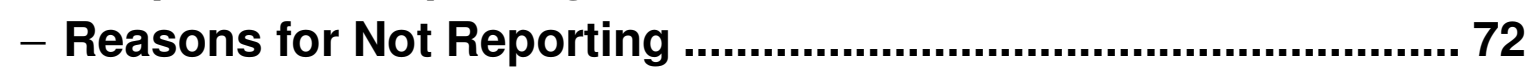

• Stalking .......................................................................................... 75

• Training ........................................................................................... 83

- Student Perceptions.................................................................. 93 


\section{Crude/Offensive Behavior Incident Rate Percent of Women}

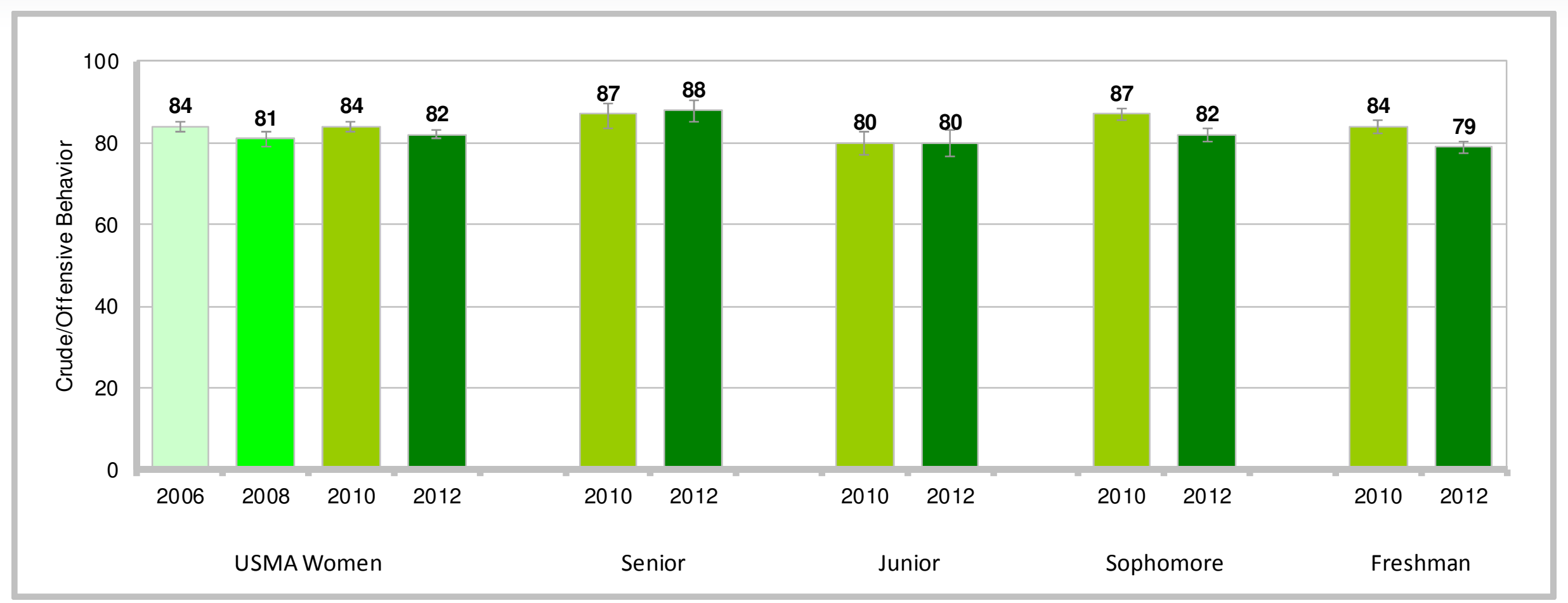

- $82 \%$ of women indicated experiencing crude/offensive behavior in 2012

- 2012 comparisons across years - lower than 2010, 2006

- Sophomores and freshmen in 2012 lower than 2010

- Class differences in $\mathbf{2 0 1 2}$ - higher response led by seniors; lower response led by freshmen 


\section{Crude/Offensive Behavior Incident Rate} Percent of Men

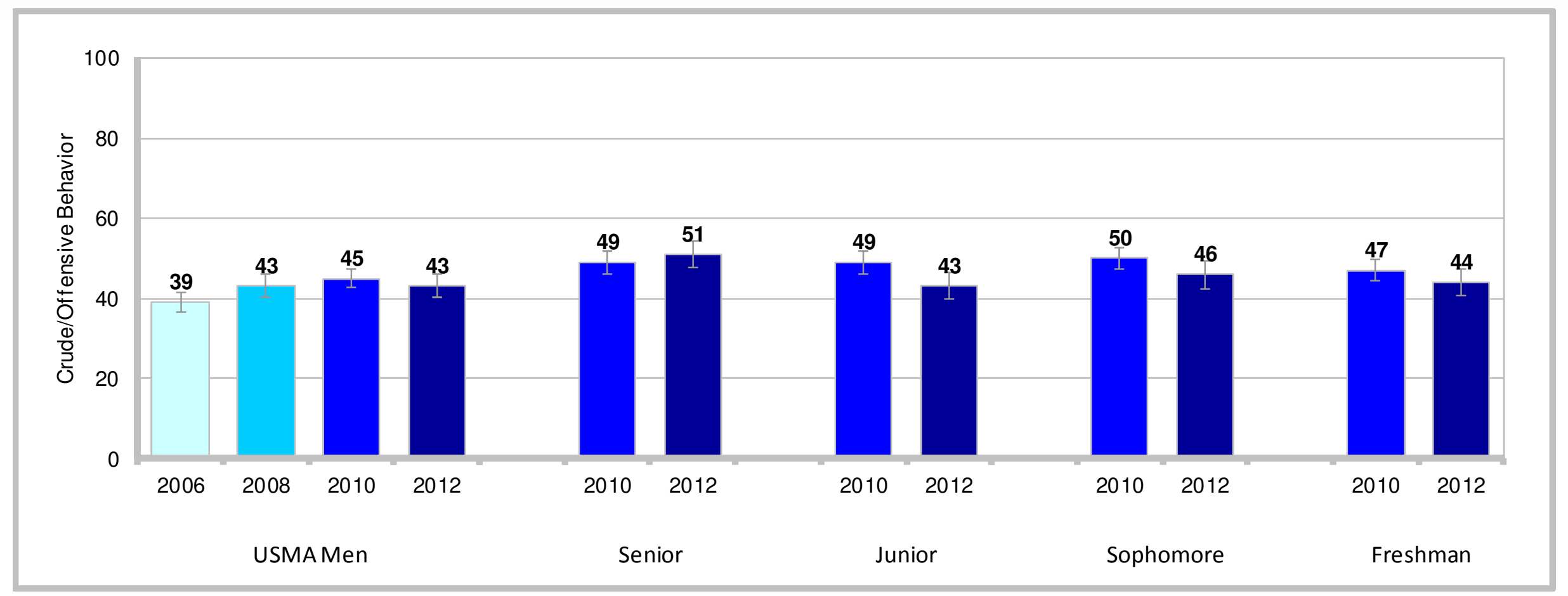

- $43 \%$ of men indicated experiencing crude/offensive behavior in 2012

- 2012 comparisons across years - higher than 2006

- Class differences in 2012 - higher response led by seniors 


\section{Unwanted Sexual Attention Incident Rate Percent of Women}

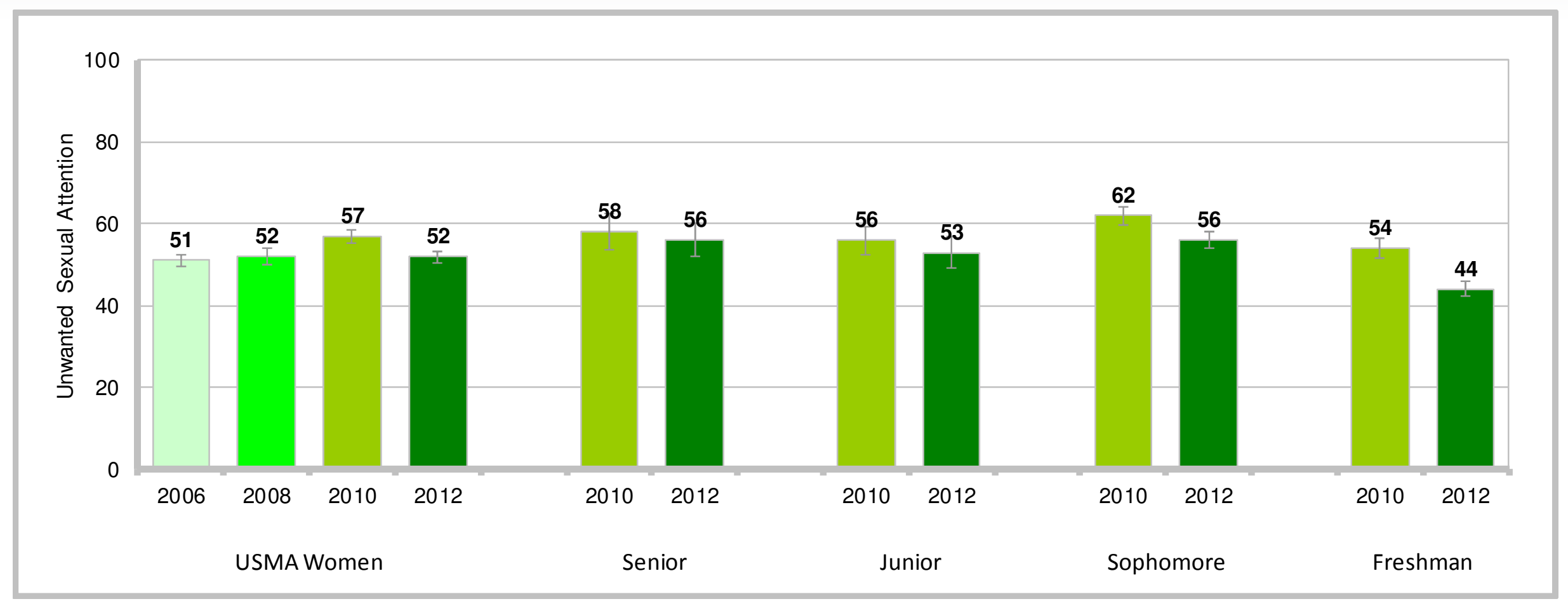

- $52 \%$ of women indicated experiencing unwanted sexual attention in 2012

- 2012 comparisons across years - lower than 2010

- Sophomores and freshmen in 2012 lower than 2010

- Class differences in 2012 - higher response led by sophomores*; lower response led by freshmen

*Note that $56 \%$ of senior women also indicated experiencing unwanted sexual attention. This percentage is not significantly higher than the average of percentages in the other class years due to a higher margin of error for senior women responding to this question. 


\section{Unwanted Sexual Attention Incident Rate Percent of Men}

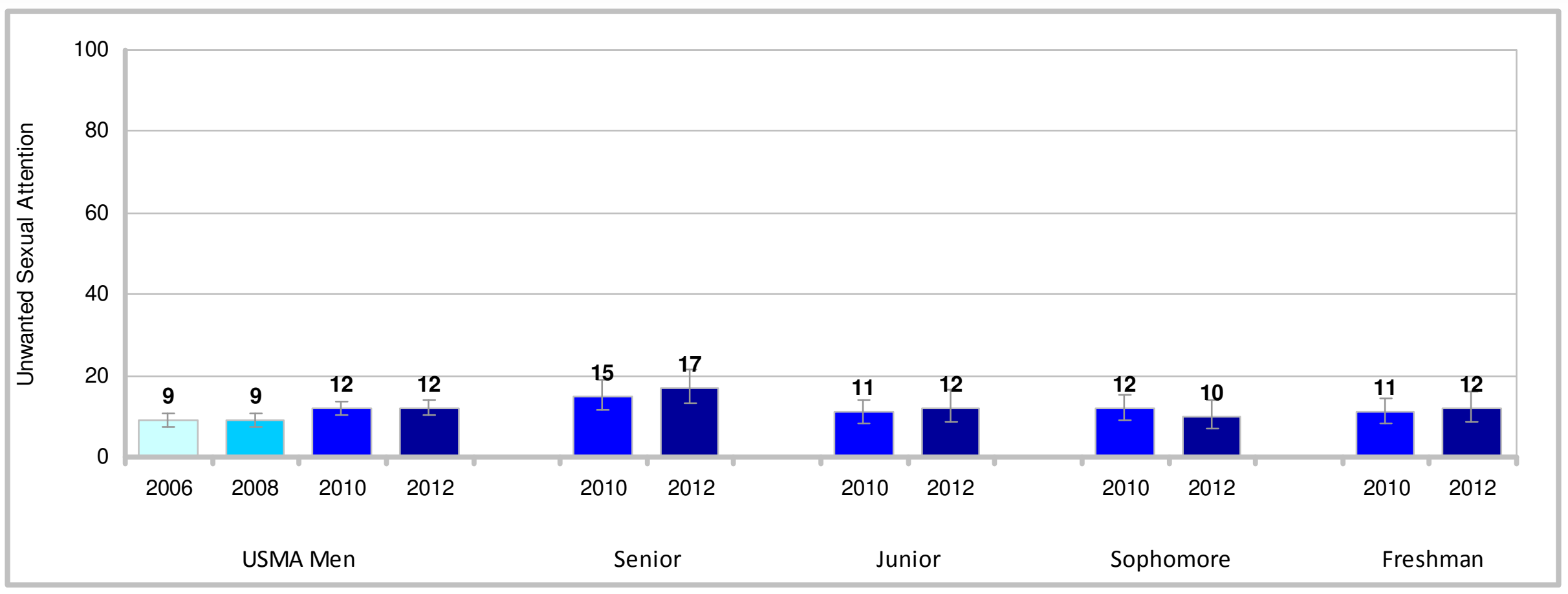

- $12 \%$ of men indicated experiencing unwanted sexual attention in 2012

- 2012 comparisons across years - higher than 2008, 2006

- Class differences in 2012 - no differences 


\section{Sexual Coercion Incident Rate}

Percent of Women

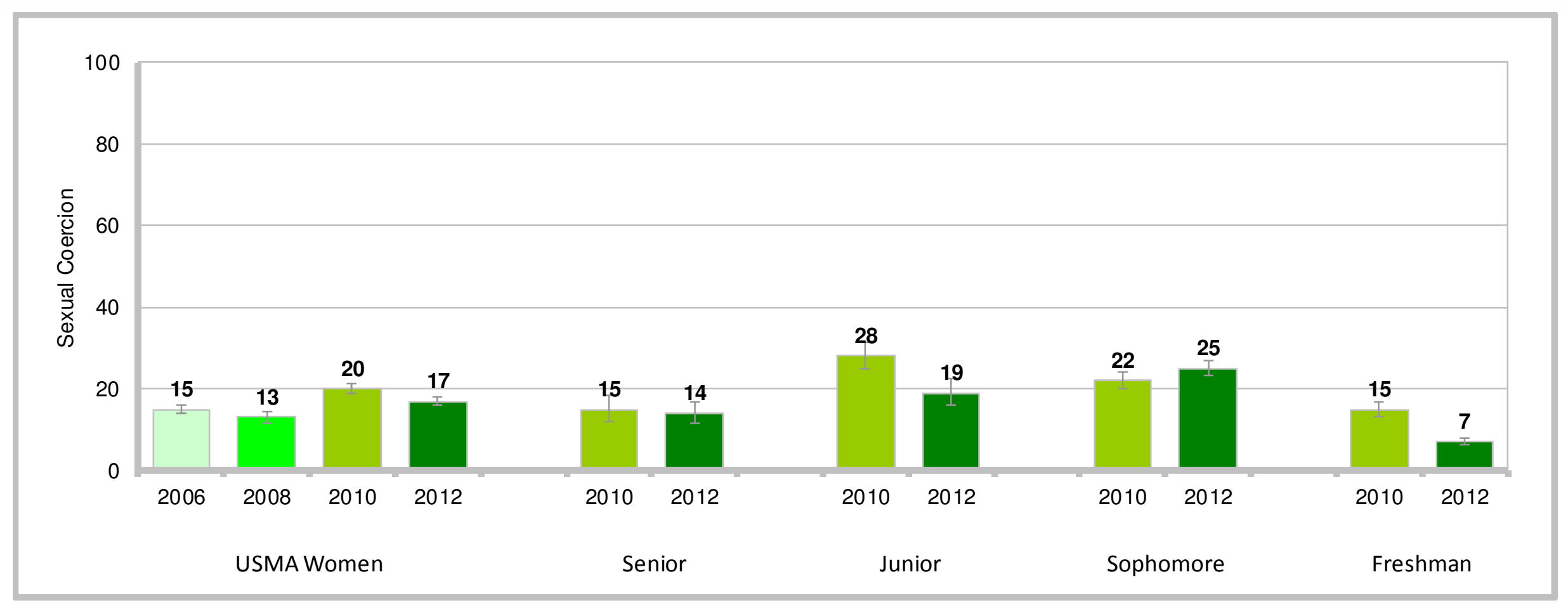

- $17 \%$ of women indicated experiencing sexual coercion in 2012

- 2012 comparisons across years - lower than 2010; higher than 2008, 2006

- Juniors and freshmen in 2012 lower than 2010, whereas sophomores higher

- Class differences in $\mathbf{2 0 1 2}$ - higher response led by sophomores; lower response led by freshmen 


\section{Sexual Coercion Incident Rate}

Percent of Men

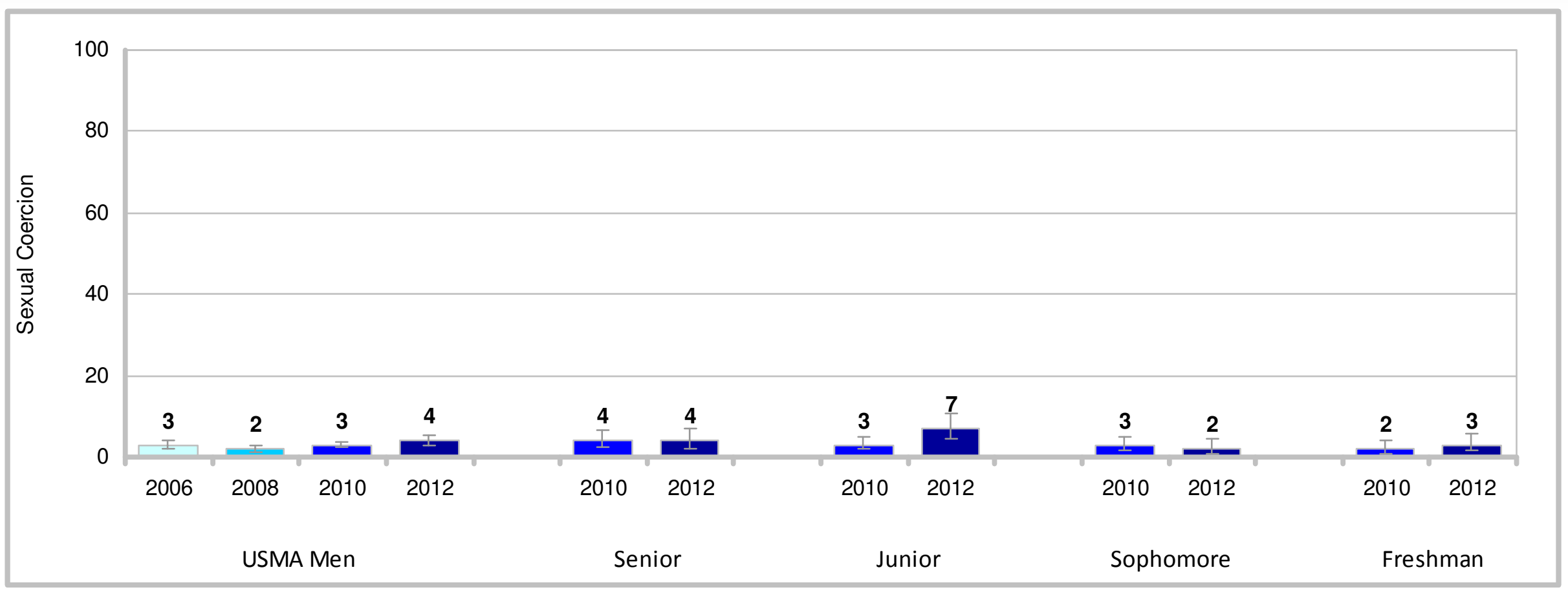

- $4 \%$ of men indicated experiencing sexual coercion in 2012

- 2012 comparisons across years - higher than 2008

- Juniors in 2012 higher than 2010

- Class differences in 2012 - no differences 


\section{Contents}

- Introduction 3

- Unwanted Sexual Contact ................................................................... 7

- Unwanted Gender-Related Behaviors ............................................... 49

- Sexual Harassment ...................................................................... 51

- Categories of Behaviors Associated with Sexual

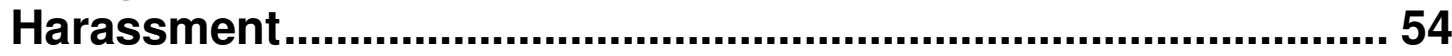

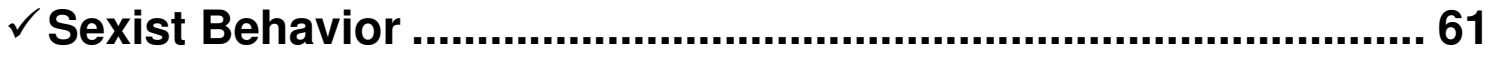

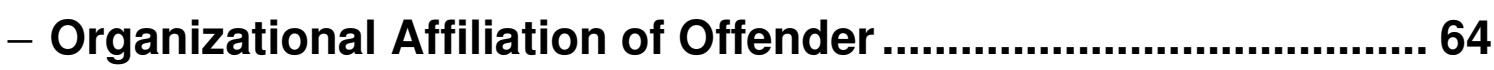

- Discussing/Reporting Experiences............................................. 67

- Response to Reporting.............................................................. 70

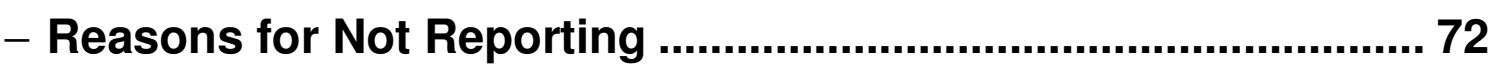

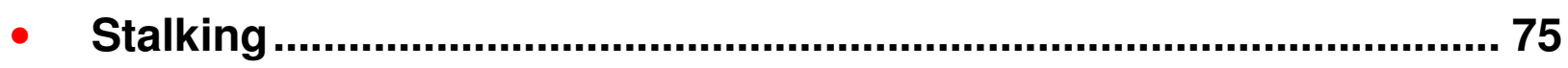

- Training................................................................................................... 83

- Student Perceptions............................................................................ 93 


\section{Sexist Behavior Incident Rate Percent of Women}

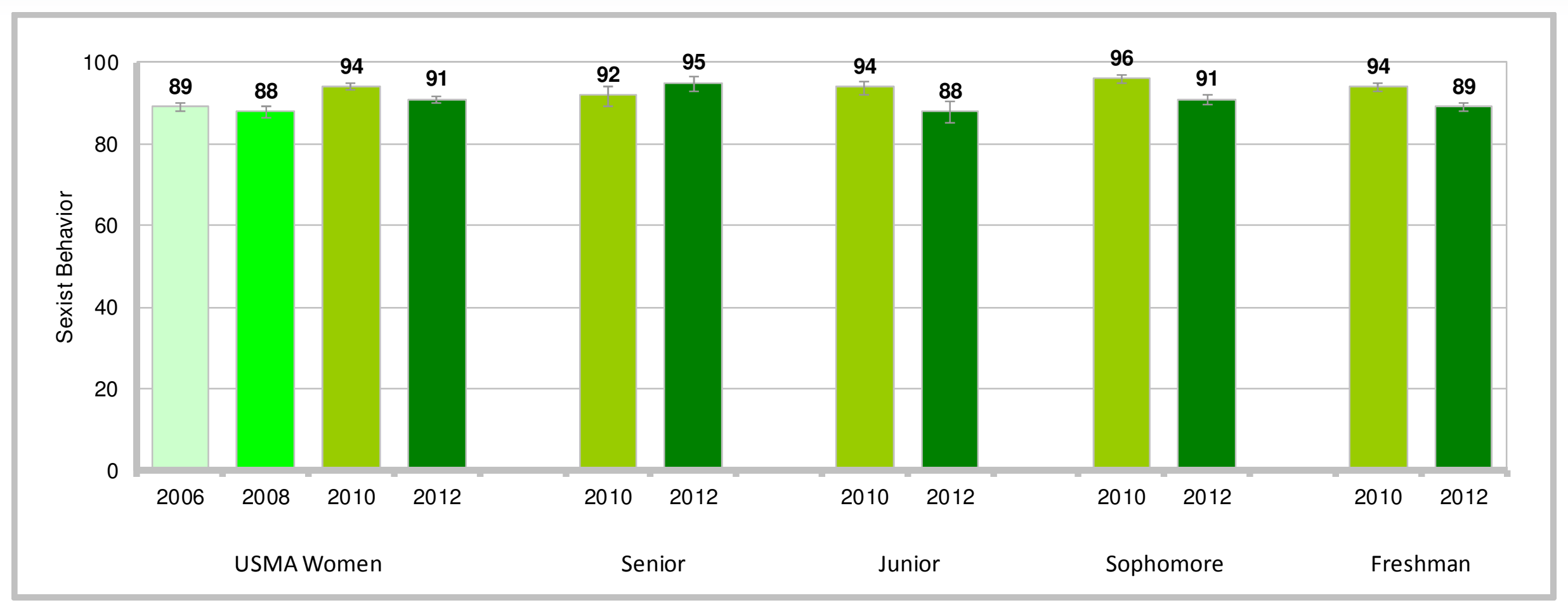

- $91 \%$ of women indicated experiencing sexist behavior in 2012

- 2012 comparisons across years - lower than 2010; higher than 2008, 2006

- Seniors in 2012 higher than 2010, whereas juniors, sophomores, and freshmen lower

- Class differences in $\mathbf{2 0 1 2}$ - higher response led by seniors; lower response led by juniors and freshmen 


\section{Sexist Behavior Incident Rate}

Percent of Men

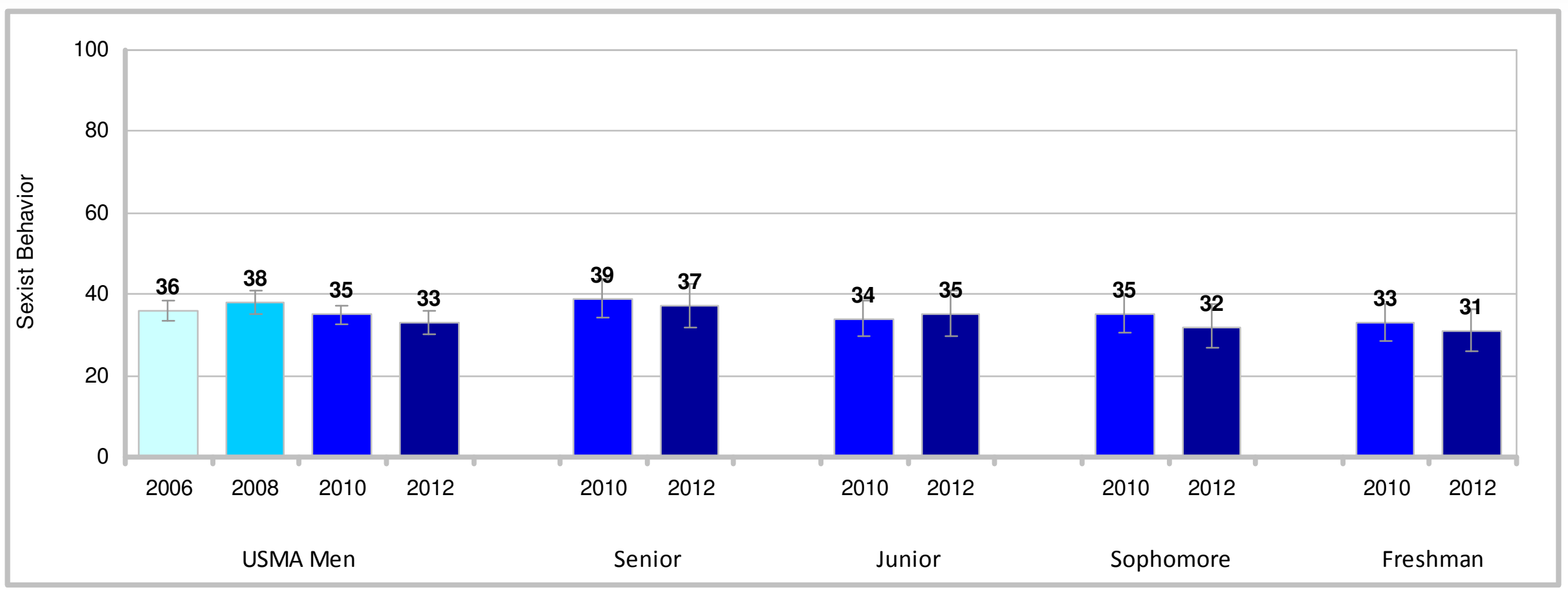

- 33\% of men indicated experiencing sexist behavior in 2012

- 2012 comparisons across years - lower than 2008

- Class differences in 2012 - no differences 


\section{Contents}

- Introduction 3

- Unwanted Sexual Contact ................................................................... 7

- Unwanted Gender-Related Behaviors ............................................... 49

- Sexual Harassment ...................................................................... 51

- Categories of Behaviors Associated with Sexual Harassment.................................................................................... 54

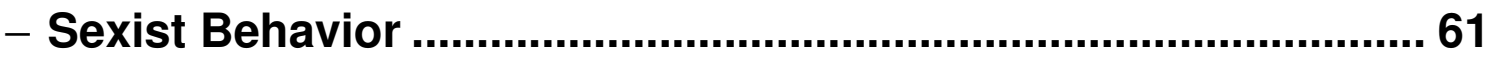

$\checkmark$ Organizational Affiliation of Offender .......................................64 64

- Discussing/Reporting Experiences............................................. 67

- Response to Reporting.............................................................. 70

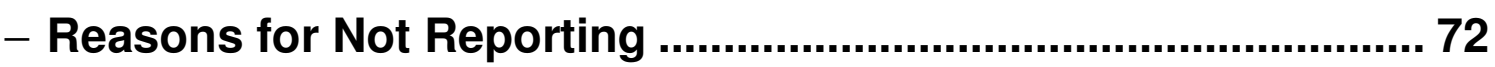

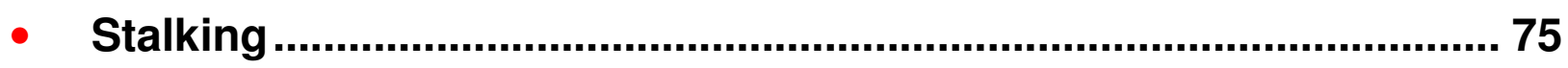

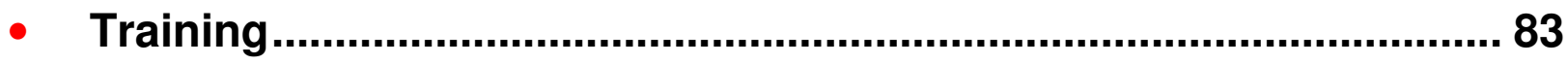

- Student Perceptions............................................................................ 93 


\section{Combinations of Offender Affiliations}

\section{Percent of Women Who Experienced Unwanted Gender-Related Behavior}

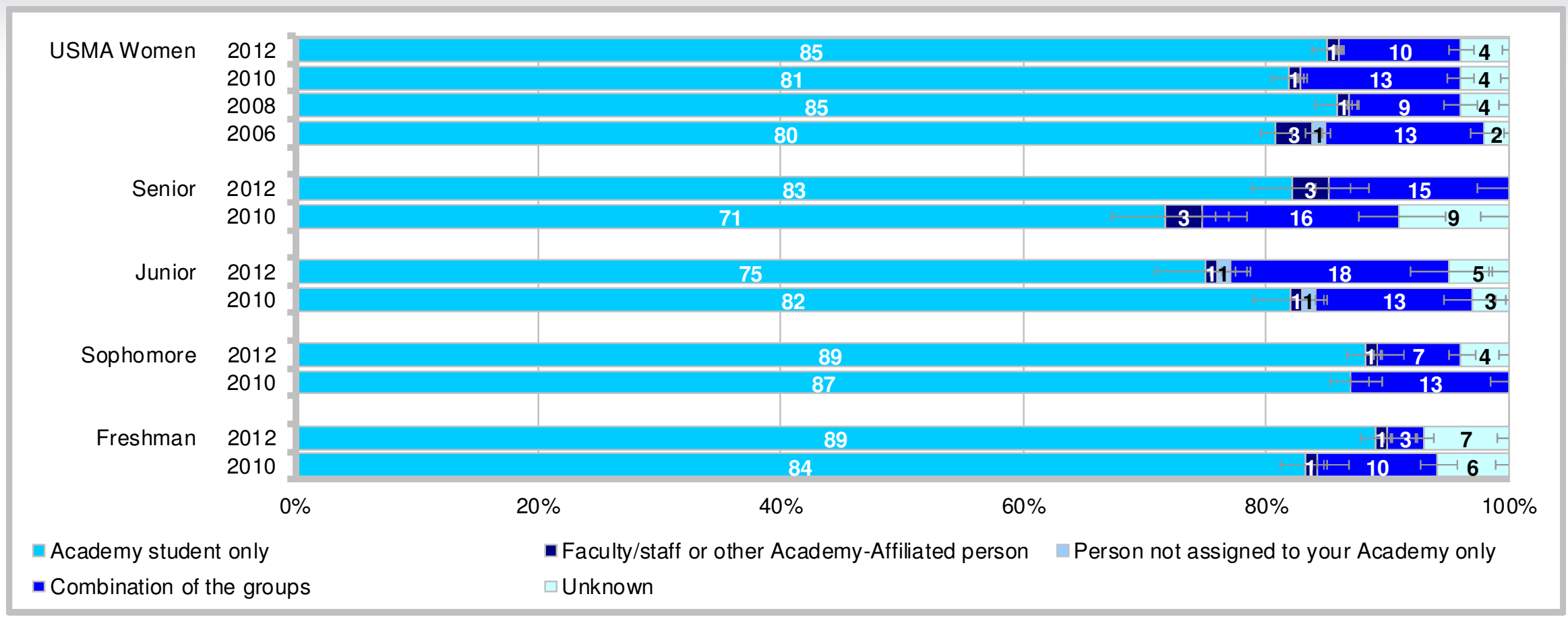

- $93 \%$ of women indicated experiencing one or more of the unwanted gender-related behaviors (i.e., crude/offensive behavior, unwanted sexual attention, sexual coercion, sexist behavior). Of those, $85 \%$ indicated the offender was an Academy student only; $1 \%$ indicated faculty/staff; $0 \%$ indicated person not assigned to the Academy; $10 \%$ indicated combination of groups; and $4 \%$ indicated unknown.

- 2012 comparisons across years - Academy student only higher than 2010, 2006; faculty/staff lower than 2006; combination of groups lower than 2010, 2006; unknown higher than 2006

- Seniors and freshmen indicating Academy student only in 2012 higher than 2010, whereas juniors lower; juniors indicating combination of groups in 2012 higher than 2010, whereas sophomores and freshmen lower; sophomores indicating unknown in 2012 higher than 2010, whereas seniors lower

- Class differences in 2012 - Academy student only led by freshmen and sophomores; faculty/staff led by seniors; combination of groups led by juniors and seniors; unknown led by freshmen 


\section{Combinations of Offender Affiliations}

Percent of Men Who Experienced Unwanted Gender-Related Behavior

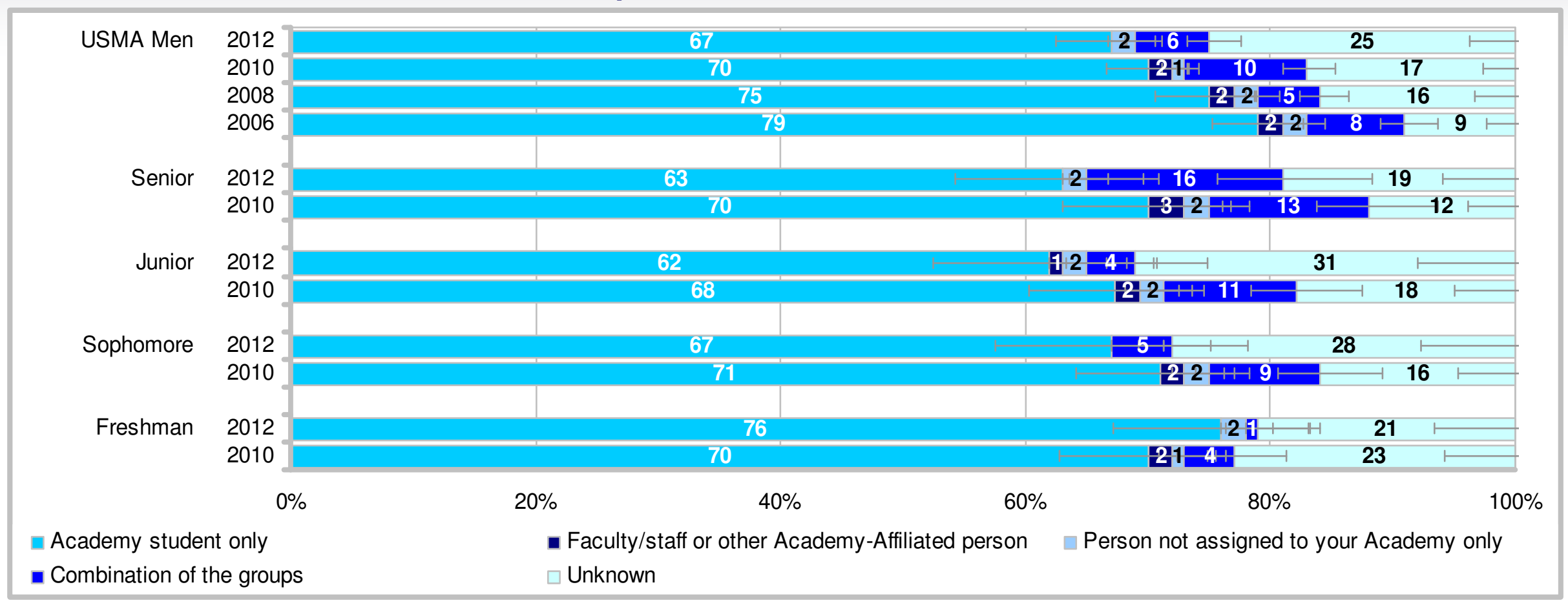

- $51 \%$ of men indicated experiencing one or more of the unwanted gender-related behaviors (i.e., crude/offensive behavior, unwanted sexual attention, sexual coercion, sexist behavior). Of those, $67 \%$ indicated the offender was Academy student only; $0 \%$ indicated faculty/staff; $2 \%$ indicated person not assigned to the Academy; $6 \%$ indicated combination of groups; and $25 \%$ indicated unknown.

- 2012 comparisons across years - Academy student only lower than 2008, 2006; faculty/stafflower than 2010, 2008, 2006; combination of groups lower than 2010; unknown higher than 2010, 2008, 2006

- Seniors, sophomores, and freshmen indicating faculty/staff in 2012 lower than 2010; sophomores indicating person not assigned to the Academy in 2012 lower than 2010; juniors indicating combination of groups in 2012 lower than 2010; juniors and sophomores indicating unknown in 2012 higher than 2010

- Class differences in 2012 - combination of groups led by seniors SAGR 2012 Q15 


\section{Contents}

- Introduction 3

- Unwanted Sexual Contact ................................................................... 7

- Unwanted Gender-Related Behaviors ............................................... 49

- Sexual Harassment ...................................................................... 51

- Categories of Behaviors Associated with Sexual Harassment...................................................................................... 54

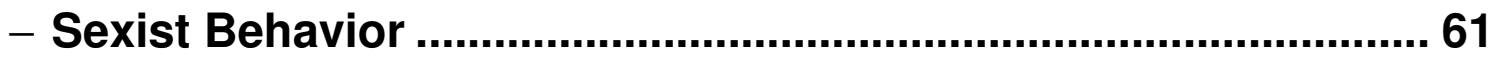

- Organizational Affiliation of Offender .........................................64 64

$\checkmark$ Discussing/Reporting Experiences...........................................6 67

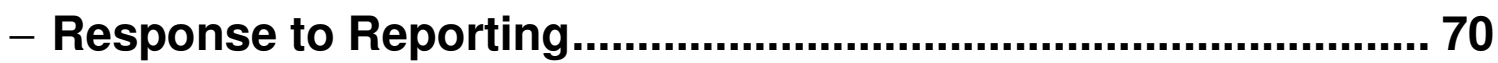

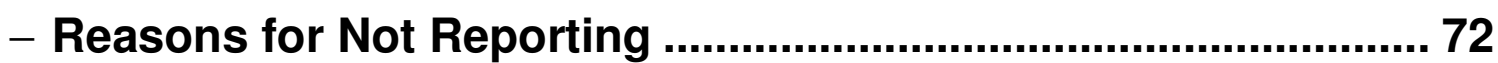

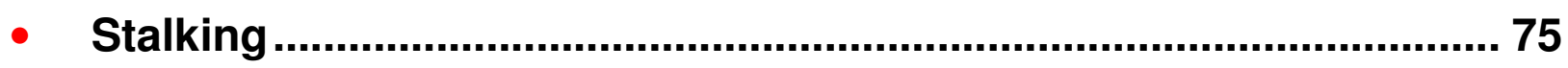

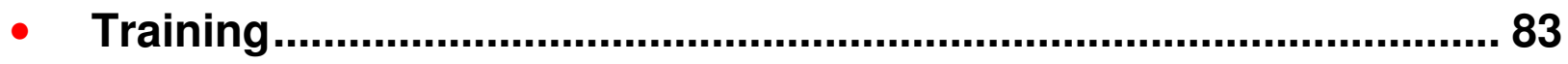

- Student Perceptions............................................................................ 93 


\section{Discuss/Report This Situation to an Authority or Organization}

Percent of Women Who Experienced Unwanted Gender-Related Behavior

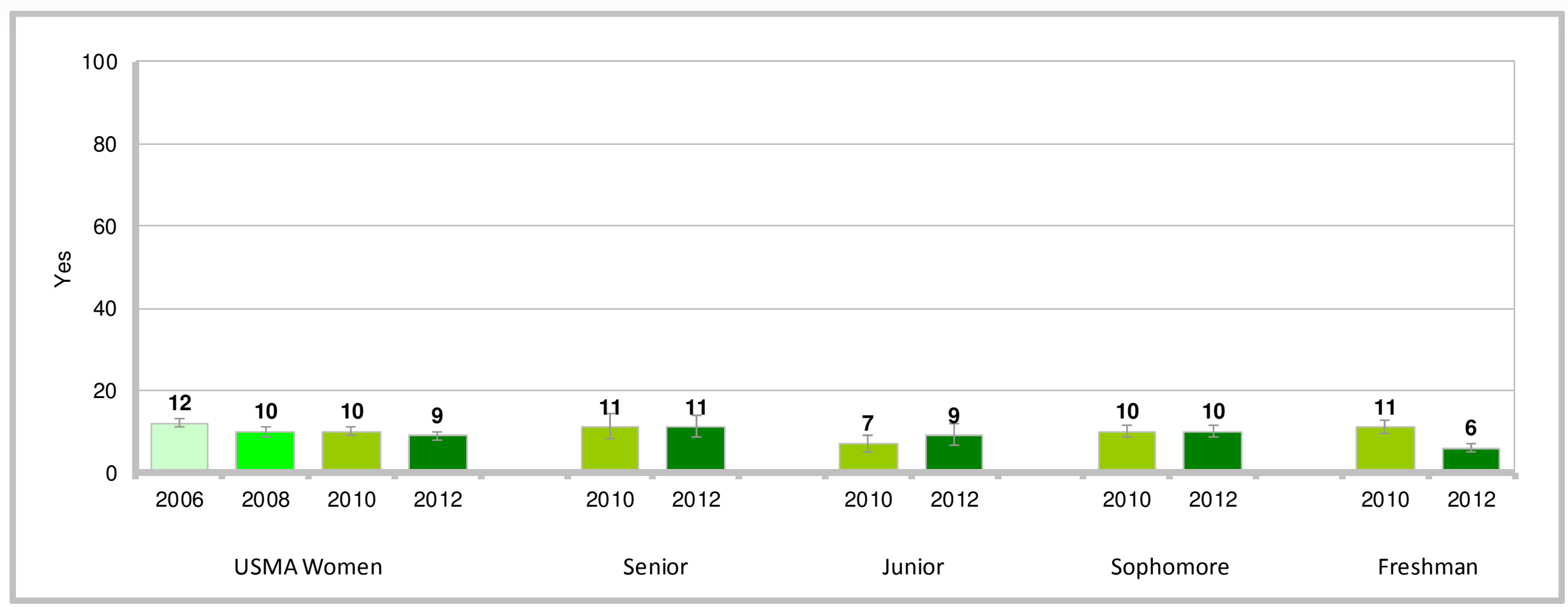

- $93 \%$ of women indicated experiencing one or more of the unwanted gender-related behaviors (i.e., crude/offensive behavior, unwanted sexual attention, sexual coercion, sexist behavior). Of those, $9 \%$ indicated they reported the situation to an authority or organization.

- 2012 comparisons across years - lower than 2006

- Freshmen in 2012 lower than 2010

- Class differences in 2012 - lower response led by freshmen 


\section{Discuss/Report This Situation to an Authority or Organization \\ Percent of Men Who Experienced Unwanted Gender-Related Behavior}

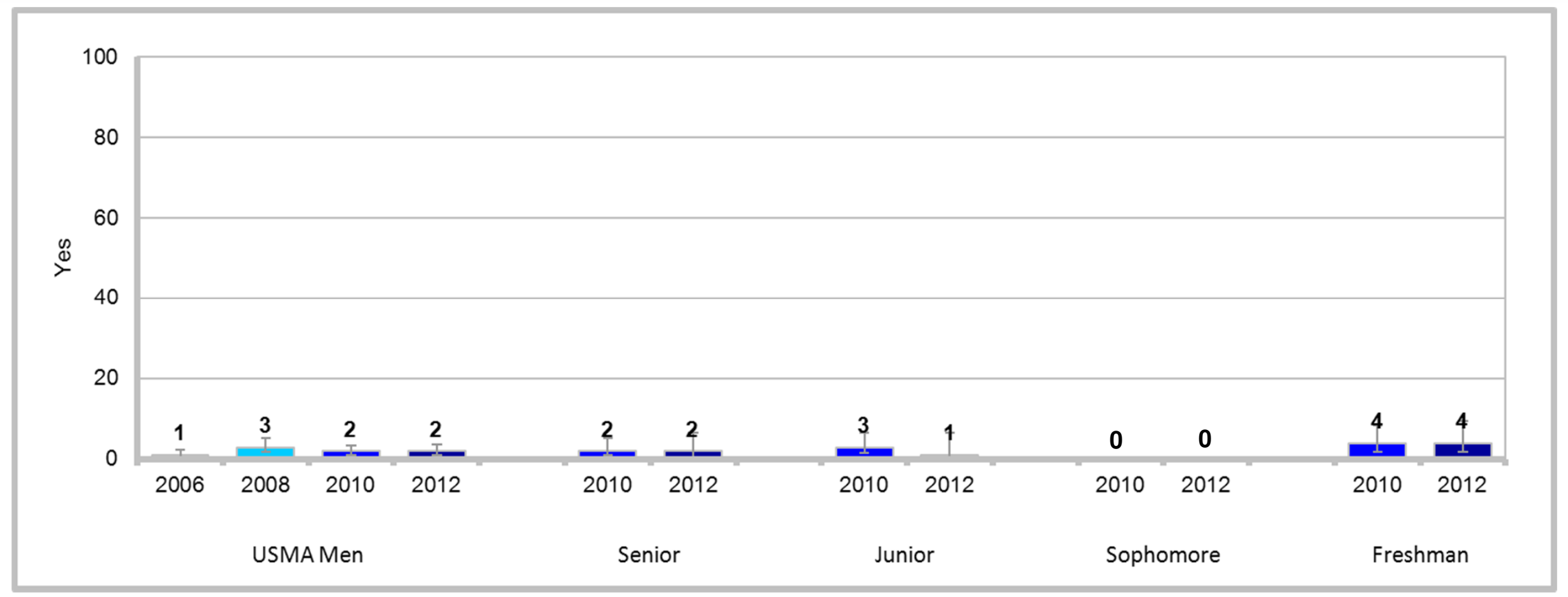

- $51 \%$ of men indicated experiencing one or more of the unwanted gender-related behaviors (i.e., crude/offensive behavior, unwanted sexual attention, sexual coercion, sexist behavior). Of those, $2 \%$ indicated they reported the situation to an authority or organization.

- 2012 comparisons across years - no differences

- Class differences in 2012 - no differences 


\section{Contents}

- Introduction ..................................................................................... 3

- Unwanted Sexual Contact ...................................................................... 7

- Unwanted Gender-Related Behaviors .............................................. 49

- Sexual Harassment ..................................................................... 51

- Categories of Behaviors Associated with Sexual Harassment........................................................................................ 54

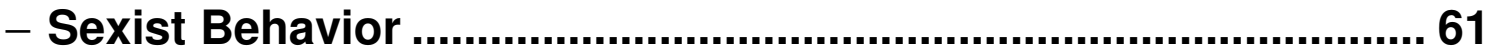

- Organizational Affiliation of Offender ...........................................64

- Discussing/Reporting Experiences...............................................67 67

$\checkmark$ Response to Reporting................................................................ 70

- Reasons for Not Reporting ........................................................... 72

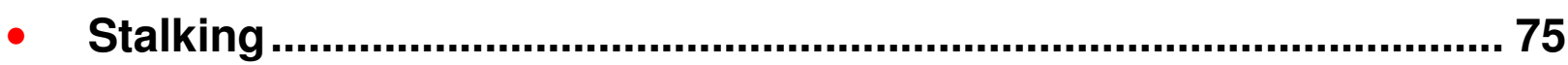

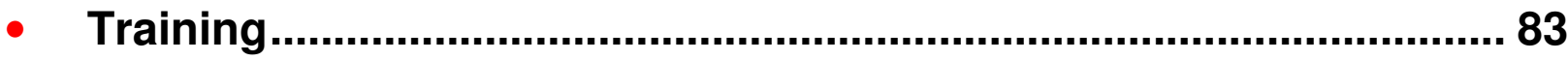

- Student Perceptions.......................................................................... 93 


\section{Actions Taken in Response to Discussing/Reporting}

Percent of Women Who Experienced Unwanted Gender-Related Behaviors and Reported It

\begin{tabular}{|c|c|c|c|c|c|c|}
\hline Response to Reporting & $\begin{array}{c}\text { Survey } \\
\text { Year }\end{array}$ & Total & Senior & Junior & Sophomore & Freshman \\
\hline \multirow{2}{*}{ The situation was corrected } & 2012 & $45 \downarrow$ & $33 \downarrow$ & 37 & $44 \downarrow$ & $78 \uparrow$ \\
\hline & 2010 & 63 & 64 & 56 & 64 & 67 \\
\hline \multirow{2}{*}{ My situation was/is being investigated } & 2012 & 35 & 42 & $25 \downarrow$ & 44 & 22 \\
\hline & 2010 & 39 & 45 & 56 & 36 & 27 \\
\hline \multirow{2}{*}{$\begin{array}{l}\text { I was kept informed of what actions were } \\
\text { being taken }\end{array}$} & 2012 & $31 \downarrow$ & $33 \downarrow$ & 38 & $25 \downarrow$ & $33 \downarrow$ \\
\hline & 2010 & 49 & 55 & 56 & 43 & 47 \\
\hline \multirow{2}{*}{ I was encouraged to "tough it out" } & 2012 & 33 & $42 \uparrow$ & 25 & 31 & $22 \downarrow$ \\
\hline & 2010 & 32 & 18 & 33 & 36 & 40 \\
\hline \multirow{2}{*}{$\begin{array}{l}\text { My situation was discounted or not taken } \\
\text { seriously }\end{array}$} & 2012 & 33 & 50 & 25 & 37 & $11 \downarrow$ \\
\hline & 2010 & 35 & 36 & 33 & 43 & 27 \\
\hline \multirow{2}{*}{ Action was taken against me } & 2012 & 7 & 17 & 13 & $0 \downarrow$ & 0 \\
\hline & 2010 & 6 & NR & 11 & 14 & 0 \\
\hline \multirow{2}{*}{$\begin{array}{l}\text { I was ridiculed or scorned by others for } \\
\text { discussing/reporting the situation }\end{array}$} & 2012 & $37 \uparrow$ & $50 \uparrow$ & 25 & 38 & $33 \uparrow$ \\
\hline & 2010 & 24 & 27 & 22 & 43 & 7 \\
\hline \multirow{2}{*}{ Some other action was taken } & 2012 & 33 & 33 & 37 & $37 \uparrow$ & $22 \downarrow$ \\
\hline & 2010 & 29 & 36 & 22 & 21 & 33 \\
\hline Margins of Error & & $\pm 3-6 \%$ & $\pm 13-15 \%$ & $\pm 12-16 \%$ & $\pm 0-8 \%$ & $\pm 0-8 \%$ \\
\hline
\end{tabular}

\section{- Results for men are not reportable}

\begin{tabular}{|l|c|}
\hline Higher Response of Yes & \\
\hline Lower Response of Yes & \\
\hline Higher in 2012 than 2010 & $\uparrow$ \\
\hline Lower in 2012 than 2010 & $\downarrow$ \\
\hline
\end{tabular}




\section{Contents}

- Introduction 3

- Unwanted Sexual Contact ................................................................... 7

- Unwanted Gender-Related Behaviors ............................................... 49

- Sexual Harassment .................................................................. 51

- Categories of Behaviors Associated with Sexual Harassment...................................................................................... 54

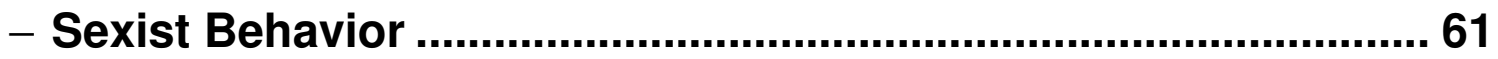

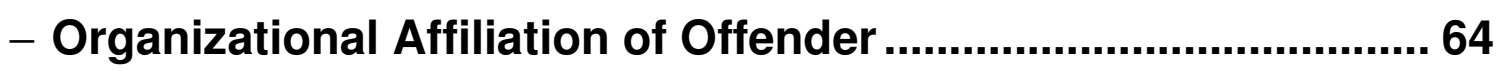

- Discussing/Reporting Experiences............................................. 67

- Response to Reporting.................................................................... 70

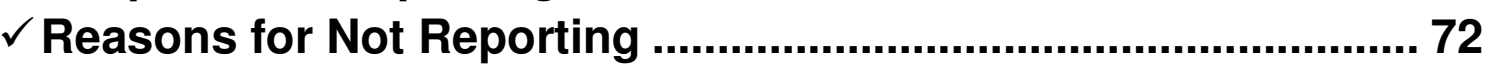

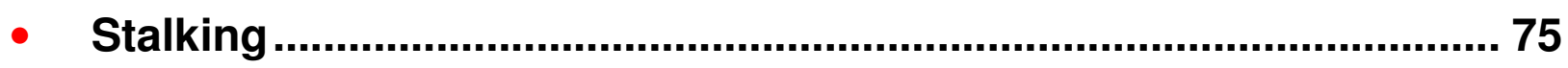

- Training................................................................................................... 83

- Student Perceptions............................................................................ 93 


\section{Most Frequently Selected Reasons for}

\section{Not Reporting Unwanted Gender-Related Situation}

Percent of Women Who Experienced Unwanted Gender-Related Behaviors and Did Not Report It

\begin{tabular}{|c|c|c|c|c|c|c|}
\hline Reasons for Not Reporting & $\begin{array}{l}\text { Survey } \\
\text { Year }\end{array}$ & Total & Senior & Junior & Sophomore & Freshman \\
\hline \multirow{2}{*}{$\begin{array}{l}\text { Thought it was not important enough to } \\
\text { report }\end{array}$} & 2012 & 84 & 83 & $79 \downarrow$ & $83 \downarrow$ & $90 \uparrow$ \\
\hline & 2010 & 85 & 81 & 84 & 91 & 85 \\
\hline \multirow{2}{*}{ Took care of the problem myself } & 2012 & $71 \uparrow$ & 69 & 76 & $71 \uparrow$ & $68 \uparrow$ \\
\hline & 2010 & 68 & 73 & 71 & 65 & 62 \\
\hline \multirow{2}{*}{ Did not want people gossiping about me } & 2012 & 38 & $30 \downarrow$ & 34 & $46 \downarrow$ & $41 \uparrow$ \\
\hline & 2010 & 40 & 37 & 38 & 54 & 31 \\
\hline \multirow{2}{*}{$\begin{array}{l}\text { Thought it would hurt my reputation and } \\
\text { standing }\end{array}$} & 2012 & 30 & 25 & $24 \downarrow$ & 40 & 28 \\
\hline & 2010 & 31 & 24 & 31 & 41 & 28 \\
\hline \multirow{2}{*}{$\begin{array}{l}\text { Thought reporting would take too much } \\
\text { time and effort }\end{array}$} & 2012 & 30 & 27 & 26 & 37 & $27 \uparrow$ \\
\hline & 2010 & 30 & 31 & 31 & 36 & 22 \\
\hline Margins of Error & & $\pm 2 \%$ & $\pm 4-6 \%$ & $\pm 4-5 \%$ & $\pm 2-3 \%$ & $\pm 2-3 \%$ \\
\hline
\end{tabular}

\begin{tabular}{|l|c|}
\hline Higher Response of Yes & \\
\hline Lower Response of Yes & \\
\hline Higher in 2012 than 2010 & $\uparrow$ \\
\hline Lower in 2012 than 2010 & $\downarrow$ \\
\hline
\end{tabular}




\section{Most Frequently Selected Reasons for Not Reporting Unwanted Gender-Related Situation} Percent of Men Who Experienced Unwanted Gender-Related Behaviors and Did Not Report It

\begin{tabular}{|c|c|c|c|c|c|c|}
\hline Reasons for Not Reporting & $\begin{array}{l}\text { Survey } \\
\text { Year }\end{array}$ & Total & Senior & Junior & Sophomore & Freshman \\
\hline \multirow{2}{*}{$\begin{array}{l}\text { Thought it was not important enough to } \\
\text { report }\end{array}$} & 2012 & 80 & 79 & 72 & 82 & 87 \\
\hline & 2010 & 79 & 74 & 81 & 80 & 80 \\
\hline \multirow{2}{*}{ Took care of the problem myself } & 2012 & $60 \uparrow$ & $63 \uparrow$ & 61 & 63 & 51 \\
\hline & 2010 & 52 & 43 & 50 & 60 & 51 \\
\hline \multirow{2}{*}{$\begin{array}{l}\text { Thought reporting would take too much } \\
\text { time and effort }\end{array}$} & 2012 & 23 & 15 & 22 & 27 & 26 \\
\hline & 2010 & 21 & 21 & 23 & 20 & 21 \\
\hline \multirow{2}{*}{ Did not want people gossiping about me } & 2012 & 16 & $7 \downarrow$ & 16 & 16 & 25 \\
\hline & 2010 & 15 & 15 & 11 & 13 & 19 \\
\hline \multirow{2}{*}{ Did not think anything would be done } & 2012 & 14 & 14 & 6 & $23 \uparrow$ & 12 \\
\hline & 2010 & 14 & 16 & 9 & 11 & 20 \\
\hline Margins of Error & & $\pm 2-5 \%$ & $\pm 7-10 \%$ & $\pm 6-11 \%$ & $\pm 6-11 \%$ & $\pm 8-11 \%$ \\
\hline
\end{tabular}

\begin{tabular}{|l|c|}
\hline Higher Response of Yes & \\
\hline Lower Response of Yes & \\
\hline Higher in 2012 than 2010 & $\uparrow$ \\
\hline Lower in 2012 than 2010 & $\downarrow$ \\
\hline
\end{tabular}




\section{Contents}

- Unwanted Sexual Contact ................................................................. 7

- Unwanted Gender-Related Behaviors ................................................ 49

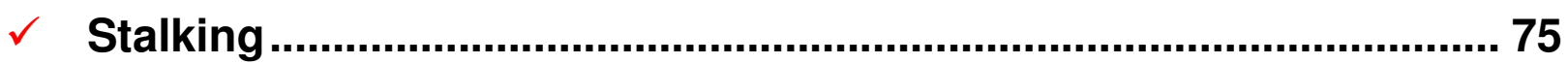

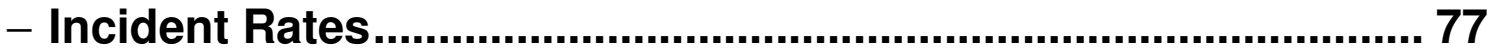

- Stalking and Sexual Harassment Related to Unwanted

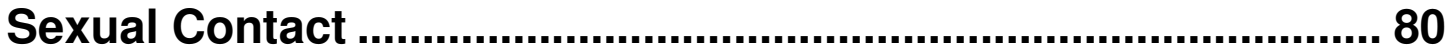

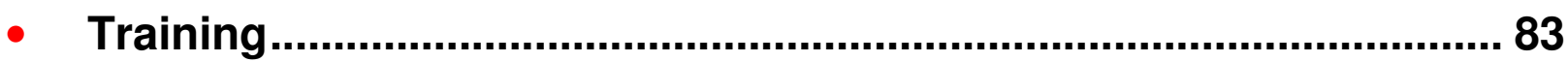

- Student Perceptions............................................................................... 93 


\section{Stalking Incident Rate}

\section{- Definition and measure of stalking:}

- Under Article 120a of the UCMJ, stalking is a crime. The UCMJ definition of stalking is "a course of conduct directed at a specific person that would cause a reasonable person to fear death or bodily harm, including sexual assault, to himself or herself or a member of his or her immediate family."

- Thus, to be punishable under the provision, stalking must be intentional, repeated (two or more occasions of such conduct), and cause unreasonable fear of physical injury. Note that this definition does not limit stalking to association with sexual harassment or sexual assault.

- The measure of stalking on the 2012 SAGR is consistent with the definition in Article 120a of the UCMJ. Students were asked (Q9) whether they had experienced any stalking behaviors (e.g., followed or spied on them in public areas; spied on them in private areas; showed up at places where they were even though he/she had no reason to be there; left unwanted items for them to find; stood outside or hung around their dorm room or classroom even though he/she had no reason to be there; and vandalized or tampered with their belongings) by someone assigned to their Academy, including students and military/civilian personnel, and whether they felt in danger of physical harm or sexual assault as a result of the experience (Q11). 


\section{Contents}

- Introduction 3

- Unwanted Sexual Contact ................................................................... 7

- Unwanted Gender-Related Behaviors ............................................... 49

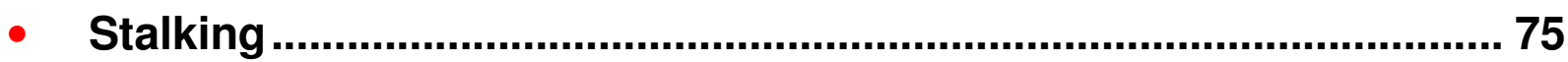

$\checkmark$ Incident Rates......................................................................... 77

- Stalking and Sexual Harassment Related to Unwanted

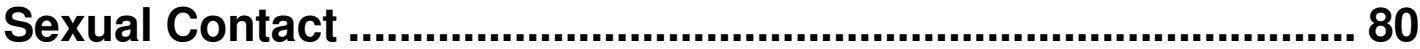

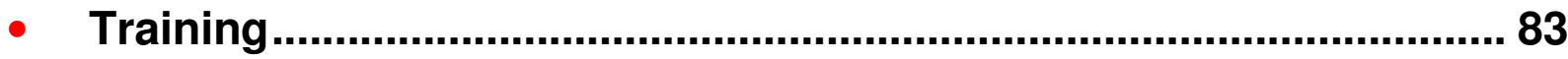

- Student Perceptions ......................................................................... 93 


\section{Stalking Incident Rate}

Percent of Women Who Experienced Stalking Behavior and Felt in Danger

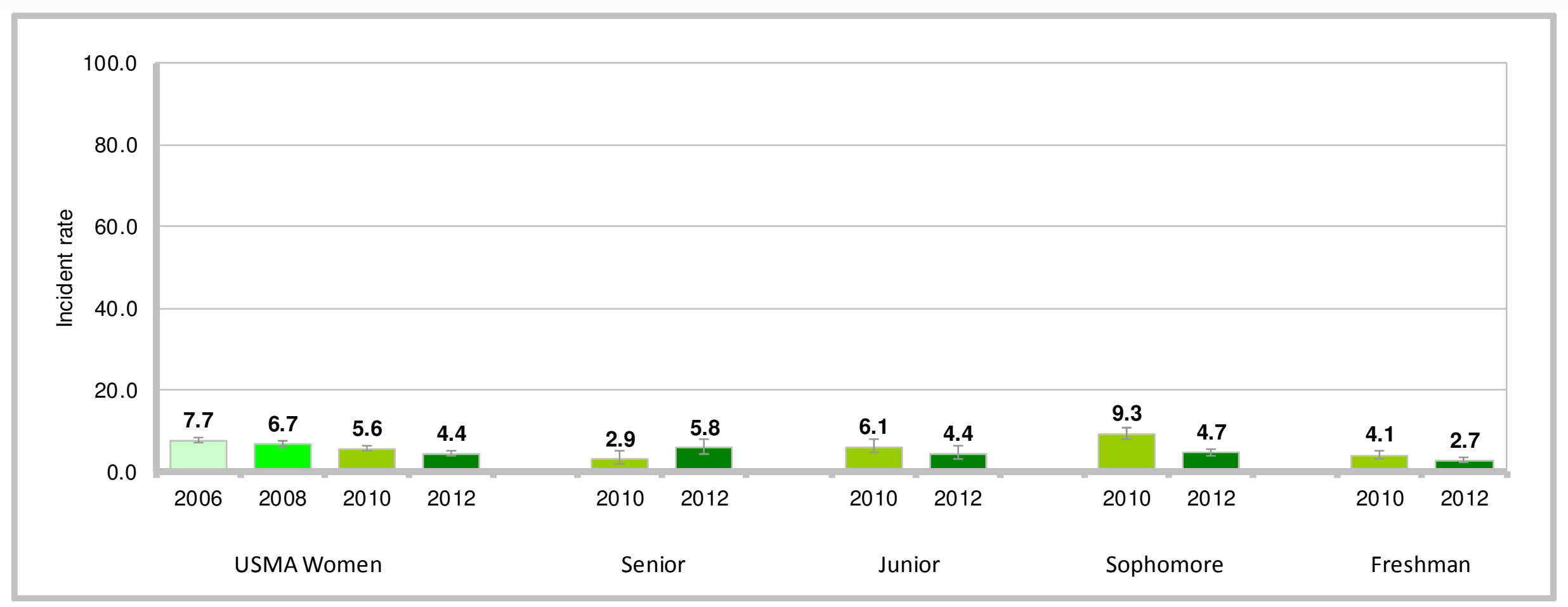

- $4.4 \%$ of women indicated experiencing stalking behavior in 2012

- 2012 comparisons across years - lower than 2010, 2008, 2006

- Seniors in 2012 higher than 2010, whereas sophomores and freshmen lower

- Class differences in 2012 - lower response led by freshmen 


\section{Stalking Incident Rate}

Percent of Men Who Experienced Stalking Behavior and Felt in Danger

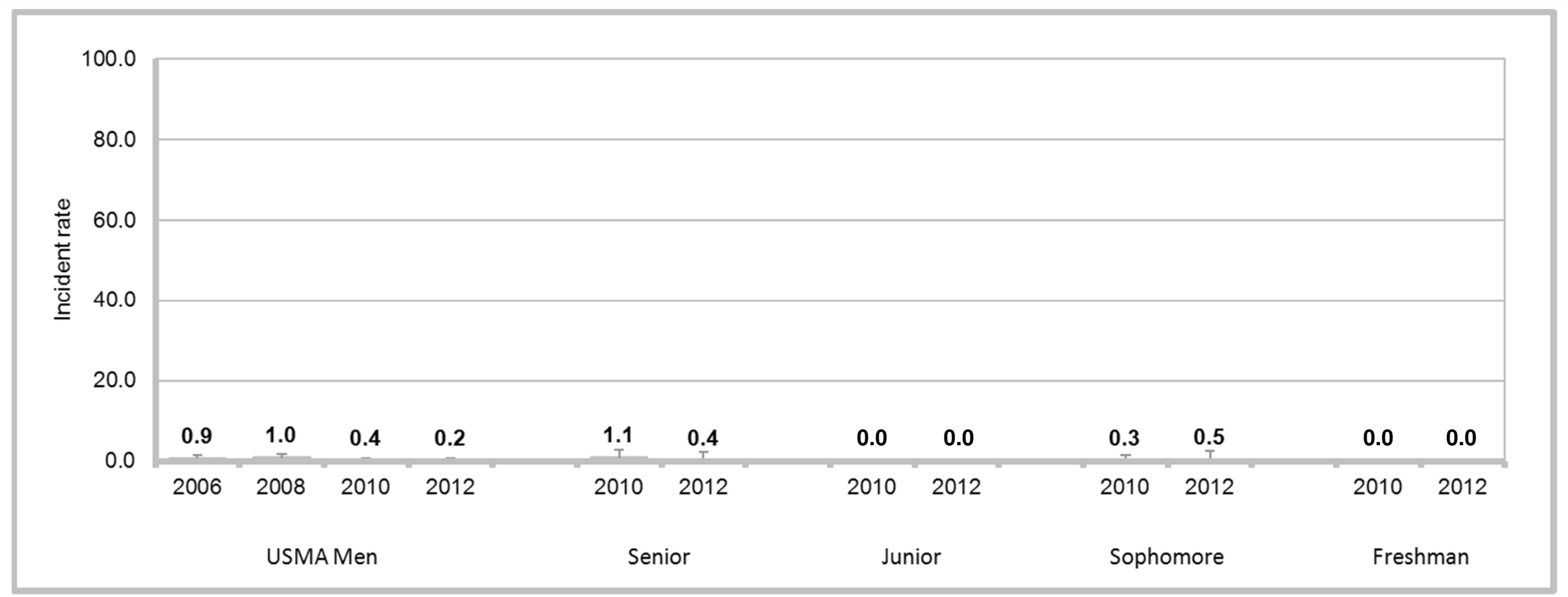

- $0.2 \%$ of men indicated experiencing stalking behavior in 2012

- 2012 comparisons across years - lower than 2008, 2006

- Class differences in 2012 - no differences 


\section{Contents}

- Introduction ..................................................................................... 3

- Unwanted Sexual Contact .............................................................. 7

- Unwanted Gender-Related Behaviors ............................................ 49

• Stalking ...................................................................................... 75

- Incident Rates............................................................................ 77

$\checkmark$ Stalking and Sexual Harassment Related to Unwanted

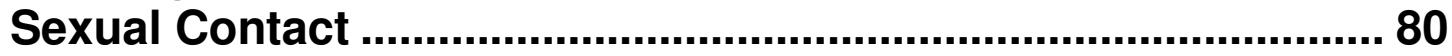

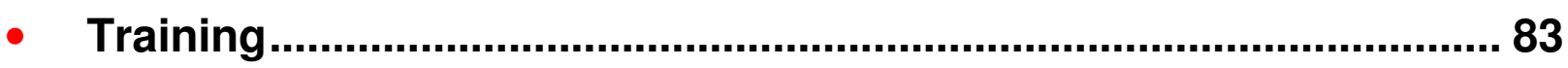

- Student Perceptions....................................................................... 93 


\section{Stalking and Sexual Harassment Possibly Lead to Sexual Assault}

\section{Percent of Women}

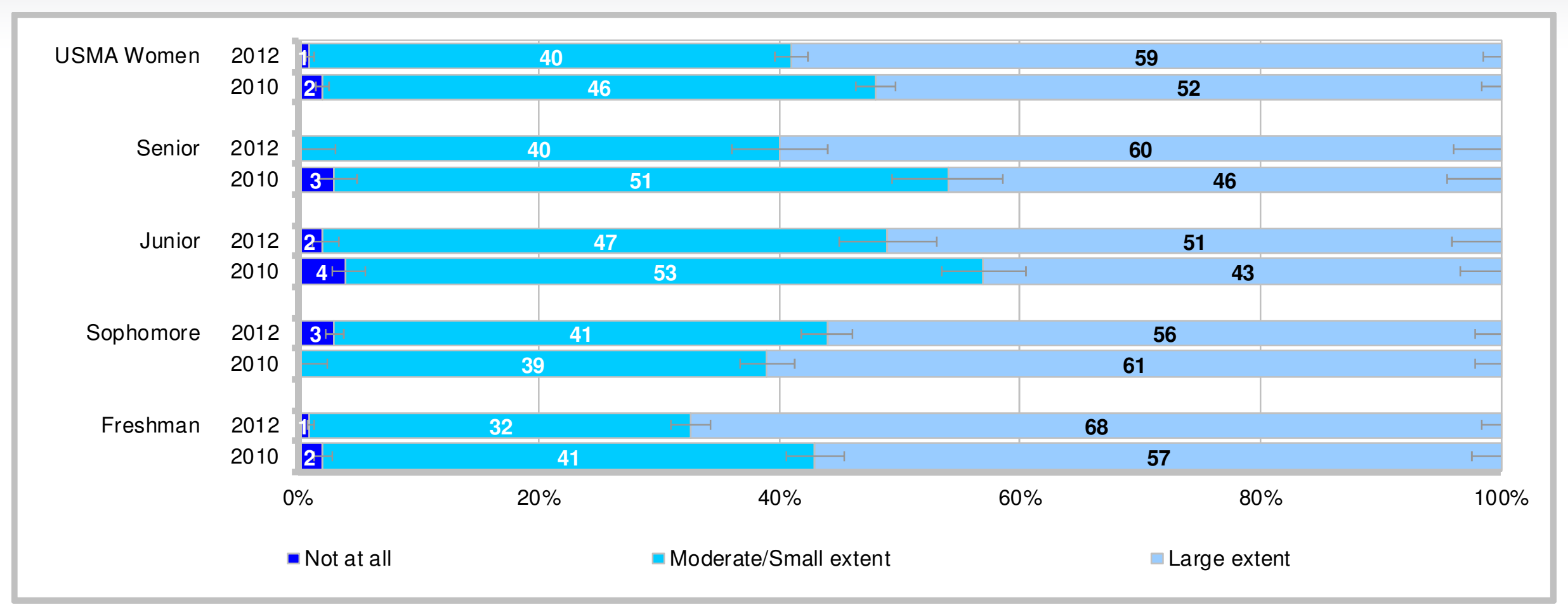

- $99 \%$ of women indicated stalking and sexual harassment possibly lead to sexual assault to at least some extent; $1 \%$ indicated stalking and sexual harassment do not lead to sexual assault

- 2012 comparisons across years - large extent higher than 2010; moderate/small extent lower than 2010

- Seniors, juniors, and freshmen indicating large extent in 2012 higher than 2010, whereas sophomores lower; seniors, juniors, and freshmen indicating moderate/small extent in 2012 lower than 2010; seniors and juniors indicating not at all in 2012 lower than 2010, whereas sophomores higher

- Class differences in 2012 - large extent led by freshmen; moderate/small extent led by juniors; not at all led by sophomores 


\section{Stalking and Sexual Harassment Possibly Lead to Sexual Assault} Percent of Men

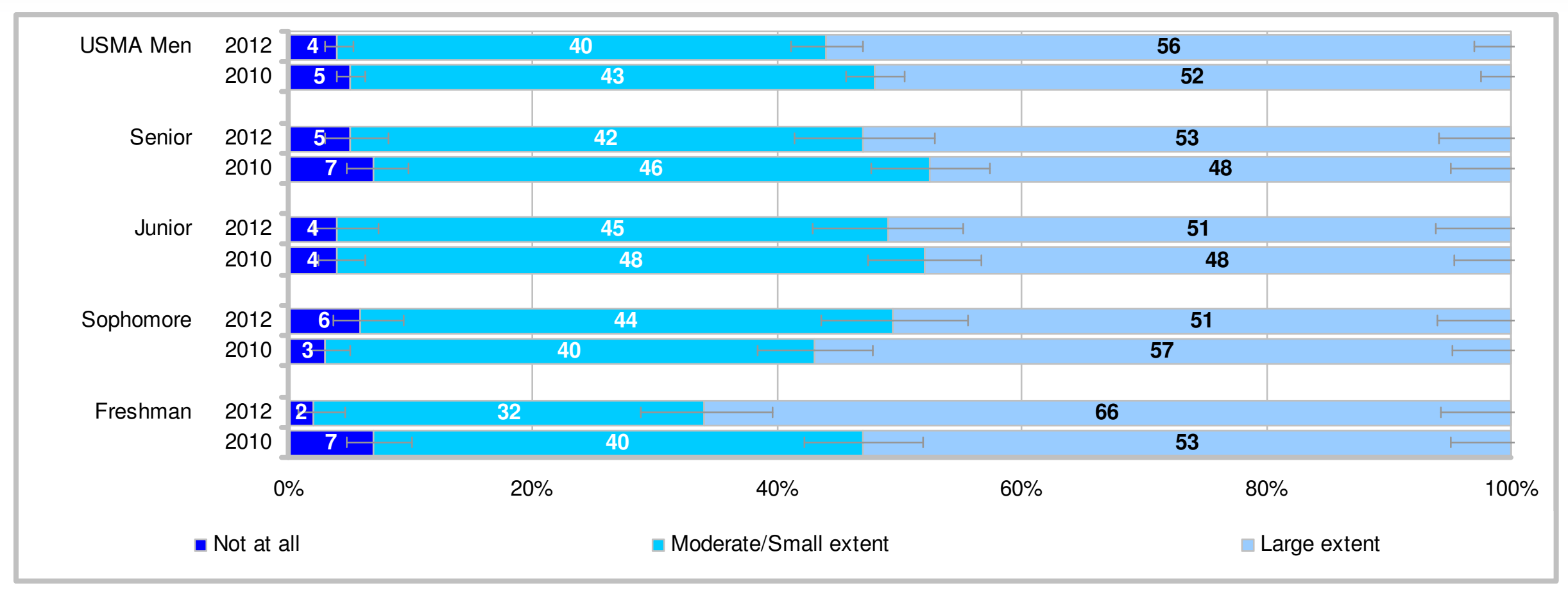

- $96 \%$ of men indicated stalking and sexual harassment possibly lead to sexual assault to at least some extent; $4 \%$ indicated stalking and sexual harassment do not lead to sexual assault

- 2012 comparisons across years - large extent higher than 2010

- Freshmen indicating large extent in 2012 higher than 2010; freshmen indicating moderate/small extent in 2012 lower than 2010; freshmen indicating not at all in 2012 lower than 2010

- Class differences in 2012 - large extent led by freshmen 


\section{Contents}

Slide

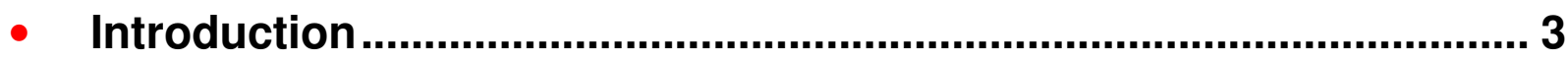

- Unwanted Sexual Contact .................................................................... 7

- Unwanted Gender-Related Behaviors .............................................. 49

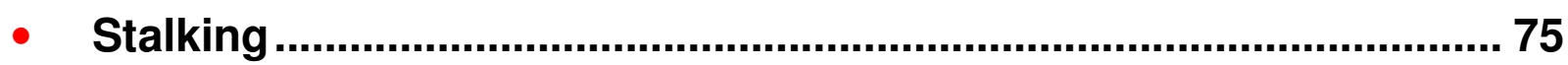

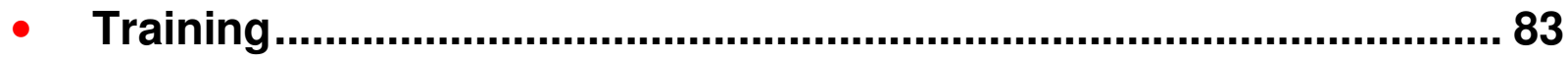

$\checkmark$ Sexual Assault Training Availability and Effectiveness ............ 83

- Sexual Harassment Training Availability and Effectiveness..... 88

- Student Perceptions....................................................................... 93 


\section{Received Training Since June 2011 on Topics Related to Sexual Assault Percent of Women}

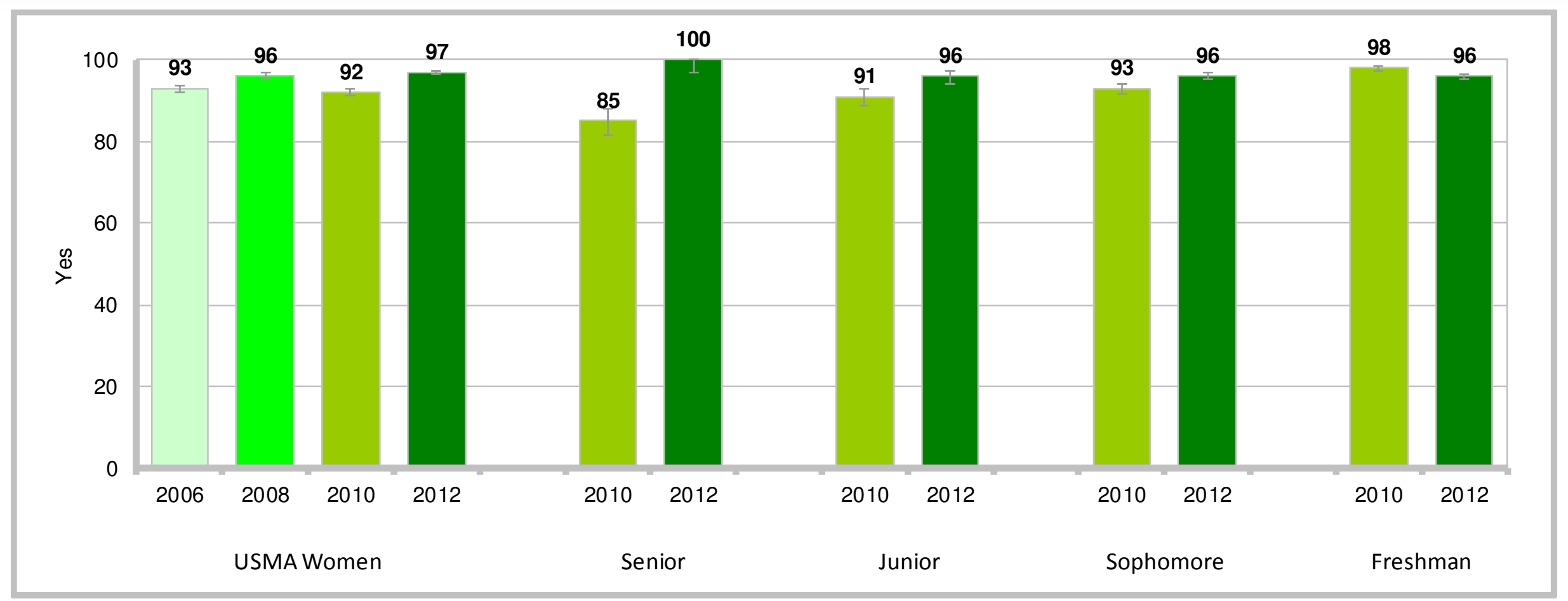

- $97 \%$ of women indicated receiving training on topics related to sexual assault in 2012

- 2012 comparisons across years - higher than 2010, 2006

- Seniors, juniors, and sophomores in 2012 higher than 2010, whereas freshmen lower

- Class differences in 2012 - higher response led by seniors 


\section{Received Training Since June 2011 on Topics Related to Sexual Assault} Percent of Men

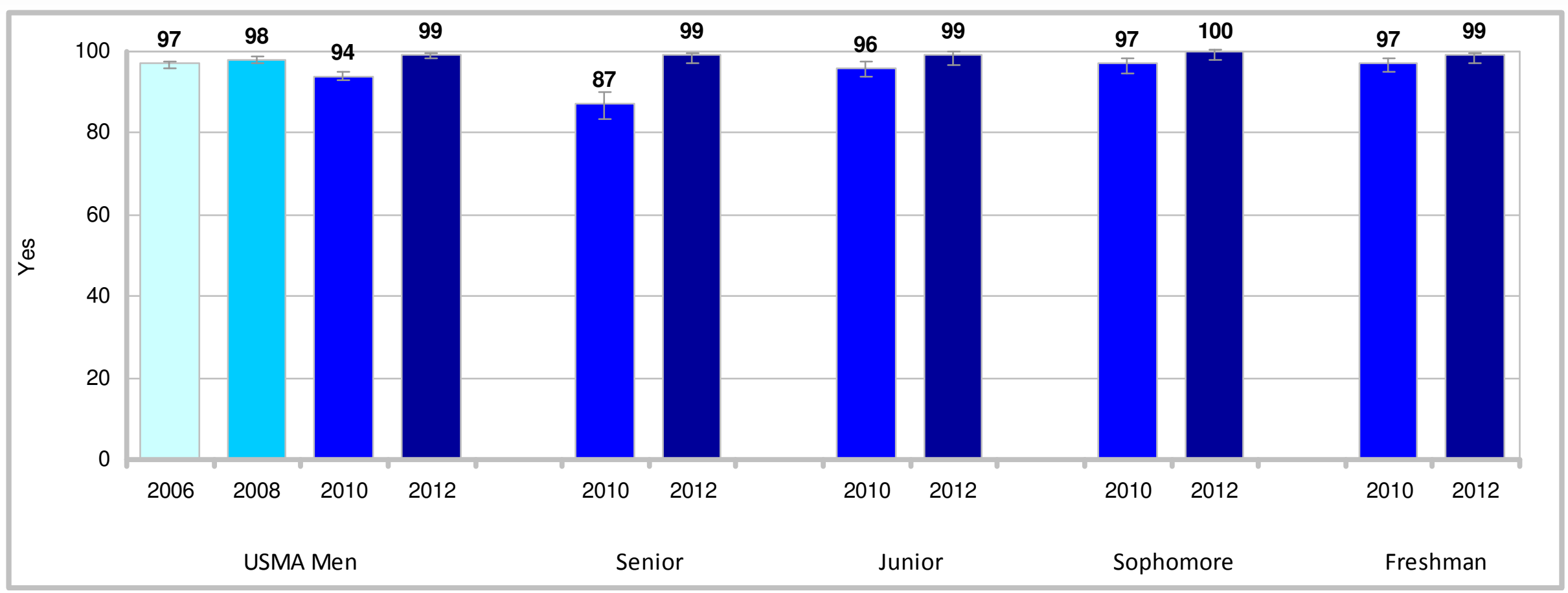

- $99 \%$ of men indicated receiving training on topics related to sexual assault in 2012

- 2012 comparisons across years - higher than 2010, 2006

- All class years in 2012 higher than 2010

- Class differences in 2012 - no differences 


\section{Effectiveness of Training in Actually Reducing/Preventing Sexual Assault Percent of Women Who Had Training on Sexual Assault}

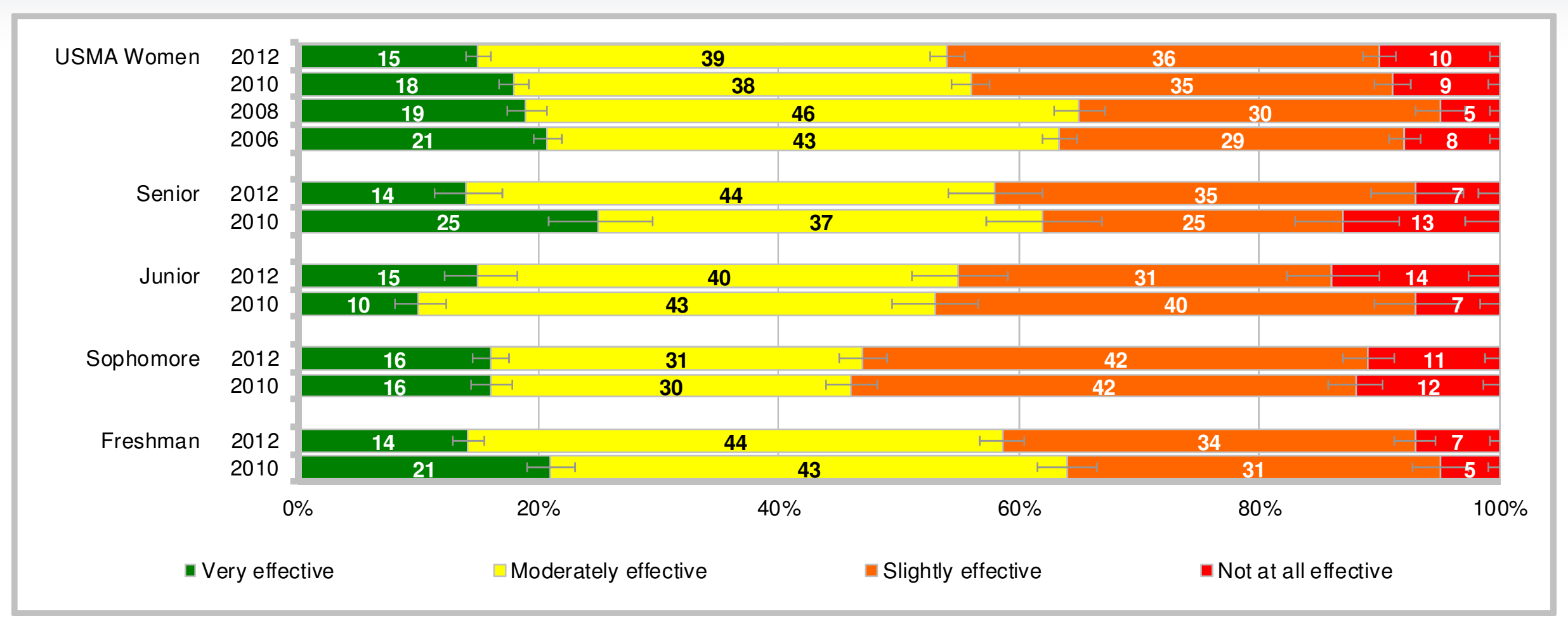

- $15 \%$ of women indicated their training was very effective in actually reducing/preventing sexual assault; $39 \%$ indicated moderately effective; $36 \%$ indicated slightly effective; and $10 \%$ indicated not at all effective

- 2012 comparisons across years - very effective lower than 2010, 2008, 2006; moderately effective lower than 2008, 2006; slightly effective higher than 2008, 2006; not at all effective higher than 2008, 2006

- Juniors indicating very effective in 2012 higher than 2010, whereas seniors and freshmen lower; seniors indicating moderately effective in 2012 higher than 2010; seniors and freshmen indicating slightly effective in 2012 higher than 2010, whereas juniors lower; juniors indicating not at all effective in 2012 higher than 2010, whereas seniors lower

- Class differences in 2012 - moderately effective led by seniors and freshmen; slightly effective led by sophomores; not at all effective led by juniors and sophomores 


\section{Effectiveness of Training in Actually Reducing/Preventing Sexual Assault} Percent of Men Who Had Training on Sexual Assault

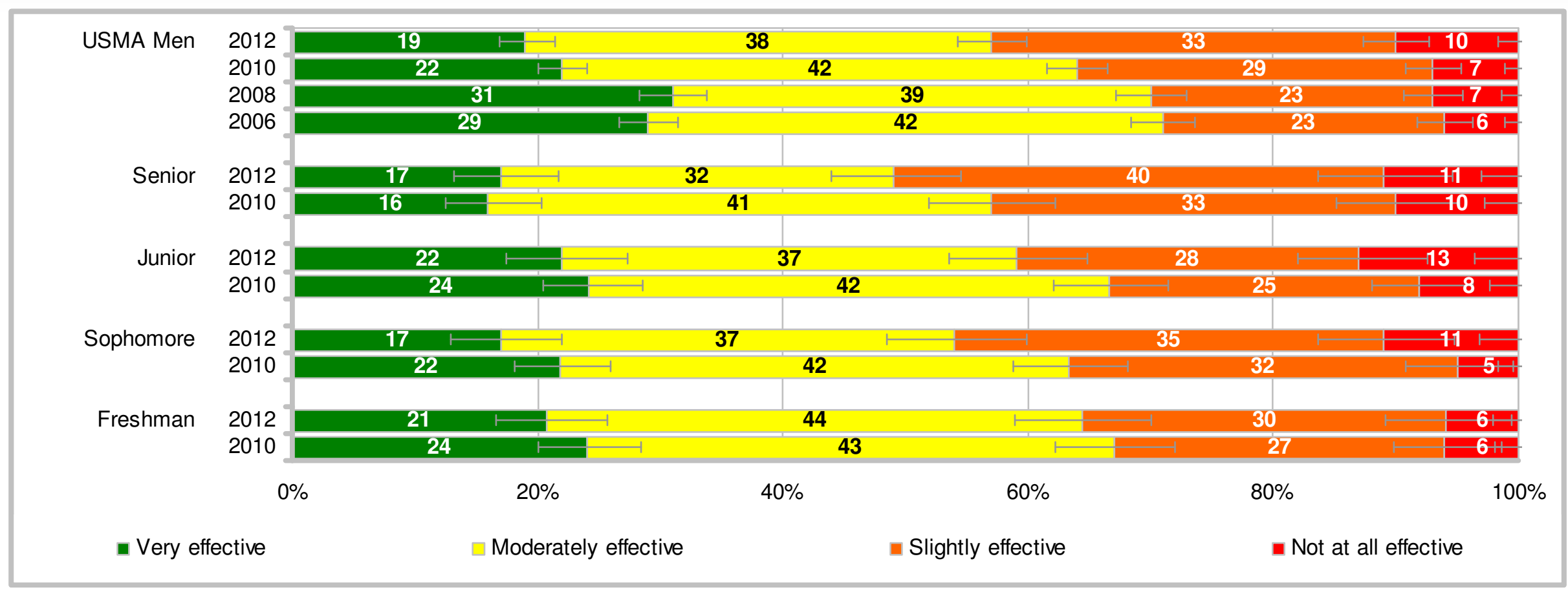

- $19 \%$ of men indicated their training was very effective in actually reducing/preventing sexual assault; $38 \%$ indicated moderately effective; $33 \%$ indicated slightly effective; and $10 \%$ indicated not at all effective

- 2012 comparisons across years - very effective lower than 2008, 2006; moderately effective lower than 2010, 2006; slightly effective higher than 2010, 2008, 2006; not at all effective higher than 2010, 2008, 2006

- Seniors indicating moderately effective in 2012 lower than 2010; juniors and sophomores indicating not at all effective in 2012 higher than 2010

- Class differences in 2012 - moderately effective led by freshmen; slightly effective led by seniors 


\section{Contents}

Slide

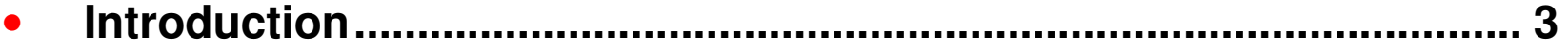

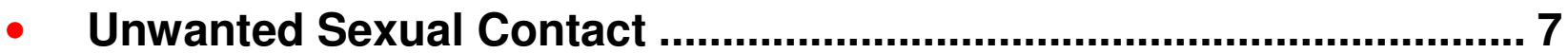

- Unwanted Gender-Related Behaviors .......................................... 49

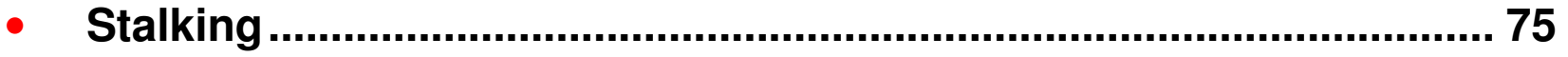

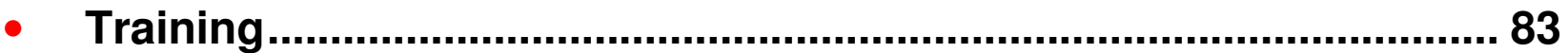

- Sexual Assault Training Availability and Effectiveness ............ 83

$\checkmark$ Sexual Harassment Training Availability and Effectiveness..... 88

- Student Perceptions..................................................................... 93 


\section{Received Training Since June 2011 on Topics Related to Sexual Harassment \\ Percent of Women}

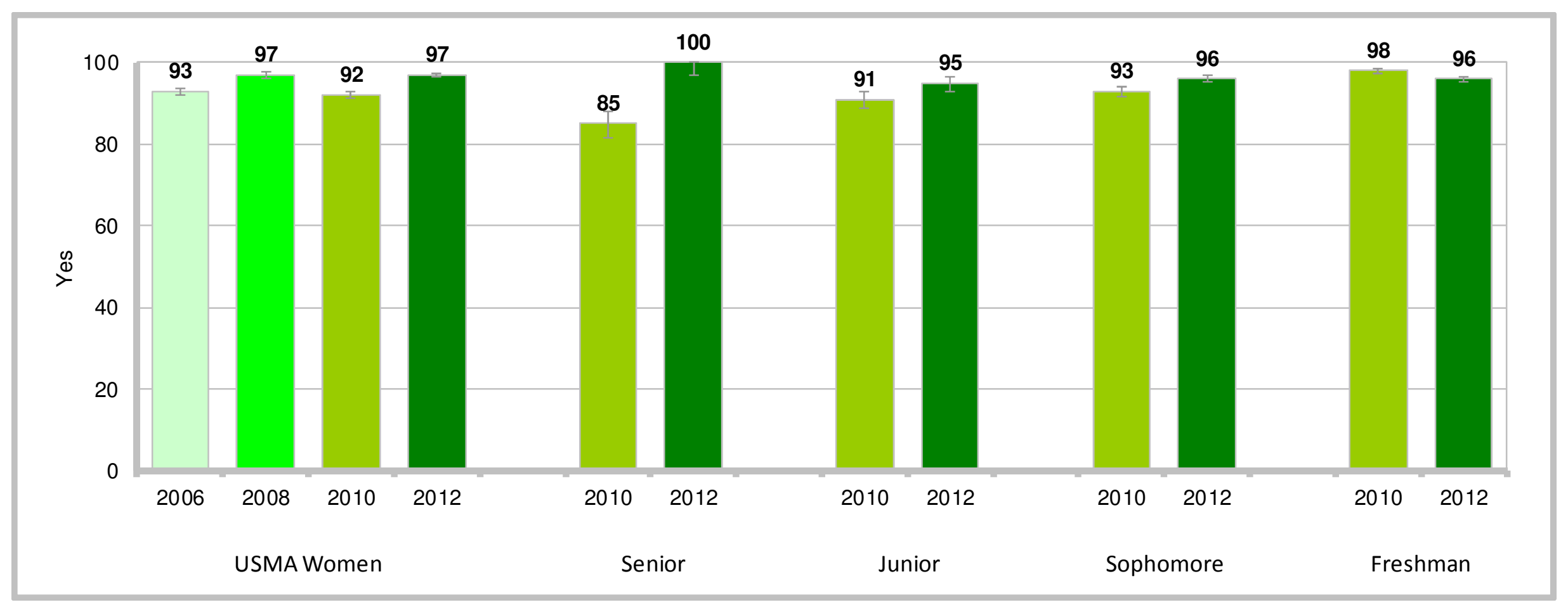

- $97 \%$ of women indicated receiving training on topics related to sexual harassment in 2012

- 2012 comparisons across years - higher than 2010, 2006

- Seniors, juniors, and sophomores in 2012 higher than 2010, whereas freshmen lower

- Class differences in $\mathbf{2 0 1 2}$ - higher response led by seniors 


\section{Received Training Since June 2011 on Topics Related to Sexual Harassment \\ Percent of Men}

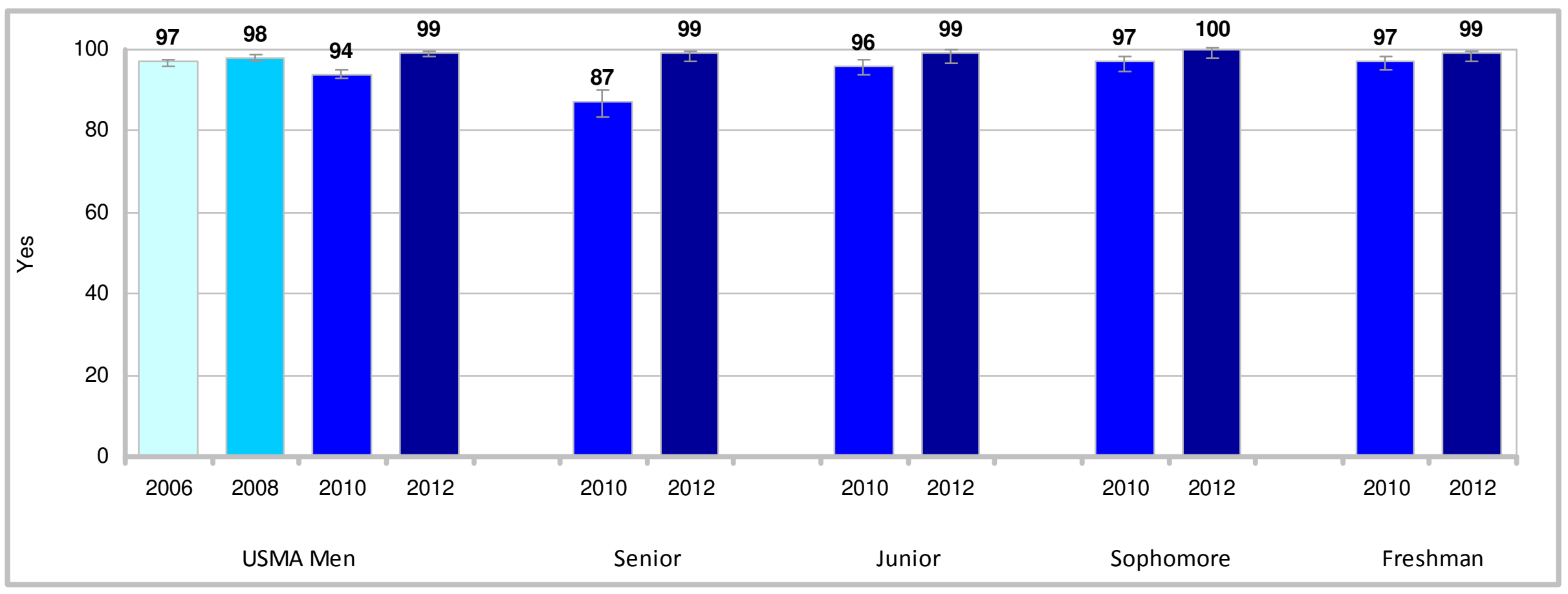

- $99 \%$ of men indicated receiving training on topics related to sexual harassment in 2012

- 2012 comparisons across years - higher than 2010, 2006

- All class years in 2012 higher than 2010

- Class differences in 2012 - no differences 


\section{Effectiveness of Training in Actually Reducing/Preventing Sexual Harassment Percent of Women Who Had Training on Sexual Harassment}

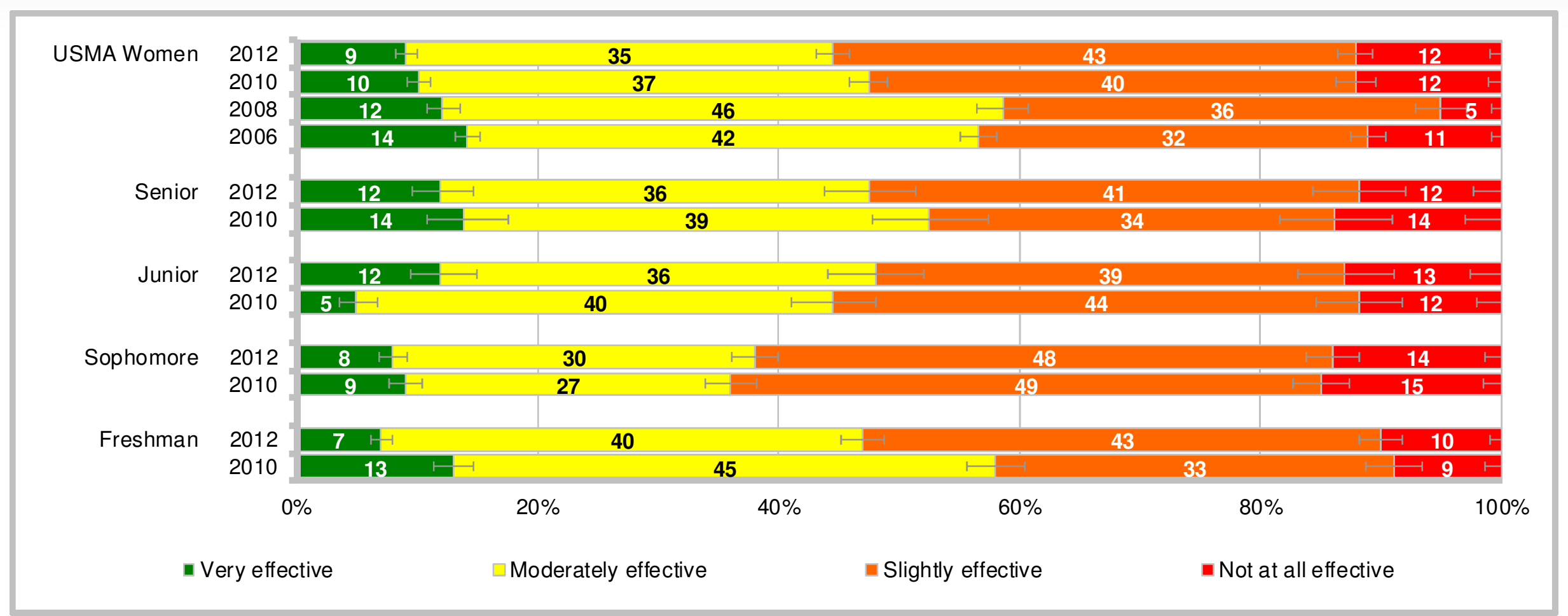

- $9 \%$ of women indicated their training was very effective in actually reducing/preventing sexual harassment; $35 \%$ indicated moderately effective; $43 \%$ indicated slightly effective; and $12 \%$ indicated not at all effective

- 2012 comparisons across years - very effective lower than 2008, 2006; moderately effective lower than 2008, 2006; slightly effective higher than 2010, 2008, 2006; not at all effective higher than 2008

- Juniors indicating very effective in 2012 higher than 2010, whereas freshmen lower; sophomores indicating moderately effective in 2012 higher than 2010, whereas freshmen lower; seniors and freshmen indicating slightly effective in 2012 higher than 2010

- Class differences in 2012 - moderately effective led by freshmen; slightly effective led by sophomores 


\section{Effectiveness of Training in Actually Reducing/Preventing Sexual Harassment \\ Percent of Men Who Had Training on Sexual Harassment}

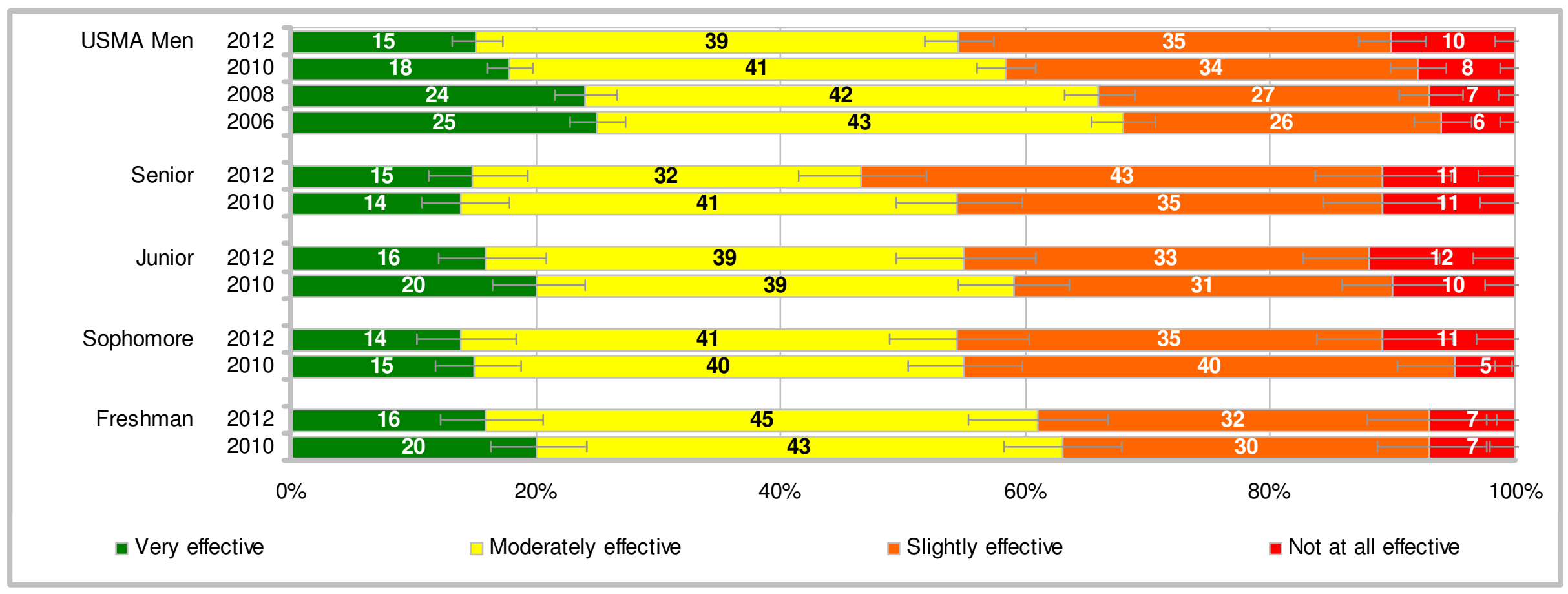

- $15 \%$ of men indicated their training was very effective in actually reducing/preventing sexual harassment; $39 \%$ indicated moderately effective; $35 \%$ indicated slightly effective; and $10 \%$ indicated not at all effective

- 2012 comparisons across years - very effective lower than 2010, 2008, 2006; moderately effective lower than 2006; slightly effective higher than 2008, 2006; not at all effective higher than 2008, 2006

- Seniors indicating moderately effective in 2012 lower than 2010; seniors indicating slightly effective in 2012 higher than 2010; sophomores indicating not at all effective in 2012 higher than 2010

- Class differences in 2012 - slightly effective led by seniors 


\section{Contents}

Slide

- Introduction .................................................................................. 3

- Unwanted Sexual Contact ................................................................... 7

- Unwanted Gender-Related Behaviors ............................................ 49

• Stalking .......................................................................................... 75

• Training ........................................................................................ 83

$\checkmark \quad$ Student Perceptions..................................................................... 93 


\section{Extent You Think Incidents of Sexual Assault Are Not Reported Percent of Women}

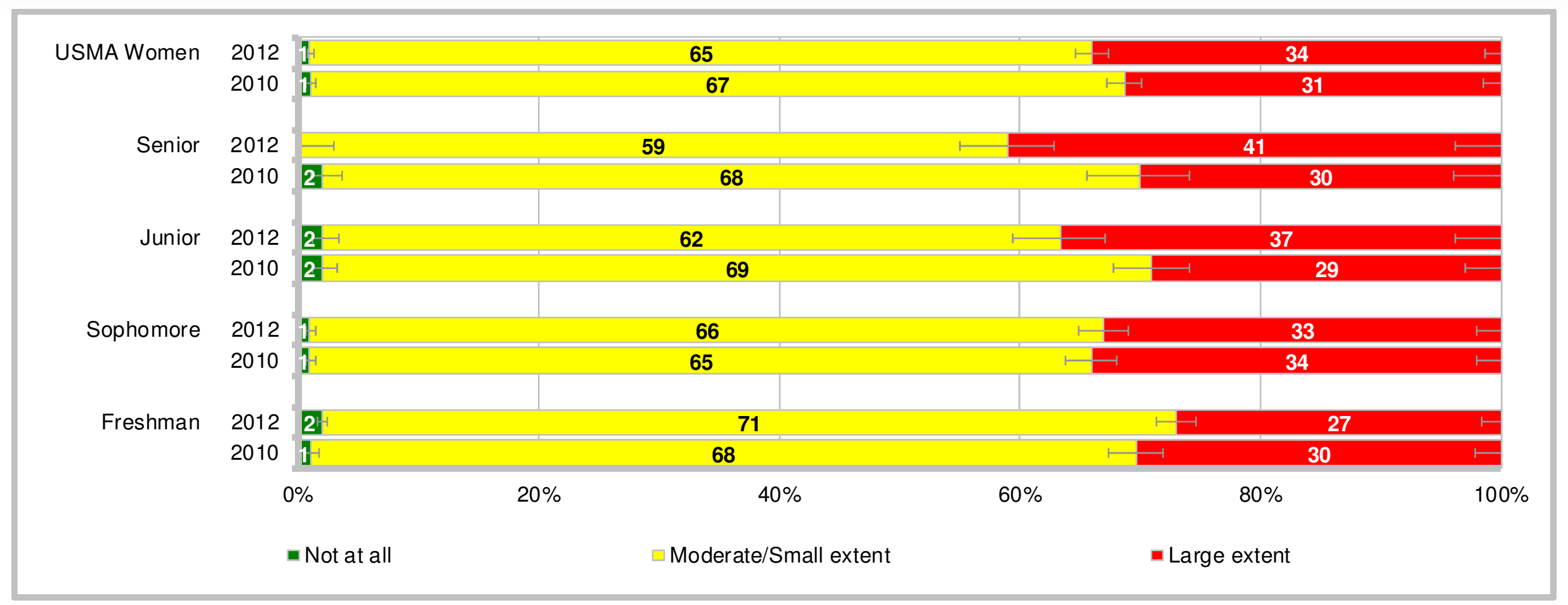

- $34 \%$ of women indicated to a large extent that incidents of sexual assault are not reported; $65 \%$ indicated moderate/small extent; and $1 \%$ indicated not at all

- 2012 comparisons across years - large extent higher than 2010

- Seniors and juniors indicating large extent in 2012 higher than 2010, whereas freshmen lower; seniors and juniors indicating moderate/small extent in 2012 lower than 2010, whereas freshmen higher; seniors indicating not at all in 2012 lower than 2010

- Class differences in 2012 - large extent led by seniors; moderate/small extent led by freshmen 


\section{Extent You Think Incidents of Sexual Assault Are Not Reported Percent of Men}

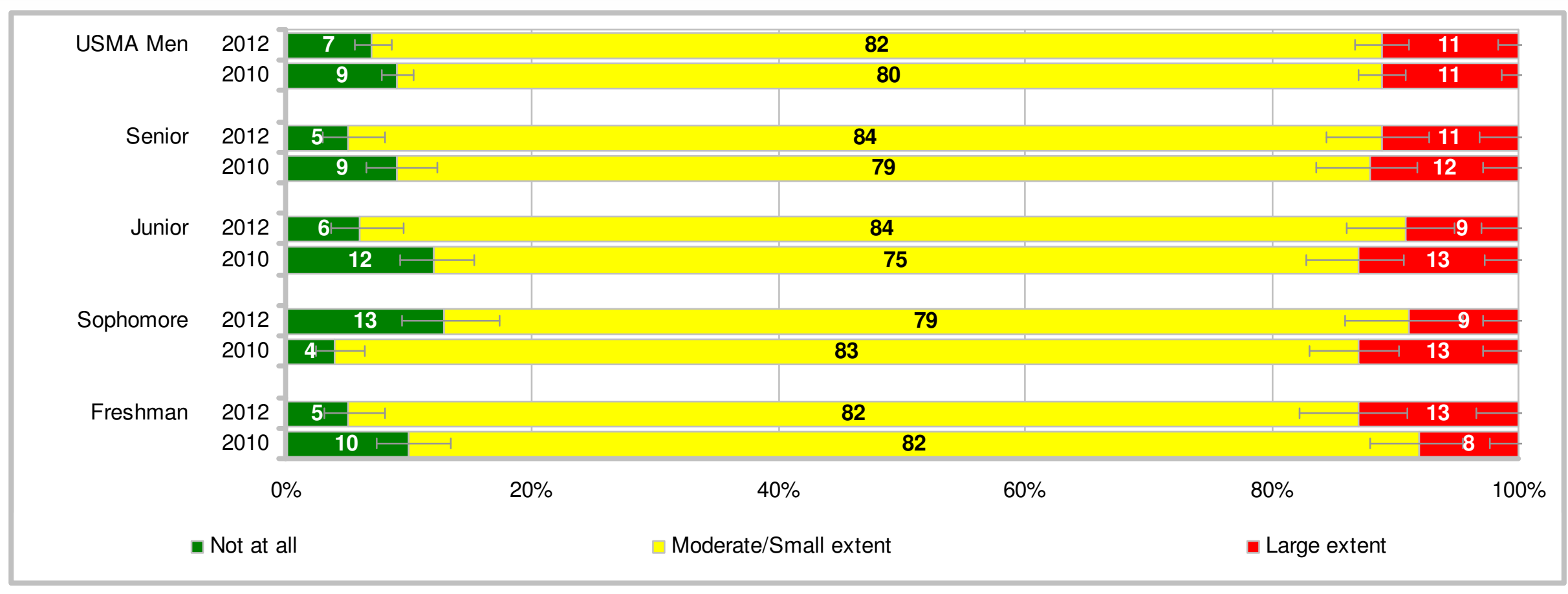

- $11 \%$ of men indicated to a large extent that incidents of sexual assault are not reported; $82 \%$ indicated moderate/small extent; and $7 \%$ indicated not at all

- 2012 comparisons across years - no differences

- Freshmen indicating large extent in 2012 higher than 2010; juniors indicating moderate/small extent in 2012 higher than 2010; sophomores indicating not at all in 2012 higher than 2010, whereas seniors, juniors, and freshmen lower

- Class differences in 2012 - not at all led by sophomores 


\section{Extent You Would Report a Sexual Assault Percent of Women}

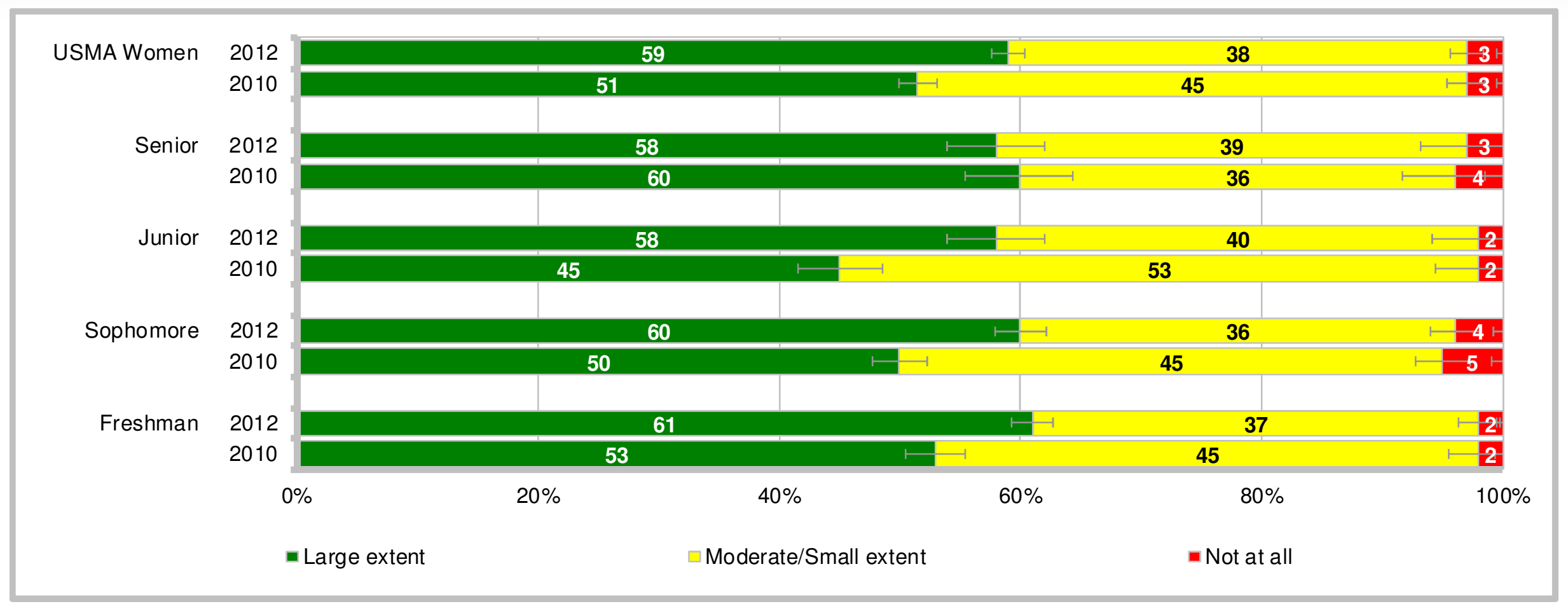

- $59 \%$ of women indicated to a large extent they would be willing to report a sexual assault; $38 \%$ indicated moderate/small extent; and $3 \%$ indicated not at all

- 2012 comparisons across years - large extent higher than 2010; moderate/small extent lower than 2010

- Juniors, sophomores, and freshmen indicating large extent in 2012 higher than 2010; juniors, sophomores, and freshmen indicating moderate/small extent in 2012 lower than 2010

- Class differences in 2012 - not at all led by sophomores 


\section{Extent You Would Report a Sexual Assault Percent of Men}

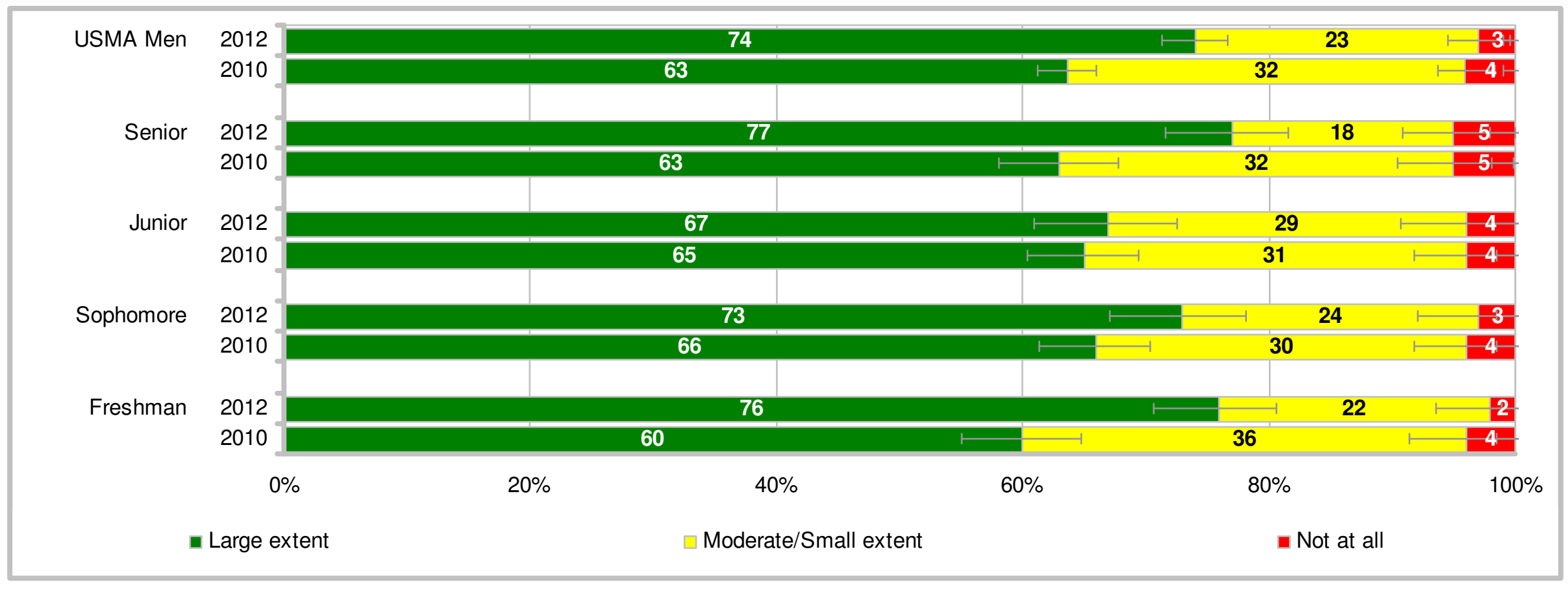

- $74 \%$ of men indicated to a large extent they would be willing to report a sexual assault; $23 \%$ indicated to a moderate/small extent; and $3 \%$ indicated not at all

- 2012 comparisons across years - large extent higher than 2010; moderate/small extent lower than 2010

- Seniors and freshmen indicating large extent in 2012 higher than 2010; seniors and freshmen indicating moderate/small extent in 2012 lower than 2010

- Class differences in 2012 - no differences 


\section{Extent You Think High-Profile Cases Deter Reporting of Sexual Assault Percent of Women}

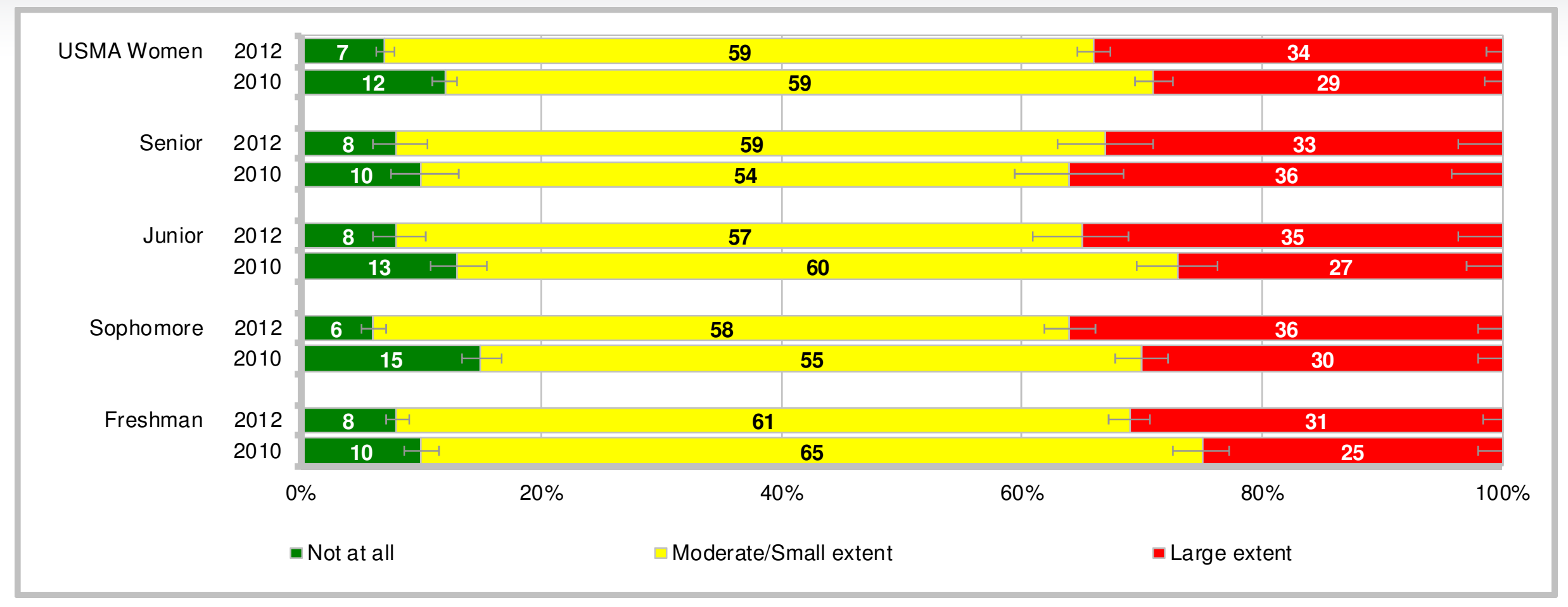

- $34 \%$ of women indicated to a large extent high-profile cases deter reporting of sexual assault; $59 \%$ indicated to a moderate/small extent; and $7 \%$ indicated not at all

- 2012 comparisons across years - not at all lower than 2010; large extent higher than 2010

- Juniors, sophomores, and freshmen indicating large extent in 2012 higher than 2010; freshmen indicating moderate/small extent in 2012 lower than 2010; juniors, sophomores, and freshmen indicating not at all in 2012 lower than 2010*

- Class differences in 2012 - large extent led by sophomores; moderate/small extent led by freshmen

*Note that both $8 \%$ of freshmen and senior women indicated not at all in 2012 and $10 \%$ in 2010 . These percentages for senior women are not significantly different due to a higher margin of error for senior women responding to this question. 


\section{Extent You Think High-Profile Cases Deter Reporting of Sexual Assault} Percent of Men

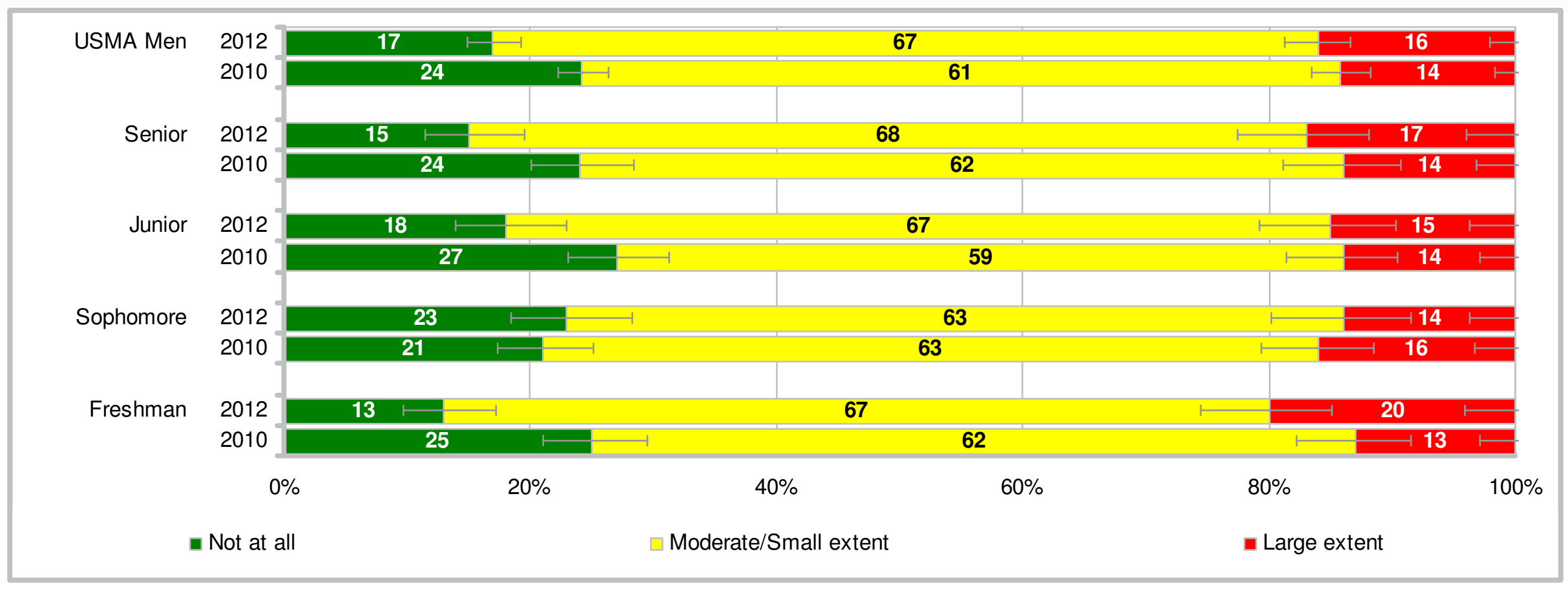

- $16 \%$ of men indicated to a large extent high-profile cases deter reporting of sexual assault; $67 \%$ indicated to a moderate/small extent; and $17 \%$ indicated not at all

- 2012 comparisons across years - moderate/small extent higher than 2010; not at all lower than 2010

- Freshmen indicating large extent in 2012 higher than 2010; juniors indicating moderate/small extent in 2012 higher than 2010; seniors, juniors, and freshmen indicating not at all in 2012 lower than 2010

- Class differences in 2012 - not at all led by sophomores 


\section{You Would Discuss an Incident With a SARC if You Were To Experience} Sexual Assault in the Future

\section{Percent of Women Who Had Not Experienced Unwanted Sexual Contact in Past 12 Months}

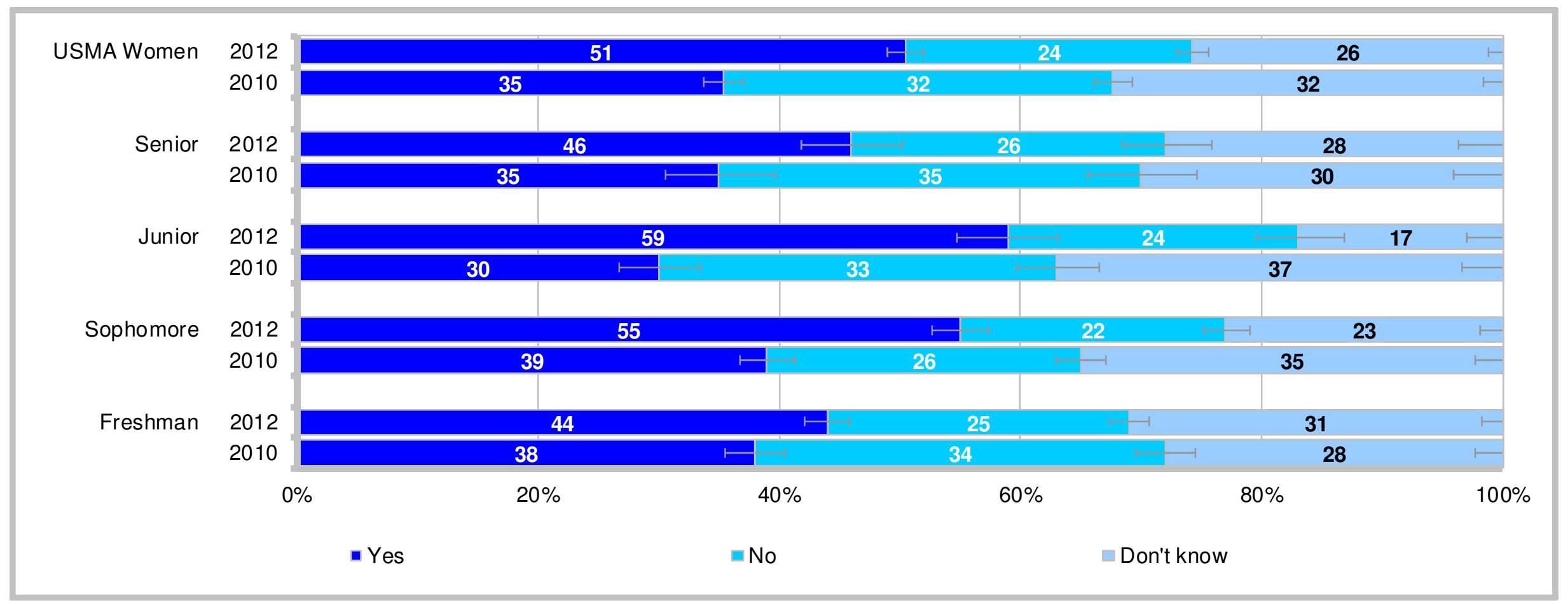

- $51 \%$ of women indicated yes, they would discuss an incident of sexual assault with a SARC; $24 \%$ indicated no; and $26 \%$ indicated don't know

- 2012 comparisons across years - yes higher than 2010; no lower than 2010; don't know lower than 2010

- All class years indicating yes in 2012 higher than 2010; all class years indicating no in 2012 lower than 2010; juniors and sophomores indicating don't know in 2012 lower than 2010, whereas freshmen higher

- Class differences in 2012 - yes led by juniors and sophomores; don't know led by freshmen 


\section{You Would Discuss an Incident With a SARC if You Were To Experience Sexual Assault in the Future \\ Percent of Men Who Had Not Experienced Unwanted Sexual Contact in Past 12 Months}

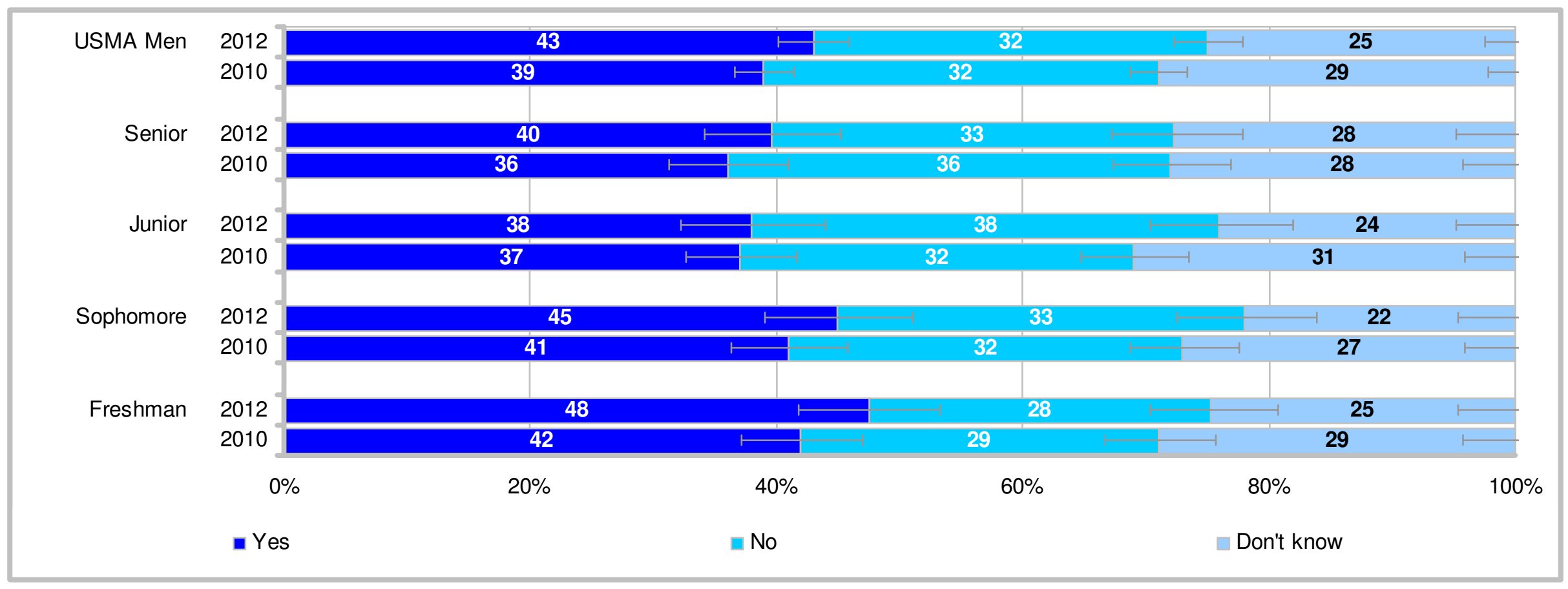

- $43 \%$ of men indicated yes, they would discuss an incident of sexual assault with a SARC; $32 \%$ indicated no; and $25 \%$ indicated don't know

- 2012 comparisons across years - yes higher than 2010; don't know lower than 2010

- Juniors indicating don't know in 2012 lower than 2010

- Class differences in 2012 - no differences 


\section{You Would Trust the System To Protect Your Privacy if You Were To Experience Sexual Assault in the Future \\ Percent of Women Who Had Not Experienced Unwanted Sexual Contact in the Past 12 Months}

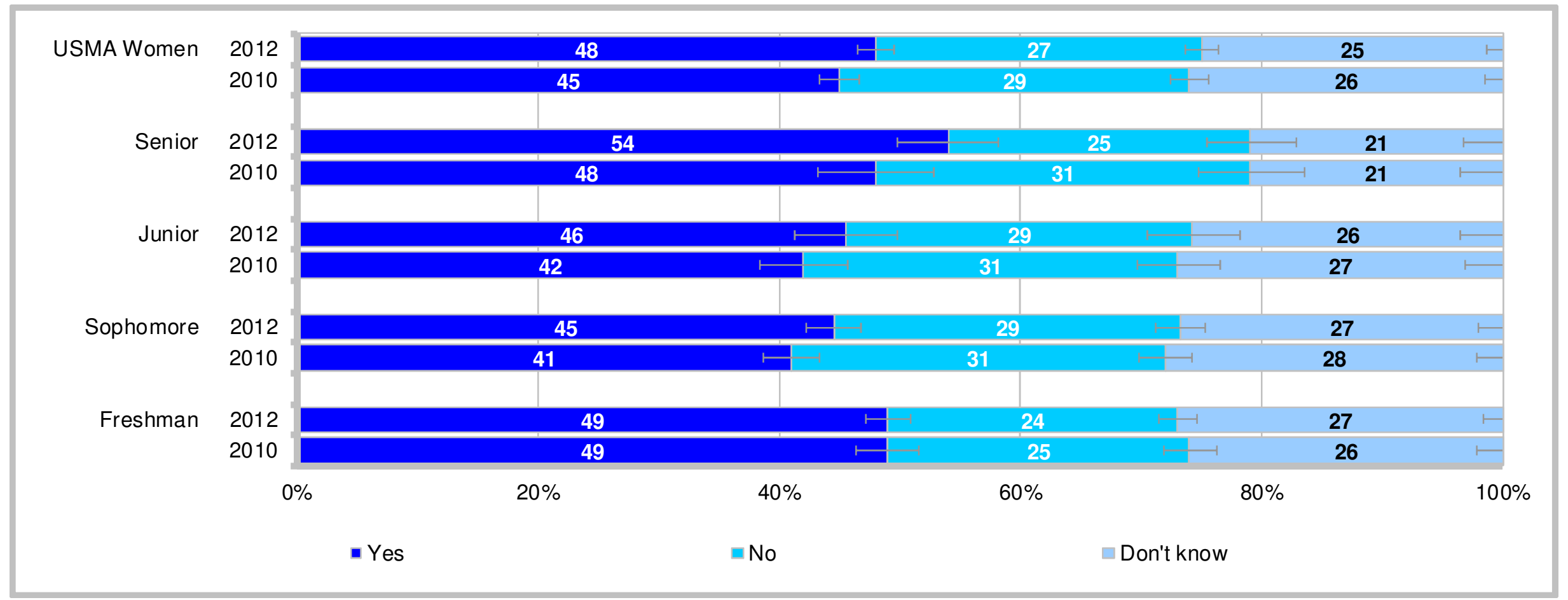

- $48 \%$ of women indicated yes, they would trust the system to protect their privacy if they experienced sexual assault; $27 \%$ indicated no; and $25 \%$ indicated don't know

- 2012 comparisons across years - yes higher than 2010

- Sophomores indicating yes in 2012 higher than 2010; seniors indicating no in 2012 lower than 2010

- Class differences in 2012 - yes led by seniors 


\section{You Would Trust the System To Protect Your Privacy if You Were To Experience Sexual Assault in the Future \\ Percent of Men Who Had Not Experienced Unwanted Sexual Contact in the Past 12 Months}

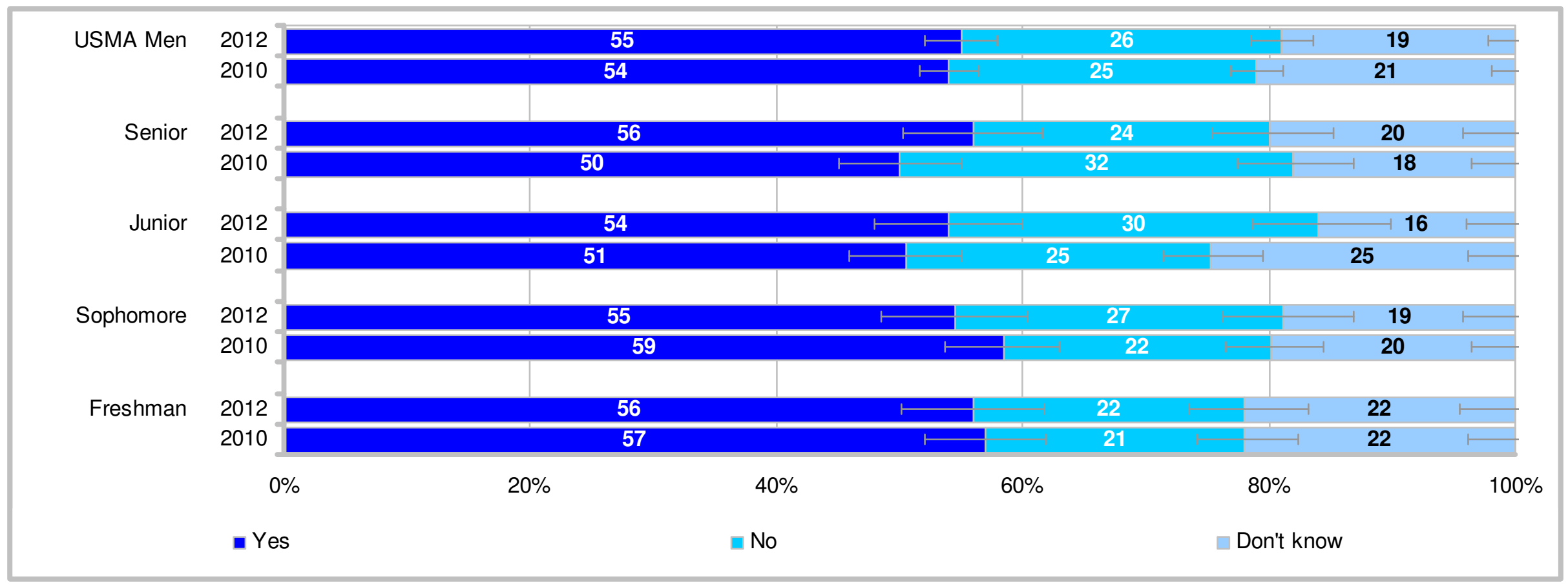

- $55 \%$ of men indicated yes, they would trust the system to protect their privacy if they experienced sexual assault; $26 \%$ indicated no; and 19\% indicated don't know

- 2012 comparisons across years - no difference

- Seniors indicating no in 2012 lower than 2010; juniors indicating don't know in 2012 lower than 2010

- Class differences in 2012 - no differences 


\section{You Would Be Willing To Encourage Someone Who Has Experienced Sexual Assault To Report It Percent of Women}

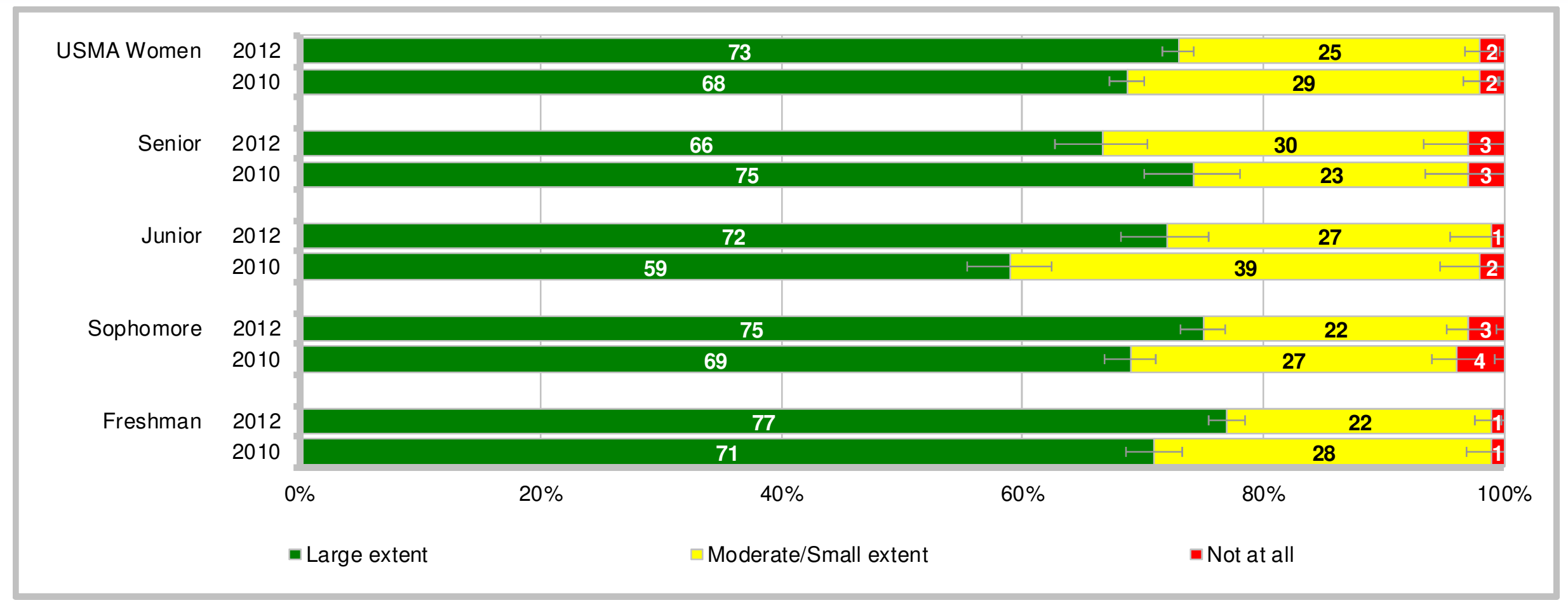

- $98 \%$ of women indicated they would encourage someone to report sexual assault to at least some extent; $2 \%$ indicated not at all

- 2012 comparisons across years - large extent higher than 2010; moderate/small extent lower than 2010

- Juniors, sophomores, and freshmen indicating large extent in 2012 higher than 2010, whereas seniors lower; juniors, sophomores, and freshmen indicating moderate/small extent in 2012 lower than 2010, whereas seniors higher

- Class differences in 2012 - large extent led by freshmen; moderate/small extent led by seniors 


\section{You Would Be Willing To Encourage Someone Who Has Experienced Sexual Assault To Report It Percent of Men}

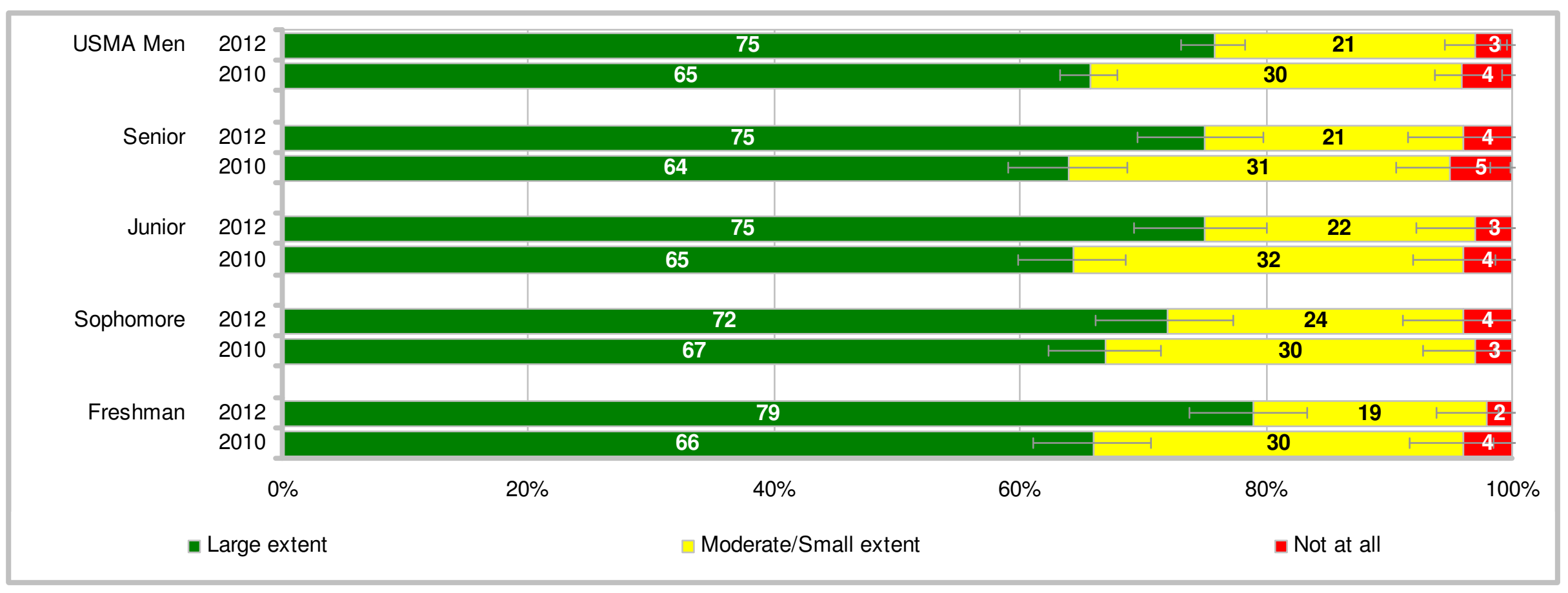

- $96 \%$ of men indicated they would encourage someone to report sexual assault to at least some extent; $3 \%$ indicated not at all

- 2012 comparisons across years - large extent higher than 2010; moderate/small extent lower than 2010

- Seniors, juniors, and freshmen indicating large extent in 2012 higher than 2010; seniors, juniors, and freshmen indicating moderate/small extent in 2012 lower than 2010

- Class differences in 2012 - no differences 


\section{You Would Step In and Stop a Situation That Might Lead to Sexual Assault} Percent of Women

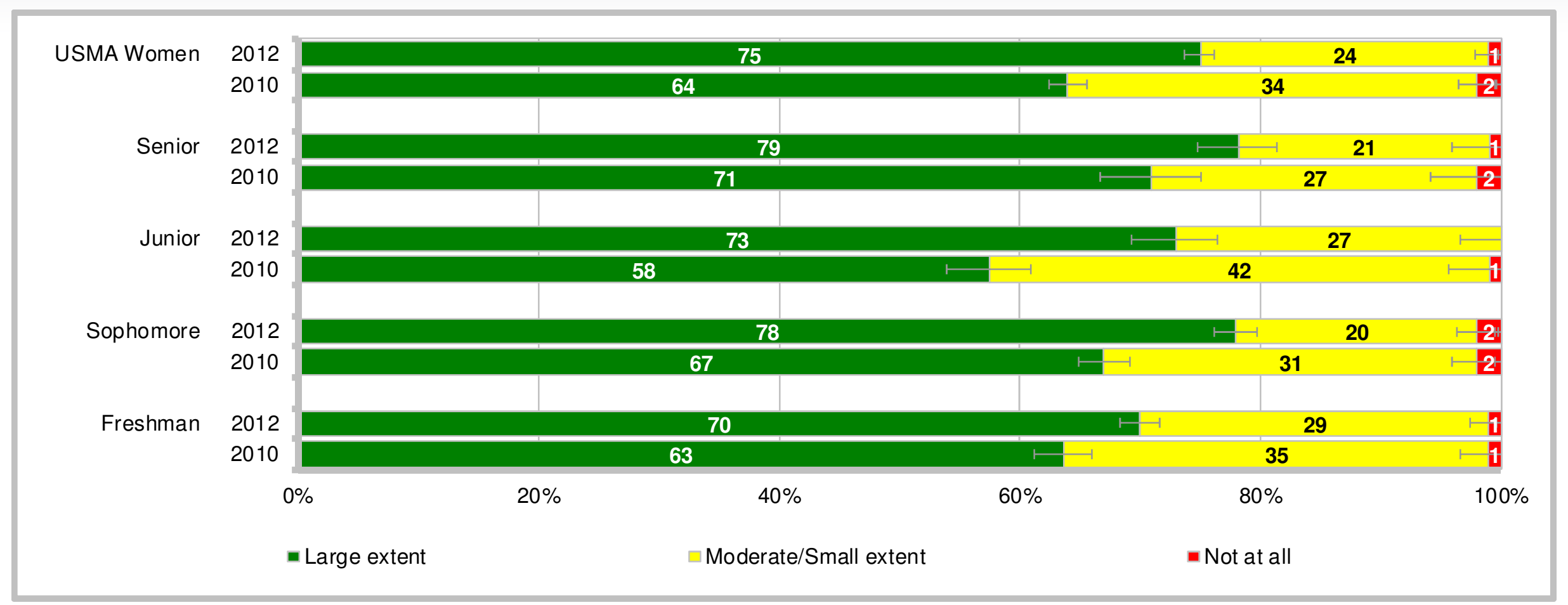

- $99 \%$ of women indicated they would step in and stop a situation that might lead to sexual assault to at least some extent; $1 \%$ indicated not at all

- 2012 comparisons across years - large extent higher than 2010; moderate/small extent lower than 2010

- All class years indicating large extent in 2012 higher than 2010; all class years indicating moderate/small extent in 2012 lower than 2010

- Class differences in 2012 - large extent led by seniors and sophomores; moderate/small extent led by freshmen; not at all led by sophomores 


\section{You Would Step In and Stop a Situation That Might Lead to Sexual Assault}

\section{Percent of Men}

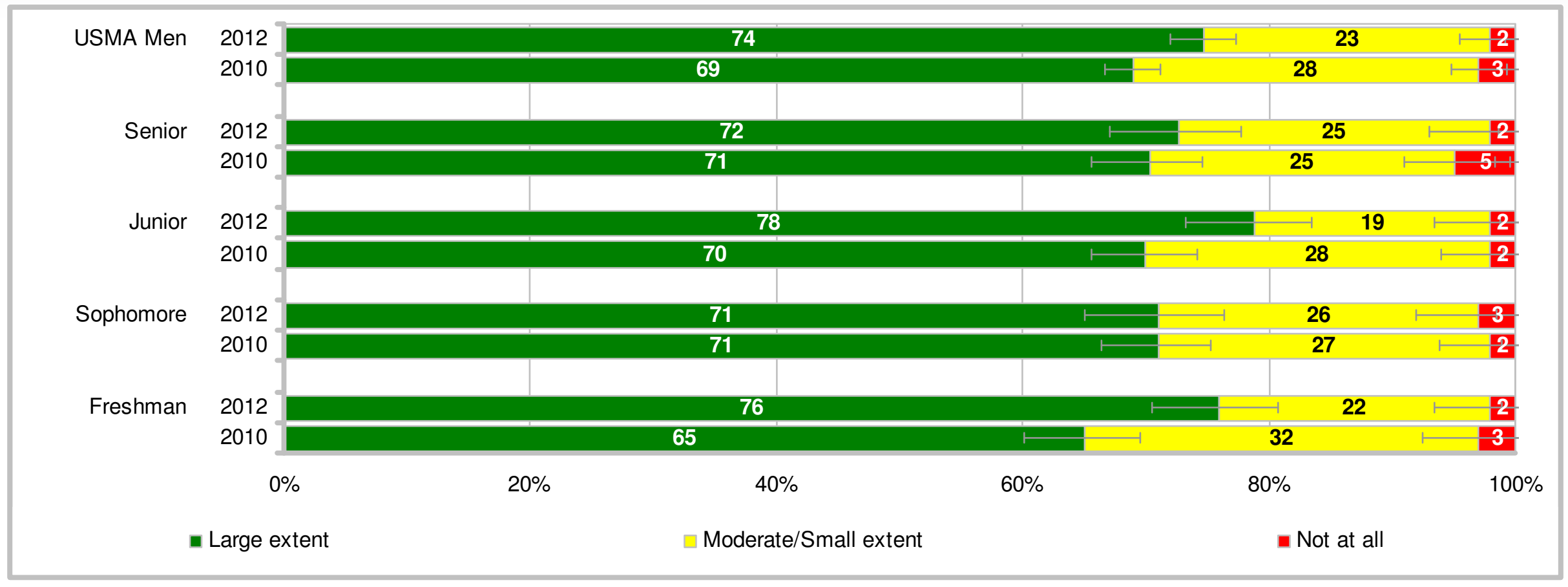

- $97 \%$ of men indicated they would step in and stop a situation that might lead to sexual assault to at least some extent; $2 \%$ indicated not at all

- 2012 comparisons across years - large extent higher than 2010; moderate/small extent lower than 2010

- Juniors and freshmen indicating large extent in 2012 higher than 2010; juniors and freshmen indicating moderate/small extent in 2012 lower than 2010; seniors indicating not at all in 2012 lower than 2010

- Class differences in 2012 - no differences 


\section{You Would Point Out to Someone That They "Crossed the Line" With Comments or Jokes \\ Percent of Women}

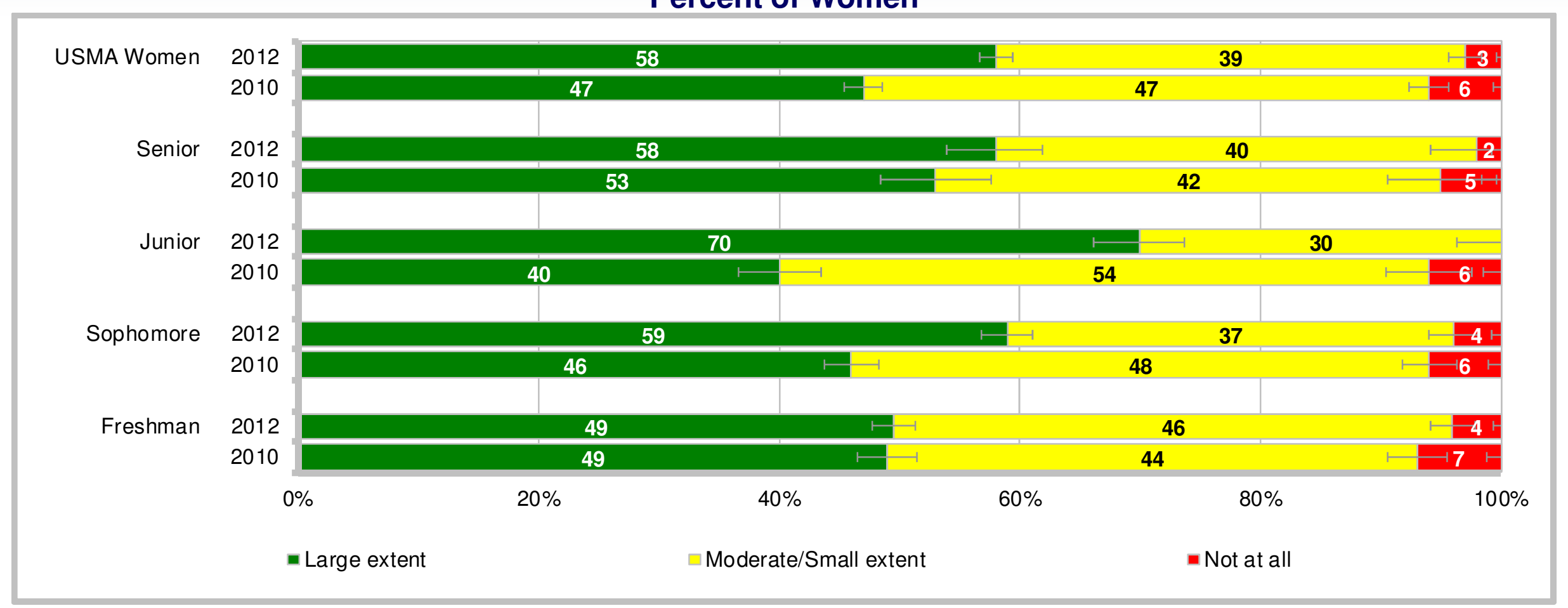

- $97 \%$ of women indicated they would point out to someone that they "crossed the line" with comments or jokes to at least some extent; $3 \%$ indicated not at all

- 2012 comparisons across years - large extent higher than 2010; moderate/small extent lower than 2010; not at all lower than 2010

- Juniors and sophomores indicating large extent in 2012 higher than 2010; juniors and sophomores indicating moderate/small extent in 2012 lower than 2010; all class years indicating not at all in 2012 lower than 2010

- Class differences in 2012 - large extent led by juniors; moderate/small extent led by freshmen; not at all led by sophomores and freshmen 


\section{You Would Point Out to Someone That They "Crossed the Line" With Comments or Jokes}

Percent of Men

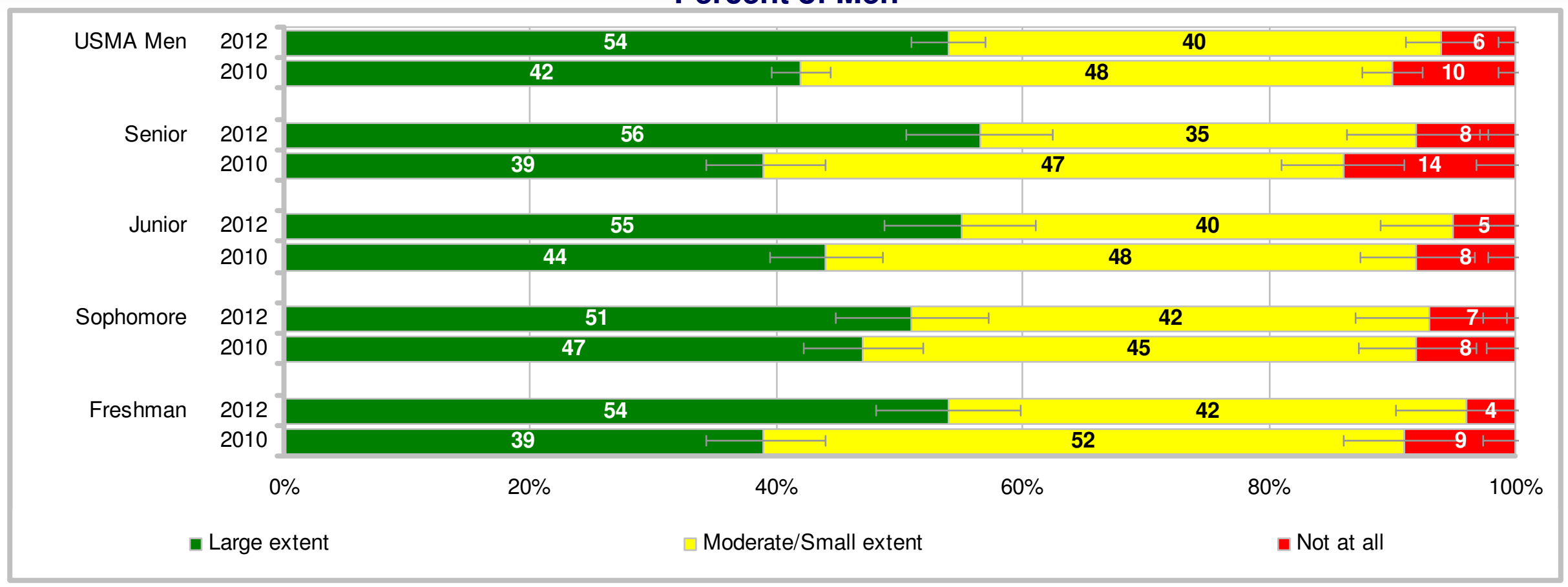

- $94 \%$ of men indicated they would point out to someone that they "crossed the line" with comments or jokes to at least some extent; $6 \%$ indicated not at all

- 2012 comparisons across years - large extent higher than 2010; moderate/small extent lower than 2010; not at all lower than 2010

- Seniors, juniors, and freshmen indicating large extent in 2012 higher than 2010; seniors, juniors, and freshmen indicating moderate/small extent in 2012 lower than 2010; seniors and freshmen indicating not at all in 2012 lower than 2010

- Class differences in 2012 - no differences 


\section{You Would Seek Help From Chain of Command in Stopping Other Students Who Continue To Engage in Sexual Harassment}

\section{Percent of Women}

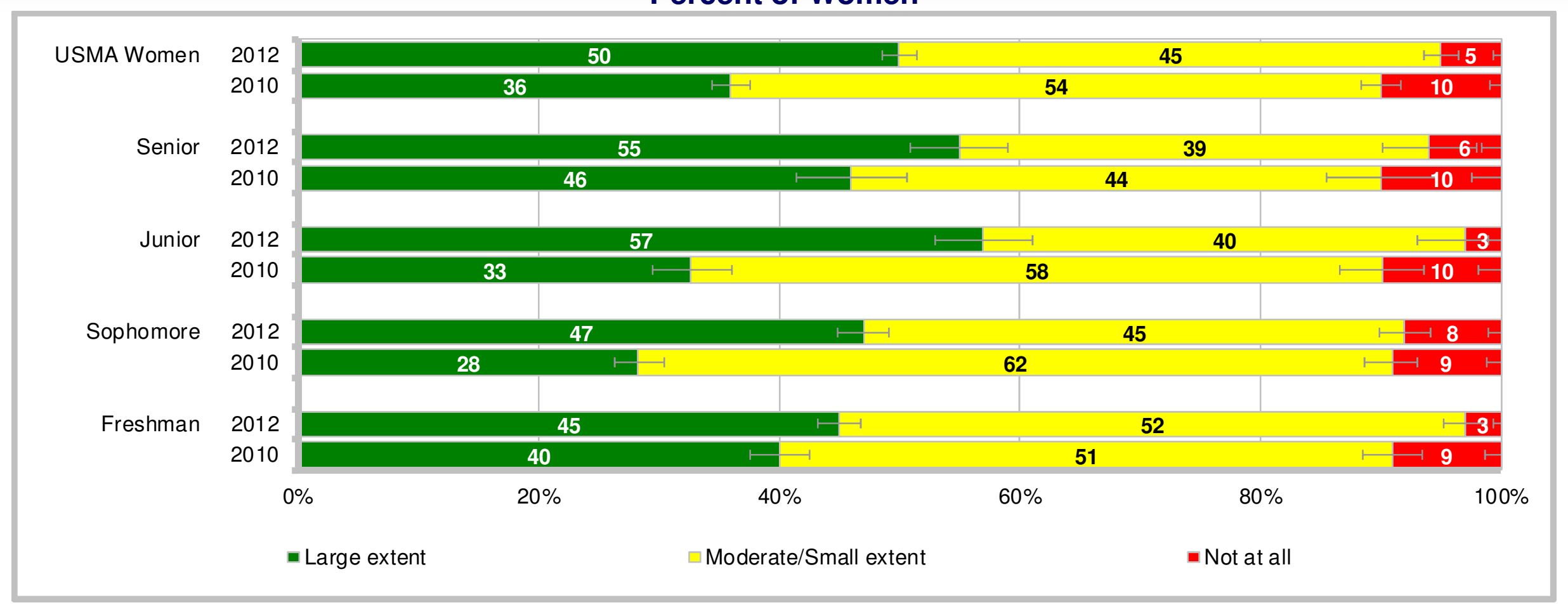

- $95 \%$ of women indicated they would seek help from the chain of command in stopping other students who continue to engage in sexual harassment to at least some extent; $5 \%$ indicated not at all

- 2012 comparisons across years - large extent higher than 2010; moderate/small extent lower than 2010; not at all lower than 2010

- All class years indicating large extent in 2012 higher than 2010; juniors and sophomores indicating moderate/small extent in 2012 lower than 2010; seniors, juniors, and freshmen indicating not at all in 2012 lower than 2010

- Class differences in 2012 - large extent led by juniors and seniors; moderate/small extent led by freshmen; not at all led by sophomores 


\section{You Would Seek Help From Chain of Command in Stopping Other Students Who Continue To Engage in Sexual Harassment \\ Percent of Men}

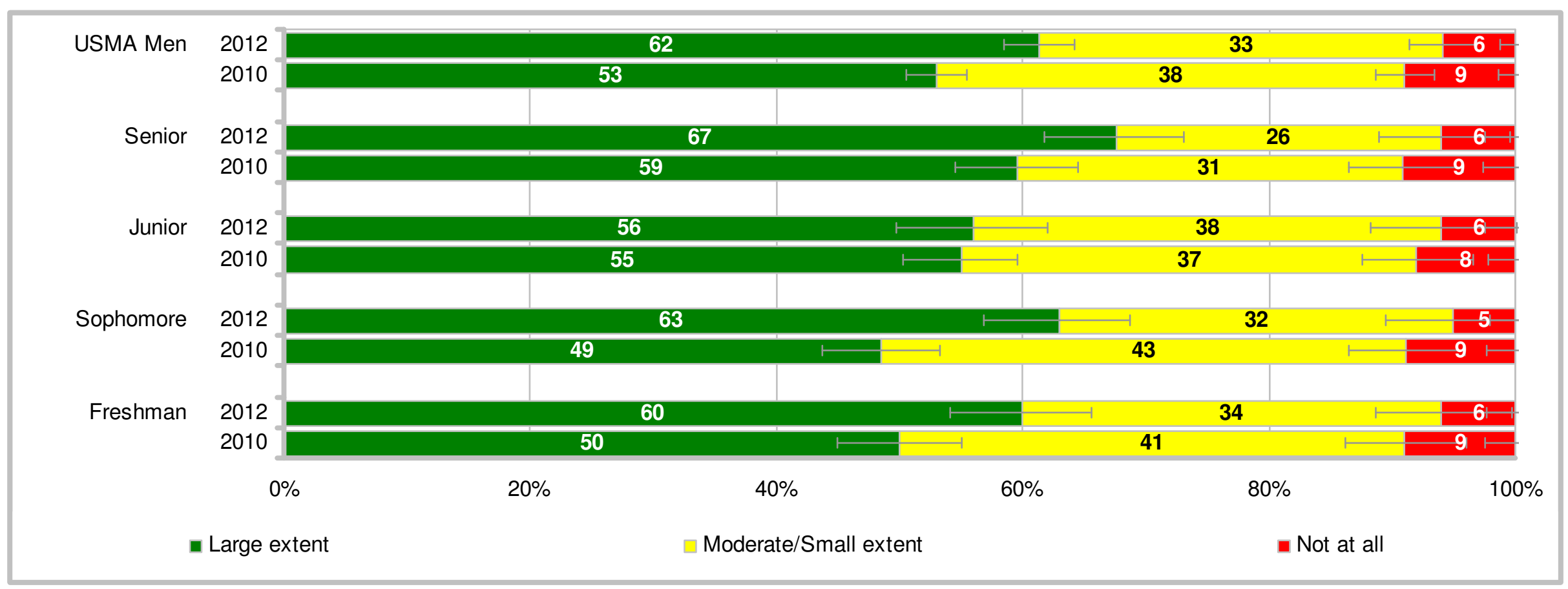

- $95 \%$ of men indicated they would seek help from the chain of command in stopping other students who continue to engage in sexual harassment to at least some extent; and $6 \%$ indicated not at all

- 2012 comparisons across years - large extent higher than 2010; moderate/small extent lower than 2010; not at all lower than 2010

- Seniors, sophomores, and freshmen indicating large extent in 2012 higher than 2010; sophomores indicating moderate/small extent in 2012 lower than 2010; sophomores indicating not at all in 2012 lower than 2010

- Class differences in 2012 - no differences 


\section{Service Academy Gender Relations Survey}

Appendix B: U.S. Naval Academy

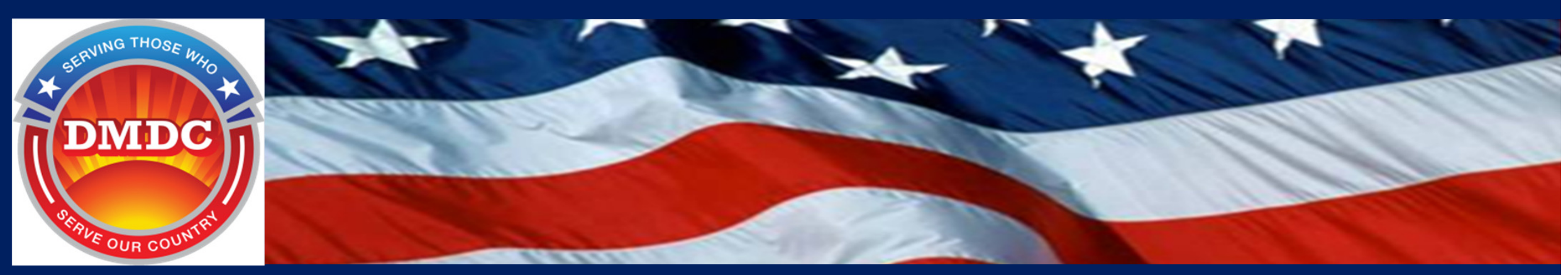




\section{Appendix B \\ U.S. Naval Academy \\ Contents}

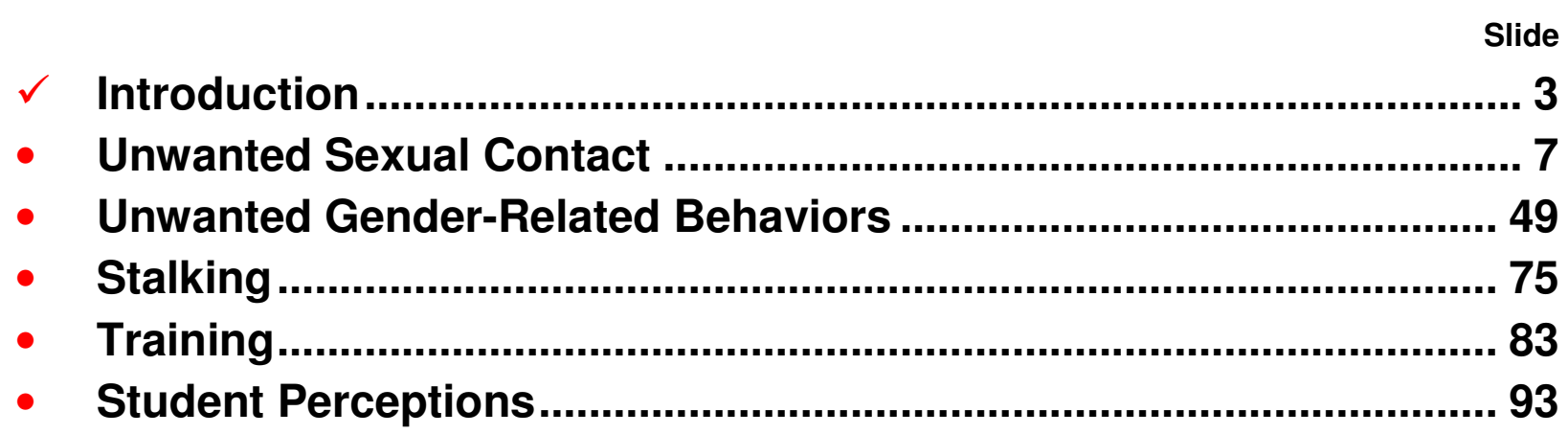




\section{Introduction}

- Paper survey administered to midshipmen in April 2012

- 874 female and 1,308 male students surveyed, weighted response rate of $80 \%$ and $67 \%$, respectively

\section{- Appendix includes results from survey items related to:}

- Results of unwanted sexual contact experienced in the past academic year

- For women who had experienced unwanted sexual contact in the past academic year, details of the incident that had the greatest impact (characteristics of the offender, location, actions taken, reporting, etc.). Details for men are not reportable due to the small number of men who experienced unwanted sexual contact.

- Unwanted sexual contact experienced prior to entering the Academy

- Unwanted gender-related behaviors experienced in the past academic year (sexual harassment, crude and offensive behavior, unwanted sexual attention, sexual coercion, and sexist behavior)

- For students who experienced unwanted gender-related behavior in the past academic year, details of the incident that had the greatest impact (characteristics of the offender, reporting)

- Stalking experienced in the past academic year

- Training received in preventing and responding to sexual assault and sexual harassment

- Student perceptions about culture and climate at the Academy

- For each survey item, briefing includes the following:

- Graphic displays of overall results by gender, survey year, and class year

- Tables showing results for grouped items by reporting categories (e.g., gender, survey year, and class year) 


\section{Introduction to Briefing Slides}

\section{- Graphics showing results by reporting categories (gender, survey year, and class year)}

- Statistical tests are used to compare each subgroup to previous survey years and other class years

- For items with trends, 2012 survey year is compared to the other survey years $(2006,2008,2010)$

- For the current 2012 survey year, each class year (e.g., freshmen) is compared to the average of the other class years (sophomore, junior, senior)

- Results are presented on the bottom of the slide for survey year and class year comparisons that are statistically significant

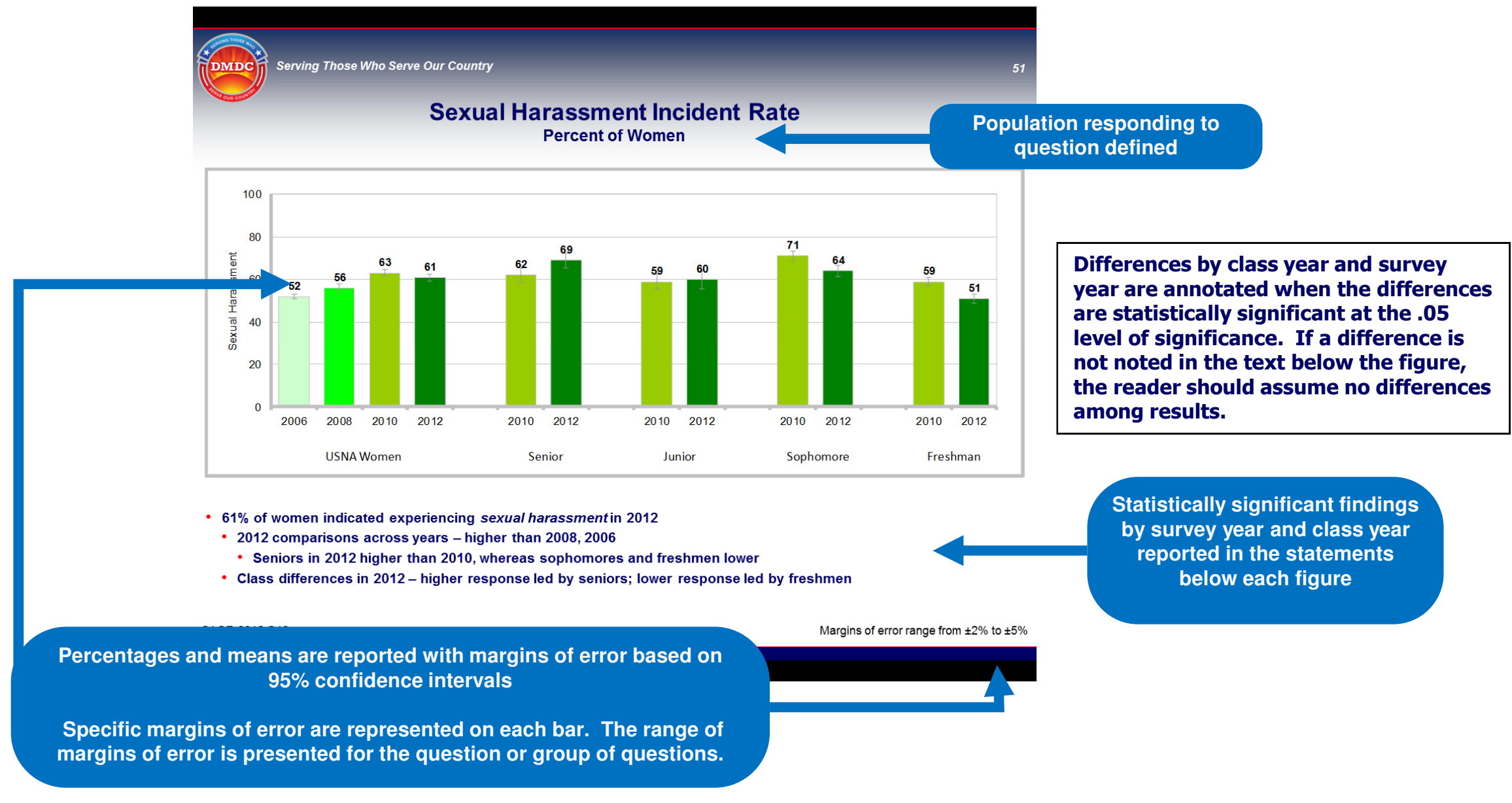




\section{Introduction to Briefing Slides}

\section{- Tables showing results for grouped items by reporting categories (gender, survey year,} and class year)

- Statistical tests are used to compare each subgroup to previous survey years and other class years

- For items with trends, 2012 survey year is compared to the other survey years $(2006,2008,2010)$

- For the current 2012 survey year, each class year (e.g., freshmen) is compared to the average of the other class years (sophomore, junior, senior)

- Results are presented within the table for survey year and class year comparisons that are statistically significant

Most Frequently Selected Reasons for Not Reporting Unwanted Sexual Contact Situation Percent of Women Who Experienced Unwanted Sexual Contact and Did Not Report It

\begin{tabular}{|c|c|c|c|c|c|c|}
\hline Reasons for Not Reporting & $\begin{array}{c}\text { Survey } \\
\text { Year }\end{array}$ & Total & Senior & Junior & Sophomore & Freshman \\
\hline \multirow{2}{*}{ Took care of it myself } & 2012 & $77 \uparrow$ & $79 \uparrow$ & 72 & $81 \uparrow$ & $80 \downarrow$ \\
\hline & 2010 & 67 & 58 & 64 & 63 & 95 \\
\hline \multirow{2}{*}{ Did not want people gossiping } & 2012 & 71 & 57 & $89 \uparrow$ & 71 & $90 \uparrow$ \\
\hline & 2010 & 71 & 63 & 68 & 76 & 68 \\
\hline \multirow{2}{*}{ Did not want anyone to know } & 2012 & $68 \uparrow$ & 50 & $83 \uparrow$ & 71 & $90 \uparrow$ \\
\hline & 2010 & 61 & 63 & 56 & 70 & 47 \\
\hline \multirow{2}{*}{$\begin{array}{l}\text { Thought it was not important enough to } \\
\text { report }\end{array}$} & 2012 & 63 & $68 \uparrow$ & 61 & 55 & 90 \\
\hline & 2010 & 64 & 53 & 68 & 59 & 84 \\
\hline \multirow{2}{*}{ Felt shame/embarrassment } & 2012 & 57 & $39 \downarrow$ & 56 & 68 & $90 \uparrow$ \\
\hline & 2010 & 56 & 63 & 48 & 61 & 53 \\
\hline \multirow{2}{*}{ Felt uncomfortable making a report } & 2012 & 55 & $43 \downarrow$ & 61 & 55 & 70 \\
\hline & 2010 & 59 & 63 & 56 & 57 & 63 \\
\hline \multirow{3}{*}{$\begin{array}{l}\text { Thought it would hurt my reputation and } \\
\text { standing }\end{array}$} & 2012 & 55 & 43 & $72 \uparrow$ & 58 & 50 \\
\hline & 2010 & 56 & 53 & 44 & 63 & 58 \\
\hline & & $\pm 4-5 \%$ & $\pm 9-11 \%$ & $\pm 9-13 \%$ & $\pm 5-8 \%$ & \\
\hline
\end{tabular}

\section{Higher Response of Yes} Lower Response of Yes \begin{tabular}{|l|l|}
\hline Higher in 2012 than 2010 & $\uparrow$ \\
\hline Lower in 2012 than 2010 & $\downarrow$ \\
\hline
\end{tabular}
Percentages and means are reported with margins of error based on $95 \%$ confidence intervals.

The range of margins of error is presented for the question or group of questions.
Population responding to question defined

Arrows indicate whether there is a statistically significant change between 2012 and 2010 .

Color coding indicates differences by class year.

Only class differences in 2012 are reported.

Differences by class year and survey

year are annotated when the

differences are statistically significant at the .05 level of significance. If a difference is not noted within the table, the reader should assume no differences among results. 


\section{Contents}

$\checkmark$ Unwanted Sexual Contact ............................................................. 7

- Most Serious Behaviors Experienced.......................................... 11

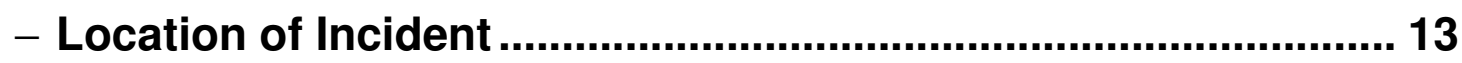

- Summer Experience......................................................................... 15

- Characteristics of Offender............................................................. 17

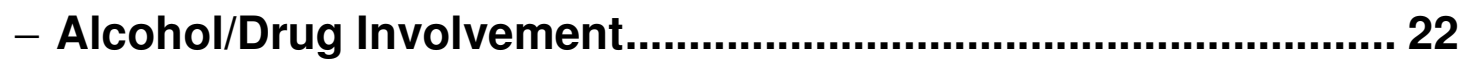

- Use of Threats/Force ................................................................ 27

- Experience of Sexual Harassment/Stalking ................................ 32

- Consequences of the Situation ..................................................... 25

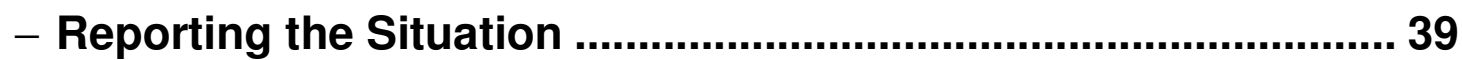

- Experience of Unwanted Sexual Contact Prior to Entering the Academy .............................................................................. 47

- Unwanted Gender-Related Behaviors ............................................. 49

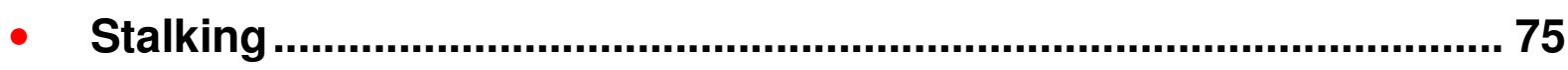

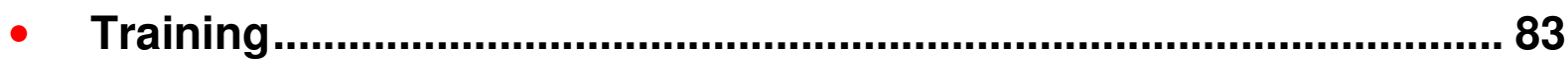

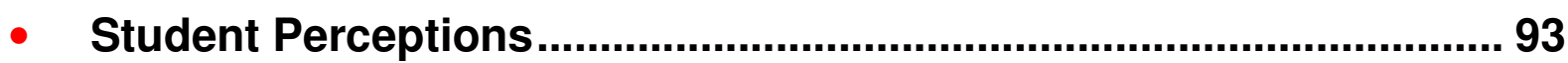




\section{Unwanted Sexual Contact Incident Rates}

\section{- Definition and measure of unwanted sexual contact:}

- The 2012 SAGR survey includes a measure of unwanted sexual contact (i.e., sexual assault). Although this term does not appear in the Uniform Code of Military Justice $(\mathrm{UCMJ})$, it is used to refer to a range of activities that is used as an umbrella term intended to include certain acts prohibited by the UCMJ.

- Unwanted sexual contact is measured in the 2012 SAGR survey by asking students to refer to experiences since June 2011 in which they experienced any of the following intentional sexual contacts that were against their will or which occurred when they did not or could not consent in which someone...

- Sexually touched them (e.g., intentional touching of genitalia, breasts, or buttocks) or made them sexually touch someone,

- Attempted to make them have sexual intercourse, but was not successful,

- Made them have sexual intercourse,

- Attempted to make them perform or receive oral sex, anal sex, or penetration by a finger or object, but was not successful, or

- Made them perform or receive oral sex, anal sex, or penetration by a finger or object.

- A student is counted in the unwanted sexual contact incident rate if he or she replied "yes" to any of the behaviors listed.

- The 2012 SAGR survey was administered in March and April 2012. The timeframe looking back to June 2011 is intended to include behaviors experienced in the majority of Academic Program Year 2011-2012 to be consistent with the timeframe for reporting incidents of sexual assault at the Academies. 


\section{Unwanted Sexual Contact Incident Rate Percent of Women}

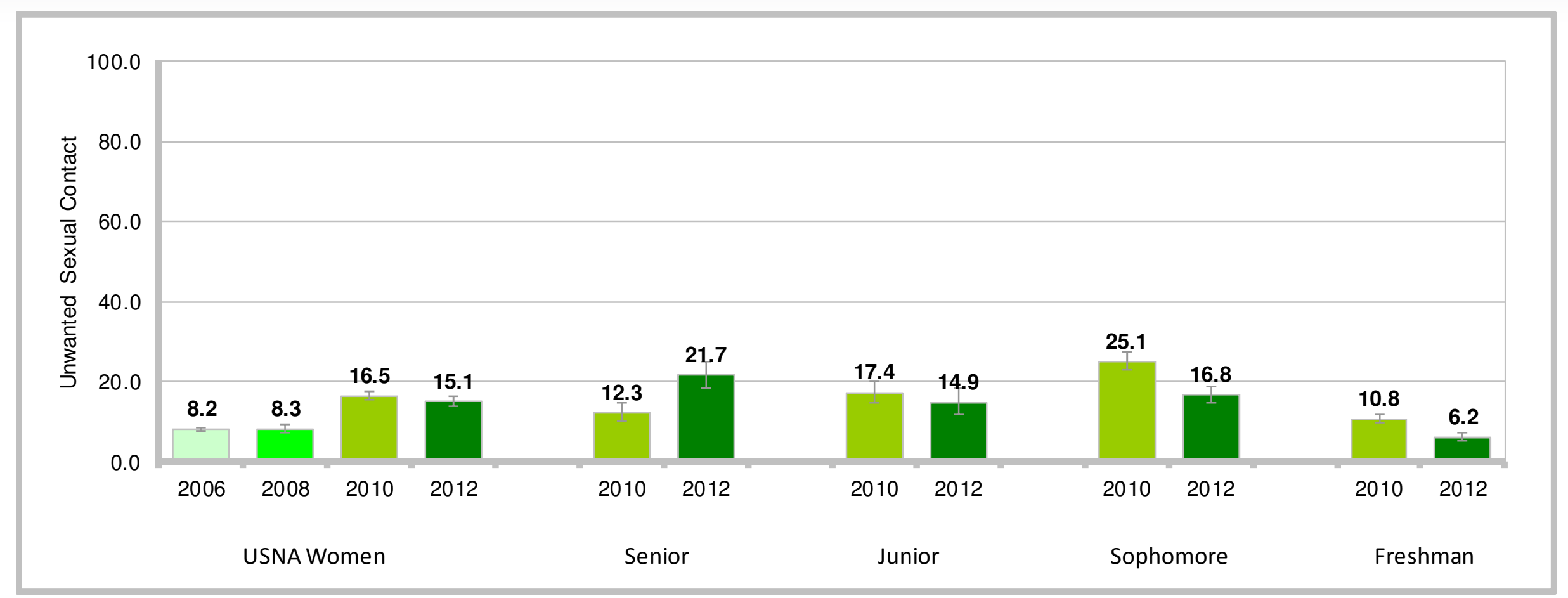

- $15.1 \%$ of women indicated experiencing unwanted sexual contact in 2012

- 2012 comparisons across years - higher than 2008, 2006

- Seniors in 2012 higher than 2010, whereas sophomores and freshmen lower

- Class differences in 2012 - higher response led by seniors; lower response led by freshmen 


\section{Unwanted Sexual Contact Incident Rate}

Percent of Men

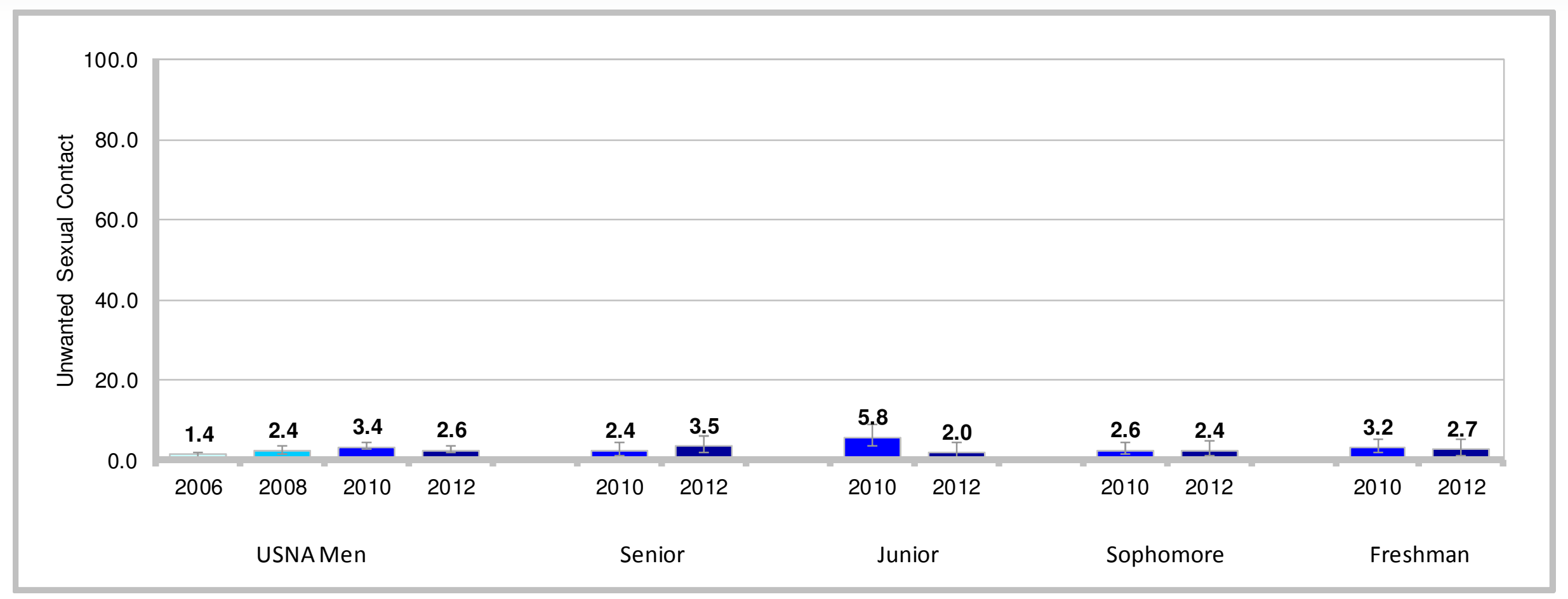

- $2.6 \%$ of men indicated experiencing unwanted sexual contact in 2012

- 2012 comparisons across years - higher than 2006

- Juniors in 2012 lower than 2010

- Class differences in 2012 - no differences 


\section{Contents}

Slide

- Introduction ............................................................................................ 3

- Unwanted Sexual Contact ................................................................ 7

$\checkmark$ Most Serious Behaviors Experienced...................................... 11

- Location of Incident ........................................................................ 13

- Summer Experience................................................................... 15

- Characteristics of Offender......................................................... 17

- Alcohol/Drug Involvement........................................................... 22

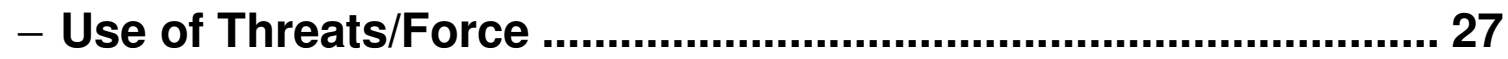

- Experience of Sexual Harassment/Stalking ............................. 32

- Consequences of the Situation .................................................. 35

- Reporting the Situation ............................................................ 39

- Experience of Unwanted Sexual Contact Prior to Entering

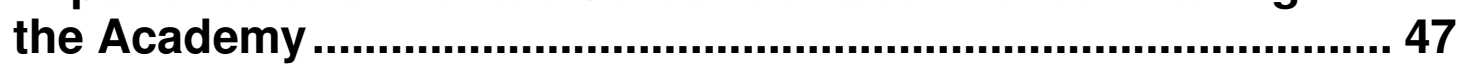

- Unwanted Gender-Related Behaviors ............................................. 49

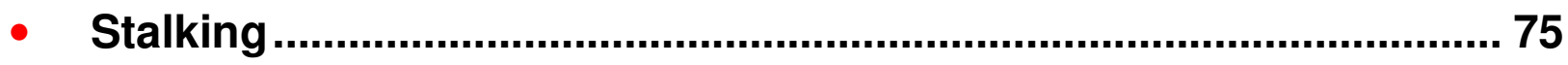

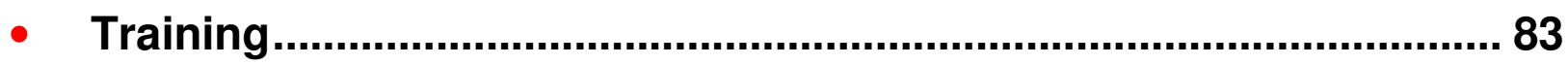

- Student Perceptions .................................................................... 93 


\section{Most Serious Unwanted Sexual Contact Behaviors Experienced Percent of Women Who Experienced Unwanted Sexual Contact}

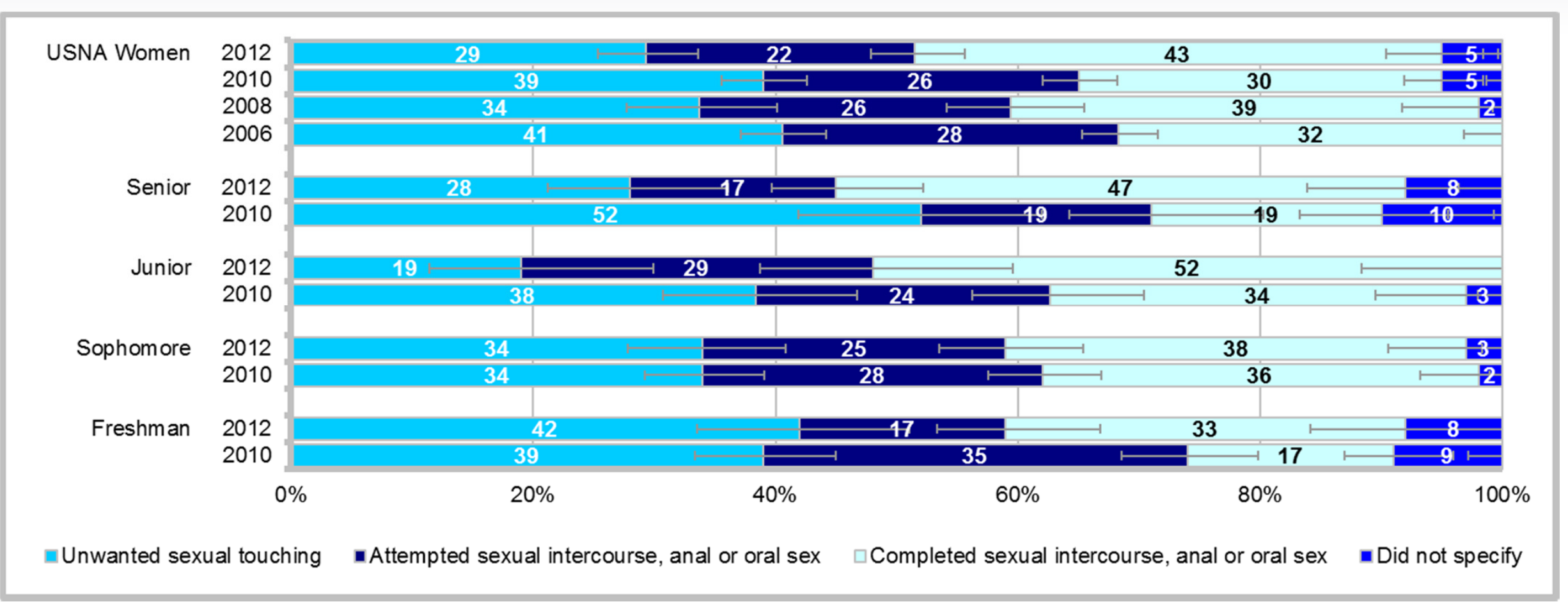

- Of the $15.1 \%$ of women who experienced USC, $29 \%$ indicated experiencing touching; $22 \%$ indicated experiencing attempted sex; $43 \%$ indicated experiencing completed sex; and $5 \%$ did not specify

- 2012 comparisons across years - touching lower than 2010, 2006; attempted sex lower than 2006; completed sex higher than 2010, 2006; did not specify higher than 2008, 2006

- Seniors and juniors indicating touching in 2012 lower than 2010; freshmen indicating attempted sex in 2012 lower than 2010; seniors, juniors, and freshmen indicating completed sex in 2012 higher than 2010

- Class differences in 2012 - higher response of touching led by freshmen 


\section{Contents}

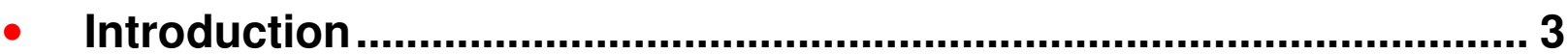

- Unwanted Sexual Contact ..................................................................... 7

- Most Serious Behaviors Experienced........................................ 11

$\checkmark$ Location of Incident ...................................................................... 13

- Summer Experience.................................................................... 15

- Characteristics of Offender...................................................... 17

- Alcohol/Drug Involvement............................................................. 22

- Use of Threats/Force ................................................................... 27

- Experience of Sexual Harassment/Stalking ............................... 32

- Consequences of the Situation ..................................................... 35

- Reporting the Situation .............................................................. 39

- Experience of Unwanted Sexual Contact Prior to Entering the Academy ...................................................................................... 47

- Unwanted Gender-Related Behaviors .............................................. 49

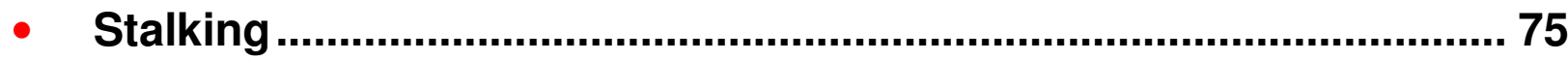

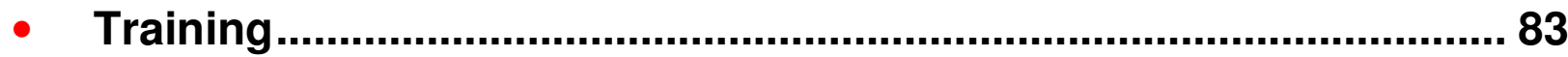

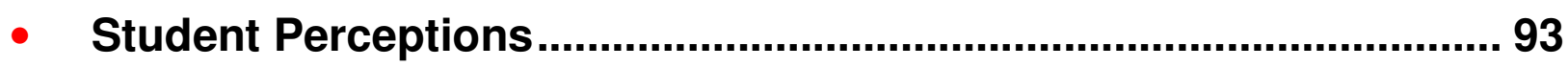




\section{Location of Unwanted Sexual Contact Incident Percent of Women Who Experienced Unwanted Sexual Contact}

\begin{tabular}{|l|c|c|c|c|c|}
\hline \multicolumn{1}{|c|}{ Location of Incident } & Total & Senior & Junior & Sophomore & Freshman \\
\hline On Academy grounds in dormitory/living area & 50 & 44 & 52 & 61 \\
\hline On Academy grounds not in dormitory/living area & 17 & 17 & 14 & 13 & 36 \\
\hline $\begin{array}{l}\text { Off Academy grounds at a social event (such as a } \\
\text { party) }\end{array}$ & 43 & 47 & 43 & 32 \\
\hline $\begin{array}{l}\text { Off Academy grounds at an Academy sponsored } \\
\text { event }\end{array}$ & 9 & 8 & 10 & 10 & 9 \\
\hline Off Academy grounds other & 25 & 36 & 24 & 16 & 18 \\
\hline Margins of Error & $\pm 4-5 \%$ & $\pm 6-9 \%$ & $\pm 10-12 \%$ & $\pm 5-7 \%$ & $\pm 8-10 \%$ \\
\hline
\end{tabular}

\begin{tabular}{|l|l|}
\hline Higher Response of Yes & \\
\hline Lower Response of Yes & \\
\hline
\end{tabular}

- New question in 2012; no trend data available 


\section{Contents}

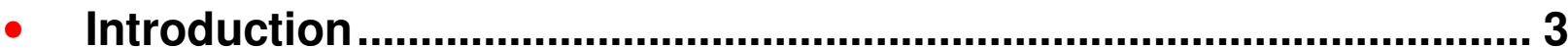

- Unwanted Sexual Contact ................................................................... 7

- Most Serious Behaviors Experienced............................................ 11

- Location of Incident ........................................................................ 13

$\checkmark$ Summer Experience...................................................................... 15

- Characteristics of Offender......................................................... 17

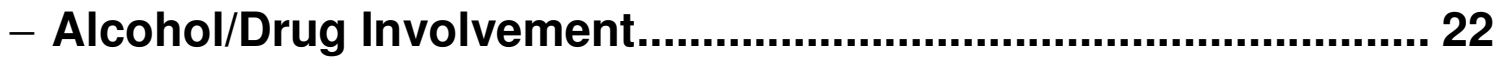

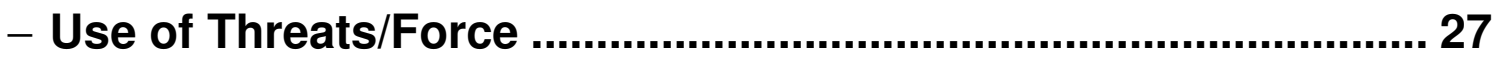

- Experience of Sexual Harassment/Stalking ............................... 32

- Consequences of the Situation ................................................ 35

- Reporting the Situation ..................................................................... 39

- Experience of Unwanted Sexual Contact Prior to Entering the Academy ...................................................................................... 47

- Unwanted Gender-Related Behaviors .............................................. 49

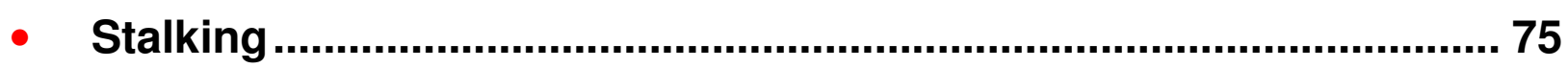

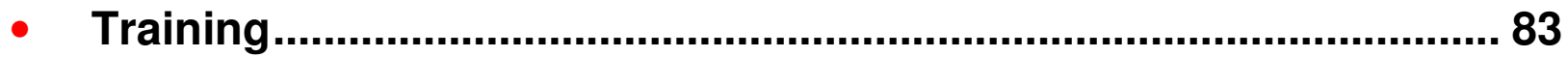

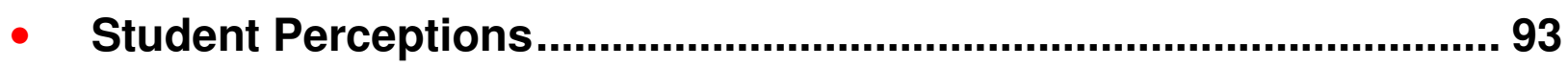




\section{Unwanted Sexual Contact Incident Occurred During Summer Experience/Training/Sea Duty Percent of Women Who Experienced Unwanted Sexual Contact}

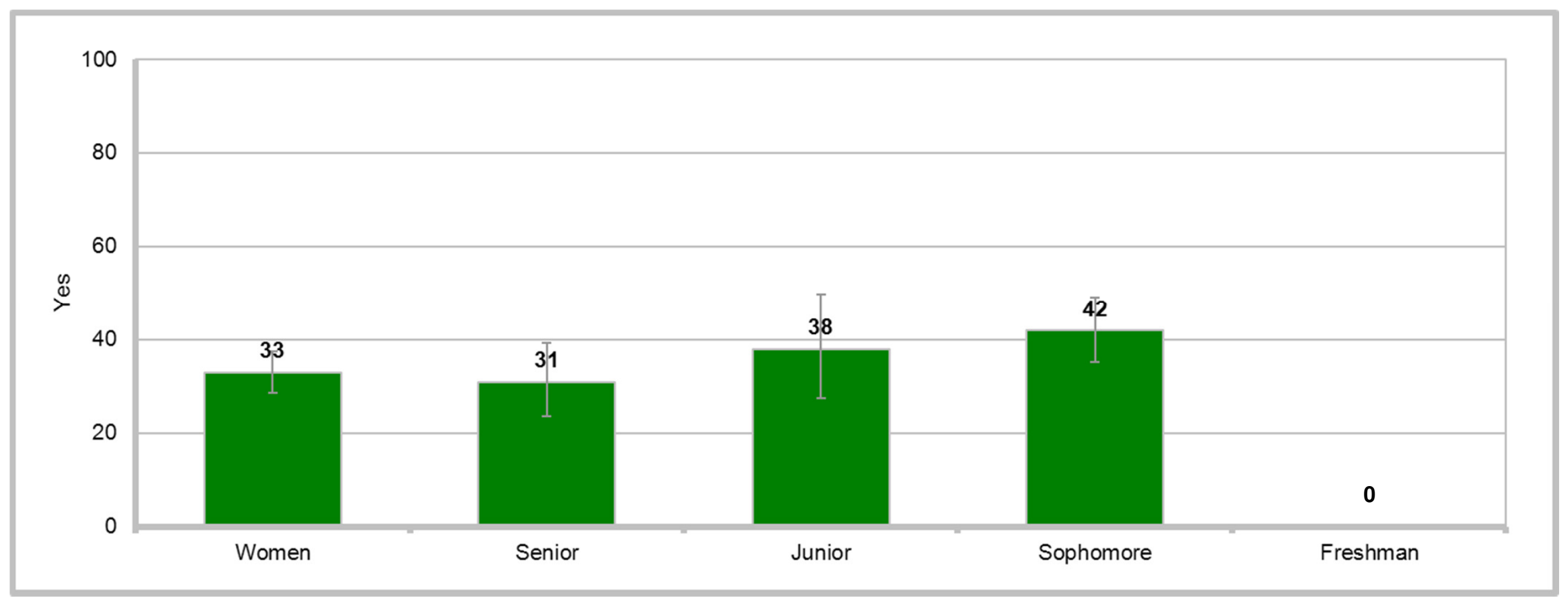

- Of the $15.1 \%$ of women who experienced USC, $33 \%$ indicated the offense occurred during summer experience/ training/sea duty

- 2012 comparisons across years - new question in 2012; no trend data available

- Class differences in 2012 - higher response led by sophomores; lower response led by freshmen 


\section{Contents}

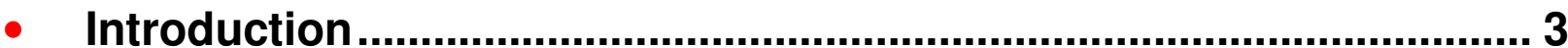

- Unwanted Sexual Contact ................................................................... 7

- Most Serious Behaviors Experienced........................................... 11

- Location of Incident ....................................................................... 13

- Summer Experience...................................................................... 15

$\checkmark$ Characteristics of Offender......................................................... 17

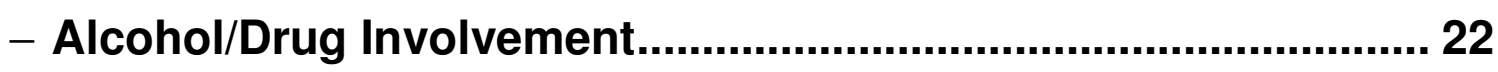

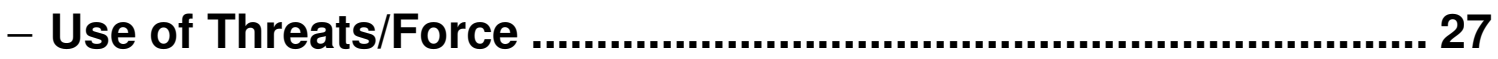

- Experience of Sexual Harassment/Stalking ............................... 32

- Consequences of the Situation ................................................ 35

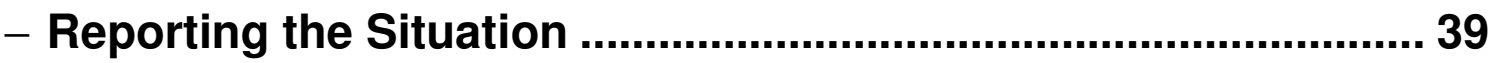

- Experience of Unwanted Sexual Contact Prior to Entering the Academy ...................................................................................... 47

- Unwanted Gender-Related Behaviors .............................................. 49

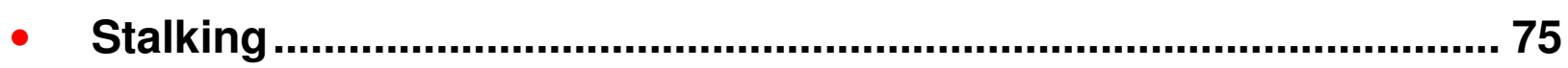

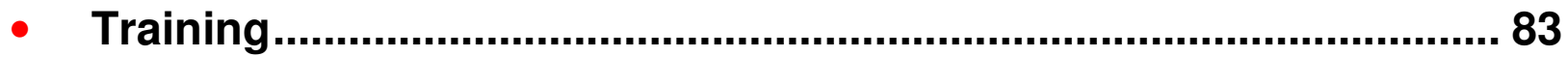

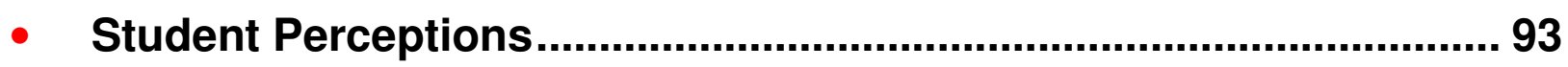




\section{Combinations of Offender Affiliations Percent of Women Who Experienced Unwanted Sexual Contact}

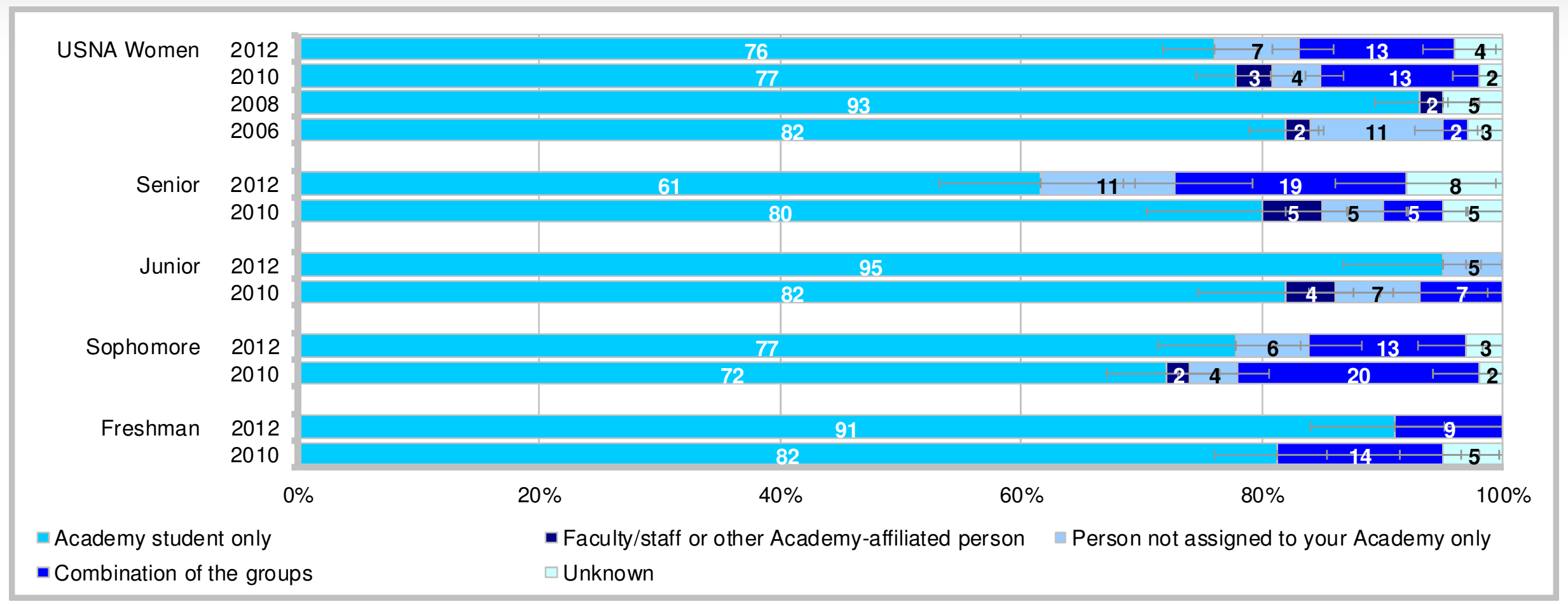

- Of the $15.1 \%$ of women who experienced USC, $76 \%$ indicated the offender was Academy student only; $0 \%$ indicated faculty/staff; $7 \%$ indicated person not assigned to the Academy; $13 \%$ indicated combination of the groups; and $4 \%$ indicated the offender was unknown

- 2012 comparisons across years - Academy student only lower than 2008, 2006; faculty/staff lower than 2010, 2008, 2006; person not assigned to the Academy higher than 2010, 2008, whereas lower than 2006; combination of the groups higher than 2008,2006

- Seniors indicating Academy student only in 2012 lower than 2010, whereas juniors and freshmen higher; seniors, juniors, and sophomores indicating faculty/staff in 2012 lower than 2010; seniors indicating combination of the groups in 2012 higher than 2010 , whereas sophomores lower; freshmen indicating unknown in 2012 lower than 2010

- Class differences in 2012 - Academy student only led by juniors and freshmen; combination of the groups led by seniors; unknown led by seniors 


\section{Gender of the Offender Percent of Women Who Experienced Unwanted Sexual Contact}

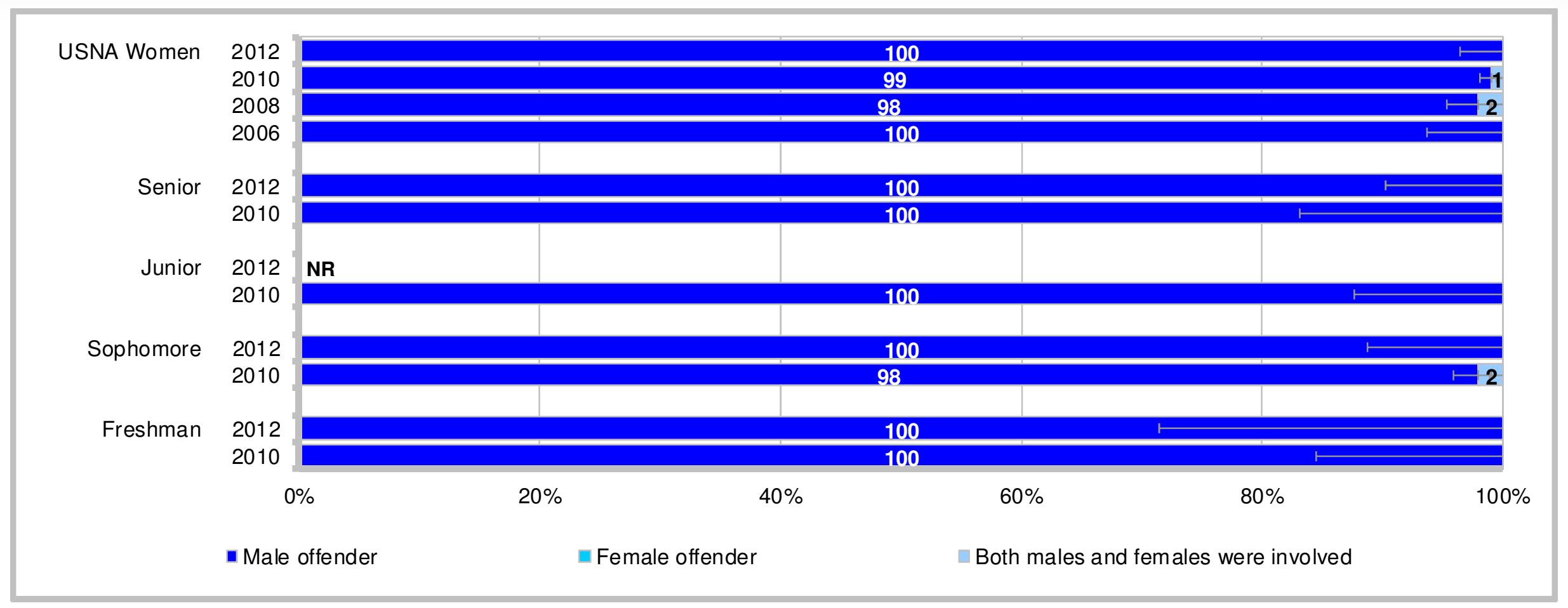

- Of the $15.1 \%$ of women who experienced USC, $100 \%$ indicated the offender was male

- 2012 comparisons across years - male offender higher than 2008; both lower than 2008

- Sophomores indicating male offender in 2012 higher than 2010; sophomores indicating both in 2012 lower than 2010

- Class differences in 2012 - no differences 


\section{Multiple Offenders Involved}

Percent of Women Who Experienced Unwanted Sexual Contact

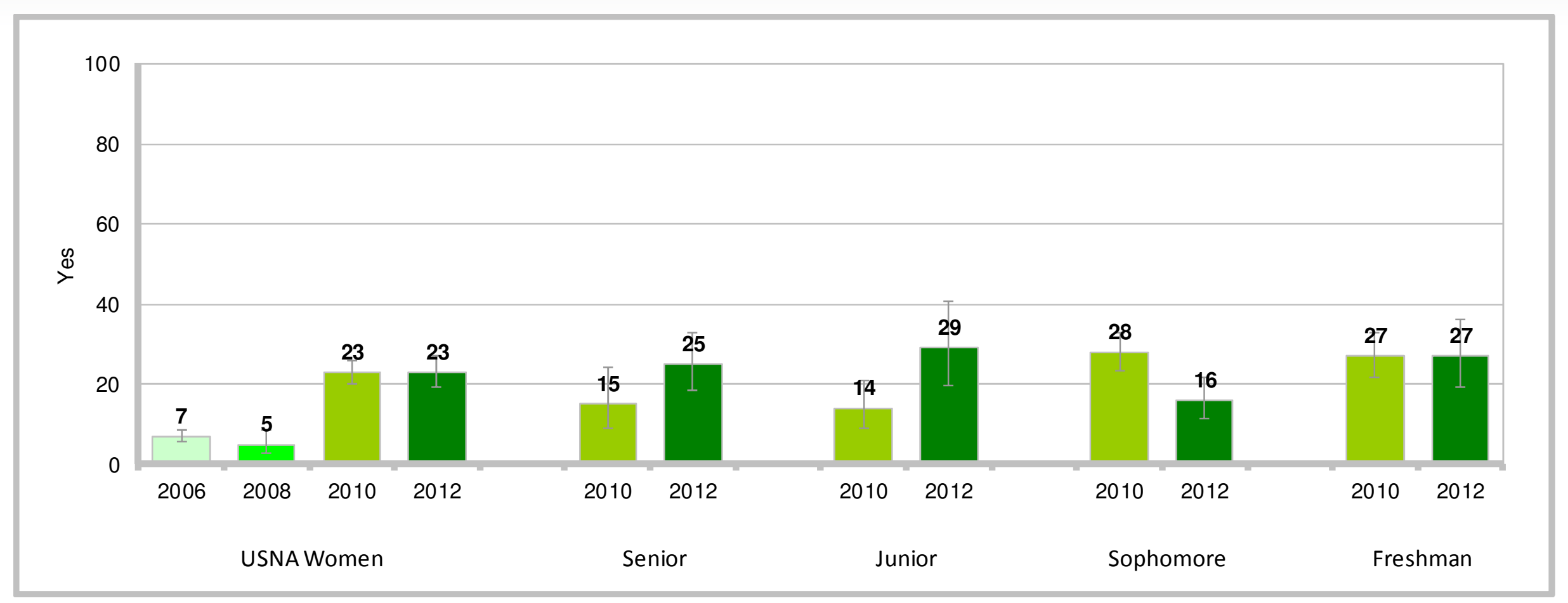

- Of the $15.1 \%$ of women who experienced USC, $23 \%$ indicated multiple offenders were involved

- 2012 comparisons across years - higher than 2008, 2006

- Juniors in 2012 higher than 2010, whereas sophomores lower

- Class differences in 2012 - lower response led by sophomores 


\section{Offender Was a "Creeper" (i.e., someone who is socially awkward)} Percent of Women Who Experienced Unwanted Sexual Contact Involving an Academy Student

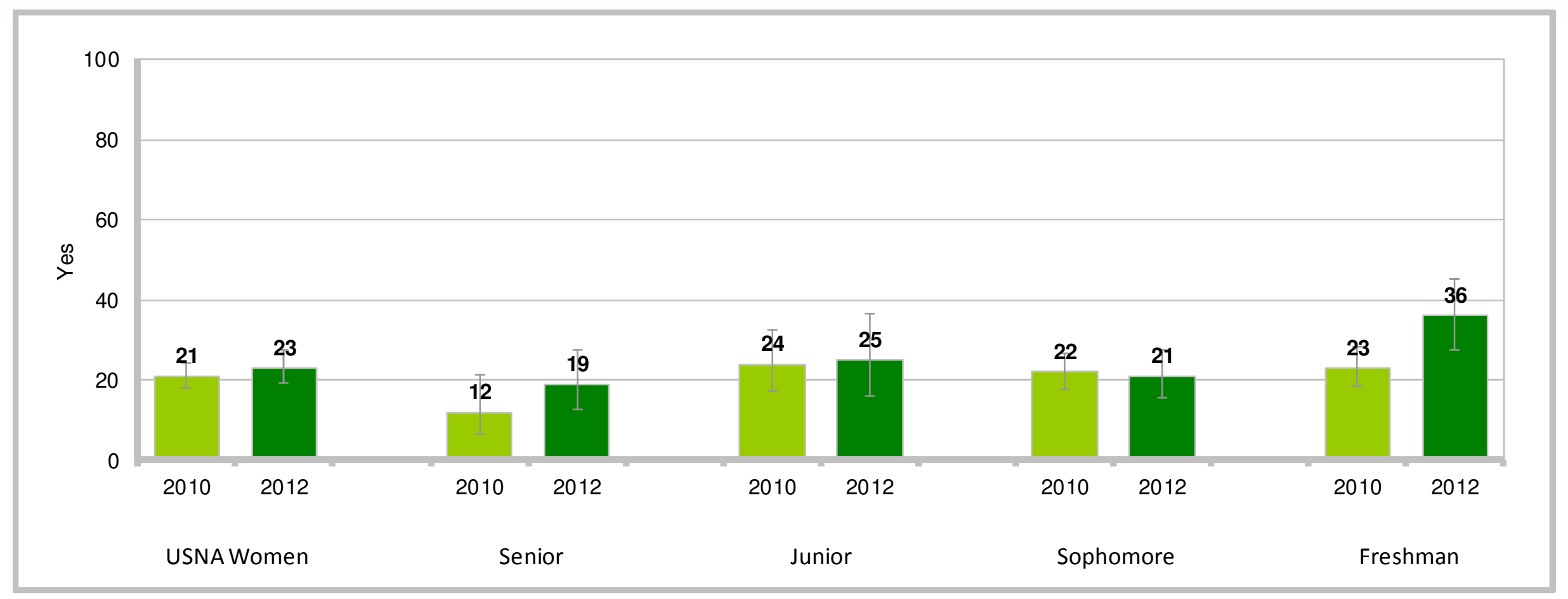

- Of the $15.1 \%$ of women who experienced USC, $23 \%$ indicated the offender was a "creeper"

- 2012 comparisons across years - no differences

- Freshmen in 2012 higher than 2010

- Class differences in 2012 - higher response led by freshmen 


\section{Contents}

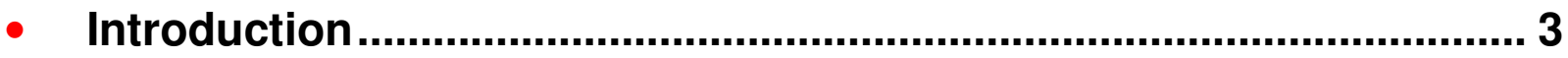

- Unwanted Sexual Contact ............................................................... 7

- Most Serious Behaviors Experienced....................................... 11

- Location of Incident ..................................................................... 13

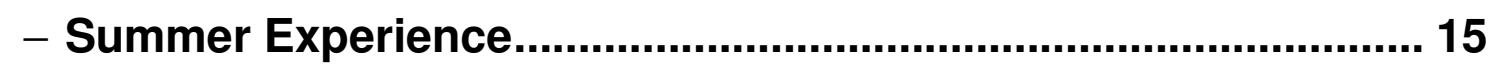

- Characteristics of Offender....................................................... 17

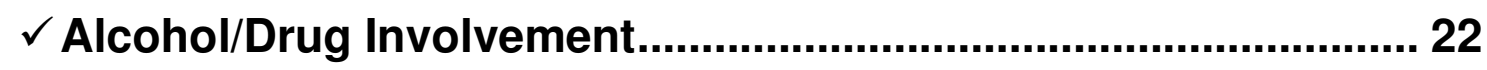

- Use of Threats/Force .............................................................. 27

- Experience of Sexual Harassment/Stalking ............................... 32

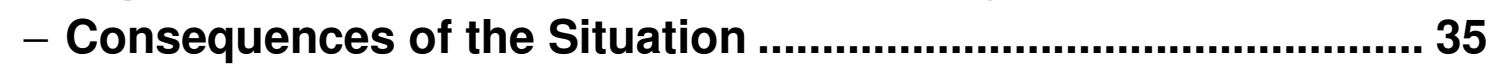

- Reporting the Situation ............................................................ 39

- Experience of Unwanted Sexual Contact Prior to Entering

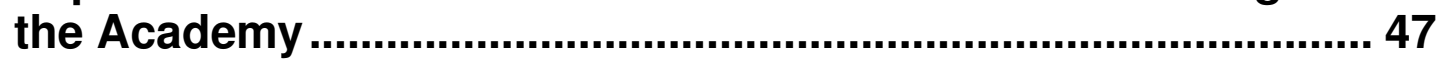

- Unwanted Gender-Related Behaviors ................................................ 49

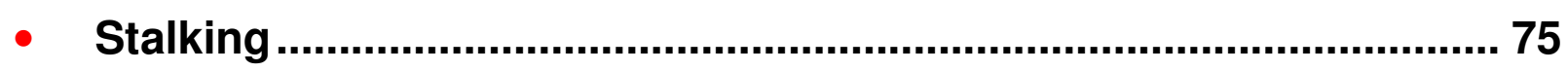

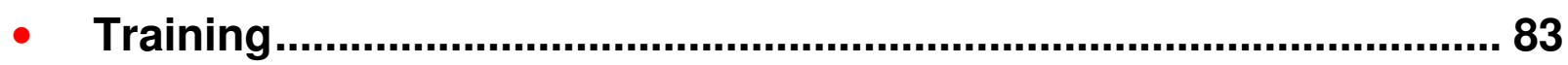

- Student Perceptions.................................................................... 93 


\section{Use of Alcohol and/or Drugs in the Incident} Percent of Women Who Experienced Unwanted Sexual Contact

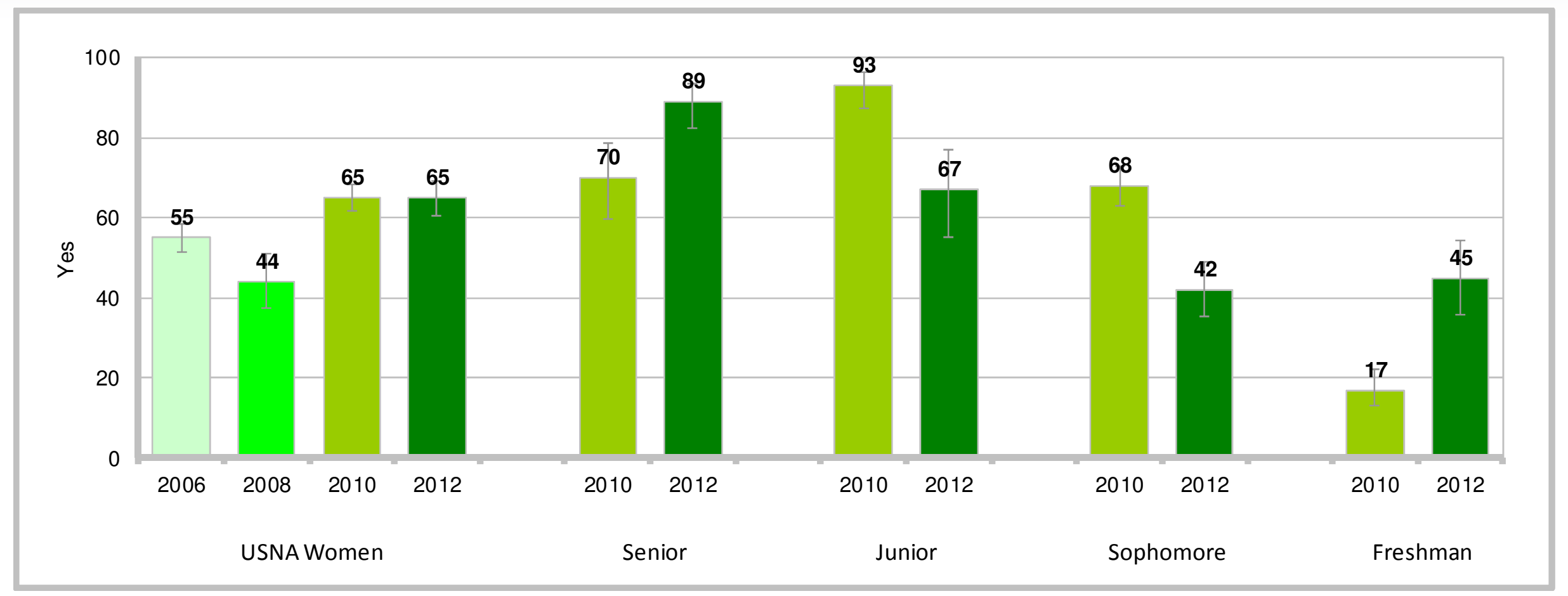

- Of the $15.1 \%$ of women who experienced USC, $65 \%$ indicated alcohol and/or drugs were involved

- 2012 comparisons across years - higher than 2008, 2006

- Seniors and freshmen in 2012 higher than 2010, whereas juniors and sophomores lower

- Class differences in $\mathbf{2 0 1 2}$ - higher response led by seniors; lower response led by sophomores and freshmen 


\section{Use of Alcohol Before the Incident} Percent of Women Who Experienced Unwanted Sexual Contact

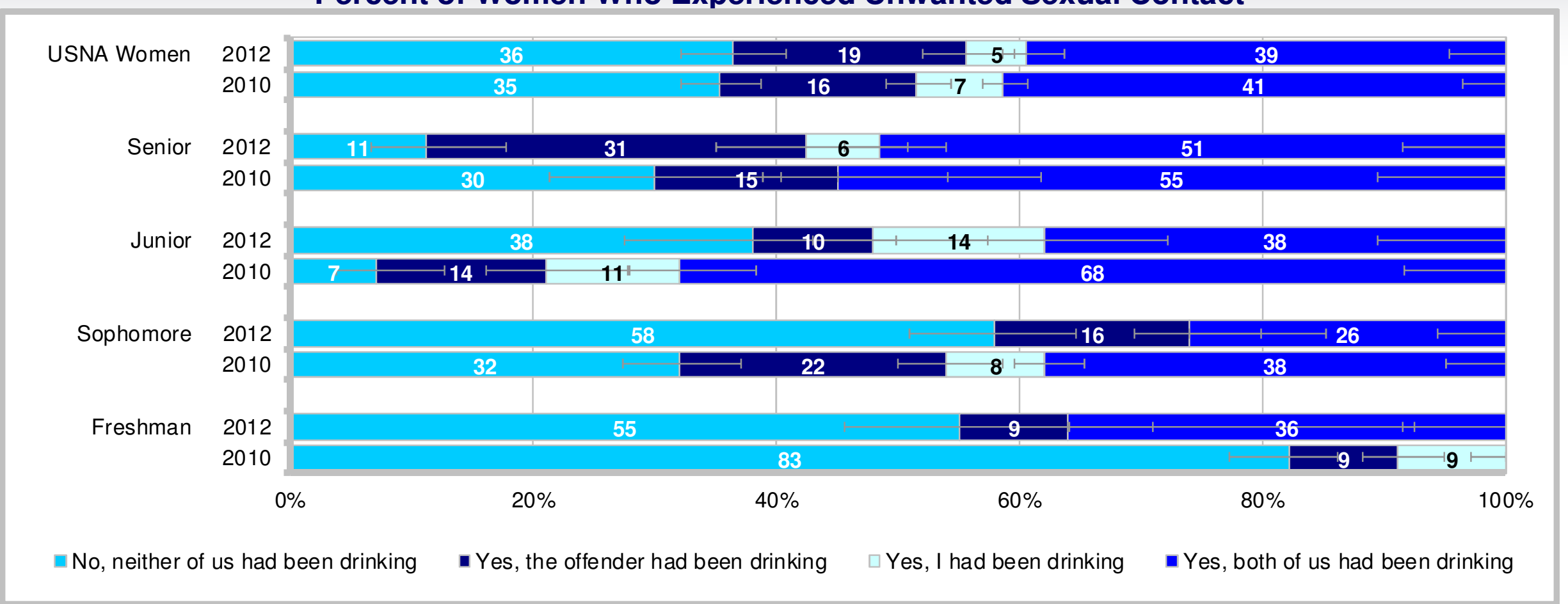

- Of the $15.1 \%$ of women who experienced USC, $36 \%$ indicated neither of them had been drinking; $19 \%$ indicated the offender had been drinking; $5 \%$ indicated they had been drinking; and 39\% indicated both had been drinking

- 2012 comparisons across years - no differences

- Seniors and freshmen indicating neither in 2012 lower than 2010, whereas juniors and sophomores higher; seniors indicating the offender in 2012 higher than 2010; seniors indicating they had in 2012 higher than 2010, whereas sophomores and freshmen lower; juniors and sophomores indicating both in 2012 lower than 2010, whereas freshmen higher

- Class differences in 2012 - neither led by freshmen and sophomores; the offender led by seniors; they had led by juniors; both led by seniors 


\section{Use of Drugs Before the Incident Percent of Women Who Experienced Unwanted Sexual Contact}

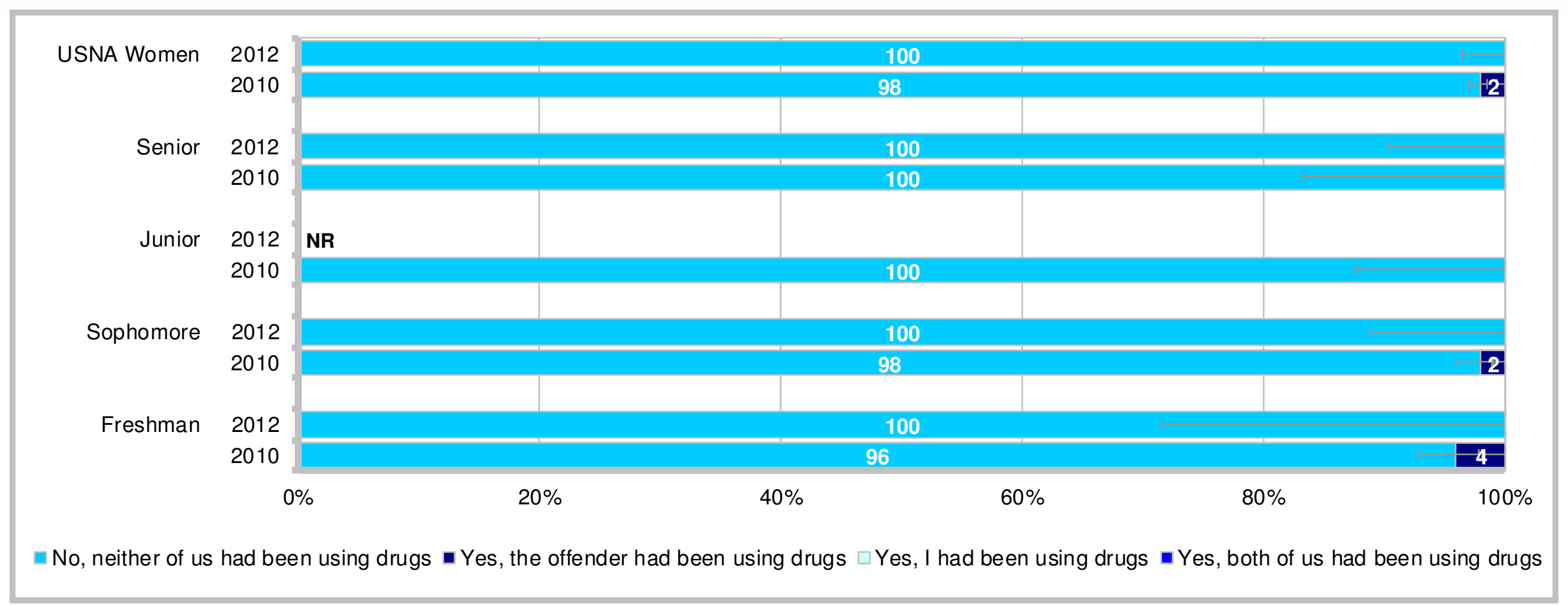

- Of the $15.1 \%$ of women who experienced USC, $100 \%$ indicated neither of them had been using drugs

- 2012 comparisons across years - neither higher than 2010; the offender lower than 2010

- Sophomores and freshmen indicating neither in 2012 higher than 2010; sophomores and freshmen indicating the offender in 2012 lower than 2010

- Class differences in 2012 - no differences 


\section{Use of Knock Out Drugs Before the Incident Percent of Women Who Experienced Unwanted Sexual Contact}

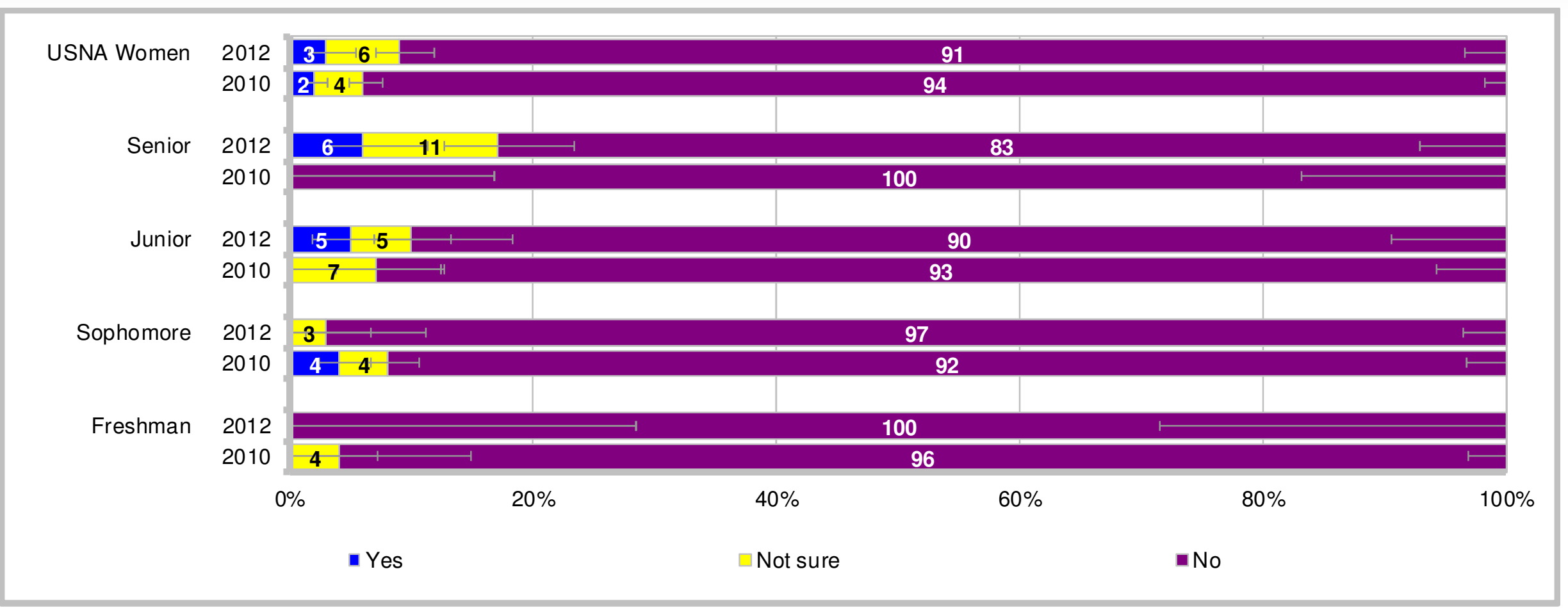

- Of the $15.1 \%$ of women who experienced USC, $91 \%$ indicated the offender did not use drugs to knock them out; $6 \%$ indicated they were not sure; $3 \%$ indicated the offender used drugs to knock them out

- 2012 comparisons across years - no differences

- Seniors indicating the offender did not use drugs to knock them out in 2012 lower than 2010, whereas sophomores and freshmen higher; seniors indicating not sure in 2012 higher than 2010, whereas freshmen lower; seniors and juniors indicating the offender used drugs to knock them out in 2012 higher than 2010, whereas sophomores lower

- Class differences in 2012 - not sure led by seniors; did not use drugs to knock them out led by freshmen and sophomores 


\section{Contents}

Slide

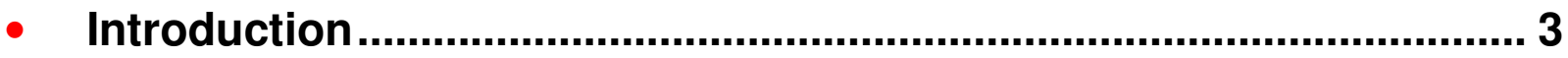

- Unwanted Sexual Contact ............................................................... 7

- Most Serious Behaviors Experienced....................................... 11

- Location of Incident ..................................................................... 13

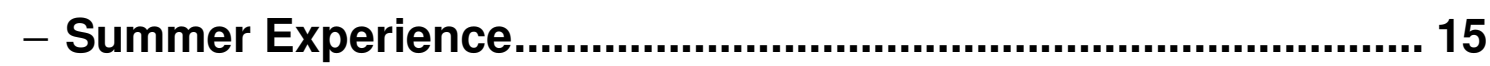

- Characteristics of Offender......................................................... 17

- Alcohol/Drug Involvement......................................................... 22

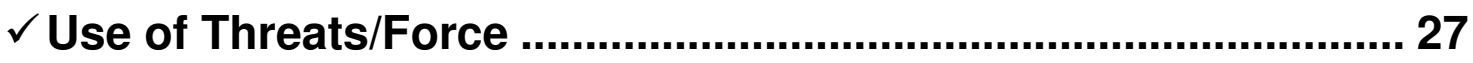

- Experience of Sexual Harassment/Stalking .............................. 32

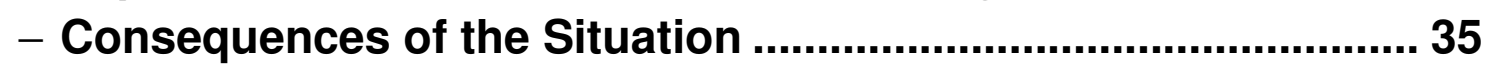

- Reporting the Situation ........................................................... 39

- Experience of Unwanted Sexual Contact Prior to Entering

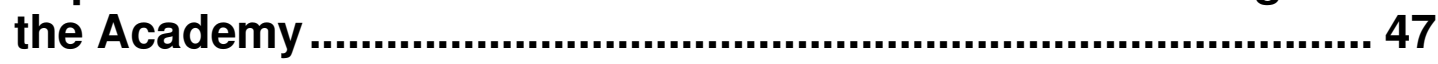

- Unwanted Gender-Related Behaviors ................................................ 49

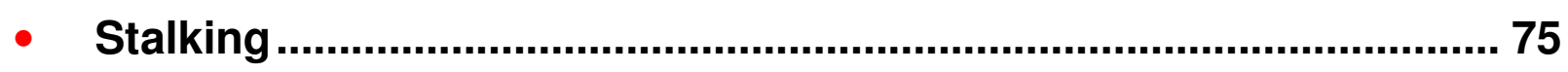

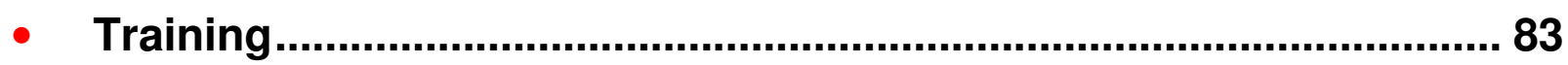

- Student Perceptions.................................................................... 93 


\section{Use of Threats and Physical Force in the Incident Percent of Women Who Experienced Unwanted Sexual Contact}

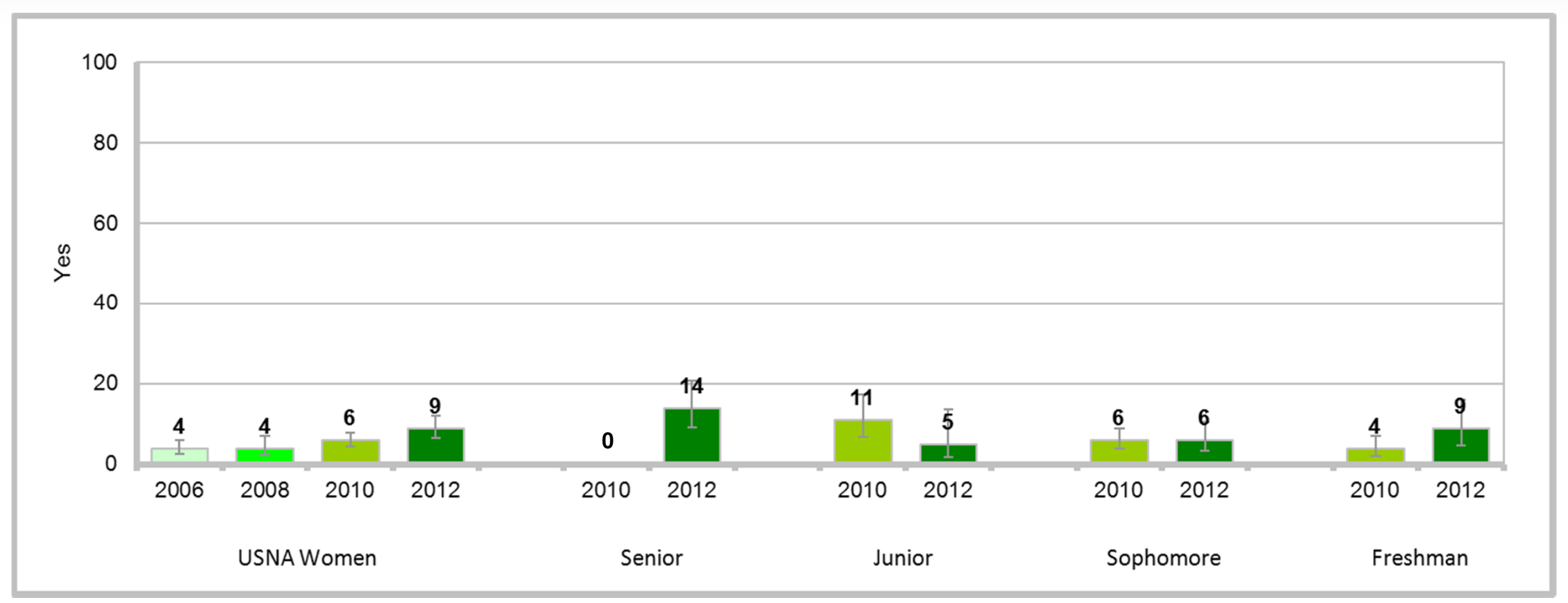

- Of the $15.1 \%$ of women who experienced USC, $9 \%$ indicated the situation involved threats and force

- 2012 comparisons across years - higher than 2008, 2006

- Seniors in 2012 higher than 2010

- Class differences in 2012 - no differences 


\section{Offender Threatened To Ruin Your Reputation if You Did Not Consent Percent of Women Who Experienced Unwanted Sexual Contact}

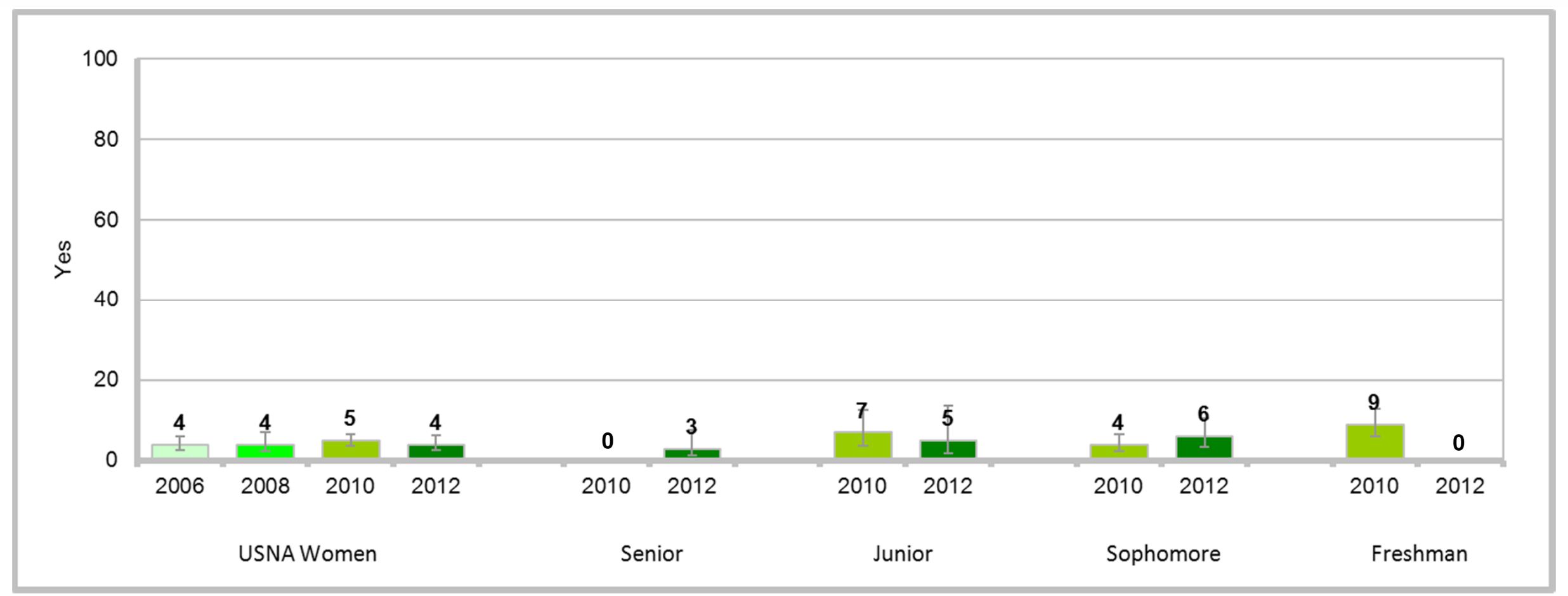

- Of the $15.1 \%$ of women who experienced USC, $4 \%$ indicated the offender threatened to ruin their reputation

- 2012 comparisons across years - no differences

- Seniors in 2012 higher than 2010, whereas freshmen lower

- Class differences in $\mathbf{2 0 1 2}$ - lower response led by freshmen 


\section{Offender Threatened To Harm You if You Did Not Consent Percent of Women Who Experienced Unwanted Sexual Contact}

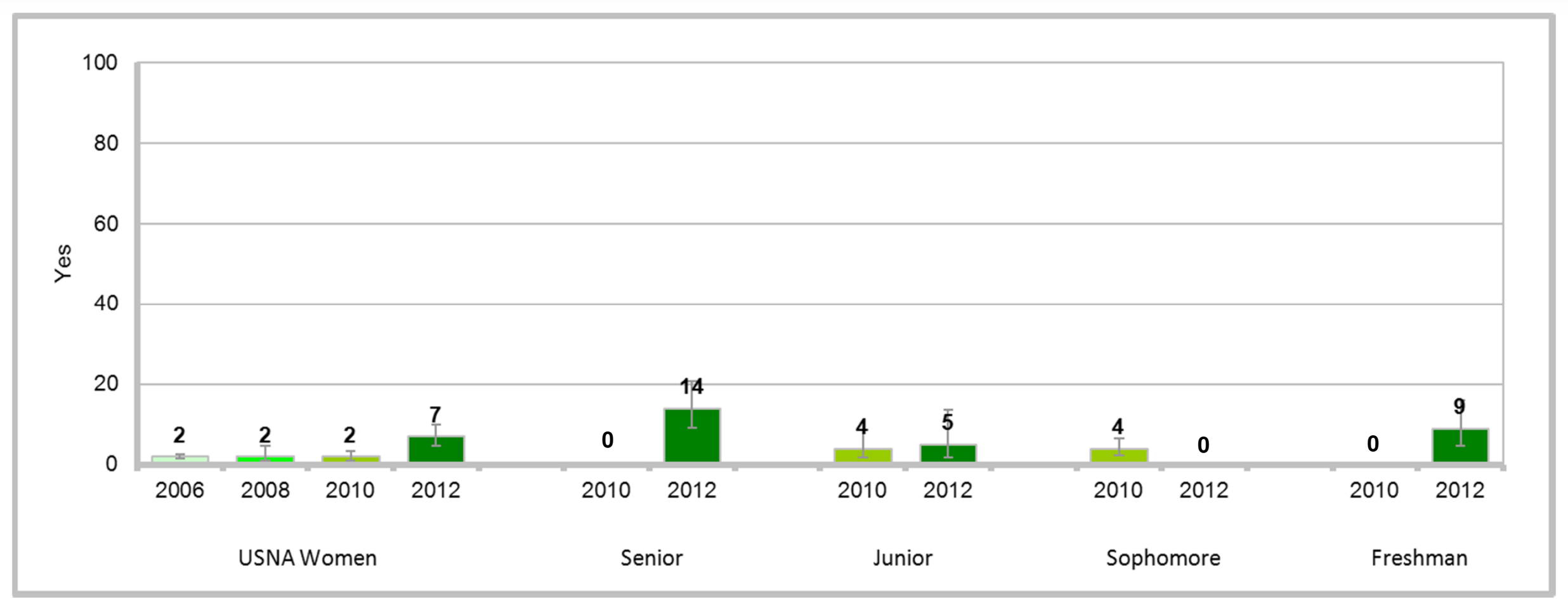

- Of the $15.1 \%$ of women who experienced USC, $7 \%$ indicated the offender threatened to harm them

- 2012 comparisons across years - higher than 2010, 2008, 2006

- Seniors and freshmen in 2012 higher than 2010, whereas sophomores lower

- Class differences in 2012 - higher response led by seniors; lower response led by sophomores 


\section{Offender Used Some Degree of Physical Force}

Percent of Women Who Experienced Unwanted Sexual Contact

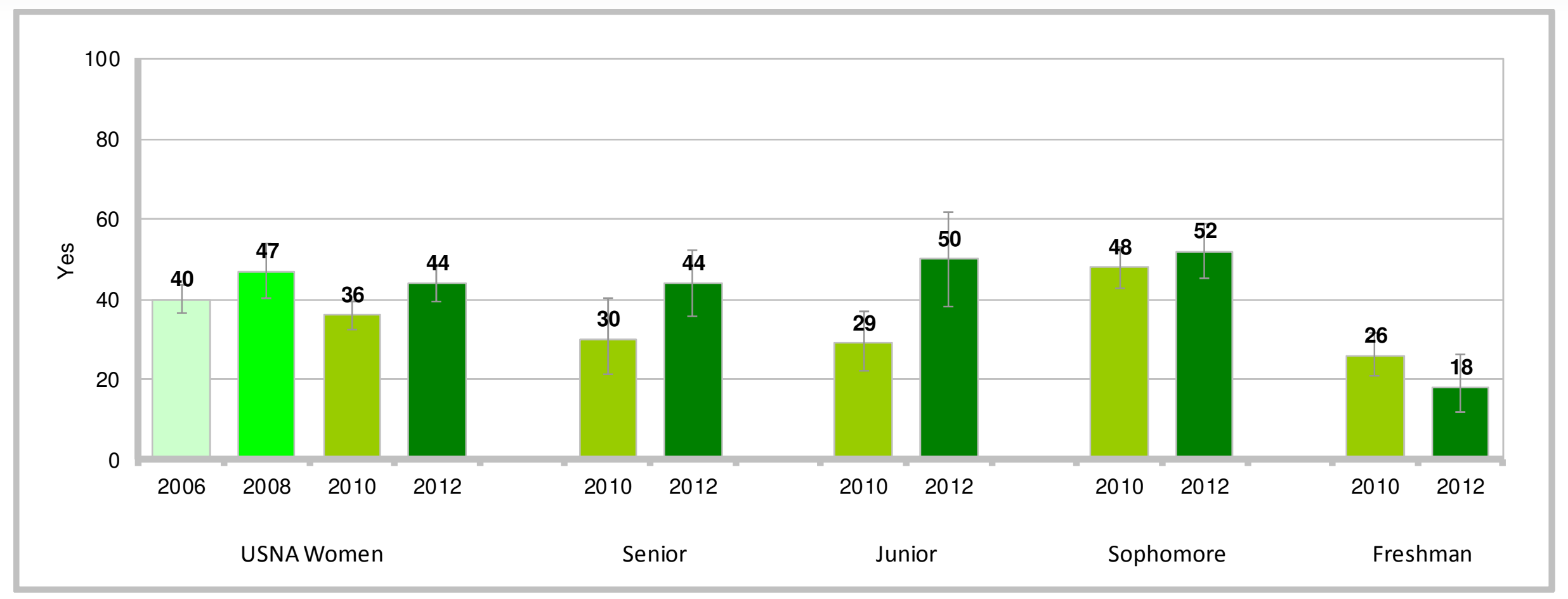

- Of the $15.1 \%$ of women who experienced USC, $44 \%$ indicated the offender threatened to use some degree of physical force

- 2012 comparisons across years - higher than 2010

- Seniors and juniors in 2012 higher than 2010

- Class differences in 2012 - lower response led by freshmen 


\section{Contents}

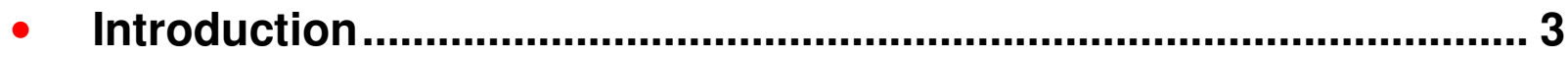

- Unwanted Sexual Contact ............................................................... 7

- Most Serious Behaviors Experienced....................................... 11

- Location of Incident ..................................................................... 13

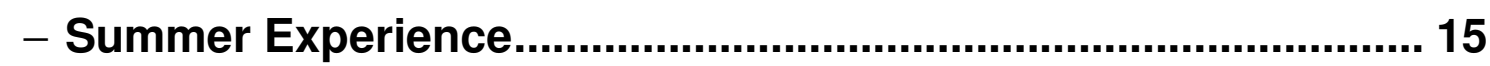

- Characteristics of Offender......................................................... 17

- Alcohol/Drug Involvement......................................................... 22

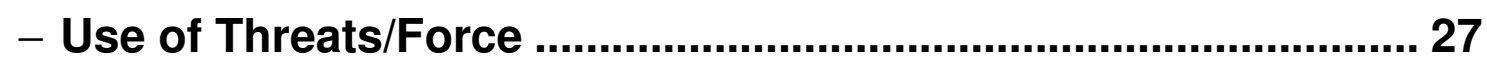

$\checkmark$ Experience of Sexual Harassment/Stalking .............................. 32

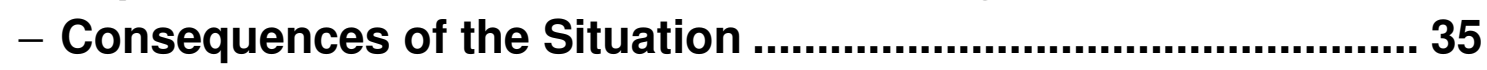

- Reporting the Situation ........................................................... 39

- Experience of Unwanted Sexual Contact Prior to Entering

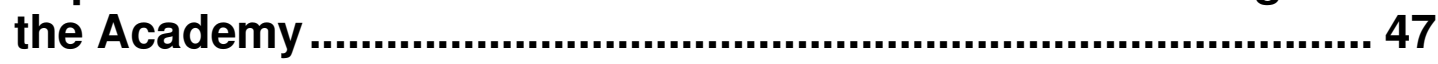

- Unwanted Gender-Related Behaviors ................................................ 49

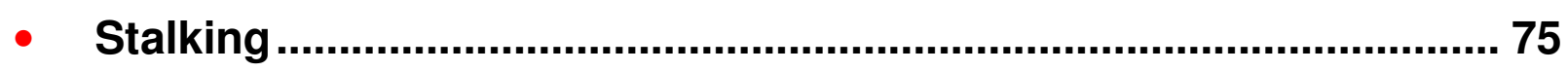

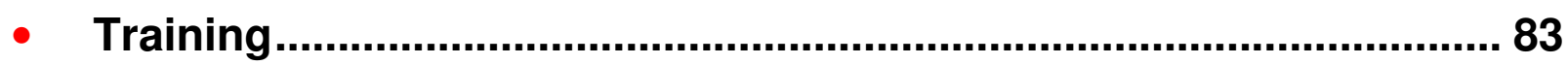

- Student Perceptions.................................................................... 93 


\section{Offender Sexually Harassed and/or Stalked You Before or After the Incident} Percent of Women Who Experienced Unwanted Sexual Contact

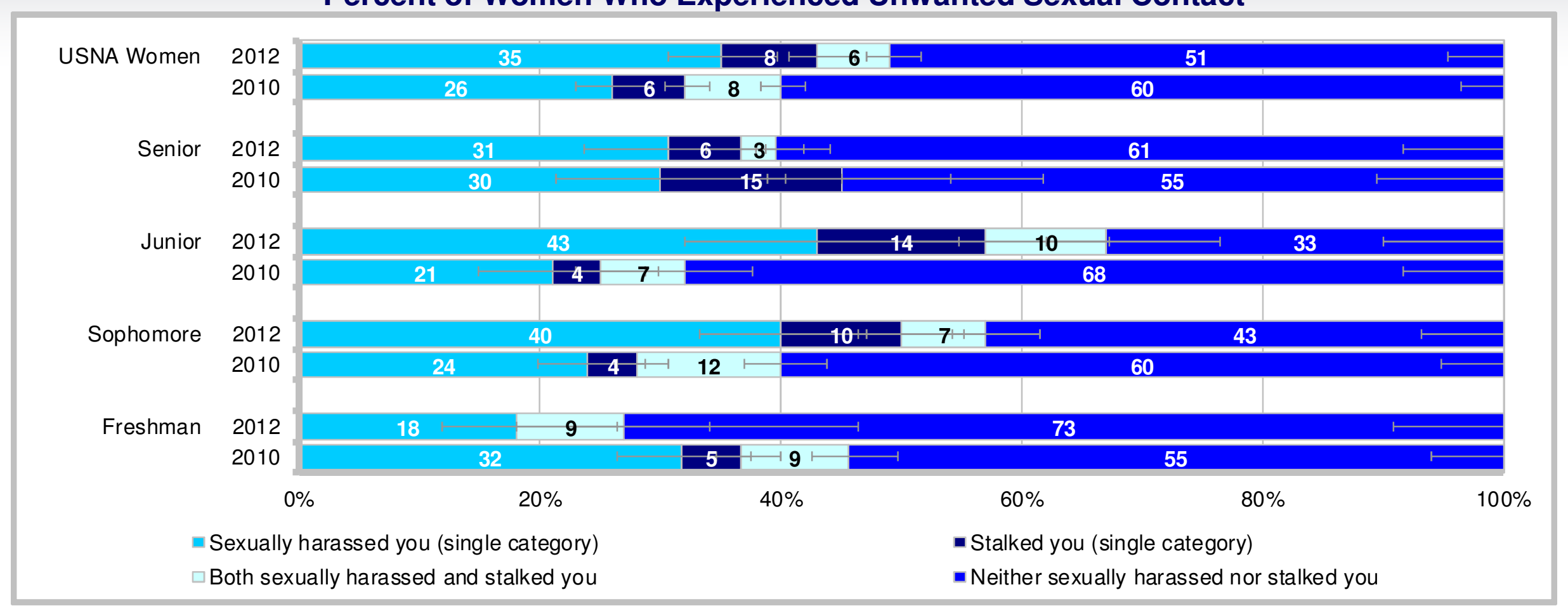

- Of the $15.1 \%$ of women who experienced USC, $35 \%$ indicated the offender sexually harassed them; $8 \%$ indicated the offender stalked them; $6 \%$ indicated the offender both sexually harassed and stalked them; and $51 \%$ indicated the offender neither sexually harassed nor stalked them

- 2012 comparisons across years - sexually harassed higher than 2010; neither lower than 2010

- Juniors and sophomores indicating sexually harassed in 2012 higher than 2010, whereas freshmen lower; seniors and freshmen indicating stalked in 2012 lower than 2010, whereas juniors and sophomores higher; seniors indicating both in 2012 higher than 2010, whereas sophomores lower; juniors and sophomores indicating neither in 2012 lower than 2010, whereas freshmen higher

- Class differences in 2012 - neither led by freshmen and seniors 


\section{When Offender Sexually Harassed and/or Stalked You Percent of Women Who Experienced Unwanted Sexual Contact}

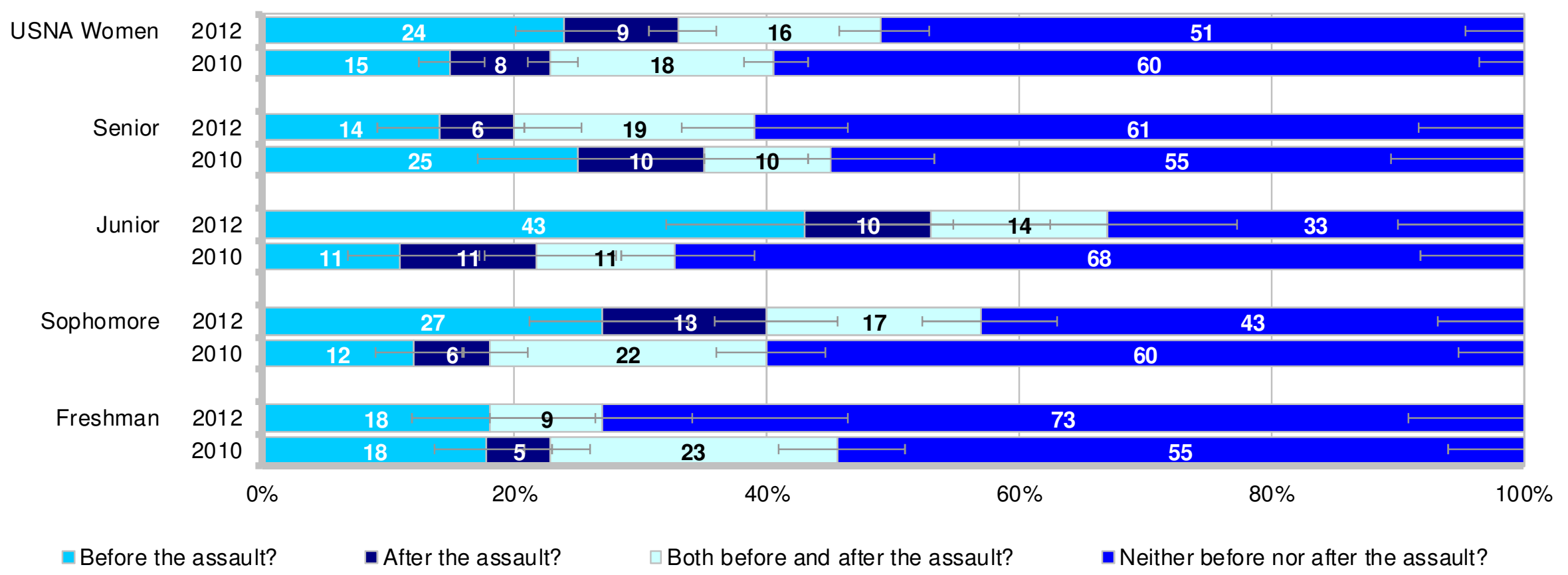

- Of the $15.1 \%$ of women who experienced USC, $24 \%$ indicated the offender sexually harassed or stalked them before the assault; $9 \%$ indicated the offender sexually harassed or stalked them after the assault; $16 \%$ indicated the offender sexually harassed or stalked them both before and after the assault; and $51 \%$ indicated neither before nor after the assault

- 2012 comparisons across years - before higher than 2010; neither lower than 2010

- Seniors indicating before in 2012 lower than 2010, whereas juniors and sophomores higher; sophomores indicating after in 2012 higher than 2010, whereas freshmen lower; freshmen indicating both in 2012 lower than 2010; juniors and sophomores indicating neither in 2012 lower than 2010, whereas freshmen higher

- Class differences in 2012 - before led by juniors; neither led by freshmen and seniors SAGR 2012 Q31 


\section{Contents}

Slide

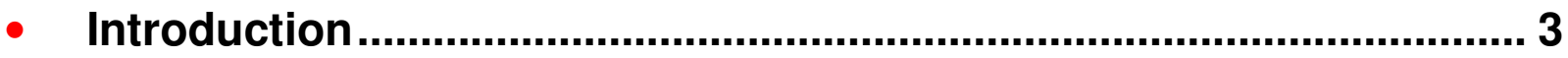

- Unwanted Sexual Contact ............................................................... 7

- Most Serious Behaviors Experienced....................................... 11

- Location of Incident ..................................................................... 13

- Summer Experience.................................................................... 15

- Characteristics of Offender......................................................... 17

- Alcohol/Drug Involvement......................................................... 22

- Use of Threats/Force ................................................................. 27

- Experience of Sexual Harassment/Stalking ............................. 32

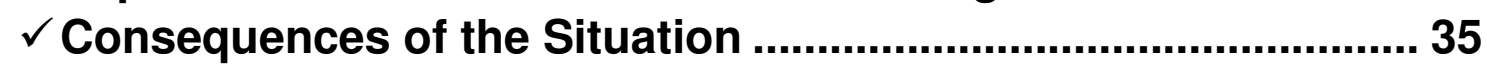

- Reporting the Situation ............................................................ 39

- Experience of Unwanted Sexual Contact Prior to Entering

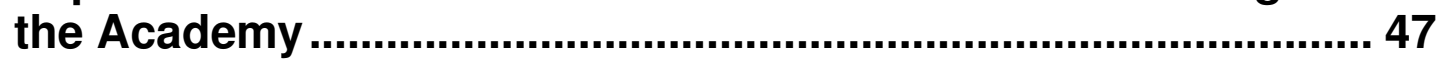

- Unwanted Gender-Related Behaviors ................................................ 49

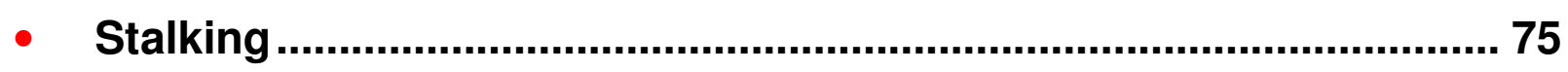

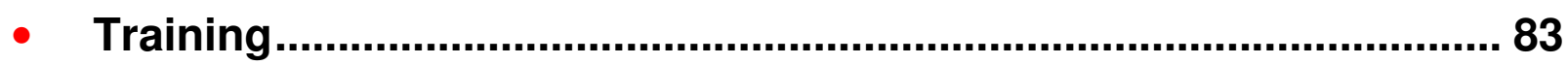

- Student Perceptions.................................................................... 93 


\section{Considered Requesting a Transfer to Another Company Percent of Women Who Experienced Unwanted Sexual Contact}

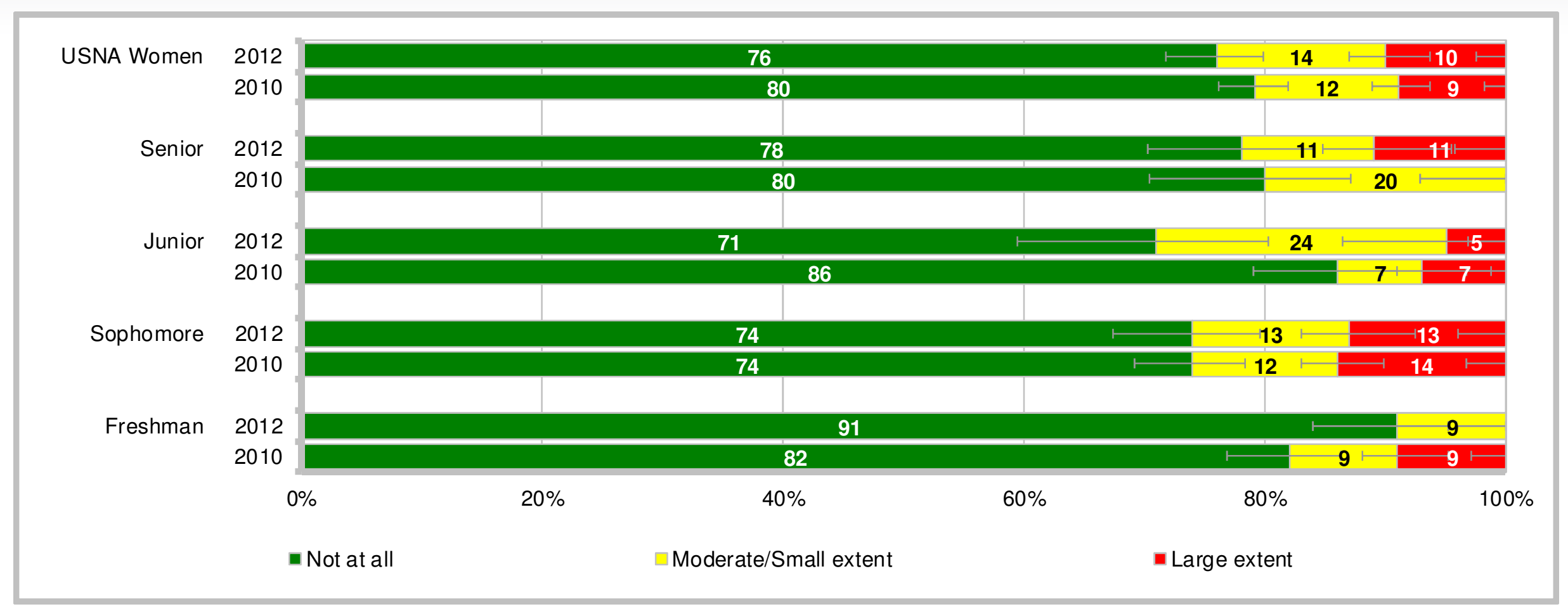

- Of the $15.1 \%$ of women who experienced USC, $24 \%$ indicated they considered a transfer to some extent; $76 \%$ indicated not at all

- 2012 comparisons across years - no differences

- Juniors indicating not at all in 2012 lower than 2010, whereas freshmen higher; juniors indicating moderate/small extent in 2012 higher than 2010; freshmen indicating large extent in 2012 lower than 2010

- Class differences in 2012 - not at all led by freshmen 


\section{Thought About Leaving Your Academy Percent of Women Who Experienced Unwanted Sexual Contact}

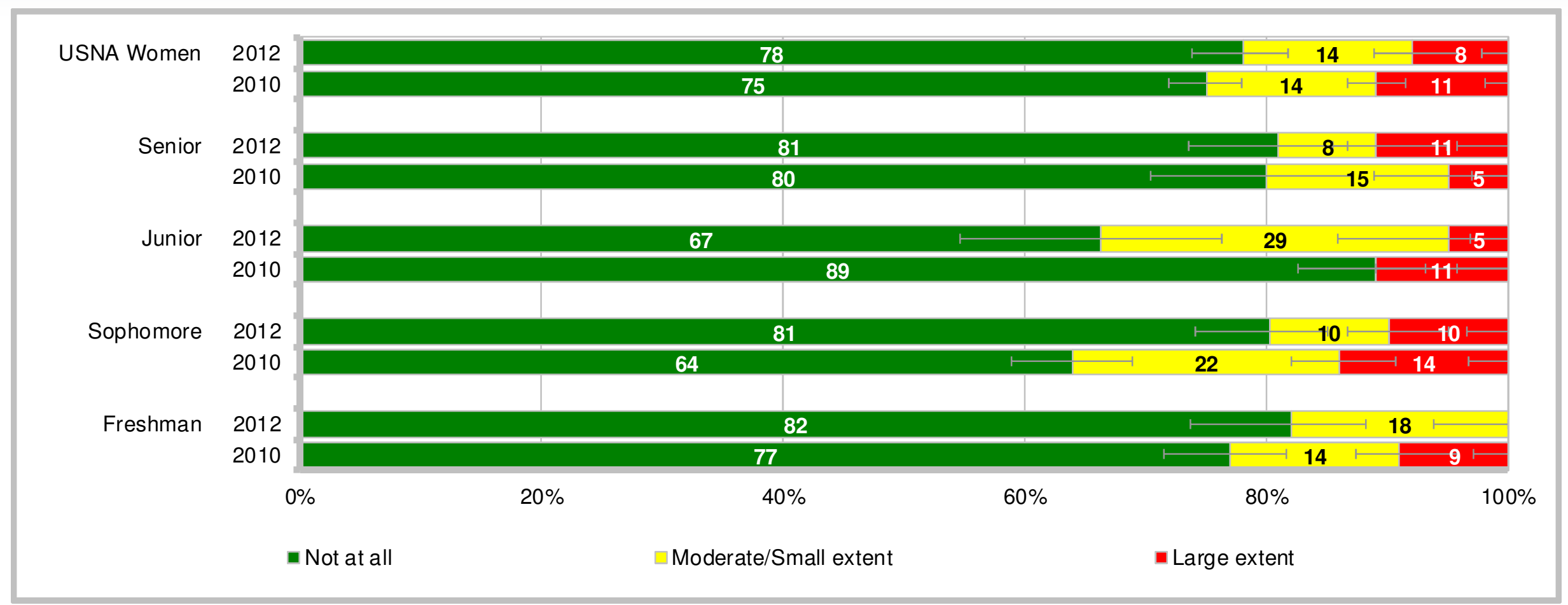

- Of the $15.1 \%$ of women who experienced USC, $22 \%$ indicated they thought about leaving the Academy to some extent; $78 \%$ indicated not at all

- 2012 comparisons across years - no differences

- Sophomores indicating not at all in 2012 higher than 2010, whereas juniors lower; juniors indicating moderate/small extent in 2012 higher than 2010, whereas sophomores lower; freshmen indicating large extent in 2012 lower than 2010

- Class differences in 2012 - moderate/small extent led by juniors SAGR 2012 Q32b 


\section{Your Academic Performance Suffered Percent of Women Who Experienced Unwanted Sexual Contact}

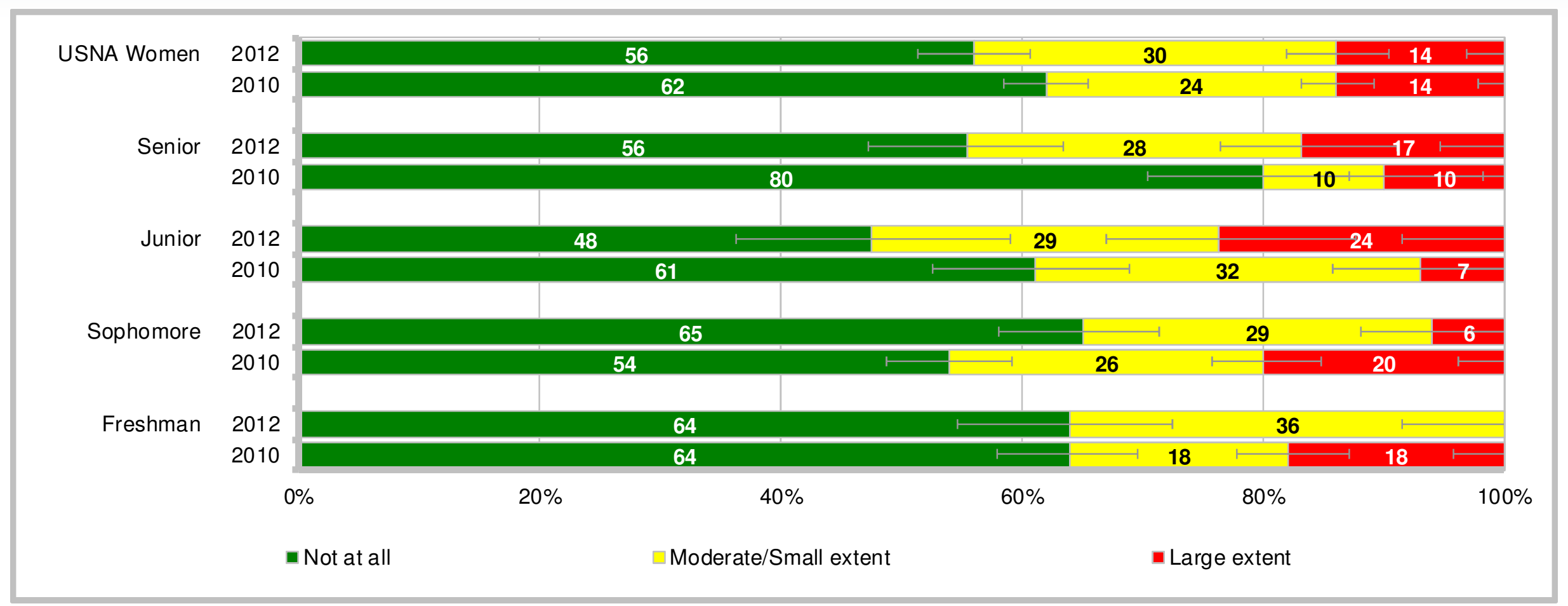

- Of the $15.1 \%$ of women who experienced USC, $44 \%$ indicated their academic performance suffered to some extent; $56 \%$ indicated not at all

- 2012 comparisons across years - not at all lower than 2010; moderate/small extent higher than 2010

- Sophomores indicating not at all in 2012 higher than 2010; seniors and freshmen indicating moderate/small extent in 2012 higher than 2010; juniors indicating large extent in 2012 higher than 2010, whereas sophomores lower

- Class differences in 2012 - no differences 


\section{Contents}

Slide

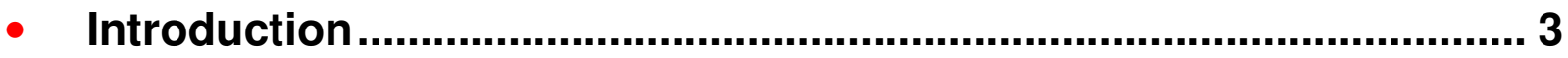

- Unwanted Sexual Contact ............................................................... 7

- Most Serious Behaviors Experienced....................................... 11

- Location of Incident ..................................................................... 13

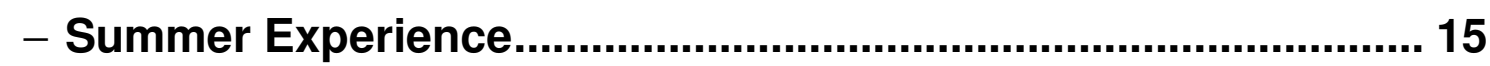

- Characteristics of Offender......................................................... 17

- Alcohol/Drug Involvement......................................................... 22

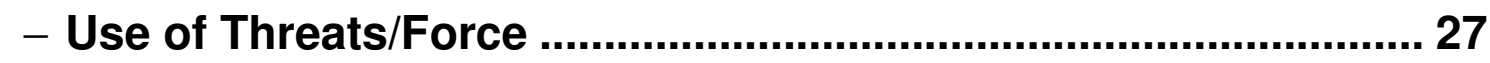

- Experience of Sexual Harassment/Stalking ............................. 32

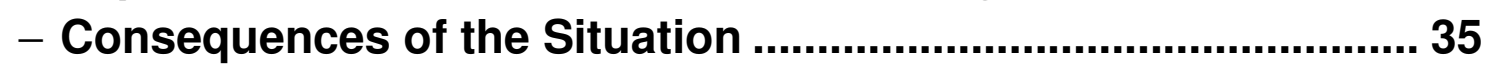

$\checkmark$ Reporting the Situation ............................................................ 39

- Experience of Unwanted Sexual Contact Prior to Entering

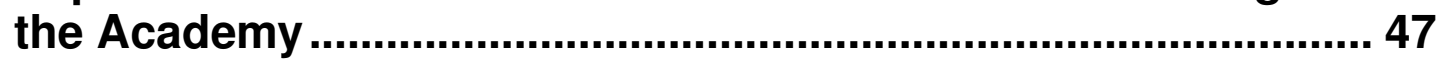

- Unwanted Gender-Related Behaviors ................................................ 49

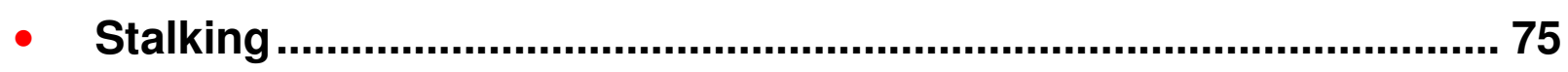

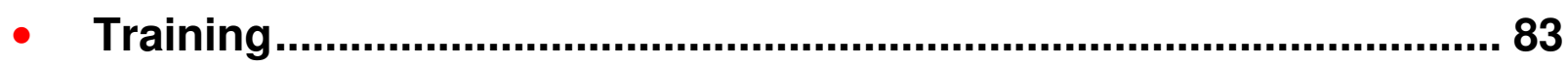

- Student Perceptions.................................................................... 93 


\section{Reported This Situation to a Military Authority or Organization Percent of Women Who Experienced Unwanted Sexual Contact}

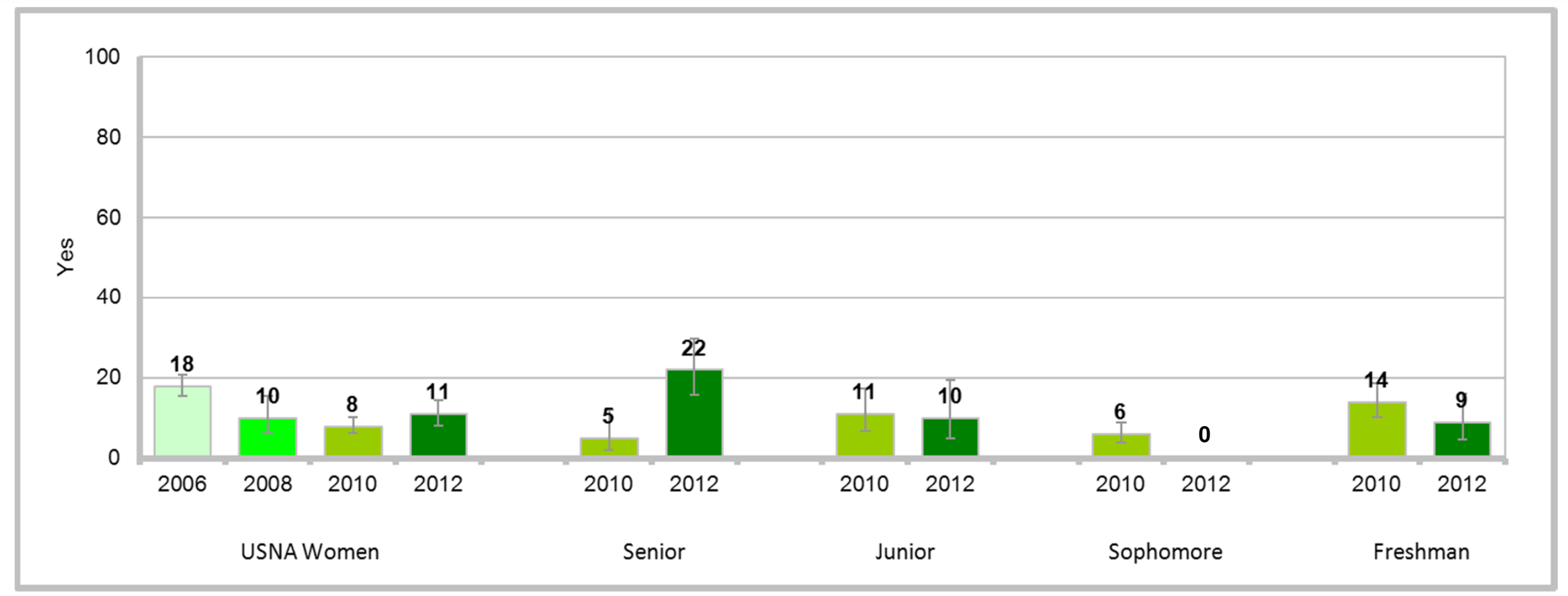

- Of the $15.1 \%$ of women who experienced USC, $11 \%$ indicated they reported the situation

- 2012 comparisons across years - lower than 2006

- Seniors in 2012 higher than 2010, whereas sophomores lower

- Class differences in 2012 - higher response led by seniors; lower response led by sophomores 


\section{Type of Report Made to a Military Authority} Percent of Women Who Experienced Unwanted Sexual Contact and Reported It

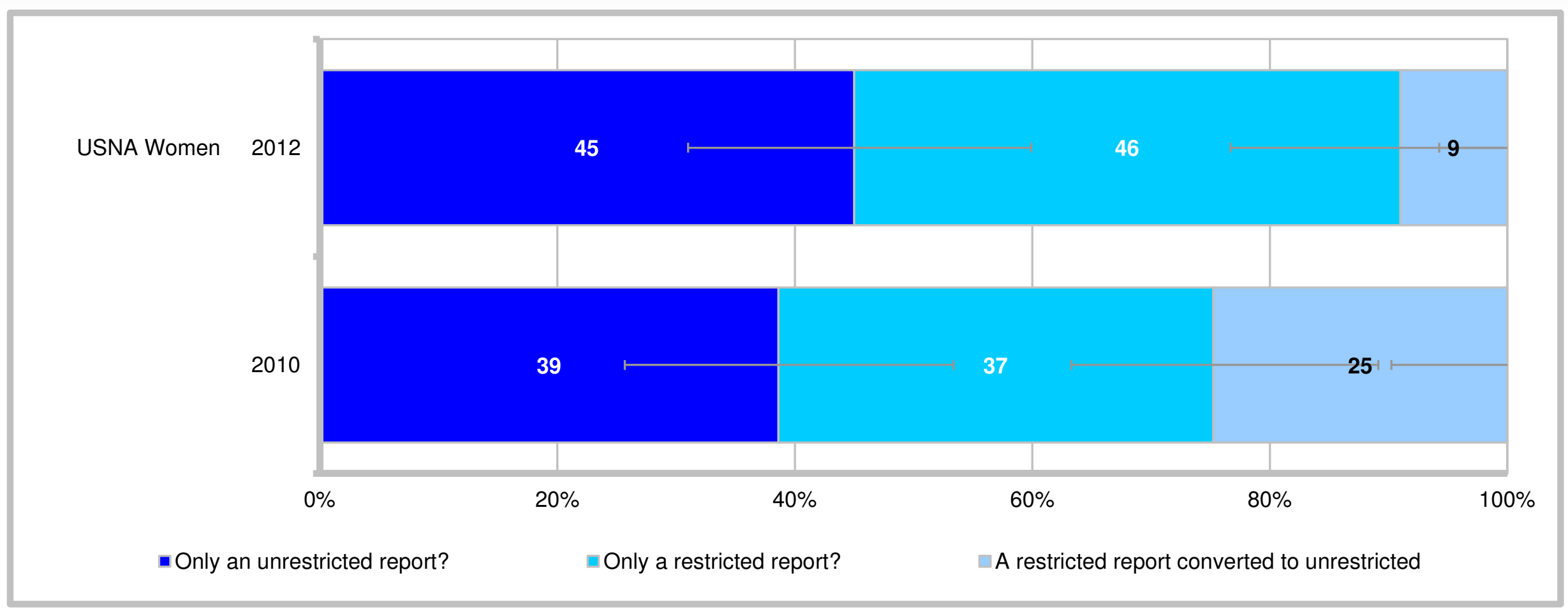

- Of the $11 \%$ of women who reported the situation, $45 \%$ indicated they made only an unrestricted report; $46 \%$ made only a restricted report; and $9 \%$ made a restricted report converted to unrestricted

- 2012 comparisons across years - converted lower than 2010

- Results by class year not reportable 


\section{When Report Made to a Military Authority}

Percent of Women Who Experienced Unwanted Sexual Contact and Reported It

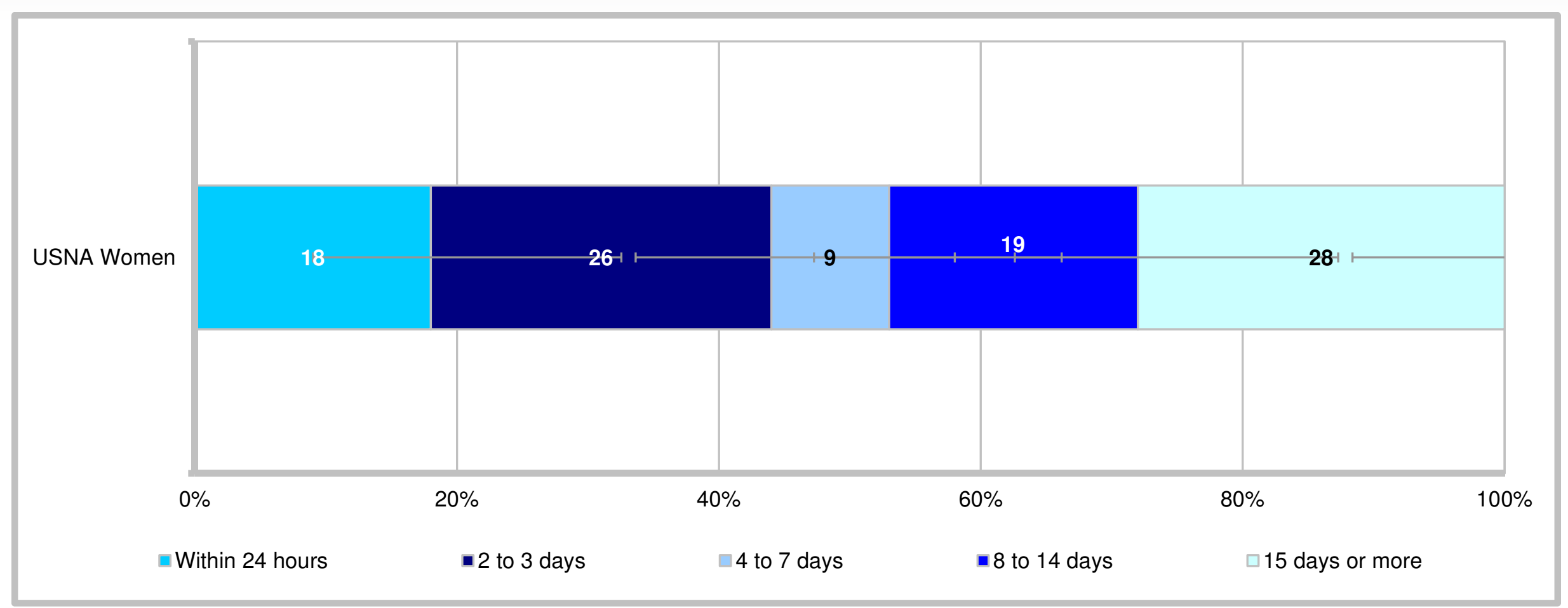

- Of the $11 \%$ of women who reported the situation, $18 \%$ indicated they made their report within 24 hours; $26 \%$ within 2 to 3 days; $9 \%$ within 4 to 7 days; $19 \%$ within 8 to 14 days; and $28 \%$ after 15 days or more

- 2012 comparisons across years - new question in 2012; no trend data available

- Results by class year not reportable 


\section{Reason for Delay in Reporting the Situation}

Percent of Women Who Experienced Unwanted Sexual Contact and Waited at Least 24 Hours to Report It

\begin{tabular}{|l|c|}
\hline \multicolumn{1}{|c|}{ Reasons for Delay in Reporting } & Total \\
\hline $\begin{array}{l}\text { Wanted to think about the situation before } \\
\text { deciding to report }\end{array}$ & 89 \\
\hline $\begin{array}{l}\text { Wanted to seek advice first from a friend or } \\
\text { family member }\end{array}$ & 45 \\
\hline Had to figure out how to report & 34 \\
\hline $\begin{array}{l}\text { Did not realize at first that the situation was a } \\
\text { crime }\end{array}$ & 22 \\
\hline $\begin{array}{l}\text { Wanted to seek advice/counseling from a } \\
\text { professional before deciding to report }\end{array}$ & 22 \\
\hline Margins of Error & $\pm 16-17 \%$ \\
\hline
\end{tabular}

- New question in 2012; no trend data available

- Results by class year not reportable 


\section{Most Frequently Selected Reasons for Reporting Unwanted Sexual Contact Situation Percent of Women Who Experienced Unwanted Sexual Contact and Reported It}

\begin{tabular}{|l|c|}
\hline \multicolumn{1}{|c|}{ Reasons for Reporting } & Total \\
\hline Seek closure on the incident & 83 \\
\hline Seek help dealing with an emotional incident & 74 \\
\hline It was the right thing to do & 72 \\
\hline Seek justice & 55 \\
\hline Stop the offender from hurting you again & 54 \\
\hline Stop the offender from hurting others & 54 \\
\hline Discourage other potential offenders & 54 \\
\hline Margins of Error & $\pm 15-16 \%$ \\
\hline
\end{tabular}

- New question in 2012; no trend data available

- Results by class year not reportable 


\section{Most Frequently Selected Reasons for} Not Reporting Unwanted Sexual Contact Situation Percent of Women Who Experienced Unwanted Sexual Contact and Did Not Report It

\begin{tabular}{|l|c|c|c|c|c|c|}
\hline \multicolumn{1}{|c}{ Reasons for Not Reporting } & $\begin{array}{c}\text { Survey } \\
\text { Year }\end{array}$ & Total & Senior & Junior & Sophomore & Freshman \\
\hline \multirow{2}{*}{ Took care of it myself } & 2012 & $77 \uparrow$ & $79 \uparrow$ & 72 & $81 \uparrow$ & $80 \downarrow$ \\
\cline { 2 - 7 } & 2010 & 67 & 58 & 64 & 63 & 95 \\
\hline \multirow{2}{*}{ Did not want people gossiping } & 2012 & 71 & 57 & $89 \uparrow$ & 71 & $90 \uparrow$ \\
\cline { 2 - 7 } & 2010 & 71 & 63 & 68 & 76 & 68 \\
\hline \multirow{2}{*}{ Did not want anyone to know } & 2012 & $68 \uparrow$ & 50 & $83 \uparrow$ & 71 & $90 \uparrow$ \\
\cline { 2 - 7 } & 2010 & 61 & 63 & 56 & 70 & 47 \\
\hline \multirow{2}{*}{$\begin{array}{l}\text { Thought it was not important enough to } \\
\text { report }\end{array}$} & 2012 & 63 & $68 \uparrow$ & 61 & 55 & 90 \\
\hline \multirow{2}{*}{ Felt shame/embarrassment } & 2010 & 64 & 53 & 68 & 59 & 84 \\
\hline \multirow{2}{*}{ Felt uncomfortable making a report } & 2012 & 57 & $39 \downarrow$ & 56 & 68 & $90 \uparrow$ \\
\cline { 2 - 7 } & 2010 & 56 & 63 & 48 & 61 & 53 \\
\hline $\begin{array}{l}\text { Thought it would hurt my reputation and } \\
\text { standing }\end{array}$ & 2012 & 55 & $43 \downarrow$ & 61 & 55 & 70 \\
\hline Margins of Error & 2010 & 59 & 63 & 56 & 57 & 63 \\
\cline { 2 - 7 }
\end{tabular}

Higher Response of Yes

Lower Response of Yes

Higher in 2012 than 2010

Lower in 2012 than 2010 


\section{Would Make the Same Decision About Reporting or Not Reporting if Could Do It Over}

Percent of Women Who Experienced Unwanted Sexual Contact and Reported or Did Not Report to a Military Authority

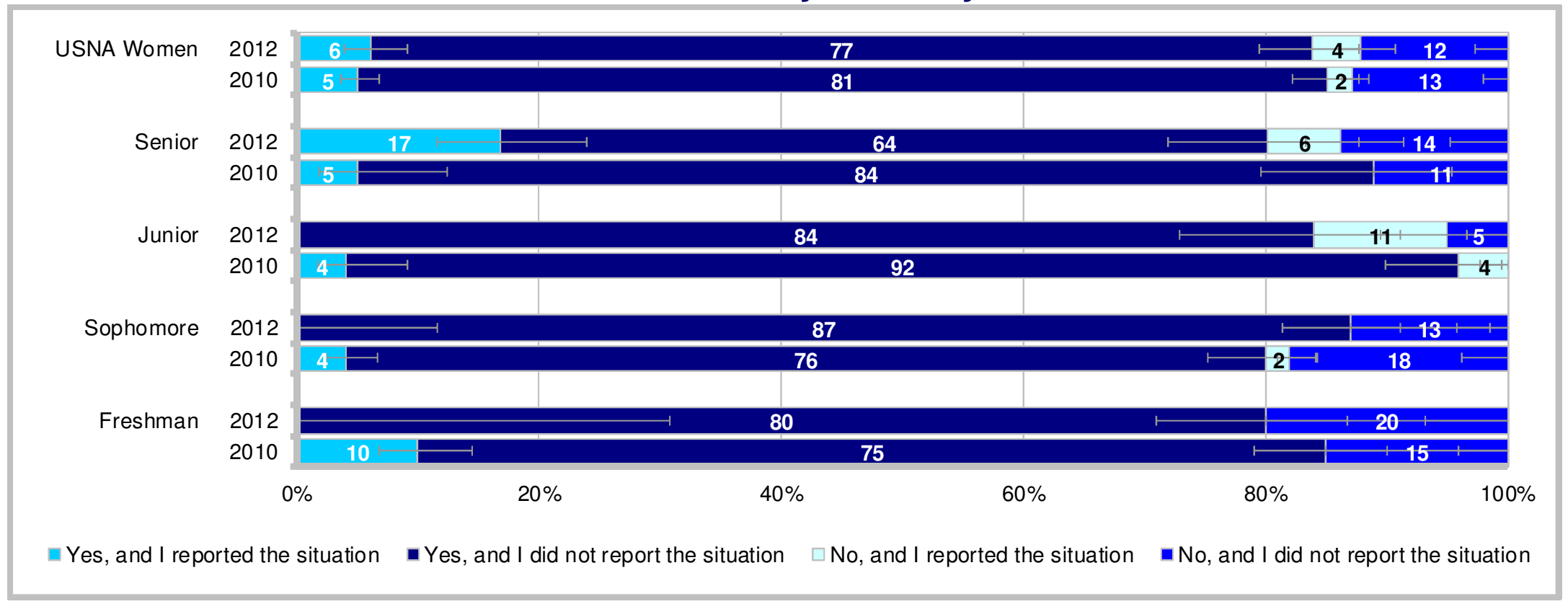

- Of the $15.1 \%$ of women who experienced USC, $6 \%$ indicated they reported the situation and would make the same decision again; $77 \%$ indicated they did not report the situation and would make the same decision again; $4 \%$ indicated they reported but would not make the same decision again; and $12 \%$ indicated they did not report the situation but would not make the same decision again

- 2012 comparisons across years - no differences

- Seniors indicating reported and would make the same decision in 2012 higher than 2010, whereas juniors, sophomores, and freshmen lower; sophomores indicating did not report and would make the same decision in 2012 higher than 2010, whereas seniors lower; seniors indicating reported but would not make the same decision in 2012 higher than 2010, whereas sophomores lower

- Class differences in 2012 - reported and would make the same decision led by seniors; did not report and would make the same decision led by sophomores 


\section{Contents}

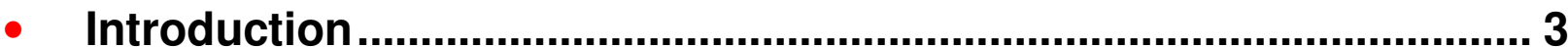

- Unwanted Sexual Contact ................................................................... 7

- Most Serious Behaviors Experienced........................................... 11

- Location of Incident ....................................................................... 13

- Summer Experience..................................................................... 15

- Characteristics of Offender....................................................... 17

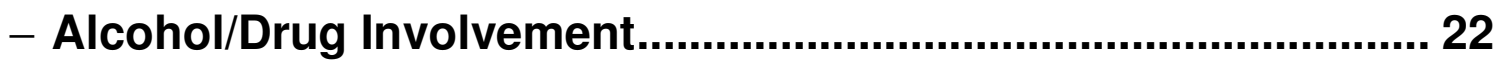

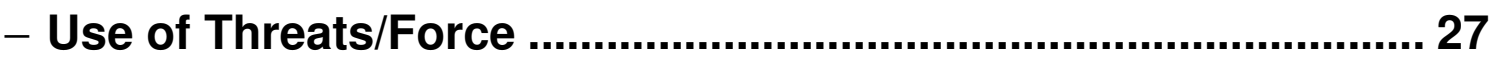

- Experience of Sexual Harassment/Stalking ............................... 32

- Consequences of the Situation ...................................................... 35

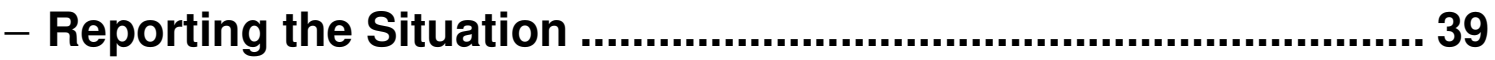

$\checkmark$ Experience of Unwanted Sexual Contact Prior to Entering the Academy .................................................................................... 47

- Unwanted Gender-Related Behaviors .............................................. 49

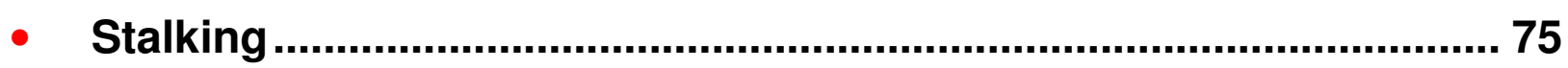

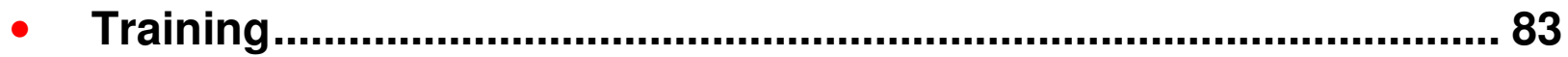

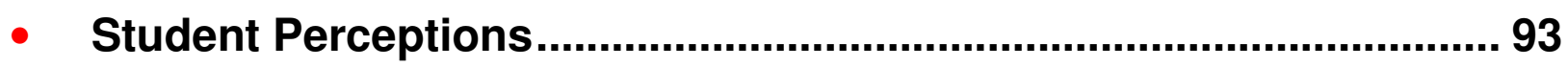




\section{Unwanted Sexual Contact Incident Rate Prior to Entering the Academy} Percent of All Students

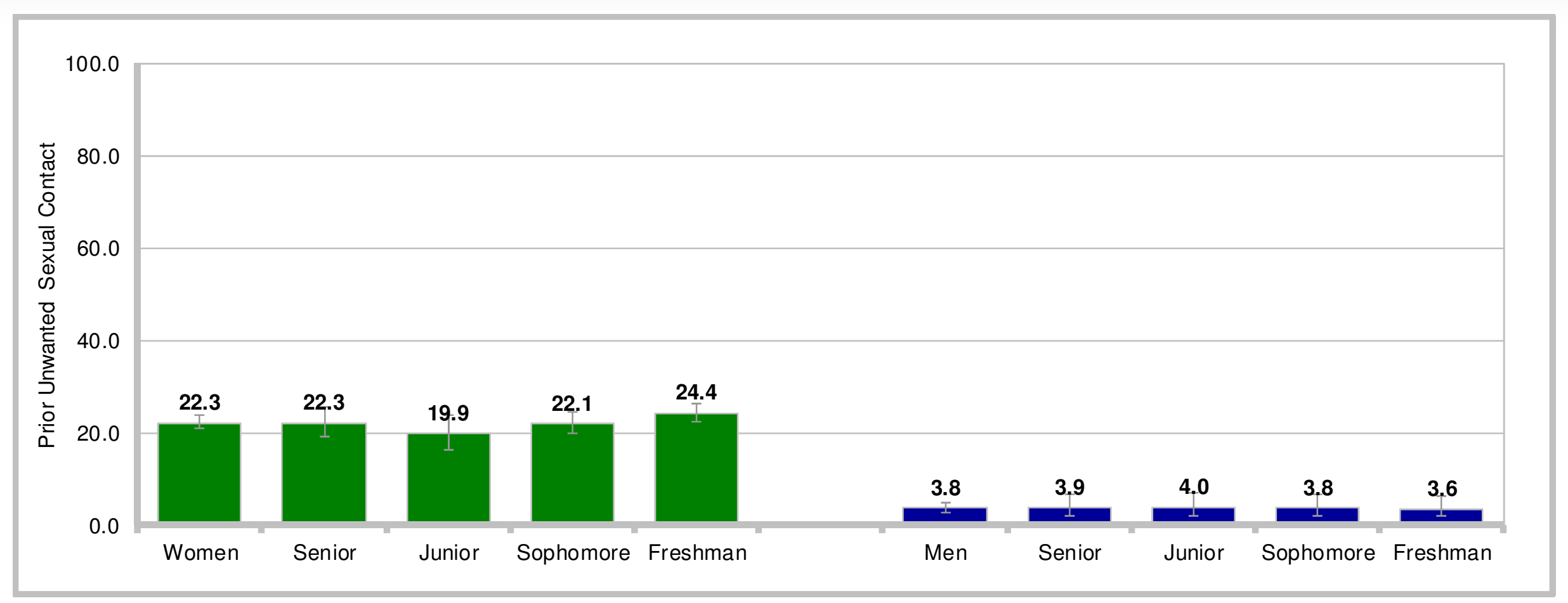

- $22.3 \%$ of women and $3.8 \%$ of men indicated experiencing unwanted sexual contact prior to entering the Academy

- 2012 comparisons across years - new question in 2012; no trend data available

- Class differences in 2012 - no differences 


\section{Contents}

Slide

- Introduction ................................................................................ 3

- Unwanted Sexual Contact ........................................................... 7

$\checkmark \quad$ Unwanted Gender-Related Behaviors ........................................... 49

- Sexual Harassment ............................................................... 51

- Categories of Behaviors Associated with Sexual Harassment.................................................................................. 54

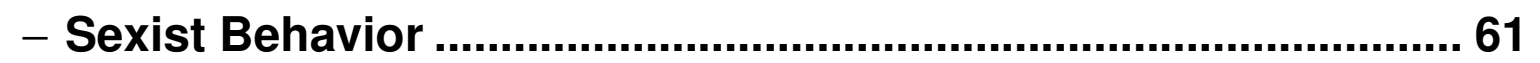

- Organizational Affiliation of Offender ..................................... 64

- Discussing/Reporting Experiences............................................6 67

- Response to Reporting.......................................................... 70

- Reasons for Not Reporting ................................................. 72

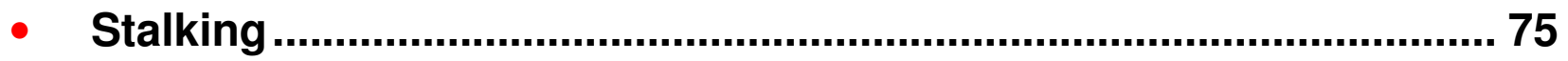

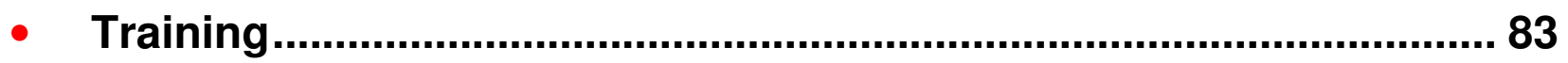

- Student Perceptions ...................................................................... 93 


\section{Unwanted Gender-Related Behaviors:}

\section{Sexual Harassment and Sexist Behavior Incident Rates}

\section{- Definition and measure of sexual harassment and sexist behavior:}

- DoD defines sexual harassment as "a form of sex discrimination that involves unwelcome sexual advances, requests for sexual favors, and other verbal or physical conduct of a sexual nature when:

- Submission to such conduct is made either explicitly or implicitly a term or condition of a person's job, pay, or career, or

- Submission to or rejection of such conduct by a person is used as a basis for career or employment decisions affecting that person, or

- Such conduct has the purpose or effect of unreasonably interfering with an individual's work performance or creates an intimidating, hostile, or offensive working environment" (Department of Defense, 1995).

- The core measure of unwanted gender-related behaviors on the 2012 SAGR consists of 17 items

- Sexual harassment measure has 12 items in a three-factor structure and a single "labeling" item

- Crude/Offensive Behavior - four items regarding offensive or embarrassing verbal/nonverbal behaviors of a sexual nature

- Unwanted Sexual Attention - four items regarding unwanted attempts to establish a sexual relationship

- Sexual Coercion - four items regarding classic quid pro quo instances of special treatment or favoritism conditioned on sexual cooperation

- One "labeling" item that asks if the student considers any of the selected behaviors to be sexual harassment

- Sexist behavior measure has four items that include verbal/nonverbal behaviors that convey insulting, offensive, and/or condescending attitudes based on gender of student

- Write-in comments analyzed separately
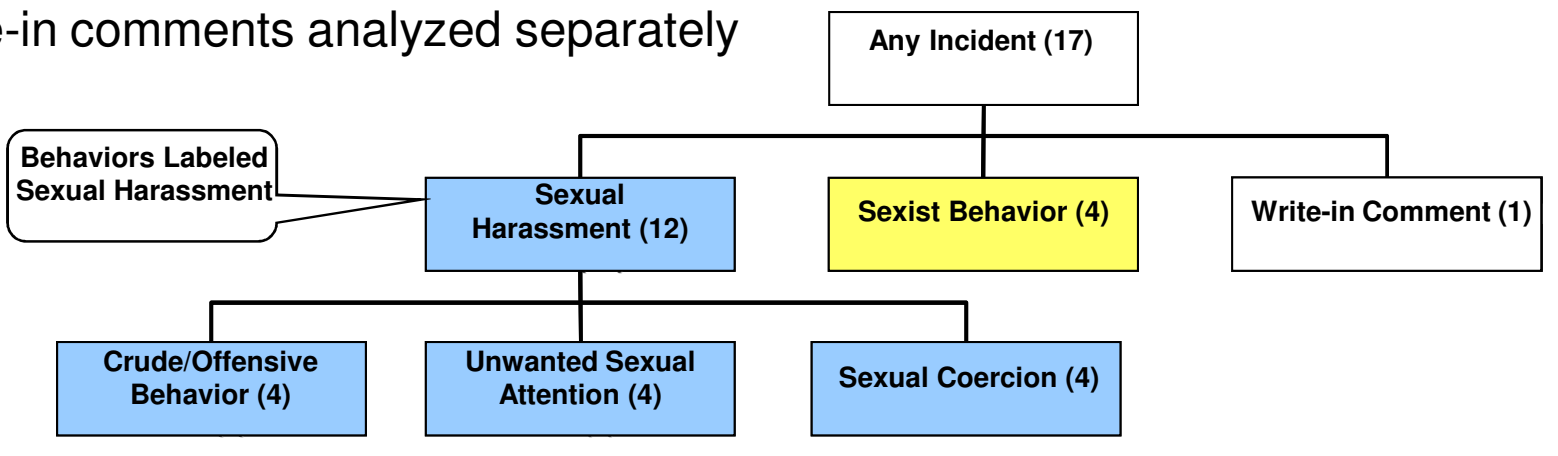


\section{Contents}

- Introduction 3

- Unwanted Sexual Contact ................................................................... 7

- Unwanted Gender-Related Behaviors .............................................. 49

$\checkmark$ Sexual Harassment ................................................................. 51

- Categories of Behaviors Associated with Sexual Harassment.................................................................................... 55

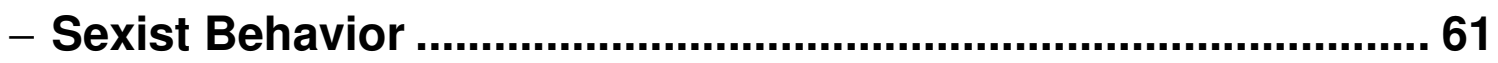

- Organizational Affiliation of Offender ........................................ 64

- Discussing/Reporting Experiences............................................. 67

- Response to Reporting.............................................................. 70

- Reasons for Not Reporting ............................................................. 72

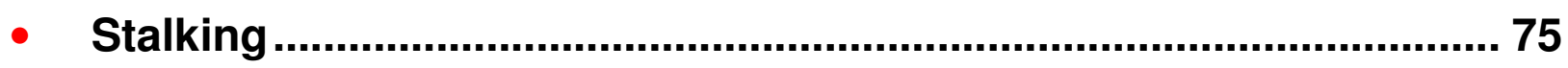

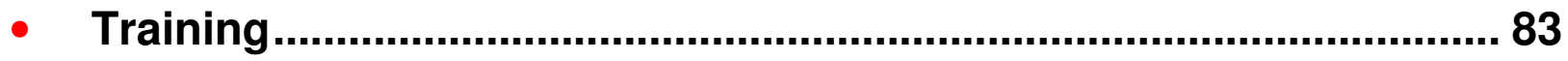

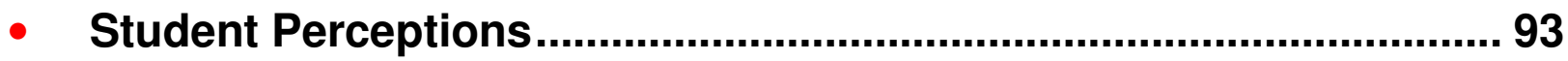




\section{Sexual Harassment Incident Rate Percent of Women}

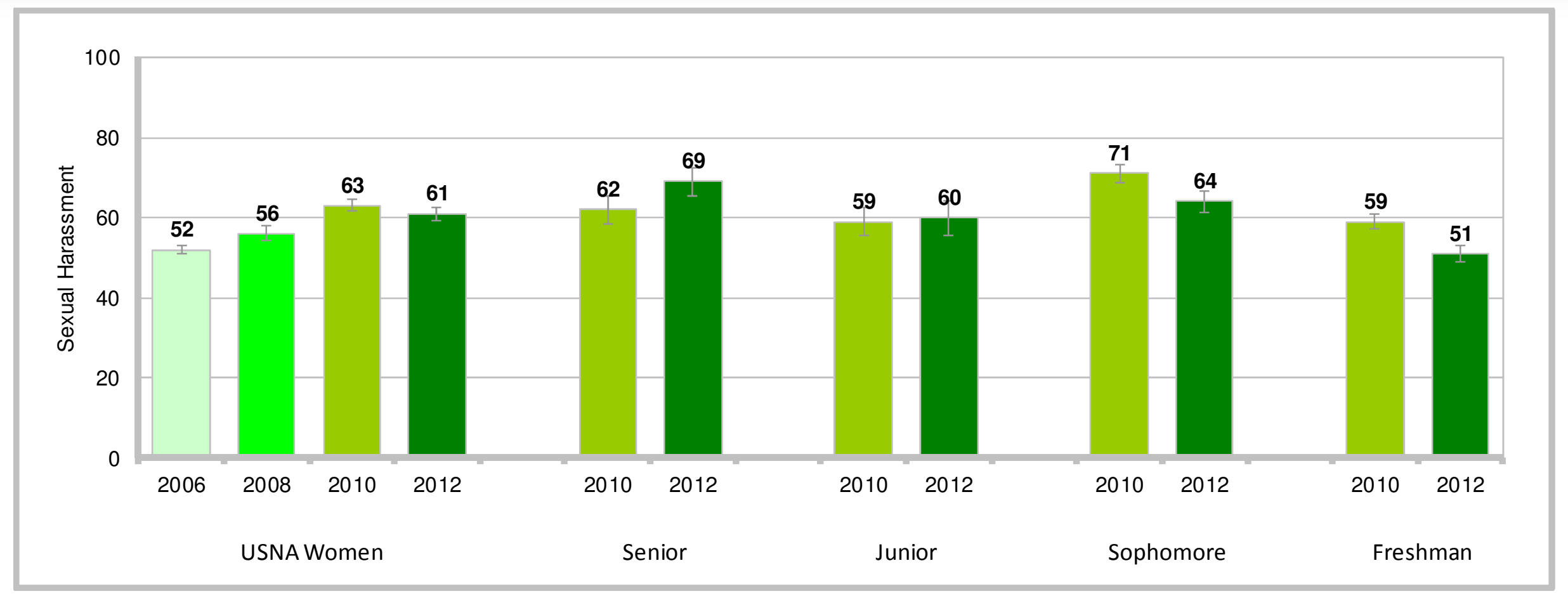

- $61 \%$ of women indicated experiencing sexual harassment in 2012

- 2012 comparisons across years - higher than 2008, 2006

- Seniors in 2012 higher than 2010, whereas sophomores and freshmen lower

- Class differences in 2012 - higher response led by seniors; lower response led by freshmen 


\section{Sexual Harassment Incident Rate}

Percent of Men

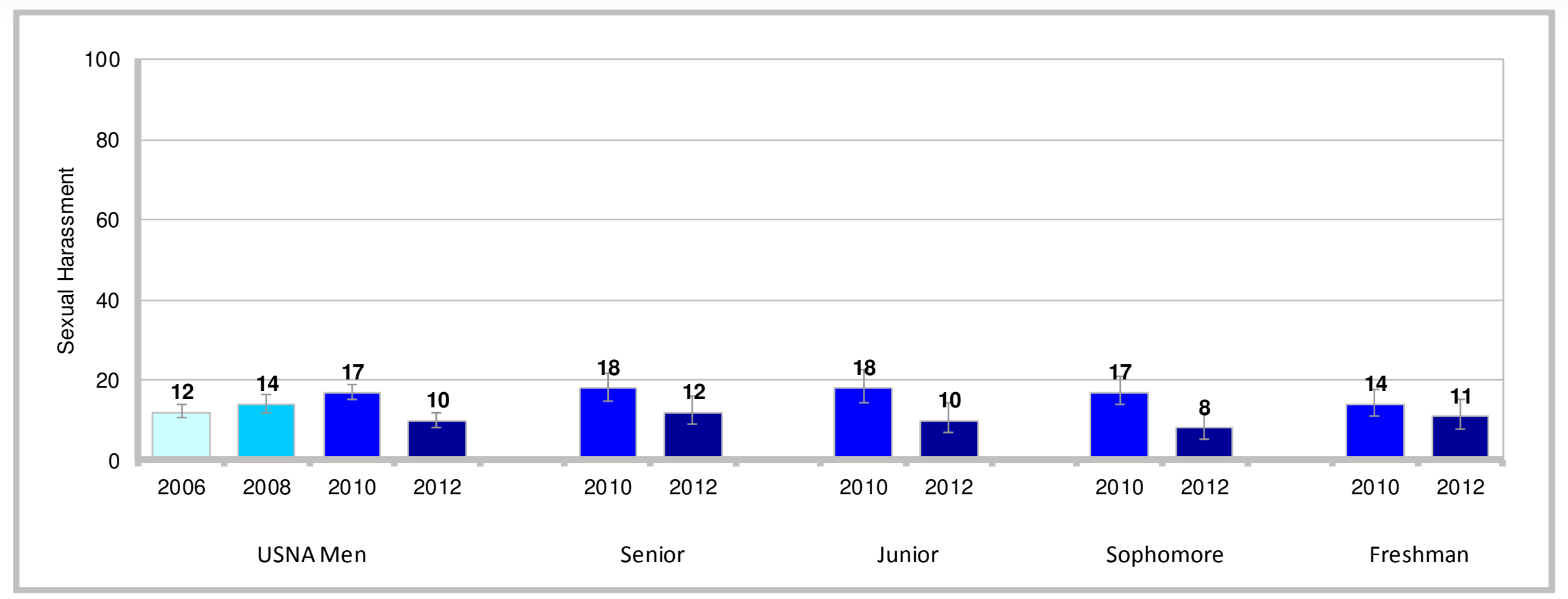

- $10 \%$ of men indicated experiencing sexual harassment in 2012

- 2012 comparisons across years - lower than 2010, 2008

- Seniors, juniors, and sophomores in 2012 lower than 2010

- Class differences in 2012 - no differences 


\section{Contents}

Slide

- Introduction ................................................................................. 3

- Unwanted Sexual Contact ........................................................... 7

- Unwanted Gender-Related Behaviors ......................................... 49

- Sexual Harassment .................................................................. 51

$\checkmark$ Categories of Behaviors Associated with Sexual Harassment.................................................................................. 54

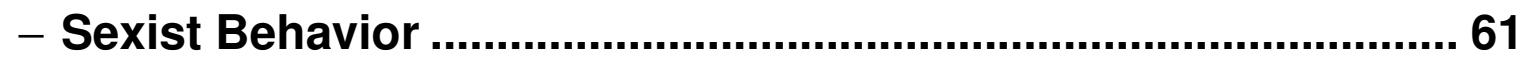

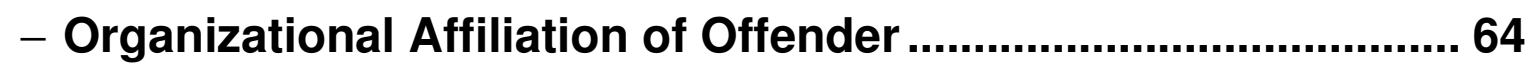

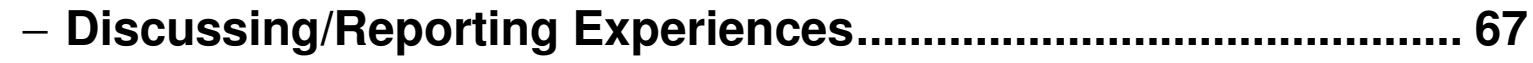

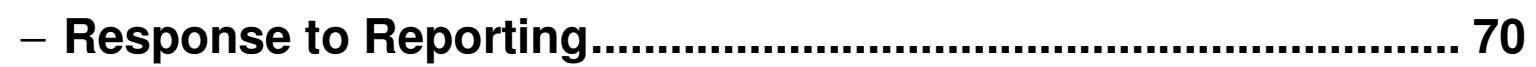

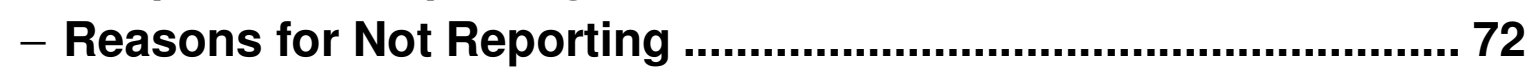

• Stalking .......................................................................................... 75

• Training ........................................................................................... 83

- Student Perceptions.................................................................. 93 


\section{Crude/Offensive Behavior Incident Rate Percent of Women}

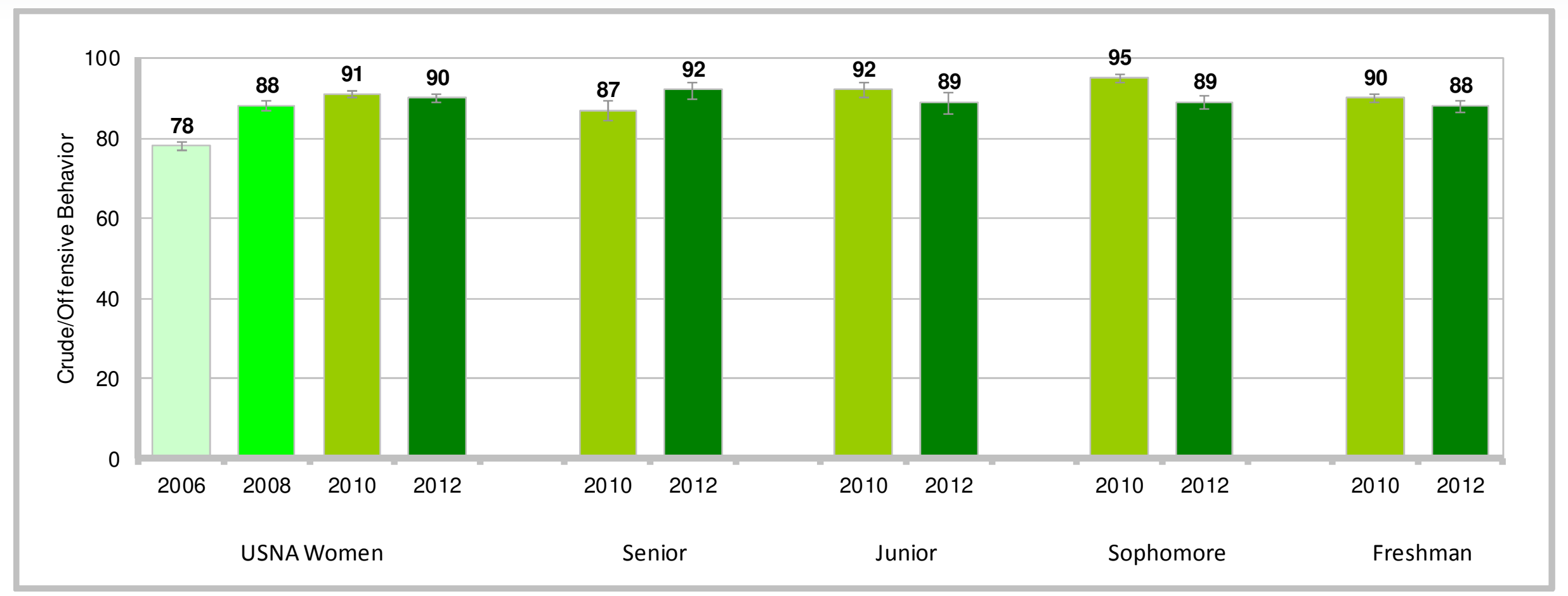

- $90 \%$ of women indicated experiencing crude/offensive behavior in 2012

- 2012 comparisons across years - higher than 2008, 2006

- Seniors in 2012 higher than 2010, whereas sophomores and freshmen lower

- Class differences in $\mathbf{2 0 1 2}$ - higher response led by seniors 


\section{Crude/Offensive Behavior Incident Rate} Percent of Men

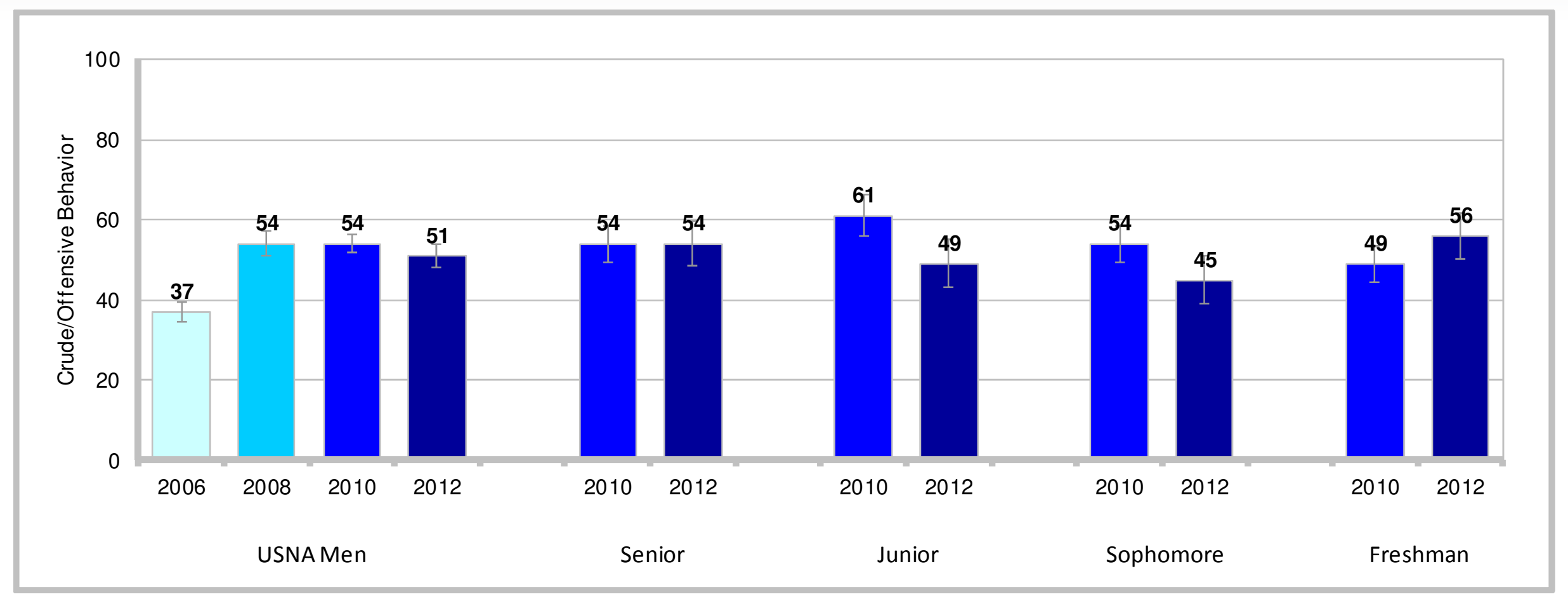

- $51 \%$ of men indicated experiencing crude/offensive behavior in 2012

- 2012 comparisons across years - higher than 2006

- Juniors and sophomores in 2012 lower than 2010

- Class differences in 2012 - no differences 


\section{Unwanted Sexual Attention Incident Rate Percent of Women}

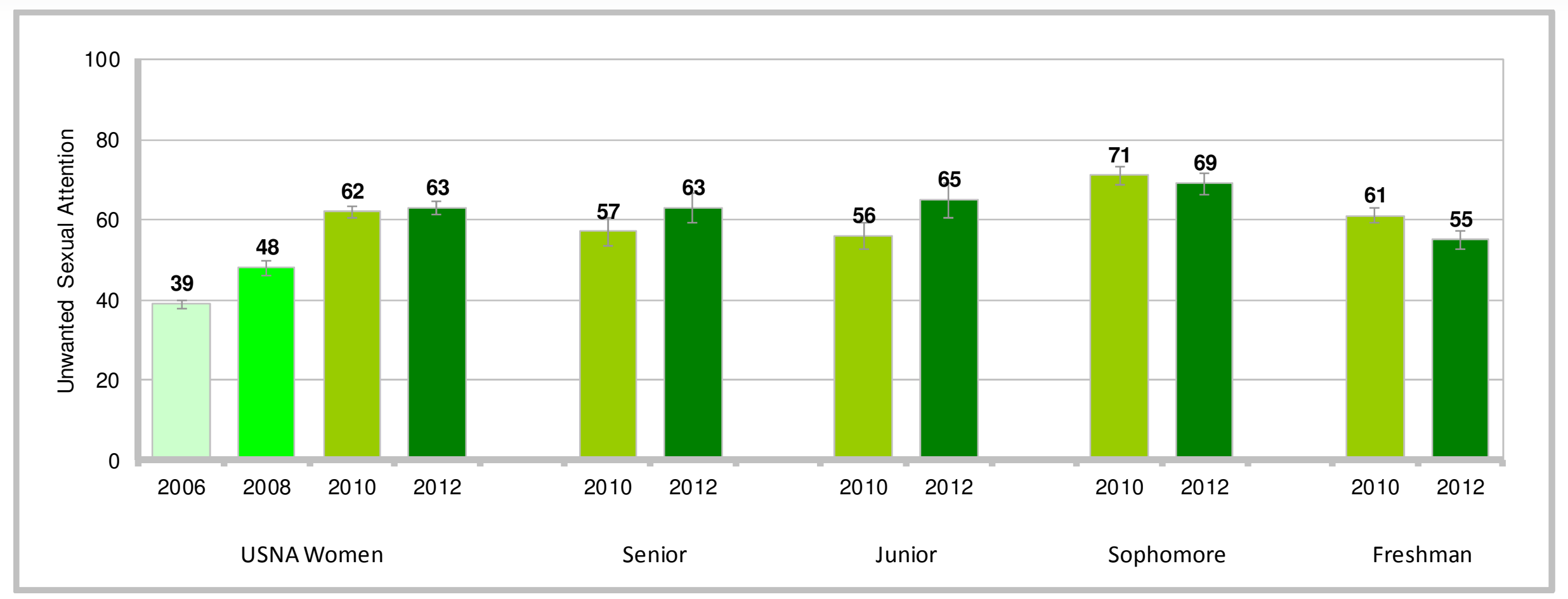

- $63 \%$ of women indicated experiencing unwanted sexual attention in 2012

- 2012 comparisons across years - higher than 2008, 2006

- Seniors and juniors in 2012 higher than 2010, whereas freshmen lower

- Class differences in $\mathbf{2 0 1 2}$ - higher response led by sophomores; lower response led by freshmen 


\section{Unwanted Sexual Attention Incident Rate Percent of Men}

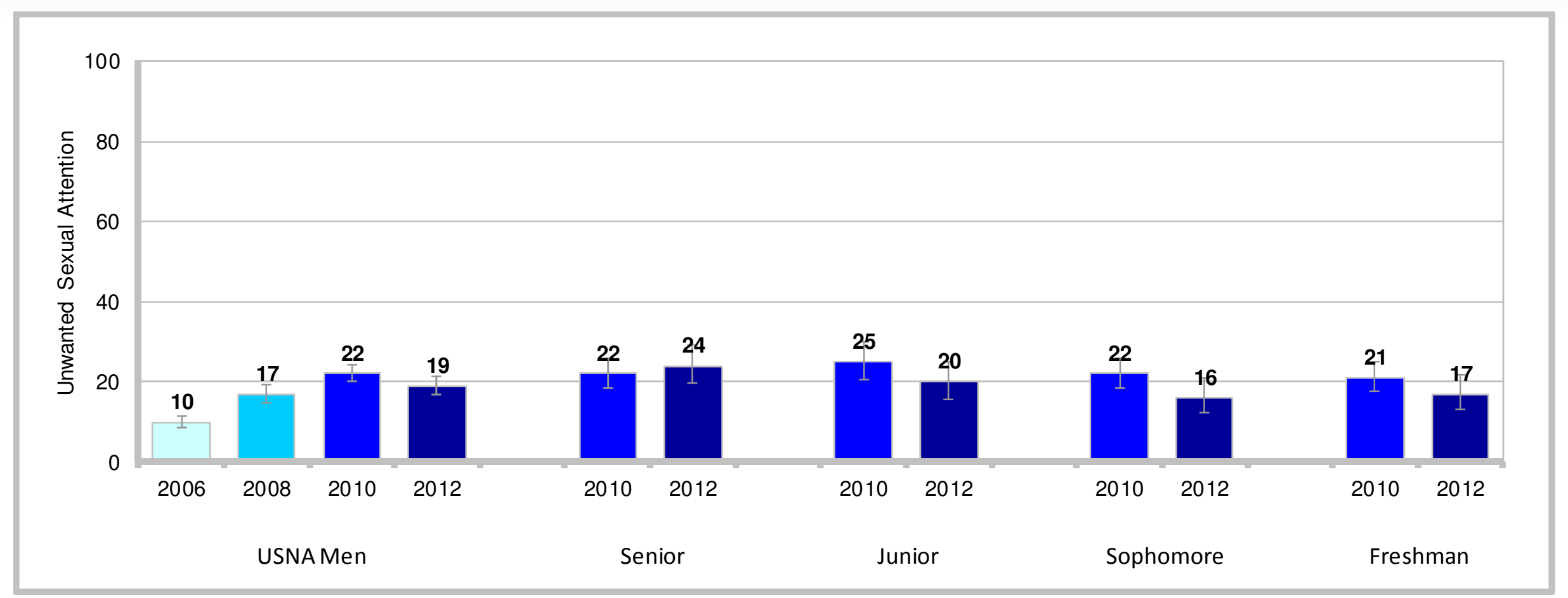

- $19 \%$ of men indicated experiencing unwanted sexual attention in 2012

- 2012 comparisons across years - higher than 2006

- Sophomores in 2012 lower than 2010

- Class differences in 2012 - no differences 


\section{Sexual Coercion Incident Rate}

Percent of Women

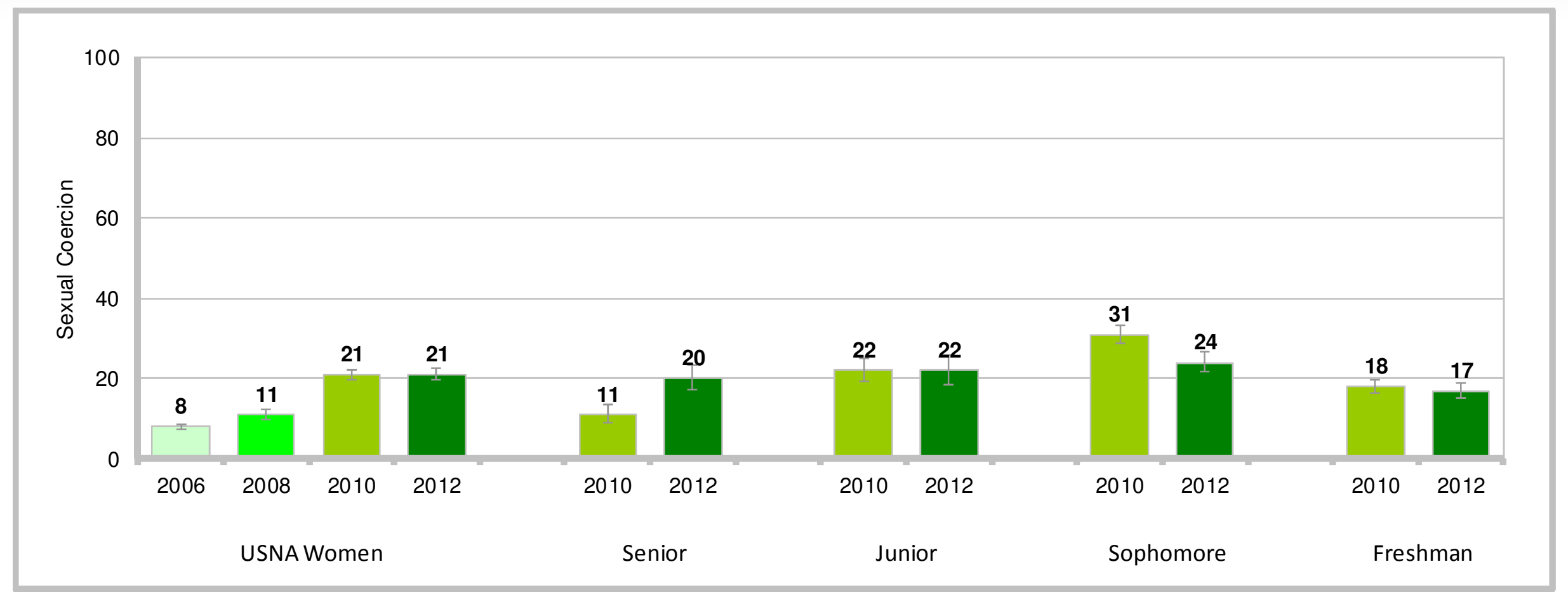

- $21 \%$ of women indicated experiencing sexual coercion in 2012

- 2012 comparisons across years - higher than 2008, 2006

- Sophomores in 2012 lower than 2010, whereas seniors higher

- Class differences in $\mathbf{2 0 1 2}$ - higher response led by sophomores; lower response led by freshmen 


\section{Sexual Coercion Incident Rate}

Percent of Men

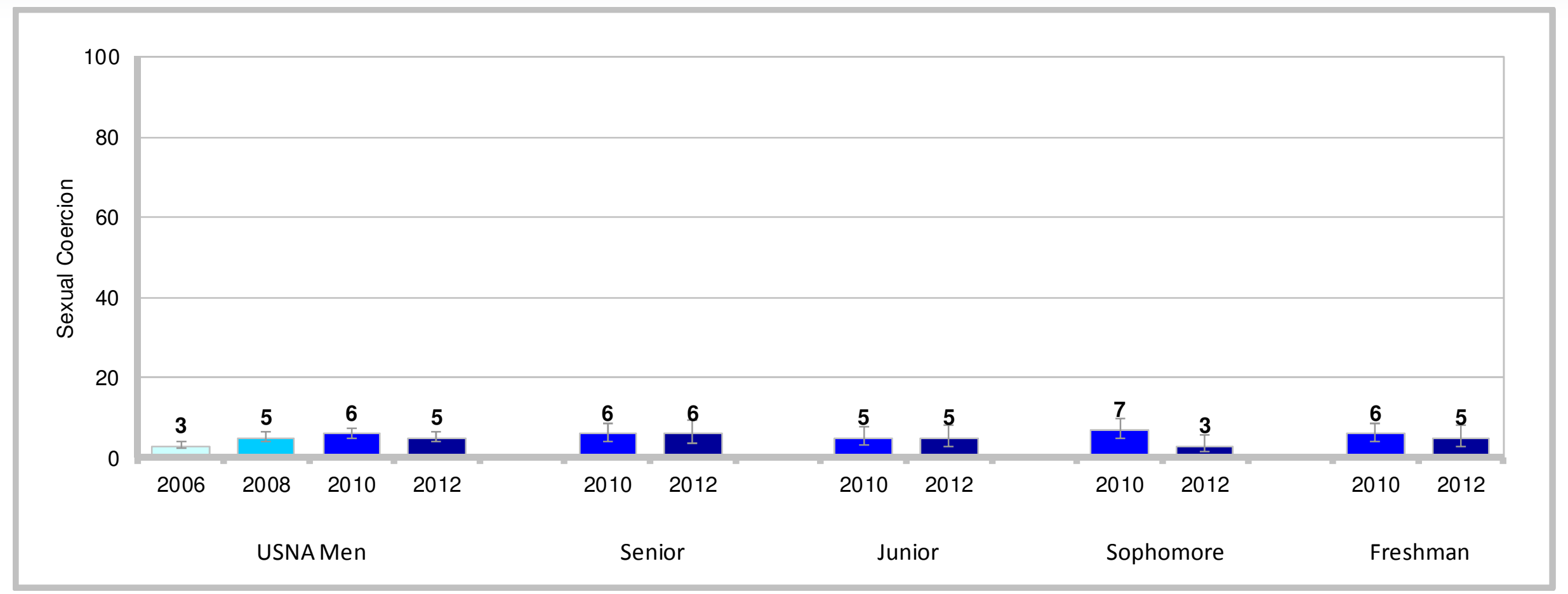

- $5 \%$ of men indicated experiencing sexual coercion in 2012

- 2012 comparisons across years - higher than 2006

- Sophomores in 2012 lower than 2010

- Class differences in 2012 - no differences 


\section{Contents}

Slide

- Introduction .................................................................................. 3

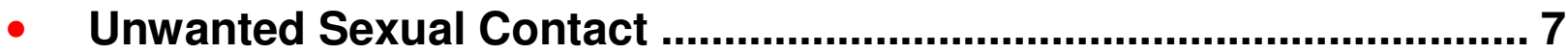

- Unwanted Gender-Related Behaviors ............................................. 49

- Sexual Harassment ..................................................................... 51

- Categories of Behaviors Associated with Sexual Harassment........................................................................................ 54

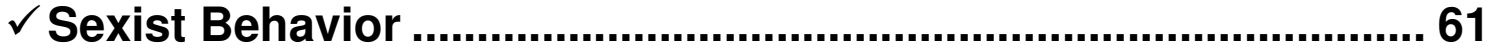

- Organizational Affiliation of Offender ........................................ 64

- Discussing/Reporting Experiences............................................ 67

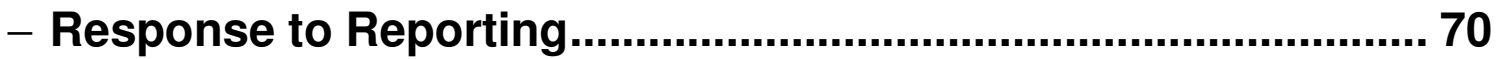

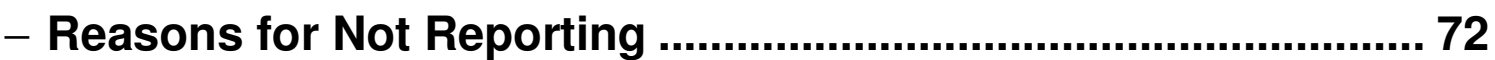

- Stalking ............................................................................................ 75

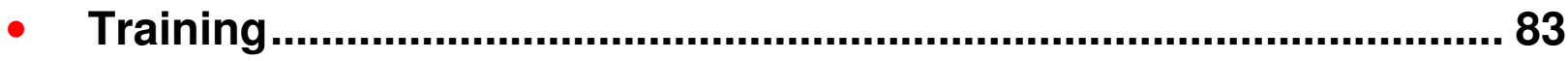

- Student Perceptions............................................................................ 93 


\section{Sexist Behavior Incident Rate Percent of Women}

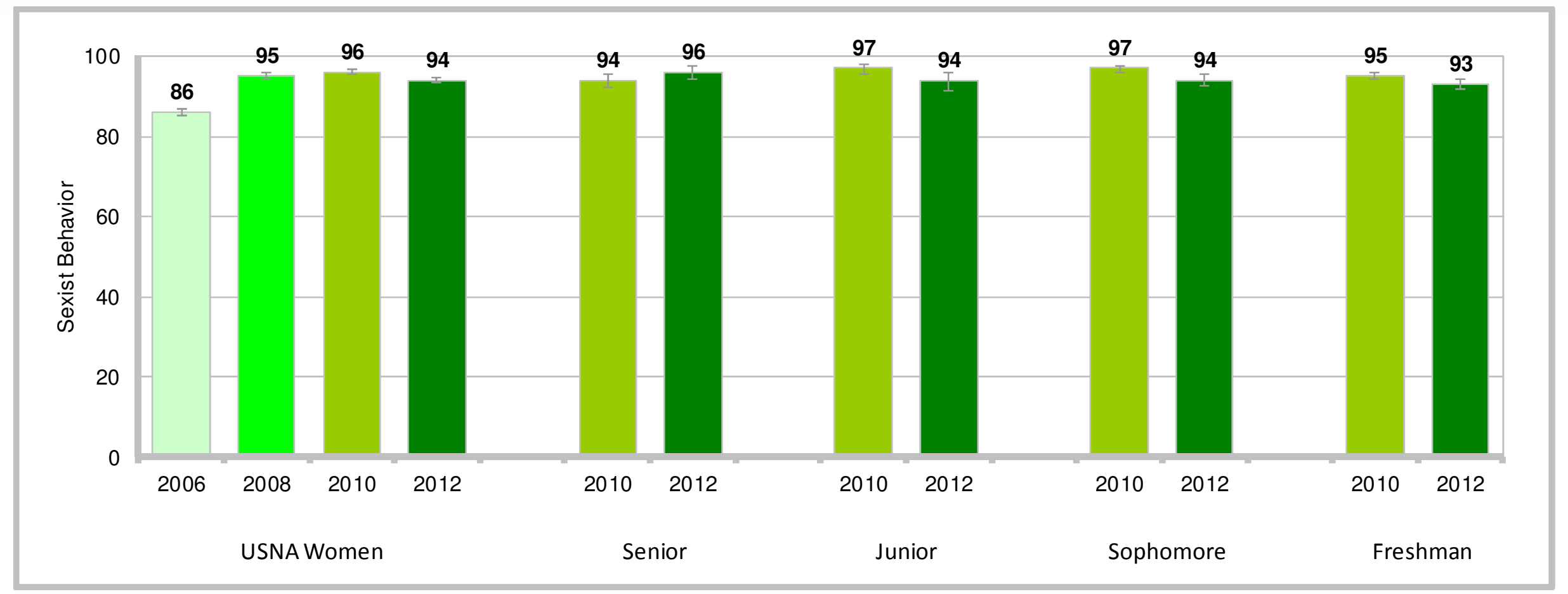

- $94 \%$ of women indicated experiencing sexist behavior in 2012

- 2012 comparisons across years - lower than 2010; higher than 2006

- Juniors, sophomores, and freshmen in 2012 lower than 2010

- Class differences in 2012 - no differences 


\section{Sexist Behavior Incident Rate}

Percent of Men

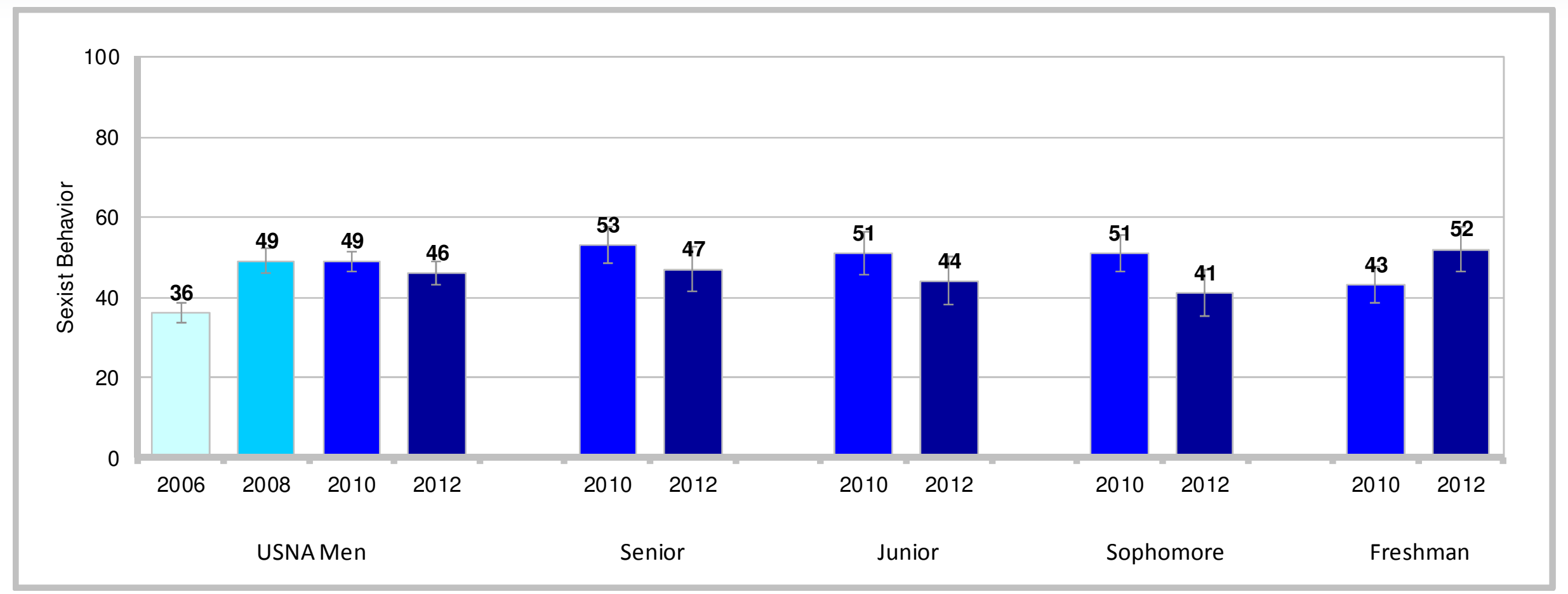

- $46 \%$ of men indicated experiencing sexist behavior in 2012

- 2012 comparisons across years - higher than 2006

- Sophomores in 2012 lower than 2010, whereas freshmen higher

- Class differences in 2012 - no differences 


\section{Contents}

- Introduction 3

- Unwanted Sexual Contact ................................................................... 7

- Unwanted Gender-Related Behaviors ............................................... 49

- Sexual Harassment ...................................................................... 51

- Categories of Behaviors Associated with Sexual Harassment.................................................................................... 54

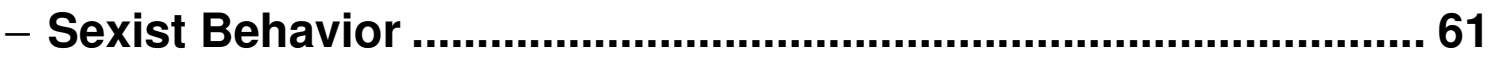

$\checkmark$ Organizational Affiliation of Offender .......................................64 64

- Discussing/Reporting Experiences............................................. 67

- Response to Reporting.............................................................. 70

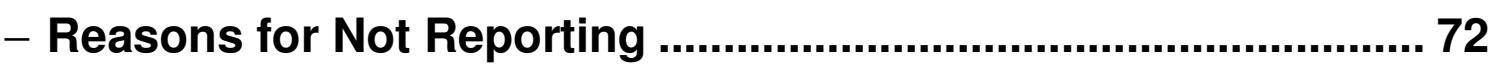

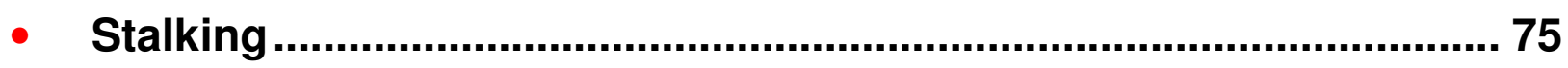

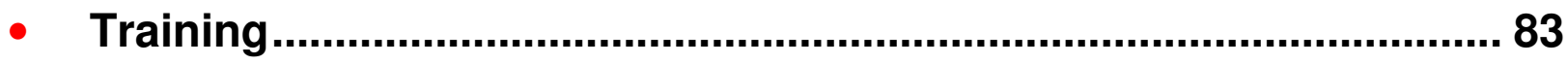

- Student Perceptions............................................................................ 93 


\section{Combinations of Offender Affiliations}

\section{Percent of Women Who Experienced Unwanted Gender-Related Behavior}

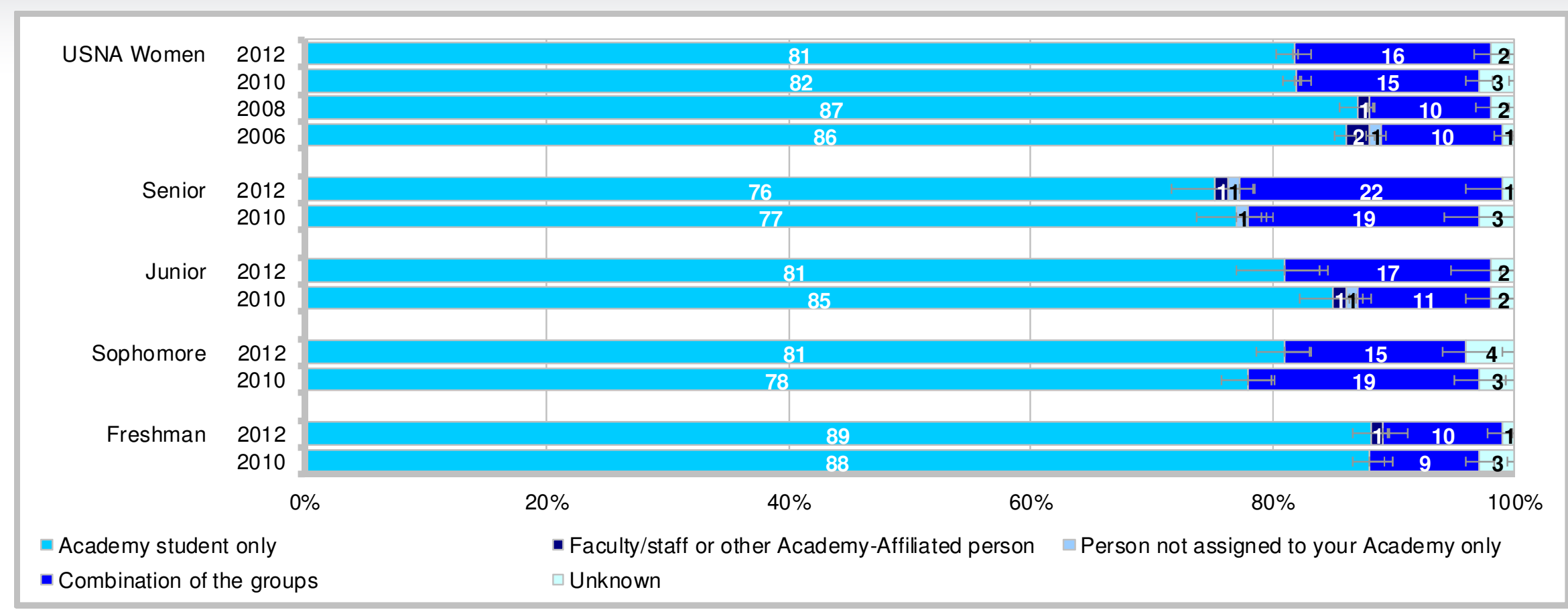

- $96 \%$ of women indicated experiencing one or more of the unwanted gender-related behaviors (i.e., crude/offensive behavior, unwanted sexual attention, sexual coercion, sexist behavior). Of those, $81 \%$ indicated the offender was an Academy student only; $0 \%$ indicated faculty/staff; $0 \%$ indicated person not assigned to the Academy; $16 \%$ indicated combination of groups; and $2 \%$ indicated unknown.

- 2012 comparisons across years - Academy student only lower than 2008, 2006; faculty/staff lower than 2006; combination of groups higher than 2008, 2006

- Juniors indicating combination of groups in 2012 higher than 2010, whereas sophomores lower; seniors and freshmen indicating unknown in 2012 lower than 2010

- Class differences in 2012 - Academy student only led by freshmen; combination of groups led by seniors; unknown led by sophomores 


\section{Combinations of Offender Affiliations \\ Percent of Men Who Experienced Unwanted Gender-Related Behavior}

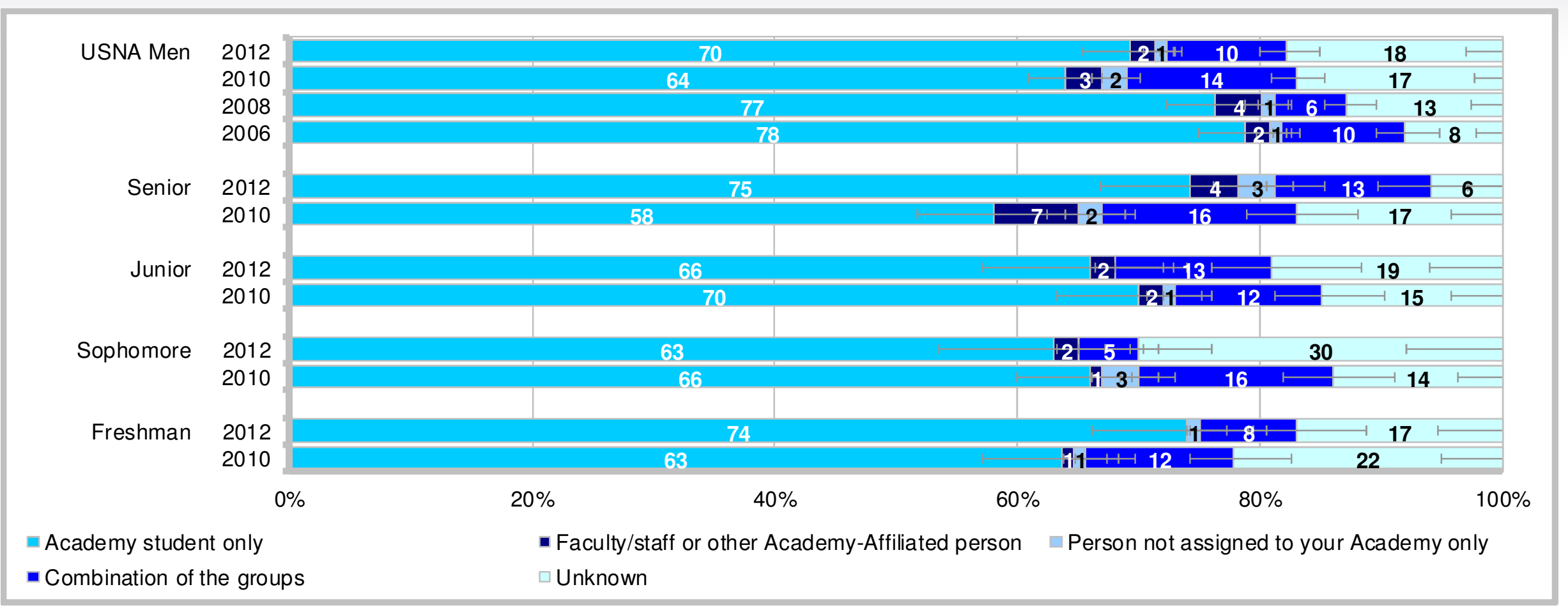

- $61 \%$ of men indicated experiencing one or more of the unwanted gender-related behaviors (i.e., crude/offensive behavior, unwanted sexual attention, sexual coercion, sexist behavior). Of those, $70 \%$ indicated the offender was Academy student only; $2 \%$ indicated faculty/staff; $1 \%$ indicated person not assigned to the Academy; $10 \%$ indicated combination of groups; and 18\% indicated unknown.

- 2012 comparisons across years - Academy student only higher than 2010, whereas lower than 2008, 2006; combination of groups lower than 2010, whereas higher than 2008; unknown higher than 2008, 2006

- Seniors and freshmen indicating Academy student only in 2012 higher than 2010; sophomores indicating person not assigned to the Academy in 2012 lower than 2010; sophomores indicating combination of groups in 2012 lower than 2010; seniors indicating unknown in 2012 lower than 2010, whereas sophomores higher

- Class differences in 2012 - unknown led by sophomores SAGR 2012 Q15 


\section{Contents}

- Introduction 3

- Unwanted Sexual Contact ................................................................... 7

- Unwanted Gender-Related Behaviors ............................................... 49

- Sexual Harassment ...................................................................... 51

- Categories of Behaviors Associated with Sexual Harassment...................................................................................... 54

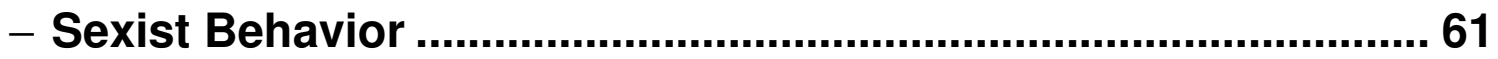

- Organizational Affiliation of Offender .........................................64 64

$\checkmark$ Discussing/Reporting Experiences...........................................6 67

- Response to Reporting.............................................................. 70

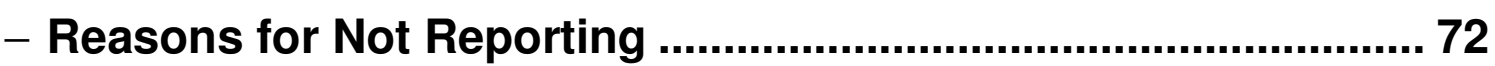

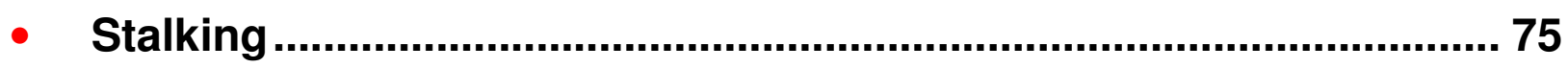

- Training................................................................................................... 83

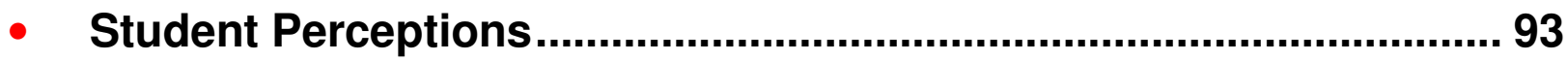




\section{Discuss/Report This Situation to an Authority or Organization}

Percent of Women Who Experienced Unwanted Gender-Related Behavior

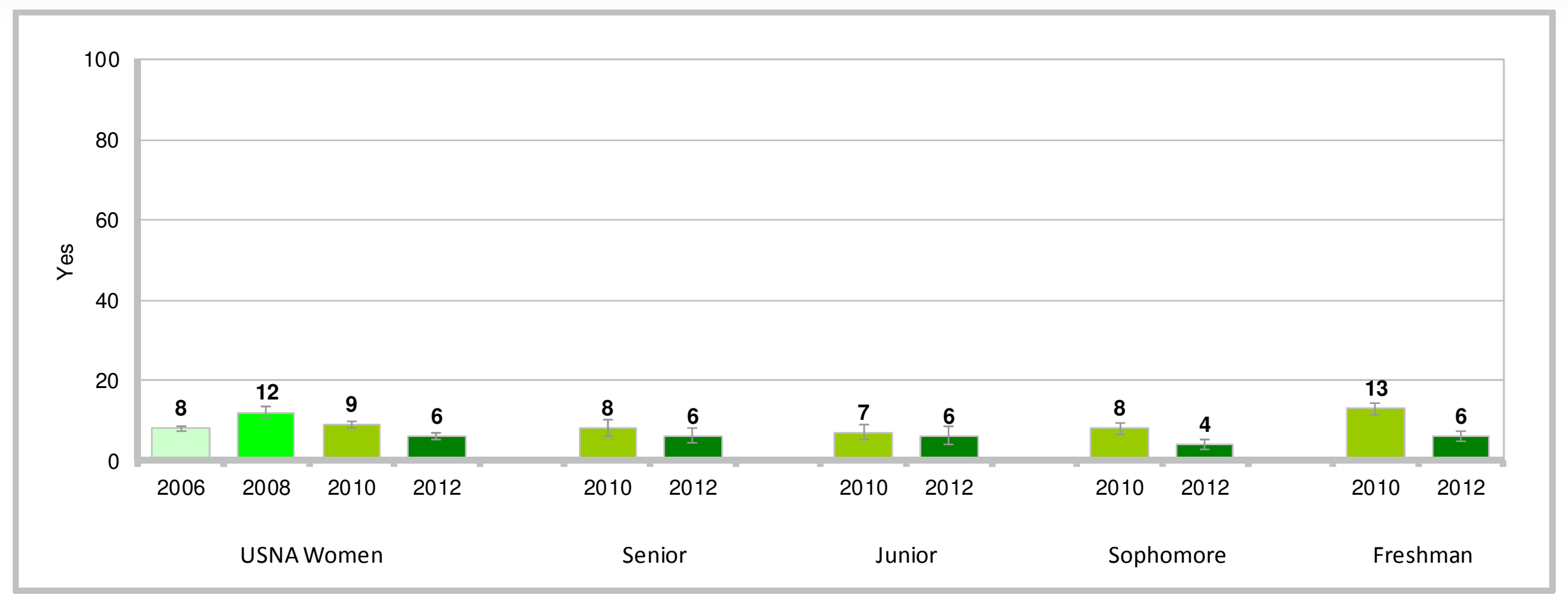

- $96 \%$ of women indicated experiencing one or more of the unwanted gender-related behaviors (i.e., crude/offensive behavior, unwanted sexual attention, sexual coercion, sexist behavior). Of those, $6 \%$ indicated they reported the situation to an authority or organization.

- 2012 comparisons across years - lower than 2010, 2008, 2006

- Sophomores and freshmen in 2012 lower than 2010

- Class differences in 2012 - lower response led by sophomores 


\section{Discuss/Report This Situation to an Authority or Organization Percent of Men Who Experienced Unwanted Gender-Related Behavior}

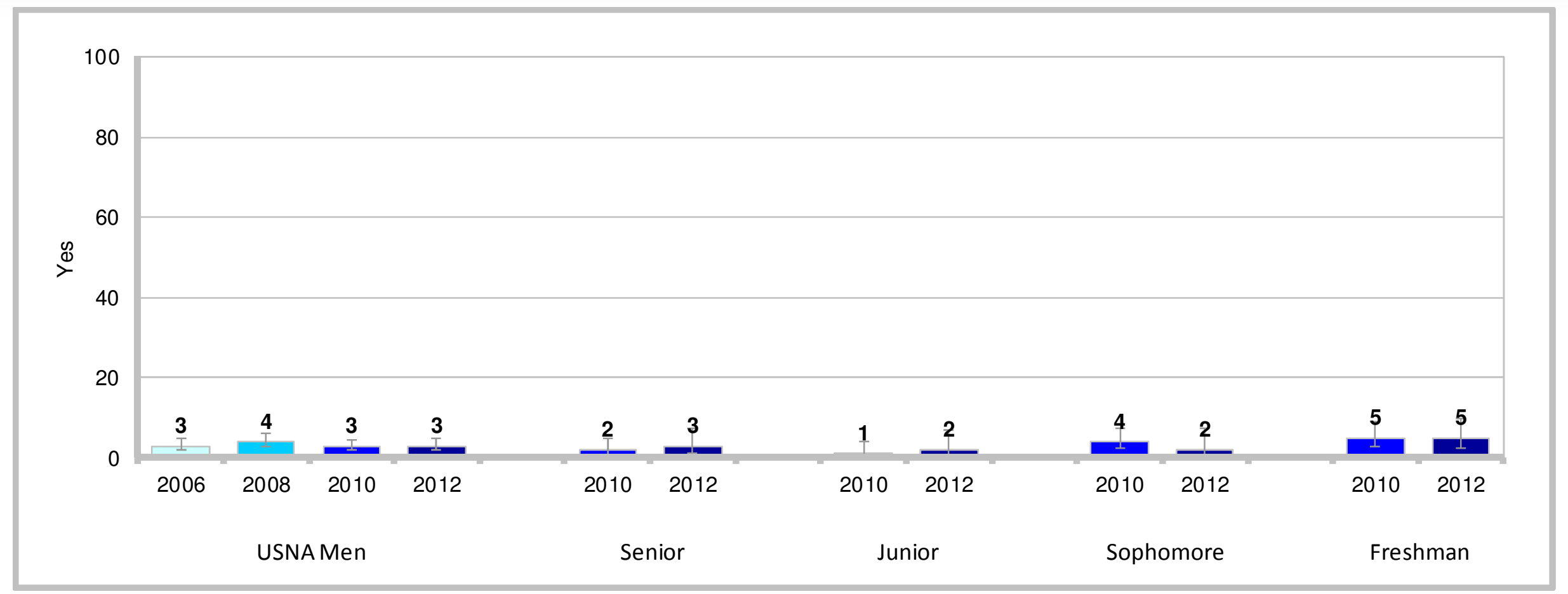

- $61 \%$ of men indicated experiencing one or more of the unwanted gender-related behaviors (i.e., crude/offensive behavior, unwanted sexual attention, sexual coercion, sexist behavior). Of those, $3 \%$ indicated they reported the situation to an authority or organization.

- 2012 comparisons across years - no differences

- Class differences in 2012 - no differences 


\section{Contents}

- Introduction ..................................................................................... 3

- Unwanted Sexual Contact ...................................................................... 7

- Unwanted Gender-Related Behaviors .............................................. 49

- Sexual Harassment ..................................................................... 51

- Categories of Behaviors Associated with Sexual Harassment........................................................................................ 54

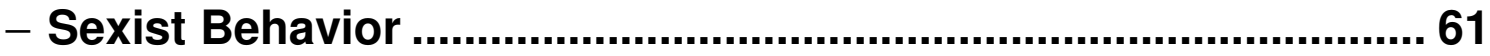

- Organizational Affiliation of Offender ...........................................64

- Discussing/Reporting Experiences...............................................67 67

$\checkmark$ Response to Reporting................................................................ 70

- Reasons for Not Reporting ........................................................... 72

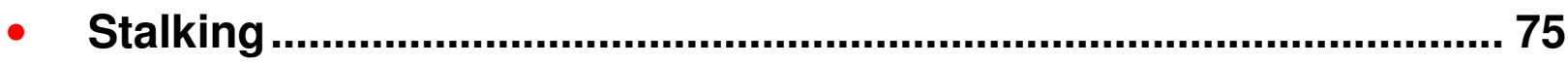

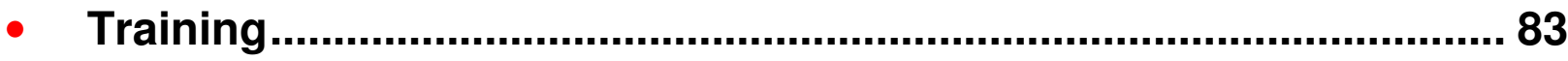

- Student Perceptions.......................................................................... 93 


\section{Actions Taken in Response to Discussing/Reporting}

Percent of Women Who Experienced Unwanted Gender-Related Behaviors and Reported It

\begin{tabular}{|c|c|c|c|c|c|c|}
\hline Response to Reporting & $\begin{array}{l}\text { Survey } \\
\text { Year }\end{array}$ & Total & Senior & Junior & Sophomore & Freshman \\
\hline \multirow{2}{*}{ The situation was corrected } & 2012 & $41 \downarrow$ & 30 & $62 \uparrow$ & $29 \downarrow$ & $45 \downarrow$ \\
\hline & 2010 & 50 & 31 & 30 & 53 & 68 \\
\hline \multirow{2}{*}{ My situation was/is being investigated } & 2012 & 35 & $30 \uparrow$ & 25 & $\overline{71 \uparrow}$ & $18 \downarrow$ \\
\hline & 2010 & 34 & 8 & 30 & 40 & 48 \\
\hline \multirow{2}{*}{$\begin{array}{l}\text { I was kept informed of what actions were } \\
\text { being taken }\end{array}$} & 2012 & 27 & $20 \downarrow$ & 38 & 43 & $18 \downarrow$ \\
\hline & 2010 & 35 & 38 & 20 & 27 & 44 \\
\hline \multirow{2}{*}{ I was encouraged to "tough it out" } & 2012 & 44 & 40 & 62 & 43 & $27 \downarrow$ \\
\hline & 2010 & 44 & 38 & 50 & 33 & 52 \\
\hline \multirow{2}{*}{$\begin{array}{l}\text { My situation was discounted or not taken } \\
\text { seriously }\end{array}$} & 2012 & 36 & 50 & 38 & $\overline{57 \uparrow}$ & $9 \downarrow$ \\
\hline & 2010 & 35 & 46 & 40 & 20 & 36 \\
\hline \multirow{2}{*}{ Action was taken against me } & 2012 & $14 \uparrow$ & 20 & 25 & $14 \uparrow$ & $0 \downarrow$ \\
\hline & 2010 & 6 & NR & NR & 0 & 16 \\
\hline \multirow{2}{*}{$\begin{array}{l}\text { I was ridiculed or scorned by others for } \\
\text { discussing/reporting the situation }\end{array}$} & 2012 & 46 & 40 & 50 & $57 \uparrow$ & $36 \downarrow$ \\
\hline & 2010 & 38 & 38 & 40 & 20 & 48 \\
\hline \multirow{2}{*}{ Some other action was taken } & 2012 & $41 \uparrow$ & 30 & $62 \uparrow$ & 29 & $45 \uparrow$ \\
\hline & 2010 & 16 & 15 & 10 & 40 & 4 \\
\hline Margins of Error & & $\pm 2-8 \%$ & $\pm 11-17 \%$ & $\pm 13-20 \%$ & $\pm 0-15 \%$ & $\pm 0-10 \%$ \\
\hline \multirow{4}{*}{ - Results for men not reportable } & & \multicolumn{3}{|c|}{ Higher Response of Yes } & & \\
\hline & & \multicolumn{3}{|c|}{ Lower Response of Yes } & & \\
\hline & & \multirow{2}{*}{\multicolumn{2}{|c|}{ Higher in 2012 than 2010}} & $\uparrow$ & & \\
\hline & & & & $\downarrow$ & & \\
\hline
\end{tabular}




\section{Contents}

- Introduction 3

- Unwanted Sexual Contact ................................................................... 7

- Unwanted Gender-Related Behaviors ............................................... 49

- Sexual Harassment ...................................................................... 51

- Categories of Behaviors Associated with Sexual Harassment...................................................................................... 54

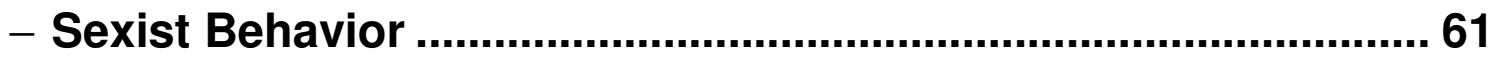

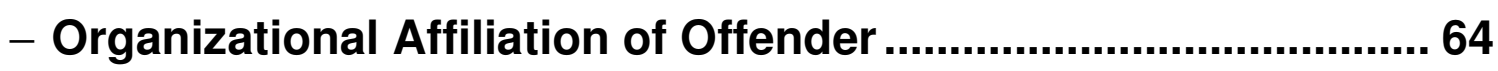

- Discussing/Reporting Experiences............................................. 67

- Response to Reporting.................................................................... 70

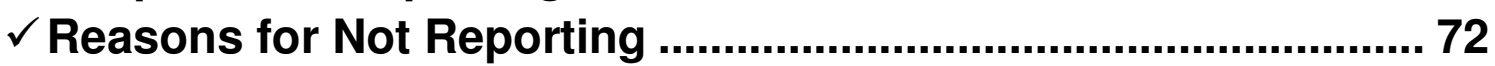

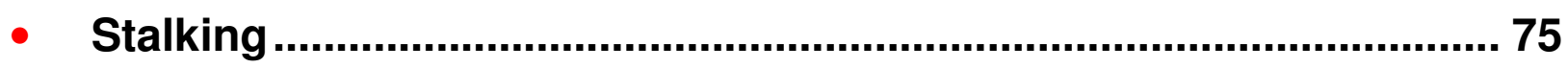

- Training.................................................................................................. 83

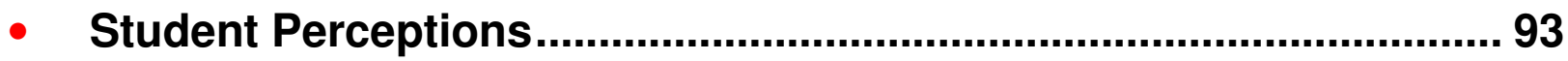




\section{Most Frequently Selected Reasons for}

\section{Not Reporting Unwanted Gender-Related Situation}

Percent of Women Who Experienced Unwanted Gender-Related Behaviors and Did Not Report It

\begin{tabular}{|c|c|c|c|c|c|c|}
\hline Reasons for Not Reporting & $\begin{array}{l}\text { Survey } \\
\text { Year }\end{array}$ & Total & Senior & Junior & Sophomore & Freshman \\
\hline \multirow{2}{*}{$\begin{array}{l}\text { Thought it was not important enough to } \\
\text { report }\end{array}$} & 2012 & $82 \uparrow$ & $79 \uparrow$ & $83 \uparrow$ & 80 & $85 \downarrow$ \\
\hline & 2010 & 79 & 73 & 75 & 81 & 89 \\
\hline \multirow{2}{*}{ Took care of the problem myself } & 2012 & $73 \downarrow$ & 79 & 77 & 69 & $68 \downarrow$ \\
\hline & 2010 & 76 & 81 & 80 & 71 & 72 \\
\hline \multirow{2}{*}{ Did not want people gossiping about me } & 2012 & 48 & $52 \uparrow$ & 48 & $45 \downarrow$ & 48 \\
\hline & 2010 & 48 & 41 & 44 & 58 & 47 \\
\hline \multirow{2}{*}{$\begin{array}{l}\text { Thought it would hurt my reputation and } \\
\text { standing }\end{array}$} & 2012 & 38 & $42 \uparrow$ & 38 & $37 \downarrow$ & 35 \\
\hline & 2010 & 35 & 26 & 34 & 42 & 36 \\
\hline \multirow{2}{*}{$\begin{array}{l}\text { Thought reporting would take too much } \\
\text { time and effort }\end{array}$} & 2012 & 31 & $34 \uparrow$ & 34 & $29 \downarrow$ & 31 \\
\hline & 2010 & 33 & 26 & 34 & 38 & 31 \\
\hline Margins of Error & & $\pm 2 \%$ & $\pm 4-5 \%$ & $\pm 4-6 \%$ & $\pm 3-4 \%$ & $\pm 3 \%$ \\
\hline
\end{tabular}

\begin{tabular}{|l|c|}
\hline Higher Response of Yes & \\
\hline Lower Response of Yes & \\
\hline Higher in 2012 than 2010 & $\uparrow$ \\
\hline Lower in 2012 than 2010 & $\downarrow$ \\
\hline
\end{tabular}




\section{Most Frequently Selected Reasons for Not Reporting Unwanted Gender-Related Situation} Percent of Men Who Experienced Unwanted Gender-Related Behaviors and Did Not Report It

\begin{tabular}{|c|c|c|c|c|c|c|}
\hline Reasons for Not Reporting & $\begin{array}{l}\text { Survey } \\
\text { Year }\end{array}$ & Total & Senior & Junior & Sophomore & Freshman \\
\hline \multirow{2}{*}{$\begin{array}{l}\text { Thought it was not important enough to } \\
\text { report }\end{array}$} & 2012 & 78 & $86 \uparrow$ & 72 & $74 \downarrow$ & $79 \uparrow$ \\
\hline & 2010 & 76 & 70 & 74 & 88 & 69 \\
\hline \multirow{2}{*}{ Took care of the problem myself } & 2012 & 57 & 66 & 56 & 53 & 51 \\
\hline & 2010 & 58 & 60 & 60 & 58 & 54 \\
\hline \multirow{2}{*}{$\begin{array}{l}\text { Thought reporting would take too much } \\
\text { time and effort }\end{array}$} & 2012 & 20 & 22 & 18 & $16 \downarrow$ & 21 \\
\hline & 2010 & 20 & 14 & 19 & 26 & 21 \\
\hline \multirow{2}{*}{ Did not think anything would be done } & 2012 & 16 & $13 \downarrow$ & 11 & 16 & 21 \\
\hline & 2010 & 17 & 21 & 16 & 15 & 16 \\
\hline \multirow{2}{*}{ Felt uncomfortable making a report } & 2012 & 12 & 11 & 10 & 9 & 17 \\
\hline & 2010 & 9 & 6 & 5 & 14 & 10 \\
\hline Margins of Error & & $\pm 3-5 \%$ & $\pm 5-9 \%$ & $\pm 5-10 \%$ & $\pm 5-11 \%$ & $\pm 6-8 \%$ \\
\hline
\end{tabular}

\begin{tabular}{|l|c|}
\hline Higher Response of Yes & \\
\hline Lower Response of Yes & \\
\hline Higher in 2012 than 2010 & $\uparrow$ \\
\hline Lower in 2012 than 2010 & $\downarrow$ \\
\hline
\end{tabular}




\section{Contents}

- Unwanted Sexual Contact ................................................................. 7

- Unwanted Gender-Related Behaviors ................................................ 49

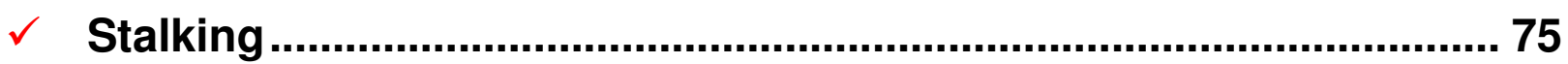

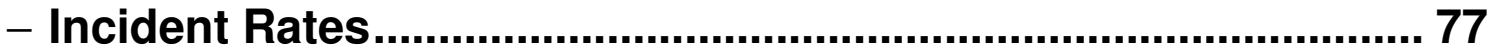

- Stalking and Sexual Harassment Related to Unwanted

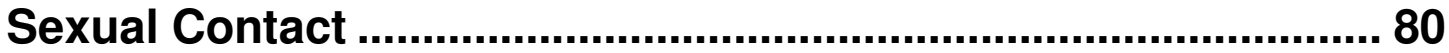

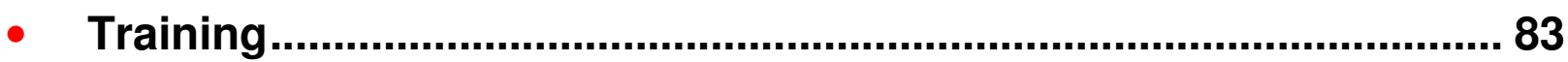

- Student Perceptions............................................................................... 93 


\section{Stalking Incident Rate}

\section{- Definition and measure of stalking:}

- Under Article 120a of the UCMJ, stalking is a crime. The UCMJ definition of stalking is "a course of conduct directed at a specific person that would cause a reasonable person to fear death or bodily harm, including sexual assault, to himself or herself or a member of his or her immediate family."

- Thus, to be punishable under the provision, stalking must be intentional, repeated (two or more occasions of such conduct), and cause unreasonable fear of physical injury. Note that this definition does not limit stalking to association with sexual harassment or sexual assault.

- The measure of stalking on the 2012 SAGR is consistent with the definition in Article 120a of the UCMJ. Students were asked (Q9) whether they had experienced any stalking behaviors (e.g., followed or spied on them in public areas; spied on them in private areas; showed up at places where they were even though he/she had no reason to be there; left unwanted items for them to find; stood outside or hung around their dorm room or classroom even though he/she had no reason to be there; and vandalized or tampered with their belongings) by someone assigned to their Academy, including students and military/civilian personnel, and whether they felt in danger of physical harm or sexual assault as a result of the experience (Q11). 


\section{Contents}

- Introduction 3

- Unwanted Sexual Contact ................................................................... 7

- Unwanted Gender-Related Behaviors ............................................... 49

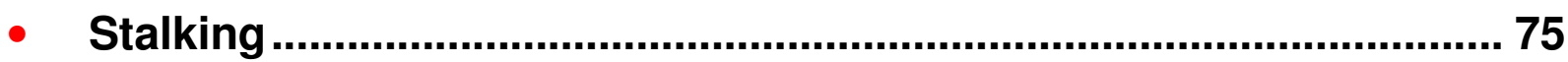

$\checkmark$ Incident Rates......................................................................... 77

- Stalking and Sexual Harassment Related to Unwanted

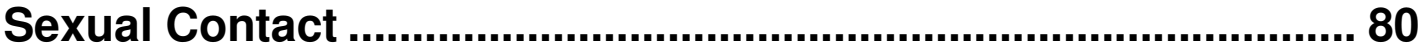

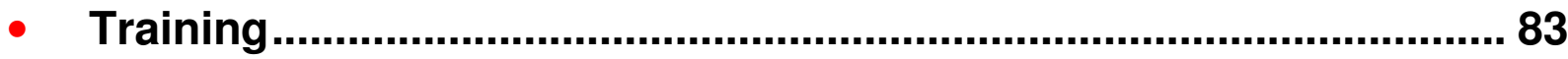

- Student Perceptions ......................................................................... 93 


\section{Stalking Incident Rate}

Percent of Women Who Experienced Stalking Behavior and Felt in Danger

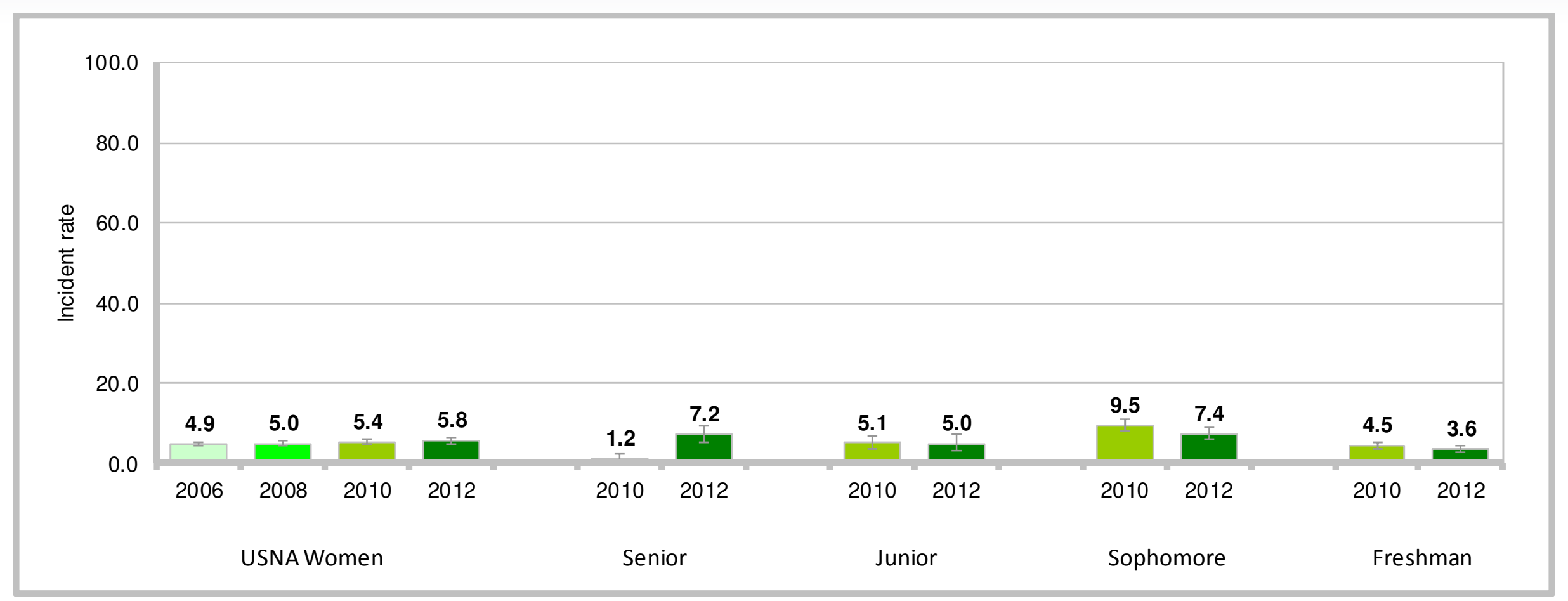

- $5.8 \%$ of women indicated experiencing stalking behavior in 2012

- 2012 comparisons across years - no differences

- Seniors in 2012 higher than 2010

- Class differences in $\mathbf{2 0 1 2}$ - lower response led by freshmen 


\section{Stalking Incident Rate}

Percent of Men Who Experienced Stalking Behavior and Felt in Danger

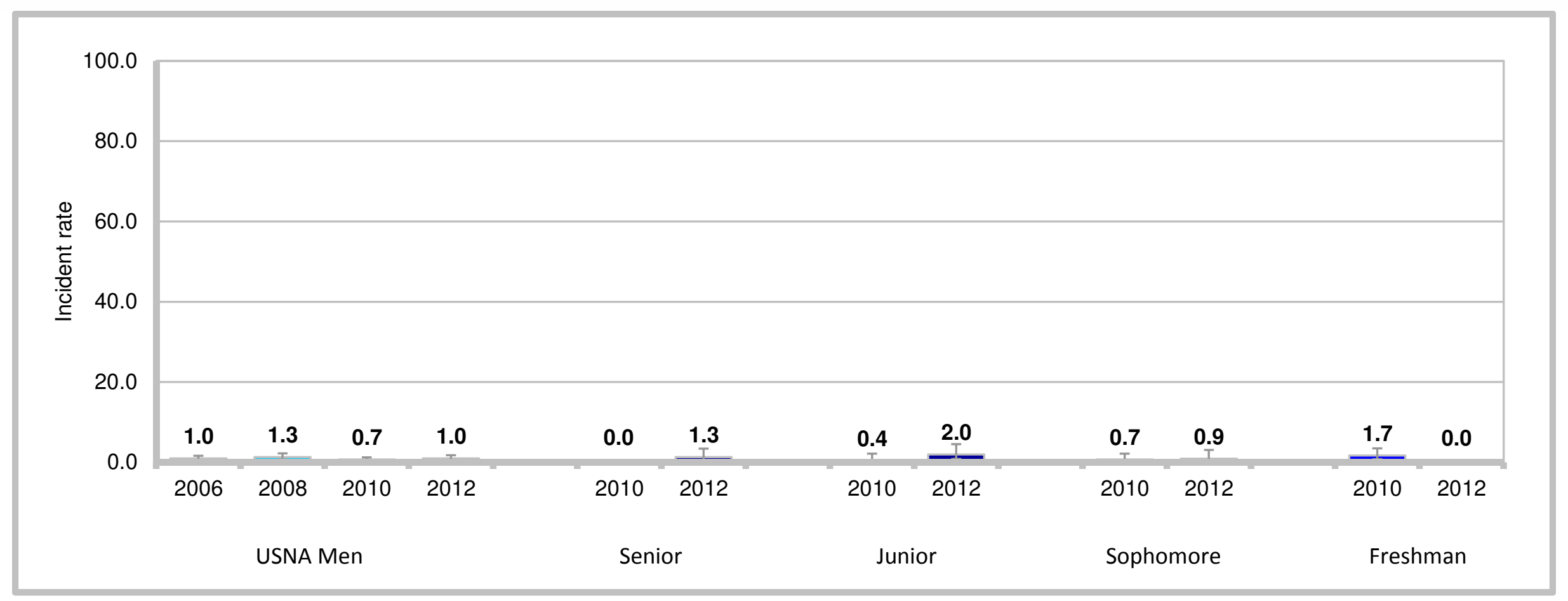

- $1 \%$ of men indicated experiencing stalking behavior in 2012

- 2012 comparisons across years - no differences

- Seniors in 2012 higher than 2010, whereas freshmen lower

- Class differences in 2012 - lower response led by freshmen 


\section{Contents}

- Introduction ..................................................................................... 3

- Unwanted Sexual Contact .............................................................. 7

- Unwanted Gender-Related Behaviors ............................................ 49

• Stalking ...................................................................................... 75

- Incident Rates............................................................................ 77

$\checkmark$ Stalking and Sexual Harassment Related to Unwanted

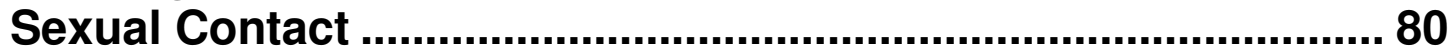

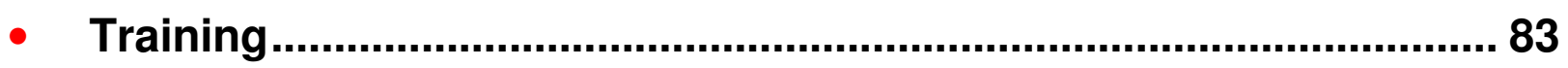

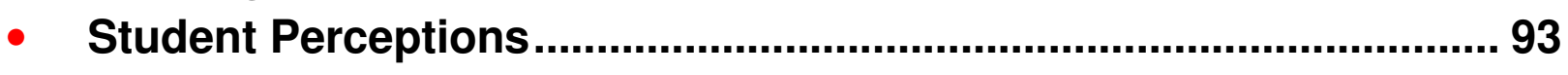




\section{Stalking and Sexual Harassment Possibly Lead to Sexual Assault} Percent of Women

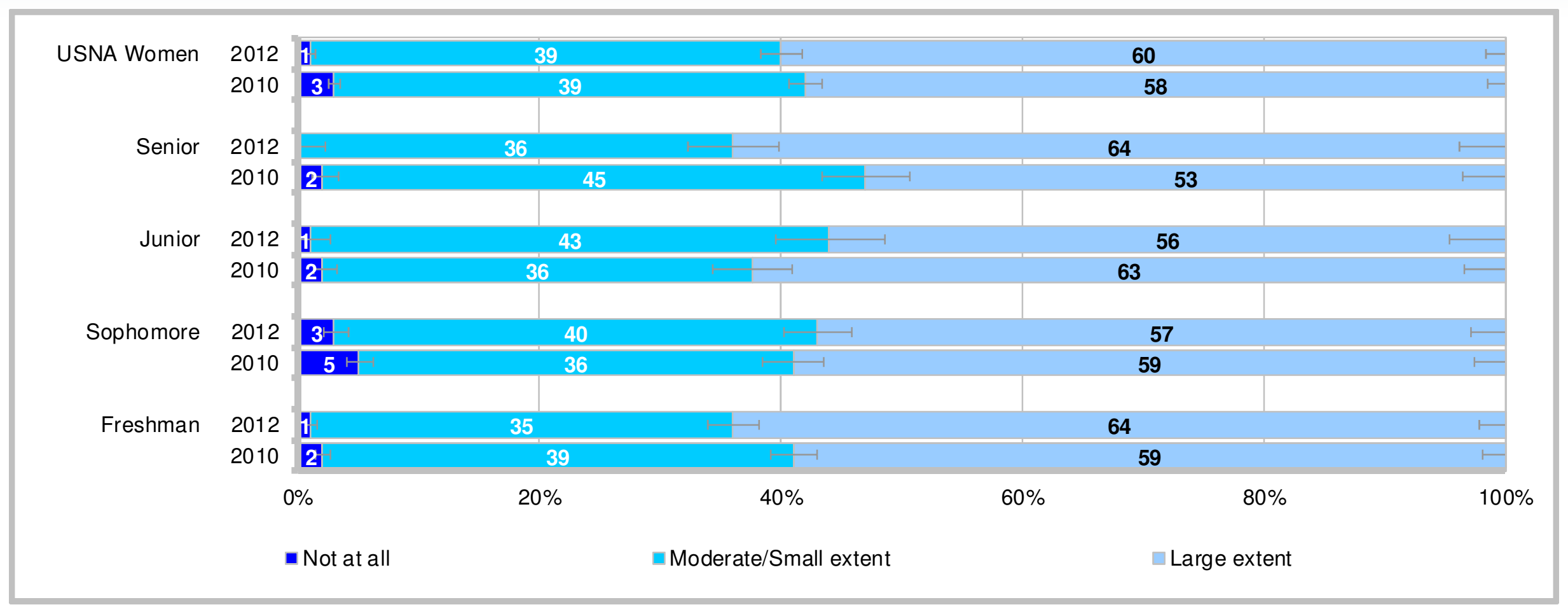

- $99 \%$ of women indicated stalking and sexual harassment possibly lead to sexual assault to at least some extent; $1 \%$ indicated stalking and sexual harassment do not lead to sexual assault

- 2012 comparisons across years - not at all lower than 2010

- Seniors and freshmen indicating large extent in 2012 higher than 2010, whereas juniors lower; seniors and freshmen indicating moderate/small extent in 2012 lower than 2010, whereas juniors and sophomores higher; seniors and sophomores indicating not at all in 2012 lower than 2010

- Class differences in 2012 - large extent led by freshmen; not at all led by sophomores 


\section{Stalking and Sexual Harassment Possibly Lead to Sexual Assault} Percent of Men

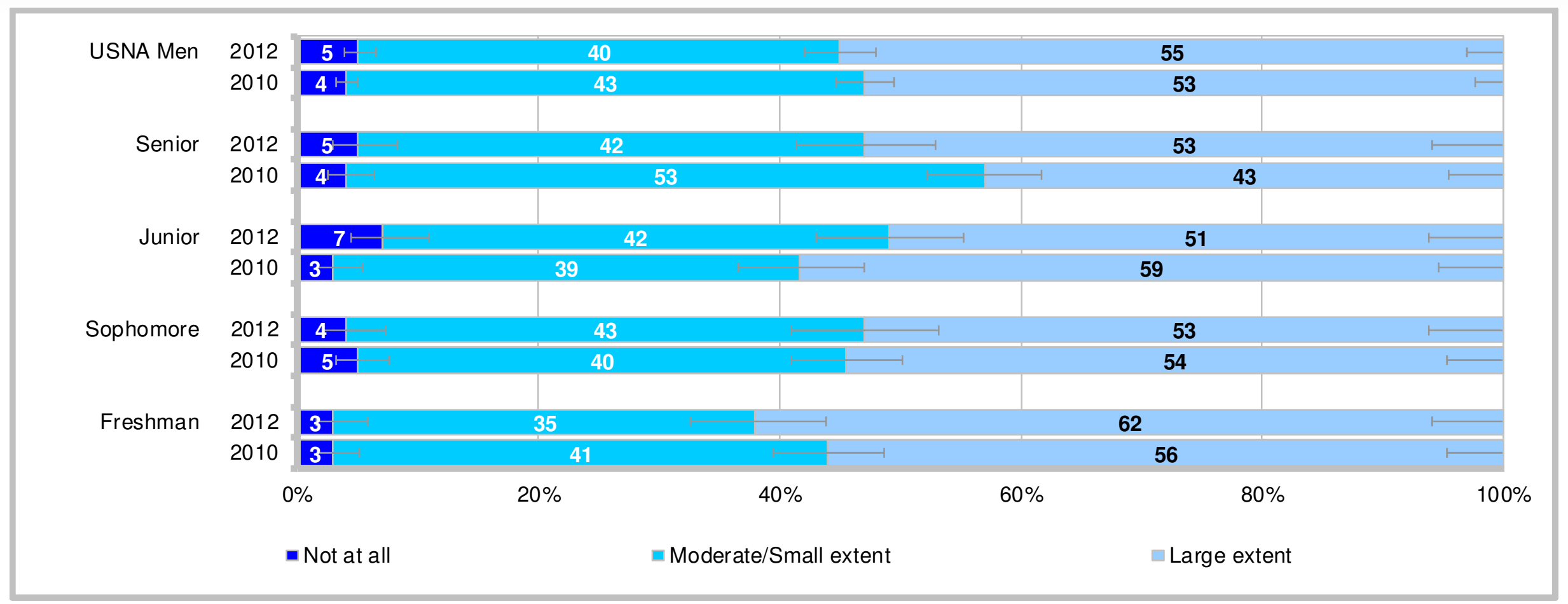

- $95 \%$ of men indicated stalking and sexual harassment possibly lead to sexual assault to at least some extent; $5 \%$ indicated stalking and sexual harassment do not lead to sexual assault

- 2012 comparisons across years - no differences

- Seniors indicating large extent in 2012 higher than 2010; seniors indicating moderate/small extent in 2012 lower than 2010; juniors indicating not at all in 2012 higher than 2010

- Class differences in 2012 - large extent led by freshmen 


\section{Contents}

Slide

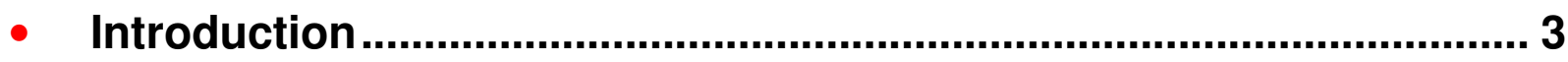

- Unwanted Sexual Contact .................................................................... 7

- Unwanted Gender-Related Behaviors .............................................. 49

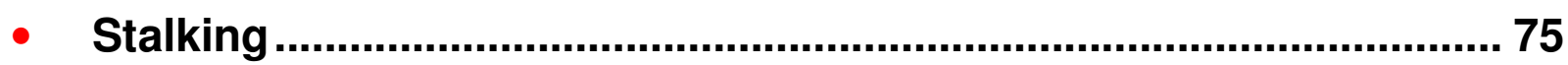

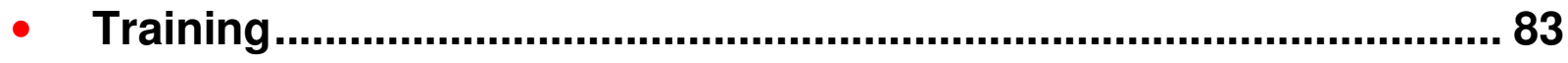

$\checkmark$ Sexual Assault Training Availability and Effectiveness ............ 83

- Sexual Harassment Training Availability and Effectiveness.... 88

- Student Perceptions....................................................................... 93 


\section{Received Training Since June 2011 on Topics Related to Sexual Assault} Percent of Women

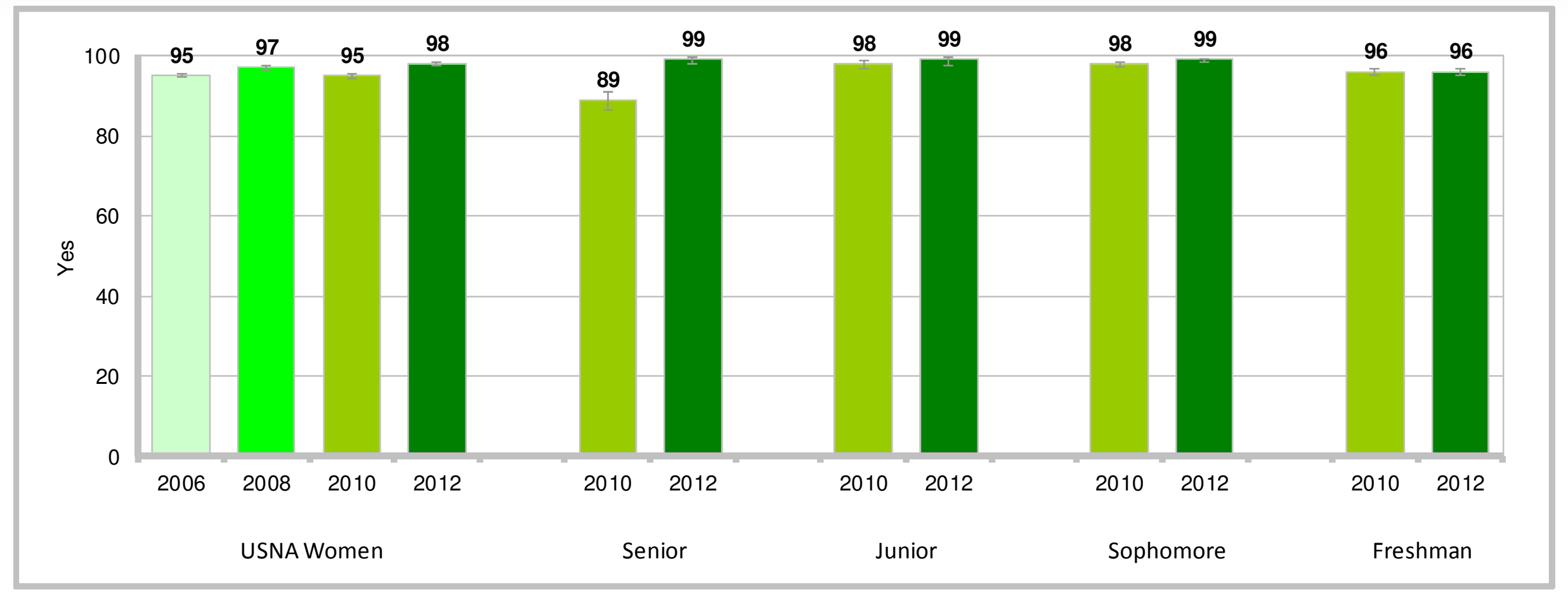

- $98 \%$ of women indicated receiving training on topics related to sexual assault in 2012

- 2012 comparisons across years - higher than 2010, 2006

- Seniors in 2012 higher than 2010

- Class differences in $\mathbf{2 0 1 2}$ - lower response led by freshmen 


\section{Received Training Since June 2011 on Topics Related to Sexual Assault} Percent of Men

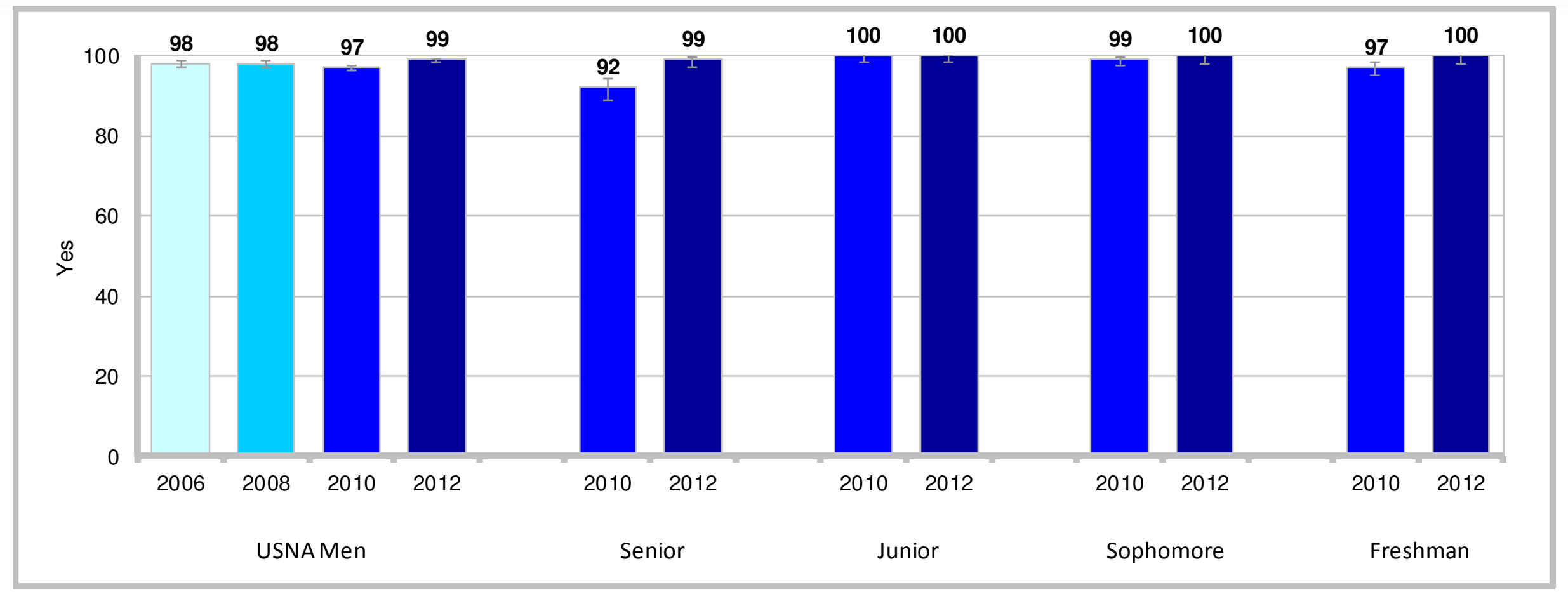

- $99 \%$ of men indicated receiving training on topics related to sexual assault in 2012

- 2012 comparisons across years - higher than 2010

- Seniors and freshmen in 2012 higher than 2010

- Class differences in 2012 - no differences 


\section{Effectiveness of Training in Actually Reducing/Preventing Sexual Assault Percent of Women Who Had Training on Sexual Assault}

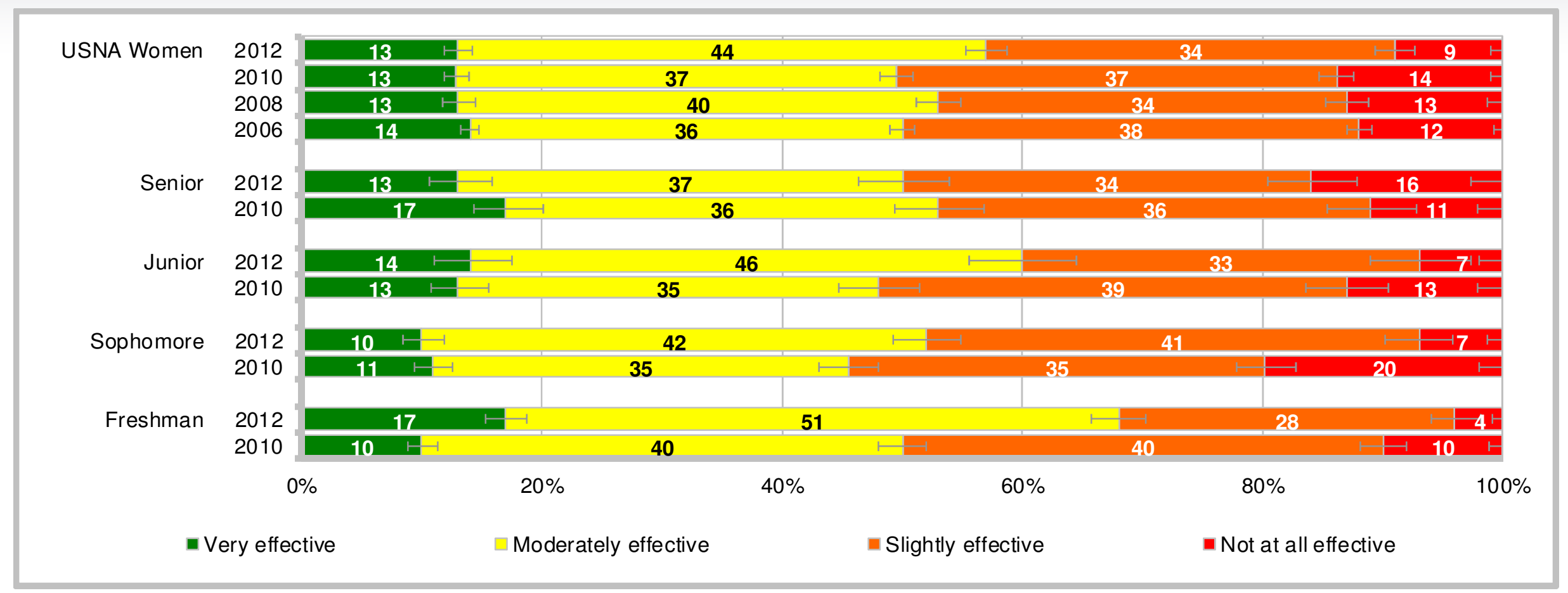

- $13 \%$ of women indicated their training was very effective in actually reducing/preventing sexual assault; $44 \%$ indicated moderately effective; $34 \%$ indicated slightly effective; and $9 \%$ indicated not at all effective

- 2012 comparisons across years - moderately effective higher than 2010, 2008, 2006; slightly effective lower than 2010, 2006; not at all effective lower than 2010, 2008, 2006

- Seniors indicating very effective in 2012 lower than 2010, whereas freshmen higher; juniors, sophomores, and freshmen indicating moderately effective in 2012 higher than 2010; juniors and freshmen indicating slightly effective in 2012 lower than 2010, whereas sophomores higher; seniors indicating not at all effective in 2012 higher than 2010, whereas juniors, sophomores, and freshmen lower

- Class differences in 2012 - very effective led by freshmen; moderately effective led by freshmen; slightly effective led by sophomores; not at all effective led by seniors 


\section{Effectiveness of Training in Actually Reducing/Preventing Sexual Assault} Percent of Men Who Had Training on Sexual Assault

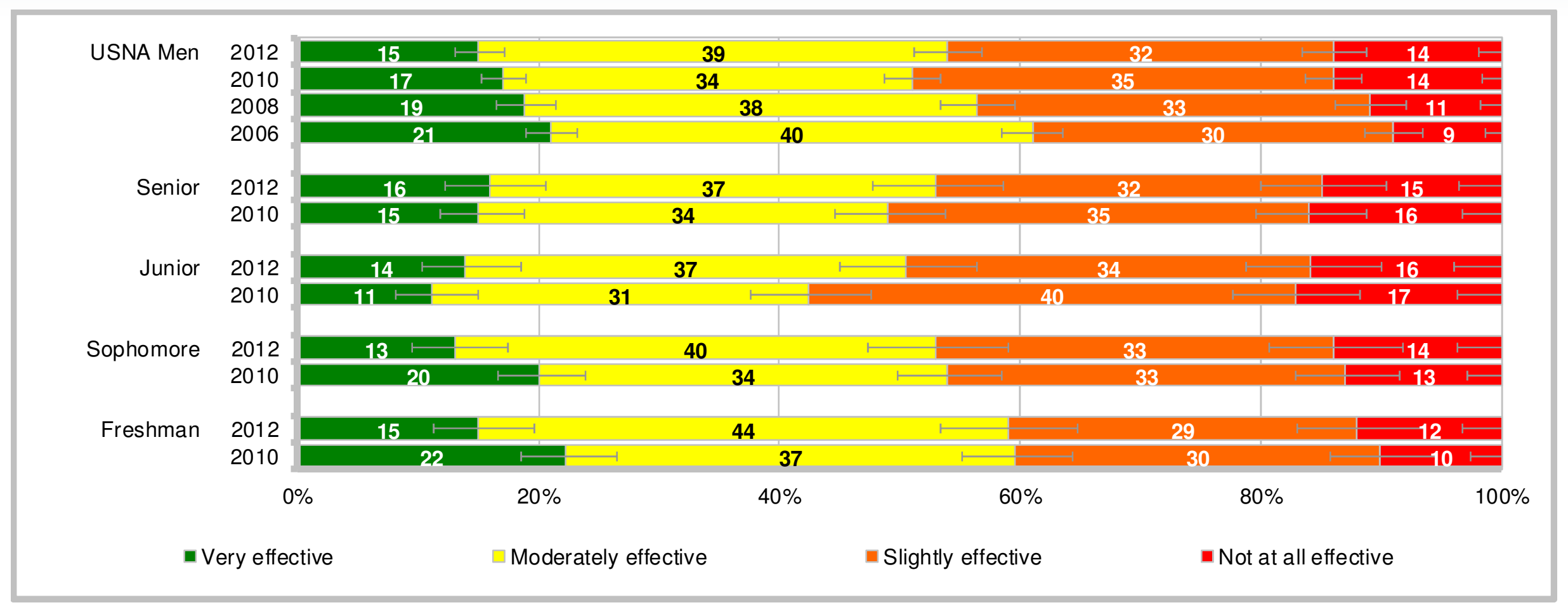

- $15 \%$ of men indicated their training was very effective in actually reducing/preventing sexual assault; $39 \%$ indicated moderately effective; $32 \%$ indicated slightly effective; and $14 \%$ indicated not at all effective

- 2012 comparisons across years - very effective lower than 2008, 2006; moderately effective higher than 2010; not at all effective higher than 2008, 2006

- Sophomores and freshmen indicating very effective in 2012 lower than 2010

- Class differences in 2012 - no differences 


\section{Contents}

Slide

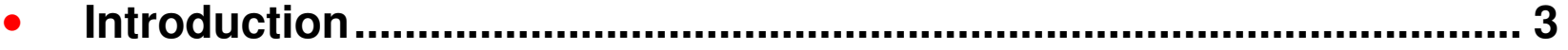

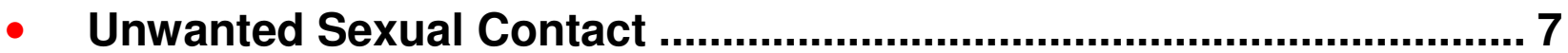

- Unwanted Gender-Related Behaviors .......................................... 49

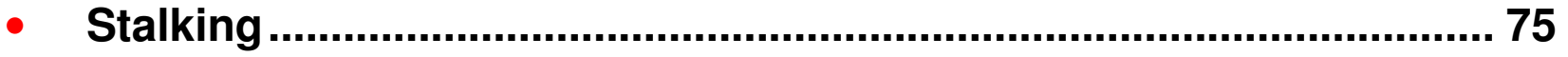

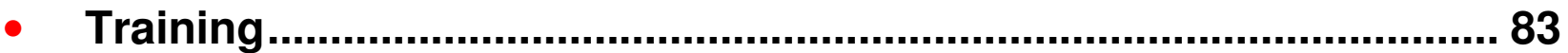

- Sexual Assault Training Availability and Effectiveness ............ 83

$\checkmark$ Sexual Harassment Training Availability and Effectiveness..... 88

- Student Perceptions..................................................................... 93 


\section{Received Training Since June 2011 on Topics Related to Sexual Harassment \\ Percent of Women}

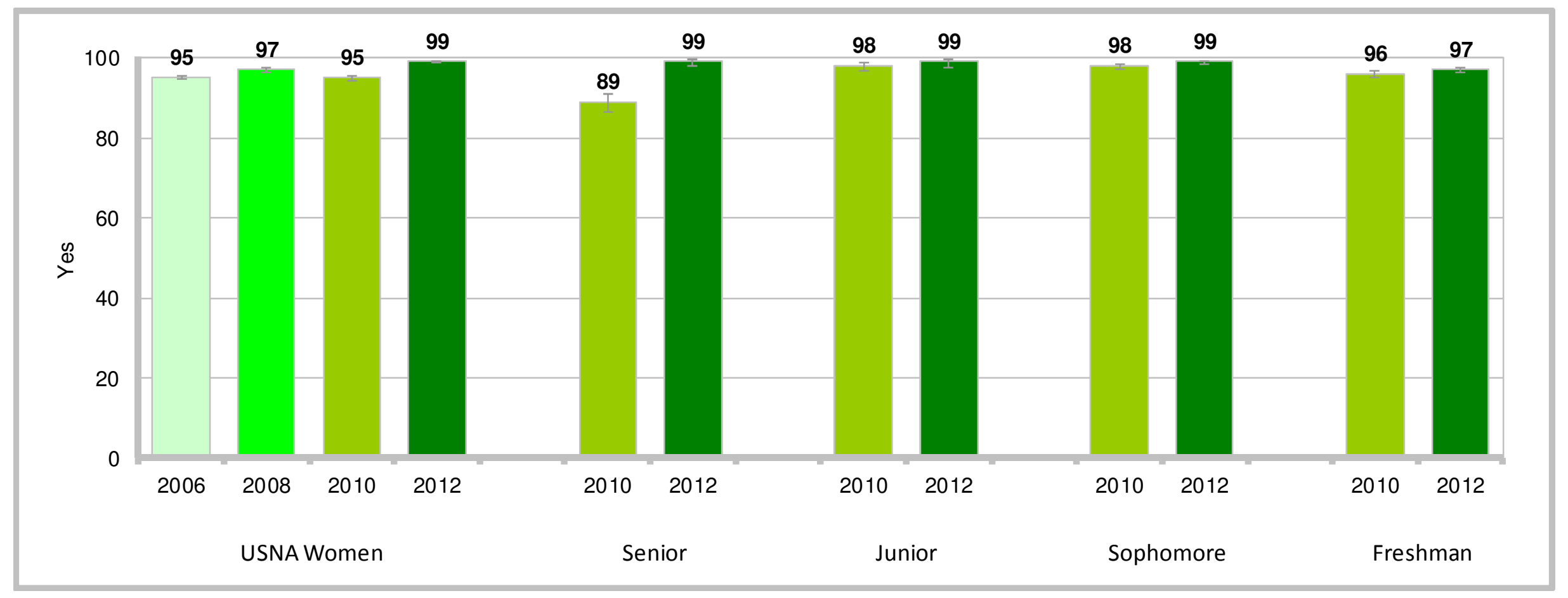

- $99 \%$ of women indicated receiving training on topics related to sexual harassment in 2012

- 2012 comparisons across years - higher than 2010, 2008, 2006

- Seniors in 2012 higher than 2010

- Class differences in 2012 - lower response led by freshmen 


\section{Received Training Since June 2011 on Topics Related to Sexual Harassment \\ Percent of Men}

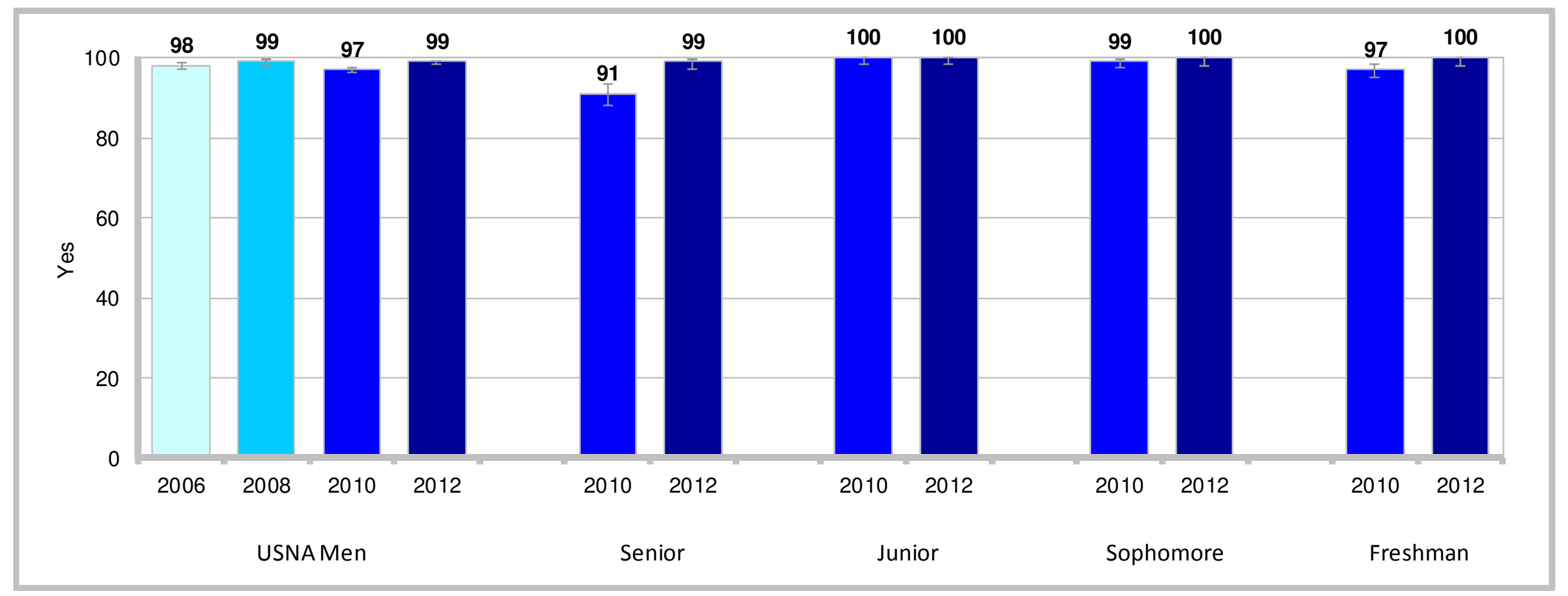

- $99 \%$ of men indicated receiving training on topics related to sexual harassment in 2012

- 2012 comparisons across years - higher than 2010

- Seniors and freshmen in 2012 higher than 2010

- Class differences in 2012 - no differences 


\section{Effectiveness of Training in Actually Reducing/Preventing Sexual Harassment \\ Percent of Women Who Had Training on Sexual Harassment}

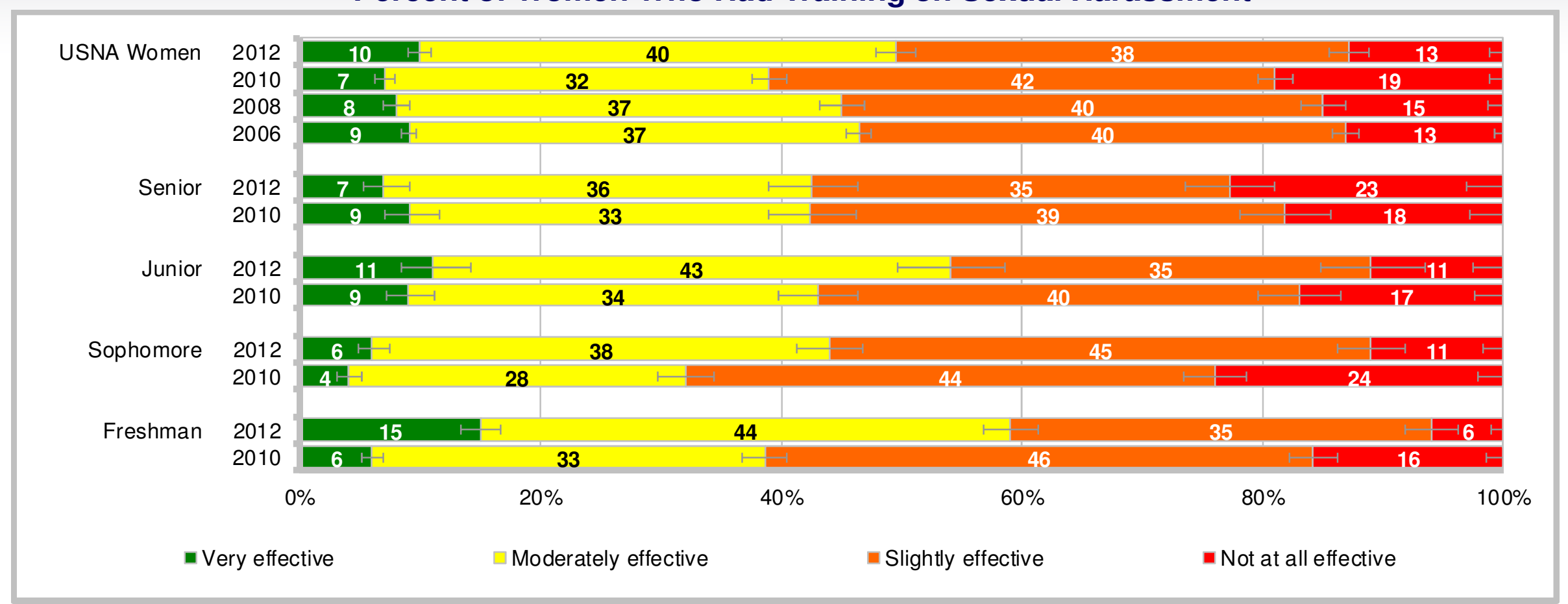

- $10 \%$ of women indicated their training was very effective in actually reducing/preventing sexual harassment; $40 \%$ indicated moderately effective; $38 \%$ indicated slightly effective; and $13 \%$ indicated not at all effective

- 2012 comparisons across years - very effective higher than 2010, 2008; moderately effective higher than 2010, 2008, 2006; slightly effective lower than 2010, 2006; not at all effective lower than 2010, 2008

- Sophomores and freshmen indicating very effective in 2012 higher than 2010; juniors, sophomores, and freshmen indicating moderately effective in 2012 higher than 2010; freshmen indicating slightly effective in 2012 lower than 2010; seniors indicating not at all effective in 2012 higher than 2010, whereas juniors, sophomores, and freshmen lower

- Class differences in 2012 - very effective led by freshmen; moderately effective led by freshmen; slightly effective led by sophomores; not at all effective led by seniors

*Note that $40 \%$ of USNA women also indicated slightly effective in 2008. The 2012 percentage is not lower 


\section{Effectiveness of Training in Actually Reducing/Preventing Sexual Harassment \\ Percent of Men Who Had Training on Sexual Harassment}

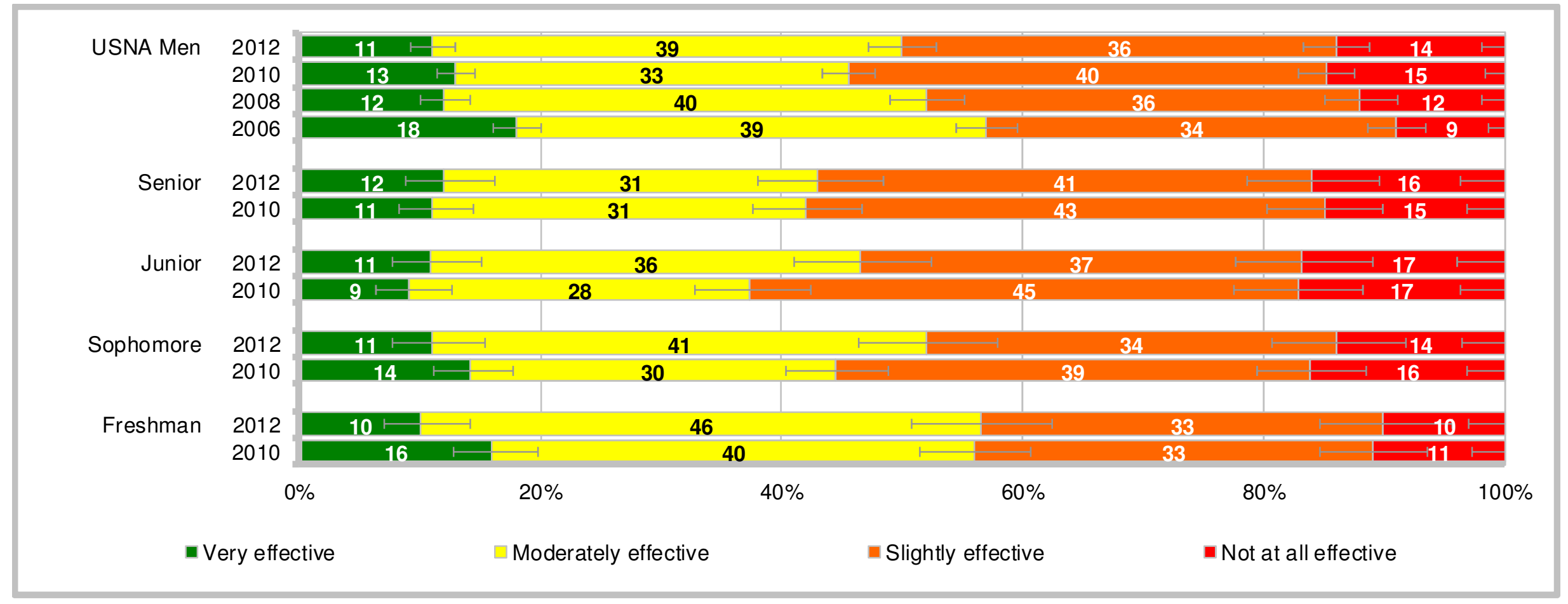

- $11 \%$ of men indicated their training was very effective in actually reducing/preventing sexual harassment; $39 \%$ indicated moderately effective; $36 \%$ indicated slightly effective; and $14 \%$ indicated not at all effective

- 2012 comparisons across years - very effective lower than 2006; moderately effective higher than 2010; slightly effective lower than 2010; not at all effective higher than 2006

- Freshmen indicating very effective in 2012 lower than 2010; juniors and sophomores indicating moderately effective in 2012 higher than 2010; juniors indicating slightly effective in 2012 lower than 2010

- Class differences in 2012 - moderately effective led by freshmen 


\section{Contents}

Slide

- Introduction .................................................................................. 3

- Unwanted Sexual Contact ................................................................... 7

- Unwanted Gender-Related Behaviors ............................................ 49

• Stalking .......................................................................................... 75

• Training ........................................................................................ 83

$\checkmark \quad$ Student Perceptions..................................................................... 93 


\section{Extent You Think Incidents of Sexual Assault Are Not Reported Percent of Women}

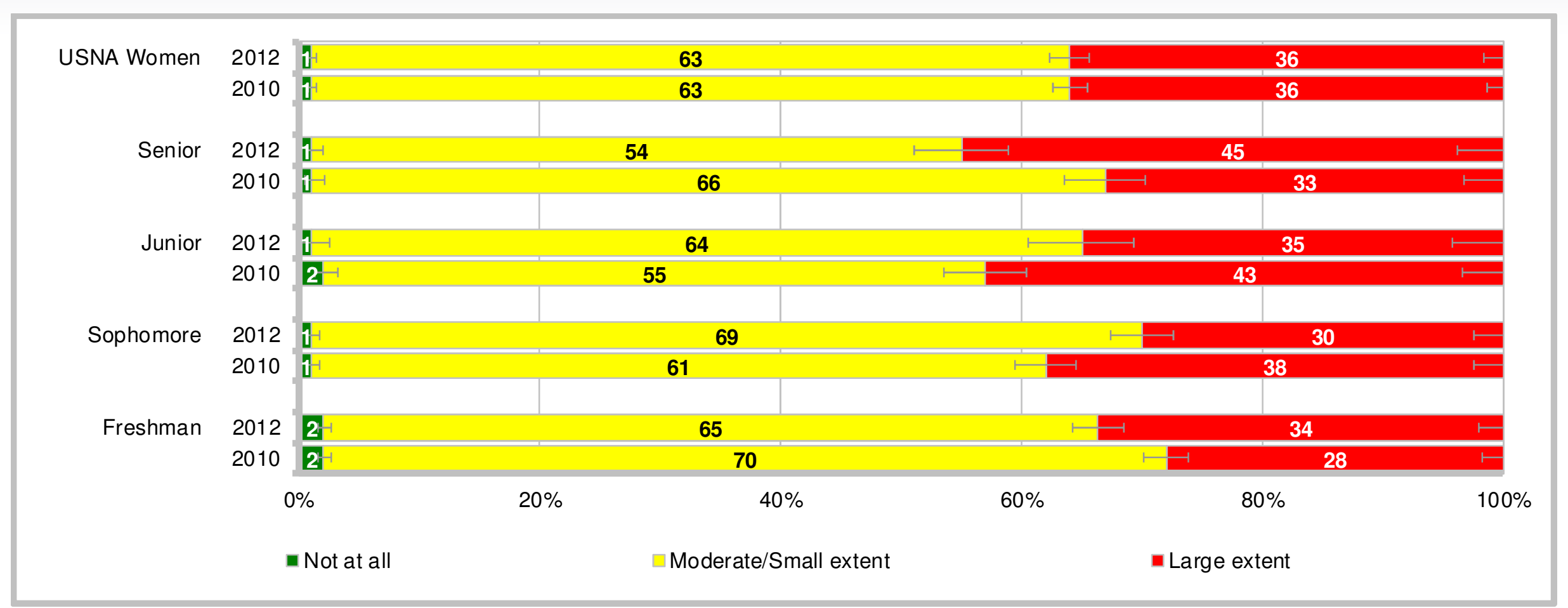

- $36 \%$ of women indicated to a large extent that incidents of sexual assault are not reported; $63 \%$ indicated moderate/small extent; and $1 \%$ indicated not at all

- 2012 comparisons across years - no differences

- Seniors and freshmen indicating moderate/small extent in 2012 lower than 2010, whereas juniors and sophomores higher; seniors and freshmen indicating large extent in 2012 higher than 2010, whereas juniors and sophomores lower

- Class differences in 2012 - large extent led by seniors; moderate/small extent led by sophomores 


\section{Extent You Think Incidents of Sexual Assault Are Not Reported Percent of Men}

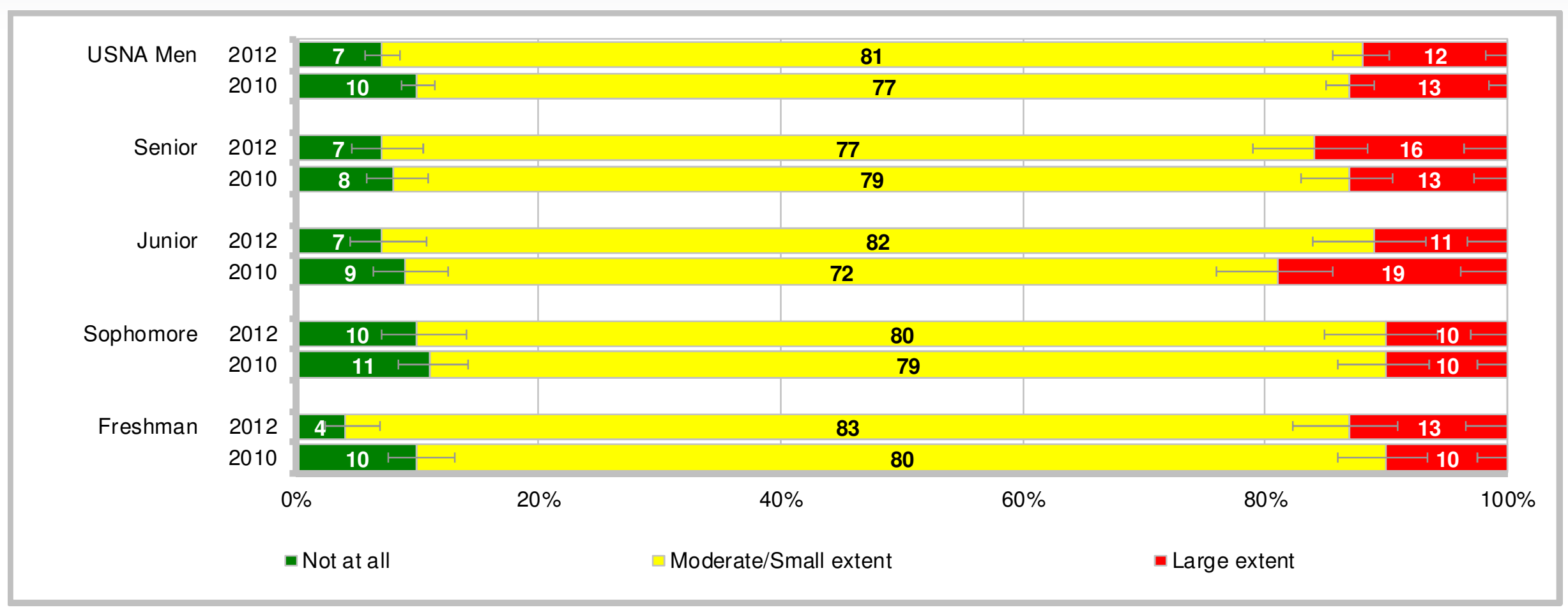

- $12 \%$ of men indicated to a large extent that incidents of sexual assault are not reported; $81 \%$ indicated moderate/small extent; and $7 \%$ indicated not at all

- 2012 comparisons across years - not at all lower than 2010; moderate/small extent higher than 2010

- Freshmen indicating not at all in 2012 lower than 2010; juniors indicating moderate/small extent in 2012 higher than 2010; and juniors indicating large extent in 2012 lower than 2010

- Class differences in 2012 - no differences 


\section{Extent You Would Report a Sexual Assault Percent of Women}

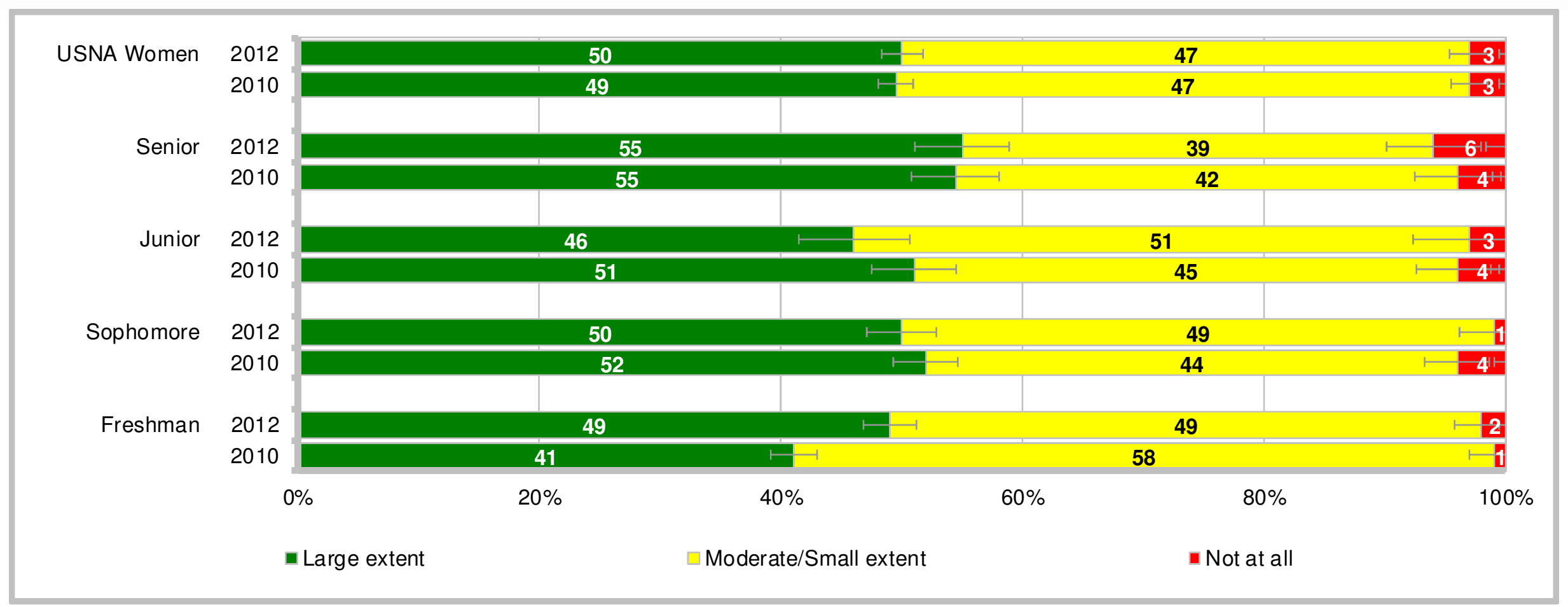

- $50 \%$ of women indicated to a large extent they would be willing to report a sexual assault; $47 \%$ indicated moderate/small extent; and $3 \%$ indicated not at all

- 2012 comparisons across years - no differences

- Freshmen indicating large extent in 2012 higher than 2010; juniors and sophomores indicating moderate/small extent in 2012 higher than 2010, whereas freshmen lower; sophomores indicating not at all in 2012 lower than 2010

- Class differences in 2012 - large extent led by seniors; not at all led by seniors 


\section{Extent You Would Report a Sexual Assault Percent of Men}

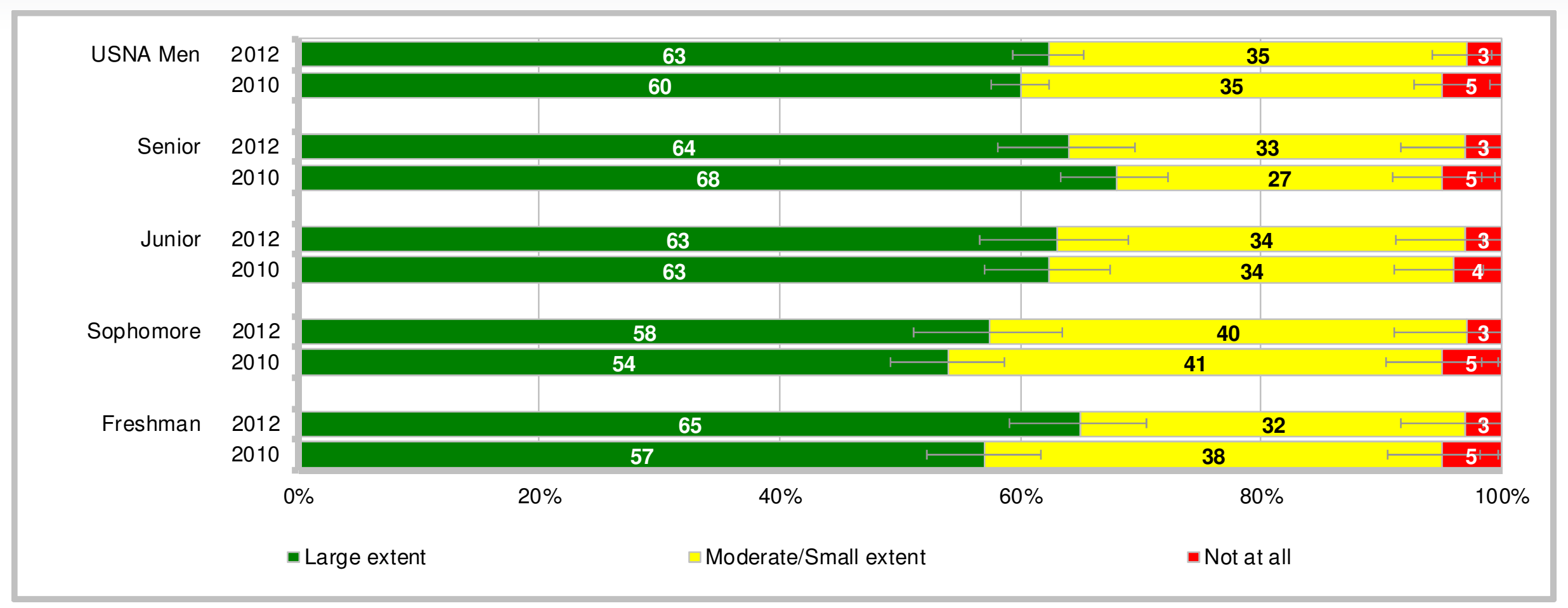

- $63 \%$ of men indicated to a large extent they would be willing to report a sexual assault; $35 \%$ indicated to a moderate/small extent; and $3 \%$ indicated not at all

- 2012 comparisons across years - not at all lower than 2010

- Freshmen indicating large extent in 2012 higher than 2010

- Class differences in 2012 - no differences 


\section{Extent You Think High-Profile Cases Deter Reporting of Sexual Assault} Percent of Women

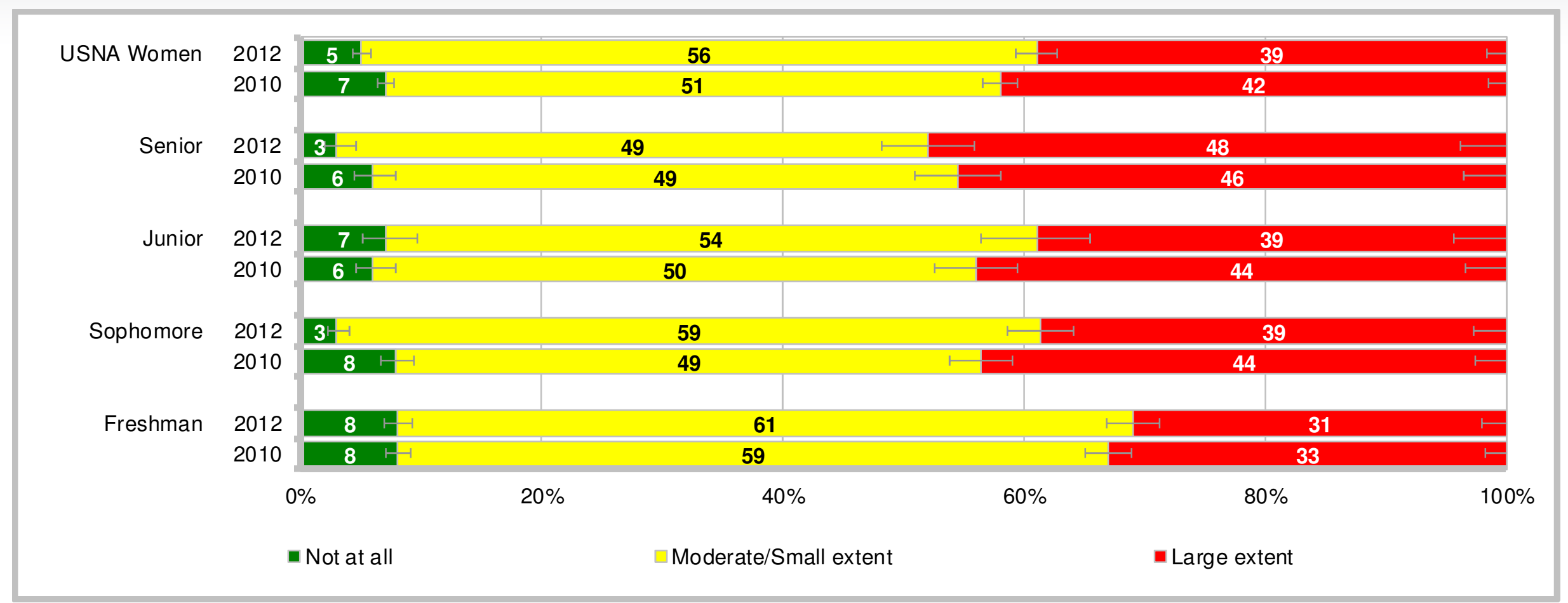

- $39 \%$ of women indicated to a large extent high-profile cases deter reporting of sexual assault; $56 \%$ indicated to a moderate/small extent; and $5 \%$ indicated not at all

- 2012 comparisons across years - not at all lower than 2010; moderate/small extent higher than 2010; large extent lower than 2010

- Sophomores indicating large extent in 2012 lower than 2010; sophomores indicating moderate/small extent in 2012 higher than 2010; seniors and sophomores indicating not at all in 2012 lower than 2010

- Class differences in 2012 - large extent led by seniors; moderate/small extent led by freshmen; not at all led by freshmen 


\section{Extent You Think High-Profile Cases Deter Reporting of Sexual Assault Percent of Men}

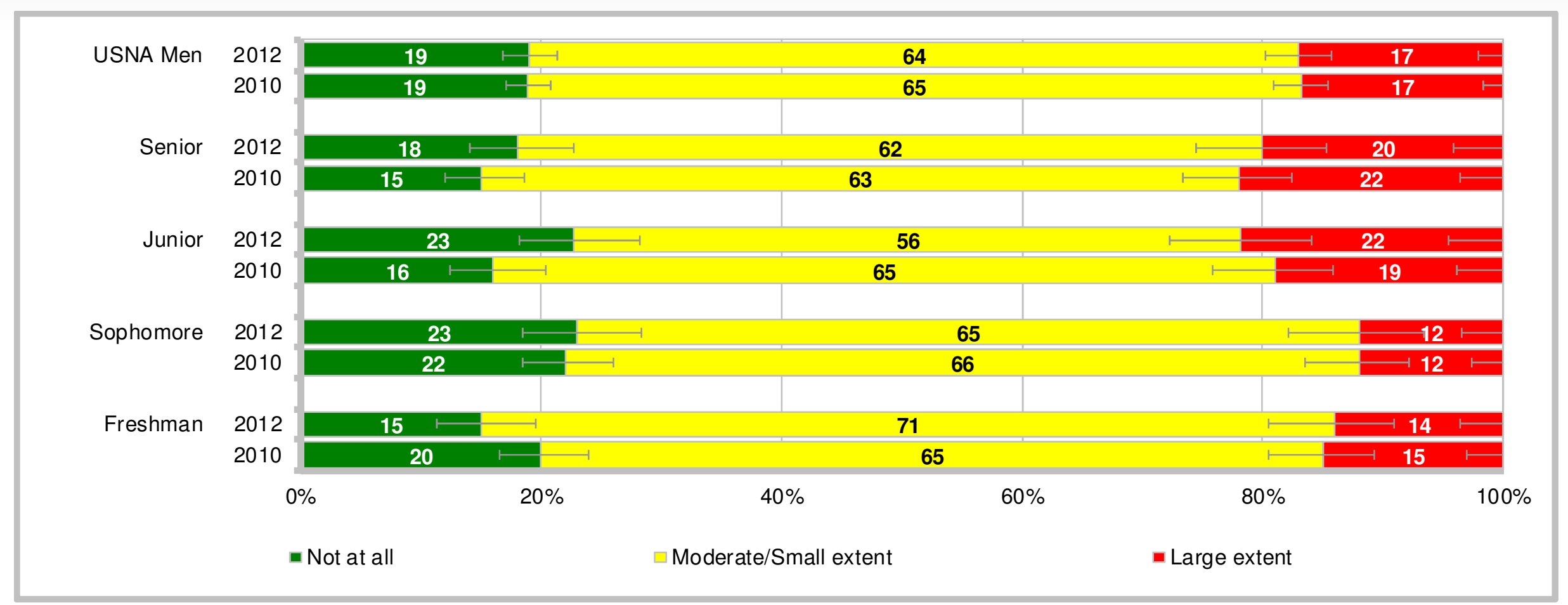

- $17 \%$ of men indicated to a large extent high-profile cases deter reporting of sexual assault; $64 \%$ indicated to a moderate/small extent; and $19 \%$ indicated not at all

- 2012 comparisons across years - no differences

- Juniors indicating moderate/small extent in 2012 lower than 2010; juniors indicating not at all in 2012 higher than 2010

- Class differences in 2012 - moderate/small extent led by freshmen 


\section{You Would Discuss an Incident With a SARC if You Were To Experience Sexual Assault in the Future \\ Percent of Women Who Had Not Experienced Unwanted Sexual Contact in Past 12 Months}

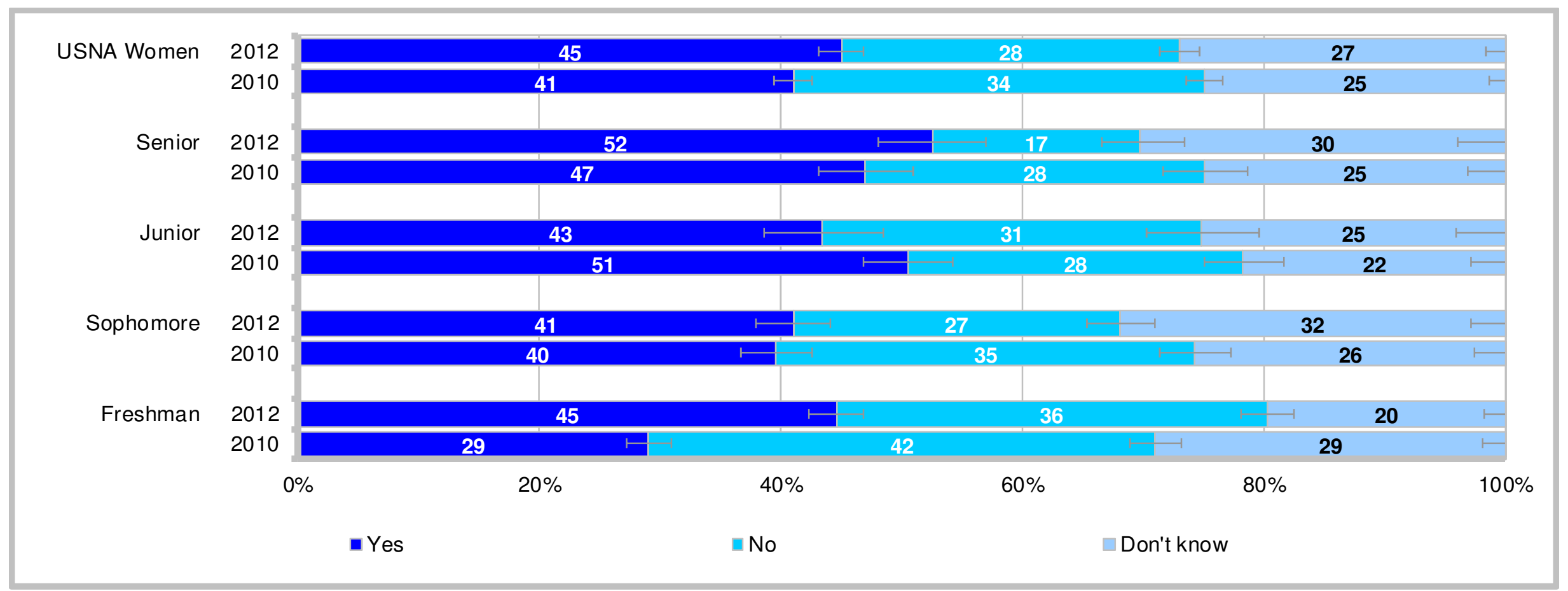

- $45 \%$ of women indicated yes, they would discuss an incident of sexual assault with a SARC; $28 \%$ indicated no; and $27 \%$ indicated don't know

- 2012 comparisons across years - yes higher than 2010; no lower than 2010

- Juniors indicating yes in 2012 lower than 2010, whereas freshmen higher; seniors, sophomores, and freshmen indicating no in 2012 lower than 2010; sophomores indicating don't know in 2012 higher than 2010, whereas freshmen lower

- Class differences in 2012 - yes led by seniors; no led by freshmen; don't know led by sophomores 


\section{You Would Discuss an Incident With a SARC if You Were To Experience Sexual Assault in the Future Percent of Men Who Had Not Experienced Unwanted Sexual Contact in Past 12 Months}

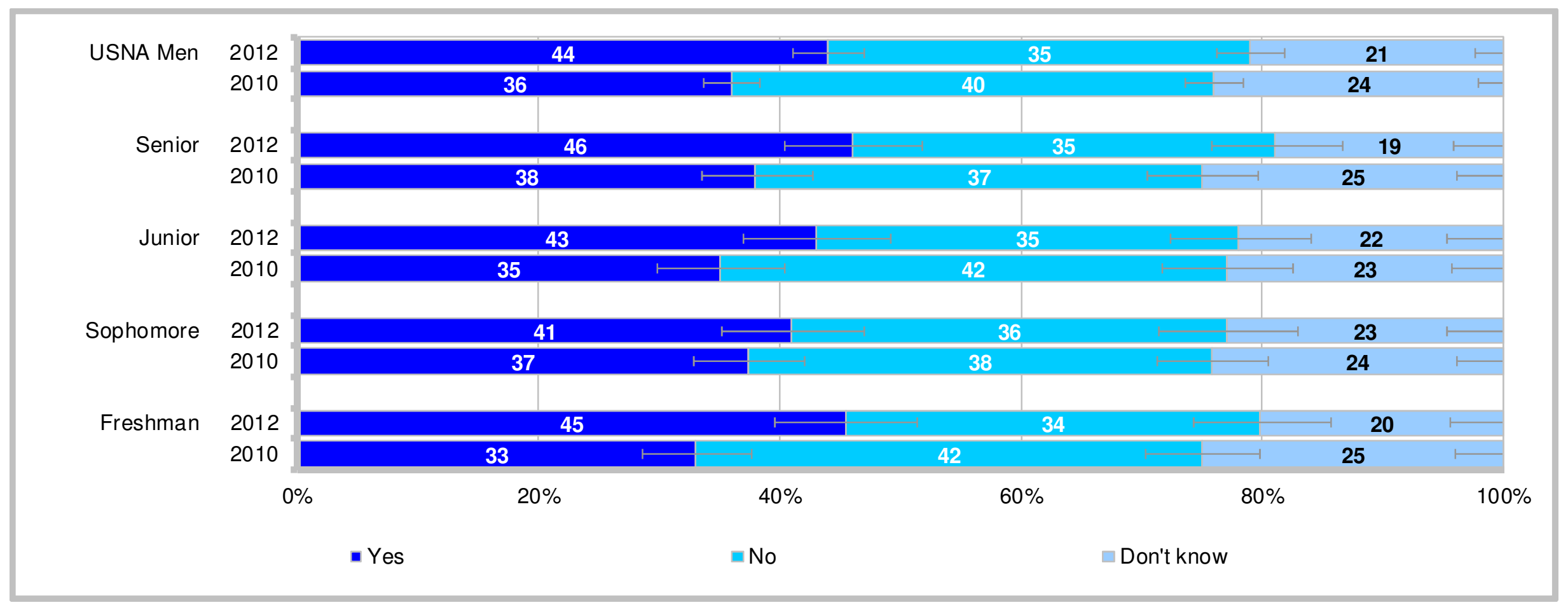

- $44 \%$ of men indicated yes, they would discuss an incident of sexual assault with a SARC; $35 \%$ indicated no; and $21 \%$ indicated don't know

- 2012 comparisons across years - yes higher than 2010; no lower than 2010

- Seniors and freshmen indicating yes in 2012 higher than 2010; freshmen indicating no in 2012 lower than 2010

- Class differences in 2012 - no differences 


\section{You Would Trust the System To Protect Your Privacy if You Were To Experience Sexual Assault in the Future \\ Percent of Women Who Had Not Experienced Unwanted Sexual Contact in the Past 12 Months}

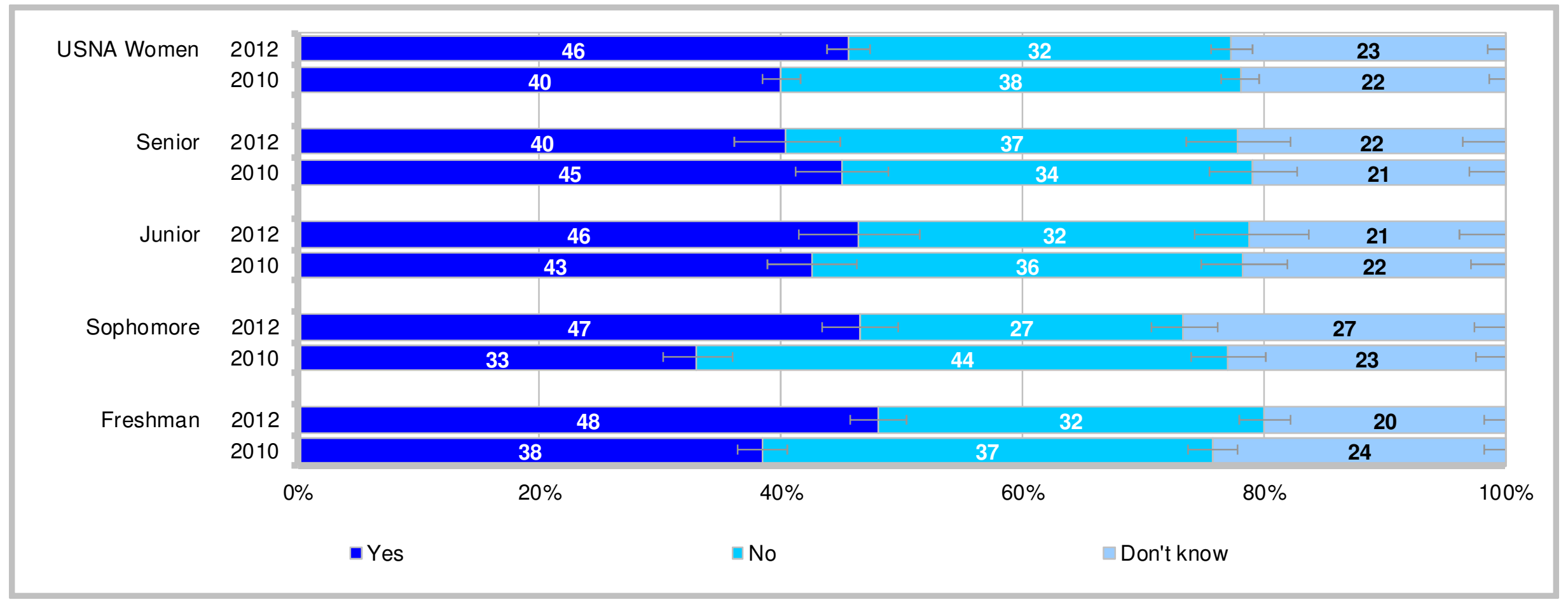

- $46 \%$ of women indicated yes, they would trust the system to protect their privacy if they experienced sexual assault; $32 \%$ indicated no; and $23 \%$ indicated don't know

- 2012 comparisons across years - yes higher than 2010; no lower than 2010

- Sophomores and freshmen indicating yes in 2012 higher than 2010; sophomores and freshmen indicating no in 2012 lower than 2010; sophomores indicating don't know in 2012 higher than 2010, whereas freshmen lower

- Class differences in 2012 - no led by seniors; don't know led by sophomores 


\section{You Would Trust the System To Protect Your Privacy if You Were To Experience Sexual Assault in the Future \\ Percent of Men Who Had Not Experienced Unwanted Sexual Contact in the Past 12 Months}

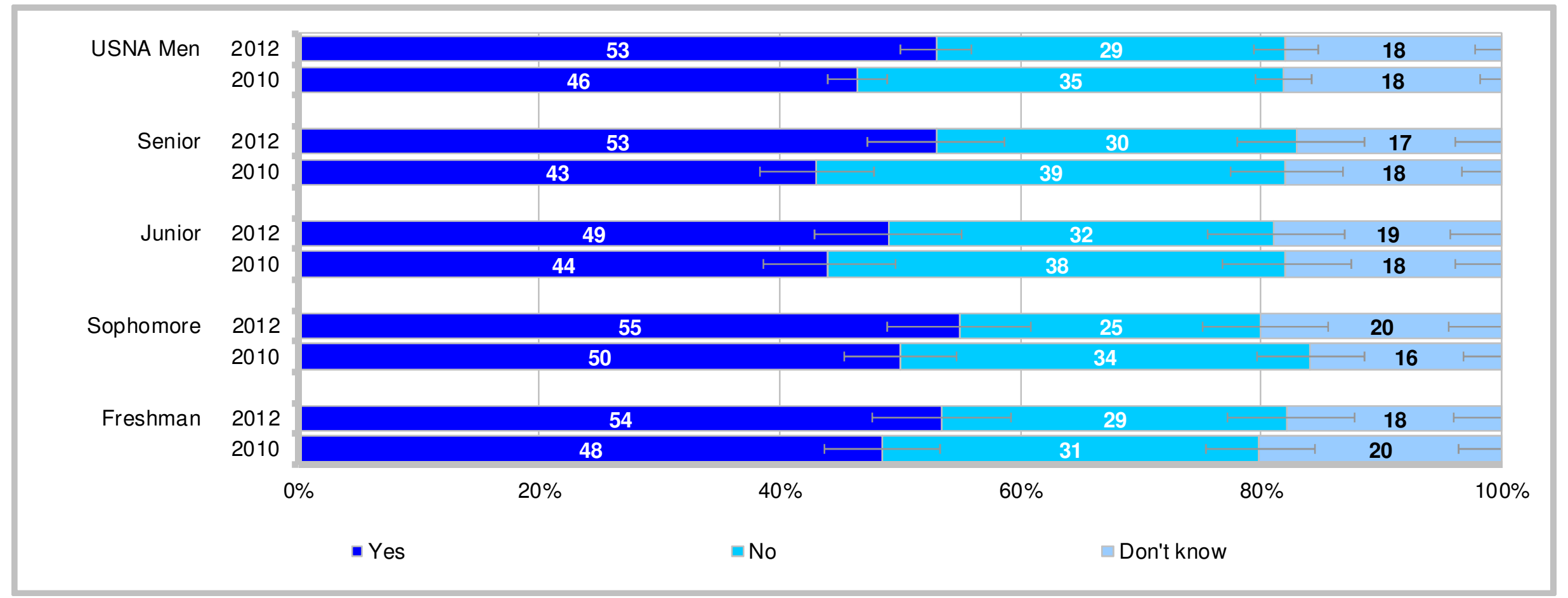

- $53 \%$ of men indicated yes, they would trust the system to protect their privacy if they experienced sexual assault; $29 \%$ indicated no; and 18\% indicated don't know

- 2012 comparisons across years - yes higher than 2010; no lower than 2010

- Seniors indicating yes in 2012 higher than 2010; seniors and sophomores indicating no in 2012 lower than 2010

- Class differences in 2012 - no differences 


\section{You Would Be Willing To Encourage Someone Who Has Experienced Sexual Assault To Report It Percent of Women}

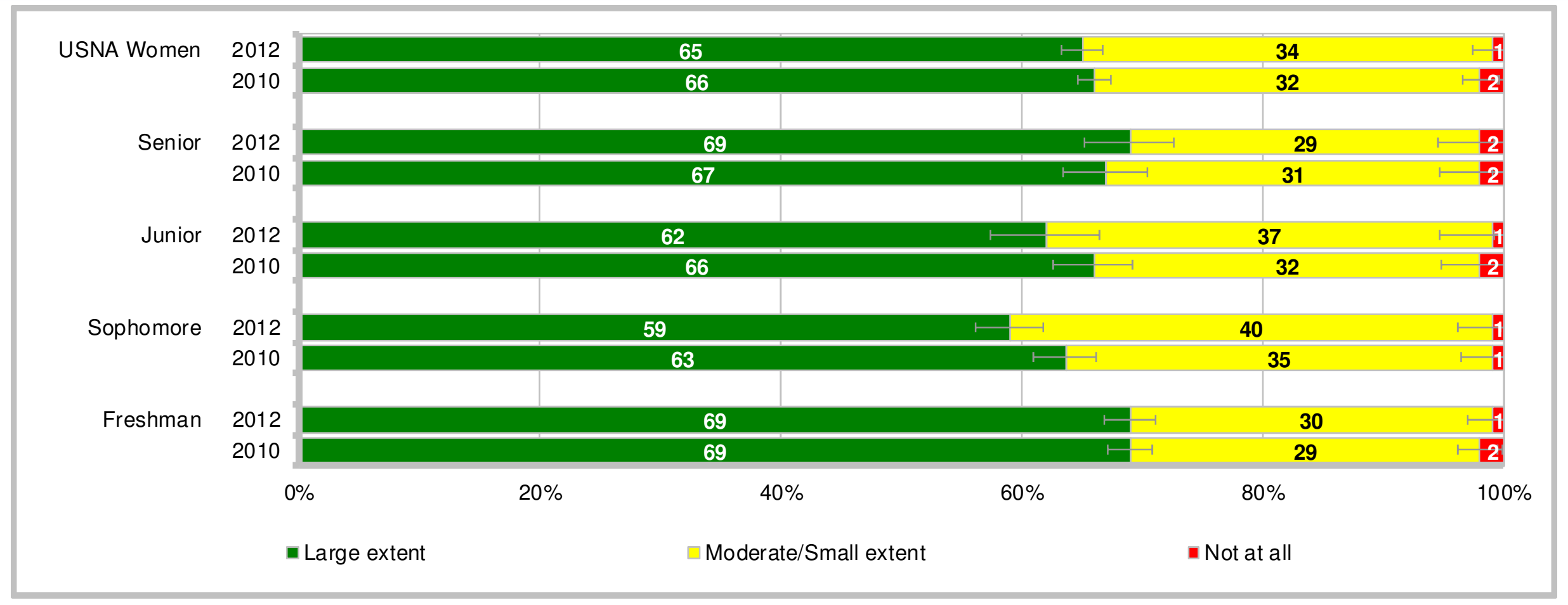

- $99 \%$ of women indicated they would encourage someone to report sexual assault to at least some extent; $1 \%$ indicated not at all

- 2012 comparisons across years - no differences

- Sophomores indicating large extent in 2012 lower than 2010; sophomores indicating moderate/small extent in 2012 higher than 2010

- Class differences in 2012 - large extent led by seniors and freshmen; moderate/small extent led by sophomores 


\section{You Would Be Willing To Encourage Someone Who Has Experienced Sexual Assault To Report It \\ Percent of Men}

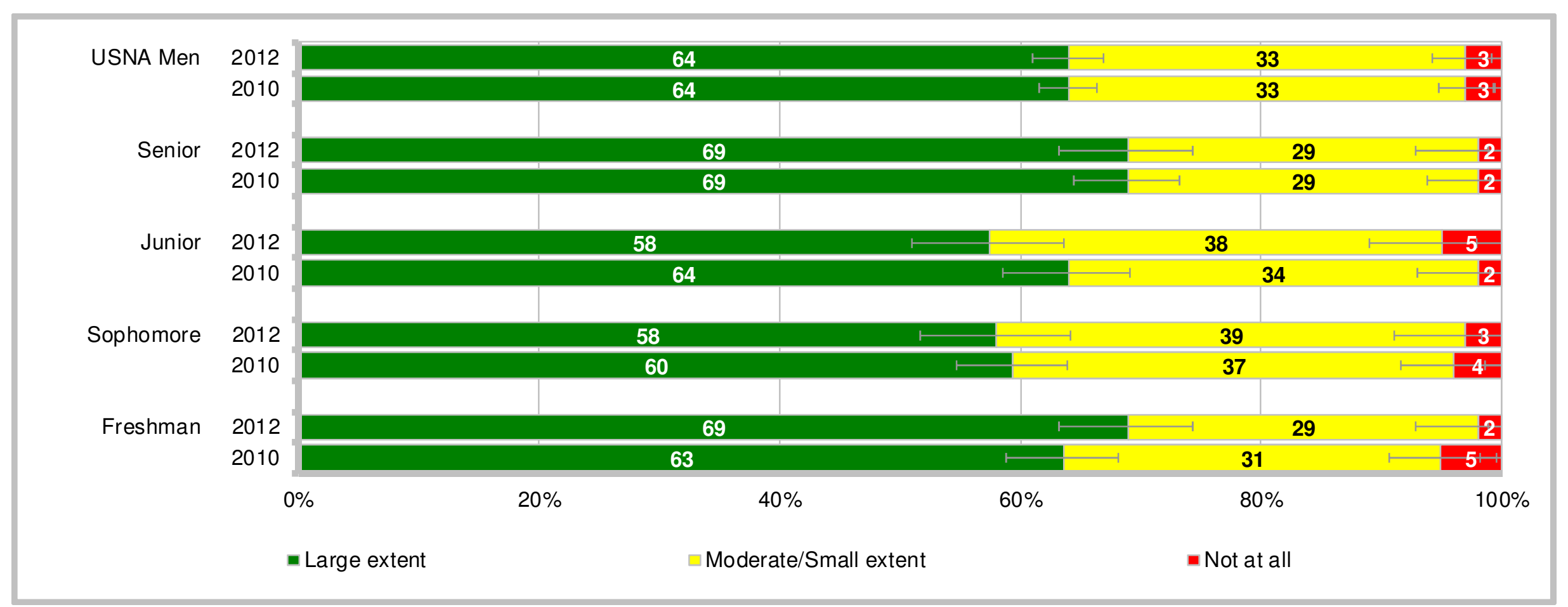

- $97 \%$ of men indicated they would encourage someone to report sexual assault to at least some extent; $3 \%$ indicated not at all

- 2012 comparisons across years - no differences

- Freshmen indicating not at all in 2012 lower than 2010

- Class differences in 2012 - no differences 


\section{You Would Step In and Stop a Situation That Might Lead to Sexual Assault} Percent of Women

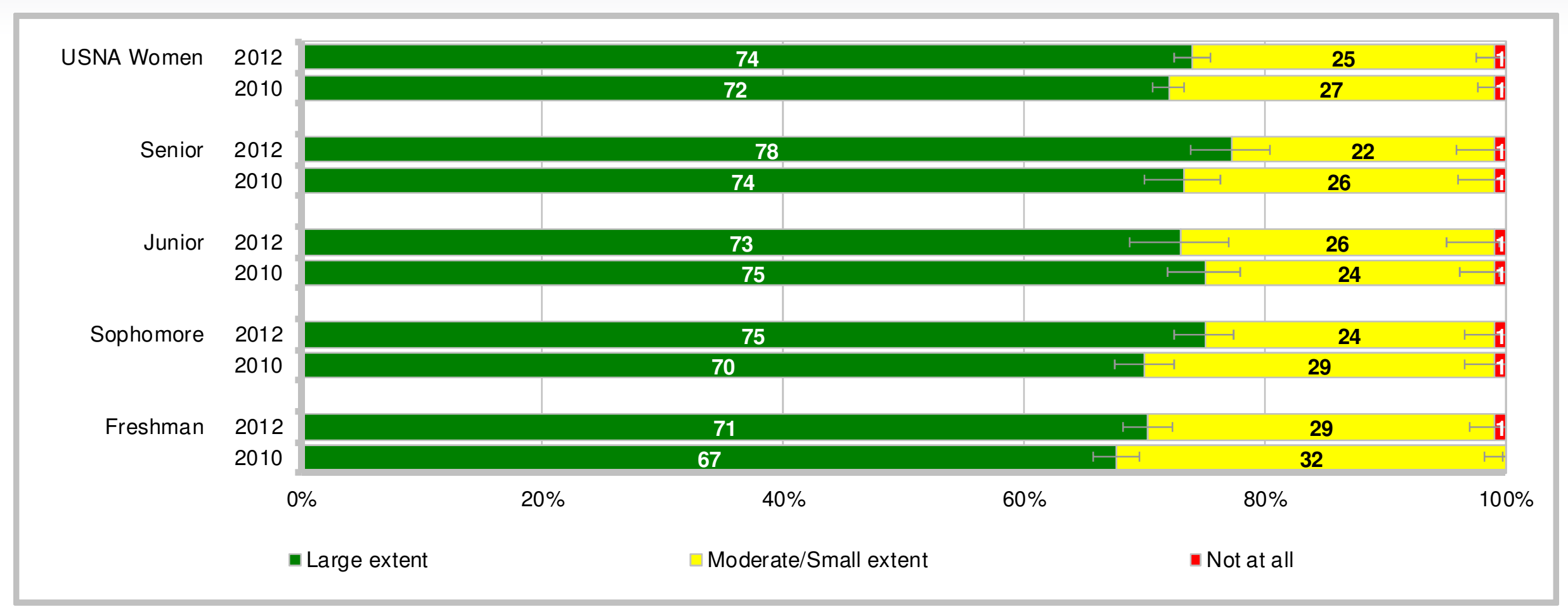

- $99 \%$ of women indicated they would step in and stop a situation that might lead to sexual assault to at least some extent; $1 \%$ indicated not at all

- 2012 comparisons across years - moderate/small extent lower than 2010

- Sophomores and freshmen indicating large extent in 2012 higher than 2010; sophomores and freshmen indicating moderate/small extent in 2012 lower than 2010

- Class differences in 2012 - moderate/small extent led by freshmen 


\section{You Would Step In and Stop a Situation That Might Lead to Sexual Assault} Percent of Men

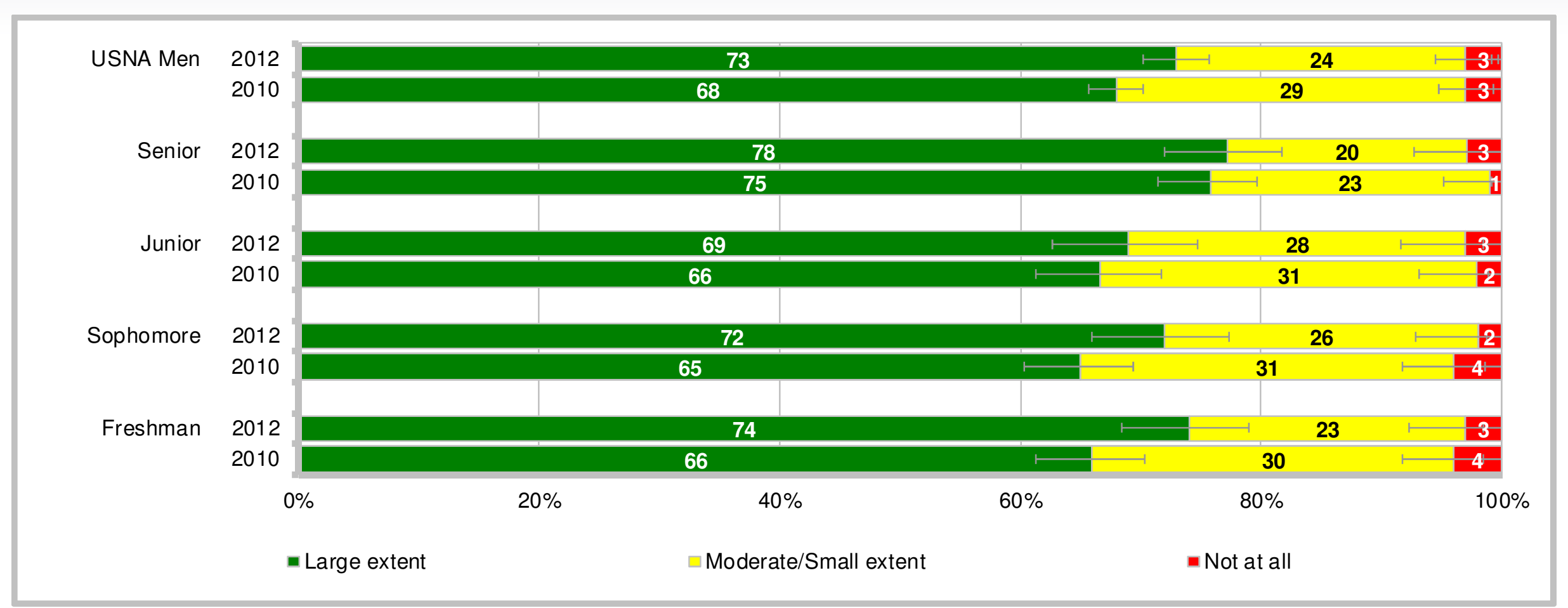

- $97 \%$ of men indicated they would step in and stop a situation that might lead to sexual assault to at least some extent; $3 \%$ indicated not at all

- 2012 comparisons across years - large extent higher than 2010; moderate/small extent lower than 2010

- Freshmen indicating large extent in 2012 higher than 2010; freshmen indicating moderate/small extent in 2012 lower than 2010

- Class differences in 2012 - no differences 


\section{You Would Point Out to Someone That They "Crossed the Line" With Comments or Jokes \\ Percent of Women}

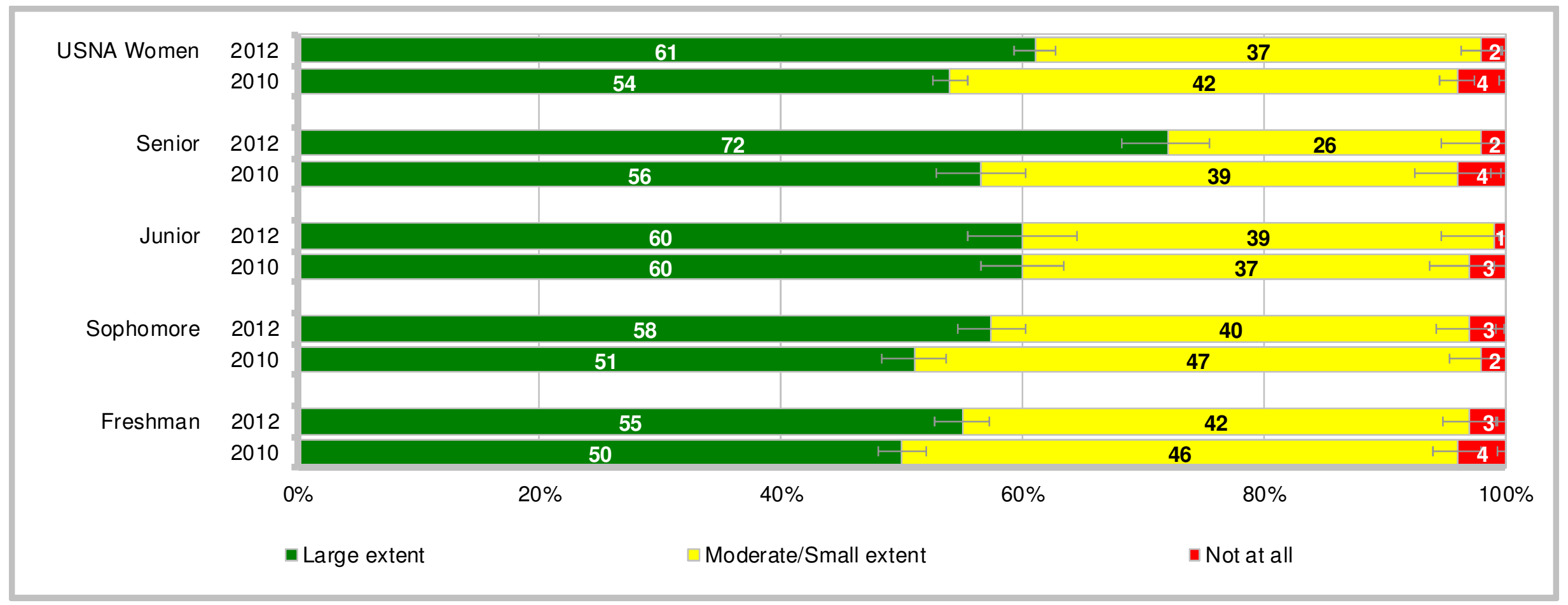

- $98 \%$ of women indicated they would point out to someone that they "crossed the line" with comments or jokes to at least some extent; $2 \%$ indicated not at all

- 2012 comparisons across years - large extent higher than 2010; moderate/small extent lower than 2010; not at all lower than 2010

- Seniors, sophomores, and freshmen indicating large extent in 2012 higher than 2010; seniors, sophomores, and freshmen indicating moderate/small extent in 2012 lower than 2010; seniors and juniors indicating not at all in 2012 lower than 2010

- Class differences in 2012 - large extent led by seniors; moderate/small extent led by freshmen 


\section{You Would Point Out to Someone That They "Crossed the Line" With Comments or Jokes}

Percent of Men

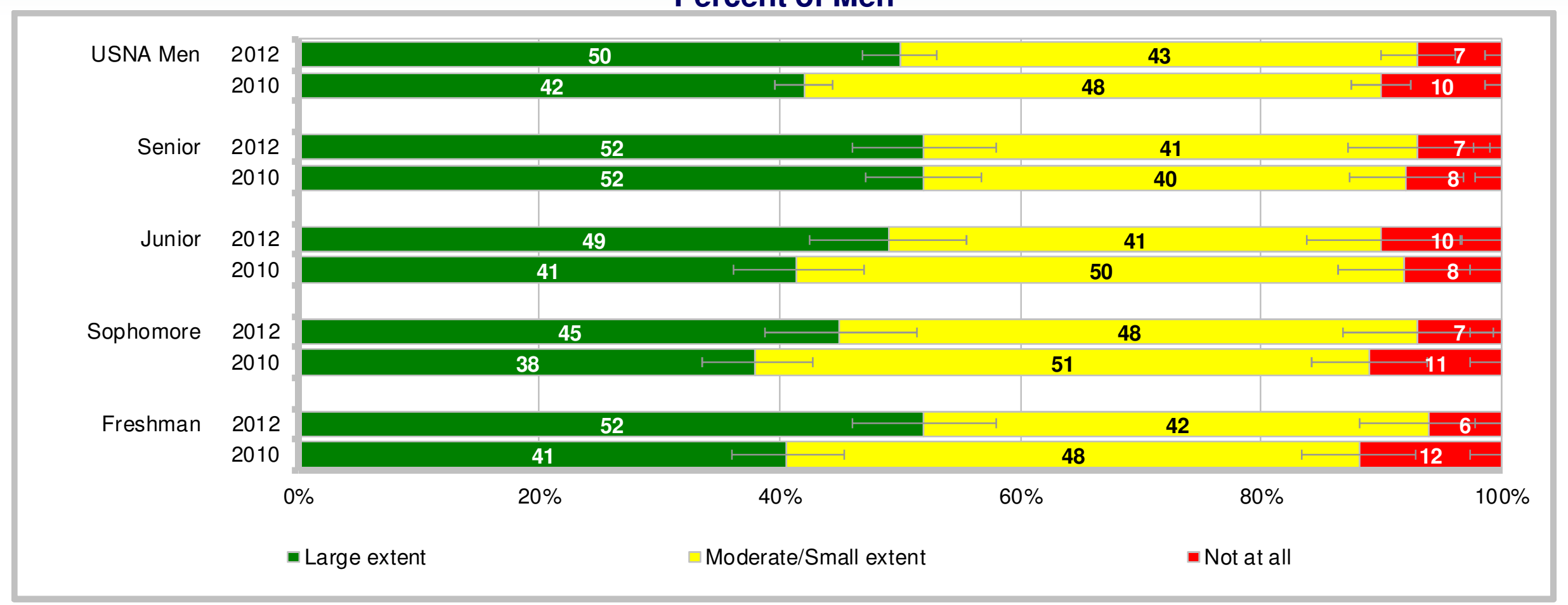

- $93 \%$ of men indicated they would point out to someone that they "crossed the line" with comments or jokes to at least some extent; $7 \%$ indicated not at all

- 2012 comparisons across years - large extent higher than 2010; moderate/small extent lower than 2010; not at all lower than 2010

- Freshmen indicating large extent in 2012 higher than 2010; juniors indicating moderate/small extent in 2012 lower than 2010; freshmen indicating not at all in 2012 lower than 2010

- Class differences in 2012 - no differences 


\section{You Would Seek Help From Chain of Command in Stopping Other Students Who Continue To Engage in Sexual Harassment}

\section{Percent of Women}

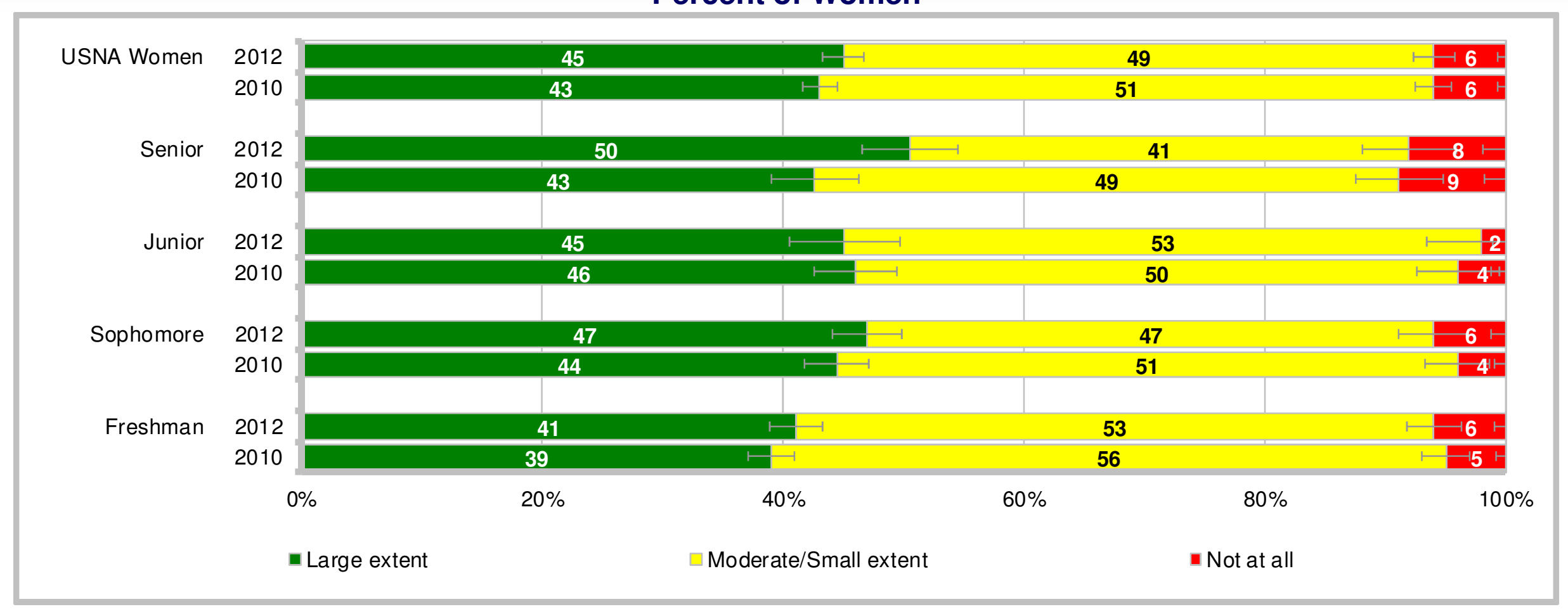

- $94 \%$ of women indicated they would seek help from the chain of command in stopping other students who continue to engage in sexual harassment to at least some extent; $6 \%$ indicated not at all

- 2012 comparisons across years - no differences

- Seniors indicating large extent in 2012 higher than 2010; seniors, sophomores, and freshmen indicating moderate/small extent in 2012 lower than 2010; juniors indicating not at all in 2012 lower than 2010, whereas sophomores higher

- Class differences in 2012 - large extent led by seniors; moderate/small extent led by juniors and freshmen; not at all led by seniors 


\section{You Would Seek Help From Chain of Command in Stopping Other Students Who Continue To Engage in Sexual Harassment \\ Percent of Men}

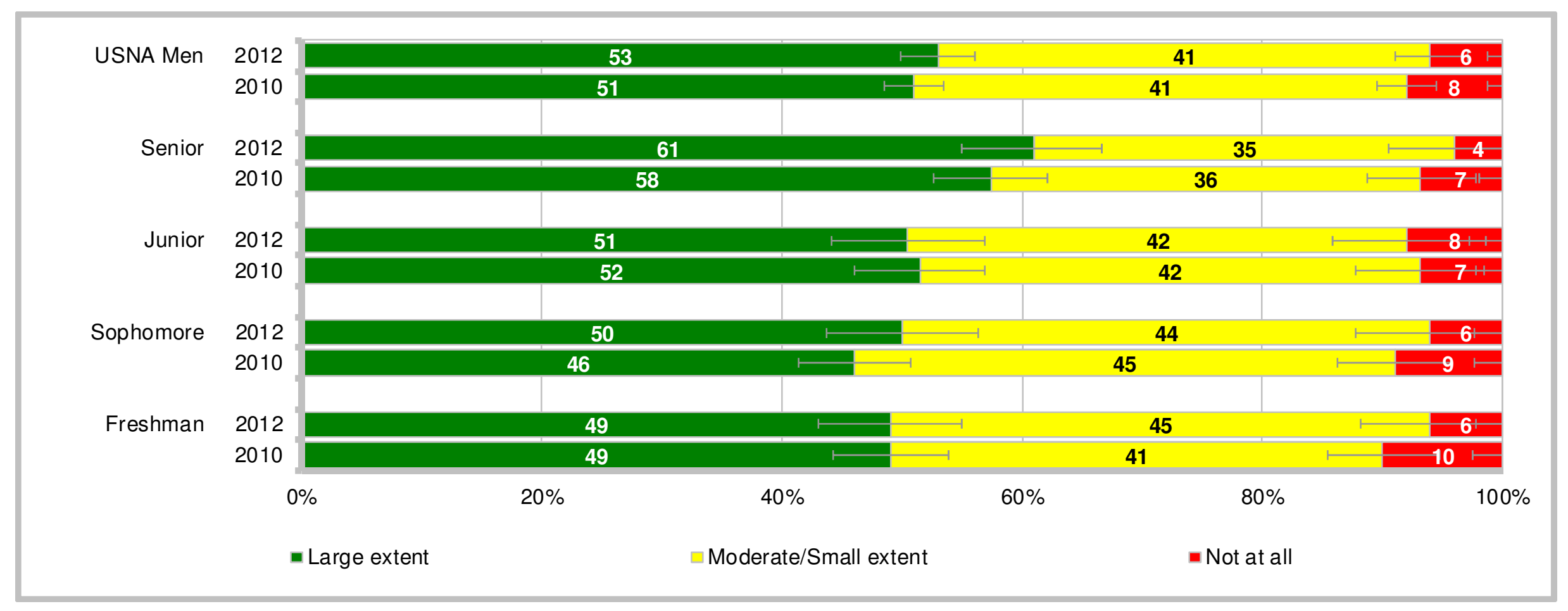

- $94 \%$ of men indicated they would seek help from the chain of command in stopping other students who continue to engage in sexual harassment to at least some extent; and 6\% indicated not at all

- 2012 comparisons across years - not at all lower than 2010

- Class differences in 2012 - large extent led by seniors 


\section{Service Academy Gender Relations Survey}

Appendix C: U.S. Air Force Academy

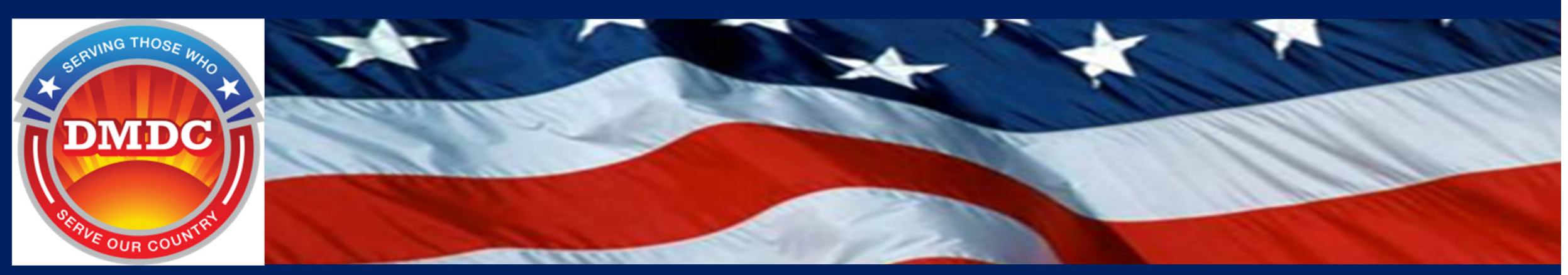




\section{Appendix A \\ U.S. Air Force Academy Contents}

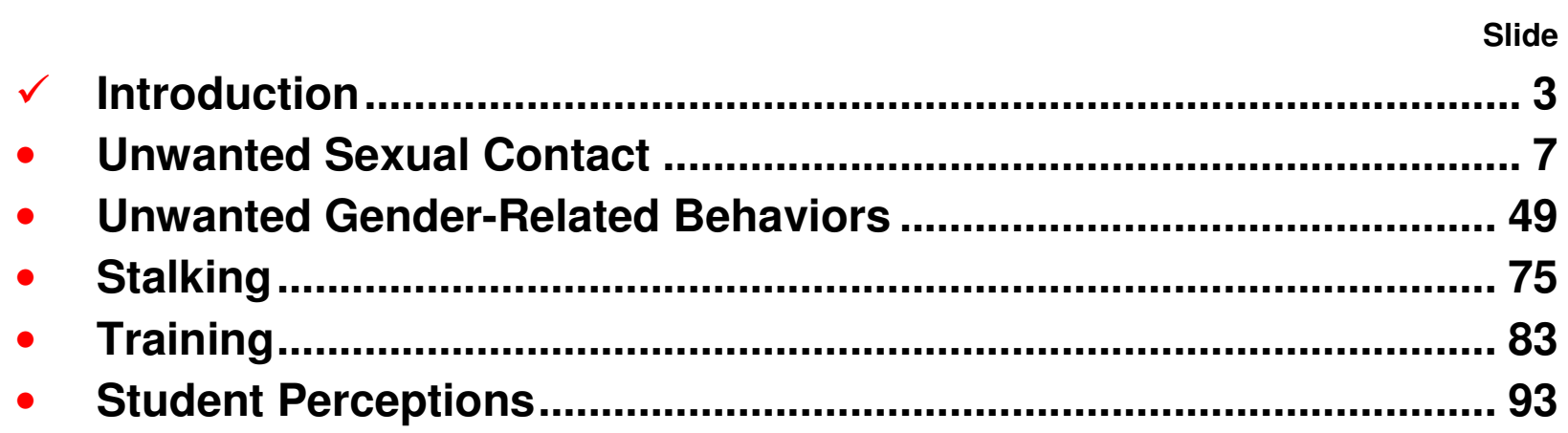




\section{Introduction}

\section{- Paper survey administered to cadets in April 2012}

- 937 female and 1,273 male students surveyed, weighted response rate of $73 \%$ and $67 \%$, respectively

\section{- Appendix includes results from survey items related to:}

- Results of unwanted sexual contact experienced in the past academic year

- For women who had experienced unwanted sexual contact in the past academic year, details of the incident that had the greatest impact (characteristics of the offender, location, actions taken, reporting, etc.). Details for men are not reportable due to the small number of men who experienced unwanted sexual contact.

- Unwanted sexual contact experienced prior to entering the Academy

- Unwanted gender-related behaviors experienced in the past academic year (sexual harassment, crude and offensive behavior, unwanted sexual attention, sexual coercion, and sexist behavior)

- For students who experienced unwanted gender-related behavior in the past academic year, details of the incident that had the greatest impact (characteristics of the offender, reporting)

- Stalking experienced in the past academic year

- Training received in preventing and responding to sexual assault and sexual harassment

- Student perceptions about culture and climate at the Academy

\section{- For each survey item, briefing includes the following:}

- Graphic displays of overall results by gender, survey year, and class year

- Tables showing results for grouped items by reporting categories (e.g., gender, survey year, and class year) 


\section{Introduction to Briefing Slides}

\section{- Graphics showing results by reporting categories (gender, survey year, and class year)}

- Statistical tests are used to compare each subgroup to previous survey years and other class years

- For items with trends, 2012 survey year is compared to the other survey years $(2006,2008,2010)$

- For the current 2012 survey year, each class year (e.g., freshmen) is compared to the average of the other class years (sophomore, junior, senior)

- Results are presented on the bottom of the slide for survey year and class year comparisons that are statistically significant

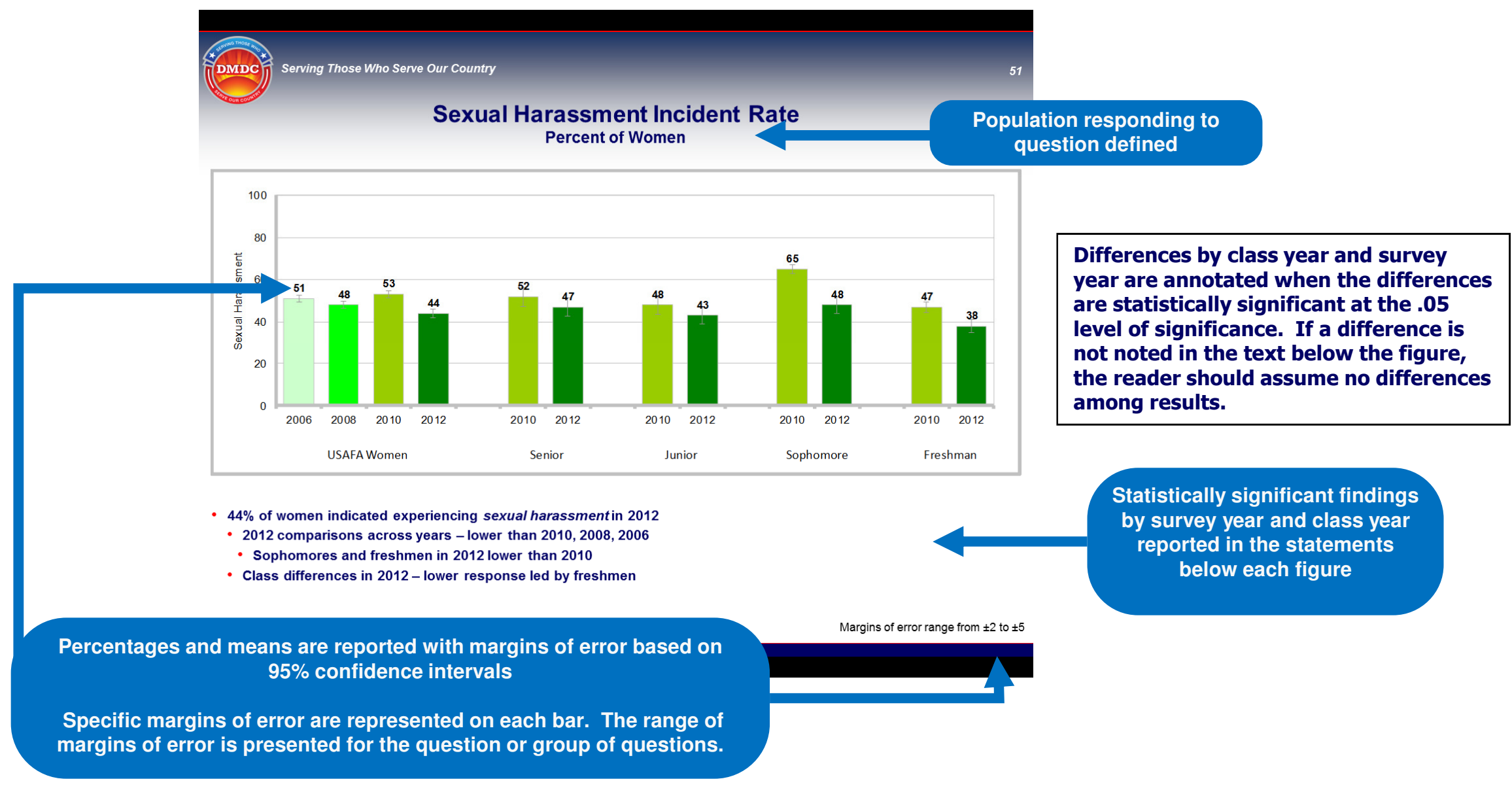




\section{Introduction to Briefing Slides}

\section{- Tables showing results for grouped items by reporting categories (gender, survey year,} and class year)

- Statistical tests are used to compare each subgroup to previous survey years and other class years

- For items with trends, 2012 survey year is compared to the other survey years $(2006,2008,2010)$

- For the current 2012 survey year, each class year (e.g., freshmen) is compared to the average of the other class years (sophomore, junior, senior)

- Results are presented within the table for survey year and class year comparisons that are statistically significant

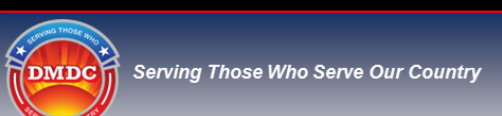

Most Frequently Selected Reasons for

Not Reporting Unwanted Sexual Contact Situation

Percent of Women Who Experienced Unwanted Sexual Contact and Did Not Report It

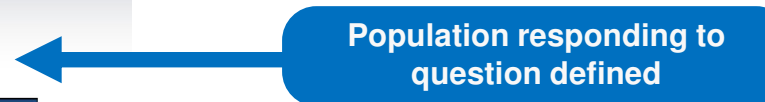

\begin{tabular}{|c|c|c|c|c|c|c|}
\hline Reasons for Not Reporting & $\begin{array}{c}\text { Survey } \\
\text { Year }\end{array}$ & Total & Senior & Junior & Sophomore & Freshman \\
\hline
\end{tabular} question defined

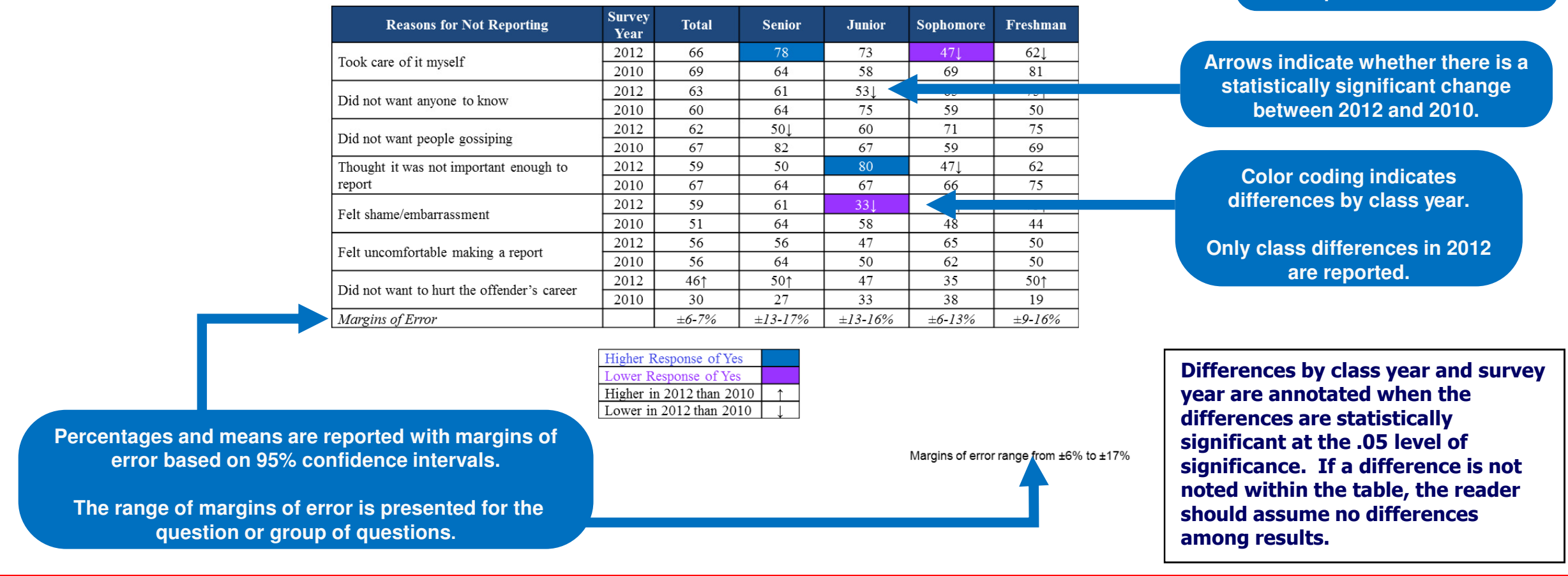




\section{Contents}

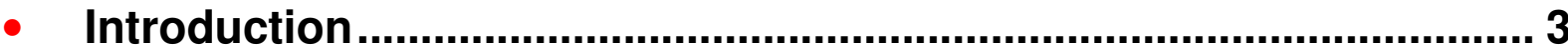

$\checkmark \quad$ Unwanted Sexual Contact .................................................................. 7

- Most Serious Behaviors Experienced......................................... 11

- Location of Incident ......................................................................... 13

- Summer Experience........................................................................... 15

- Characteristics of Offender............................................................ 17

- Alcohol/Drug Involvement........................................................... 22

- Use of Threats/Force ................................................................... 27

- Experience of Sexual Harassment/Stalking ................................. 32

- Consequences of the Situation .................................................. 25

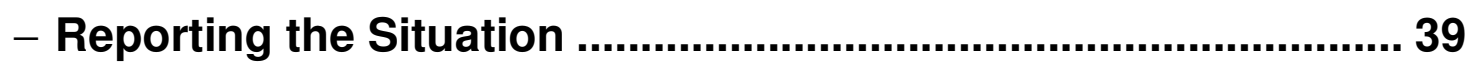

- Experience of Unwanted Sexual Contact Prior to Entering

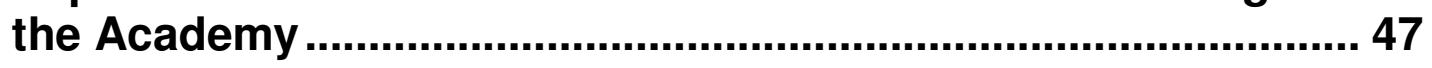

- Unwanted Gender-Related Behaviors ................................................ 49

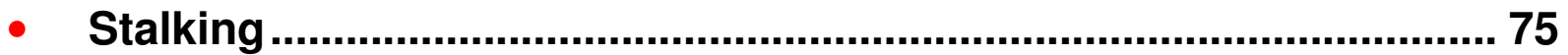

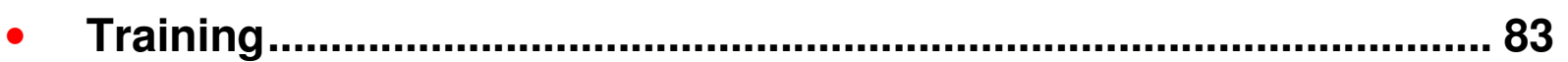

- Student Perceptions ..................................................................... 93 


\section{Unwanted Sexual Contact Incident Rates}

\section{- Definition and measure of unwanted sexual contact:}

- The 2012 SAGR survey includes a measure of unwanted sexual contact (i.e., sexual assault). Although this term does not appear in the Uniform Code of Military Justice $(\mathrm{UCMJ})$, it is used to refer to a range of activities that is used as an umbrella term intended to include certain acts prohibited by the UCMJ.

- Unwanted sexual contact is measured in the 2012 SAGR survey by asking students to refer to experiences since June 2011 in which they experienced any of the following intentional sexual contacts that were against their will or which occurred when they did not or could not consent in which someone...

- Sexually touched them (e.g., intentional touching of genitalia, breasts, or buttocks) or made them sexually touch someone,

- Attempted to make them have sexual intercourse, but was not successful,

- Made them have sexual intercourse,

- Attempted to make them perform or receive oral sex, anal sex, or penetration by a finger or object, but was not successful, or

- Made them perform or receive oral sex, anal sex, or penetration by a finger or object.

- A student is counted in the unwanted sexual contact incident rate if he or she replied "yes" to any of the behaviors listed.

- The 2012 SAGR survey was administered in March and April 2012. The timeframe looking back to June 2011 is intended to include behaviors experienced in the majority of Academic Program Year 2011-2012 to be consistent with the timeframe for reporting incidents of sexual assault at the Academies. 


\section{Unwanted Sexual Contact Incident Rate Percent of Women}

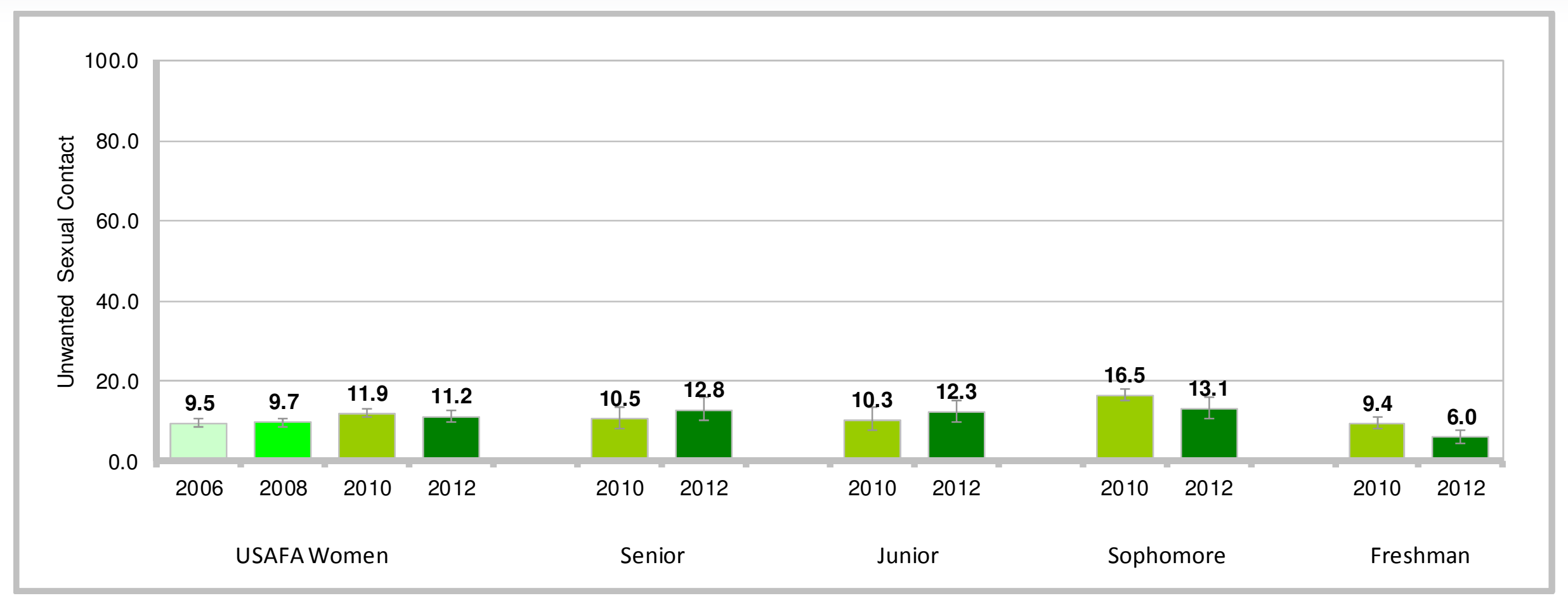

- $11.2 \%$ of women indicated experiencing unwanted sexual contact in 2012

- 2012 comparisons across years - higher than 2006

- Sophomores and freshmen in 2012 lower than 2010

- Class differences in 2012 - lower response led by freshmen 


\section{Unwanted Sexual Contact Incident Rate}

Percent of Men

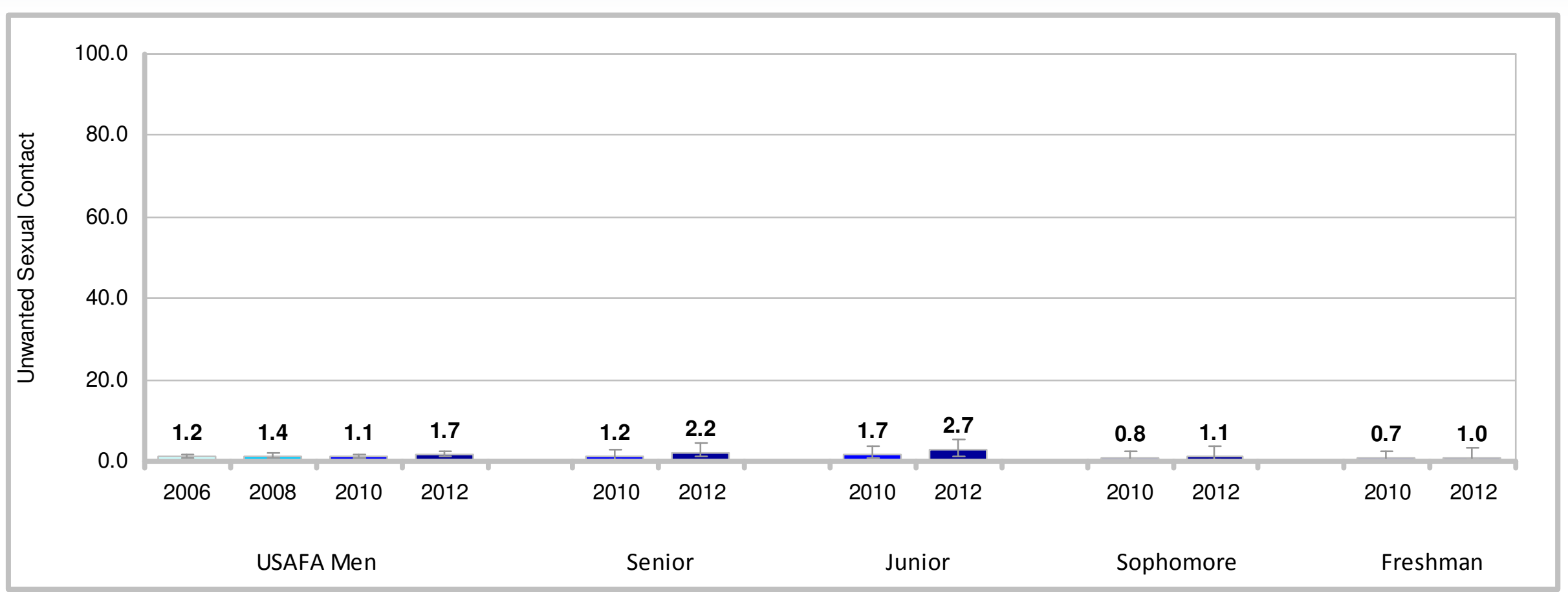

- $1.7 \%$ of men indicated experiencing unwanted sexual contact in 2012

- 2012 comparisons across years - no differences

- Class differences in 2012 - no differences 


\section{Contents}

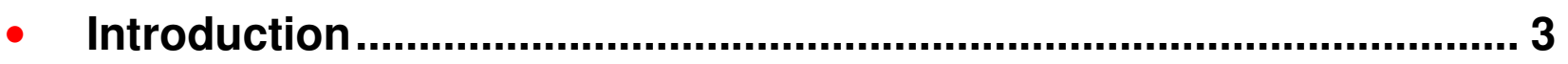

- Unwanted Sexual Contact .................................................................. 7

$\checkmark$ Most Serious Behaviors Experienced...................................... 11

- Location of Incident ..................................................................... 13

- Summer Experience.................................................................... 15

- Characteristics of Offender....................................................... 17

- Alcohol/Drug Involvement.......................................................... 22

- Use of Threats/Force ................................................................ 27

- Experience of Sexual Harassment/Stalking .............................. 32

- Consequences of the Situation ................................................... 35

- Reporting the Situation ............................................................ 39

- Experience of Unwanted Sexual Contact Prior to Entering

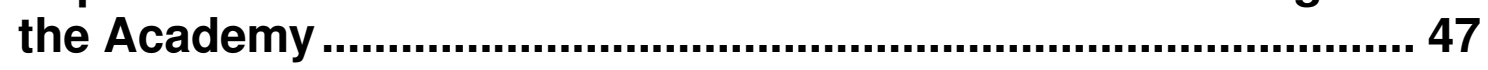

- Unwanted Gender-Related Behaviors ................................................ 49

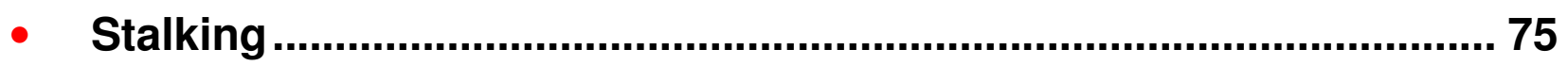

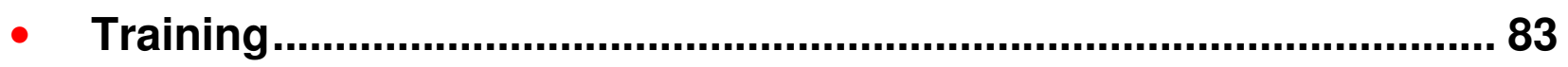

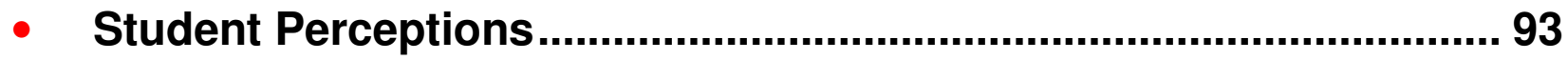




\section{Most Serious Unwanted Sexual Contact Behaviors Experienced Percent of Women Who Experienced Unwanted Sexual Contact}

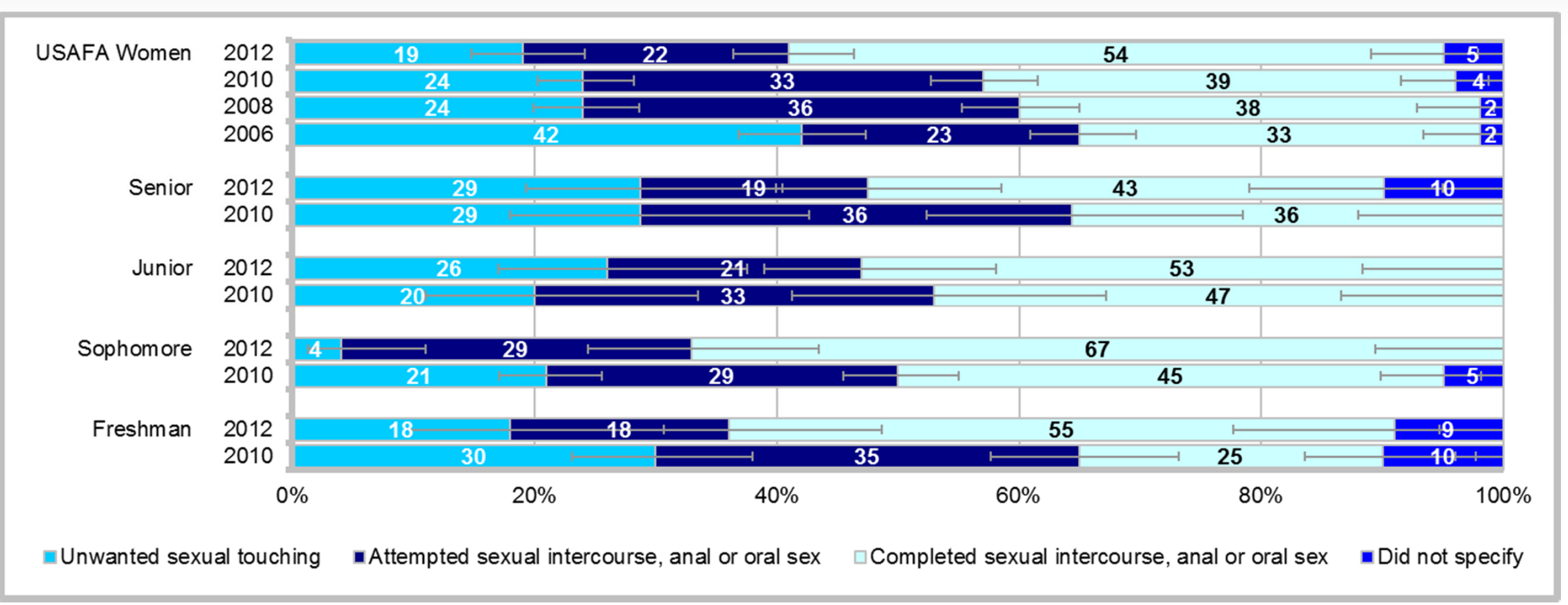

- Of the $11.2 \%$ of women who experienced USC, $19 \%$ indicated experiencing touching; $22 \%$ indicated experiencing attempted sex; $54 \%$ indicated experiencing completed sex; and $5 \%$ did not specify

- 2012 comparisons across years - touching lower than 2006; attempted sex lower than 2010, 2008; completed sex higher than 2010, 2008, 2006; did not specify higher than 2008, 2006

- Sophomores indicating touching in 2012 lower than 2010; seniors and freshmen indicating attempted sex in 2012 lower than 2010; sophomores and freshmen indicating completed sex in 2012 higher than 2010; sophomores indicating did not specify in 2012 lower than 2010

- Class differences in 2012 - higher response of completed sex led by sophomores 


\section{Contents}

- Introduction ......................................................................................... 3

- Unwanted Sexual Contact ................................................................ 7

- Most Serious Behaviors Experienced....................................... 11

$\checkmark$ Location of Incident ........................................................................ 13

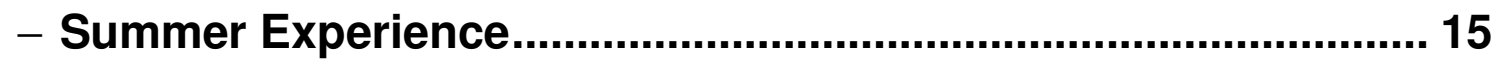

- Characteristics of Offender...................................................... 17

- Alcohol/Drug Involvement........................................................ 22

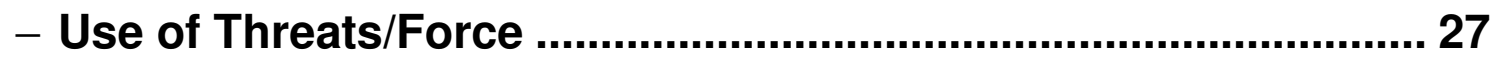

- Experience of Sexual Harassment/Stalking ................................ 32

- Consequences of the Situation .................................................. 35

- Reporting the Situation .............................................................. 39

- Experience of Unwanted Sexual Contact Prior to Entering

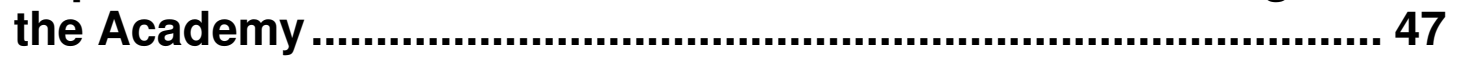

- Unwanted Gender-Related Behaviors ............................................. 49

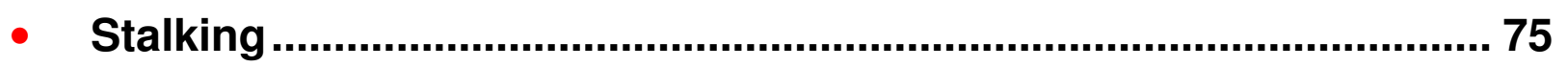

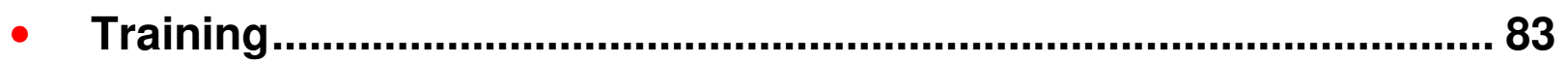

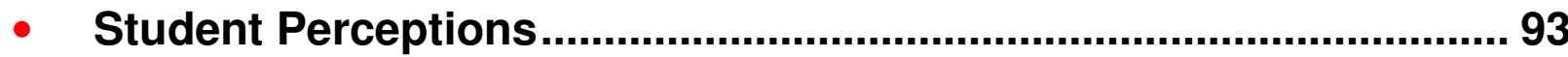




\section{Location of Unwanted Sexual Contact Incident Percent of Women Who Experienced Unwanted Sexual Contact}

\begin{tabular}{|c|c|c|c|c|c|}
\hline Location of Incident & Total & Senior & Junior & Sophomore & Freshman \\
\hline On Academy grounds in dormitory/living area & 59 & 63 & 63 & 50 & 60 \\
\hline On Academy grounds not in dormitory/living area & 14 & 5 & 11 & 4 & 60 \\
\hline $\begin{array}{l}\text { Off Academy grounds at a social event (such as a } \\
\text { party) }\end{array}$ & 32 & 47 & 16 & 29 & 30 \\
\hline $\begin{array}{l}\text { Off Academy grounds at an Academy sponsored } \\
\text { event }\end{array}$ & 3 & NR & 5 & 0 & NR \\
\hline Off Academy grounds other & 30 & 26 & 37 & 29 & 20 \\
\hline Margins of Error & $\pm 3-7 \%$ & $\pm 10-13 \%$ & $\pm 9-12 \%$ & $\pm 0-11 \%$ & $\pm 14-15 \%$ \\
\hline
\end{tabular}

\begin{tabular}{|l|l|}
\hline Higher Response of Yes & \\
\hline Lower Response of Yes & \\
\hline
\end{tabular}

- New question in 2012; no trend data available 


\section{Contents}

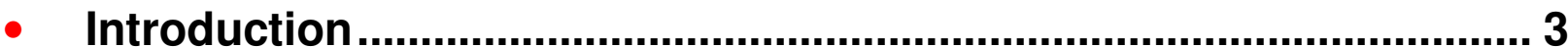

- Unwanted Sexual Contact ................................................................... 7

- Most Serious Behaviors Experienced........................................... 11

- Location of Incident ......................................................................... 13

$\checkmark$ Summer Experience...................................................................... 15

- Characteristics of Offender......................................................... 17

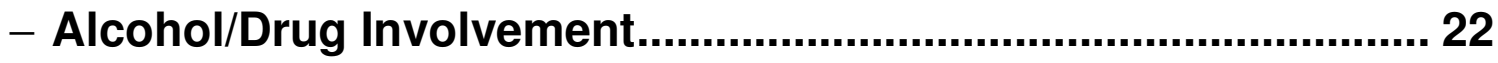

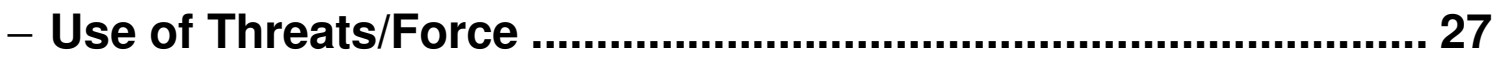

- Experience of Sexual Harassment/Stalking ............................... 32

- Consequences of the Situation ................................................ 35

- Reporting the Situation ..................................................................... 39

- Experience of Unwanted Sexual Contact Prior to Entering the Academy ...................................................................................... 47

- Unwanted Gender-Related Behaviors .............................................. 49

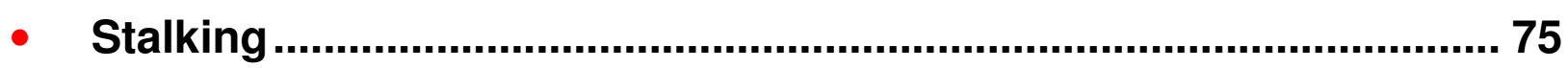

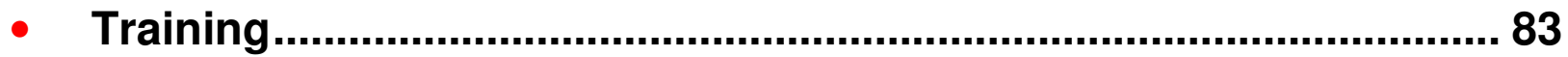

- Student Perceptions................................................................... 93 


\section{Unwanted Sexual Contact Incident Occurred During Summer Experience/Training \\ Percent of Women Who Experienced Unwanted Sexual Contact}

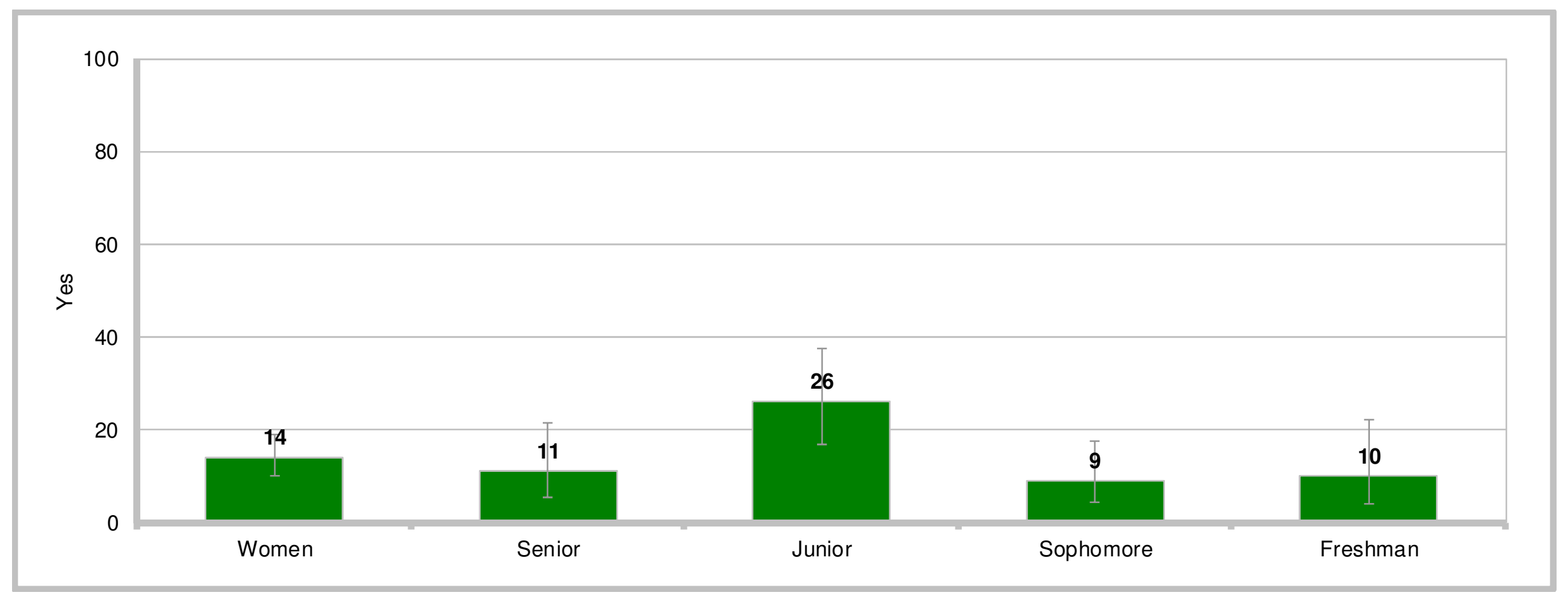

- Of the $11.2 \%$ of women who experienced USC, $14 \%$ indicated the offense occurred during summer experience/training

- 2012 comparisons across years - new question in 2012; no trend data available

- Class differences in 2012 - no differences 


\section{Contents}

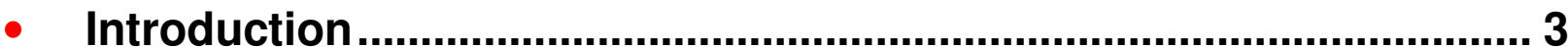

- Unwanted Sexual Contact ................................................................... 7

- Most Serious Behaviors Experienced........................................... 11

- Location of Incident ....................................................................... 13

- Summer Experience...................................................................... 15

$\checkmark$ Characteristics of Offender......................................................... 17

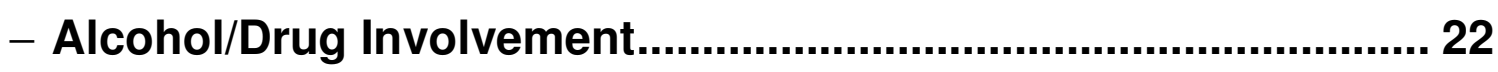

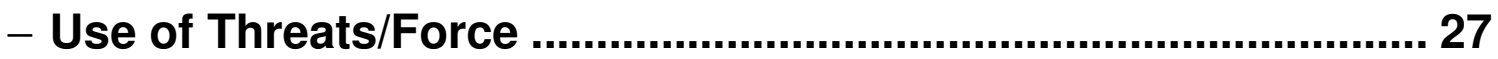

- Experience of Sexual Harassment/Stalking ............................... 32

- Consequences of the Situation ................................................ 35

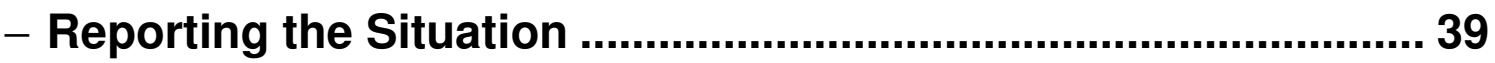

- Experience of Unwanted Sexual Contact Prior to Entering the Academy ...................................................................................... 47

- Unwanted Gender-Related Behaviors .............................................. 49

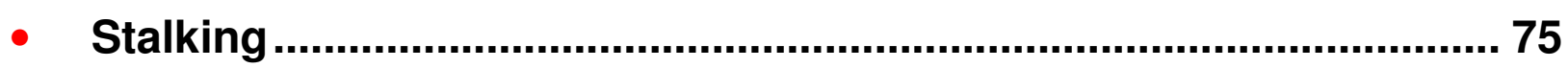

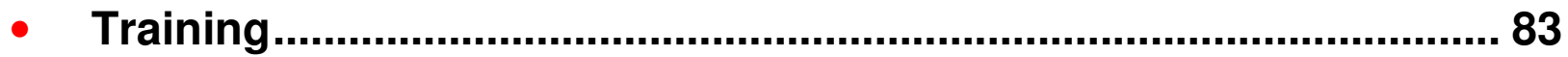

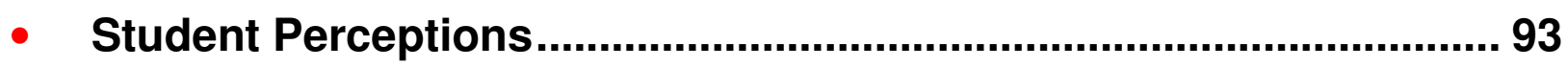




\section{Combinations of Offender Affiliations Percent of Women Who Experienced Unwanted Sexual Contact}

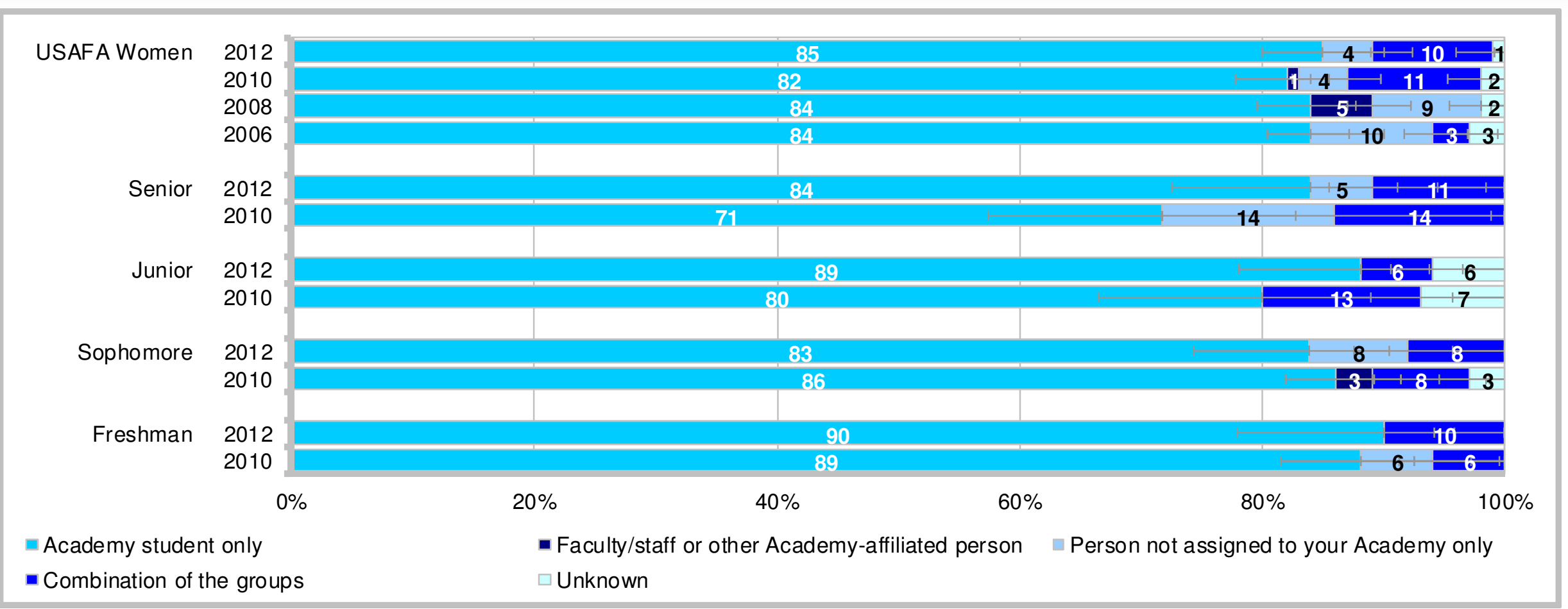

- Of the $11.2 \%$ of women who experienced USC, $85 \%$ indicated the offender was Academy student only; $0 \%$ indicated faculty/staff; $4 \%$ indicated person not assigned to the Academy; $10 \%$ indicated combination of the groups; and 1\% indicated the offender was unknown

- 2012 comparisons across years - faculty/staff lower than 2008; person not assigned to the Academy lower than 2008, 2006; combination of the groups higher than 2008, 2006

- Sophomores indicating faculty/staff in 2012 lower than 2010; sophomores indicating person not assigned to the Academy in 2012 higher than 2010; sophomores indicating unknown in 2012 lower than 2010

- Class differences in 2012 - no differences 


\section{Gender of the Offender \\ Percent of Women Who Experienced Unwanted Sexual Contact}

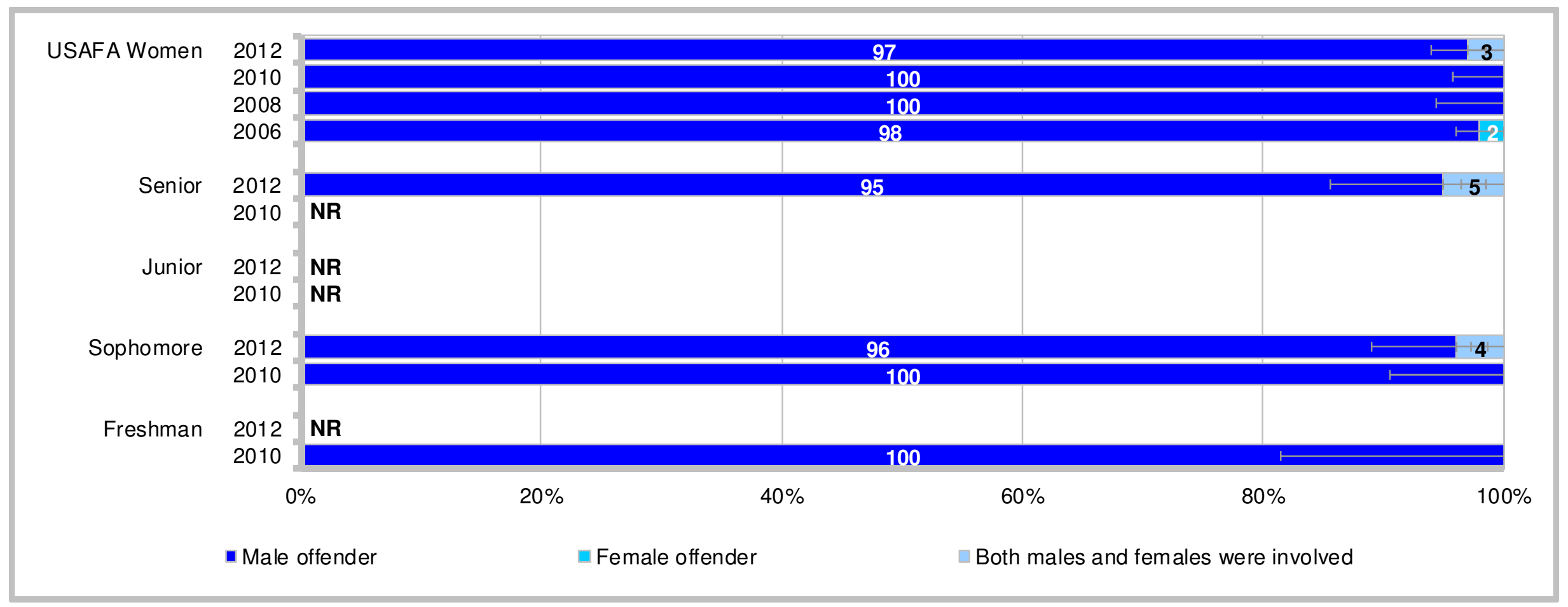

- Of the $11.2 \%$ of women who experienced USC, $97 \%$ indicated the offender was male; $0 \%$ indicated the offender was female; $3 \%$ indicated the offenders were both males and females

- 2012 comparisons across years - male offender lower than 2010, 2008; female offender lower than 2006; both male and female offenders higher than 2010, 2008, 2006

- Class differences in 2012 - no differences 


\section{Multiple Offenders Involved}

Percent of Women Who Experienced Unwanted Sexual Contact

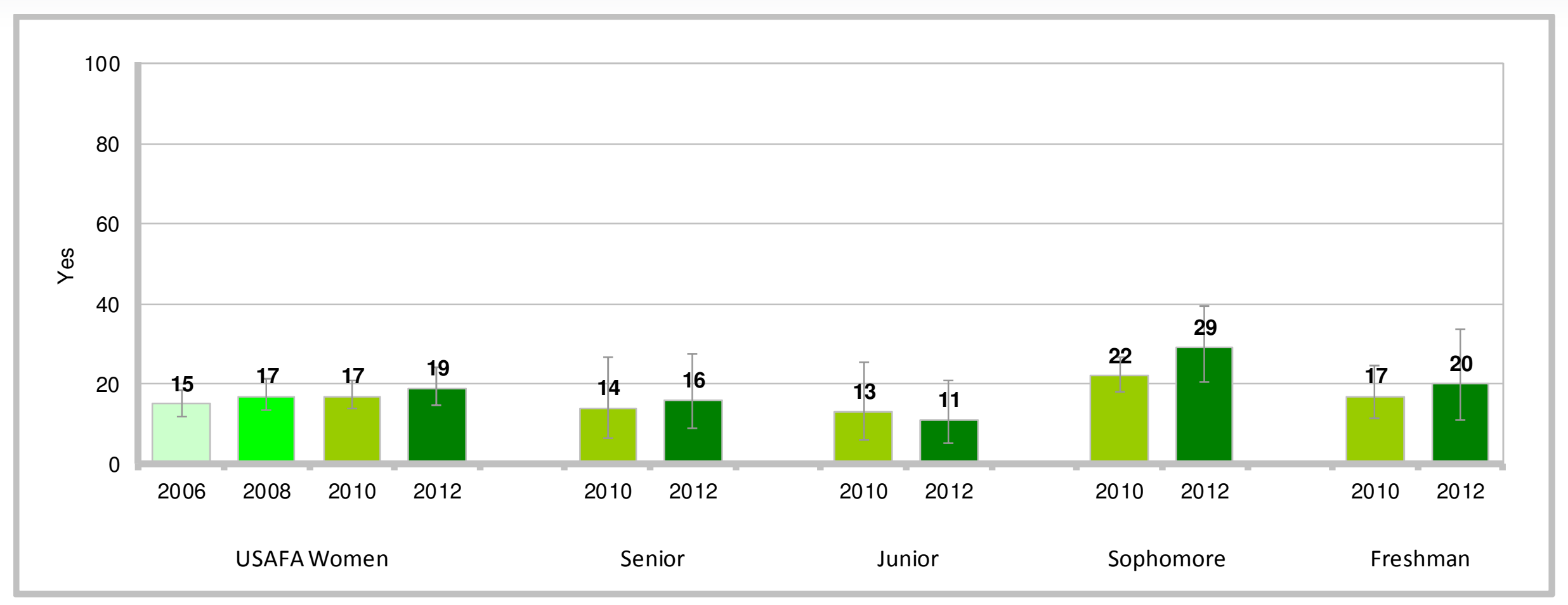

- Of the $11.2 \%$ of women who experienced USC, $19 \%$ indicated multiple offenders were involved

- 2012 comparisons across years - no differences

- Class differences in 2012 - no differences 


\section{Offender Was a "Creeper" (i.e., someone who is socially awkward)} Percent of Women Who Experienced Unwanted Sexual Contact Involving an Academy Student

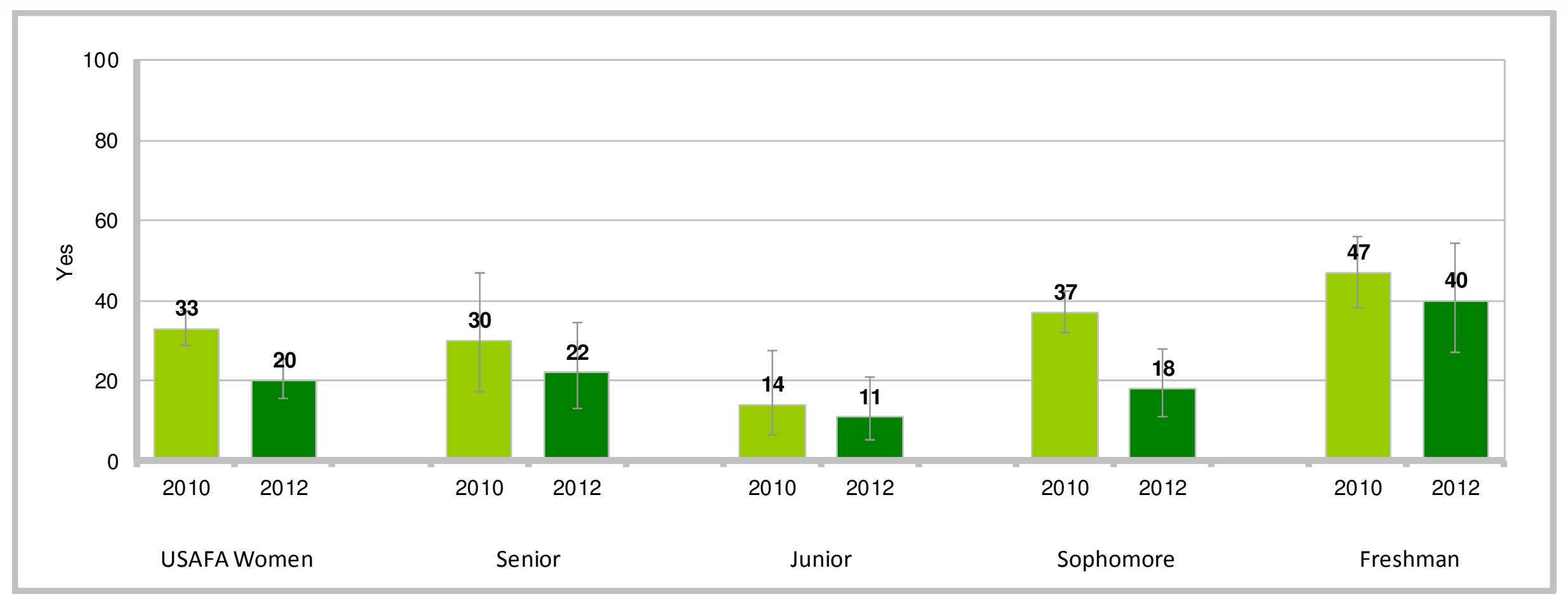

- Of the $11.2 \%$ of women who experienced USC, $20 \%$ indicated the offender was a "creeper"

- 2012 comparisons across years - lower than 2010

- Sophomores in 2012 lower than 2010

- Class differences in 2012 - higher response led by freshmen 


\section{Contents}

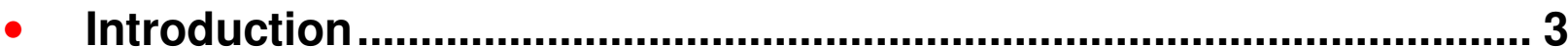

- Unwanted Sexual Contact ................................................................... 7

- Most Serious Behaviors Experienced.......................................... 11

- Location of Incident ....................................................................... 13

- Summer Experience..................................................................... 15

- Characteristics of Offender........................................................ 17

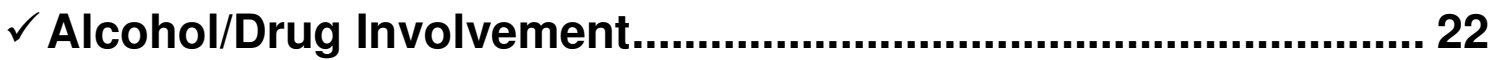

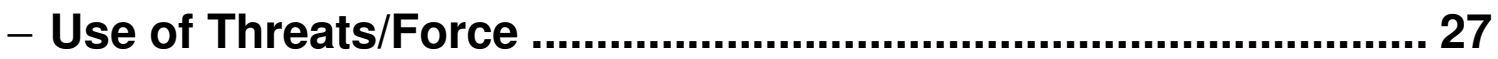

- Experience of Sexual Harassment/Stalking ............................... 32

- Consequences of the Situation ................................................ 35

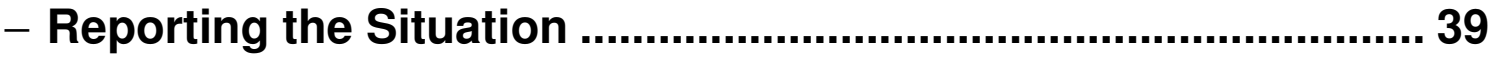

- Experience of Unwanted Sexual Contact Prior to Entering the Academy ...................................................................................... 47

- Unwanted Gender-Related Behaviors .............................................. 49

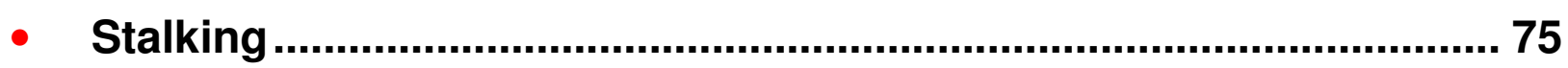

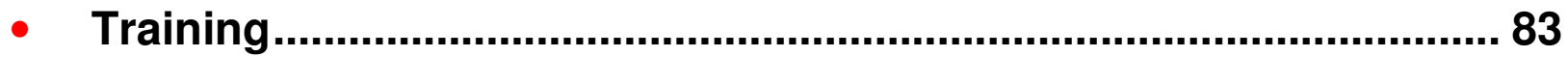

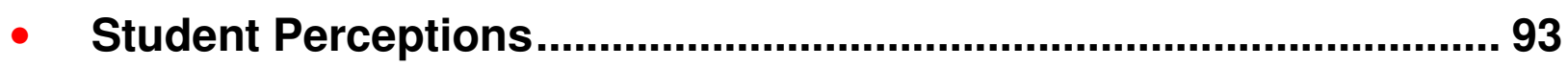




\section{Use of Alcohol and/or Drugs in the Incident Percent of Women Who Experienced Unwanted Sexual Contact}

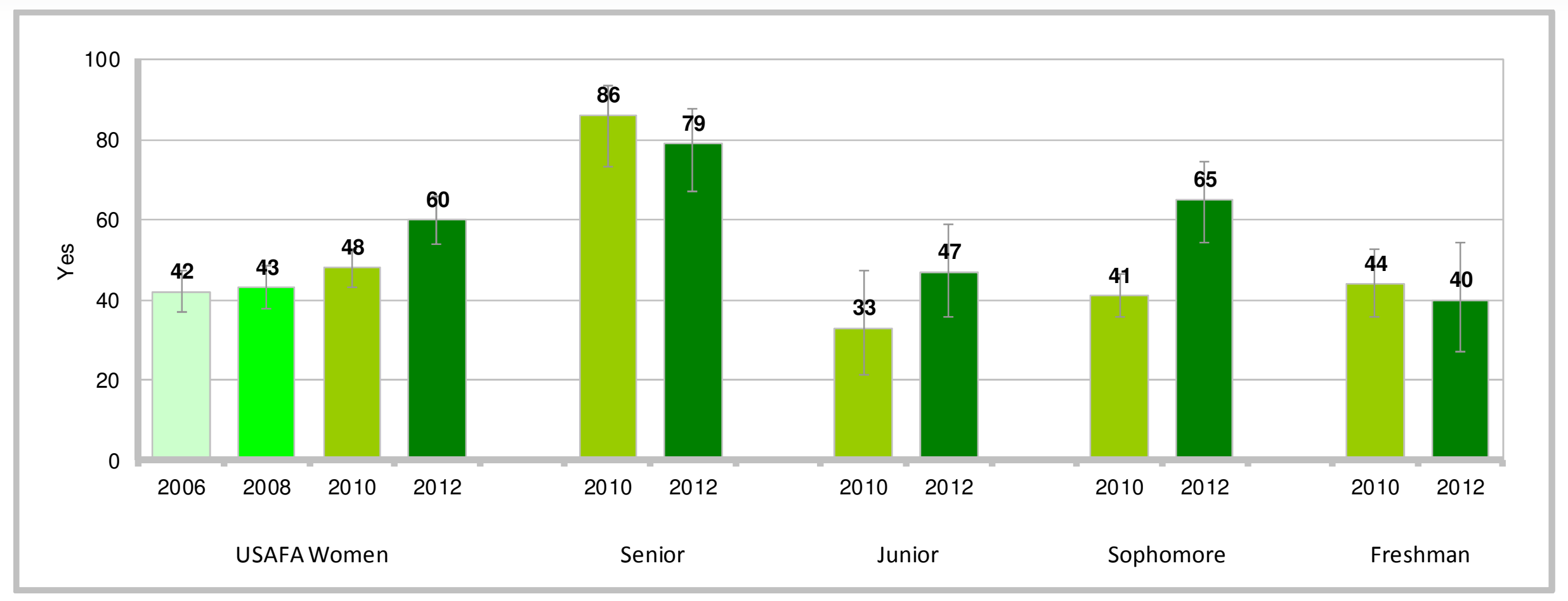

- Of the $11.2 \%$ of women who experienced USC, $60 \%$ indicated alcohol and/or drugs were involved

- 2012 comparisons across years - higher than 2010, 2008, 2006

- Sophomores in 2012 higher than 2010

- Class differences in 2012 - higher response led by seniors; lower response led by juniors and freshmen 


\section{Use of Alcohol Before the Incident Percent of Women Who Experienced Unwanted Sexual Contact}

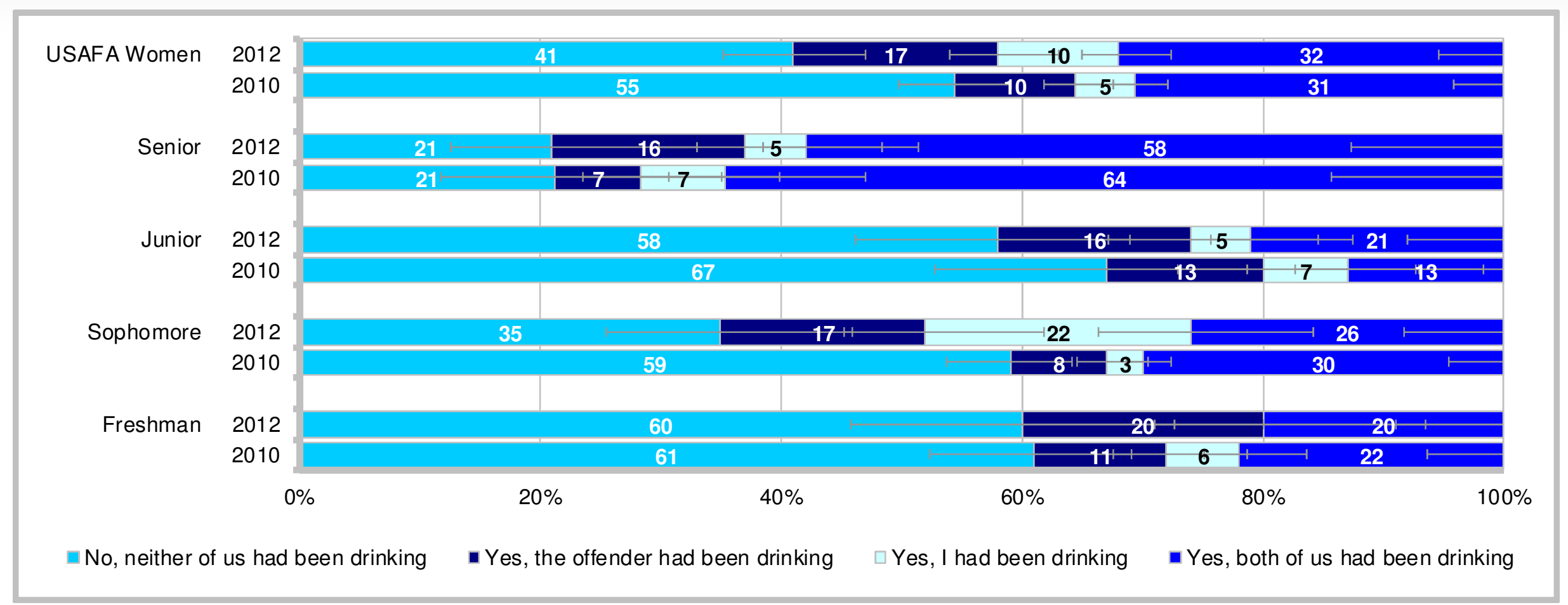

- Of the $11.2 \%$ of women who experienced USC, $41 \%$ indicated neither of them had been drinking; $17 \%$ indicated the offender had been drinking; $10 \%$ indicated they had been drinking; and $32 \%$ indicated both had been drinking

- 2012 comparisons across years - neither of them had been drinking lower than 2010; the offender had been drinking higher than 2010; they had been drinking higher than 2010

- Sophomores indicating neither of them had been drinking in 2012 lower than 2010; sophomores indicating the offender had been drinking in 2012 higher than 2010; sophomores indicating they had been drinking in 2012 higher than 2010

- Class differences in 2012 - neither of them had been drinking led by juniors and freshmen; they had been drinking led by sophomores; both had been drinking led by seniors 


\section{Use of Drugs Before the Incident Percent of Women Who Experienced Unwanted Sexual Contact}

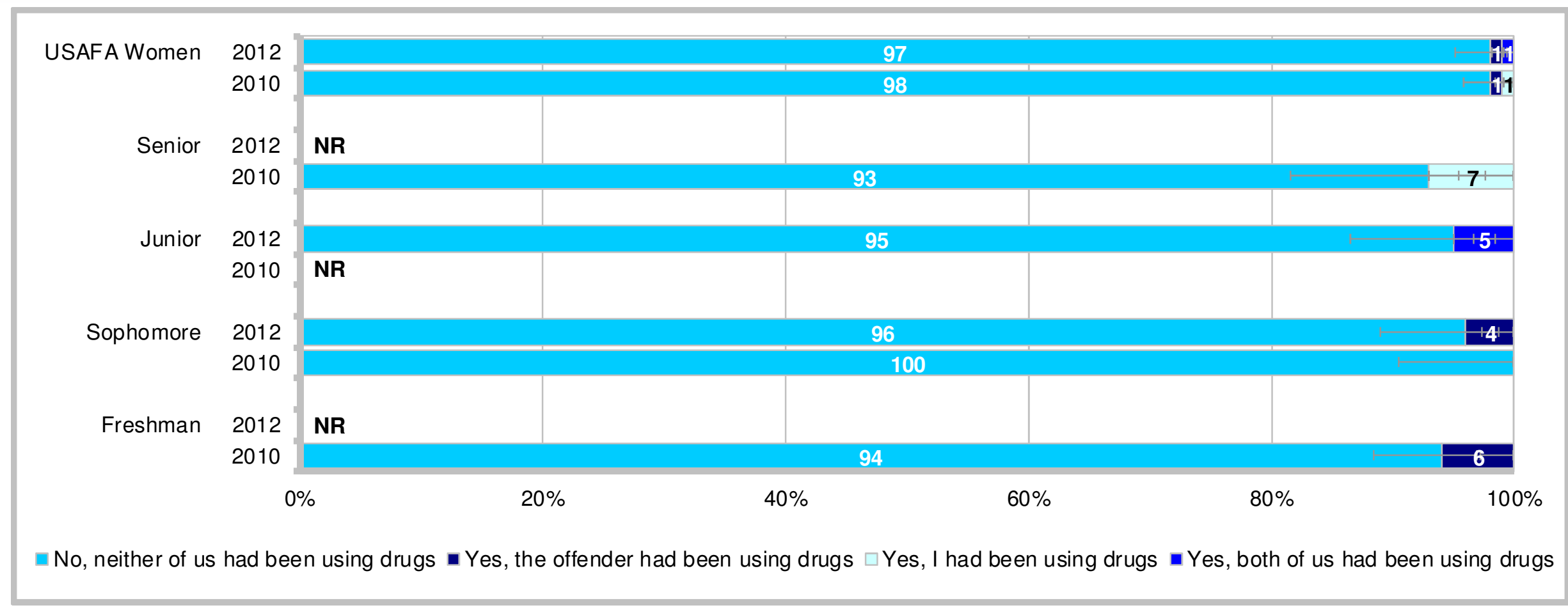

- Of the $11.2 \%$ of women who experienced USC, $97 \%$ indicated neither of them had been using drugs; $1 \%$ indicated the offender had been using drugs; $0 \%$ indicated they had been using drugs; and $1 \%$ indicated both had been using drugs

- 2012 comparisons across years - no differences

- Class differences in 2012 - no differences 


\section{Use of Knock Out Drugs Before the Incident Percent of Women Who Experienced Unwanted Sexual Contact}

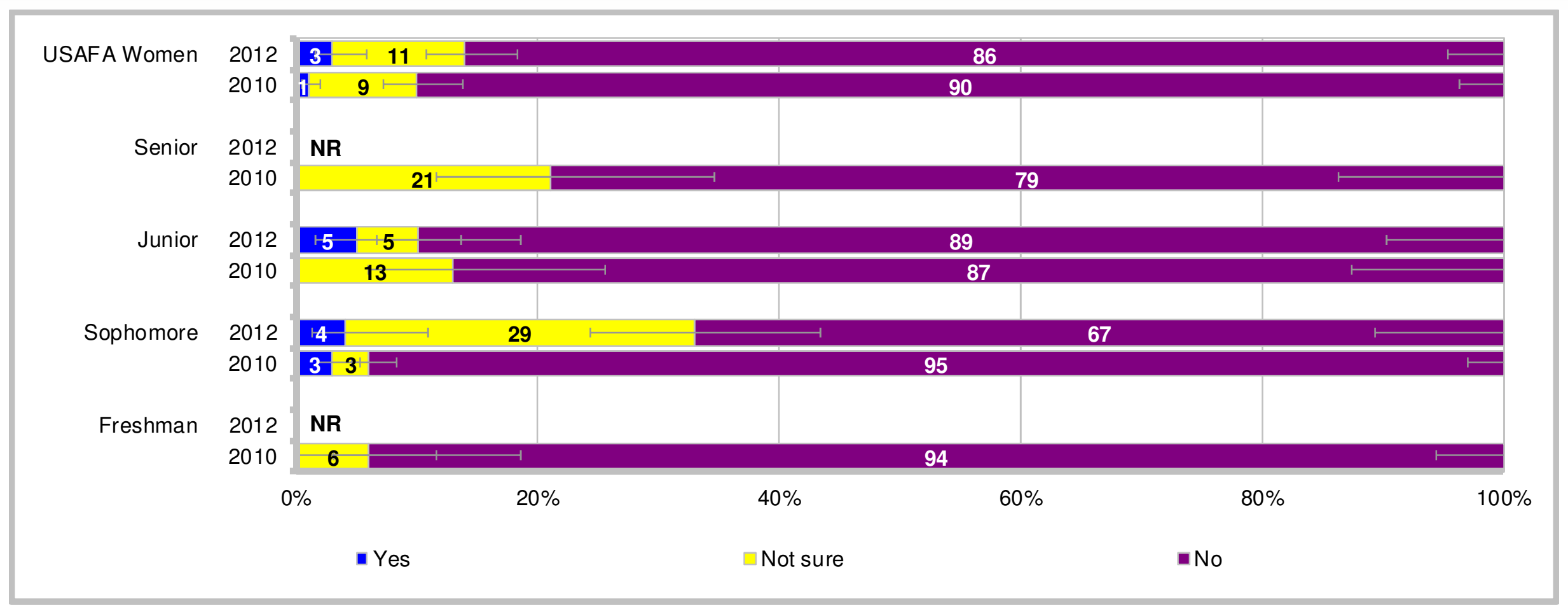

- Of the $11.2 \%$ of women who experienced USC, $86 \%$ indicated the offender did not use drugs to knock them out; $11 \%$ indicated they were not sure; $3 \%$ indicated the offender used drugs to knock them out

- 2012 comparisons across years - no differences

- Sophomores indicating the offender did not use drugs to knock them out in 2012 lower than 2010; sophomores indicating not sure in 2012 higher than 2010

- Class differences in 2012 - not sure led by sophomores 


\section{Contents}

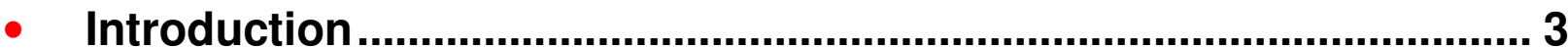

- Unwanted Sexual Contact ................................................................... 7

- Most Serious Behaviors Experienced.......................................... 11

- Location of Incident ....................................................................... 13

- Summer Experience..................................................................... 15

- Characteristics of Offender....................................................... 17

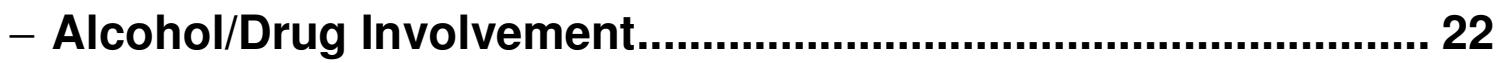

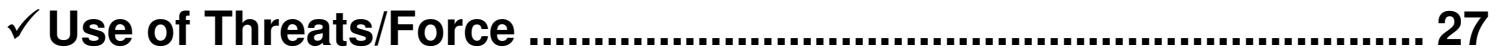

- Experience of Sexual Harassment/Stalking ............................... 32

- Consequences of the Situation ................................................ 35

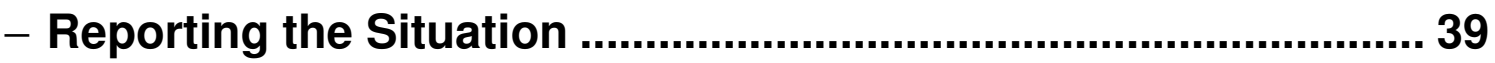

- Experience of Unwanted Sexual Contact Prior to Entering the Academy ..................................................................................... 47

- Unwanted Gender-Related Behaviors .............................................. 49

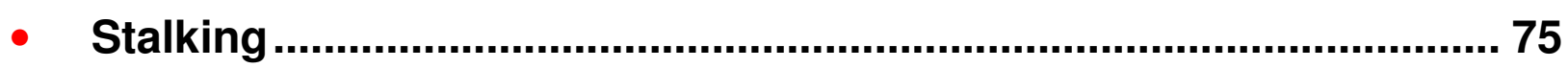

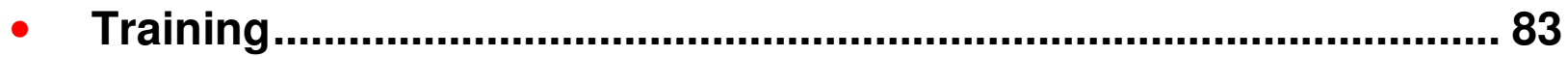

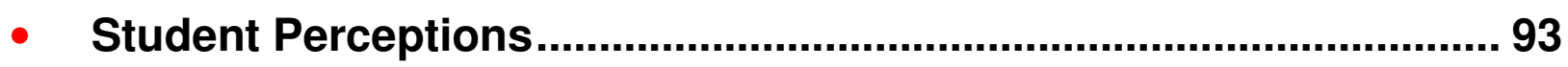




\section{Use of Threats and Physical Force in the Incident Percent of Women Who Experienced Unwanted Sexual Contact}

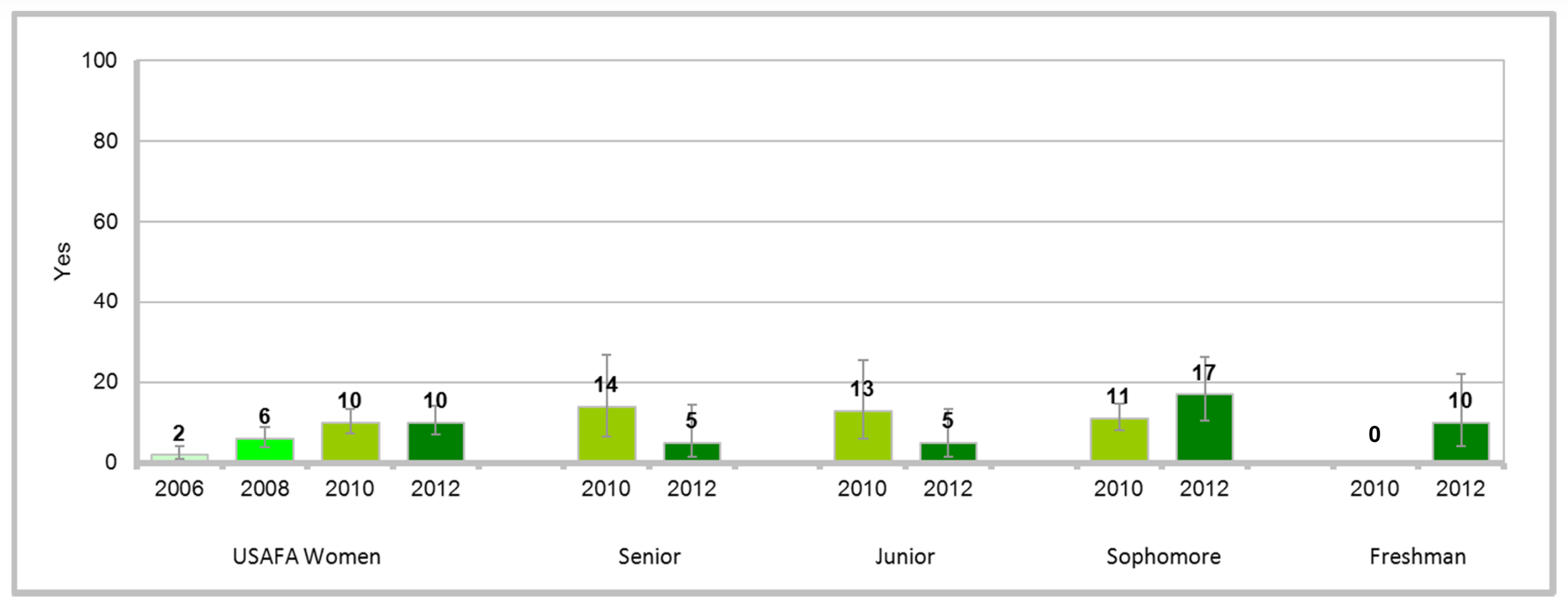

- Of the $11.2 \%$ of women who experienced USC, $10 \%$ indicated the situation involved threats and force

- 2012 comparisons across years - higher than 2006

- Freshmen in 2012 higher than 2010

- Class differences in 2012 - no differences 


\section{Offender Threatened To Ruin Your Reputation if You Did Not Consent \\ Percent of Women Who Experienced Unwanted Sexual Contact}

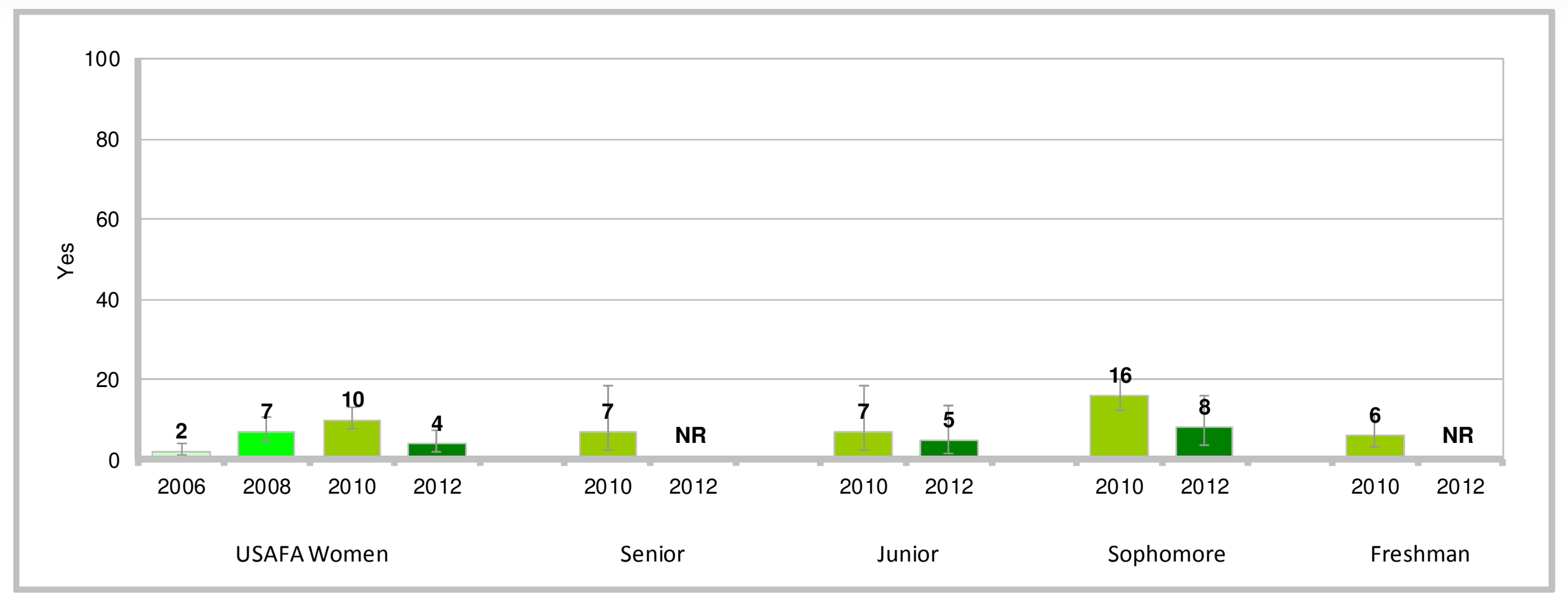

- Of the $11.2 \%$ of women who experienced USC, $4 \%$ indicated the offender threatened to ruin their reputation

- 2012 comparisons across years - lower than 2010

- Sophomores in 2012 lower than 2010

- Class differences in 2012 - no differences 


\section{Offender Threatened To Harm You if You Did Not Consent \\ Percent of Women Who Experienced Unwanted Sexual Contact}

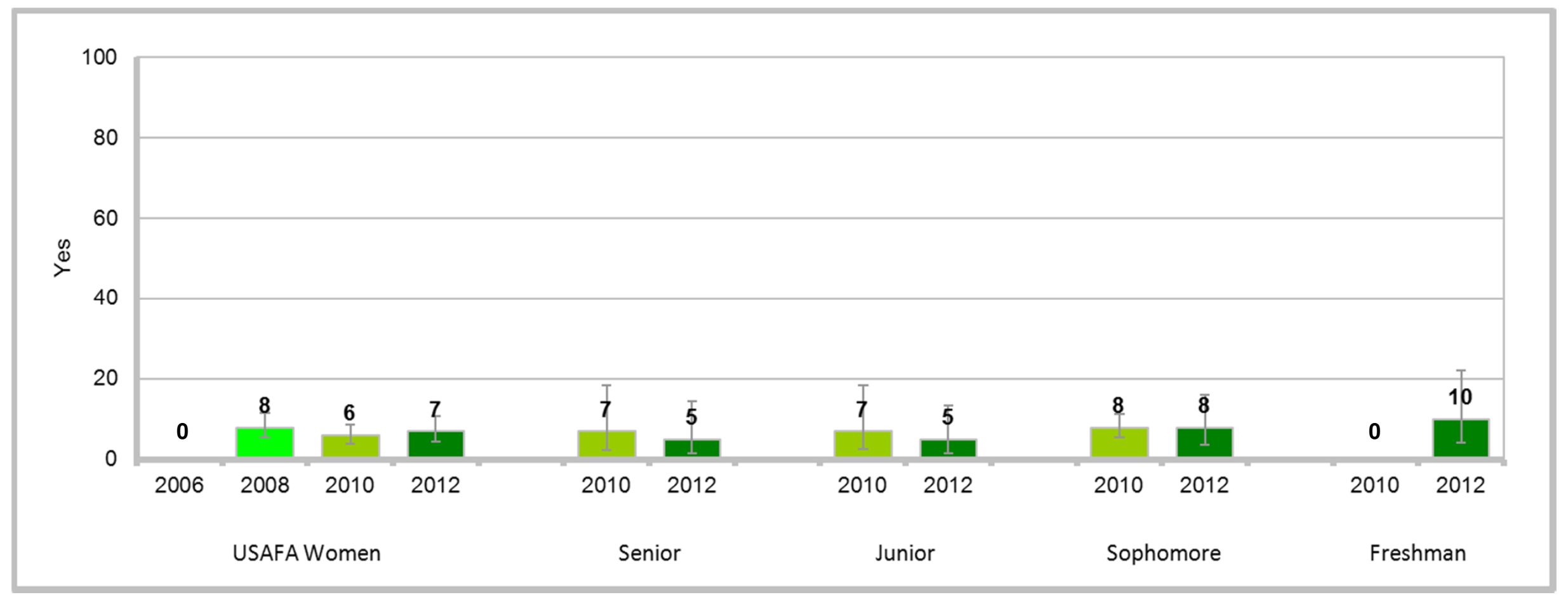

- Of the $11.2 \%$ of women who experienced USC, $7 \%$ indicated the offender threatened to harm them

- 2012 comparisons across years - higher than 2006

- Freshmen in 2012 higher than 2010

- Class differences in 2012 - no differences 


\section{Offender Used Some Degree of Physical Force}

Percent of Women Who Experienced Unwanted Sexual Contact

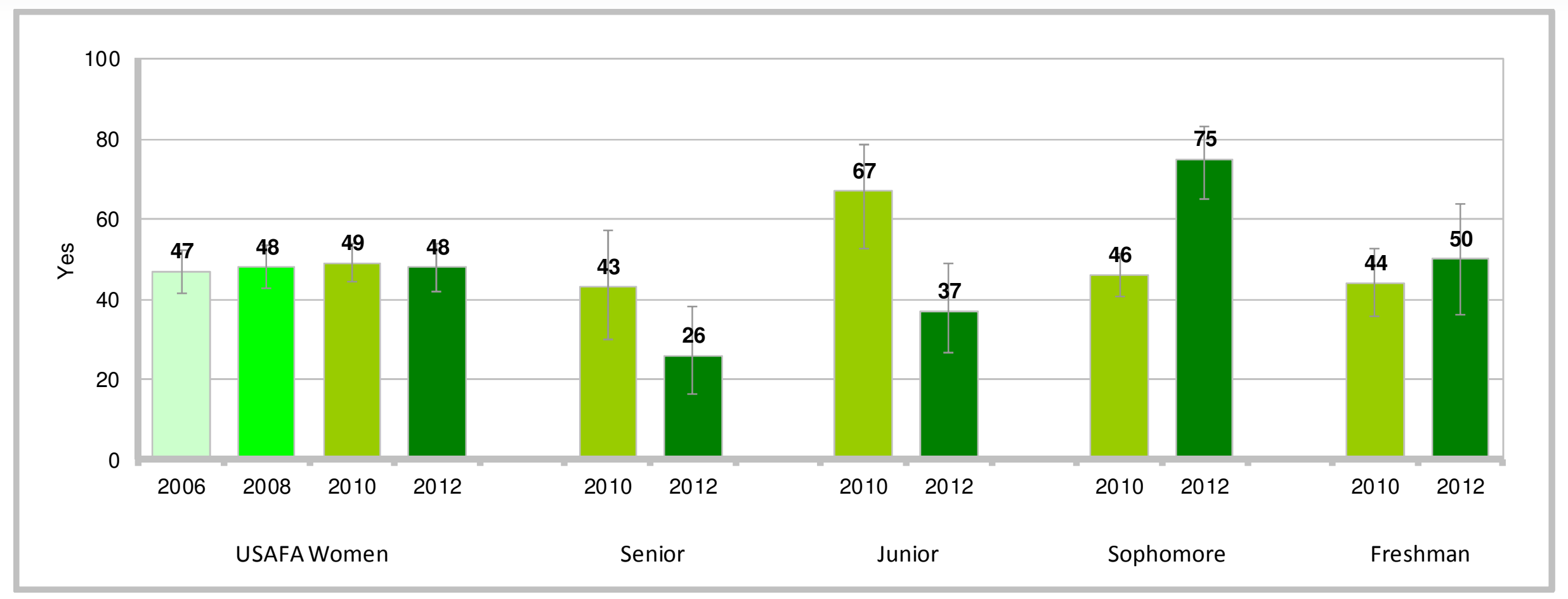

- Of the $11.2 \%$ of women who experienced USC, $48 \%$ indicated the offender threatened to use some degree of physical force

- 2012 comparisons across years - no differences

- Juniors in 2012 lower than 2010; sophomores in 2012 higher than 2010

- Class differences in 2012 - higher response led by sophomores; lower response led by seniors 


\section{Contents}

- Introduction ............................................................................ 3

- Unwanted Sexual Contact ................................................................... 7

- Most Serious Behaviors Experienced...................................... 11

- Location of Incident ........................................................................... 13

- Summer Experience......................................................................... 15

- Characteristics of Offender......................................................... 17

- Alcohol/Drug Involvement........................................................... 22

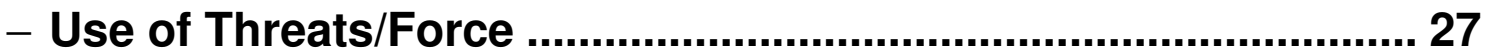

$\checkmark$ Experience of Sexual Harassment/Stalking ............................... 32

- Consequences of the Situation ................................................... 35

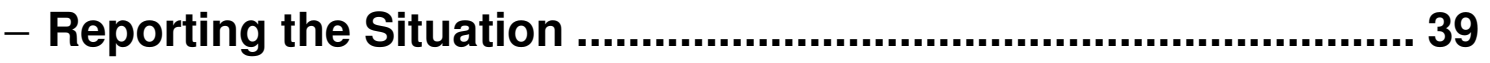

- Experience of Unwanted Sexual Contact Prior to Entering

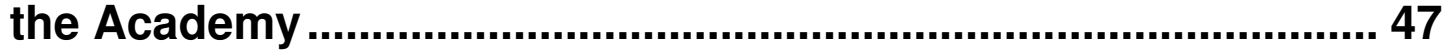

- Unwanted Gender-Related Behaviors ............................................. 49

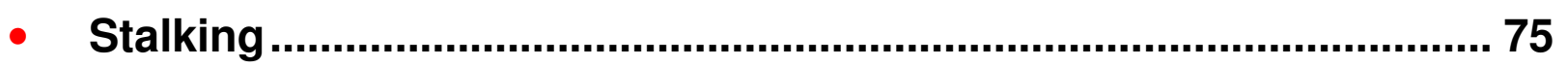

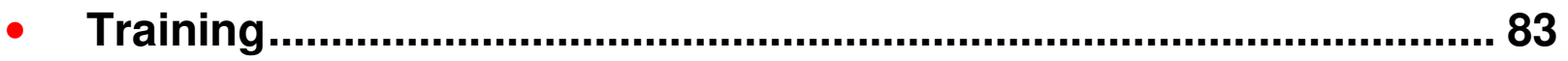

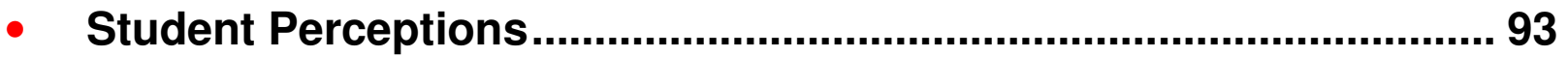




\section{Offender Sexually Harassed and/or Stalked You Before or After the Incident} Percent of Women Who Experienced Unwanted Sexual Contact

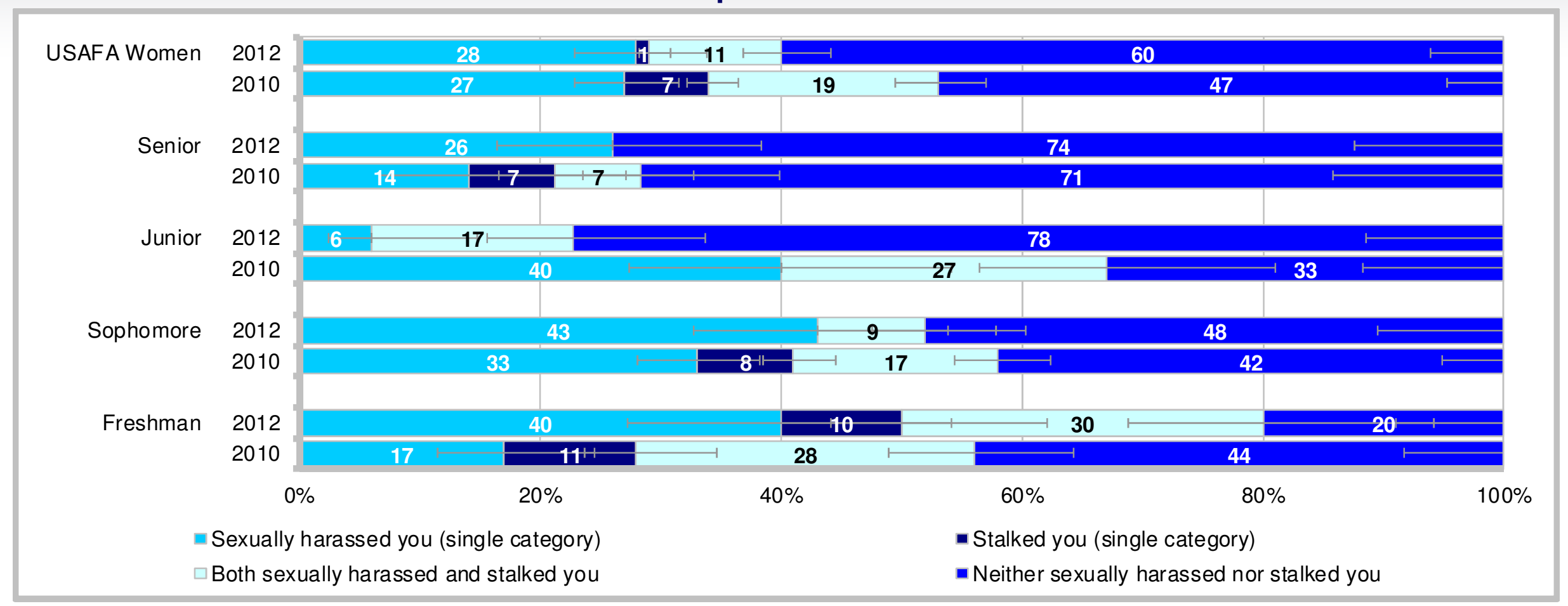

- Of the $11.2 \%$ of women who experienced USC, $28 \%$ indicated the offender sexually harassed them; $1 \%$ indicated the offender stalked them; $11 \%$ indicated the offender both sexually harassed and stalked them; and $60 \%$ indicated the offender neither sexually harassed nor stalked them

- 2012 comparisons across years - stalked lower than 2010; both sexually harassed and stalked lower than 2010; neither sexually harassed nor stalked higher than 2010

- Juniors indicating sexually harassed in 2012 lower than 2010, whereas freshmen higher; sophomores indicating stalked in 2012 lower than 2010; sophomores indicating both sexually harassed and stalked in 2012 lower than 2010; juniors indicating neither sexually harassed nor stalked in 2012 higher than 2010, whereas freshmen lower

- Class differences in 2012 - sexually harassed led by sophomores; both sexually harassed and stalked led by freshmen; neither sexually harassed nor stalked led by juniors and seniors 


\section{When Offender Sexually Harassed and/or Stalked You Percent of Women Who Experienced Unwanted Sexual Contact}

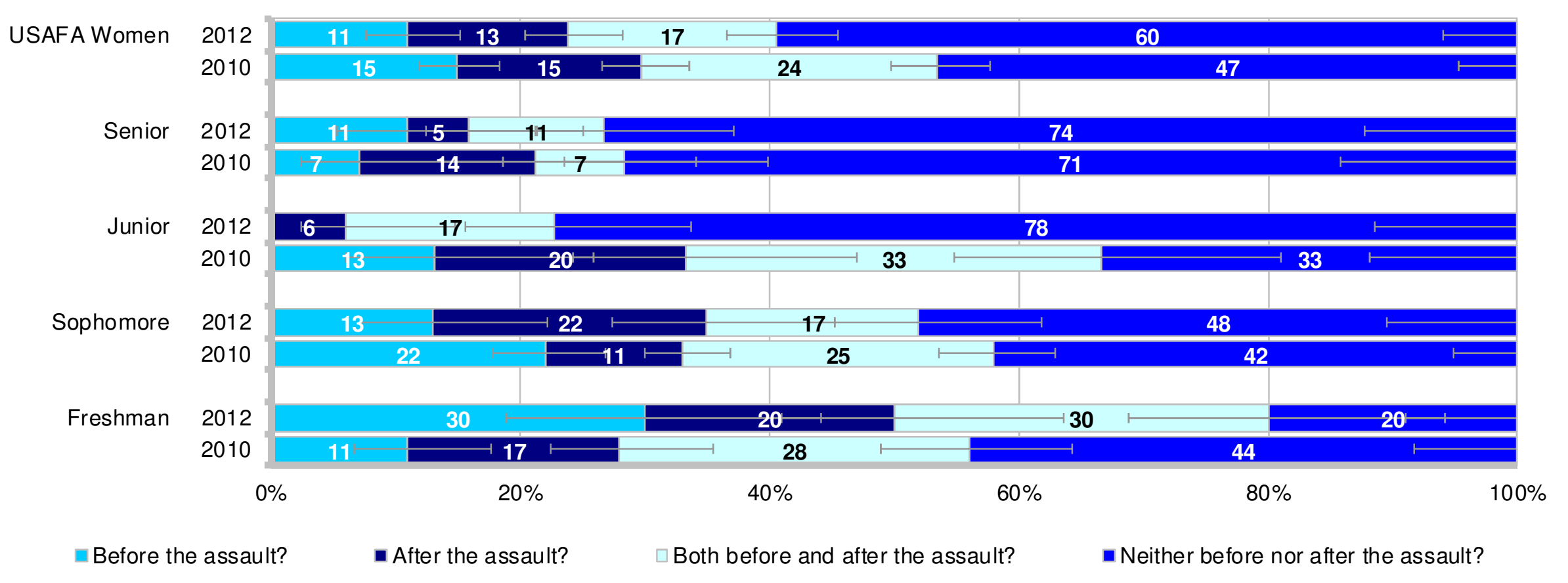

- Of the $11.2 \%$ of women who experienced USC, $11 \%$ indicated the offender sexually harassed or stalked them before the assault; $13 \%$ indicated the offender sexually harassed or stalked them after the assault; $17 \%$ indicated the offender sexually harassed or stalked them both before and after the assault; and $60 \%$ indicated the offender did not sexually harass or stalk them

- 2012 comparisons across years - both before and after the assault lower than 2010; neither higher than 2010

- Sophomores indicating before in 2012 lower than 2010, whereas freshmen higher; juniors indicating after in 2012 lower than 2010, whereas sophomores higher; juniors indicating both in 2012 lower than 2010; juniors indicating neither in 2012 higher than 2010, whereas freshmen lower

- Class differences in 2012 - before led by freshmen; after led by sophomores; neither led by juniors and seniors 


\section{Contents}

- Introduction ........................................................................................ 3

- Unwanted Sexual Contact ................................................................... 7

- Most Serious Behaviors Experienced...................................... 11

- Location of Incident ........................................................................... 13

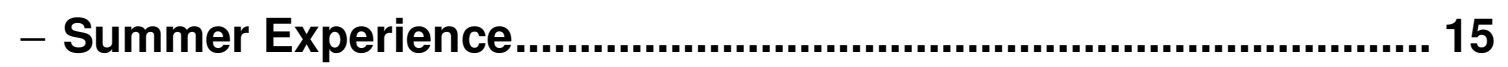

- Characteristics of Offender......................................................... 17

- Alcohol/Drug Involvement........................................................... 22

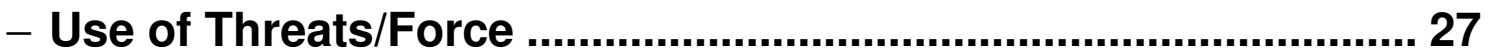

- Experience of Sexual Harassment/Stalking ............................... 32

$\checkmark$ Consequences of the Situation ................................................. 35

- Reporting the Situation ............................................................. 39

- Experience of Unwanted Sexual Contact Prior to Entering the Academy ..................................................................................... 47

- Unwanted Gender-Related Behaviors ............................................. 49

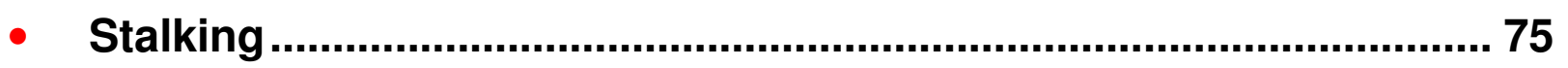

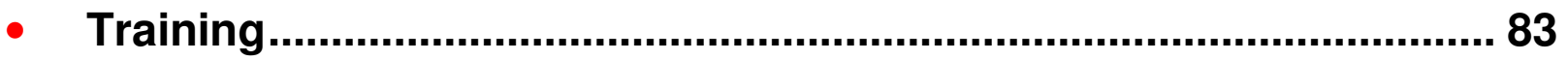

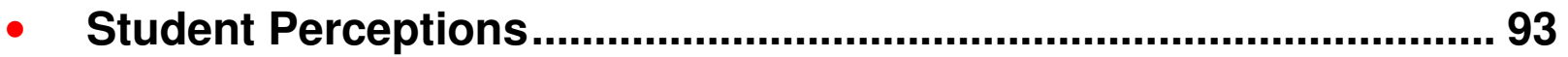




\section{Considered Requesting a Transfer to Another Squadron Percent of Women Who Experienced Unwanted Sexual Contact}

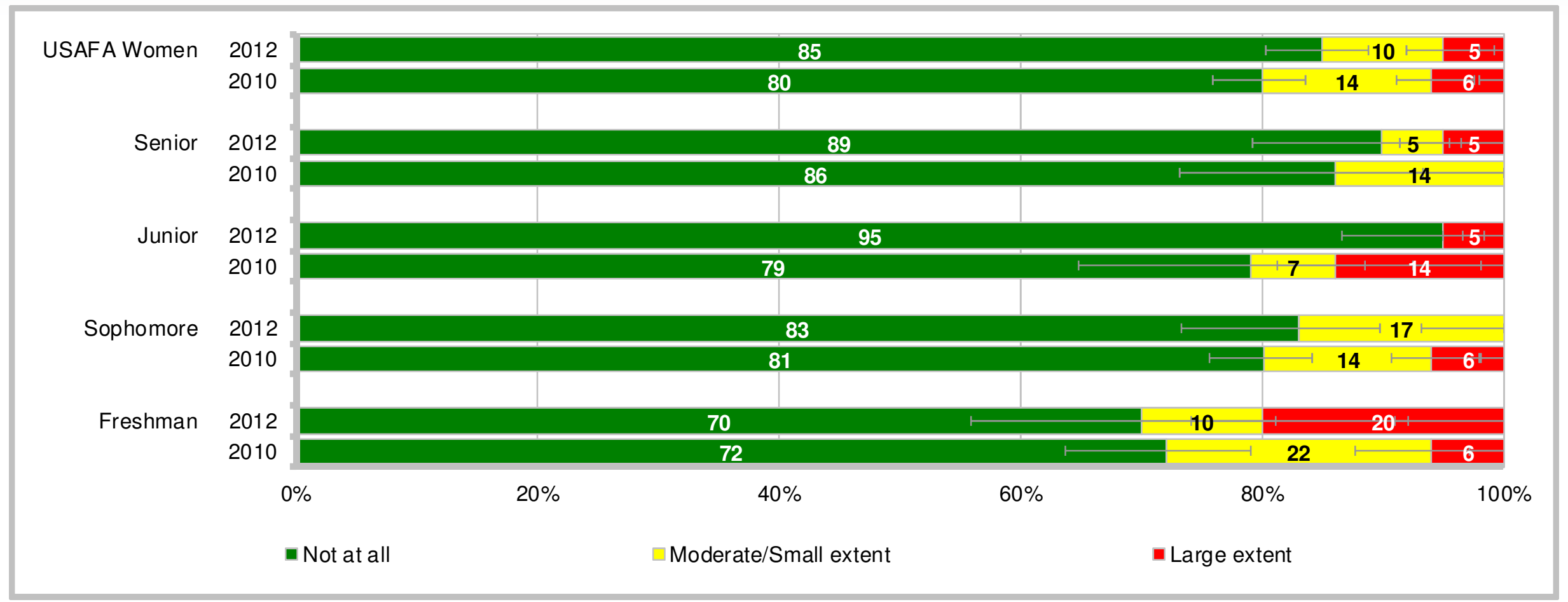

- Of the $11.2 \%$ of women who experienced USC, $15 \%$ indicated they considered a transfer to some extent; $85 \%$ indicated not at all

- 2012 comparisons across years - no differences

- Juniors indicating not at all in 2012 higher than 2010

- Class differences in 2012 - not at all led by juniors 


\section{Thought About Leaving Your Academy Percent of Women Who Experienced Unwanted Sexual Contact}

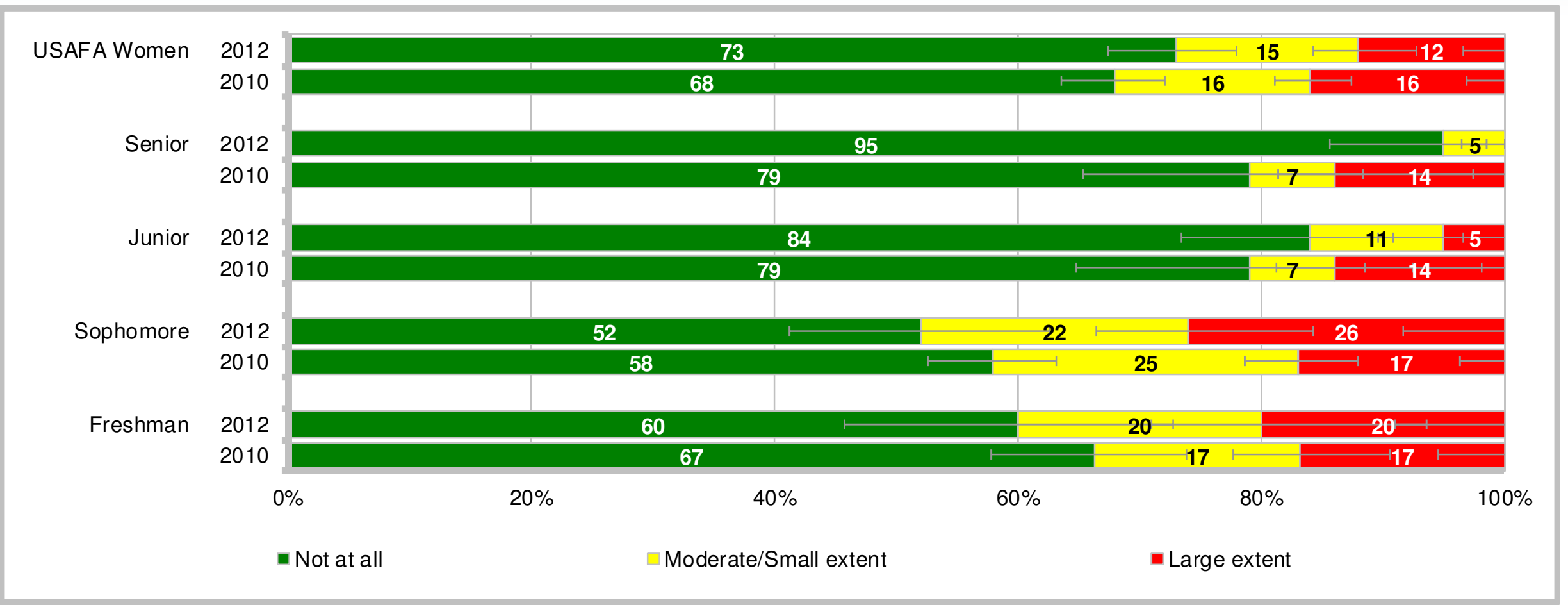

- Of the $11.2 \%$ of women who experienced USC, $27 \%$ indicated they thought about leaving the Academy to some extent; $73 \%$ indicated not at all

- 2012 comparisons across years - no differences

- Seniors indicating not at all in 2012 higher than 2010

- Class differences in 2012 - not at all led by seniors and juniors 


\section{Your Academic Performance Suffered Percent of Women Who Experienced Unwanted Sexual Contact}

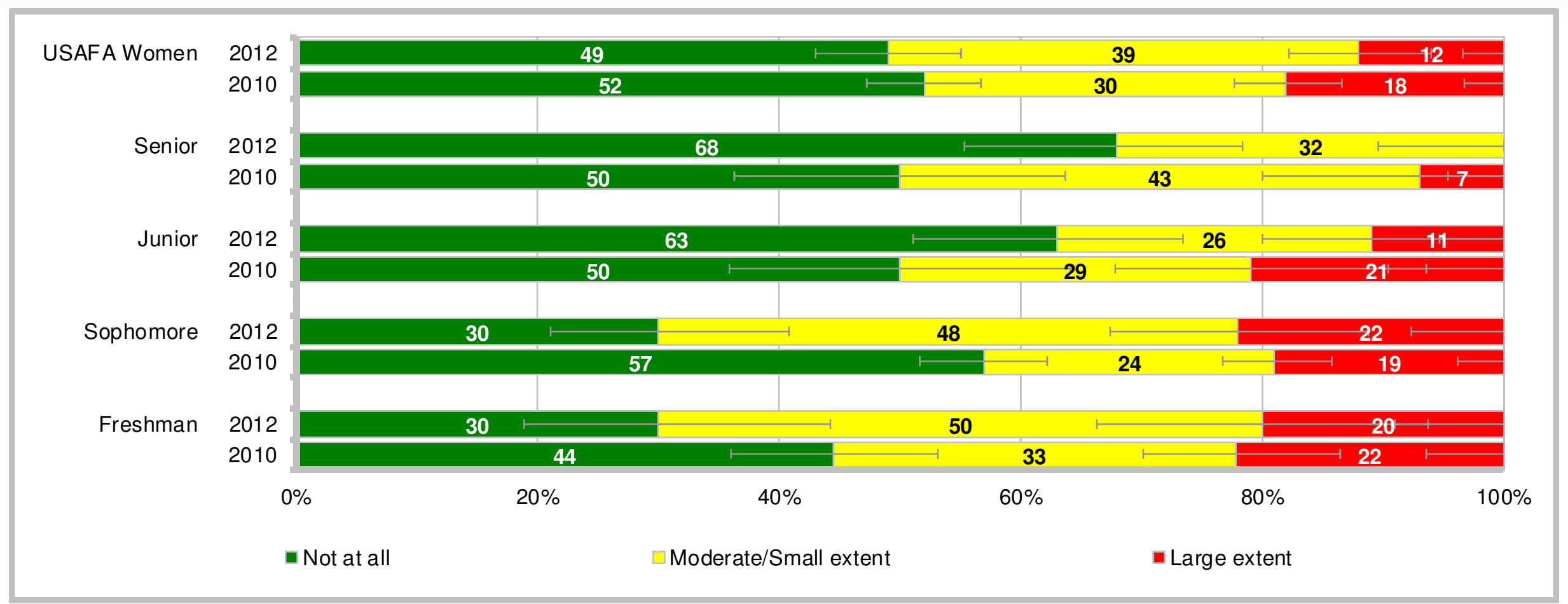

- Of the $11.2 \%$ of women who experienced USC, $51 \%$ indicated their academic performance suffered to some extent; $49 \%$ indicated not at all

- 2012 comparisons across years - no differences

- Sophomores indicating not at all in 2012 lower than 2010

- Class differences in 2012 - not at all led by seniors and juniors 


\section{Contents}

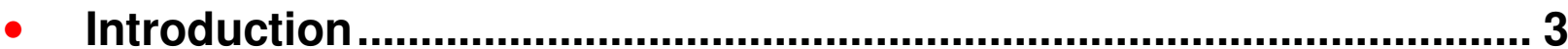

- Unwanted Sexual Contact ................................................................... 7

- Most Serious Behaviors Experienced.......................................... 11

- Location of Incident ....................................................................... 13

- Summer Experience..................................................................... 15

- Characteristics of Offender....................................................... 17

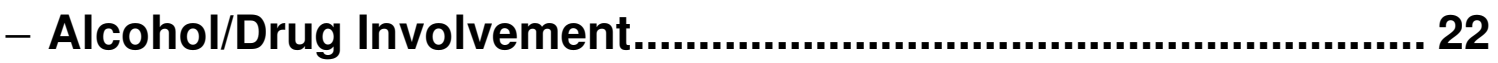

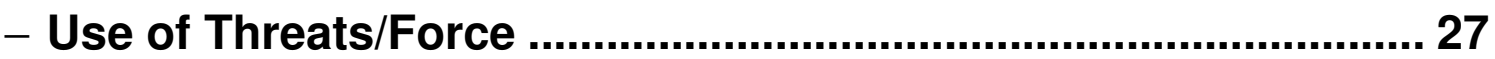

- Experience of Sexual Harassment/Stalking ............................... 32

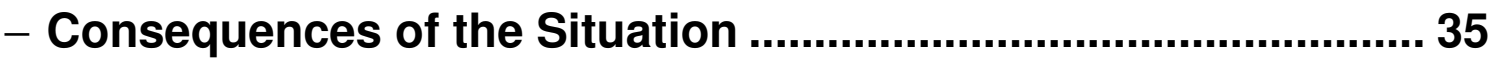

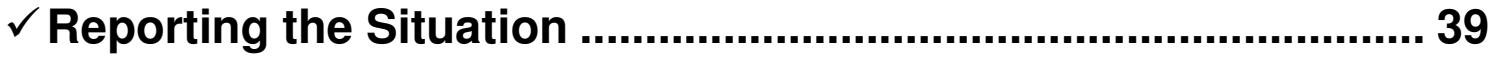

- Experience of Unwanted Sexual Contact Prior to Entering the Academy ..................................................................................... 47

- Unwanted Gender-Related Behaviors .............................................. 49

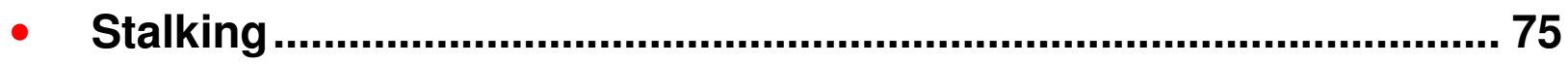

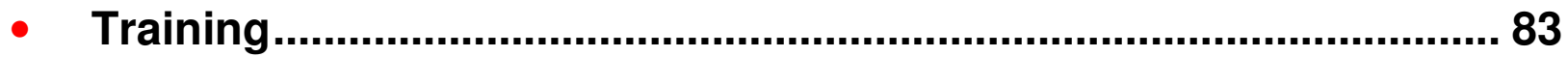

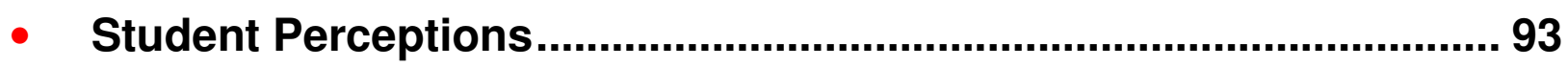




\section{Reported This Situation to a Military Authority or Organization Percent of Women Who Experienced Unwanted Sexual Contact}

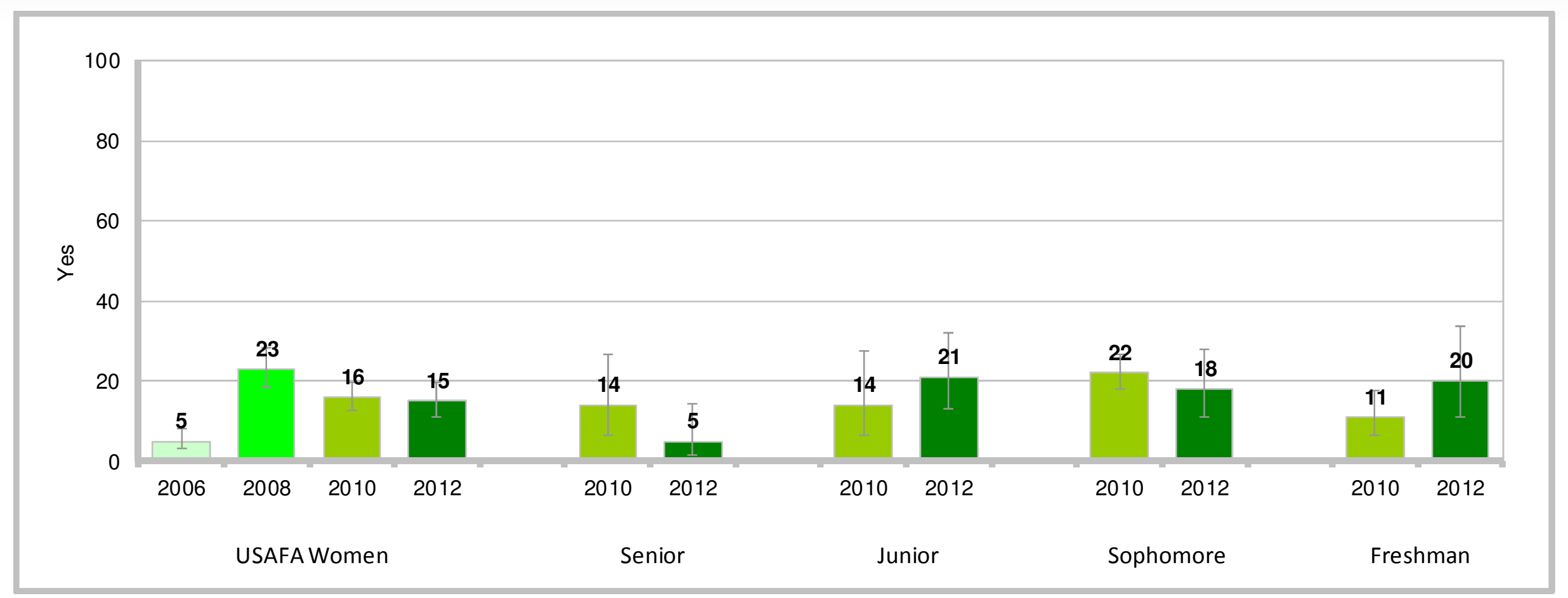

- Of the $11.2 \%$ of women who experienced USC, $15 \%$ indicated they reported the situation

- 2012 comparisons across years - lower than 2008; higher than 2006

- Class differences in 2012 - lower response led by seniors 


\section{Type of Report Made to a Military Authority}

Percent of Women Who Experienced Unwanted Sexual Contact and Reported It

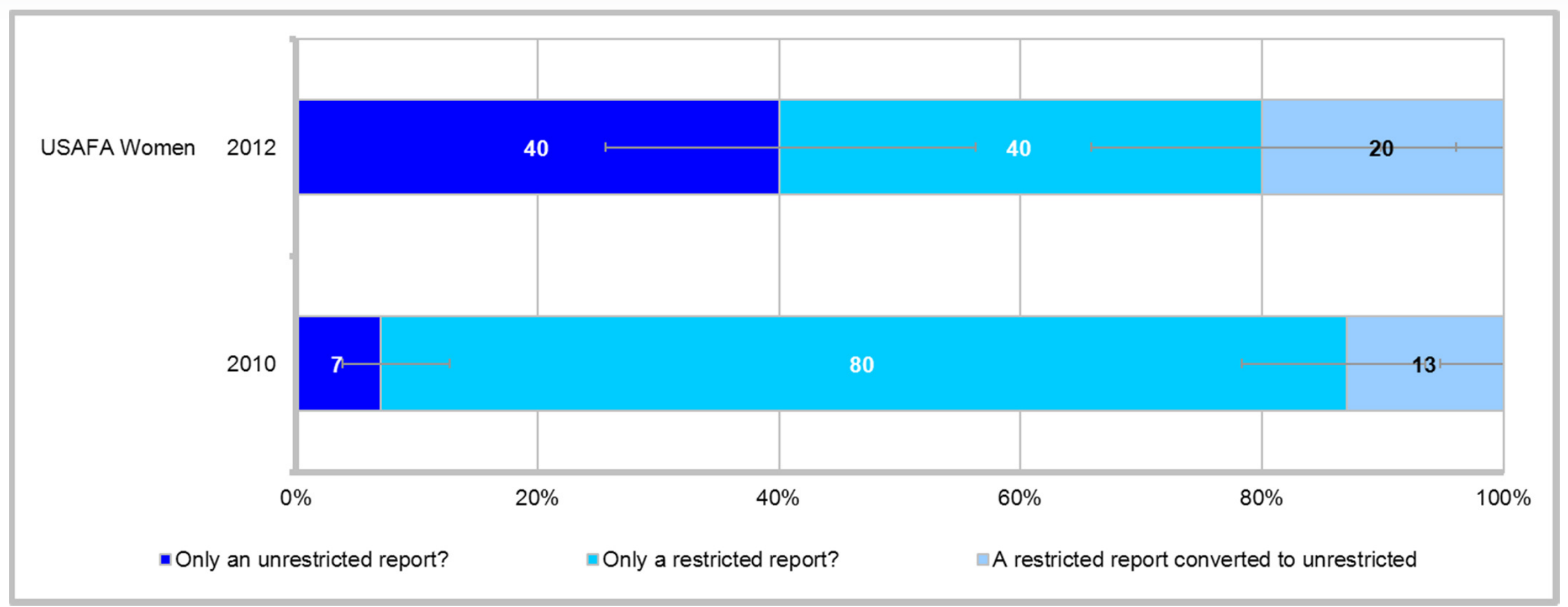

- Of the $15 \%$ of women who reported the situation, $40 \%$ indicated they made only an unrestricted report; $40 \%$ made only a restricted report; and $20 \%$ made a restricted report converted to unrestricted

- 2012 comparisons across years - only an unrestricted report higher than 2010; only an unrestricted report lower than 2010

- Results by class year not reportable 


\section{When Report Made to a Military Authority}

Percent of Women Who Experienced Unwanted Sexual Contact and Reported It

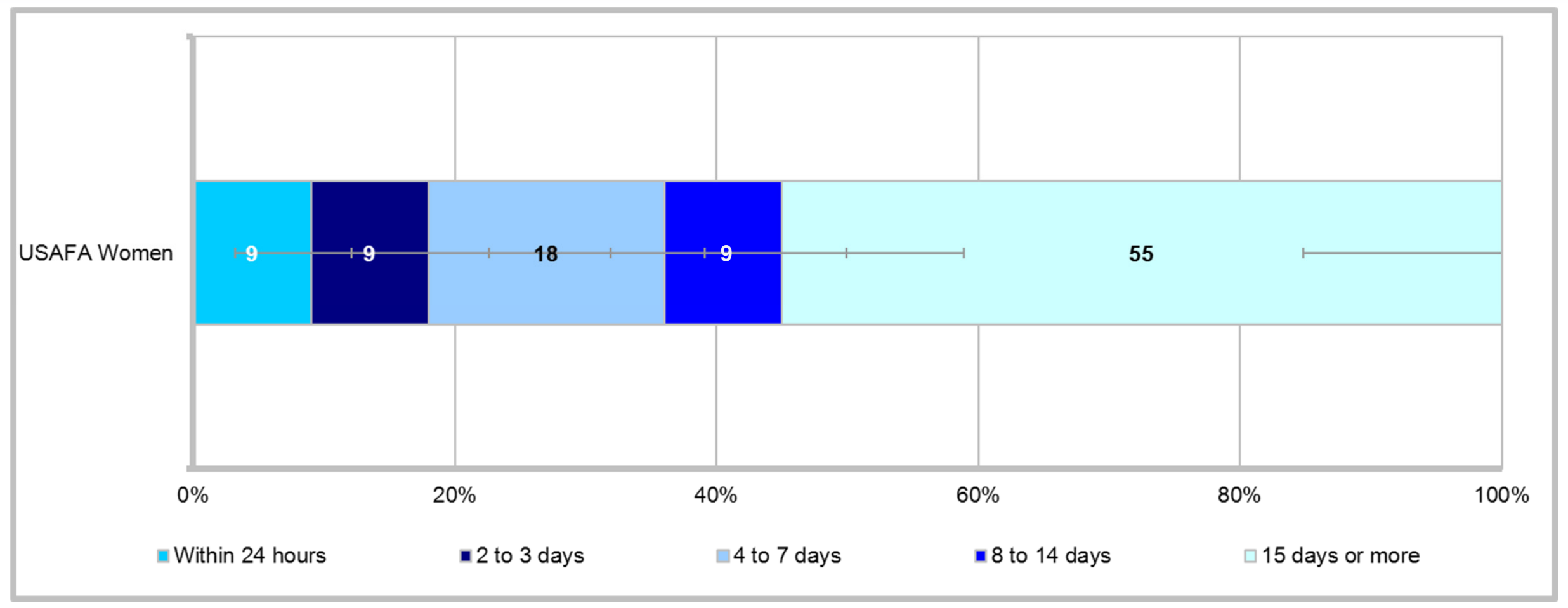

- Of the $15 \%$ of women who reported the situation, $9 \%$ indicated they made their report within 24 hours; $9 \%$ within 2 to 3 days; $18 \%$ within 4 to 7 days; $9 \%$ within 8 to 14 days; and $55 \%$ after 15 days or more

- 2012 comparisons across years - no differences

- Results by class year not reportable 


\section{Reason for Delay in Reporting the Situation}

Percent of Women Who Experienced Unwanted Sexual Contact and Waited at Least 24 Hours to Report It

\begin{tabular}{|l|c|}
\hline \multicolumn{1}{|c|}{ Reasons for Delay in Reporting } & Total \\
\hline $\begin{array}{l}\text { Wanted to think about the situation before } \\
\text { deciding to report }\end{array}$ & 59 \\
\hline $\begin{array}{l}\text { Did not realize at first that the situation was a } \\
\text { crime }\end{array}$ & 50 \\
\hline $\begin{array}{l}\text { Wanted to seek advice first from a friend or } \\
\text { family member }\end{array}$ & 40 \\
\hline Had to figure out how to report & 30 \\
\hline Margins of Error & $\pm 16-17 \%$ \\
\hline
\end{tabular}

- New question in 2012; no trend data available

- Results by class year not reportable 


\section{Most Frequently Selected Reasons for Reporting Unwanted Sexual Contact Situation Percent of Women Who Experienced Unwanted Sexual Contact and Reported It}

\begin{tabular}{|l|c|}
\hline \multicolumn{1}{|c|}{ Reasons for Reporting } & Total \\
\hline Seek help dealing with an emotional incident & 82 \\
\hline Stop the offender from hurting others & 74 \\
\hline Seek justice & 64 \\
\hline It was the right thing to do & 64 \\
\hline Stop the offender from hurting you again & 54 \\
\hline Seek closure on the incident & 54 \\
\hline Margins of Error & $\pm 15-16 \%$ \\
\hline
\end{tabular}

- New question in 2012; no trend data available

- Results by class year not reportable 


\section{Most Frequently Selected Reasons for} Not Reporting Unwanted Sexual Contact Situation Percent of Women Who Experienced Unwanted Sexual Contact and Did Not Report It

\begin{tabular}{|c|c|c|c|c|c|c|}
\hline Reasons for Not Reporting & $\begin{array}{c}\text { Survey } \\
\text { Year }\end{array}$ & Total & Senior & Junior & Sophomore & Freshman \\
\hline \multirow{2}{*}{ Took care of it myself } & 2012 & 66 & 78 & 73 & $47 \downarrow$ & $62 \downarrow$ \\
\hline & 2010 & 69 & 64 & 58 & 69 & 81 \\
\hline \multirow{2}{*}{ Did not want anyone to know } & 2012 & 63 & 61 & $53 \downarrow$ & 65 & $75 \uparrow$ \\
\hline & 2010 & 60 & 64 & 75 & 59 & 50 \\
\hline \multirow{2}{*}{ Did not want people gossiping } & 2012 & 62 & $50 \downarrow$ & 60 & 71 & 75 \\
\hline & 2010 & 67 & 82 & 67 & 59 & 69 \\
\hline \multirow{2}{*}{$\begin{array}{l}\text { Thought it was not important enough to } \\
\text { report }\end{array}$} & 2012 & 59 & 50 & 80 & $47 \downarrow$ & 62 \\
\hline & 2010 & 67 & 64 & 67 & 66 & 75 \\
\hline \multirow{2}{*}{ Felt shame/embarrassment } & 2012 & 59 & 61 & $33 \downarrow$ & $71 \uparrow$ & $75 \uparrow$ \\
\hline & 2010 & 51 & 64 & 58 & 48 & 44 \\
\hline \multirow{2}{*}{ Felt uncomfortable making a report } & 2012 & 56 & 56 & 47 & 65 & 50 \\
\hline & 2010 & 56 & 64 & 50 & 62 & 50 \\
\hline \multirow{2}{*}{ Did not want to hurt the offender's career } & 2012 & $46 \uparrow$ & $50 \uparrow$ & 47 & 35 & $50 \uparrow$ \\
\hline & 2010 & 30 & 27 & 33 & 38 & 19 \\
\hline Margins of Error & & $\pm 6-7 \%$ & $\pm 13-17 \%$ & $\pm 13-16 \%$ & $\pm 6-13 \%$ & $\pm 9-16 \%$ \\
\hline
\end{tabular}

Higher Response of Yes

Lower Response of Yes

Higher in 2012 than 2010

Lower in 2012 than 2010 


\section{Would Make the Same Decision About Reporting or Not Reporting if Could Do It Over}

Percent of Women Who Experienced Unwanted Sexual Contact and Reported or Did Not Report to a Military Authority

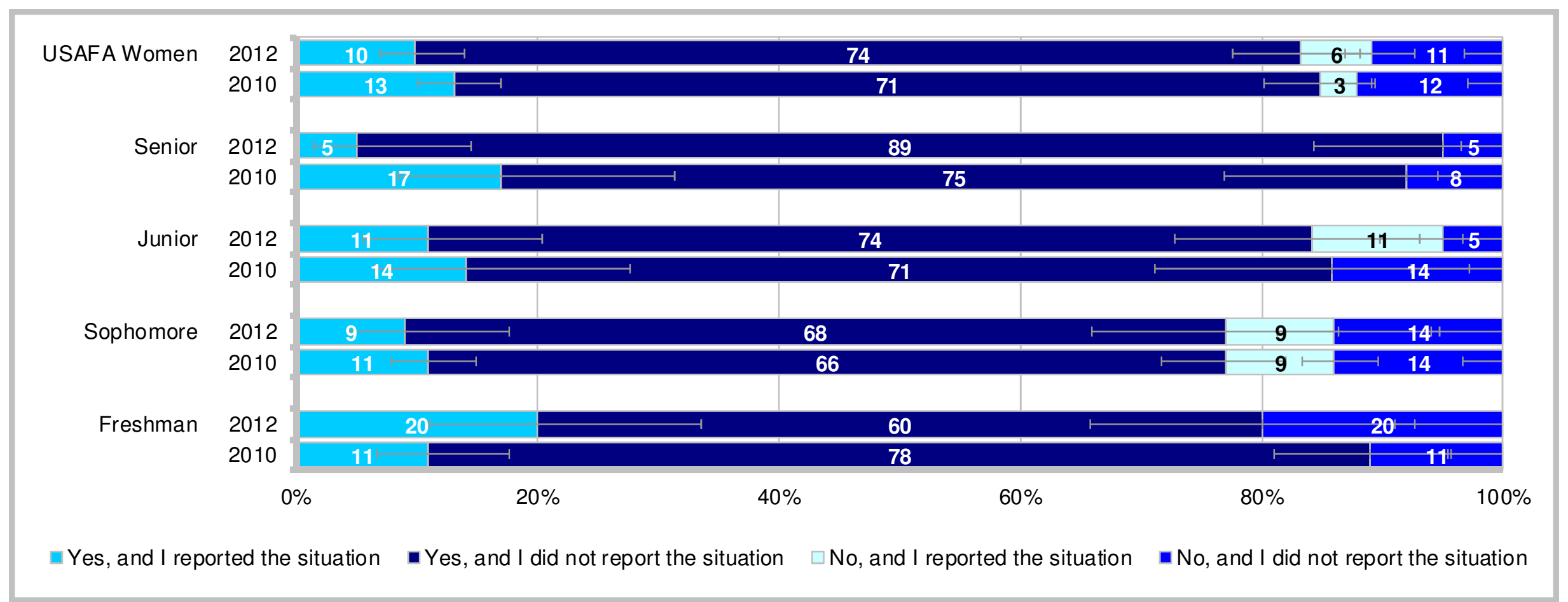

- Of the $11.2 \%$ of women who experienced USC, $10 \%$ indicated they reported the situation and would make the same decision again; $74 \%$ indicated they did not report the situation and would make the same decision again; $6 \%$ indicated they reported but would not make the same decision again; and $11 \%$ indicated they did not report the situation but would not make the same decision again

- 2012 comparisons across years - no differences

- Freshmen indicating did not report the situation and would make the same decision again in 2012 lower than 2010

- Class differences in 2012 - did not report the situation and would make the same decision led by seniors 


\section{Contents}

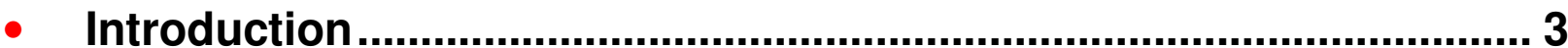

- Unwanted Sexual Contact ................................................................... 7

- Most Serious Behaviors Experienced........................................... 11

- Location of Incident ....................................................................... 13

- Summer Experience..................................................................... 15

- Characteristics of Offender....................................................... 17

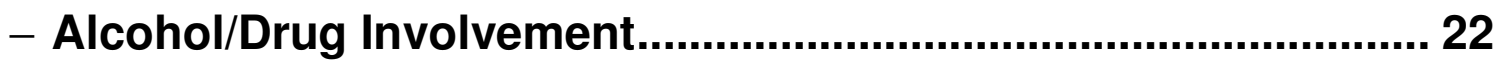

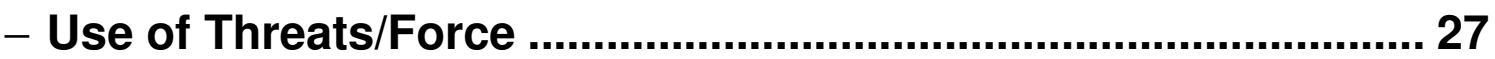

- Experience of Sexual Harassment/Stalking ............................... 32

- Consequences of the Situation ...................................................... 35

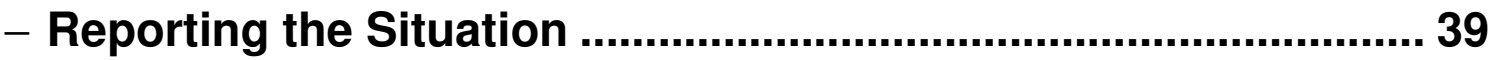

$\checkmark$ Experience of Unwanted Sexual Contact Prior to Entering the Academy .................................................................................... 47

- Unwanted Gender-Related Behaviors .............................................. 49

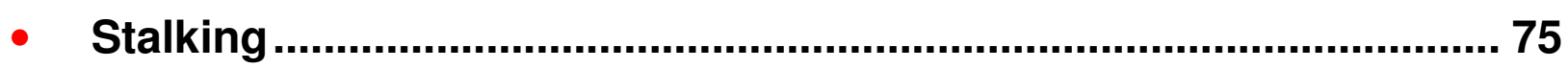

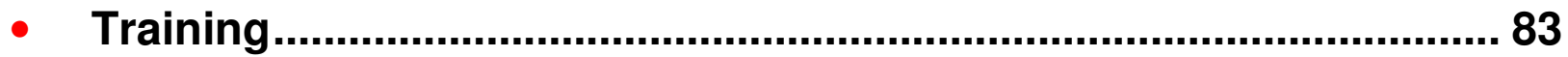

- Student Perceptions................................................................... 93 


\section{Unwanted Sexual Contact Incident Rate Prior to Entering the Academy} Percent of All Students

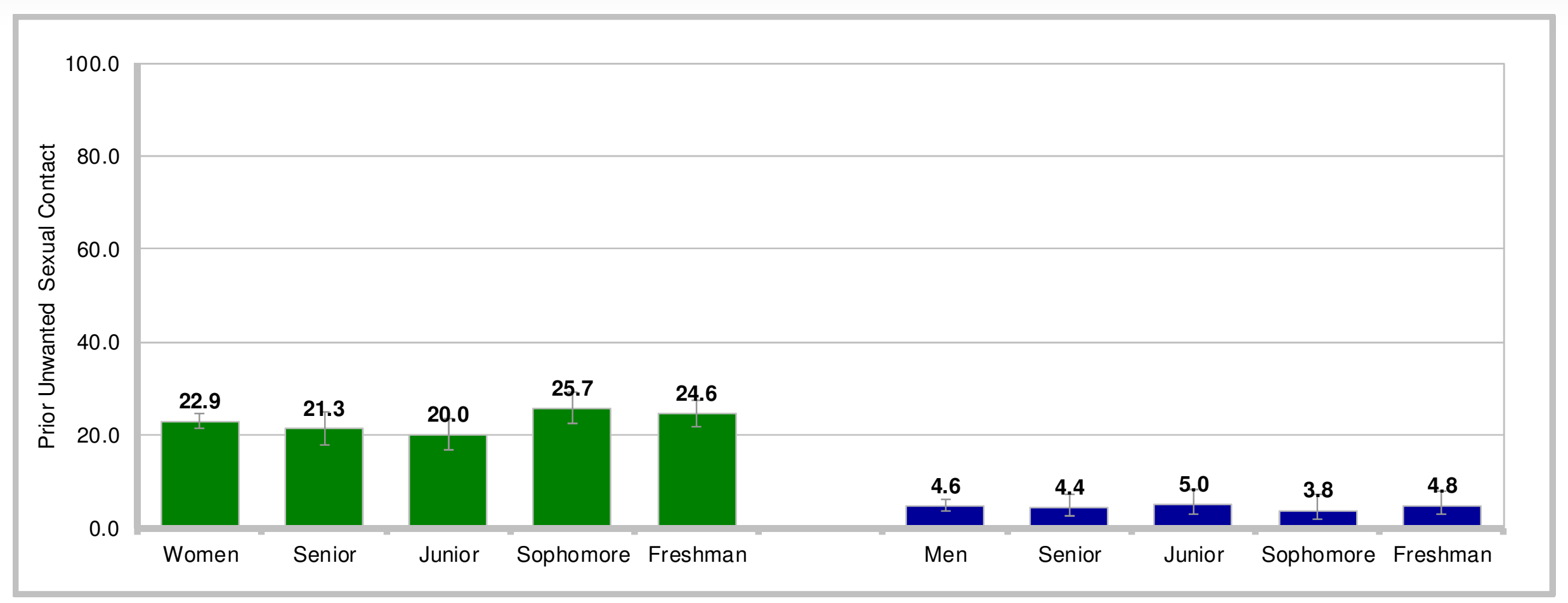

- $22.9 \%$ of women and $4.6 \%$ of men indicated experiencing unwanted sexual contact prior to entering the Academy

- 2012 comparisons across years - new question in 2012; no trend data available

- Class differences in 2012 - no differences 


\section{Contents}

Slide

- Introduction ................................................................................ 3

- Unwanted Sexual Contact ........................................................... 7

$\checkmark \quad$ Unwanted Gender-Related Behaviors ........................................... 49

- Sexual Harassment ............................................................... 51

- Categories of Behaviors Associated with Sexual Harassment.................................................................................. 54

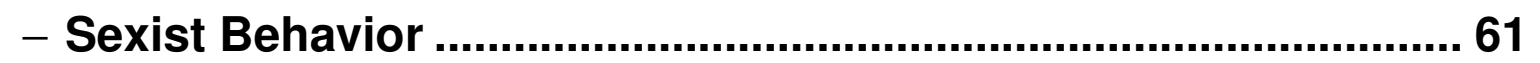

- Organizational Affiliation of Offender ..................................... 64

- Discussing/Reporting Experiences............................................6 67

- Response to Reporting.......................................................... 70

- Reasons for Not Reporting ................................................. 72

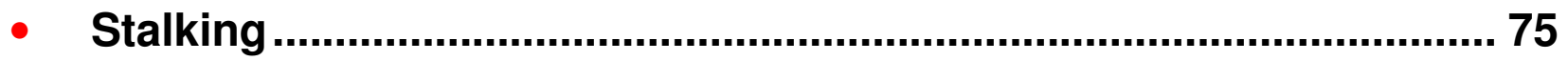

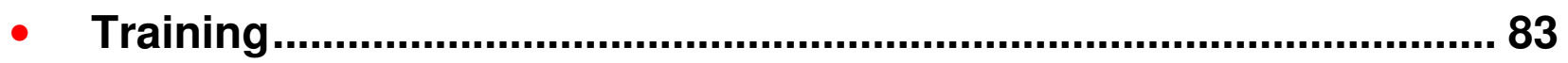

- Student Perceptions ...................................................................... 93 


\section{Unwanted Gender-Related Behaviors:}

\section{Sexual Harassment and Sexist Behavior Incident Rates}

\section{- Definition and measure of sexual harassment and sexist behavior:}

- DoD defines sexual harassment as "a form of sex discrimination that involves unwelcome sexual advances, requests for sexual favors, and other verbal or physical conduct of a sexual nature when:

- Submission to such conduct is made either explicitly or implicitly a term or condition of a person's job, pay, or career, or

- Submission to or rejection of such conduct by a person is used as a basis for career or employment decisions affecting that person, or

- Such conduct has the purpose or effect of unreasonably interfering with an individual's work performance or creates an intimidating, hostile, or offensive working environment" (Department of Defense, 1995).

- The core measure of unwanted gender-related behaviors on the 2012 SAGR consists of 17 items

- Sexual harassment measure has 12 items in a three-factor structure and a single "labeling" item

- Crude/Offensive Behavior - four items regarding offensive or embarrassing verbal/nonverbal behaviors of a sexual nature

- Unwanted Sexual Attention - four items regarding unwanted attempts to establish a sexual relationship

- Sexual Coercion - four items regarding classic quid pro quo instances of special treatment or favoritism conditioned on sexual cooperation

- One "labeling" item that asks if the student considers any of the selected behaviors to be sexual harassment

- Sexist behavior measure has four items that include verbal/nonverbal behaviors that convey insulting, offensive, and/or condescending attitudes based on gender of student

- Write-in comments analyzed separately

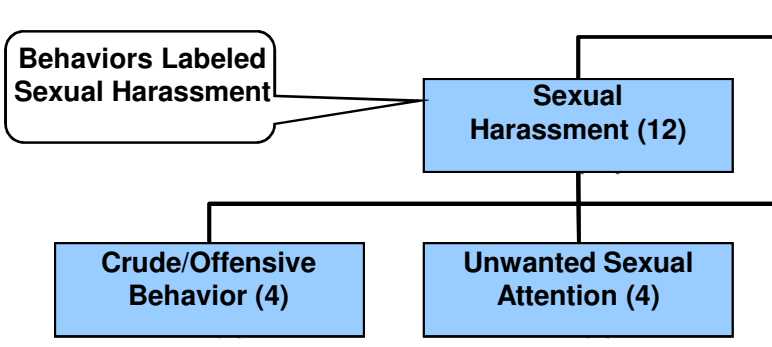

Write-in Comment (1)

Sexual Coercion (4) 


\section{Contents}

- Introduction 3

- Unwanted Sexual Contact ................................................................... 7

- Unwanted Gender-Related Behaviors .............................................. 49

$\checkmark$ Sexual Harassment ................................................................. 51

- Categories of Behaviors Associated with Sexual Harassment.................................................................................... 55

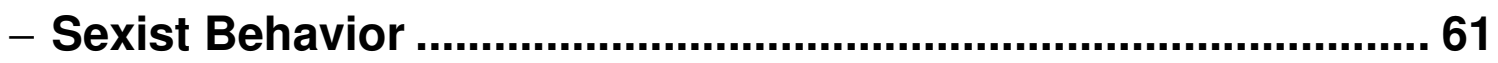

- Organizational Affiliation of Offender ........................................ 64

- Discussing/Reporting Experiences............................................. 67

- Response to Reporting.............................................................. 70

- Reasons for Not Reporting ............................................................. 72

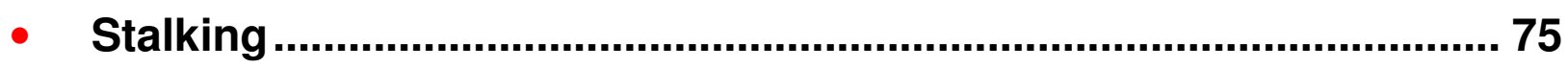

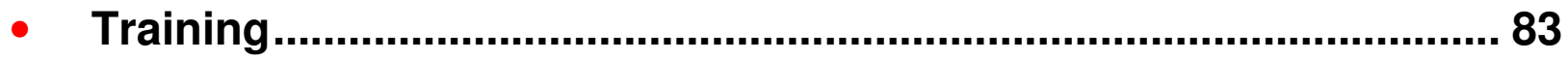

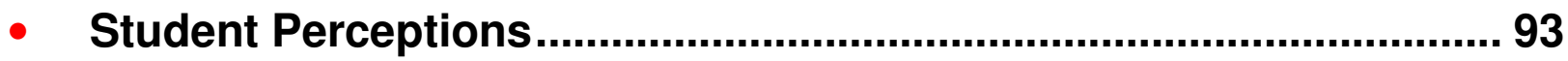




\section{Sexual Harassment Incident Rate Percent of Women}

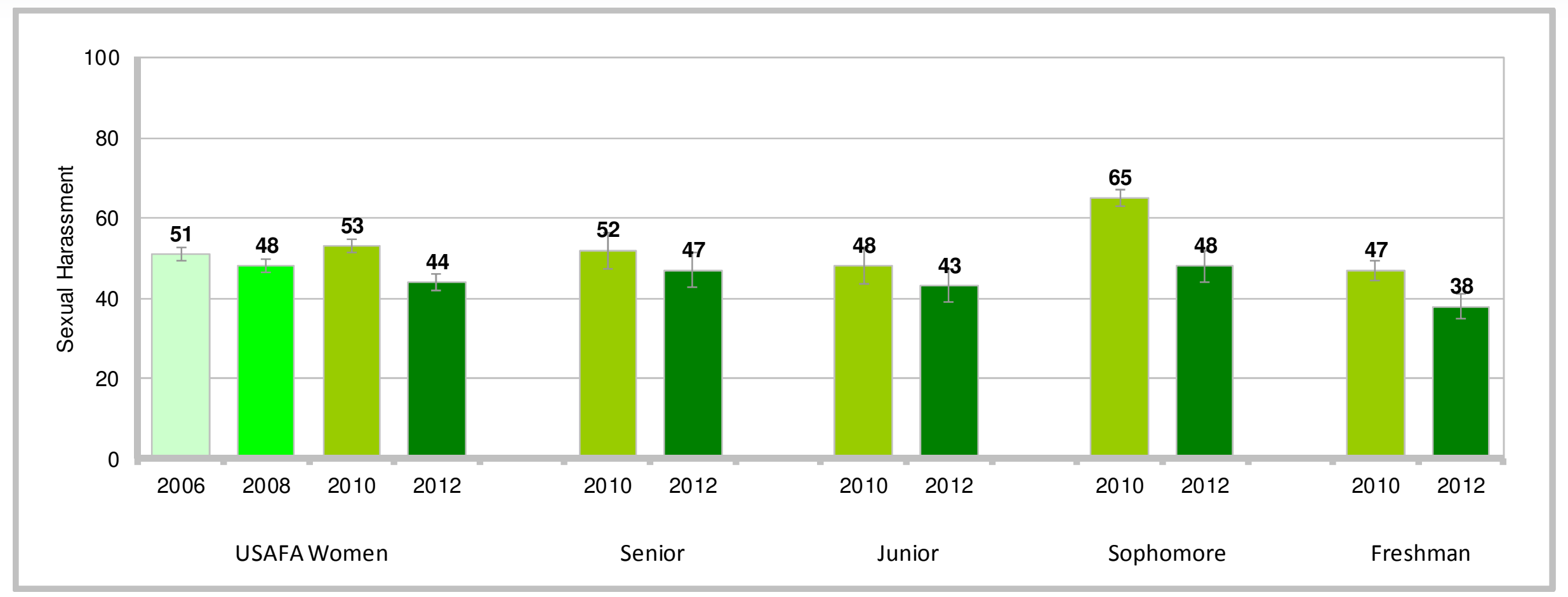

- $44 \%$ of women indicated experiencing sexual harassment in 2012

- 2012 comparisons across years - lower than 2010, 2008, 2006

- Sophomores and freshmen in 2012 lower than 2010

- Class differences in $\mathbf{2 0 1 2}$ - lower response led by freshmen 


\section{Sexual Harassment Incident Rate}

Percent of Men

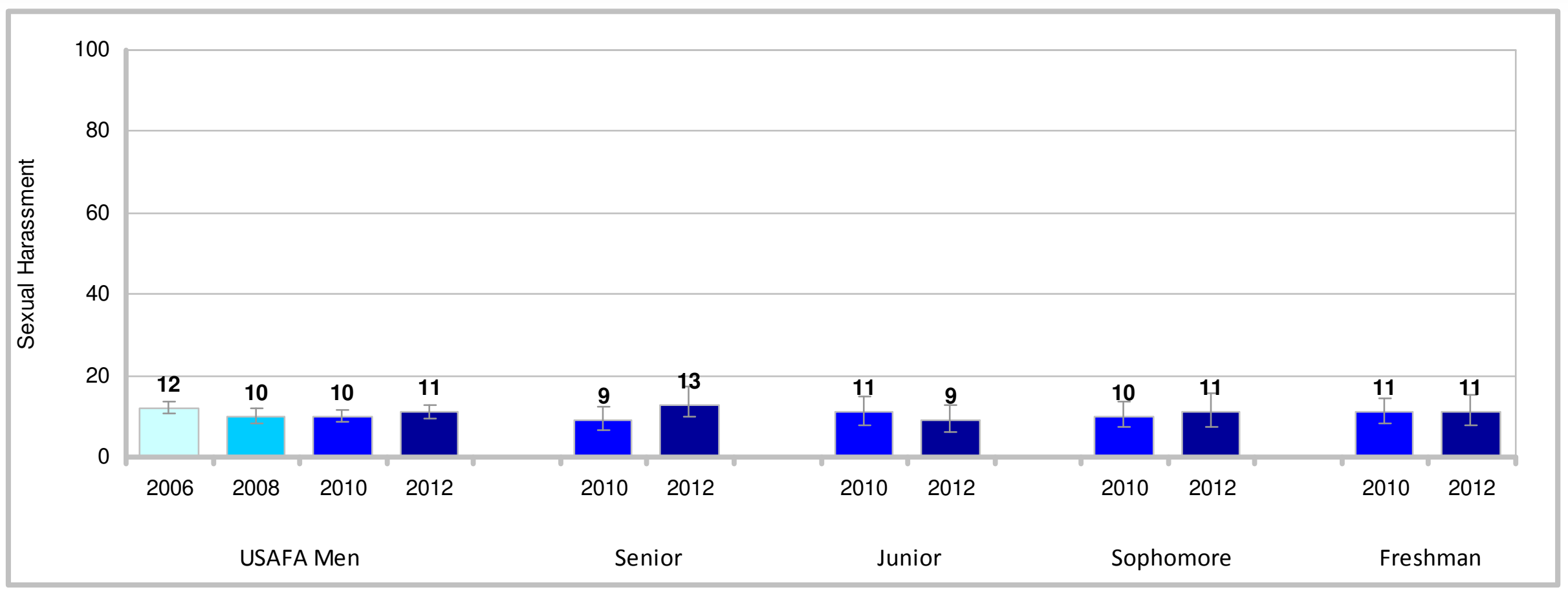

- $11 \%$ of men indicated experiencing sexual harassment in 2012

- No differences by survey year or class year 


\section{Contents}

Slide

- Introduction ................................................................................. 3

- Unwanted Sexual Contact ........................................................... 7

- Unwanted Gender-Related Behaviors ......................................... 49

- Sexual Harassment .................................................................. 51

$\checkmark$ Categories of Behaviors Associated with Sexual Harassment.................................................................................. 54

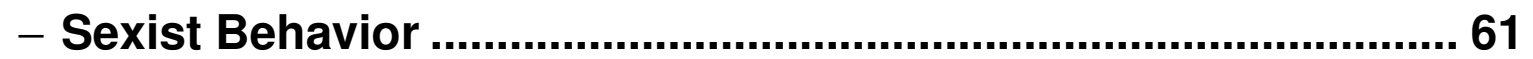

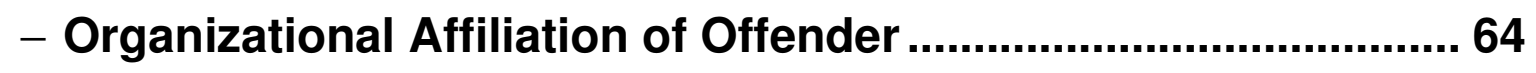

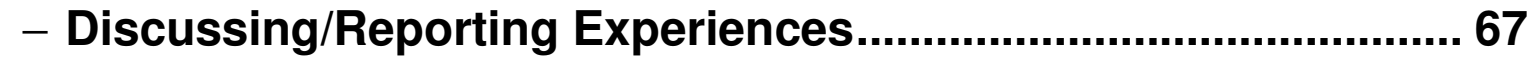

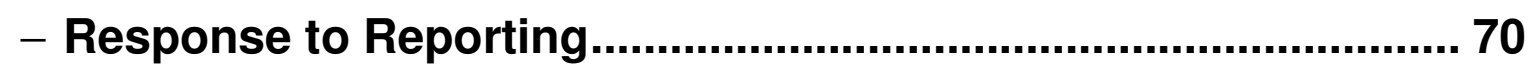

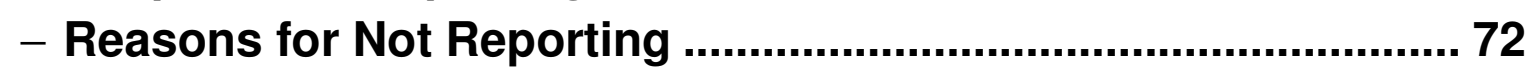

• Stalking .......................................................................................... 75

• Training ........................................................................................... 83

- Student Perceptions.................................................................. 93 


\section{Crude/Offensive Behavior Incident Rate Percent of Women}

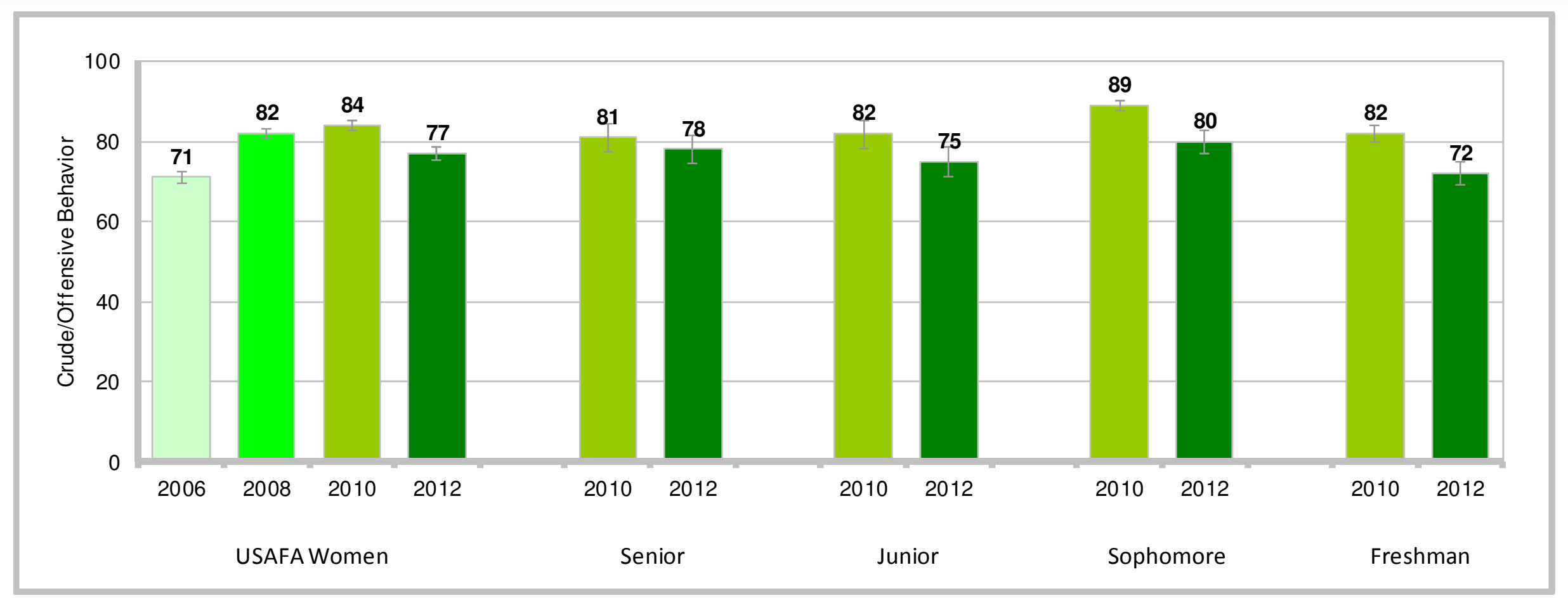

- $77 \%$ of women indicated experiencing crude/offensive behavior in 2012

- 2012 comparisons across years - lower than 2010, 2008; higher than 2006

- Juniors, sophomores, and freshmen in 2012 lower than 2010

- Class differences in 2012 - higher response led by sophomores; lower response led by freshmen 


\section{Crude/Offensive Behavior Incident Rate} Percent of Men

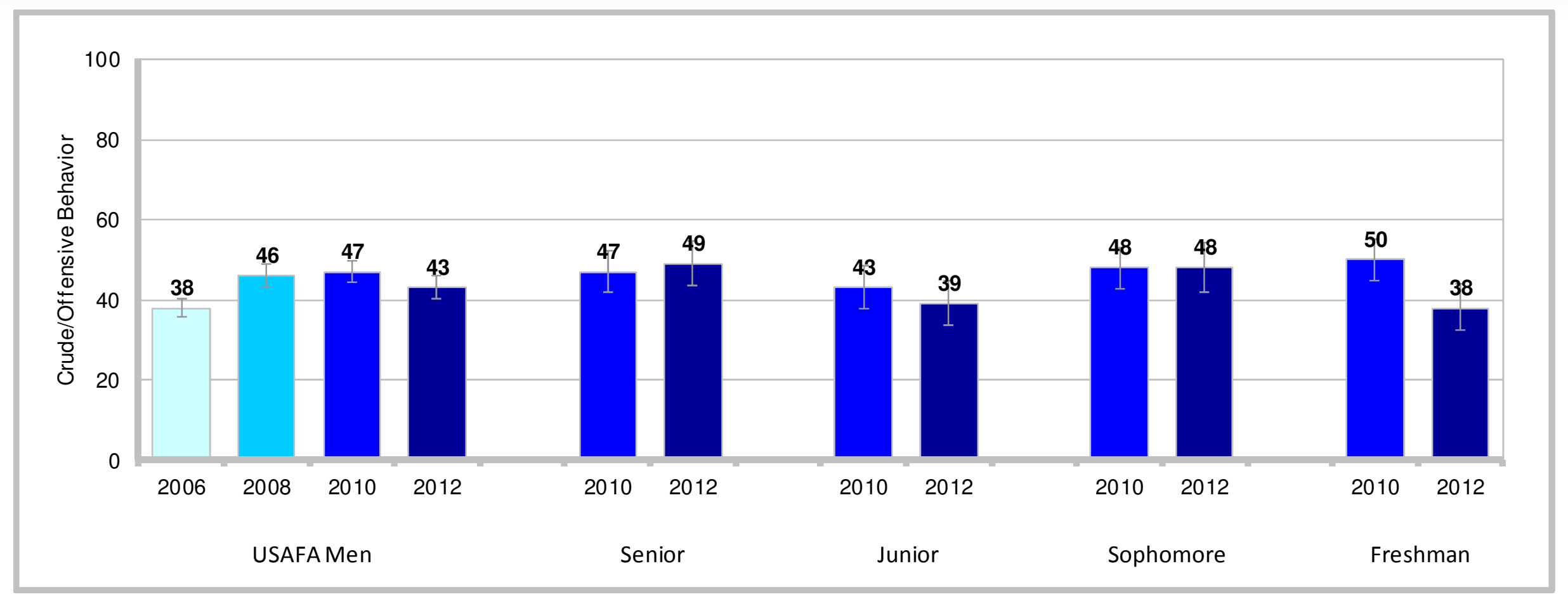

- $43 \%$ of men indicated experiencing crude/offensive behavior in 2012

- 2012 comparisons across years - lower than 2010; higher than 2006

- Freshmen in 2012 lower than 2010

- Class differences in 2012 - no differences 


\section{Unwanted Sexual Attention Incident Rate Percent of Women}

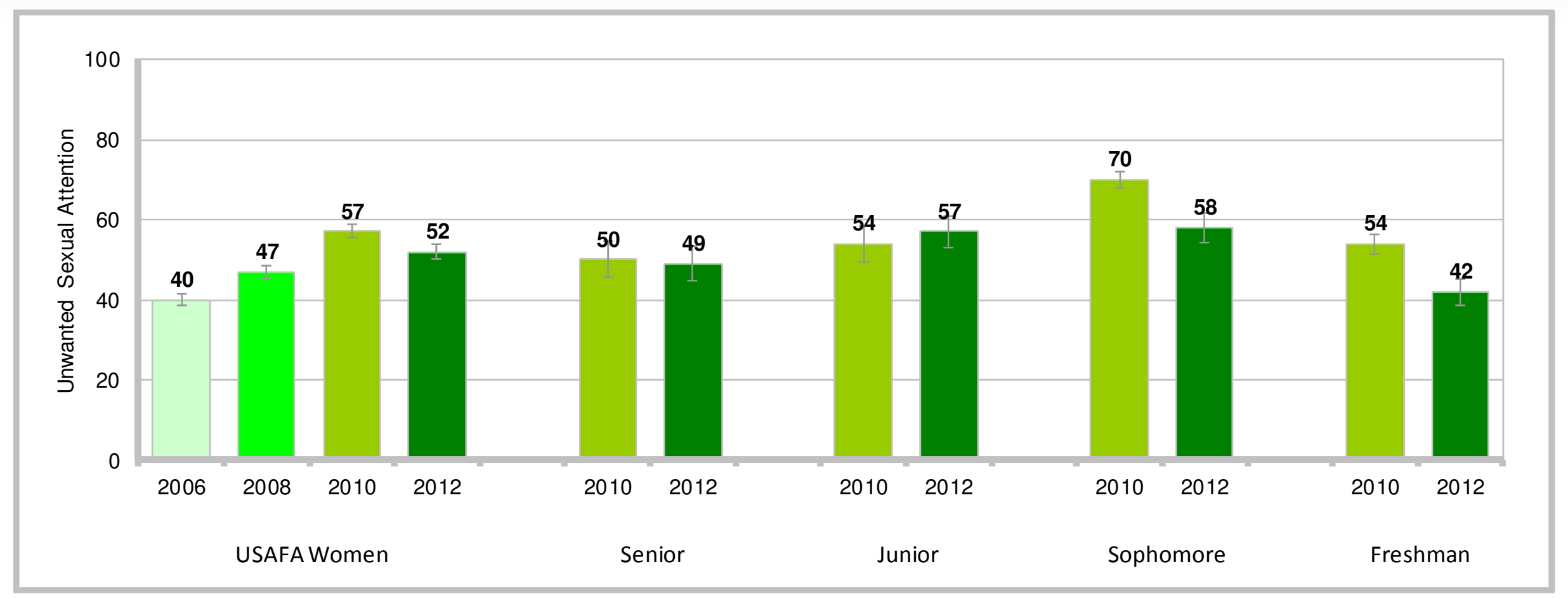

- 52\% of women indicated experiencing unwanted sexual attention in 2012

- 2012 comparisons across years - lower than 2010; higher than 2008, 2006

- Sophomores and freshmen in 2012 lower than 2010

- Class differences in 2012 - higher response led by juniors and sophomores; lower response led by freshmen 


\section{Unwanted Sexual Attention Incident Rate Percent of Men}

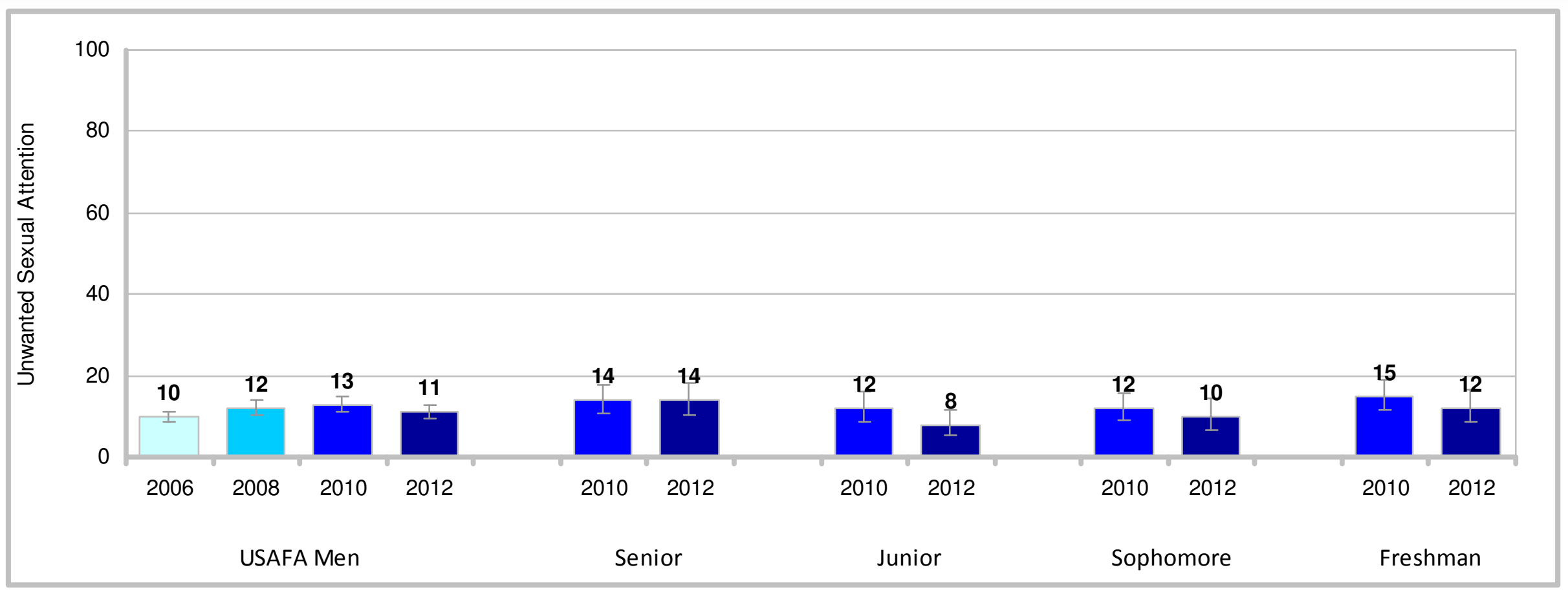

- $11 \%$ of men indicated experiencing unwanted sexual attention in 2012

- 2012 comparisons across years - no differences

- Class differences in 2012 - no differences 


\section{Sexual Coercion Incident Rate}

Percent of Women

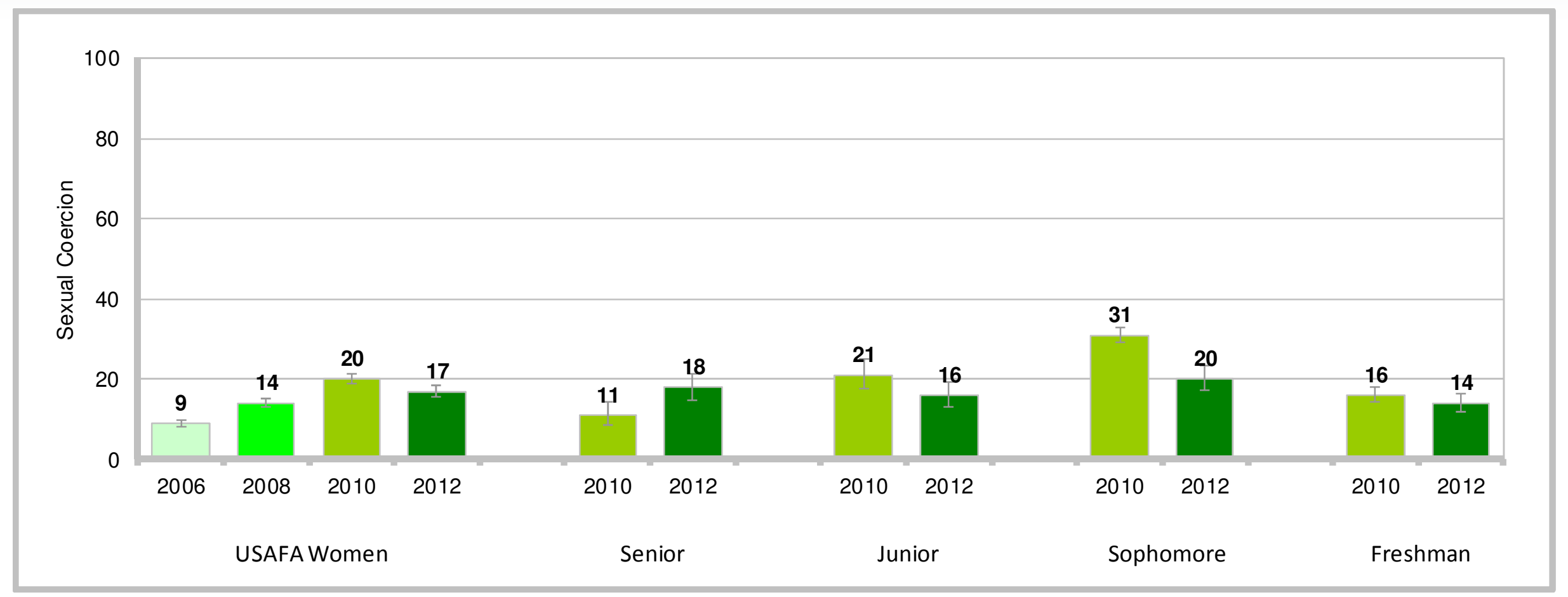

- $17 \%$ of women indicated experiencing sexual coercion in 2012

- 2012 comparisons across years - lower than 2010; higher than 2008, 2006

- Juniors and sophomores in 2012 lower than 2010, whereas seniors higher

- Class differences in 2012 - no differences 


\section{Sexual Coercion Incident Rate}

Percent of Men

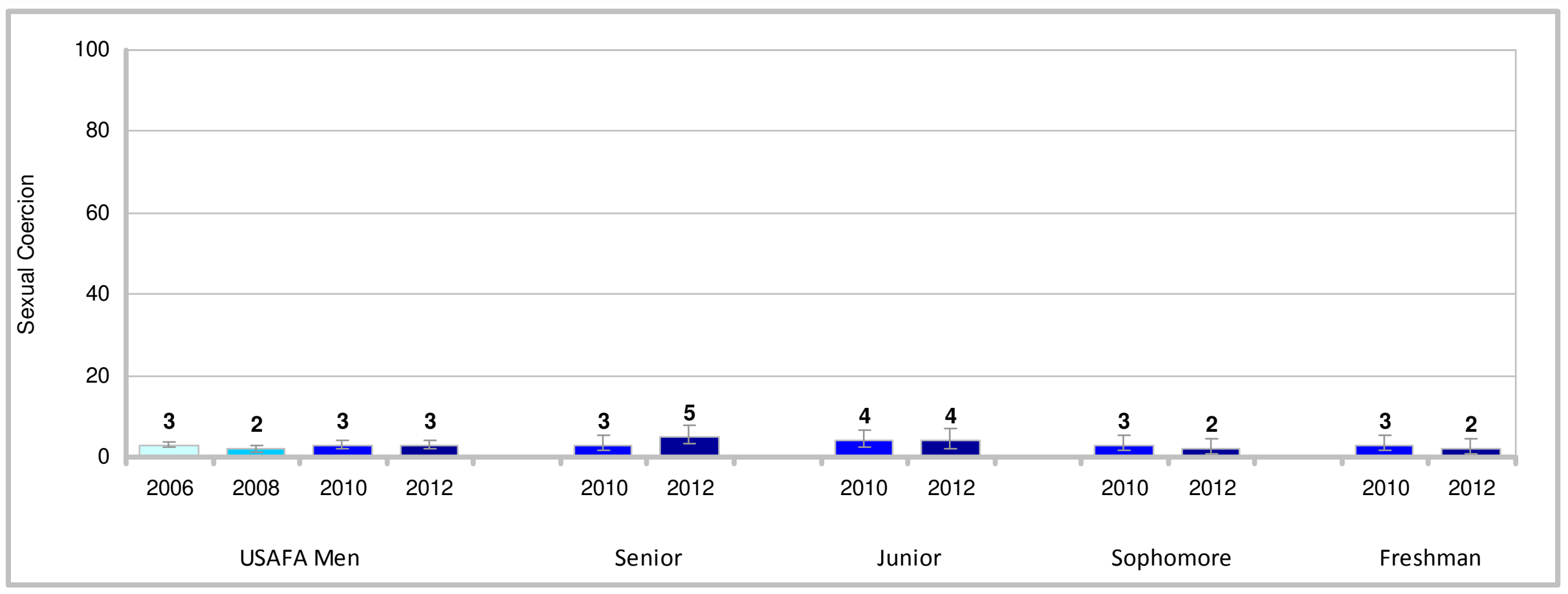

- $3 \%$ of men indicated experiencing sexual coercion in 2012

- 2012 comparisons across years - no differences

- Class differences in 2012 - no differences 


\section{Contents}

- Introduction 3

- Unwanted Sexual Contact ................................................................... 7

- Unwanted Gender-Related Behaviors ............................................... 49

- Sexual Harassment ...................................................................... 51

- Categories of Behaviors Associated with Sexual

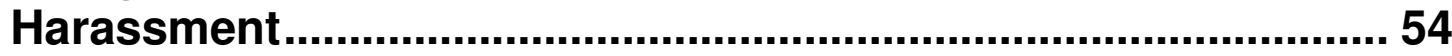

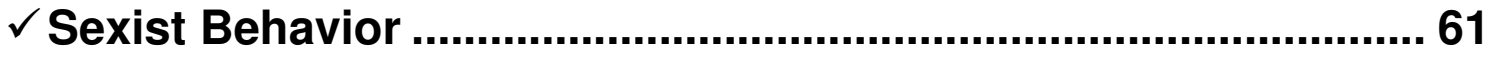

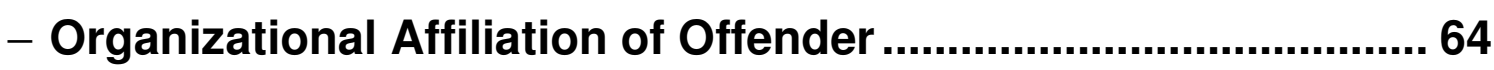

- Discussing/Reporting Experiences............................................. 67

- Response to Reporting.............................................................. 70

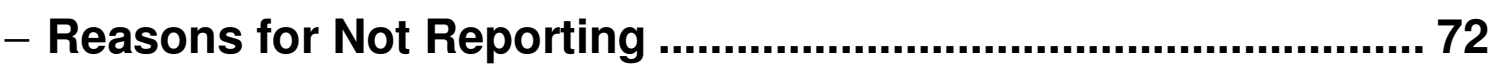

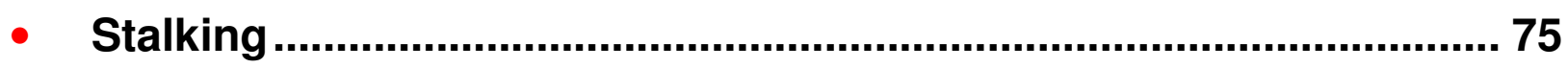

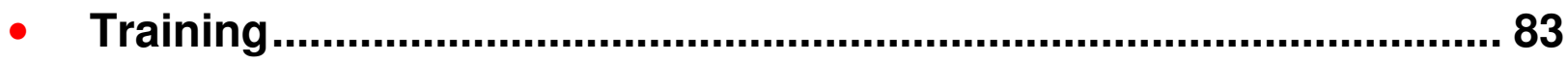

- Student Perceptions............................................................................ 93 


\section{Sexist Behavior Incident Rate Percent of Women}

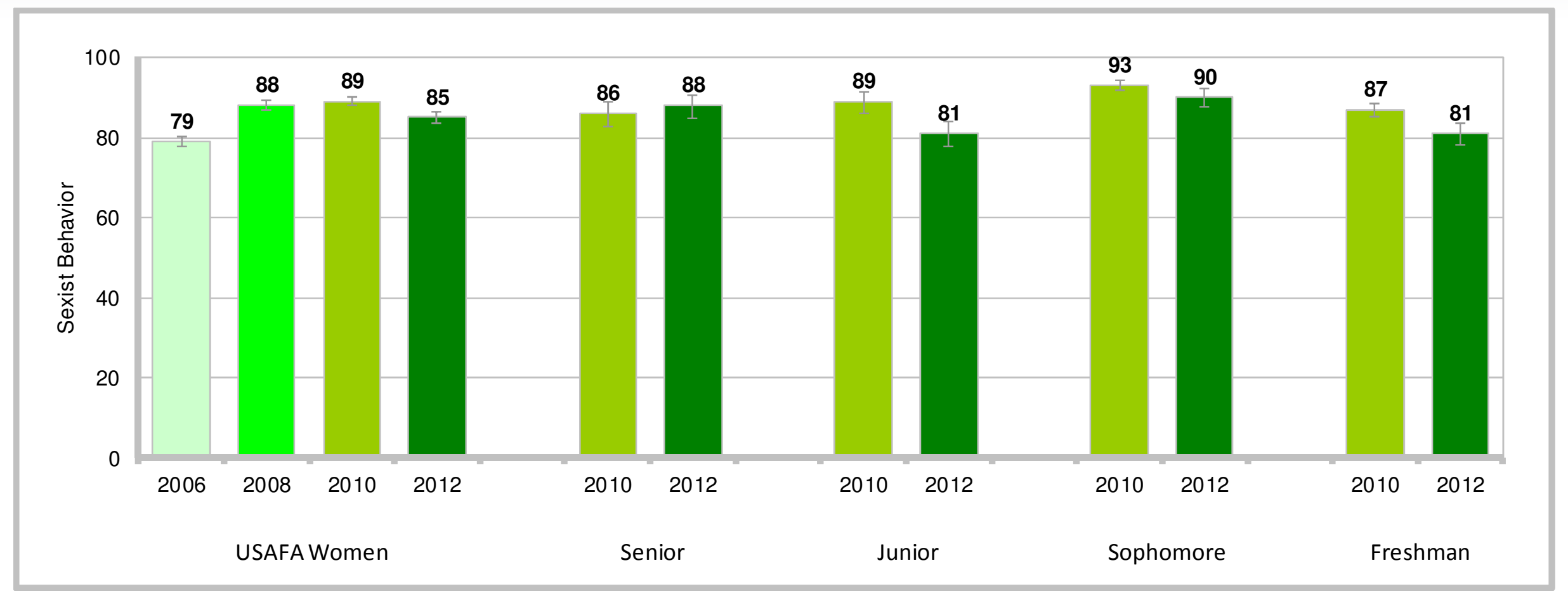

- $85 \%$ of women indicated experiencing sexist behavior in 2012

- 2012 comparisons across years - lower than 2010, 2008; higher than 2006

- Juniors, sophomores, and freshmen in 2012 lower than 2010

- Class differences in 2012 - higher response led by sophomores; lower response led by juniors and freshmen 


\section{Sexist Behavior Incident Rate}

Percent of Men

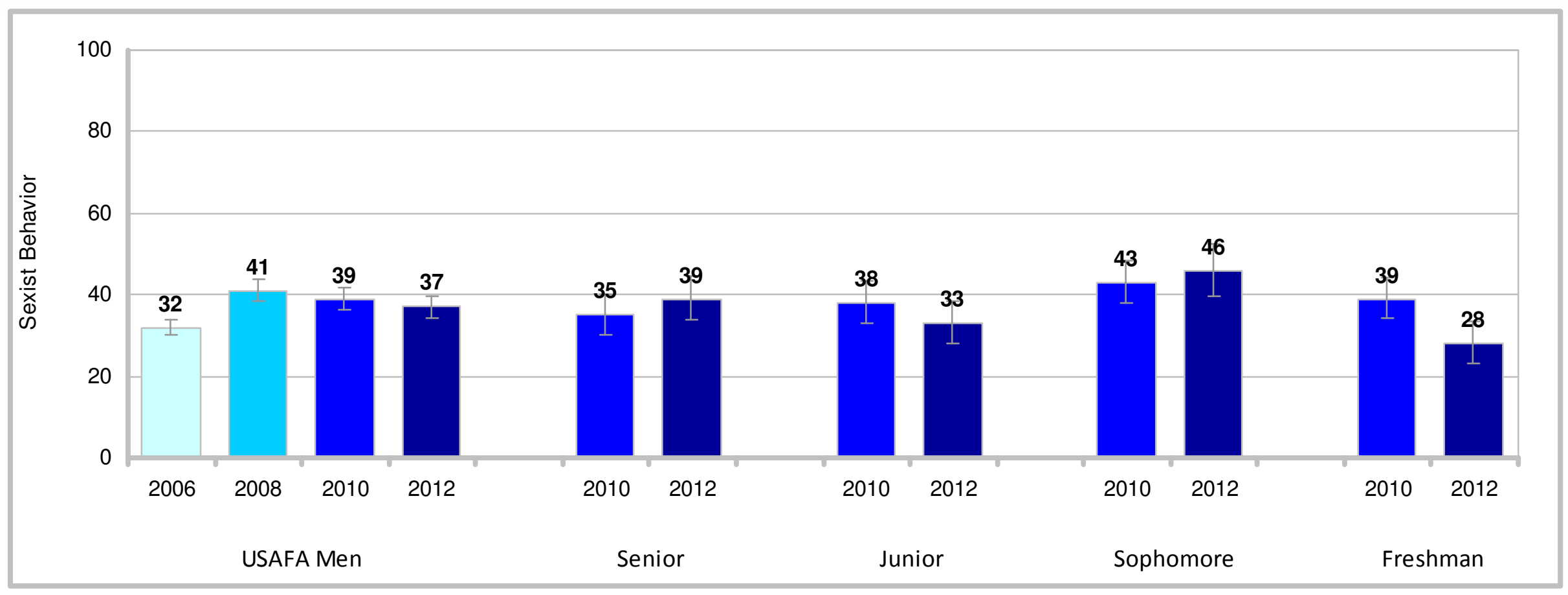

- $37 \%$ of men indicated experiencing sexist behavior in 2012

- 2012 comparisons across years - lower than 2008; higher than 2006

- Freshmen in 2012 lower than 2010

- Class differences in $\mathbf{2 0 1 2}$ - higher response led by sophomores; lower response led by freshmen 


\section{Contents}

- Introduction 3

- Unwanted Sexual Contact ................................................................... 7

- Unwanted Gender-Related Behaviors ............................................... 49

- Sexual Harassment ...................................................................... 51

- Categories of Behaviors Associated with Sexual Harassment.................................................................................... 54

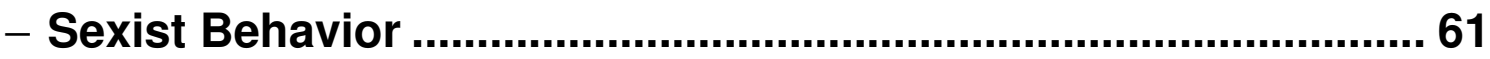

$\checkmark$ Organizational Affiliation of Offender .......................................64 64

- Discussing/Reporting Experiences............................................. 67

- Response to Reporting.............................................................. 70

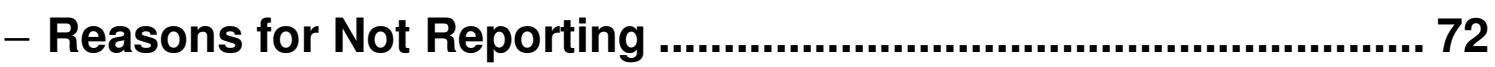

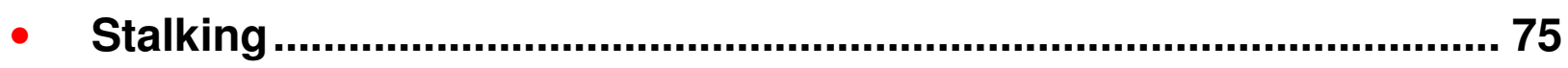

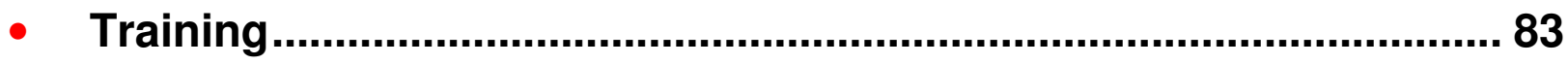

- Student Perceptions............................................................................ 93 


\section{Combinations of Offender Affiliations}

\section{Percent of Women Who Experienced Unwanted Gender-Related Behavior}

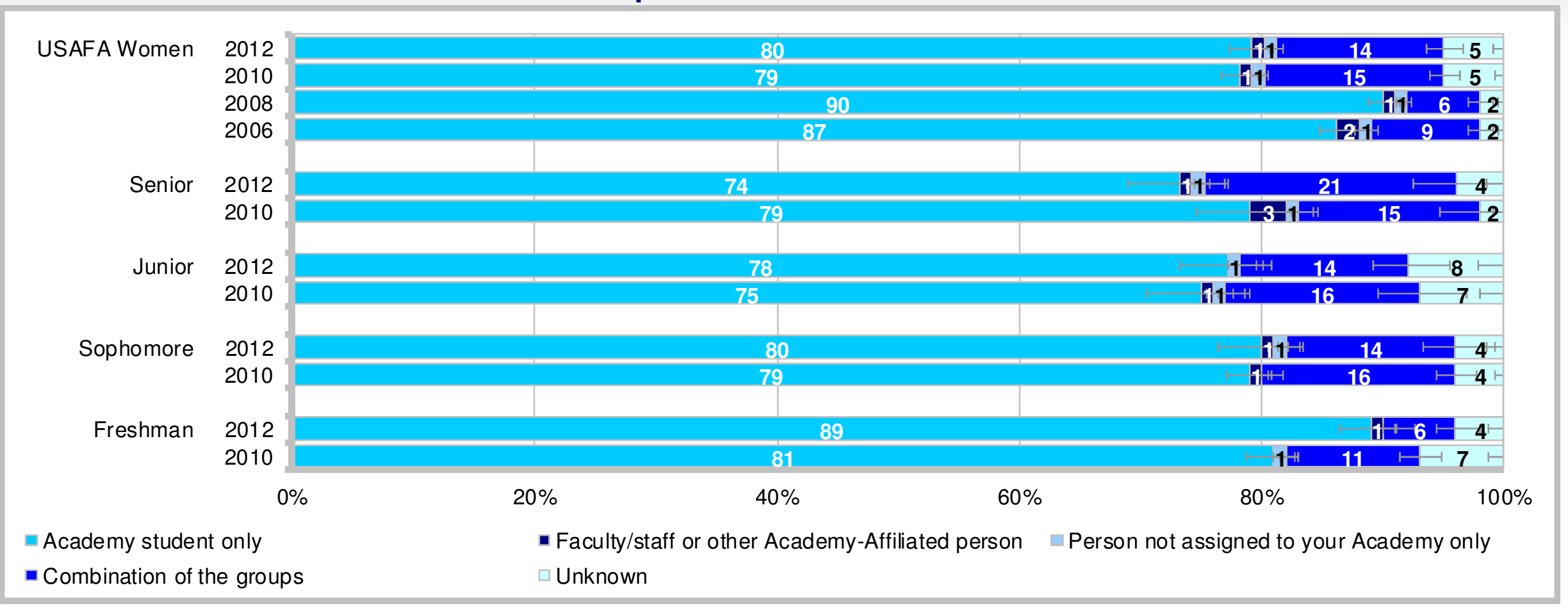

- $89 \%$ of women indicated experiencing one or more of the unwanted gender-related behaviors (i.e., crude/offensive behavior, unwanted sexual attention, sexual coercion, sexist behavior). Of those, $80 \%$ indicated the offender was an Academy student only; $1 \%$ indicated faculty/staff; $1 \%$ indicated person not assigned to the Academy; $14 \%$ indicated combination of groups; and 5\% indicated unknown.

- 2012 comparisons across years - Academy student only lower than 2008, 2006; combination of groups higher than 2008, 2006; unknown higher than 2008, 2006

- Freshmen indicating Academy student only in 2012 higher than 2010; seniors indicating faculty/staff in 2012 lower than 2010; seniors indicating combinations of groups in 2012 higher than 2010, whereas freshmen lower; freshmen indicating unknown in 2012 lower than 2010

- Class differences in 2012 - Academy student only led by freshmen; combination of groups led by seniors; unknown led by juniors 


\section{Combinations of Offender Affiliations Percent of Men Who Experienced Unwanted Gender-Related Behavior}

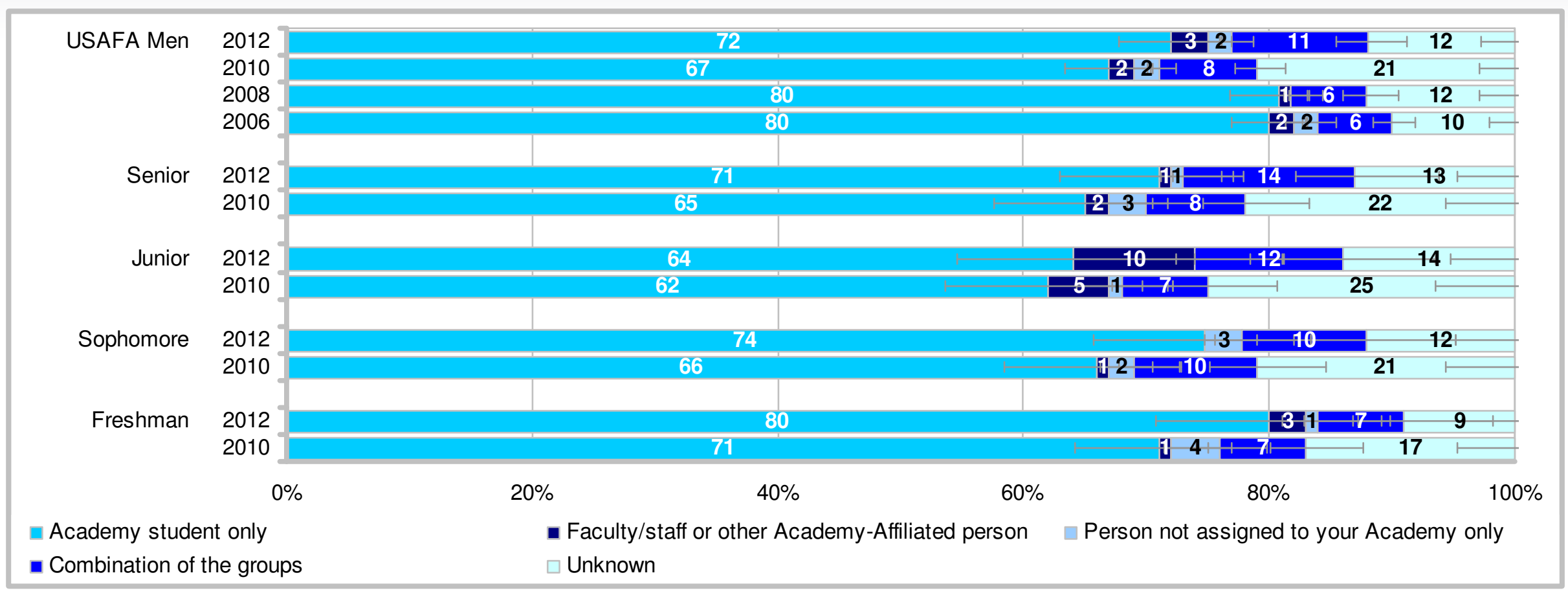

- $51 \%$ of men indicated experiencing one or more of the unwanted gender-related behaviors (i.e., crude/offensive behavior, unwanted sexual attention, sexual coercion, sexist behavior). Of those, $72 \%$ indicated the offender was Academy student only; $3 \%$ indicated faculty/staff; $2 \%$ indicated person not assigned to the Academy; $11 \%$ indicated combination of groups; and $12 \%$ indicated unknown.

- 2012 comparisons across years - Academy student only lower than 2008, 2006; faculty/staff higher than 2008; person not assigned to the Academy higher than 2008; combination of groups higher than 2010, 2008, 2006; unknown lower than 2010

- All class years indicating unknown in 2012 lower than 2010

- Class differences in 2012 - faculty/staff led by juniors 


\section{Contents}

- Introduction 3

- Unwanted Sexual Contact ................................................................... 7

- Unwanted Gender-Related Behaviors ............................................... 49

- Sexual Harassment ...................................................................... 51

- Categories of Behaviors Associated with Sexual Harassment...................................................................................... 54

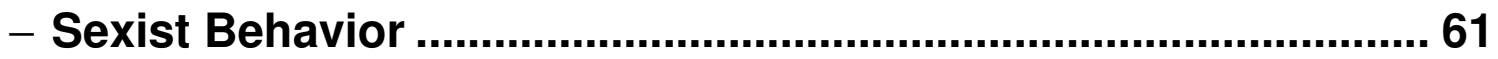

- Organizational Affiliation of Offender .........................................64 64

$\checkmark$ Discussing/Reporting Experiences...........................................6 67

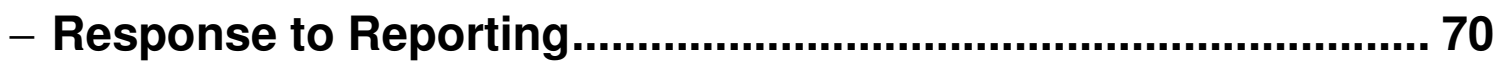

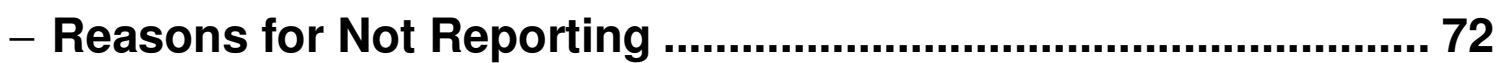

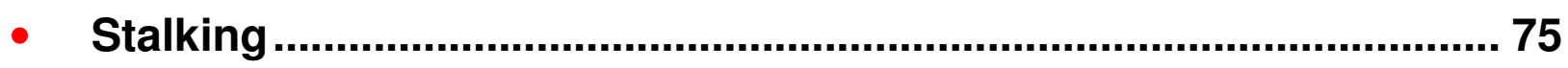

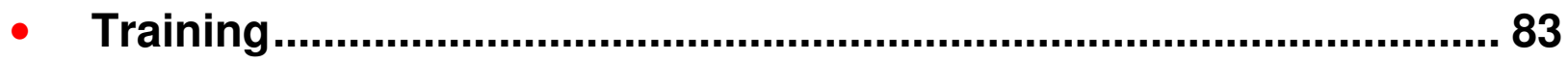

- Student Perceptions............................................................................ 93 


\section{Discuss/Report This Situation to an Authority or Organization}

Percent of Women Who Experienced Unwanted Gender-Related Behavior

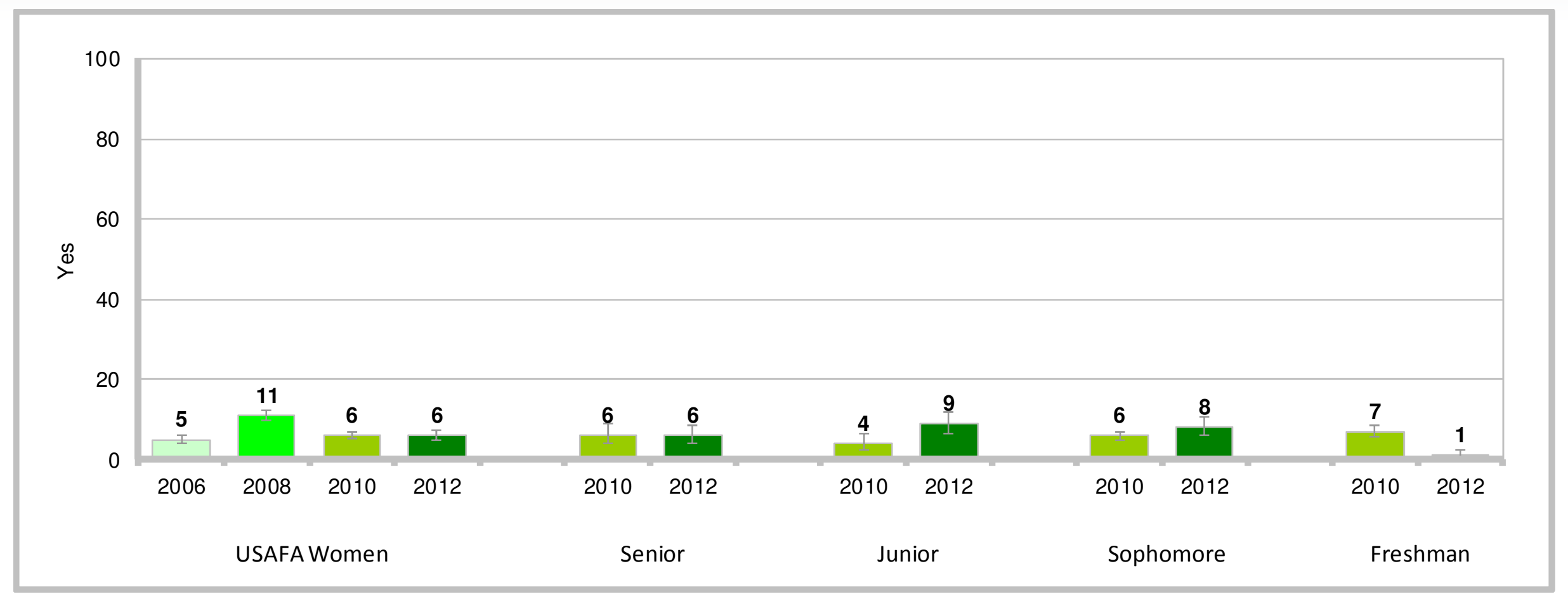

- $89 \%$ of women indicated experiencing one or more of the unwanted gender-related behaviors (i.e., crude/offensive behavior, unwanted sexual attention, sexual coercion, sexist behavior). Of those, $6 \%$ indicated they reported the situation to an authority or organization.

- 2012 comparisons across years - lower than 2008

- Juniors in 2012 higher than 2010, whereas freshmen lower

- Class differences in $\mathbf{2 0 1 2}$ - lower response led by freshmen 


\section{Discuss/Report This Situation to an Authority or Organization Percent of Men Who Experienced Unwanted Gender-Related Behavior}

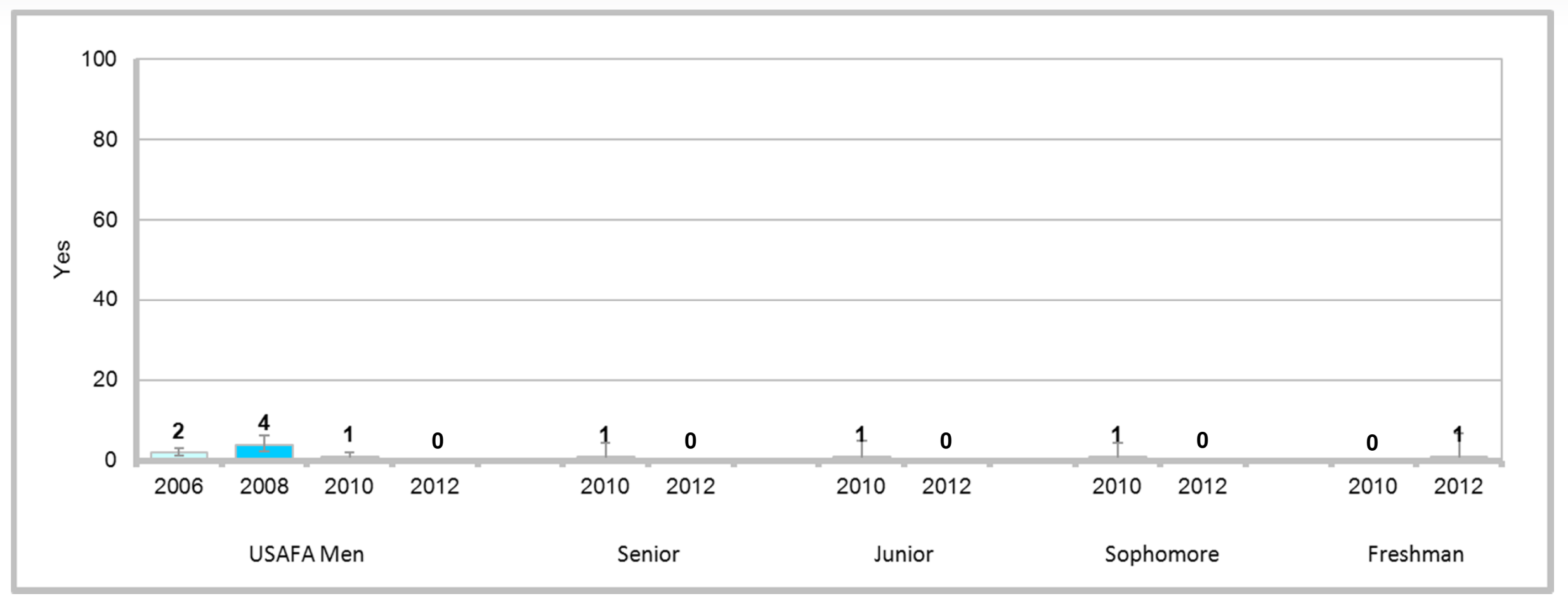

- $51 \%$ of men indicated experiencing one or more of the unwanted gender-related behaviors (i.e., crude/offensive behavior, unwanted sexual attention, sexual coercion, sexist behavior). Of those, $0 \%$ indicated they reported the situation to an authority or organization.

- 2012 comparisons across years - lower than 2008, 2006

- Class differences in 2012 - no differences 


\section{Contents}

- Introduction ..................................................................................... 3

- Unwanted Sexual Contact ...................................................................... 7

- Unwanted Gender-Related Behaviors .............................................. 49

- Sexual Harassment ..................................................................... 51

- Categories of Behaviors Associated with Sexual Harassment........................................................................................ 54

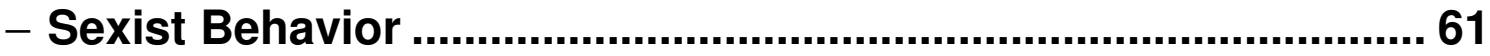

- Organizational Affiliation of Offender ...........................................64

- Discussing/Reporting Experiences...............................................67 67

$\checkmark$ Response to Reporting................................................................ 70

- Reasons for Not Reporting ........................................................... 72

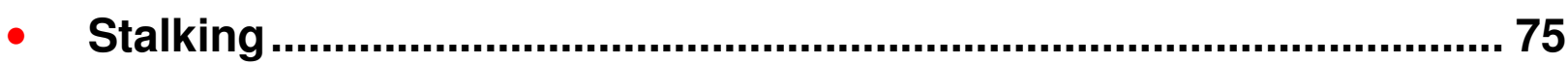

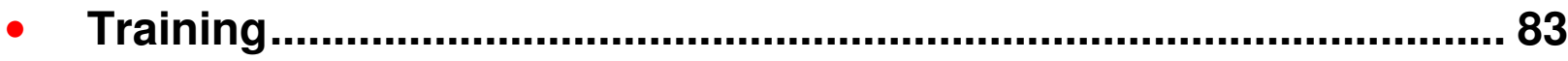

- Student Perceptions.......................................................................... 93 


\section{Actions Taken in Response to Discussing/Reporting}

Percent of Women Who Experienced Unwanted Gender-Related Behaviors and Reported It

\begin{tabular}{|c|c|c|c|c|c|c|}
\hline Response to Reporting & $\begin{array}{l}\text { Survey } \\
\text { Year }\end{array}$ & Total & Senior & Junior & Sophomore & Freshman \\
\hline \multirow{2}{*}{ The situation was corrected } & 2012 & 53 & $50 \uparrow$ & 55 & 58 & NR \\
\hline & 2010 & 48 & 14 & NR & 50 & 62 \\
\hline \multirow{2}{*}{ My situation was/is being investigated } & 2012 & $38 \uparrow$ & 25 & 18 & $58 \uparrow$ & NR \\
\hline & 2010 & 18 & NR & NR & 25 & 31 \\
\hline \multirow{2}{*}{$\begin{array}{l}\text { I was kept informed of what actions were } \\
\text { being taken }\end{array}$} & 2012 & 26 & NR & 18 & $50 \uparrow$ & NR \\
\hline & 2010 & 29 & 29 & NR & 25 & 38 \\
\hline \multirow{2}{*}{ I was encouraged to "tough it out" } & 2012 & 29 & $25 \downarrow$ & 18 & $33 \downarrow$ & NR \\
\hline & 2010 & 38 & 57 & NR & 50 & 23 \\
\hline \multirow{2}{*}{$\begin{array}{l}\text { My situation was discounted or not taken } \\
\text { seriously }\end{array}$} & 2012 & 18 & 25 & 18 & 8 & NR \\
\hline & 2010 & 11 & 14 & NR & 8 & 15 \\
\hline \multirow{2}{*}{ Action was taken against me } & 2012 & $3 \downarrow$ & 0 & 0 & $0 \downarrow$ & NR \\
\hline & 2010 & 8 & NR & NR & 8 & 15 \\
\hline \multirow{2}{*}{$\begin{array}{l}\text { I was ridiculed or scorned by others for } \\
\text { discussing/reporting the situation }\end{array}$} & 2012 & 26 & $12 \downarrow$ & 9 & $42 \uparrow$ & NR \\
\hline & 2010 & 22 & 43 & NR & 25 & 15 \\
\hline \multirow{2}{*}{ Some other action was taken } & 2012 & 26 & $12 \downarrow$ & 27 & 17 & NR \\
\hline & 2010 & 30 & 43 & $\mathrm{NR}$ & 25 & 31 \\
\hline Margins of Error & & $\pm 4-10 \%$ & $\pm 0-21 \%$ & $\pm 0-16 \%$ & $\pm 0-16 \%$ & $\pm 9-11 \%$ \\
\hline
\end{tabular}

\section{- Results for men not reportable}

\begin{tabular}{|l|c|}
\hline Higher Response of Yes & \\
\hline Lower Response of Yes & \\
\hline Higher in 2012 than 2010 & $\uparrow$ \\
\hline Lower in 2012 than 2010 & $\downarrow$ \\
\hline
\end{tabular}




\section{Contents}

- Introduction 3

- Unwanted Sexual Contact ................................................................... 7

- Unwanted Gender-Related Behaviors ............................................... 49

- Sexual Harassment ...................................................................... 51

- Categories of Behaviors Associated with Sexual Harassment...................................................................................... 54

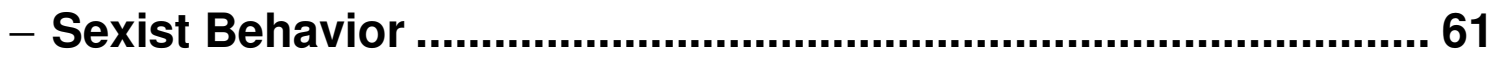

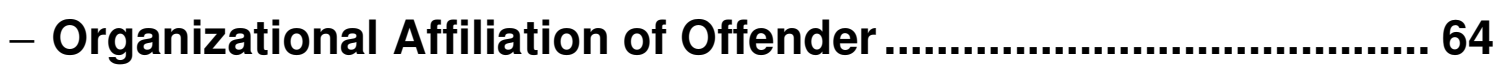

- Discussing/Reporting Experiences............................................. 67

- Response to Reporting.................................................................... 70

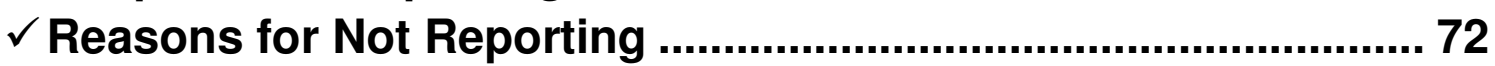

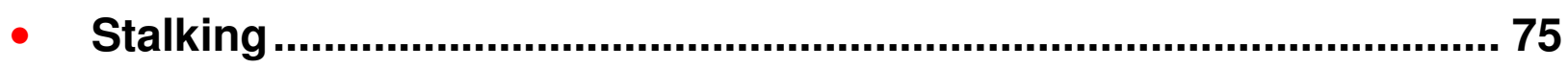

- Training.................................................................................................. 83

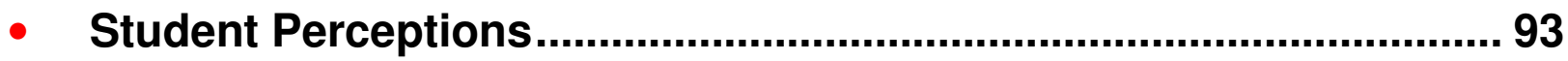




\section{Most Frequently Selected Reasons for}

\section{Not Reporting Unwanted Gender-Related Situation}

Percent of Women Who Experienced Unwanted Gender-Related Behaviors and Did Not Report It

\begin{tabular}{|c|c|c|c|c|c|c|}
\hline Reasons for Not Reporting & $\begin{array}{l}\text { Survey } \\
\text { Year }\end{array}$ & Total & Senior & Junior & Sophomore & Freshman \\
\hline \multirow{2}{*}{$\begin{array}{l}\text { Thought it was not important enough to } \\
\text { report }\end{array}$} & 2012 & 81 & $71 \downarrow$ & 84 & 83 & 86 \\
\hline & 2010 & 83 & 82 & 79 & 85 & 86 \\
\hline \multirow{2}{*}{ Took care of the problem myself } & 2012 & $70 \downarrow$ & $70 \downarrow$ & 74 & 70 & 69 \\
\hline & 2010 & 73 & 82 & 78 & 68 & 69 \\
\hline \multirow{2}{*}{ Did not want people gossiping about me } & 2012 & 35 & 36 & 35 & $33 \downarrow$ & 35 \\
\hline & 2010 & 36 & 31 & 35 & 42 & 32 \\
\hline \multirow{2}{*}{$\begin{array}{l}\text { Thought reporting would take too much } \\
\text { time and effort }\end{array}$} & 2012 & 28 & 31 & 29 & 26 & 27 \\
\hline & 2010 & 28 & 26 & 28 & 30 & 28 \\
\hline \multirow{2}{*}{ Felt uncomfortable making a report } & 2012 & 24 & 28 & 19 & $24 \downarrow$ & $27 \uparrow$ \\
\hline & 2010 & 25 & 27 & 21 & 33 & 20 \\
\hline Margins of Error & & $\pm 2-3 \%$ & $\pm 5-6 \%$ & $\pm 4-5 \%$ & $\pm 2-5 \%$ & $\pm 3-4 \%$ \\
\hline
\end{tabular}

\begin{tabular}{|l|c|}
\hline Higher Response of Yes & \\
\hline Lower Response of Yes & \\
\hline Higher in 2012 than 2010 & $\uparrow$ \\
\hline Lower in 2012 than 2010 & $\downarrow$ \\
\hline
\end{tabular}




\section{Most Frequently Selected Reasons for}

\section{Not Reporting Unwanted Gender-Related Situation}

\section{Percent of Men Who Experienced Unwanted Gender-Related Behaviors and Did Not Report It}

\begin{tabular}{|l|c|c|c|c|c|c|}
\hline \multicolumn{1}{|c|}{ Reasons for Not Reporting } & $\begin{array}{c}\text { Survey } \\
\text { Year }\end{array}$ & Total & Senior & Junior & Sophomore & Freshman \\
\hline \multirow{2}{*}{$\begin{array}{l}\text { Thought it was not important enough to } \\
\text { report }\end{array}$} & 2012 & $84 \uparrow$ & 83 & 80 & 86 & $92 \uparrow$ \\
\cline { 2 - 7 } & 2010 & 78 & 80 & 70 & 79 & 81 \\
\hline \multirow{2}{*}{ Took care of the problem myself } & 2012 & 63 & 68 & 51 & 67 & 63 \\
\cline { 2 - 7 } & 2010 & 61 & 63 & 62 & 60 & 61 \\
\hline \multirow{2}{*}{$\begin{array}{l}\text { Thought reporting would take too much } \\
\text { time and effort }\end{array}$} & 2012 & 22 & 24 & 22 & 20 & 21 \\
\cline { 2 - 7 } & 2010 & 19 & 15 & 17 & 20 & 23 \\
\hline \multirow{2}{*}{ Did not want people gossiping about me } & 2012 & 13 & 5 & 14 & 13 & $23 \uparrow$ \\
\cline { 2 - 7 } & 2010 & 10 & 7 & 9 & 11 & 10 \\
\hline \multirow{2}{*}{ Did not think anything would be done } & 2012 & 11 & 7 & 19 & $5 \downarrow$ & 18 \\
\cline { 2 - 7 } & 2010 & 9 & 4 & 10 & 12 & 9 \\
\hline $\begin{array}{l}\text { Thought it would hurt my reputation and } \\
\text { standing }\end{array}$ & 2012 & 11 & 9 & 12 & 10 & 15 \\
\cline { 2 - 7 } & 2010 & 7 & 5 & 8 & 6 & 10 \\
\hline Margins of Error & & $\pm 3-5 \%$ & $\pm 5-9 \%$ & $\pm 6-10 \%$ & $\pm 6-10 \%$ & $\pm 6-11 \%$ \\
\hline
\end{tabular}

\begin{tabular}{|l|c|}
\hline Higher Response of Yes & \\
\hline Lower Response of Yes & \\
\hline Higher in 2012 than 2010 & $\uparrow$ \\
\hline Lower in 2012 than 2010 & $\downarrow$ \\
\hline
\end{tabular}




\section{Contents}

- Unwanted Sexual Contact ................................................................. 7

- Unwanted Gender-Related Behaviors ................................................ 49

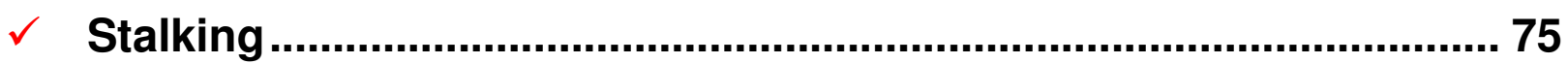

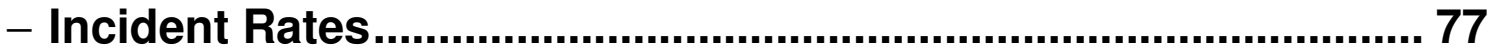

- Stalking and Sexual Harassment Related to Unwanted

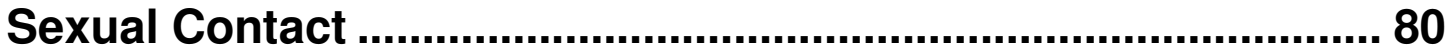

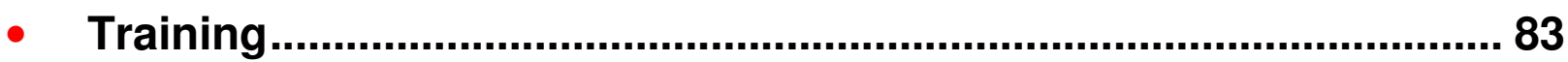

- Student Perceptions............................................................................... 93 


\section{Stalking Incident Rate}

\section{- Definition and measure of stalking:}

- Under Article 120a of the UCMJ, stalking is a crime. The UCMJ definition of stalking is "a course of conduct directed at a specific person that would cause a reasonable person to fear death or bodily harm, including sexual assault, to himself or herself or a member of his or her immediate family."

- Thus, to be punishable under the provision, stalking must be intentional, repeated (two or more occasions of such conduct), and cause unreasonable fear of physical injury. Note that this definition does not limit stalking to association with sexual harassment or sexual assault.

- The measure of stalking on the 2012 SAGR is consistent with the definition in Article 120a of the UCMJ. Students were asked (Q9) whether they had experienced any stalking behaviors (e.g., followed or spied on them in public areas; spied on them in private areas; showed up at places where they were even though he/she had no reason to be there; left unwanted items for them to find; stood outside or hung around their dorm room or classroom even though he/she had no reason to be there; and vandalized or tampered with their belongings) by someone assigned to their Academy, including students and military/civilian personnel, and whether they felt in danger of physical harm or sexual assault as a result of the experience (Q11). 


\section{Contents}

- Introduction 3

- Unwanted Sexual Contact ................................................................... 7

- Unwanted Gender-Related Behaviors ............................................... 49

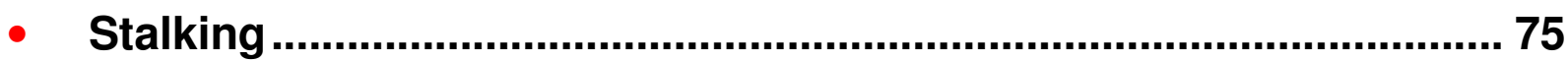

$\checkmark$ Incident Rates......................................................................... 77

- Stalking and Sexual Harassment Related to Unwanted

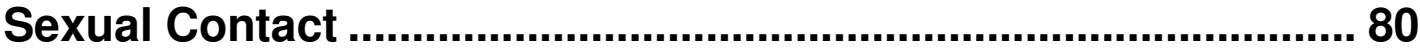

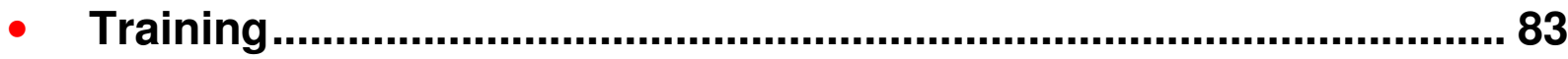

- Student Perceptions ......................................................................... 93 


\section{Stalking Incident Rate}

Percent of Women Who Experienced Stalking Behavior and Felt in Danger

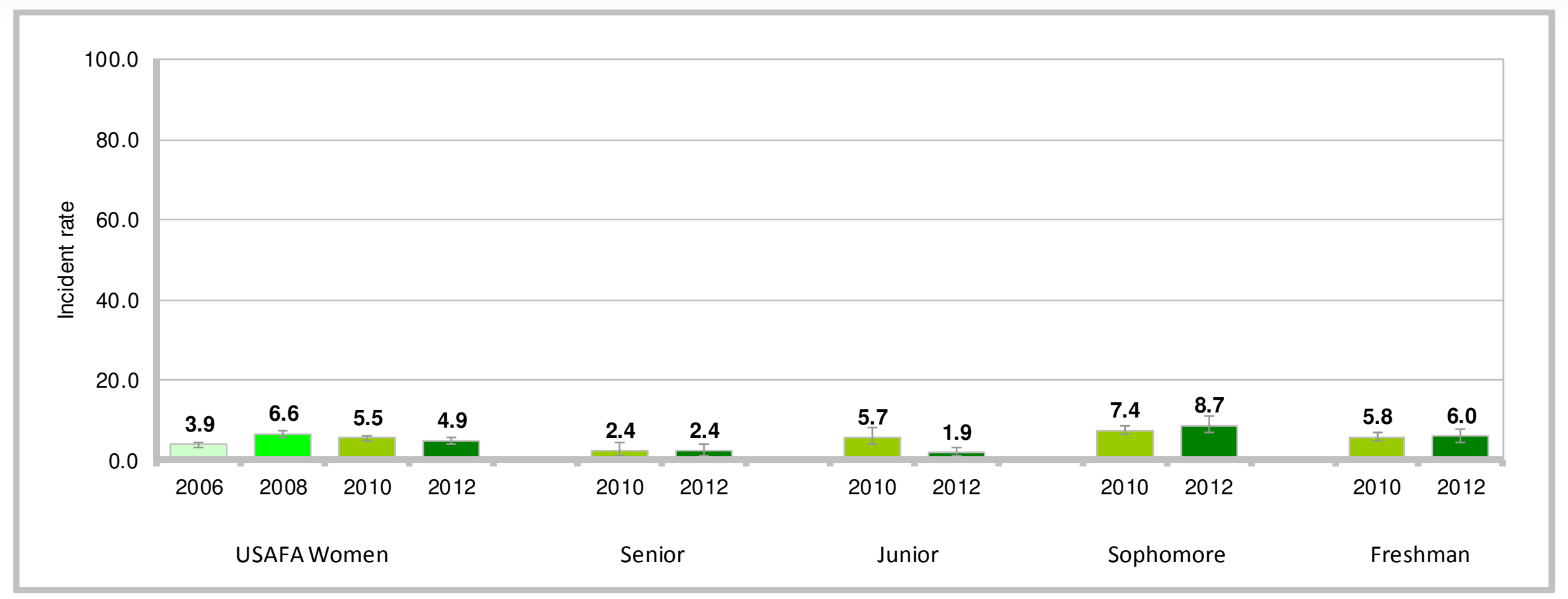

- $4.9 \%$ of women indicated experiencing stalking behavior in 2012

- 2012 comparisons across years - lower than 2008

- Juniors in 2012 lower than 2010

- Class differences in 2012 - higher response led by sophomores; lower response led by seniors and juniors 


\section{Stalking Incident Rate}

Percent of Men Who Experienced Stalking Behavior and Felt in Danger

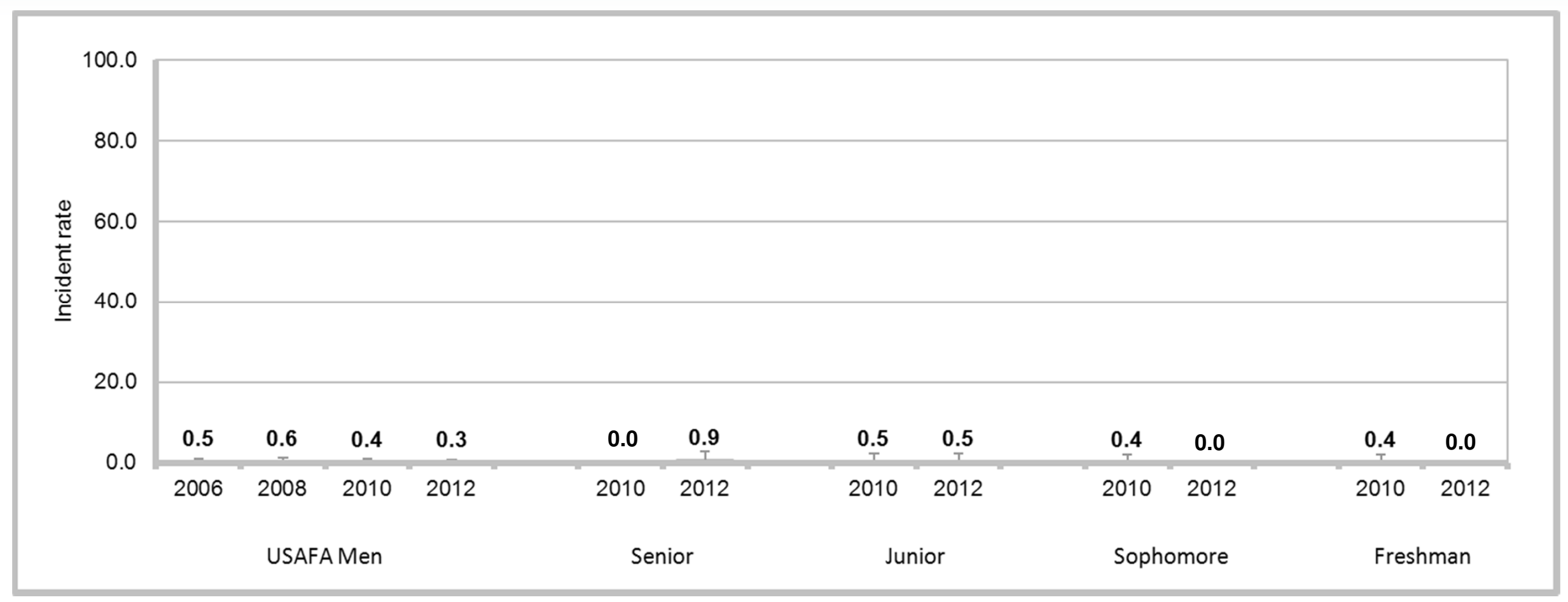

- $0.3 \%$ of men indicated experiencing stalking behavior in 2012

- 2012 comparisons across years - no differences

- Class differences in 2012 - no differences 


\section{Contents}

- Introduction ...................................................................................... 3

- Unwanted Sexual Contact .............................................................. 7

- Unwanted Gender-Related Behaviors ............................................ 49

• Stalking ...................................................................................... 75

- Incident Rates............................................................................ 77

$\checkmark$ Stalking and Sexual Harassment Related to Unwanted

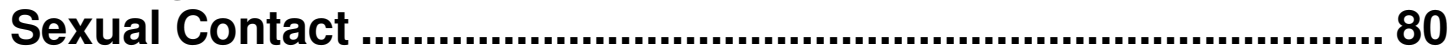

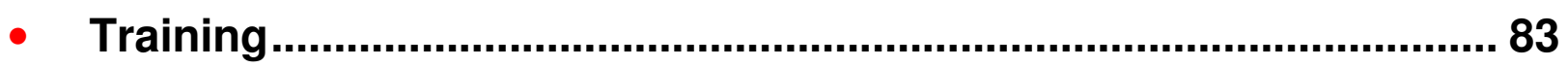

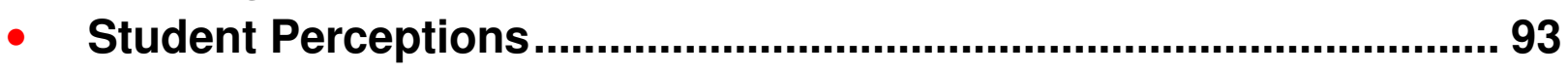




\section{Stalking and Sexual Harassment Possibly Lead to Sexual Assault} Percent of Women

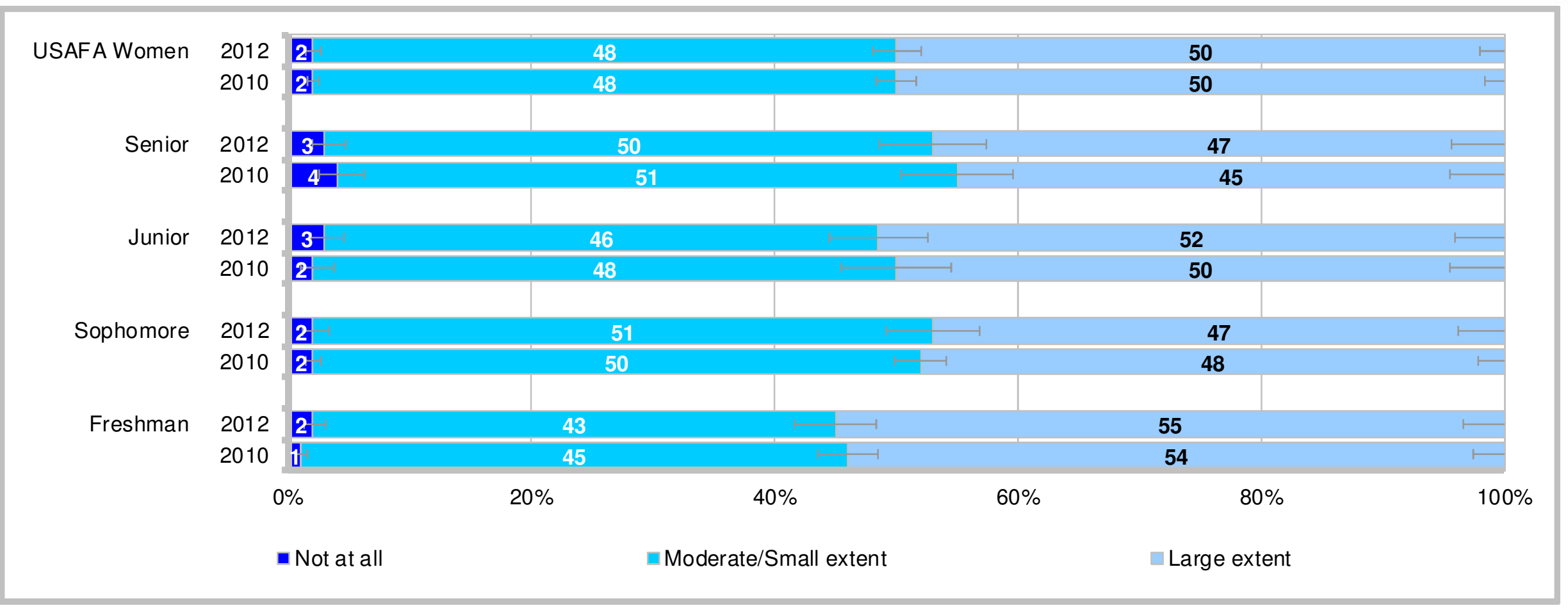

- $98 \%$ of women indicated stalking and sexual harassment possibly lead to sexual assault to at least some extent; $2 \%$ indicated stalking and sexual harassment do not lead to sexual assault

- 2012 comparisons across years - no differences

- Class differences in 2012 - large extent led by freshmen 


\section{Stalking and Sexual Harassment Possibly Lead to Sexual Assault} Percent of Men

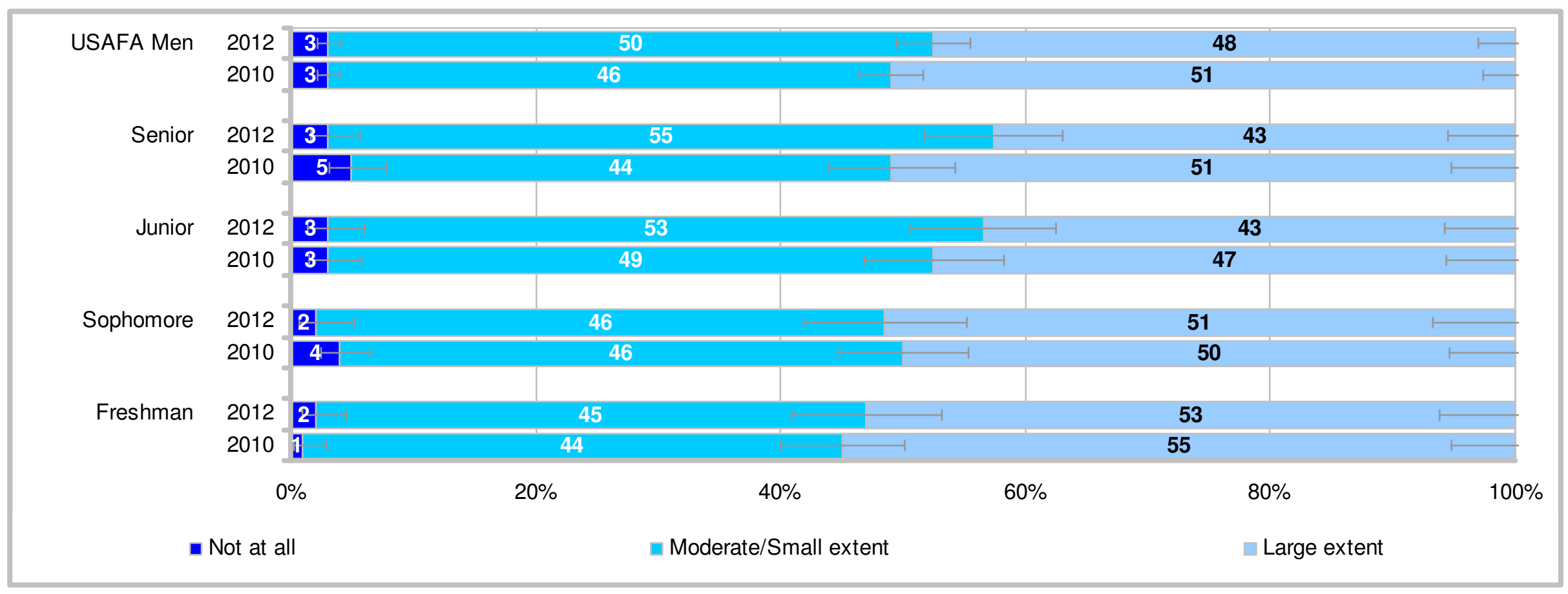

- $98 \%$ of men indicated stalking and sexual harassment possibly lead to sexual assault to at least some extent; $3 \%$ indicated stalking and sexual harassment do not lead to sexual assault

- 2012 comparisons across years - no differences

- Seniors indicating large extent in 2012 lower than 2010; seniors indicating moderate/small extent in 2012 higher than 2010

- Class differences in 2012 - no differences 


\section{Contents}

Slide

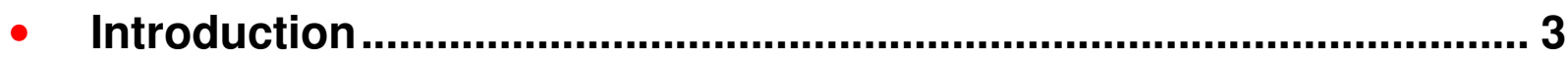

- Unwanted Sexual Contact .................................................................... 7

- Unwanted Gender-Related Behaviors .............................................. 49

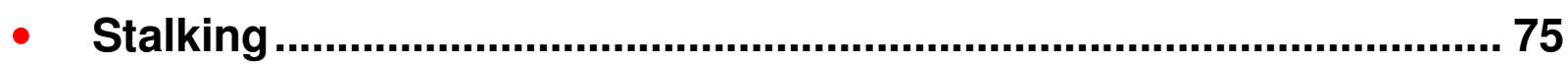

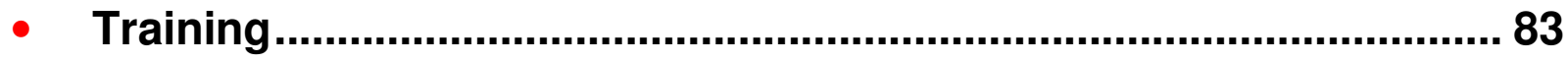

$\checkmark$ Sexual Assault Training Availability and Effectiveness ............ 83

- Sexual Harassment Training Availability and Effectiveness..... 88

- Student Perceptions....................................................................... 93 


\section{Received Training Since June 2011 on Topics Related to Sexual Assault} Percent of Women

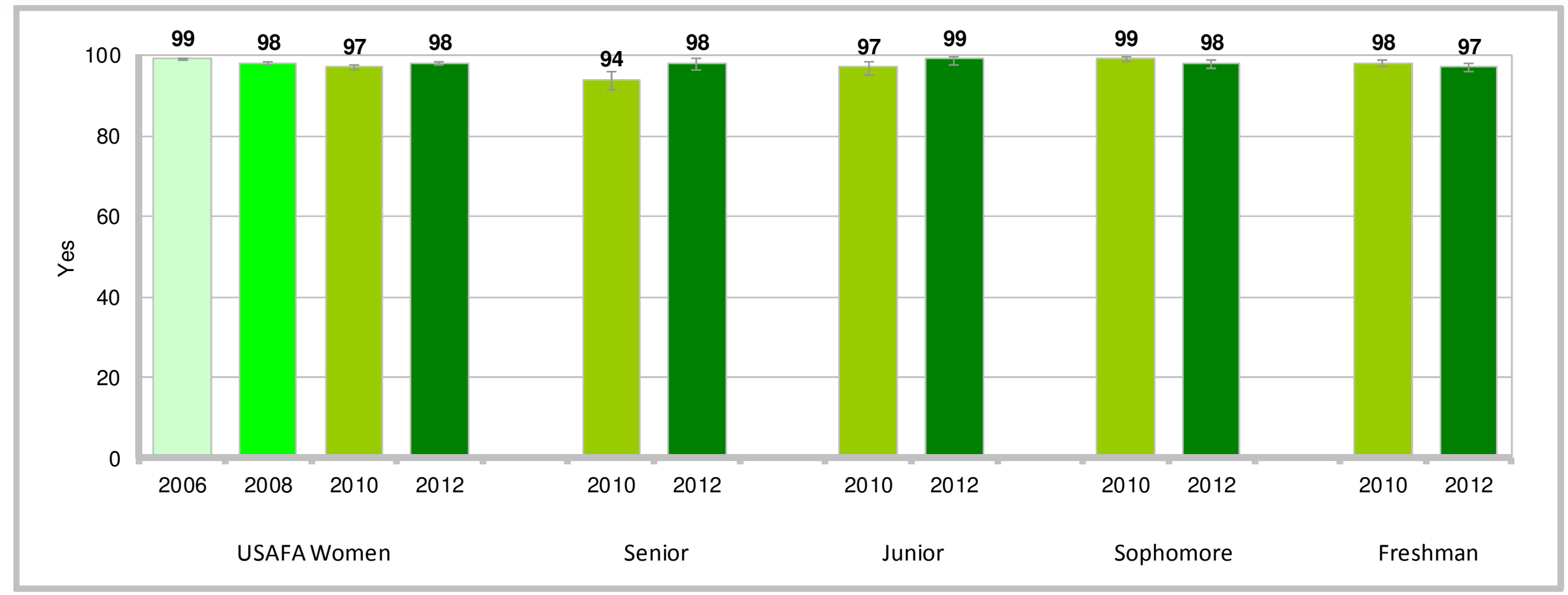

- $98 \%$ of women indicated receiving training on topics related to sexual assault in 2012

- 2012 comparisons across years - no differences

- Seniors and juniors in 2012 higher than 2010

- Class differences in 2012 - no differences 


\section{Received Training Since June 2011 on Topics Related to Sexual Assault} Percent of Men

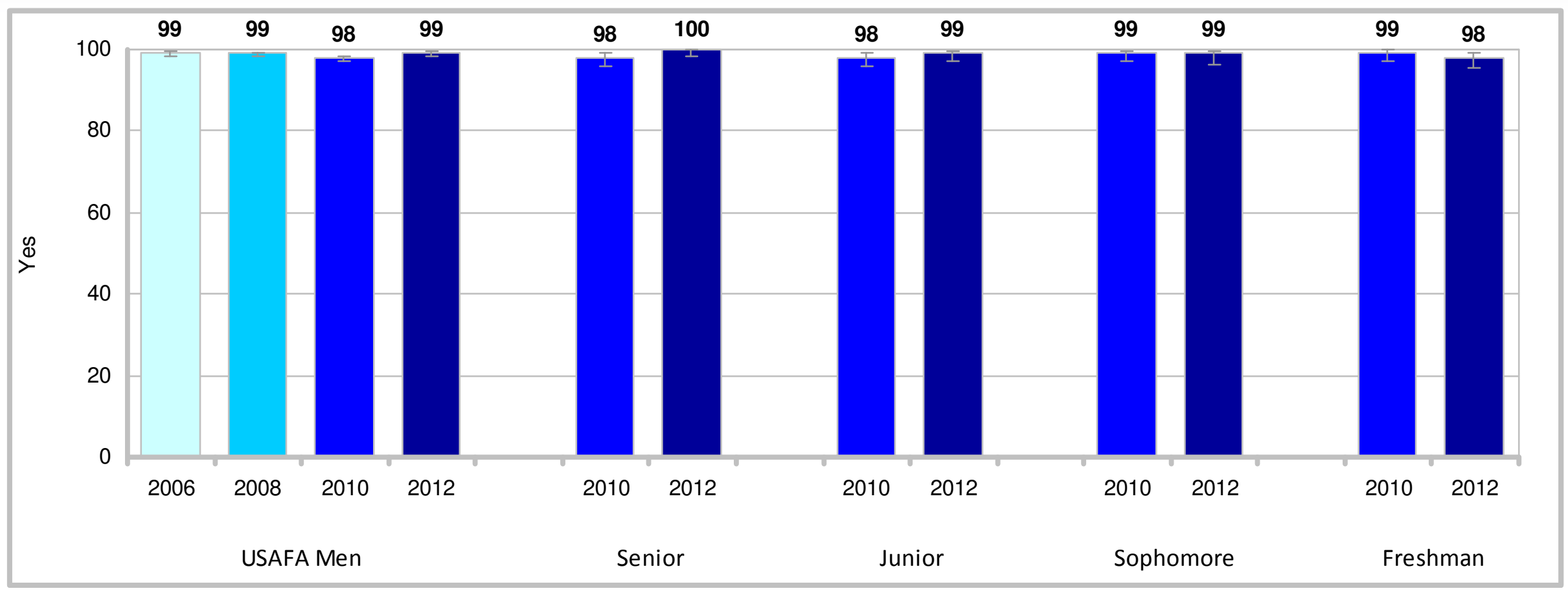

- $99 \%$ of men indicated receiving training on topics related to sexual assault in 2012

- 2012 comparisons across years - no differences

- Seniors in 2012 higher than 2010

- Class differences in 2012 - higher response led by seniors 


\section{Effectiveness of Training in Actually Reducing/Preventing Sexual Assault Percent of Women Who Had Training on Sexual Assault}

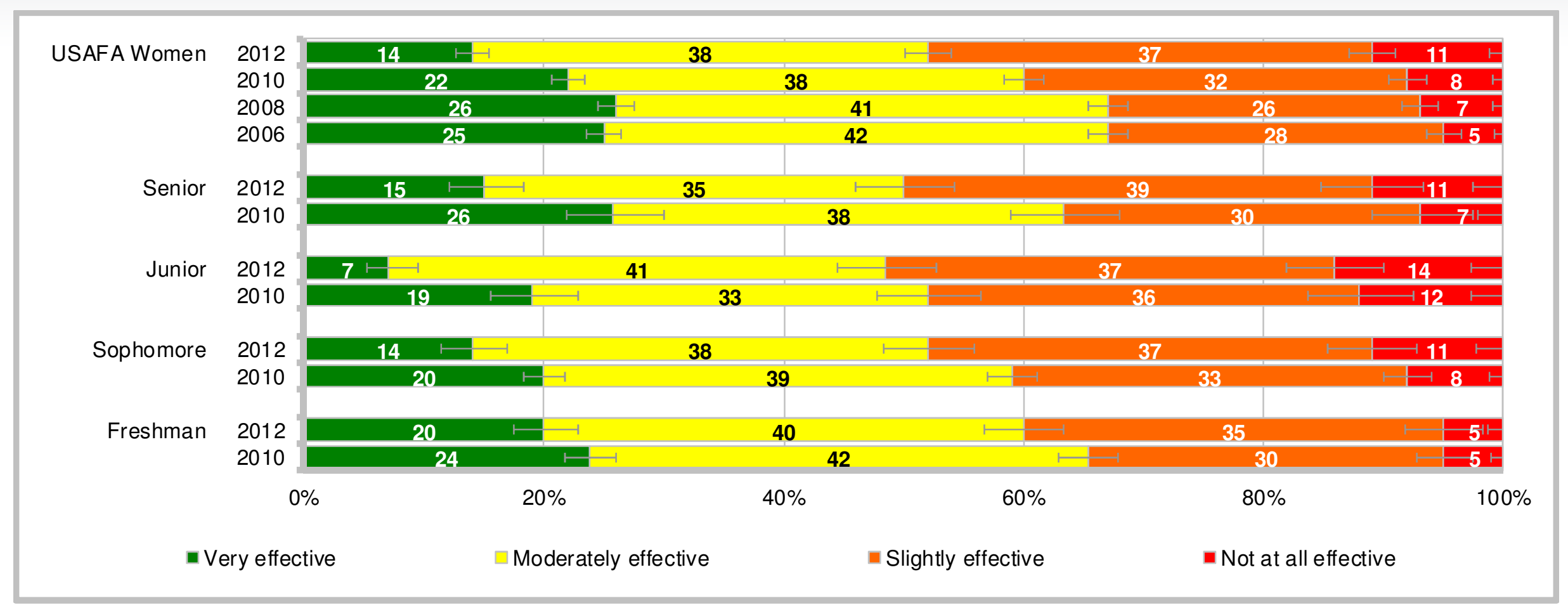

- $14 \%$ of women indicated their training was very effective in actually reducing/preventing sexual assault; $38 \%$ indicated moderately effective; $37 \%$ indicated slightly effective; and $11 \%$ indicated not at all effective

- 2012 comparisons across years - very effective lower than 2010, 2008, 2006; moderately effective lower than 2008, 2006; slightly effective higher than 2010, 2008, 2006; not at all effective higher than 2010, 2008, 2006

- All class years indicating very effective in 2012 lower than 2010; juniors indicating moderately effective in 2012 higher than 2010; seniors and freshmen indicating slightly effective in 2012 higher than 2010; seniors and sophomores indicating not at all effective in 2012 higher than 2010

- Class differences in 2012 - very effective led by freshmen; not at all effective led by juniors 


\section{Effectiveness of Training in Actually Reducing/Preventing Sexual Assault} Percent of Men Who Had Training on Sexual Assault

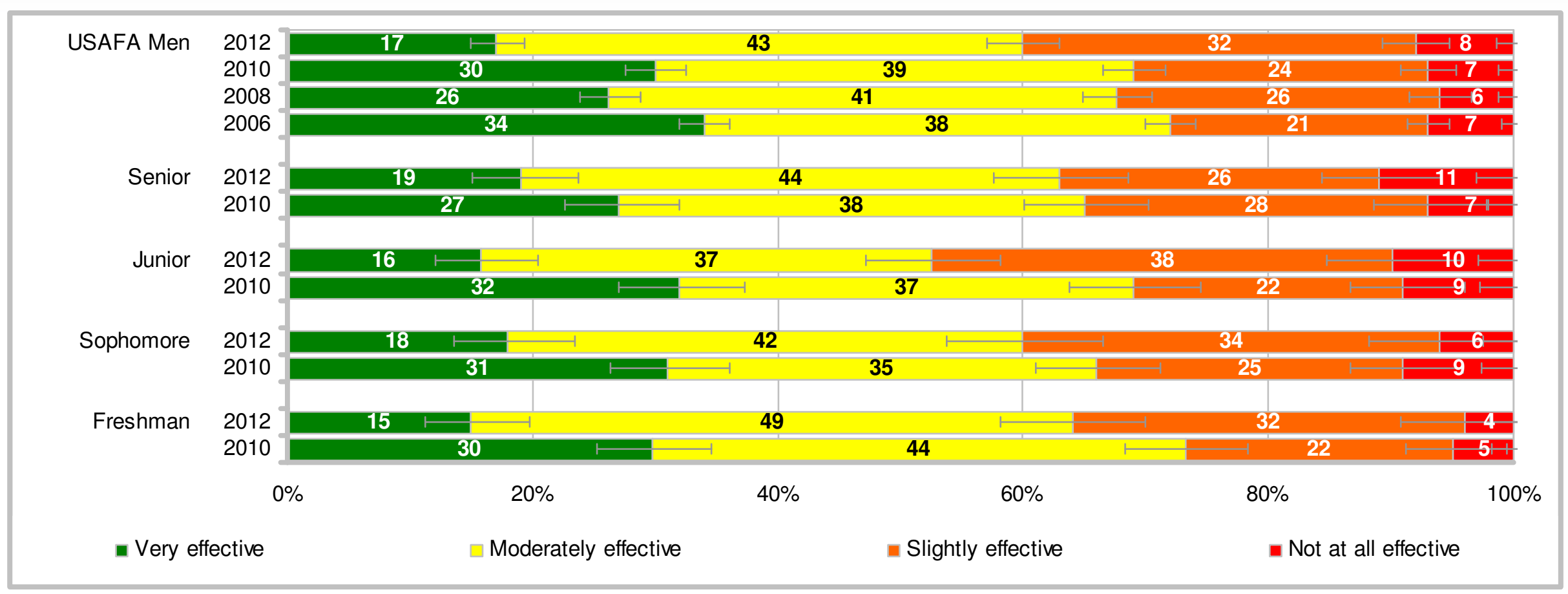

- $17 \%$ of men indicated their training was very effective in actually reducing/preventing sexual assault; $43 \%$ indicated moderately effective; $32 \%$ indicated slightly effective; and $8 \%$ indicated not at all effective

- 2012 comparisons across years - very effective lower than 2010, 2008, 2006; moderately effective higher than 2010, 2006; slightly effective higher than 2010, 2008, 2006

- All class years indicating very effective in 2012 lower than 2010; juniors, sophomores, and freshmen indicating slightly effective in 2012 higher than 2010

- Class differences in 2012 - no differences 


\section{Contents}

Slide

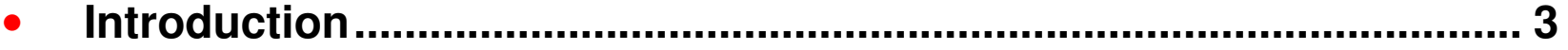

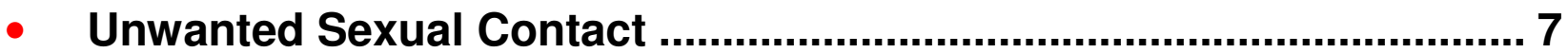

- Unwanted Gender-Related Behaviors .......................................... 49

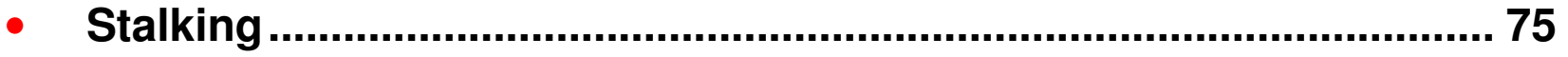

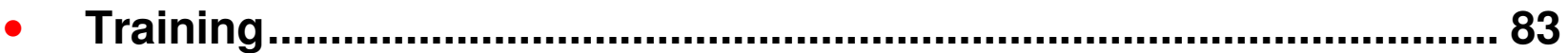

- Sexual Assault Training Availability and Effectiveness ............ 83

$\checkmark$ Sexual Harassment Training Availability and Effectiveness..... 88

- Student Perceptions..................................................................... 93 


\section{Received Training Since June 2011 on Topics Related to Sexual Harassment \\ Percent of Women}

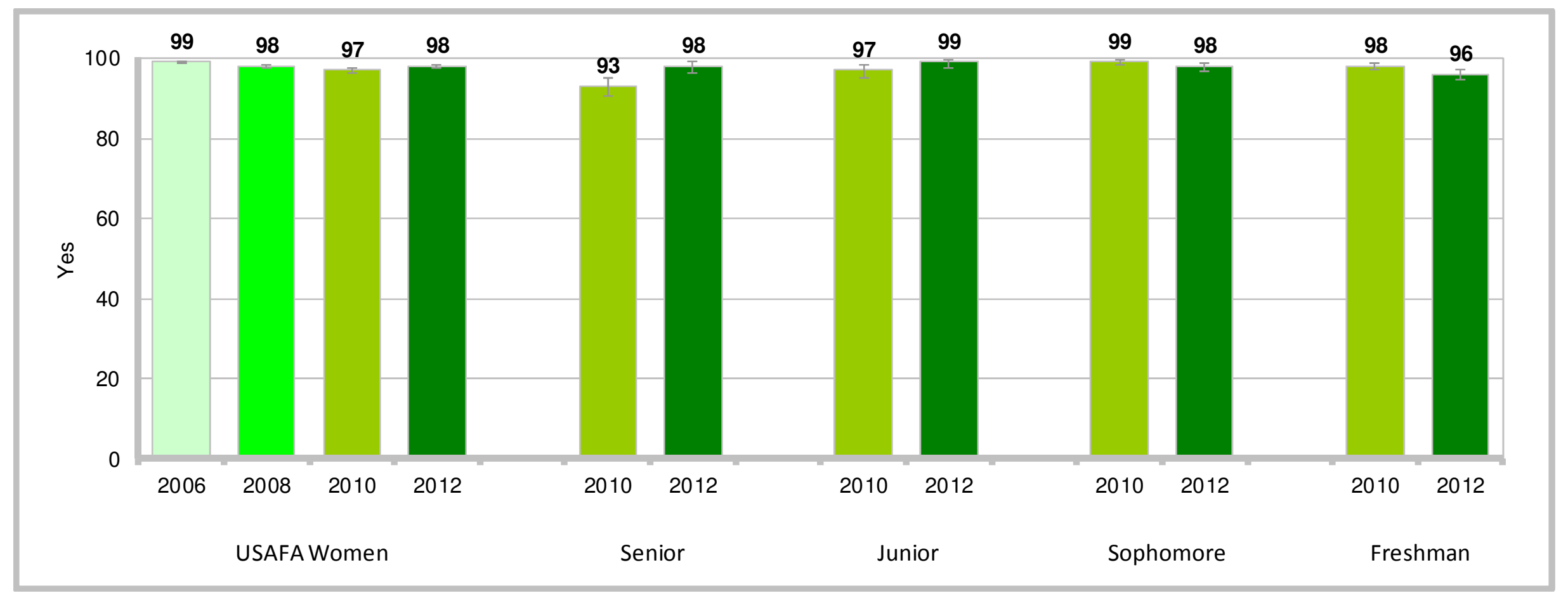

- $98 \%$ of women indicated receiving training on topics related to sexual harassment in 2012

- 2012 comparisons across years - no differences

- Seniors and juniors in 2012 higher than 2010, whereas freshmen lower

- Class differences in 2012 - no differences 


\section{Received Training Since June 2011 on Topics Related to Sexual Harassment \\ Percent of Men}

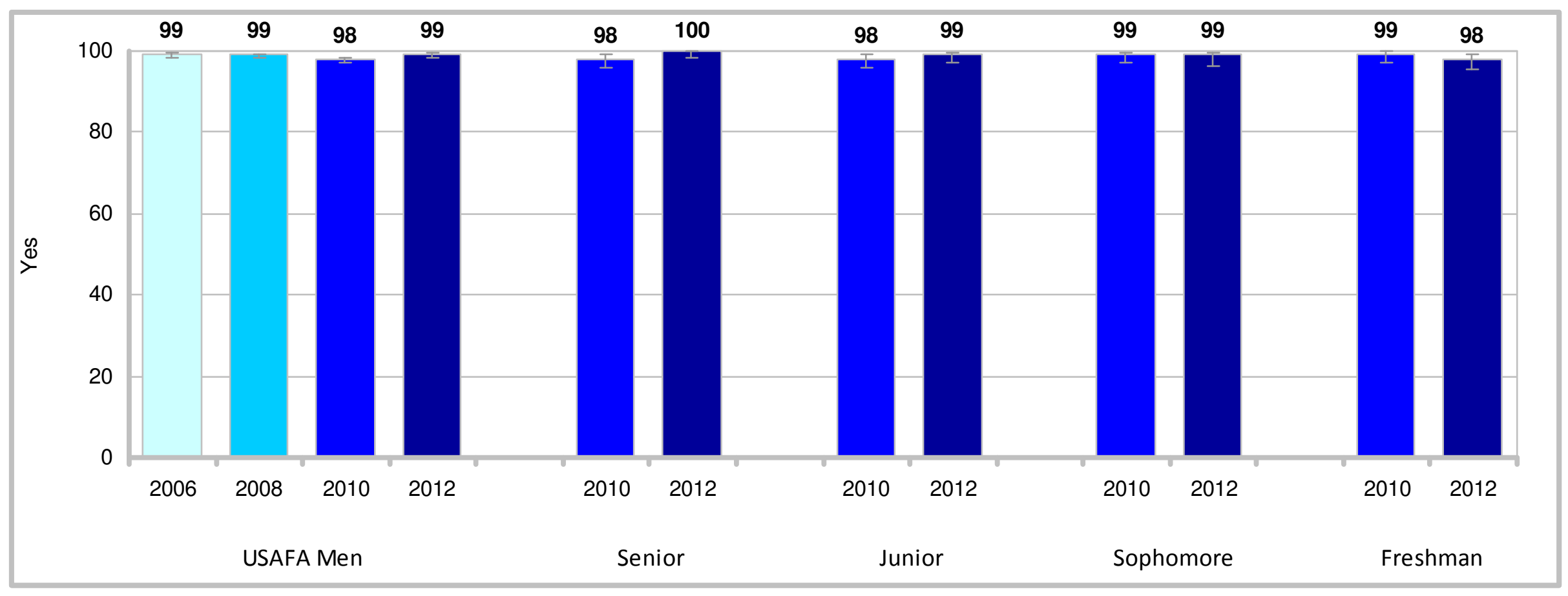

- $99 \%$ of men indicated receiving training on topics related to sexual harassment in 2012

- 2012 comparisons across years - no differences

- Seniors in 2012 higher than 2010

- Class differences in 2012 - higher response led by seniors 


\section{Effectiveness of Training in Actually Reducing/Preventing Sexual Harassment \\ Percent of Women Who Had Training on Sexual Harassment}

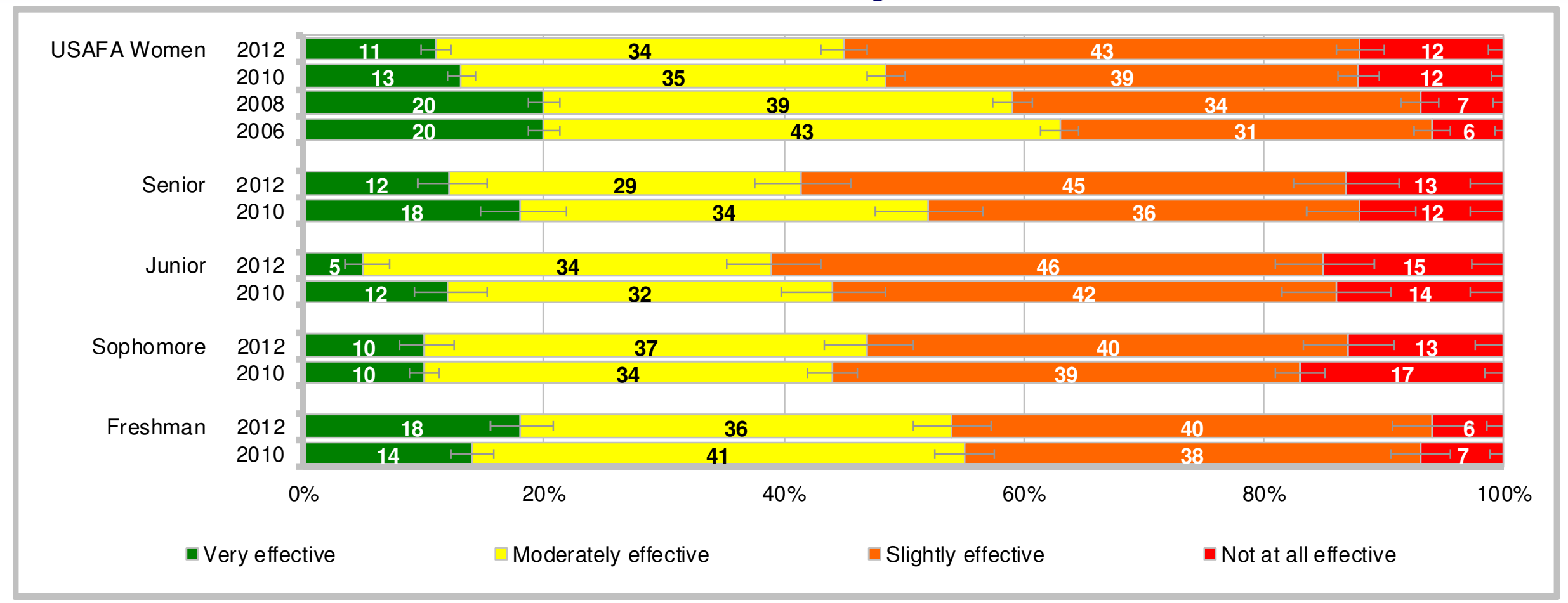

- $11 \%$ of women indicated their training was very effective in actually reducing/preventing sexual harassment; $34 \%$ indicated moderately effective; $43 \%$ indicated slightly effective; and $12 \%$ indicated not at all effective

- 2012 comparisons across years - very effective lower than 2010, 2008, 2006; moderately effective lower than 2008, 2006; slightly effective higher than 2010, 2008, 2006; not at all effective higher than 2008, 2006

- Seniors and juniors indicating very effective in 2012 lower than 2010, whereas freshmen higher; freshmen indicating moderately effective in 2012 lower than 2010; seniors indicating slightly effective in 2012 higher than 2010; sophomores indicating not at all in 2012 lower than 2010

- Class differences in 2012 - very effective led by freshmen 


\section{Effectiveness of Training in Actually Reducing/Preventing Sexual Harassment \\ Percent of Men Who Had Training on Sexual Harassment}

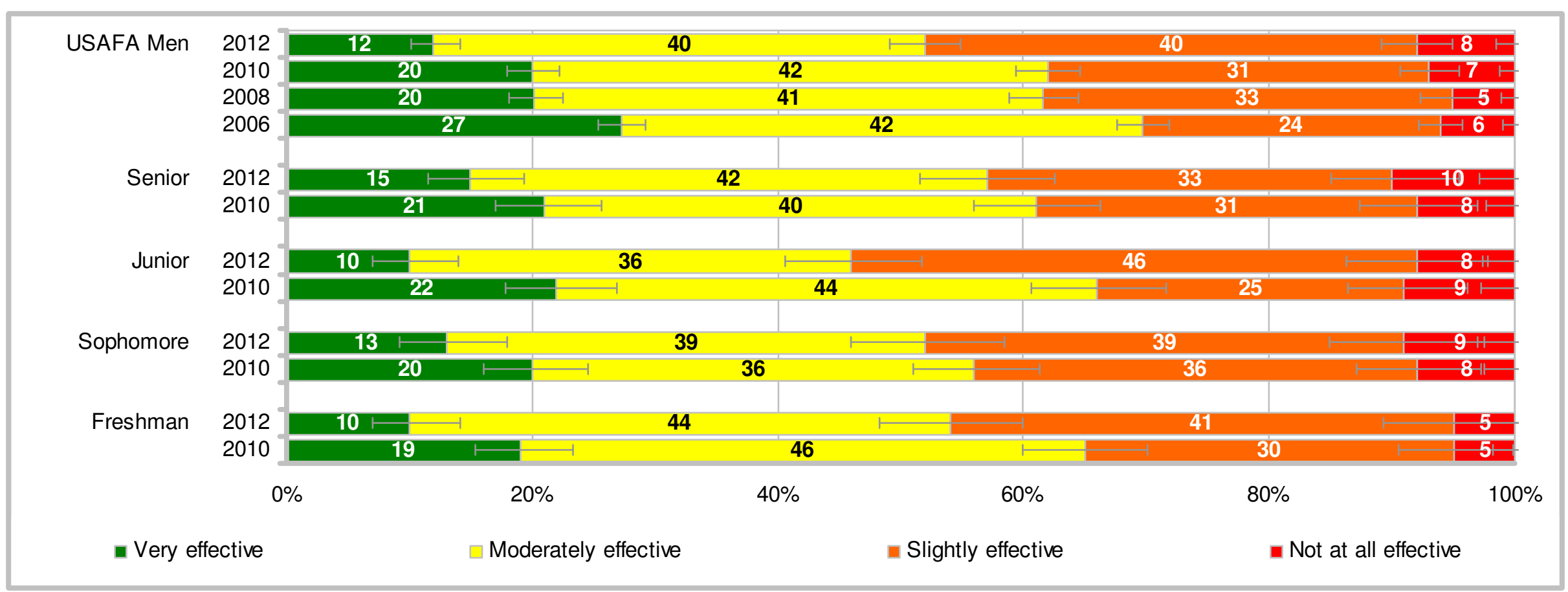

- $12 \%$ of men indicated their training was very effective in actually reducing/preventing sexual harassment; $40 \%$ indicated moderately effective; $40 \%$ indicated slightly effective; and $8 \%$ indicated not at all effective

- 2012 comparisons across years - very effective lower than 2010, 2008, 2006; slightly effective higher than 2010, 2008, 2006; not at all effective higher than 2008, 2006

- All class years indicating very effective in 2012 lower than 2010; juniors indicating moderately effective in 2012 lower than 2010; juniors and freshmen indicating slightly effective in 2012 higher than 2010

- Class differences in 2012 - no differences 


\section{Contents}

Slide

- Introduction .................................................................................. 3

- Unwanted Sexual Contact ................................................................... 7

- Unwanted Gender-Related Behaviors ............................................ 49

• Stalking .......................................................................................... 75

- Training ............................................................................................... 83

$\checkmark \quad$ Student Perceptions..................................................................... 93 


\section{Extent You Think Incidents of Sexual Assault Are Not Reported Percent of Women}

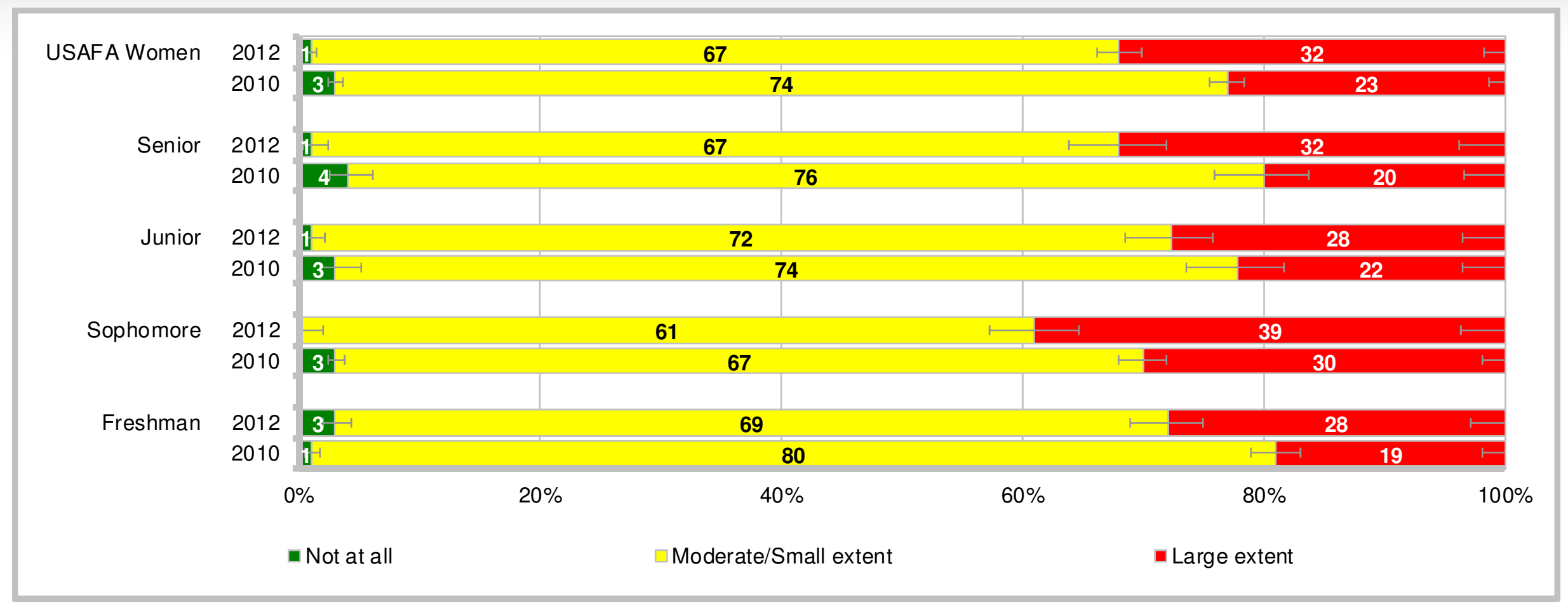

$32 \%$ of women indicated to a large extent that incidents of sexual assault are not reported; $67 \%$ indicated moderate/small extent; and $1 \%$ indicated not at all

- 2012 comparisons across years - large extent higher than 2010; moderate/small extent lower than 2010; not at all lower than 2010

- All class years indicating large extent in 2012 higher than 2010; seniors, sophomores, and freshmen indicating moderate/small extent in 2012 lower than 2010; seniors, juniors, and sophomores indicating not at all in 2012 lower than 2010, whereas freshmen higher

- Class differences in 2012 - large extent led by sophomores; moderate/small extent led by juniors; not at all led by freshmen 


\section{Extent You Think Incidents of Sexual Assault Are Not Reported Percent of Men}

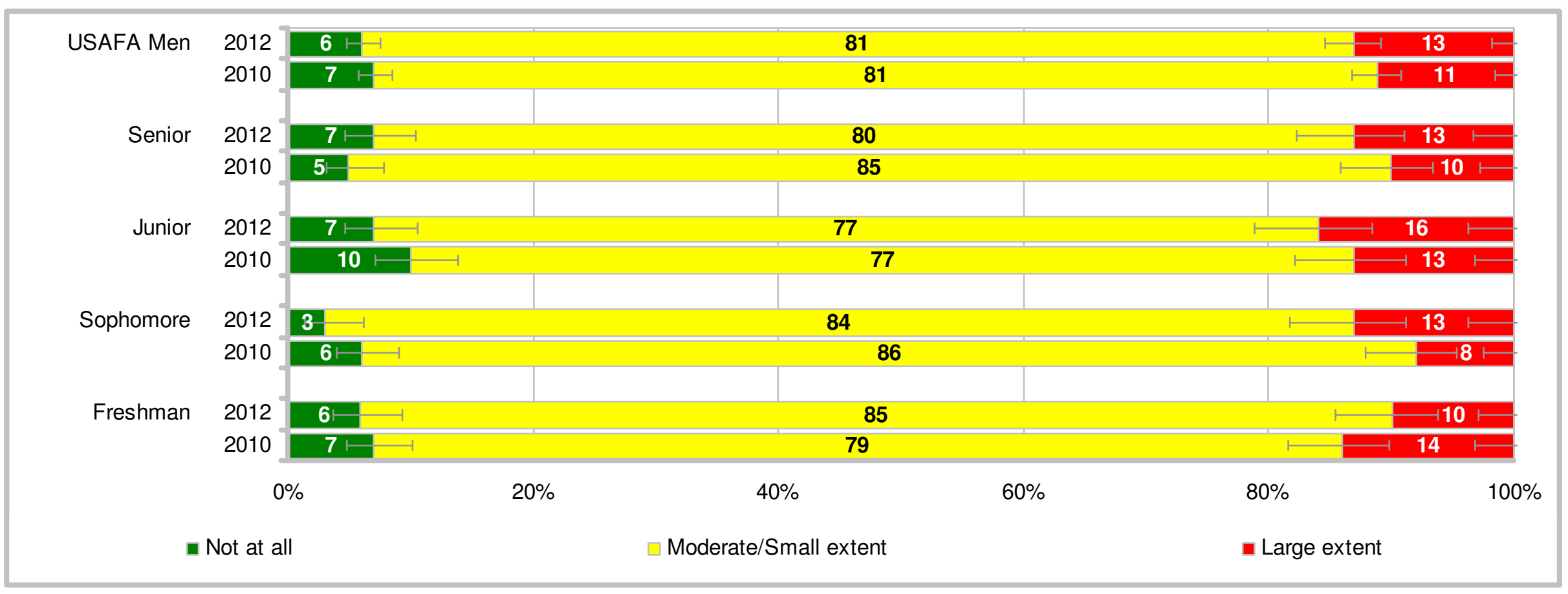

- $13 \%$ of men indicated to a large extent that incidents of sexual assault are not reported; $81 \%$ indicated moderate/small extent; and $6 \%$ indicated not at all

- 2012 comparisons across years - no differences

- Freshmen indicating moderate/small extent in 2012 higher than 2010

- Class differences in 2012 - no differences 


\section{Extent You Would Report a Sexual Assault Percent of Women}

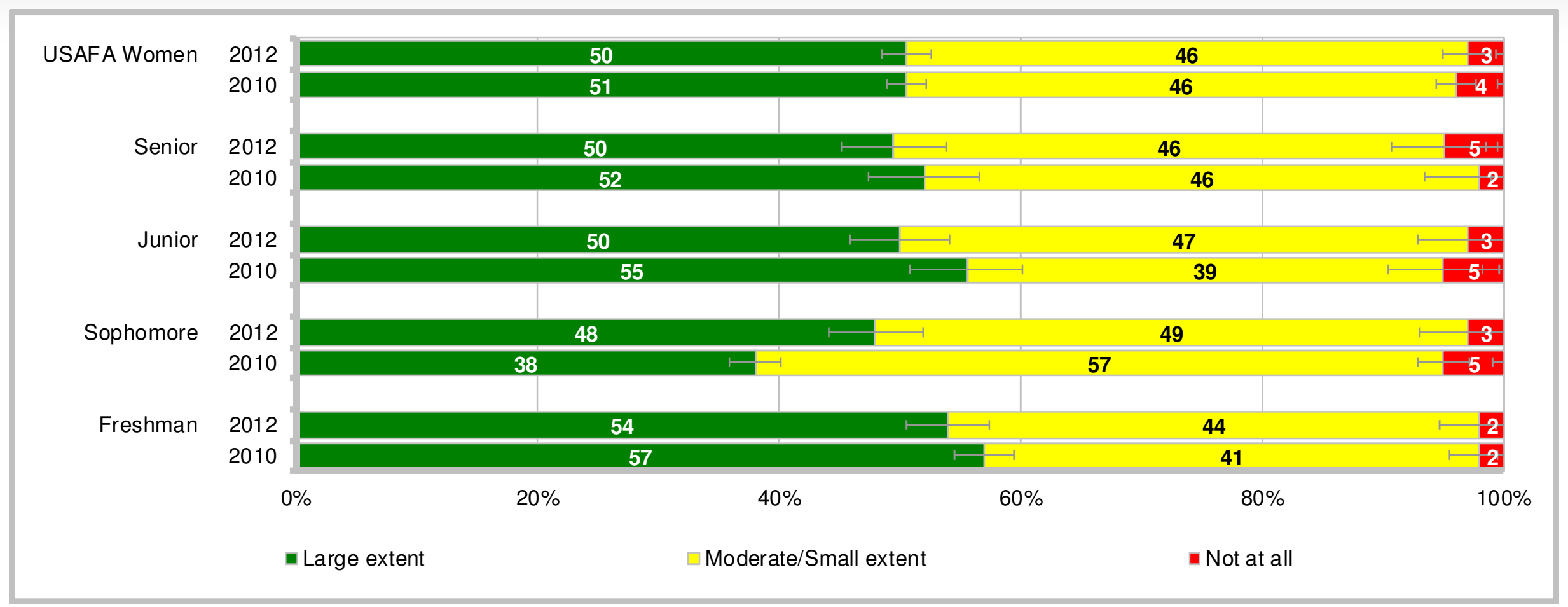

- $50 \%$ of women indicated to a large extent they would be willing to report a sexual assault; $46 \%$ indicated moderate/small extent; and $3 \%$ indicated not at all

- 2012 comparisons across years - no differences

- Sophomores indicating large extent in 2012 higher than 2010; juniors indicating moderate/small extent in 2012 higher than 2010, whereas sophomores lower; seniors indicating not at all in 2012 higher than 2010, whereas sophomores lower*

- Class differences in 2012 - no differences

*Note that similar to sophomore women, $3 \%$ of junior women indicated not at all in 2012 and $5 \%$ in 2010. These percentages for junior women are not significantly different due to a higher margin of error for junior women responding to this question. 


\section{Extent You Would Report a Sexual Assault Percent of Men}

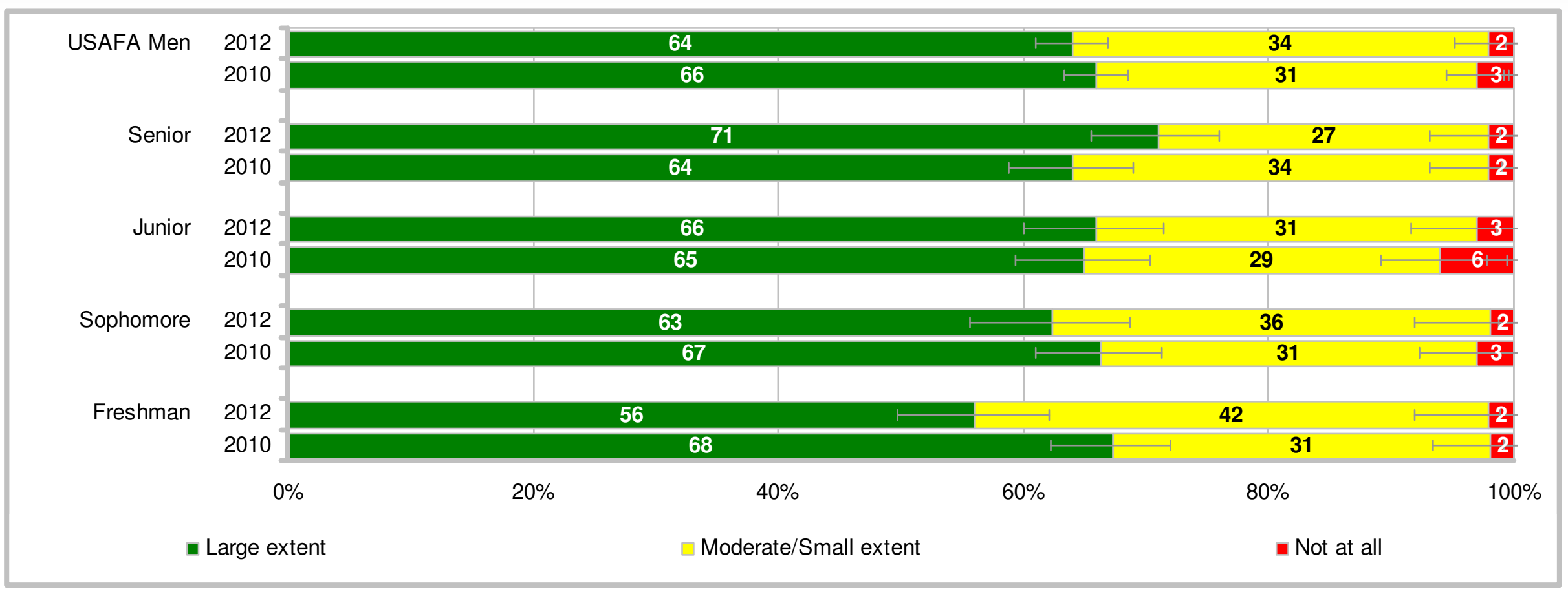

- $64 \%$ of men indicated to a large extent they would be willing to report a sexual assault; $34 \%$ indicated to a moderate/small extent; and $2 \%$ indicated not at all

- 2012 comparisons across years - no differences

- Freshmen indicating large extent in 2012 lower than 2010; freshmen indicating moderate/small extent in 2012 higher than 2010

- Class differences in 2012 - large extent led by seniors 


\section{Extent You Think High-Profile Cases Deter Reporting of Sexual Assault} Percent of Women

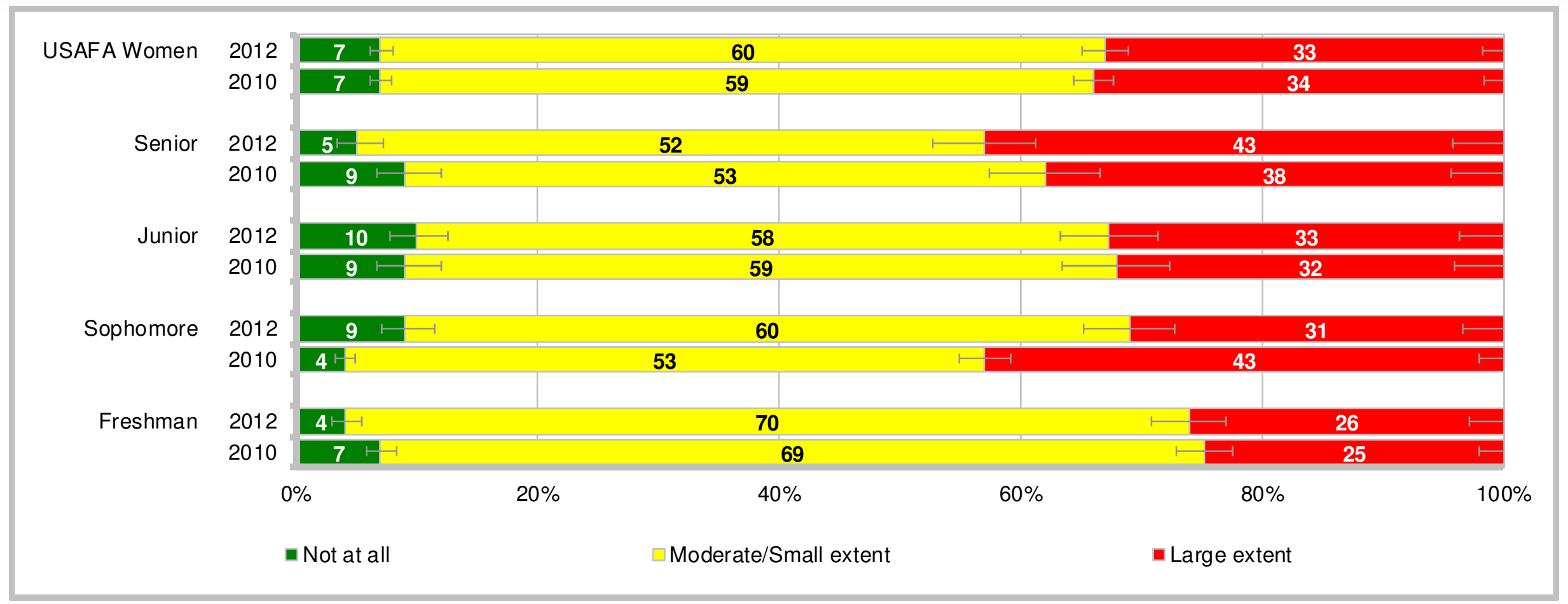

- $33 \%$ of women indicated to a large extent high-profile cases deter reporting of sexual assault; $60 \%$ indicated to a moderate/small extent; and $7 \%$ indicated not at all

- 2012 comparisons across years - no differences

- Sophomores indicating large extent in 2012 lower than 2010; sophomores indicating moderate/small extent in 2012 higher than 2010; seniors and freshmen indicating not at all in 2012 lower than 2010, whereas sophomores higher

- Class differences in 2012 - large extent led by seniors; moderate/small extent led by freshmen; not at all led by juniors 


\section{Extent You Think High-Profile Cases Deter Reporting of Sexual Assault} Percent of Men

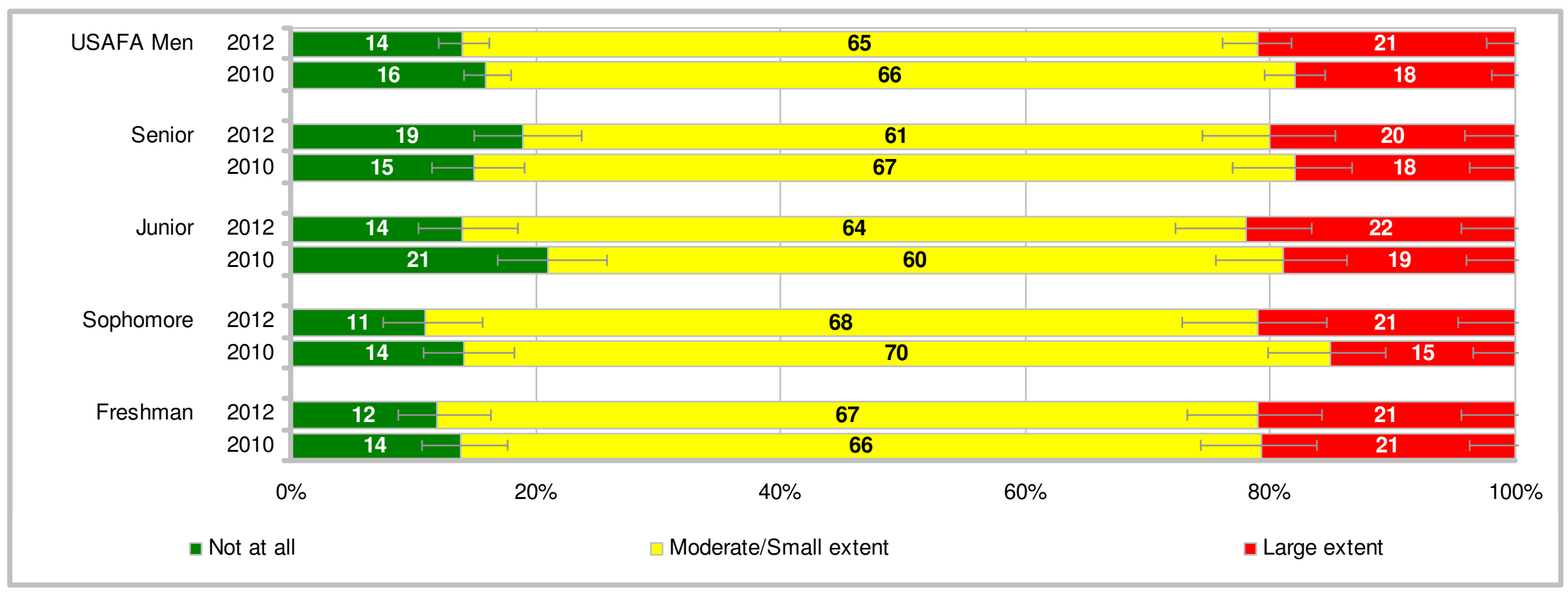

- $21 \%$ of men indicated to a large extent high-profile cases deter reporting of sexual assault; $65 \%$ indicated to a moderate/small extent; and $14 \%$ indicated not at all

- 2012 comparisons across years - no differences

- Juniors indicating not at all in 2012 lower than 2010

- Class differences in 2012 - not at all led by seniors 


\section{You Would Discuss an Incident With a SARC if You Were To Experience} Sexual Assault in the Future

\section{Percent of Women Who Had Not Experienced Unwanted Sexual Contact in Past 12 Months}

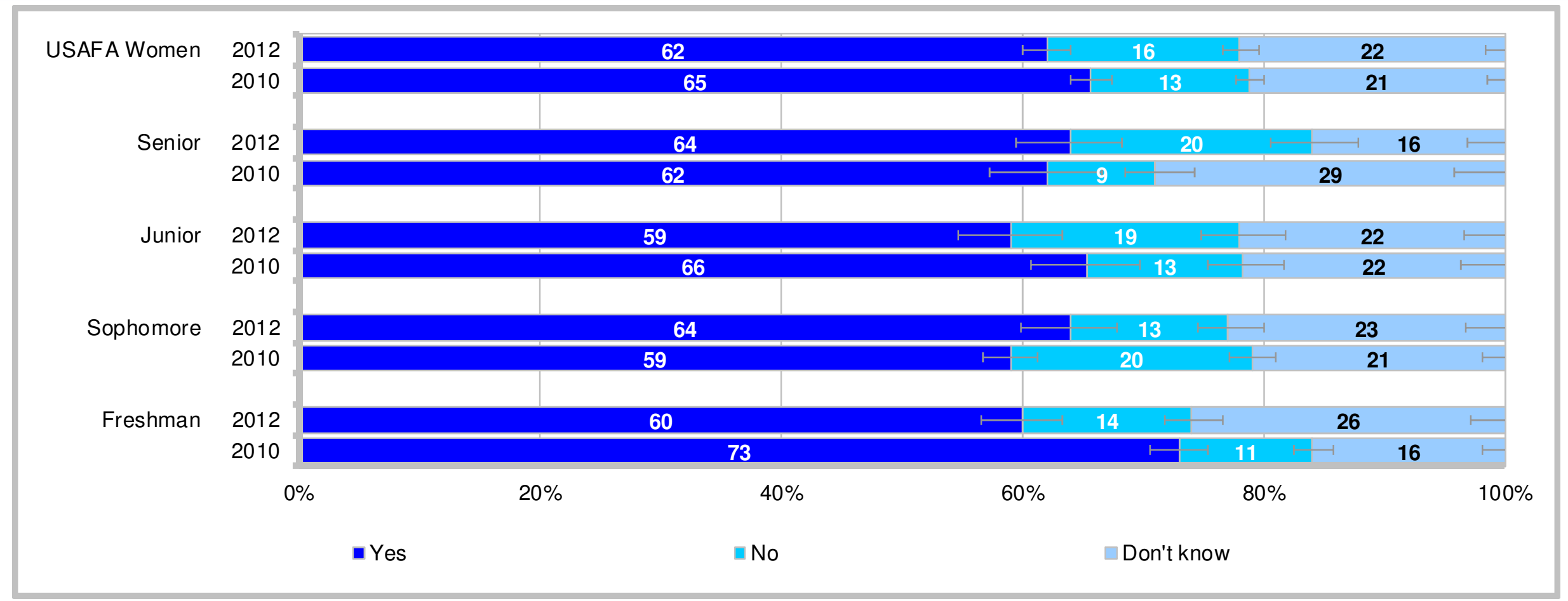

- $62 \%$ of women indicated yes, they would discuss an incident of sexual assault with a SARC; $16 \%$ indicated no; and $22 \%$ indicated don't know

- 2012 comparisons across years - yes lower than 2010; no higher than 2010

- Sophomores indicating yes in 2012 higher than 2010, whereas juniors and freshmen lower; seniors, juniors, and freshmen indicating no in 2012 higher than 2010, whereas sophomores lower; freshmen indicating don't know in 2012 higher than 2010, whereas seniors lower

- Class differences in 2012 - don't know led by freshmen 


\section{You Would Discuss an Incident With a SARC if You Were To Experience} Sexual Assault in the Future

Percent of Men Who Had Not Experienced Unwanted Sexual Contact in Past 12 Months

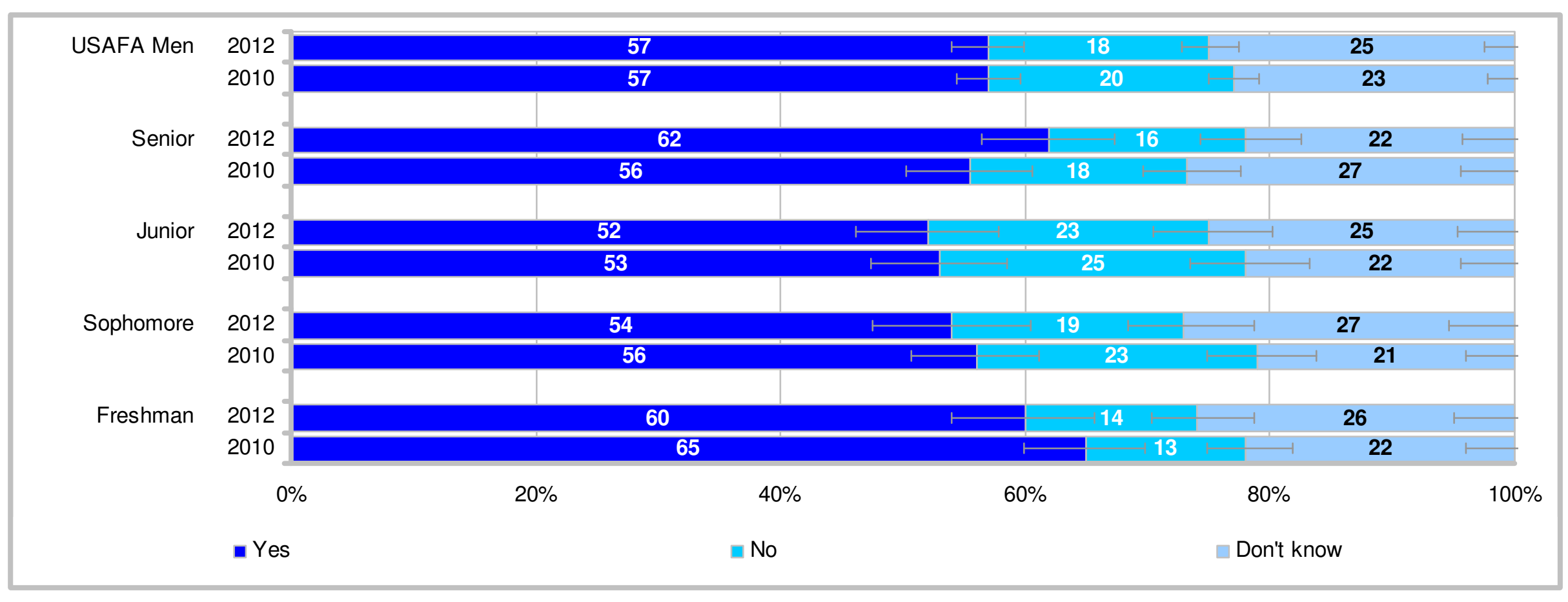

- $57 \%$ of men indicated yes, they would discuss an incident of sexual assault with a SARC; $18 \%$ indicated no; and $25 \%$ indicated don't know

- 2012 comparisons across years - no differences

- Class differences in 2012 - no differences 


\section{You Would Trust the System To Protect Your Privacy if You Were To Experience Sexual Assault in the Future Percent of Women Who Had Not Experienced Unwanted Sexual Contact}

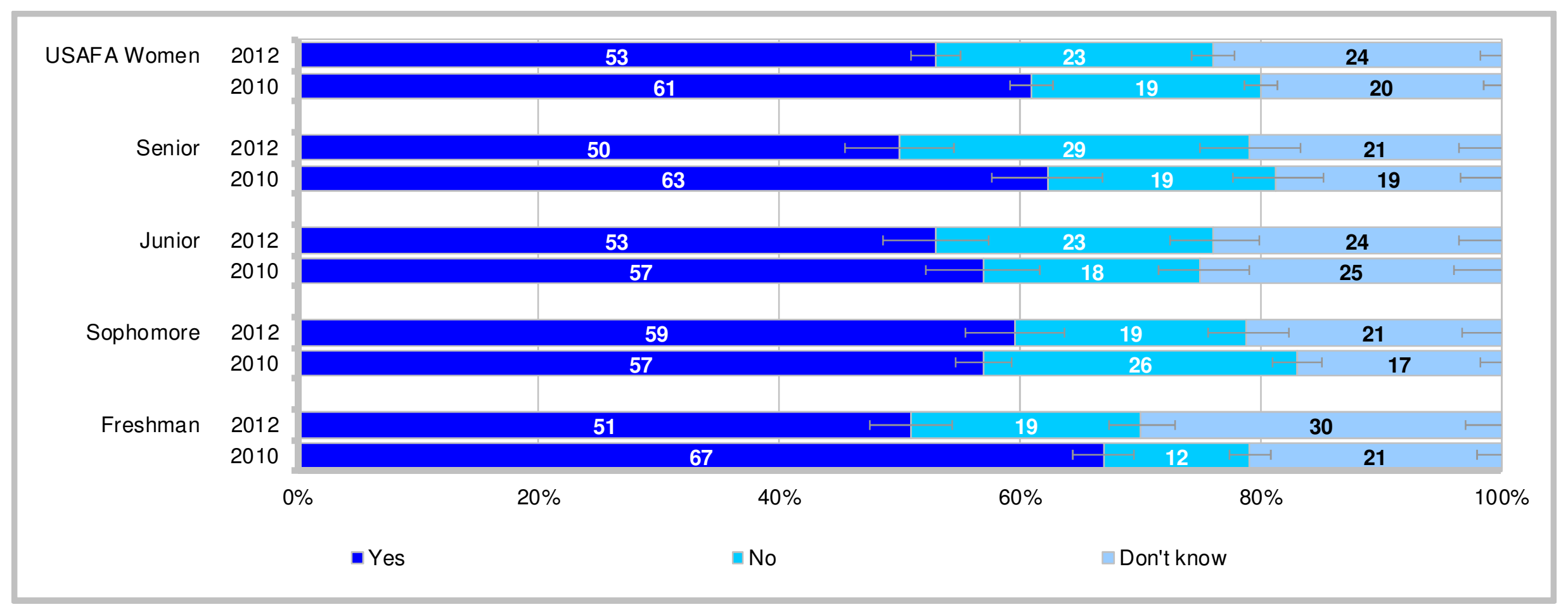

- $53 \%$ of women indicated yes, they would trust the system to protect their privacy if they experienced sexual assault; $23 \%$ indicated they no; and $24 \%$ indicated don't know

- 2012 comparisons across years - yes lower than 2010; no higher than 2010; don't know higher than 2010

- Seniors and freshmen indicating yes in 2012 lower than 2010; seniors and freshmen indicating no in 2012 higher than 2010, whereas sophomores lower; sophomores and freshmen indicating don't know in 2012 higher than 2010

- Class differences in 2012 - yes led by sophomores; no led by seniors; don't know led by freshmen 


\section{You Would Trust the System To Protect Your Privacy if You Were To Experience Sexual Assault in the Future Percent of Men Who Had Not Experienced Unwanted Sexual Contact}

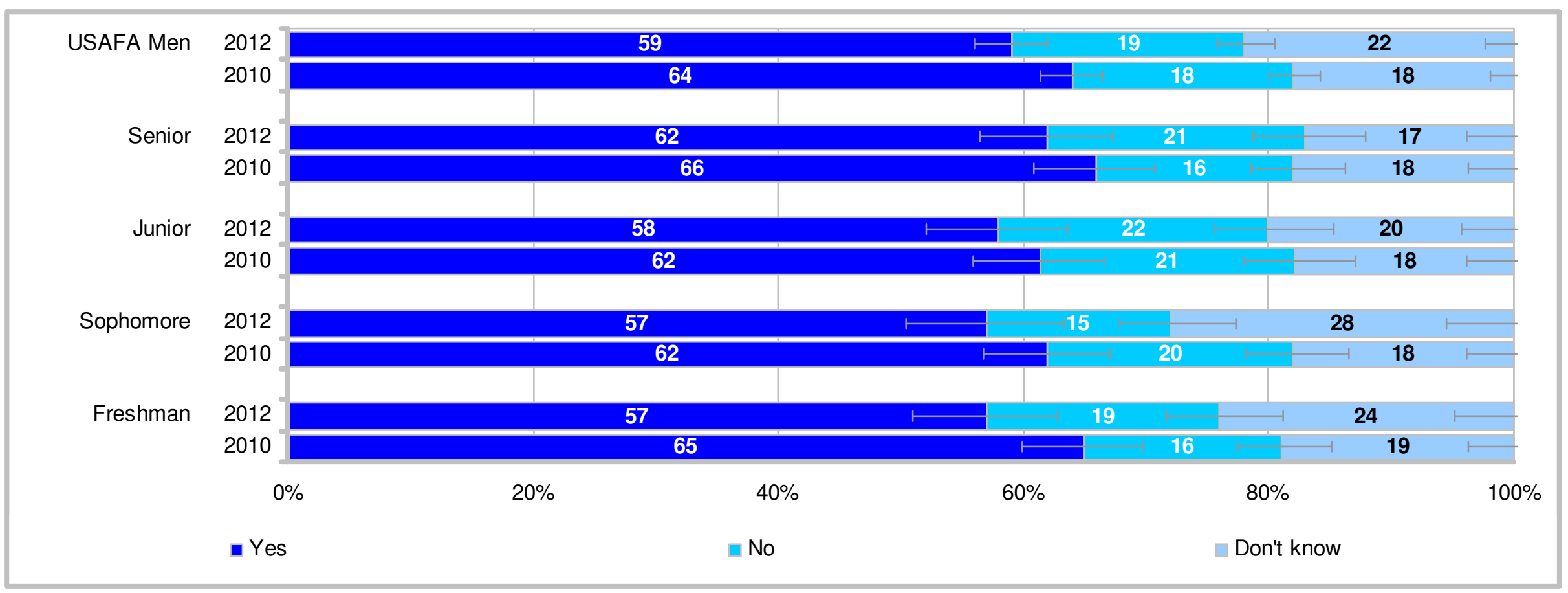

- $59 \%$ of men indicated yes, they would trust the system to protect their privacy if they experienced sexual assault; $19 \%$ indicated no; and 22\% indicated don't know

- 2012 comparisons across years - yes lower than 2010; don't know higher than 2010

- Freshmen indicating yes in 2012 lower than 2010; sophomores indicating don't know in 2012 higher than 2010

- Class differences in 2012 - no differences 


\section{You Would Be Willing To Encourage Someone Who Has Experienced Sexual Assault To Report It Percent of Women}

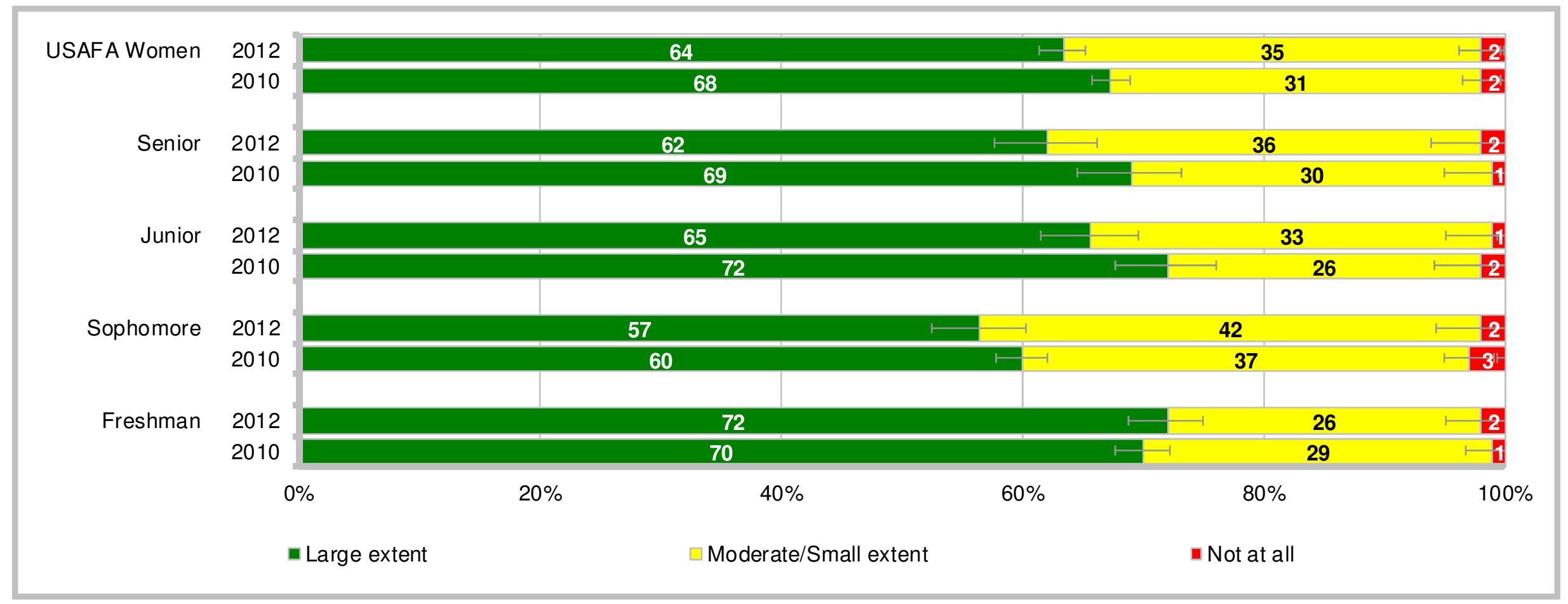

- $99 \%$ of women indicated they would encourage someone to report sexual assault to at least some extent; $2 \%$ indicated not at all

- 2012 comparisons across years - large extent lower than 2010; moderate/small extent higher than 2010

- Seniors and juniors indicating large extent in 2012 lower than 2010; juniors and sophomores indicating moderate/small extent in 2012 higher than 2010

- Class differences in 2012 - large extent led by freshmen; moderate/small extent led by sophomores 


\section{You Would Be Willing To Encourage Someone Who Has Experienced Sexual Assault To Report It \\ Percent of Men}

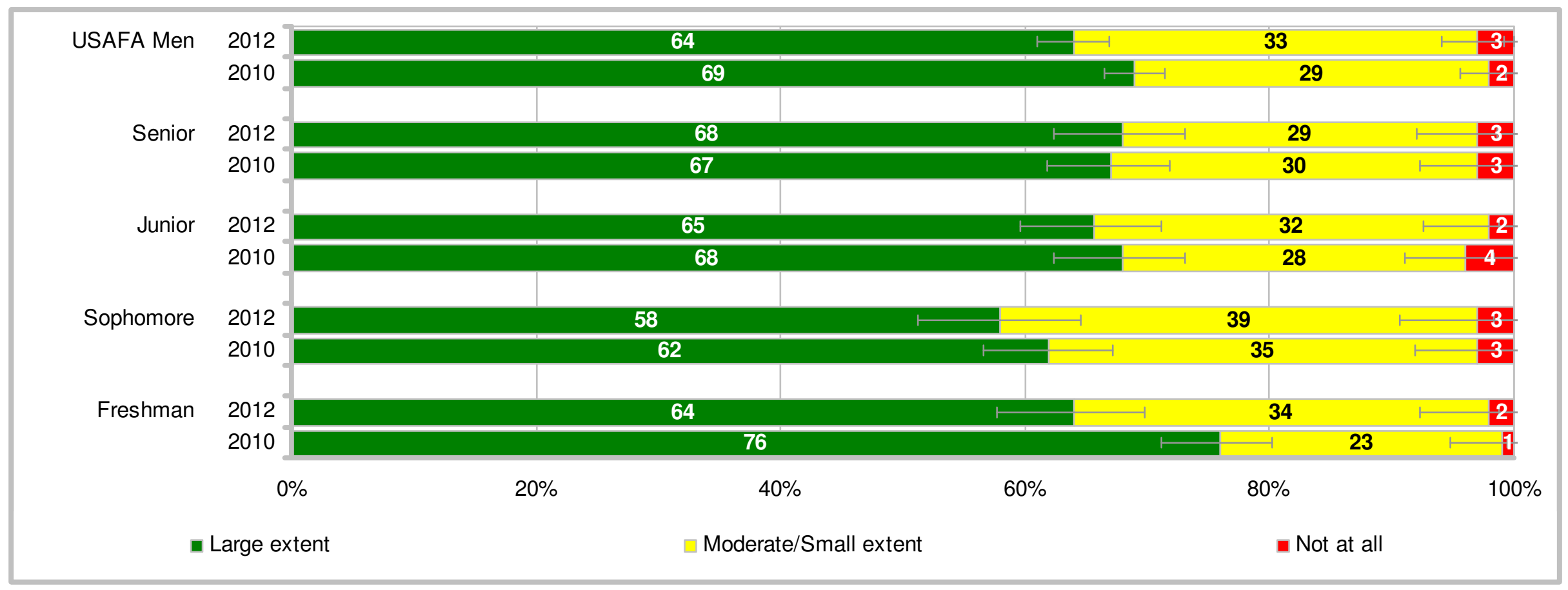

- $97 \%$ of men indicated they would encourage someone to report sexual assault to at least some extent; $3 \%$ indicated not at all

- 2012 comparisons across years - large extent lower than 2010; moderate/small extent higher than 2010

- Freshmen indicating large extent in 2012 lower than 2010; freshmen indicating moderate/small extent in 2012 higher than 2010

- Class differences in 2012 - no differences 


\section{You Would Step In and Stop a Situation That Might Lead to Sexual Assault} Percent of Women

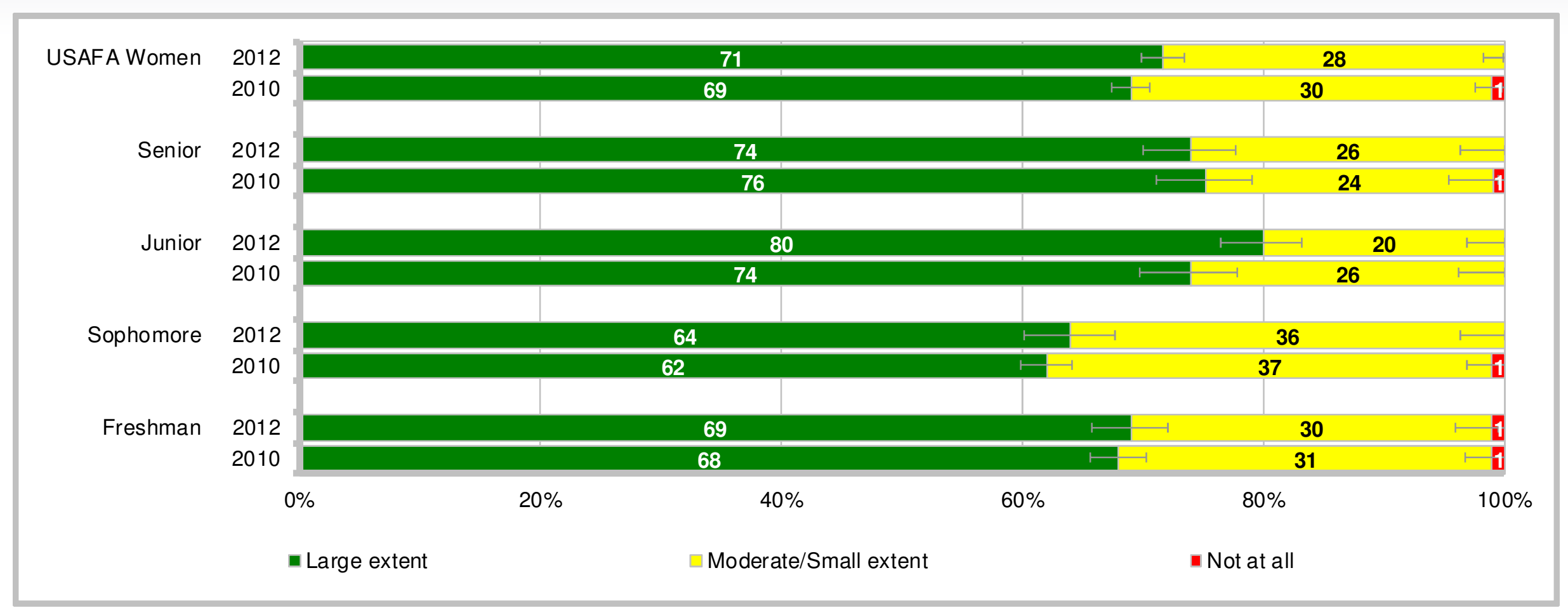

- $99 \%$ of women indicated they would step in and stop a situation that might lead to sexual assault to at least some extent; $<1 \%$ indicated not at all

- 2012 comparisons across years - no differences

- Juniors indicating large extent in 2012 higher than 2010; juniors indicating moderate/small extent in 2012 lower than 2010

- Class differences in 2012 - large extent led by juniors; moderate/small extent led by sophomores; not at all led by freshmen 


\section{You Would Step In and Stop a Situation That Might Lead to Sexual Assault} Percent of Men

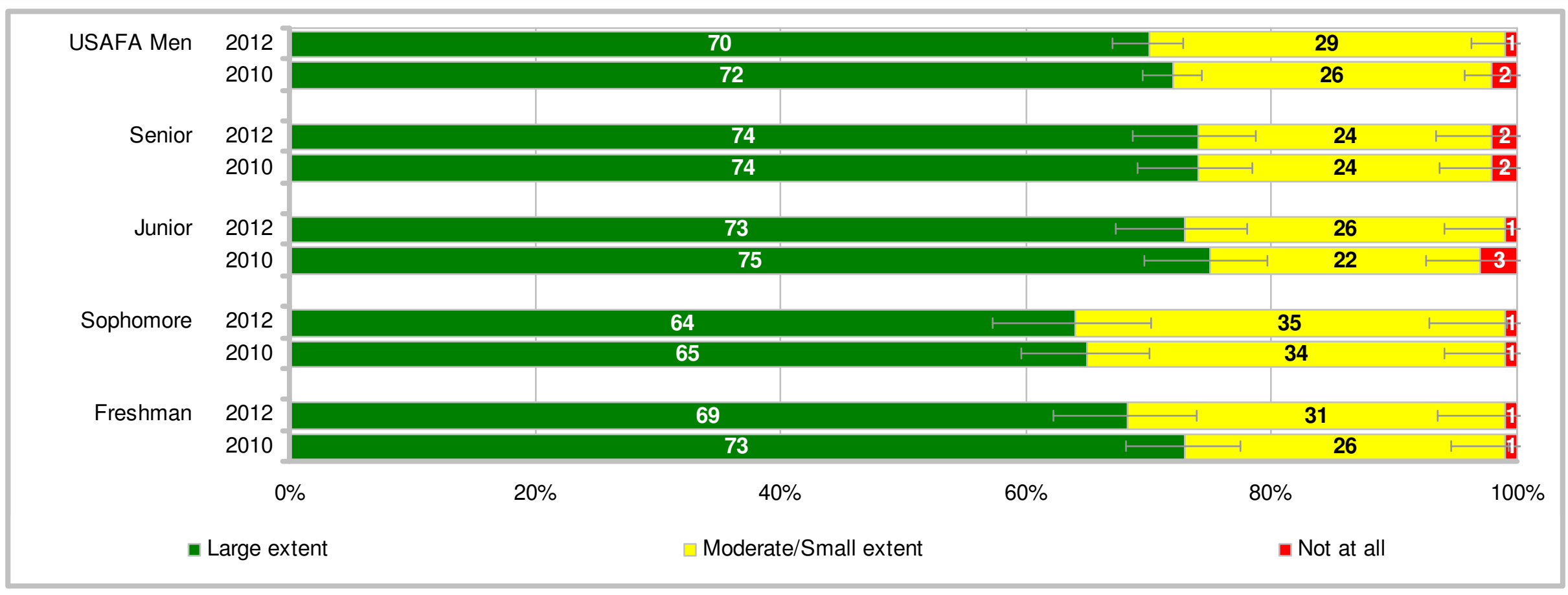

- $99 \%$ of men indicated they would step in and stop a situation that might lead to sexual assault to at least some extent; $1 \%$ indicated not at all

- 2012 comparisons across years - no differences

- Class differences in 2012 - no differences 


\section{You Would Point Out to Someone That They "Crossed the Line" With Comments or Jokes \\ Percent of Women}

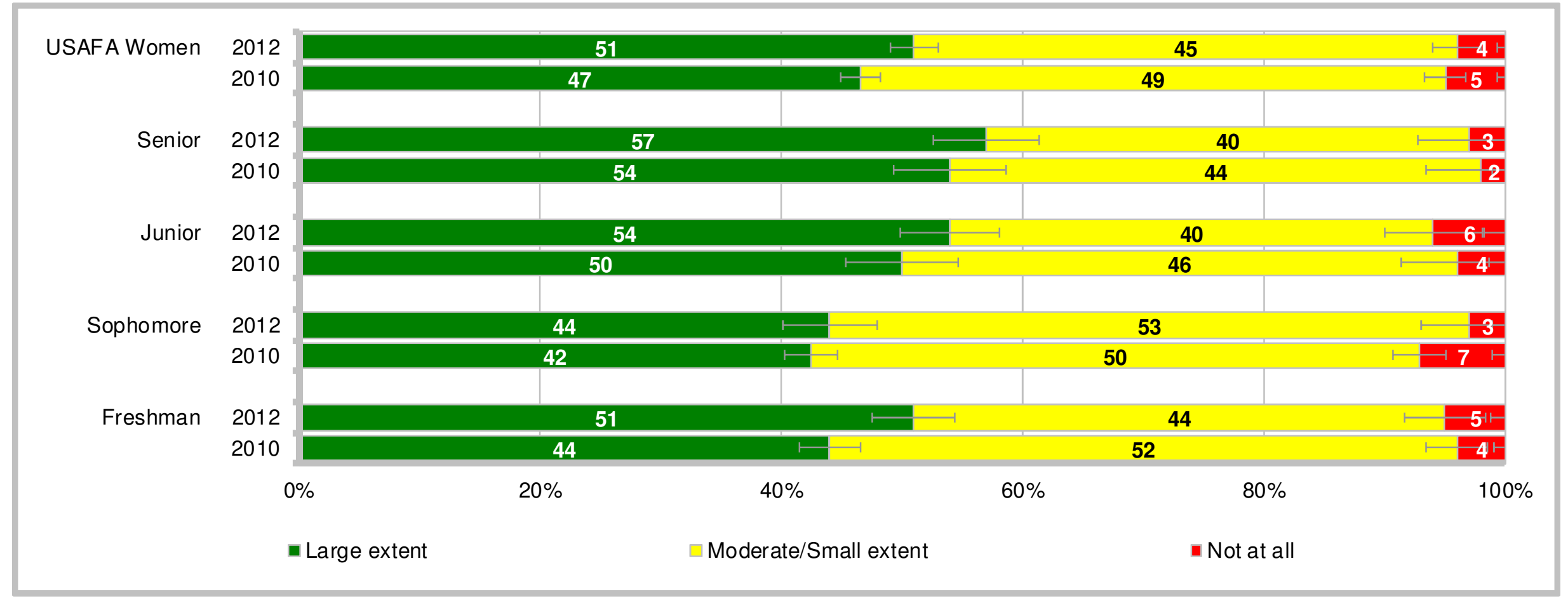

- $96 \%$ of women indicated they would point out to someone that they "crossed the line" with comments or jokes to at least some extent; $4 \%$ indicated not at all

- 2012 comparisons across years - large extent higher than 2010; moderate/small extent lower than 2010

- Freshmen indicating large extent in 2012 higher than 2010; freshmen indicating moderate/small extent in 2012 lower than 2010; sophomores indicating not at all in 2012 lower than 2010

- Class differences in 2012 - large extent led by seniors; moderate/small extent led by sophomores 


\section{You Would Point Out to Someone That They "Crossed the Line" With Comments or Jokes \\ Percent of Men}

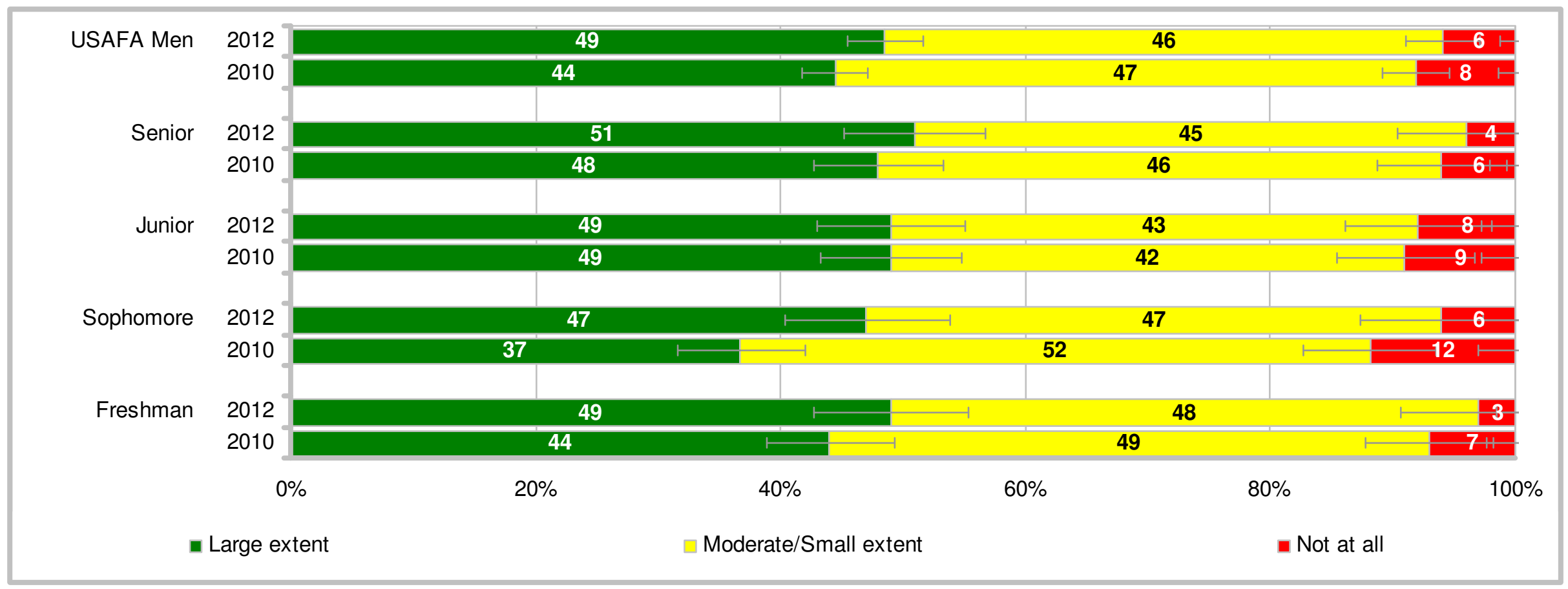

- $94 \%$ of men indicated they would point out to someone that they "crossed the line" with comments or jokes to at least some extent; $6 \%$ indicated not at all

- 2012 comparisons across years - large extent higher than 2010

- Sophomores indicating large extent in 2012 higher than 2010; sophomores and freshmen indicating not at all in 2012 lower than 2010

- Class differences in 2012 - no differences 


\section{You Would Seek Help From Chain of Command in Stopping Other Students Who Continue To Engage in Sexual Harassment}

\section{Percent of Women}

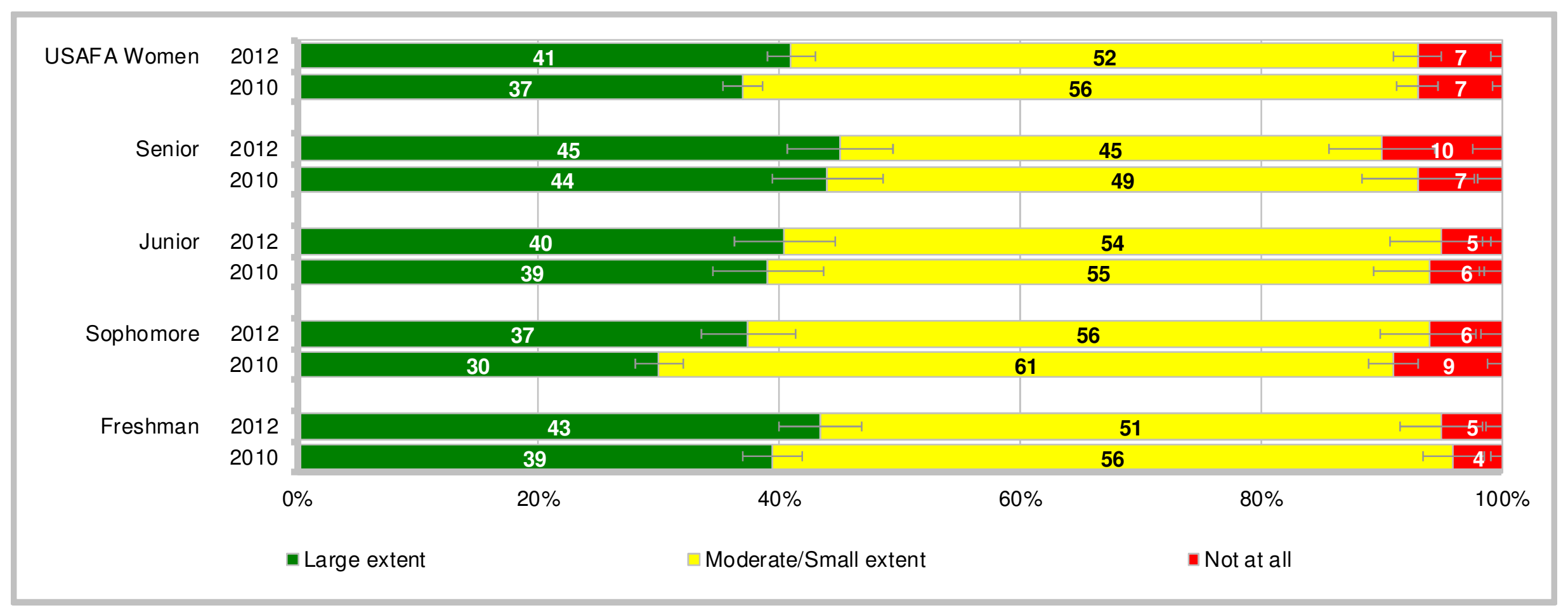

- $93 \%$ of women indicated they would seek help from the chain of command in stopping other students who continue to engage in sexual harassment to at least some extent; $7 \%$ indicated not at all

- 2012 comparisons across years - large extent higher than 2010; moderate/small extent lower than 2010

- Sophomores indicating large extent in 2012 higher than 2010; sophomores and freshmen indicating moderate/small extent in 2012 lower than 2010; sophomores indicating not at all in 2012 lower than 2010

- Class differences in 2012 - moderate/small extent led by sophomores; not at all led by seniors 


\section{You Would Seek Help From Chain of Command in Stopping Other Students Who Continue To Engage in Sexual Harassment \\ Percent of Men}

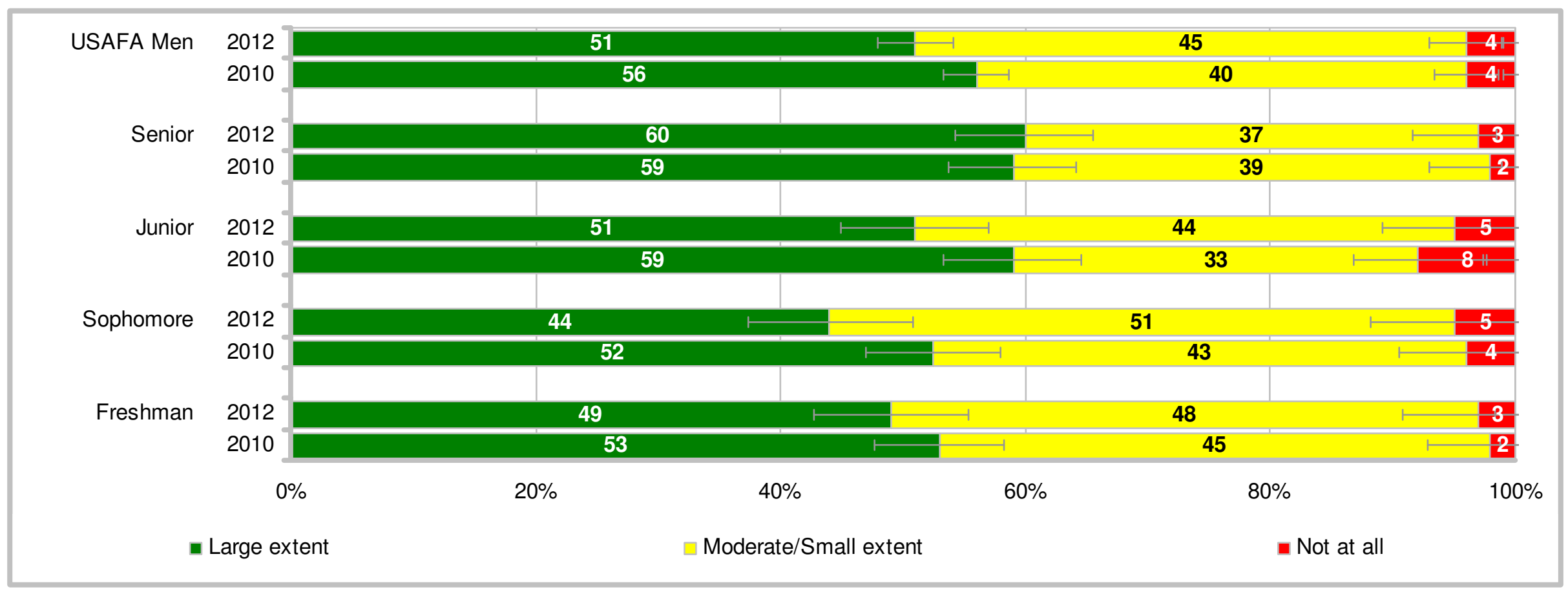

- $96 \%$ of men indicated they would seek help from the chain of command in stopping other students who continue to engage in sexual harassment to at least some extent; and $4 \%$ indicated not at all

- 2012 comparisons across years - large extent lower than 2010; moderate/small extent higher than 2010

- Juniors indicating moderate/small extent in 2012 higher than 2010

- Class differences in 2012 - large extent led by seniors 


\section{Service Academy Gender Relations Survey}

Appendix D: U.S. Coast Guard Academy

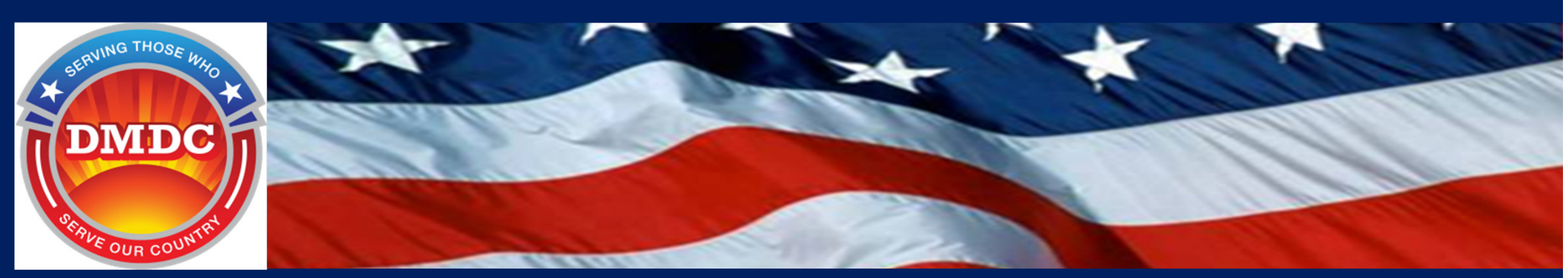




\section{Appendix D \\ U.S. Coast Guard Academy \\ Contents}

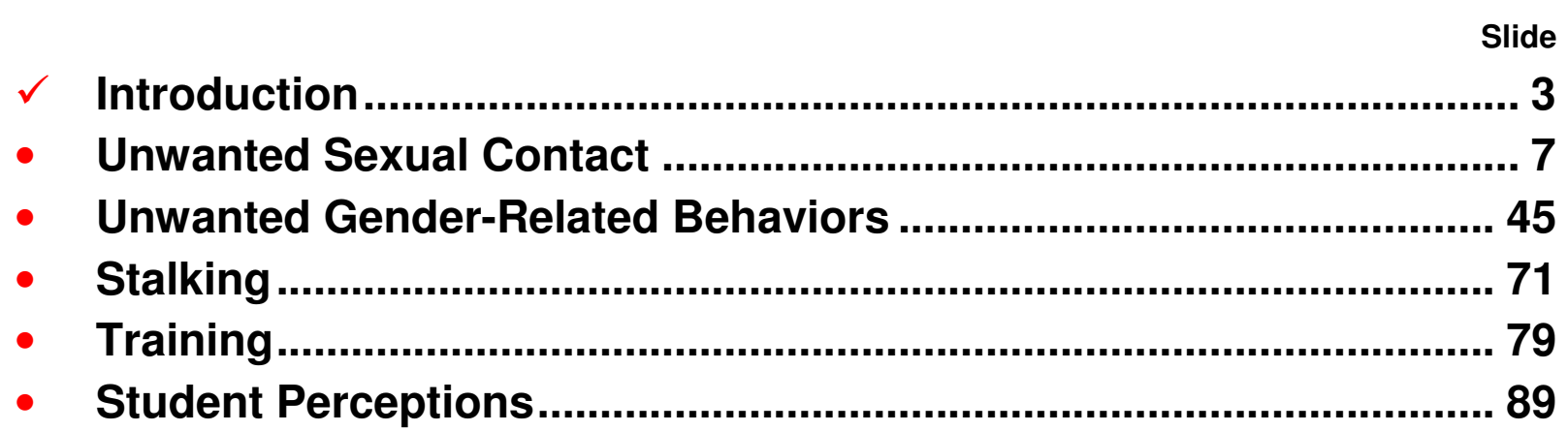




\section{Introduction}

\section{- Paper survey administered to cadets in March 2012}

- 307 female and 697 male students surveyed, weighted response rate of $88 \%$ and $76 \%$, respectively

\section{- Appendix includes results from survey items related to:}

- Results of unwanted sexual contact experienced in the past academic year

- For women who had experienced unwanted sexual contact in the past academic year, details of the incident that had the greatest impact (characteristics of the offender, location, actions taken, reporting, etc.). Details for men are not reportable due to the small number of men who experienced unwanted sexual contact.

- Unwanted sexual contact experienced prior to entering the Academy

- Unwanted gender-related behaviors experienced in the past academic year (sexual harassment, crude and offensive behavior, unwanted sexual attention, sexual coercion, and sexist behavior)

- For students who experienced unwanted gender-related behavior in the past academic year, details of the incident that had the greatest impact (characteristics of the offender, reporting)

- Stalking experienced in the past academic year

- Training received in preventing and responding to sexual assault and sexual harassment

- Student perceptions about culture and climate at the Academy

\section{- For each survey item, briefing includes the following:}

- Graphic displays of overall results by gender, survey year, and class year

- Tables showing results for grouped items by reporting categories (e.g., gender, survey year, and class year) 


\section{Introduction to Briefing Slides}

\section{- Graphics showing results by reporting categories (gender, survey year, and class year)}

- Statistical tests are used to compare each subgroup to previous survey years and other class years

- For items with trends, 2012 survey year is compared to the other survey years $(2006,2008,2010)$

- For the current 2012 survey year, each class year (e.g., freshmen) is compared to the average of the other class years (sophomore, junior, senior)

- Results are presented on the bottom of the slide for survey year and class year comparisons that are statistically significant

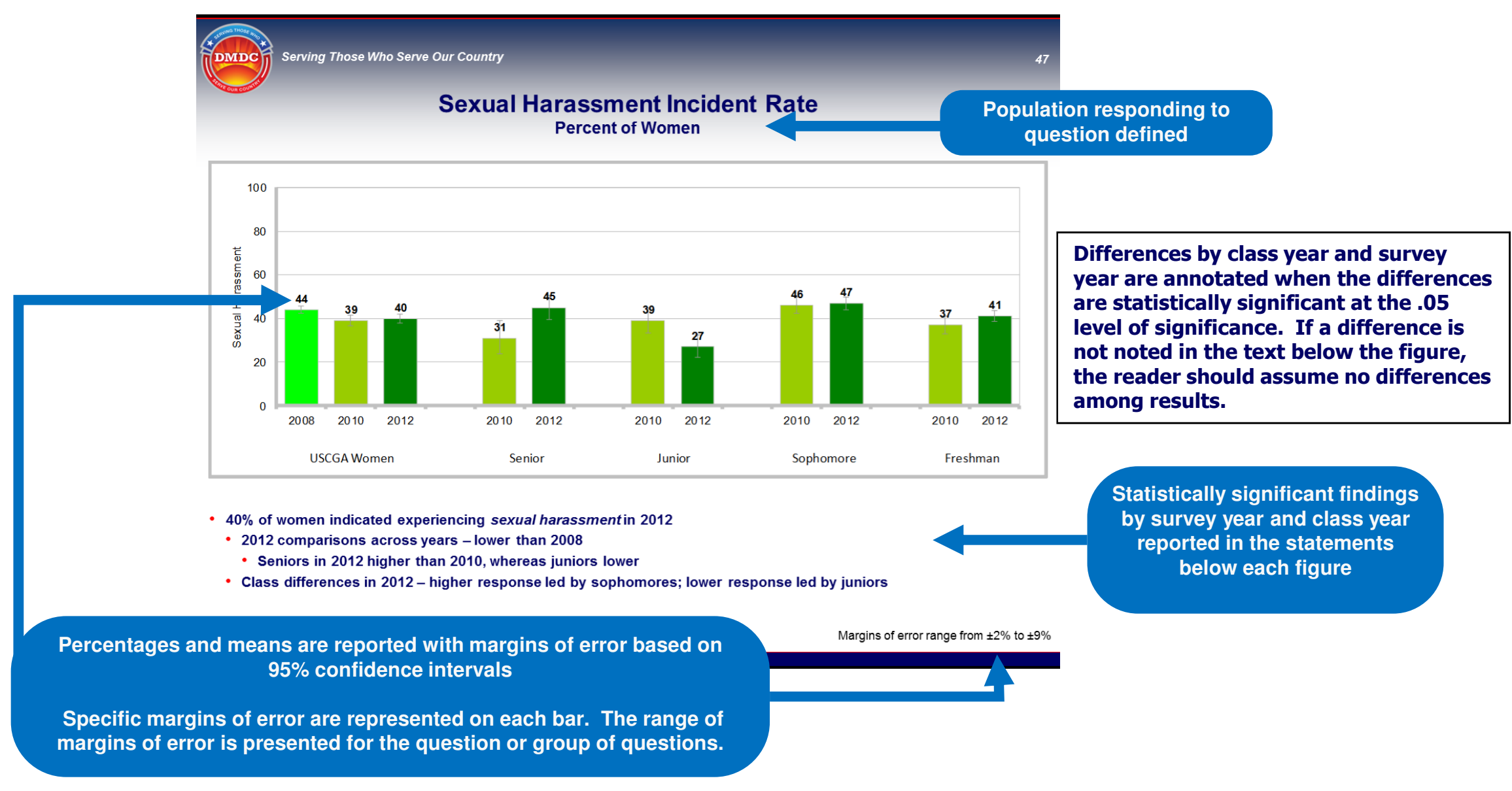




\section{Introduction to Briefing Slides}

\section{- Tables showing results for grouped items by reporting categories (gender, survey year, and class year)}

- Statistical tests are used to compare each subgroup to previous survey years and other class years

- For items with trends, 2012 survey year is compared to the other survey years $(2006,2008,2010)$

- For the current 2012 survey year, each class year (e.g., freshmen) is compared to the average of the other class years (sophomore, junior, senior)

- Results are presented within the table for survey year and class year comparisons that are statistically significant

Most Frequently Selected Reasons for

Not Reporting Unwanted Sexual Contact Situation Percent of Women Who Experienced Unwanted Sexual Contact and Did Not Report It

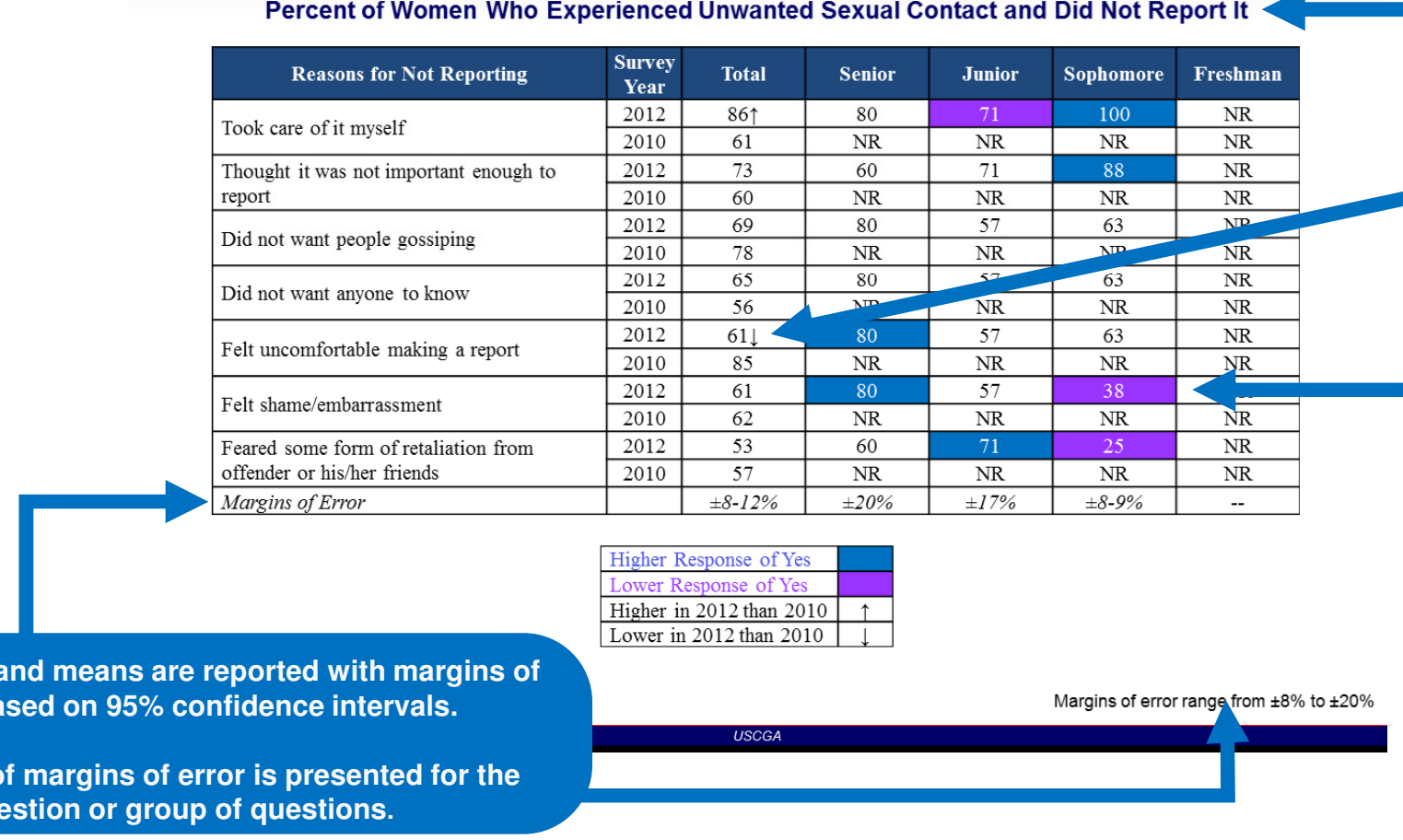

Population responding to question defined

Arrows indicate whether there is a statistically significant change between 2012 and 2010.

Color coding indicates differences by class year.

Only class differences in 2012 are reported.

Differences by class year and survey year are annotated when the differences are statistically significant at the .05 level of significance. If a difference is not noted within the table, the reader should assume no differences among results. 


\section{Contents}

$\checkmark$ Unwanted Sexual Contact ............................................................. 7

- Most Serious Behaviors Experienced.......................................... 11

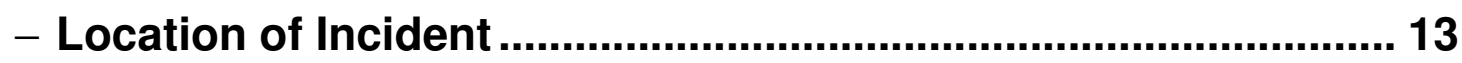

- Summer Experience....................................................................... 15

- Characteristics of Offender............................................................. 17

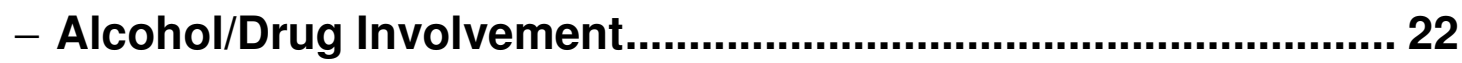

- Use of Threats/Force ................................................................ 27

- Experience of Sexual Harassment/Stalking ................................ 32

- Consequences of the Situation ..................................................... 25

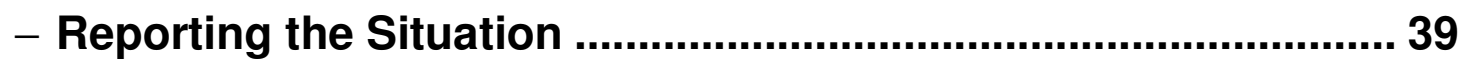

- Experience of Unwanted Sexual Contact Prior to Entering the Academy ............................................................................... 47

- Unwanted Gender-Related Behaviors ............................................. 45

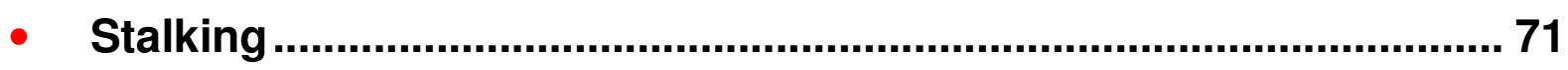

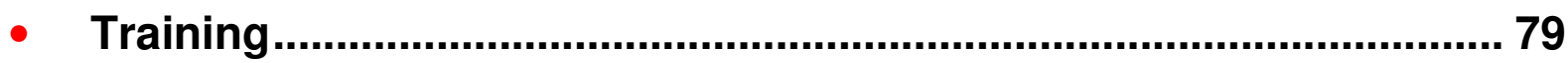

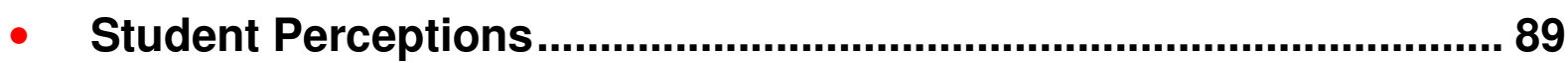




\section{Unwanted Sexual Contact Incident Rates}

\section{- Definition and measure of unwanted sexual contact:}

- The 2012 SAGR survey includes a measure of unwanted sexual contact (i.e., sexual assault). Although this term does not appear in the Uniform Code of Military Justice $(\mathrm{UCMJ})$, it is used to refer to a range of activities that is used as an umbrella term intended to include certain acts prohibited by the UCMJ.

- Unwanted sexual contact is measured in the 2012 SAGR survey by asking students to refer to experiences since June 2011 in which they experienced any of the following intentional sexual contacts that were against their will or which occurred when they did not or could not consent in which someone...

- Sexually touched them (e.g., intentional touching of genitalia, breasts, or buttocks) or made them sexually touch someone,

- Attempted to make them have sexual intercourse, but was not successful,

- Made them have sexual intercourse,

- Attempted to make them perform or receive oral sex, anal sex, or penetration by a finger or object, but was not successful, or

- Made them perform or receive oral sex, anal sex, or penetration by a finger or object.

- A student is counted in the unwanted sexual contact incident rate if he or she replied "yes" to any of the behaviors listed.

- The 2012 SAGR survey was administered in March and April 2012. The timeframe looking back to June 2011 is intended to include behaviors experienced in the majority of Academic Program Year 2011-2012 to be consistent with the timeframe for reporting incidents of sexual assault at the Academies. 


\section{Unwanted Sexual Contact Incident Rate Percent of Women}

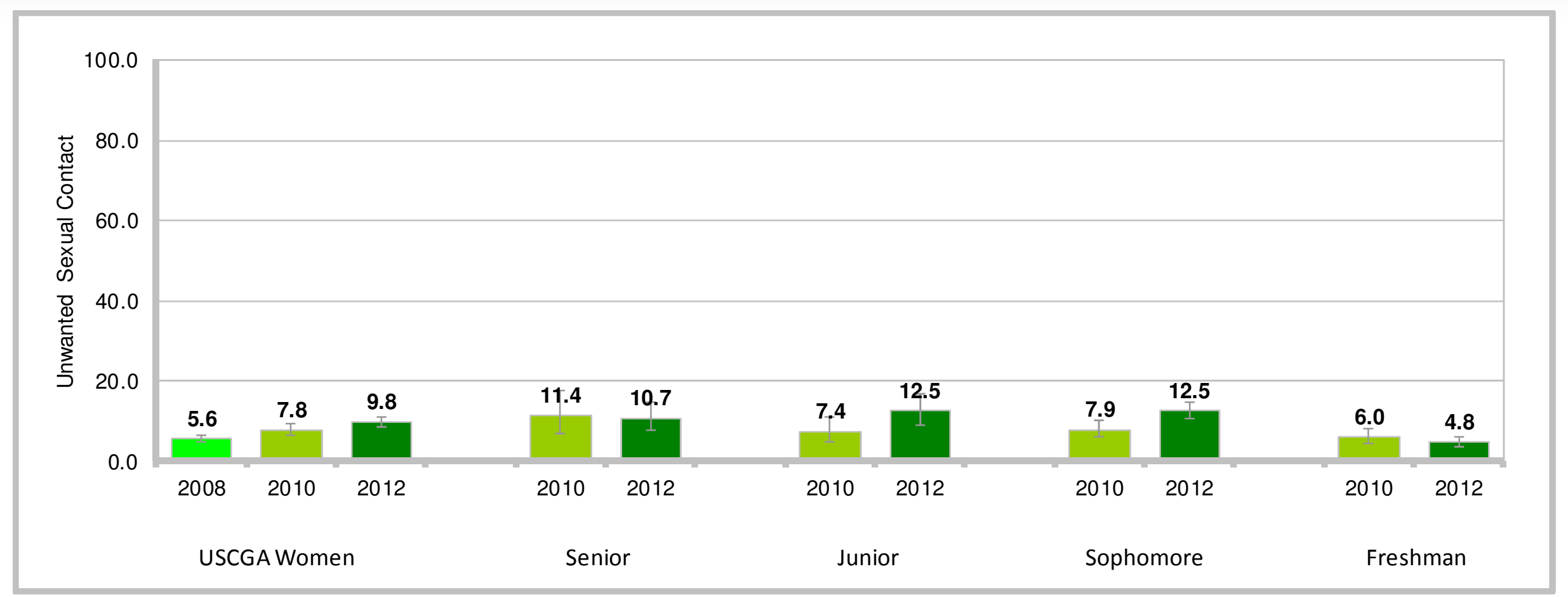

- $9.8 \%$ of women indicated experiencing unwanted sexual contact in 2012

- 2012 comparisons across years - higher than 2010, 2008

- Juniors and sophomores in 2012 higher than 2010

- Class differences in 2012 - higher response led by sophomores*; lower response led by freshmen

*Note that both $12.5 \%$ of junior and sophomore women indicated experiencing unwanted sexual contact in 2012. The percentage for junior women is not significantly different from the average of the other class years due to a higher margin of error for junior women responding to this question. 


\section{Unwanted Sexual Contact Incident Rate}

Percent of Men

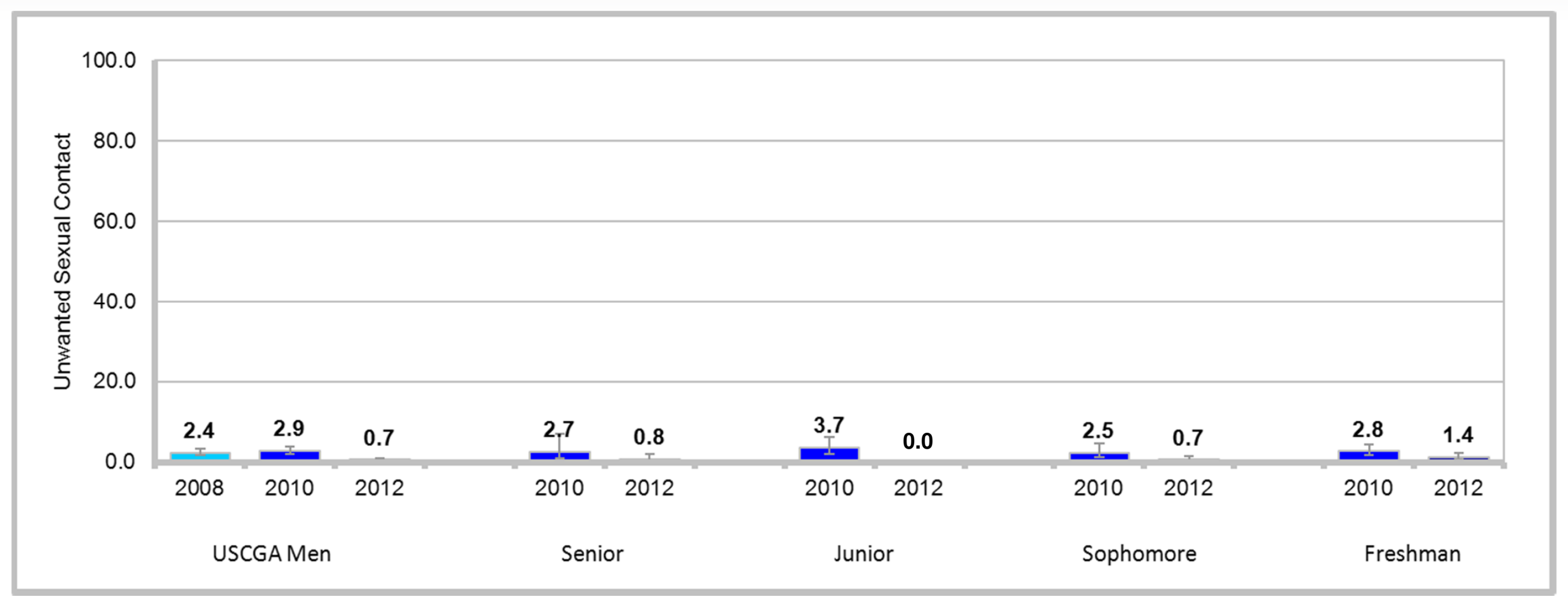

- $0.7 \%$ of men indicated experiencing unwanted sexual contact in 2012

- 2012 comparisons across years - lower than 2010, 2008

- Juniors and sophomores in 2012 lower than 2010

- Class differences in 2012 - lower response led by juniors 


\section{Contents}

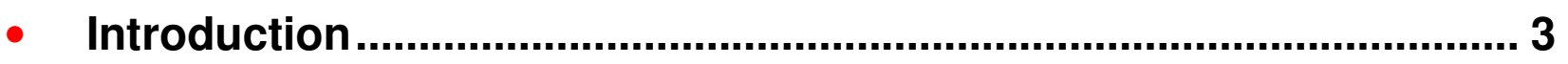

- Unwanted Sexual Contact .................................................................. 7

$\checkmark$ Most Serious Behaviors Experienced...................................... 11

- Location of Incident ..................................................................... 13

- Summer Experience.................................................................... 15

- Characteristics of Offender....................................................... 17

- Alcohol/Drug Involvement.......................................................... 22

- Use of Threats/Force ................................................................ 27

- Experience of Sexual Harassment/Stalking .............................. 32

- Consequences of the Situation ................................................. 35

- Reporting the Situation ............................................................ 39

- Experience of Unwanted Sexual Contact Prior to Entering

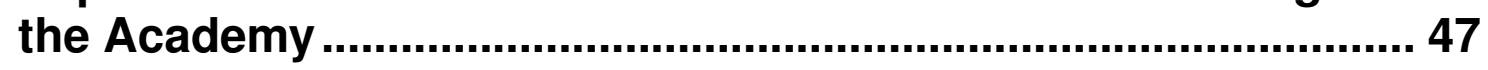

- Unwanted Gender-Related Behaviors ............................................... 49

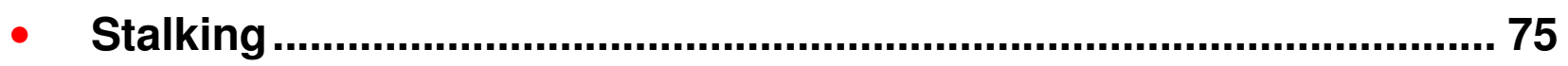

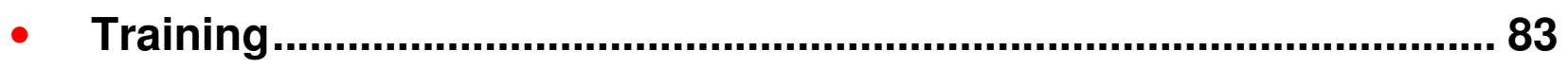

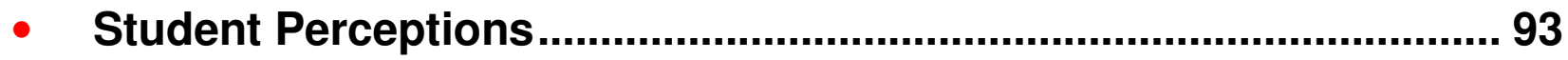




\section{Most Serious Unwanted Sexual Contact Behaviors Experienced Percent of Women Who Experienced Unwanted Sexual Contact}

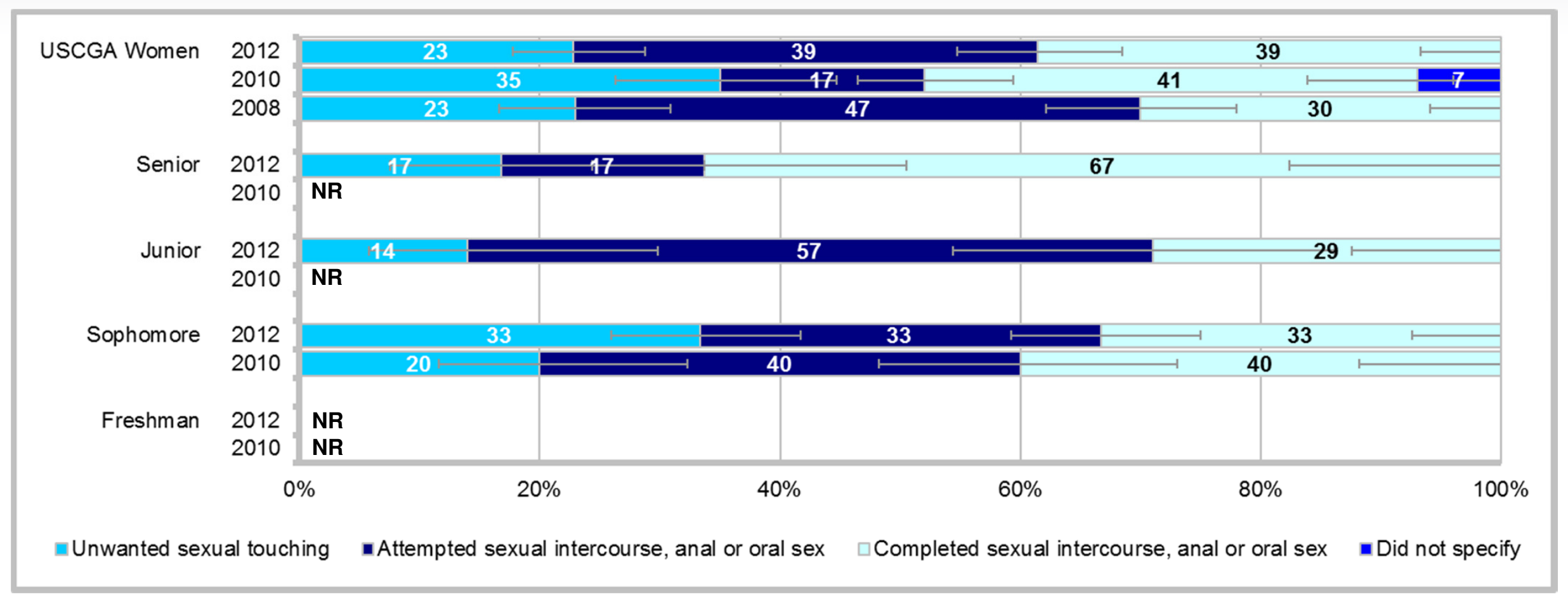

- Of the $9.8 \%$ of women who experienced USC, $23 \%$ indicated experiencing touching; $39 \%$ indicated experiencing attempted sex; $39 \%$ indicated experiencing completed sex; and $0 \%$ did not specify

- 2012 comparisons across years - touching lower than 2010; attempted sex higher than 2010; did not specify lower than 2010

- Sophomores indicating touching only in 2012 higher than 2010

- Class differences in $\mathbf{2 0 1 2}$ - higher response of touching led by sophomores; higher response of attempted sex led by juniors; higher response of completed sex led by seniors 


\section{Contents}

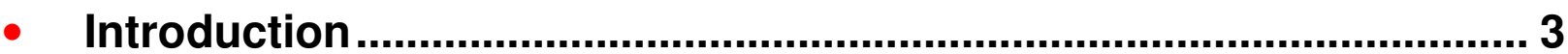

- Unwanted Sexual Contact ..................................................................... 7

- Most Serious Behaviors Experienced........................................ 11

$\checkmark$ Location of Incident ...................................................................... 13

- Summer Experience.................................................................... 15

- Characteristics of Offender...................................................... 17

- Alcohol/Drug Involvement............................................................. 22

- Use of Threats/Force ................................................................... 27

- Experience of Sexual Harassment/Stalking ............................... 32

- Consequences of the Situation ..................................................... 35

- Reporting the Situation .............................................................. 39

- Experience of Unwanted Sexual Contact Prior to Entering the Academy ...................................................................................... 47

- Unwanted Gender-Related Behaviors .............................................. 49

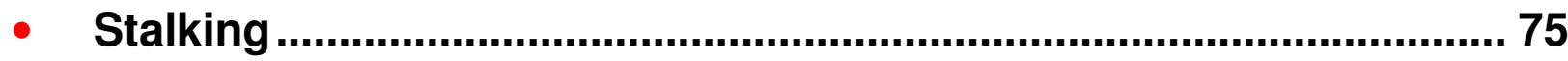

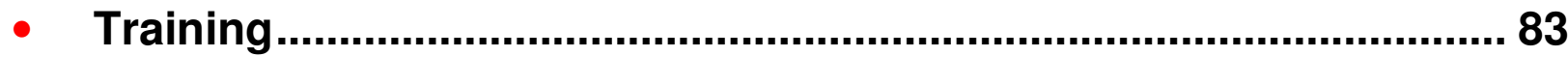

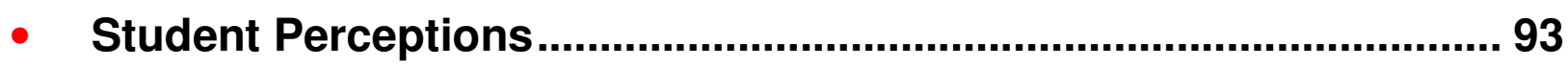




\section{Location of Unwanted Sexual Contact Incident Percent of Women Who Experienced Unwanted Sexual Contact}

\begin{tabular}{|c|c|c|c|c|c|}
\hline Location of Incident & Total & Senior & Junior & Sophomore & Freshman \\
\hline On Academy grounds in dormitory/living area & 66 & 33 & 50 & 89 & NR \\
\hline On Academy grounds not in dormitory/living area & 9 & 17 & 17 & 0 & NR \\
\hline $\begin{array}{l}\text { Off Academy grounds at a social event (such as a } \\
\text { party) }\end{array}$ & 21 & 50 & 17 & 11 & NR \\
\hline $\begin{array}{l}\text { Off Academy grounds at an Academy sponsored } \\
\text { event }\end{array}$ & 8 & 17 & NR & 11 & NR \\
\hline Off Academy grounds other & 20 & 17 & 17 & 22 & NR \\
\hline Margins of Error & $\pm 5-8 \%$ & $\pm 17-18 \%$ & $\pm 18 \%$ & $\pm 0-8 \%$ & -- \\
\hline
\end{tabular}

\begin{tabular}{|l|l|}
\hline Higher Response of Yes & \\
\hline Lower Response of Yes & \\
\hline
\end{tabular}

- New question in 2012; no trend data available 


\section{Contents}

Slide

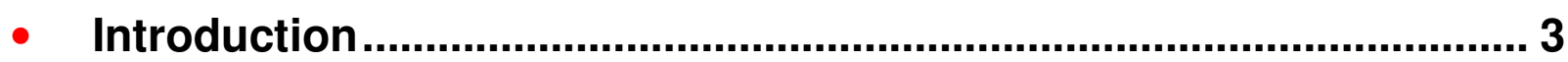

- Unwanted Sexual Contact ............................................................... 7

- Most Serious Behaviors Experienced....................................... 11

- Location of Incident ...................................................................... 13

$\checkmark$ Summer Experience.................................................................... 15

- Characteristics of Offender........................................................ 17

- Alcohol/Drug Involvement.......................................................... 22

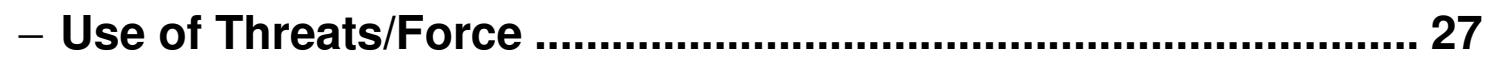

- Experience of Sexual Harassment/Stalking ............................... 32

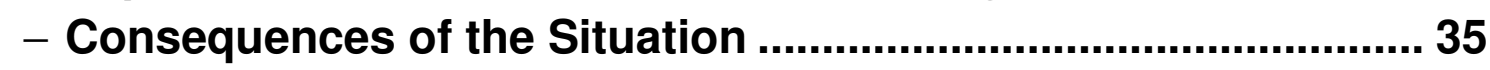

- Reporting the Situation .............................................................. 39

- Experience of Unwanted Sexual Contact Prior to Entering

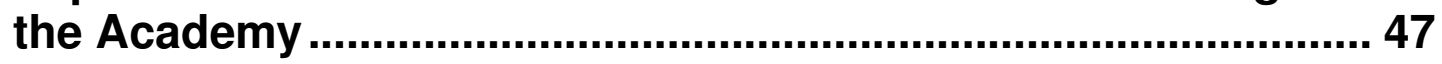

- Unwanted Gender-Related Behaviors ................................................ 49

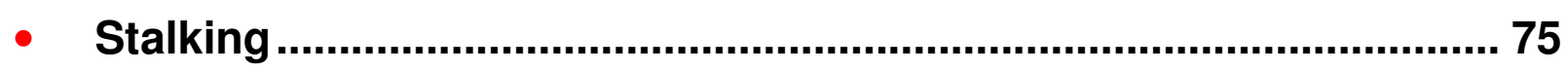

- Training ................................................................................................. 83

- Student Perceptions.................................................................... 93 


\section{Unwanted Sexual Contact Incident Occurred During Summer Experience/Training/Sea Duty \\ Percent of Women Who Experienced Unwanted Sexual Contact}

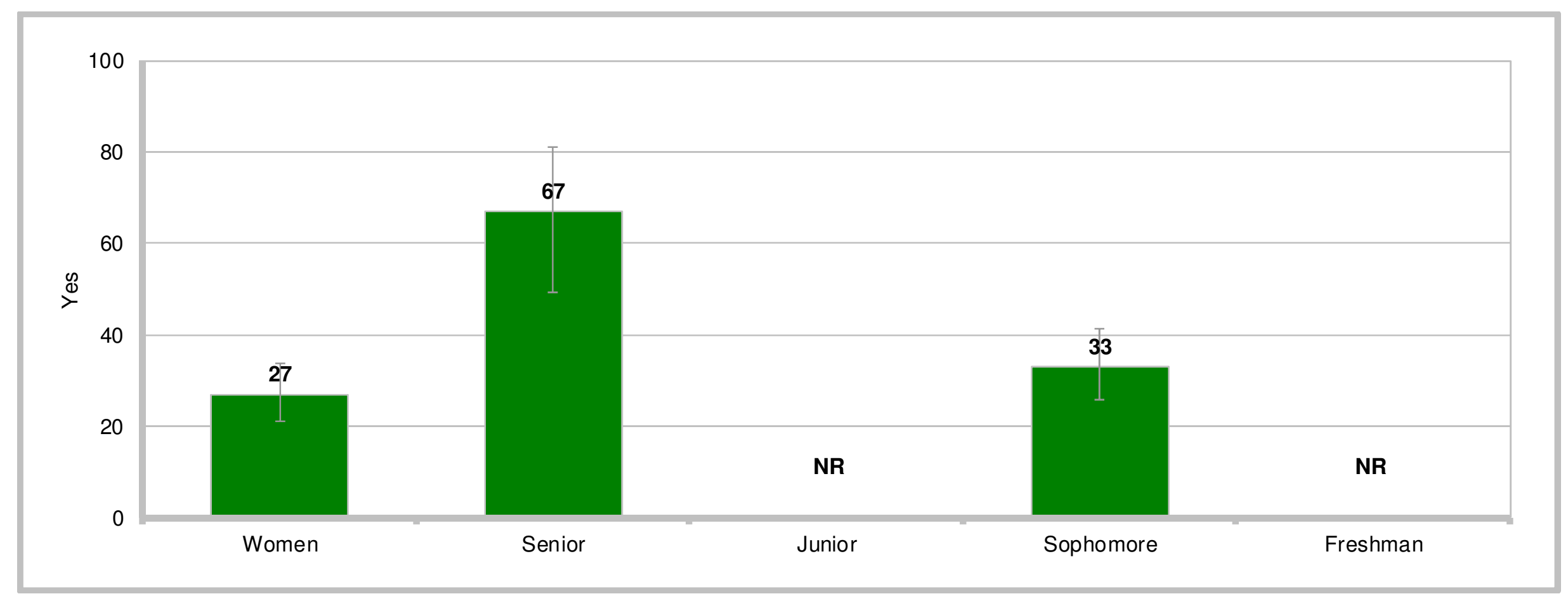

- Of the $9.8 \%$ of women who experienced USC, $27 \%$ indicated the offense occurred during summer experience/ training/sea duty

- 2012 comparisons across years - new question in 2012; no trend data available

- Class differences in $\mathbf{2 0 1 2}$ - higher response led by seniors 


\section{Contents}

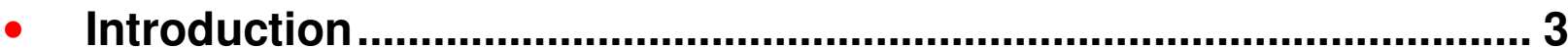

- Unwanted Sexual Contact ................................................................... 7

- Most Serious Behaviors Experienced........................................... 11

- Location of Incident ....................................................................... 13

- Summer Experience...................................................................... 15

$\checkmark$ Characteristics of Offender......................................................... 17

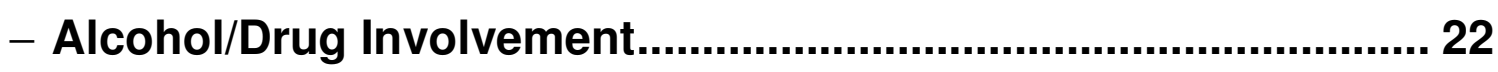

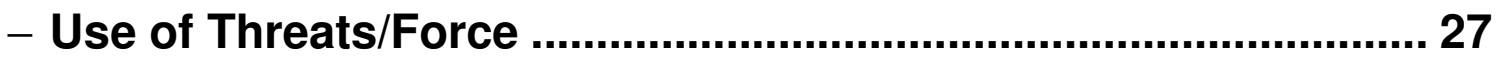

- Experience of Sexual Harassment/Stalking ............................... 32

- Consequences of the Situation ................................................ 35

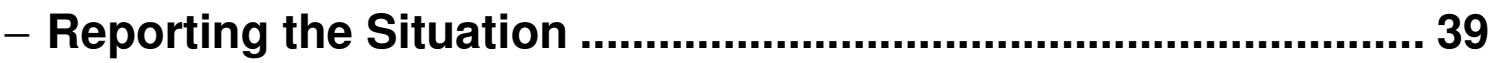

- Experience of Unwanted Sexual Contact Prior to Entering the Academy ...................................................................................... 47

- Unwanted Gender-Related Behaviors .............................................. 49

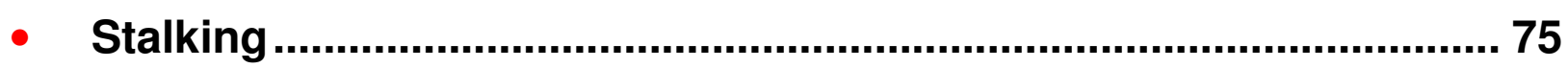

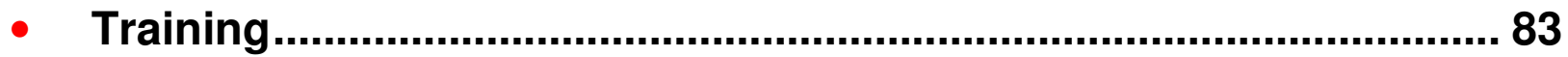

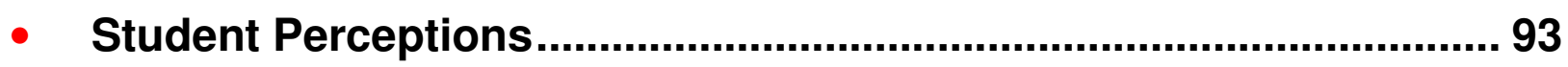




\section{Combinations of Offender Affiliations} Percent of Women Who Experienced Unwanted Sexual Contact

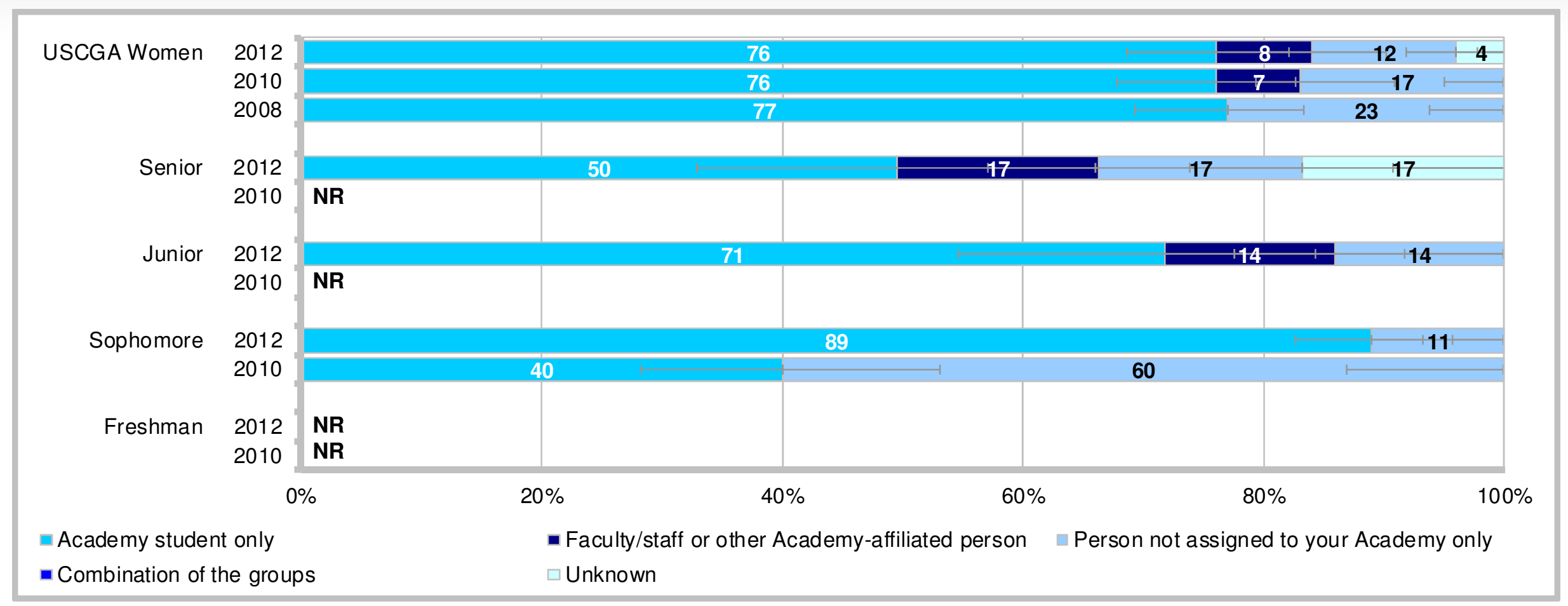

- Of the $9.8 \%$ of women who experienced USC, $76 \%$ indicated the offender was Academy student only; $8 \%$ indicated faculty/staff; $12 \%$ indicated person not assigned to the Academy; $0 \%$ indicated combination of the groups; and $4 \%$ indicated the offender was unknown

- 2012 comparisons across years - faculty/staff higher than 2008; person not assigned to the Academy lower than 2008; unknown higher than 2010, 2008

- Sophomores indicating Academy student only in 2012 higher than 2010; sophomores indicating person not assigned to the Academy in 2012 lower than 2010

- Class differences in 2012 - Academy student only led by sophomores 


\section{Gender of the Offender Percent of Women Who Experienced Unwanted Sexual Contact}

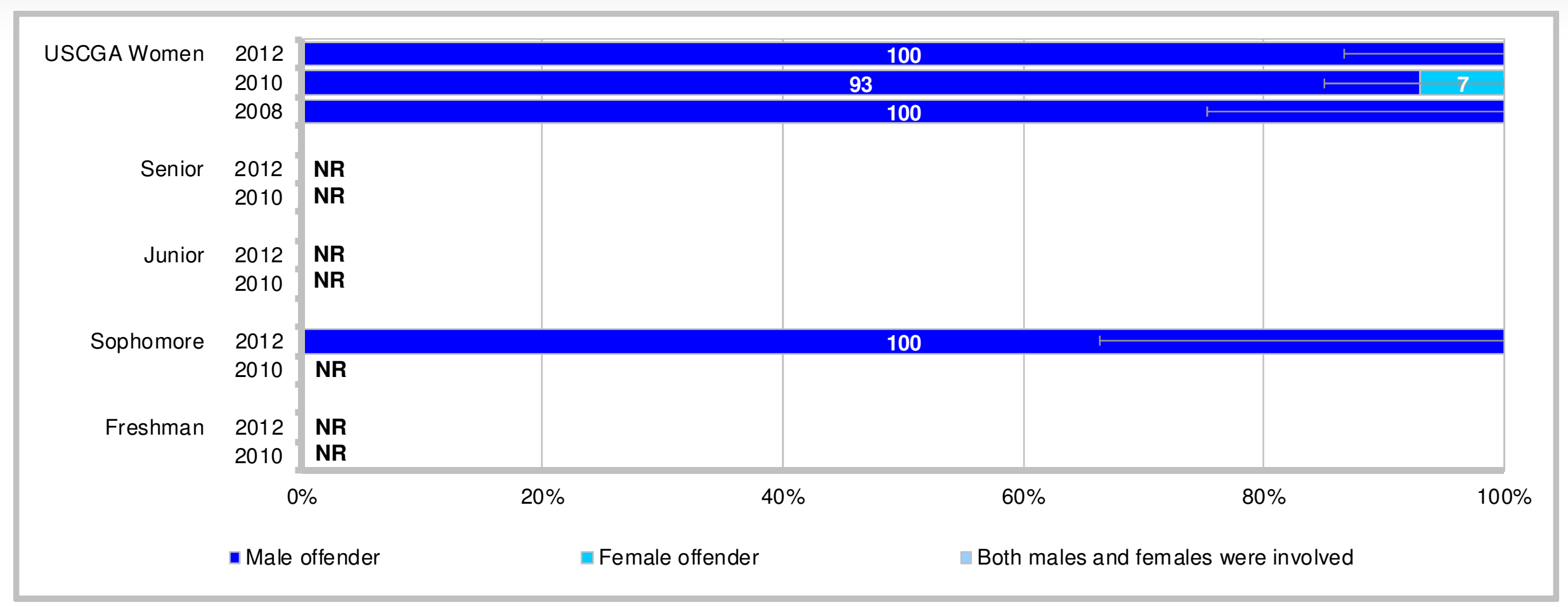

- Of the $9.8 \%$ of women who experienced USC, $100 \%$ indicated the offender was male - 2012 comparisons across years - male higher than 2010; female lower than 2010

- Class differences in 2012 - no differences 


\section{Multiple Offenders Involved}

Percent of Women Who Experienced Unwanted Sexual Contact

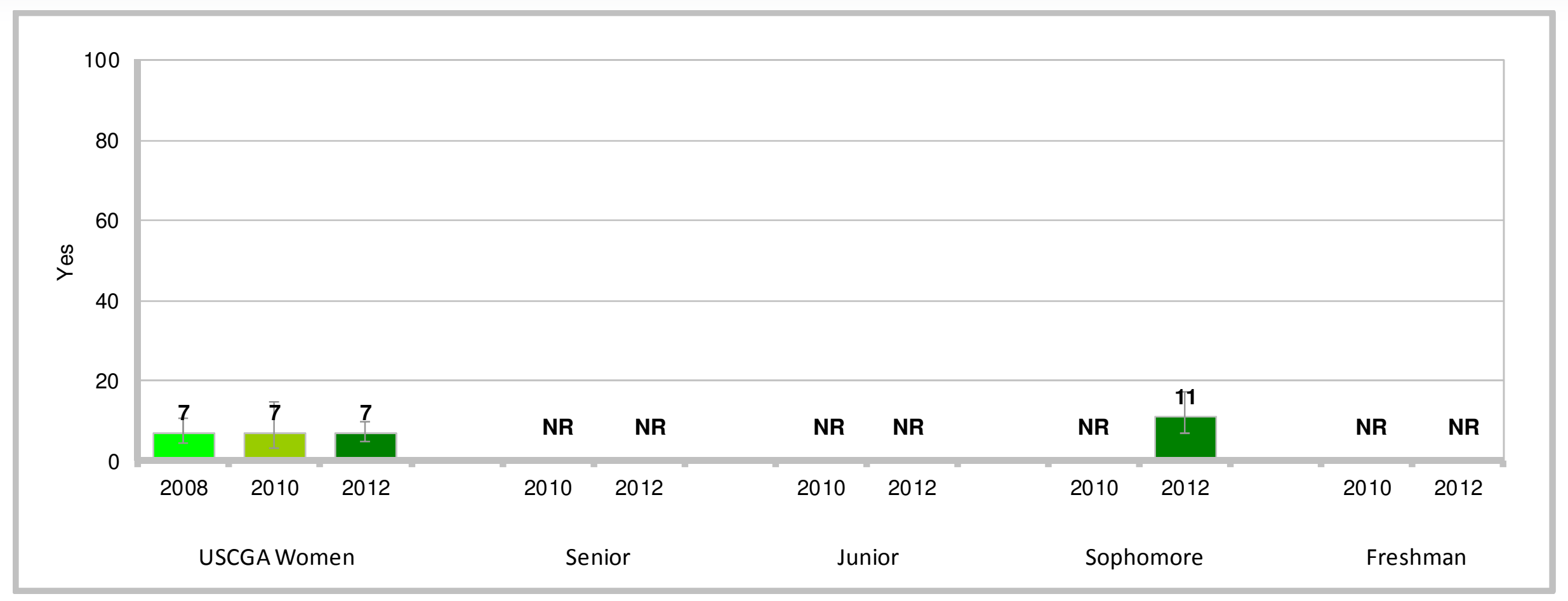

- Of the $9.8 \%$ of women who experienced USC, $7 \%$ indicated multiple offenders were involved

- 2012 comparisons across years - no differences

- Class differences in 2012 - no differences 


\section{Offender Was a "Creeper" (i.e., someone who is socially awkward)} Percent of Women Who Experienced Unwanted Sexual Contact Involving an Academy Student

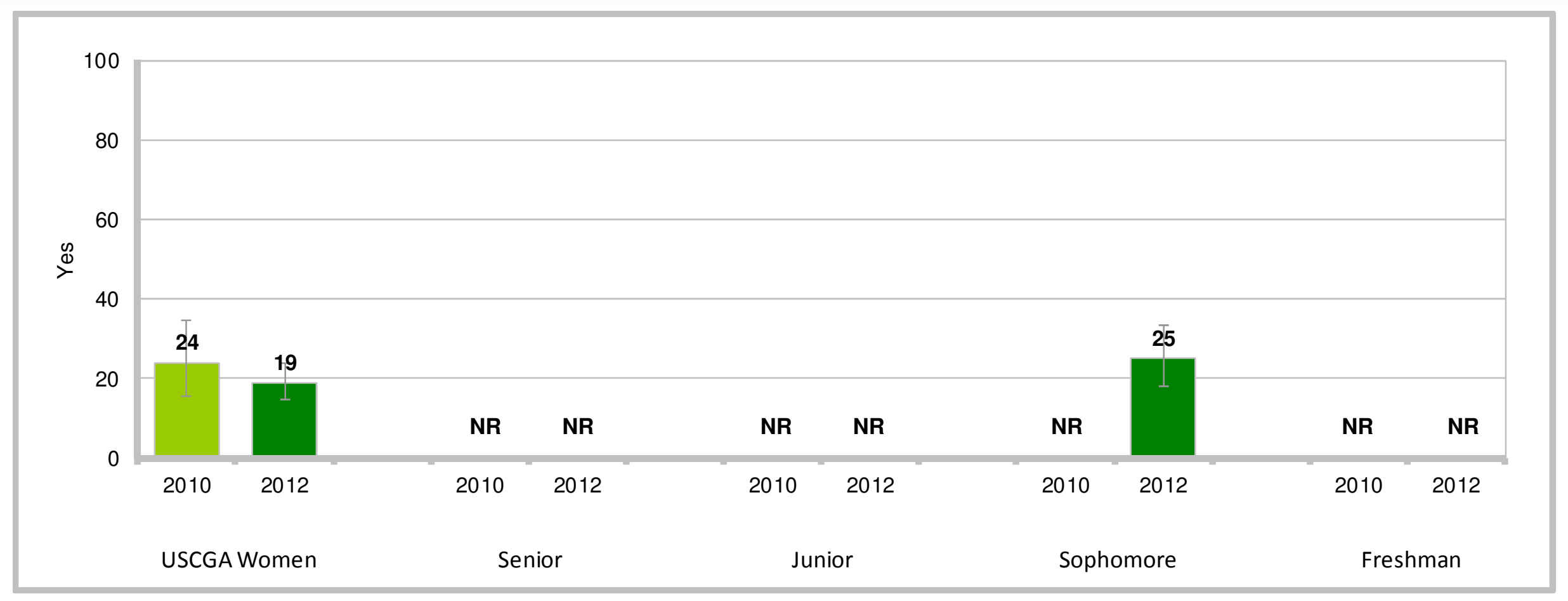

- Of the $9.8 \%$ of women who experienced USC, $19 \%$ indicated the offender was a "creeper"

- 2012 comparisons across years - no differences

- Class differences in 2012 - no differences 


\section{Contents}

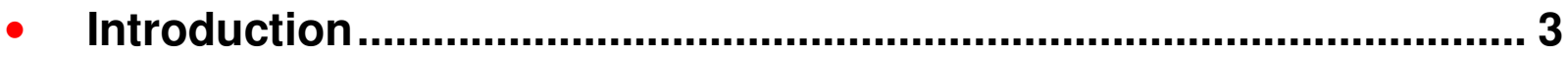

- Unwanted Sexual Contact ............................................................... 7

- Most Serious Behaviors Experienced....................................... 11

- Location of Incident ..................................................................... 13

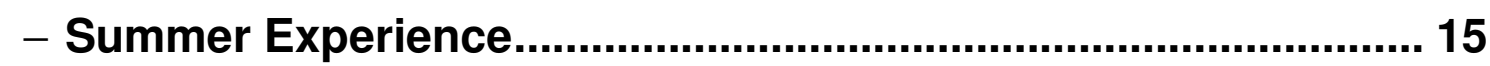

- Characteristics of Offender....................................................... 17

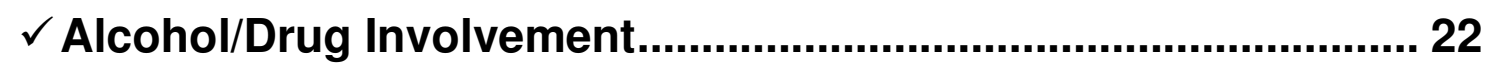

- Use of Threats/Force .............................................................. 27

- Experience of Sexual Harassment/Stalking ............................... 32

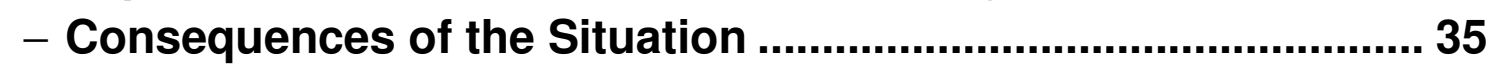

- Reporting the Situation ............................................................ 39

- Experience of Unwanted Sexual Contact Prior to Entering

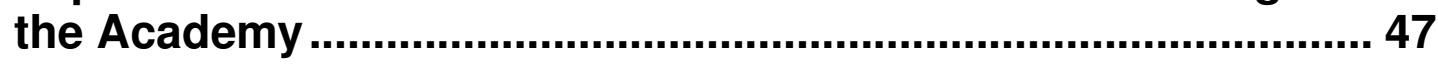

- Unwanted Gender-Related Behaviors ................................................ 49

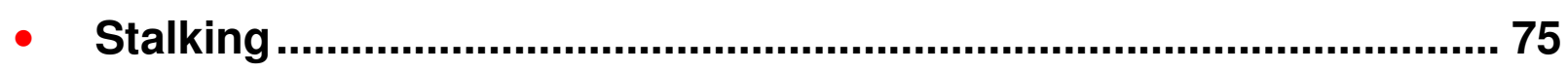

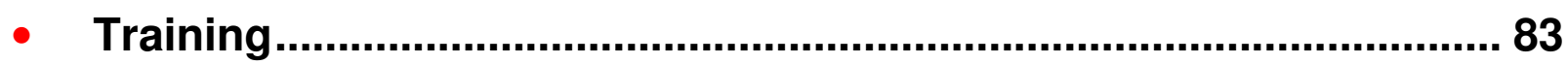

- Student Perceptions.................................................................... 93 


\section{Use of Alcohol and/or Drugs in the Incident Percent of Women Who Experienced Unwanted Sexual Contact}

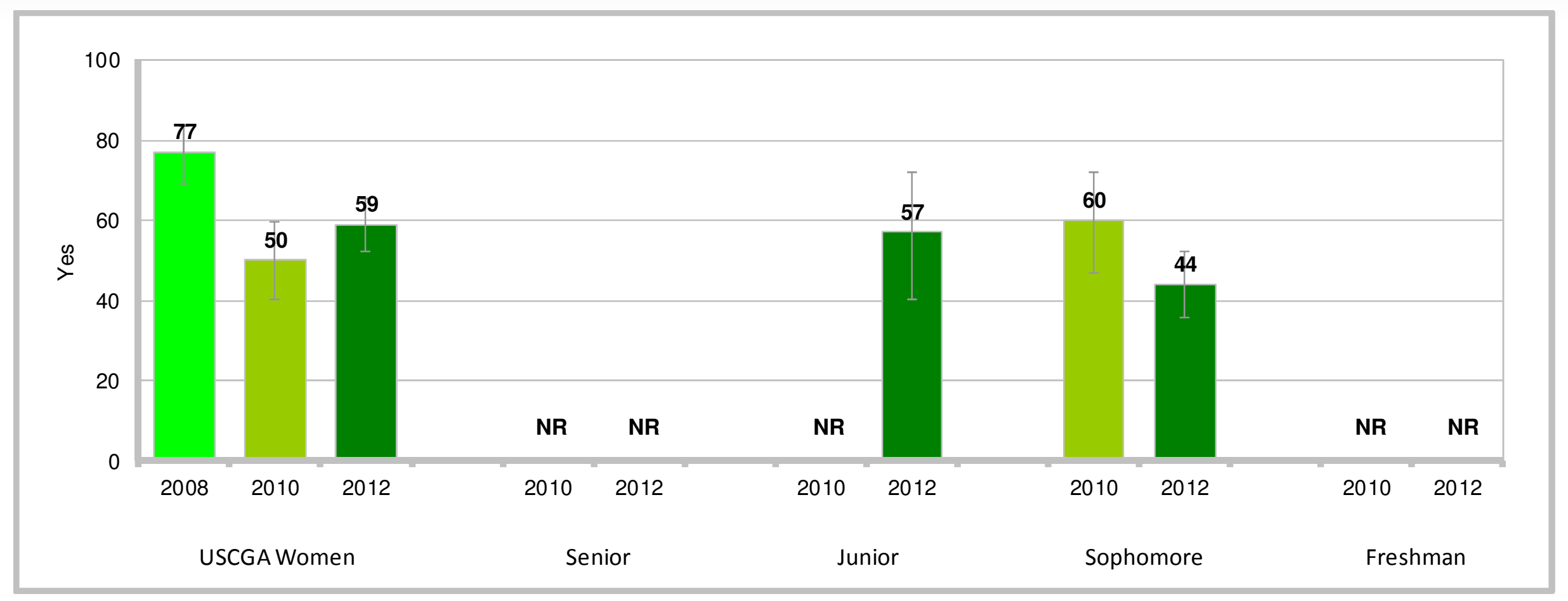

- Of the $9.8 \%$ of women who experienced USC, $59 \%$ indicated alcohol and/or drugs were involved

- 2012 comparisons across years - lower than 2008

- Sophomores in 2012 lower than 2010

- Class differences in 2012 - lower response led by sophomores 


\section{Use of Alcohol Before the Incident}

\section{Percent of Women Who Experienced Unwanted Sexual Contact}

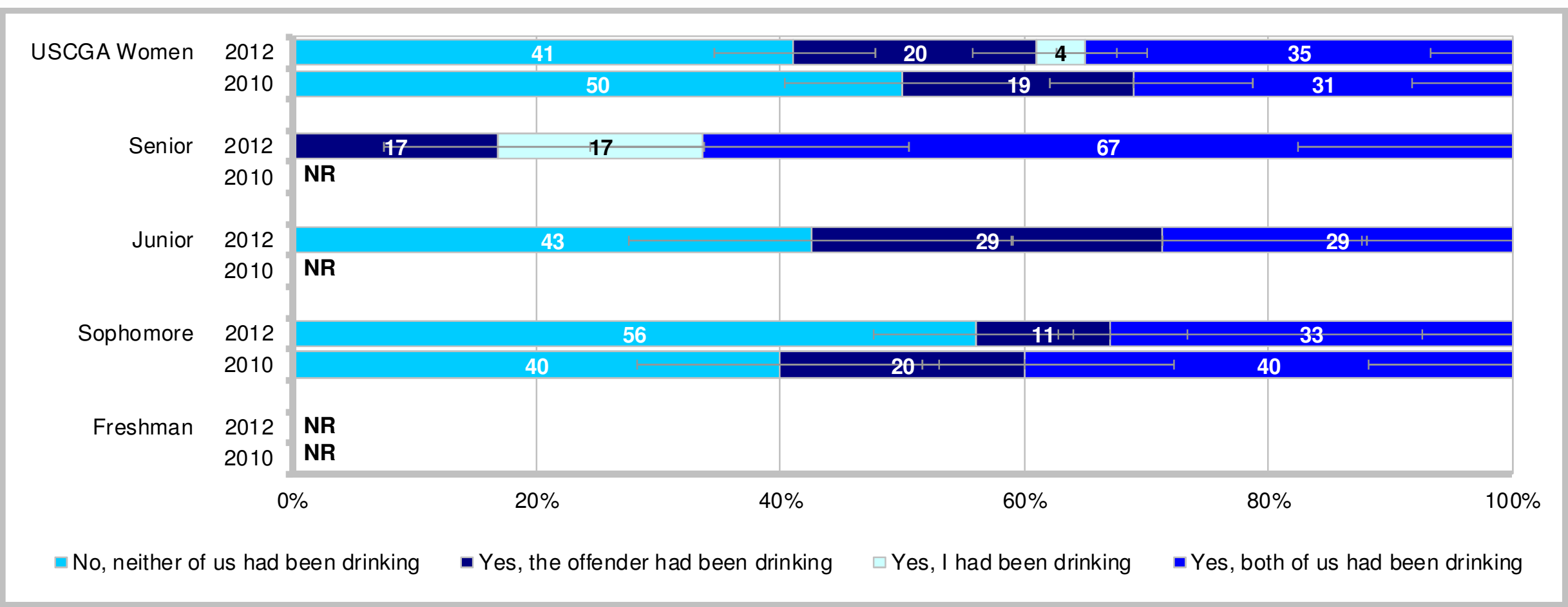

- Of the $9.8 \%$ of women who experienced USC, $41 \%$ indicated neither of them had been drinking; $20 \%$ indicated the offender had been drinking; 4\% indicated they had been drinking; and 35\% indicated both had been drinking

- 2012 comparisons across years - they had higher than 2010

- Sophomores indicating neither in 2012 higher than 2010

- Class differences in 2012 - neither led by sophomores; both led by seniors 


\section{Use of Drugs Before the Incident} Percent of Women Who Experienced Unwanted Sexual Contact

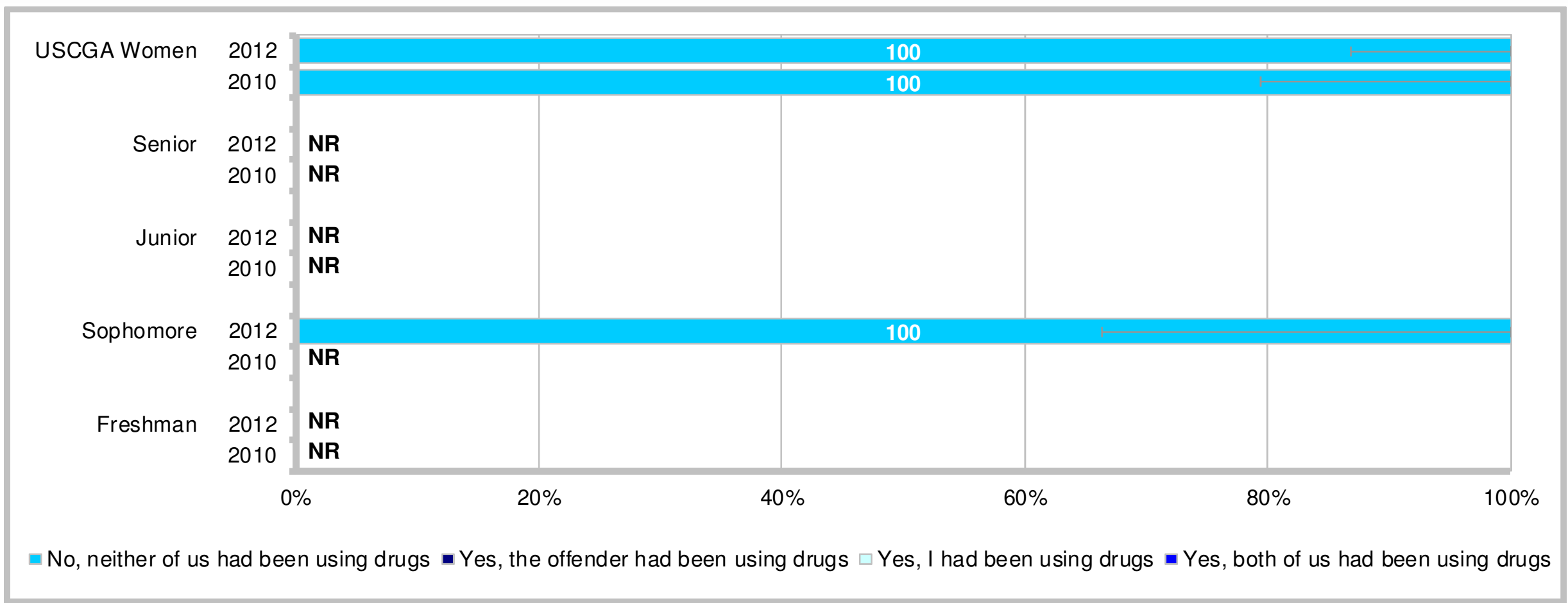

- Of the $9.8 \%$ of women who experienced USC, $100 \%$ indicated neither of them had been using drugs

- 2012 comparisons across years - no differences

- Class differences in 2012 - no differences 


\section{Use of Knock Out Drugs Before the Incident Percent of Women Who Experienced Unwanted Sexual Contact}

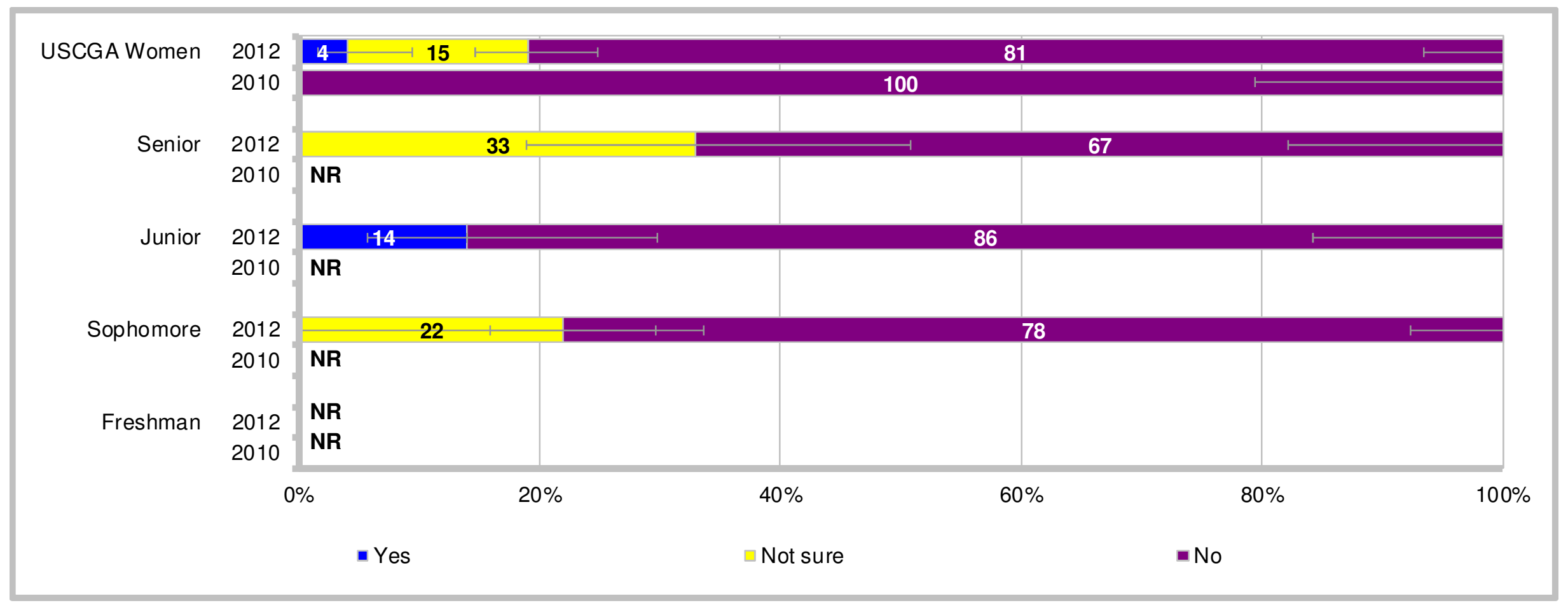

- Of the $9.8 \%$ of women who experienced USC, $81 \%$ indicated the offender did not use drugs to knock them out; $15 \%$ indicated they were not sure; $4 \%$ indicated offender used drugs to knock them out

- 2012 comparisons across years - the offender used drugs to knock them out higher than 2010; not sure higher than 2010; the offender did not use drugs to knock them out lower than 2010

- Class differences in 2012 - not sure led by seniors 


\section{Contents}

Slide

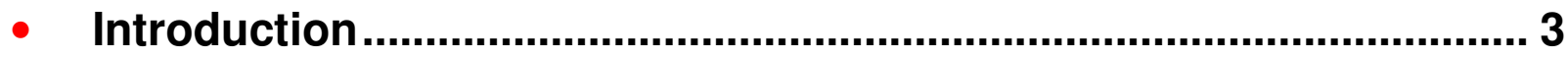

- Unwanted Sexual Contact ............................................................... 7

- Most Serious Behaviors Experienced....................................... 11

- Location of Incident ..................................................................... 13

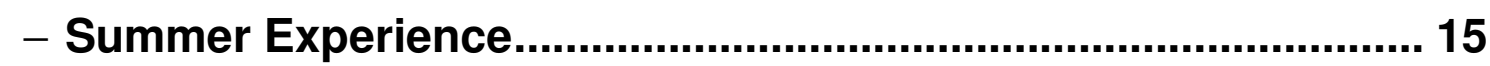

- Characteristics of Offender......................................................... 17

- Alcohol/Drug Involvement......................................................... 22

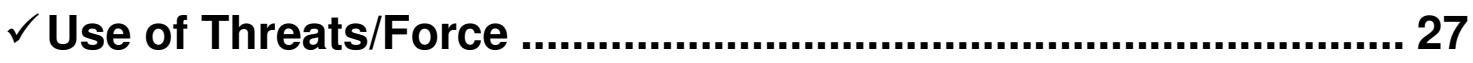

- Experience of Sexual Harassment/Stalking .............................. 32

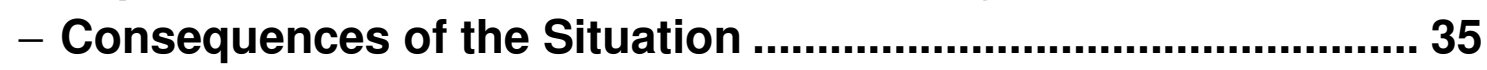

- Reporting the Situation ........................................................... 39

- Experience of Unwanted Sexual Contact Prior to Entering

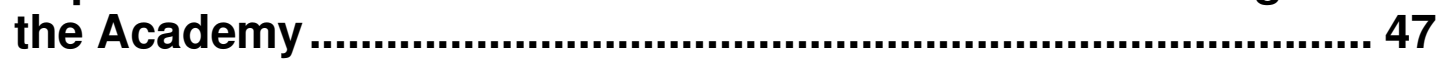

- Unwanted Gender-Related Behaviors ................................................ 49

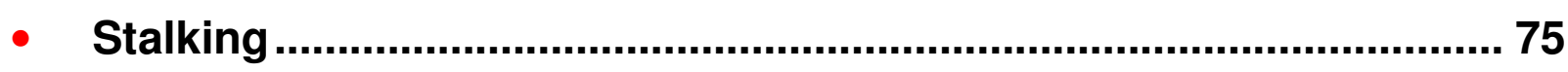

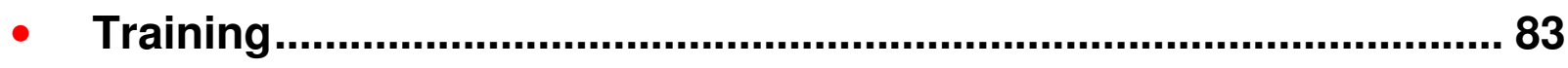

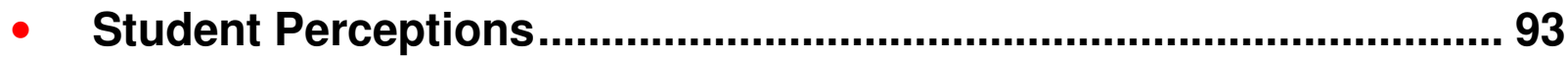




\section{Use of Threats and Physical Force in the Incident \\ Percent of Women Who Experienced Unwanted Sexual Contact}

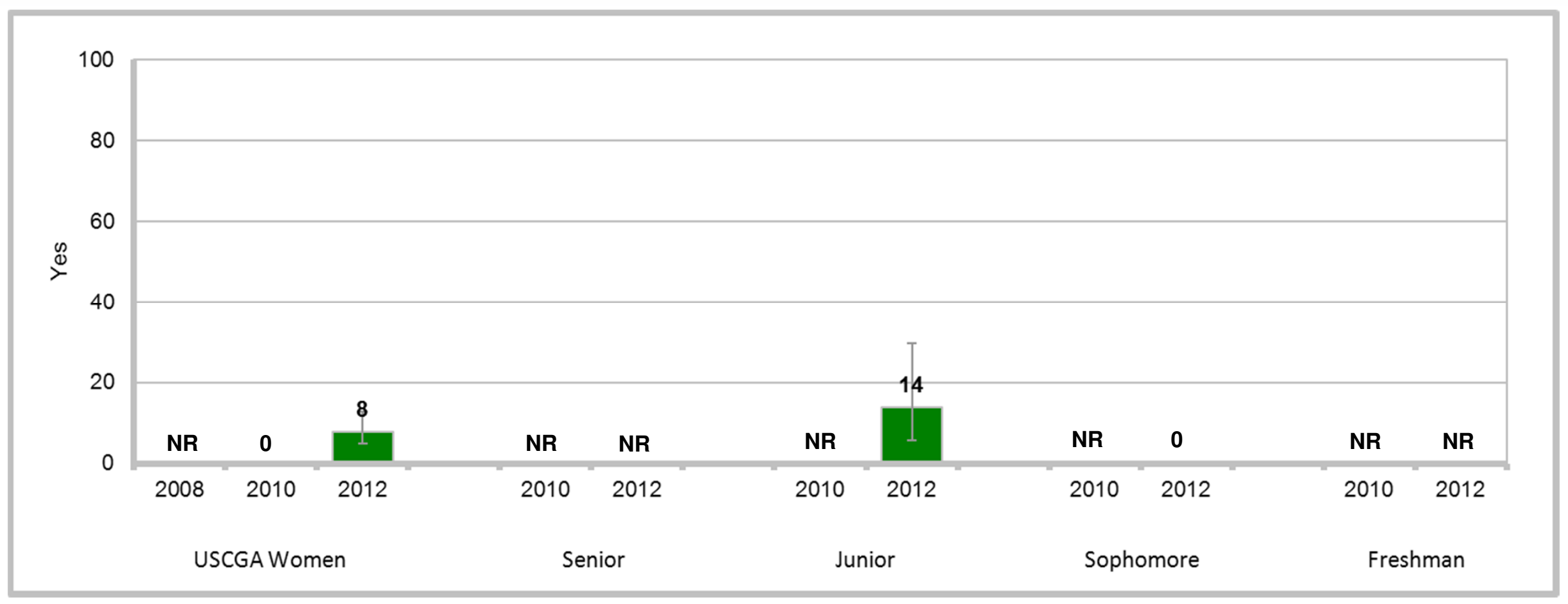

- Of the $9.8 \%$ of women who experienced USC, $8 \%$ indicated the situation involved threats and force

- 2012 comparisons across years - higher than 2010

- Class differences in 2012 - lower response led by sophomores 


\section{Offender Threatened To Ruin Your Reputation if You Did Not Consent Percent of Women Who Experienced Unwanted Sexual Contact}

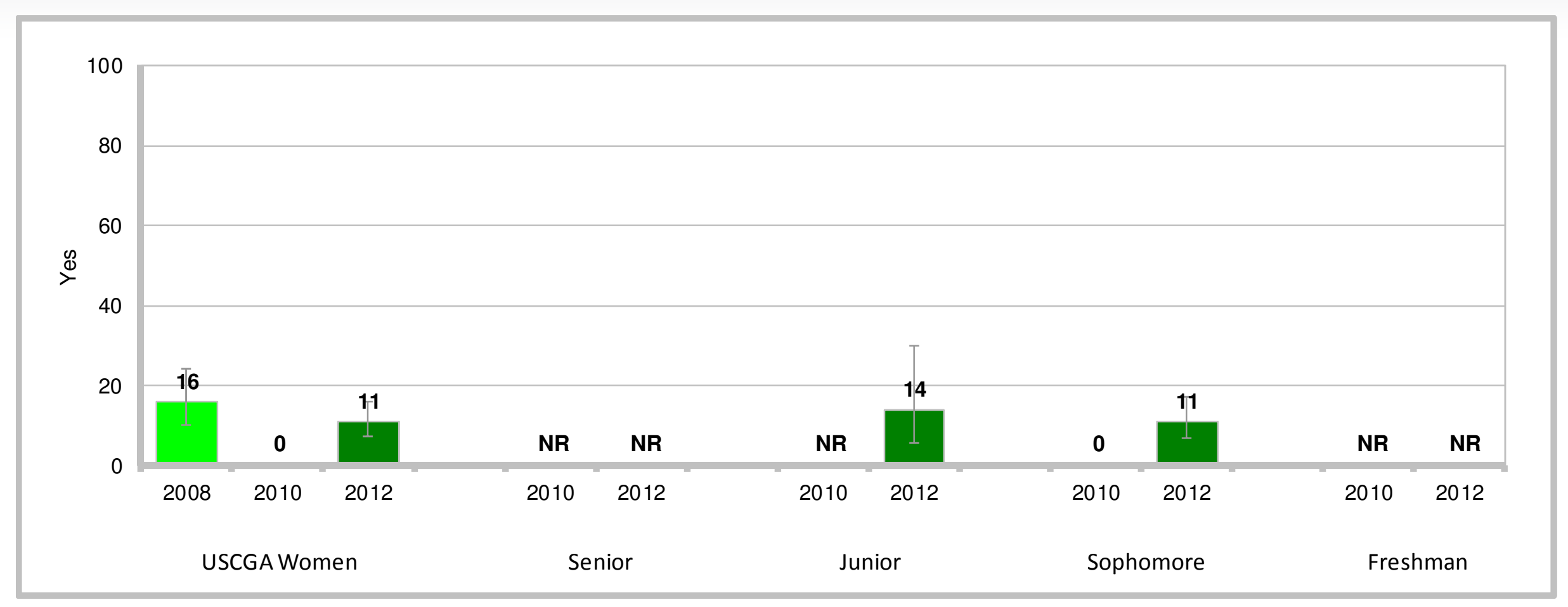

- Of the $9.8 \%$ of women who experienced USC, $11 \%$ indicated the offender threatened to ruin their reputation

- 2012 comparisons across years - higher than 2010

- Sophomores in 2012 higher than 2010

- Class differences in 2012 - no differences 


\section{Offender Threatened To Harm You if You Did Not Consent Percent of Women Who Experienced Unwanted Sexual Contact}

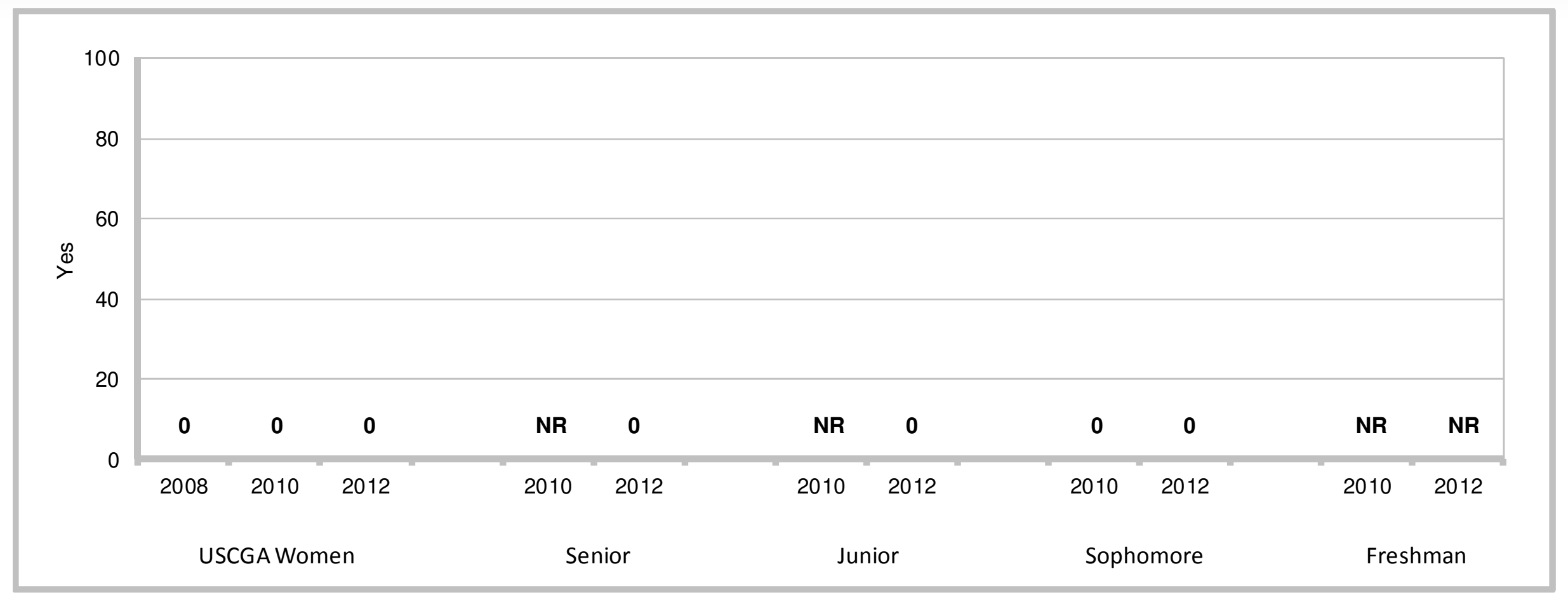

- Of the $9.8 \%$ of women who experienced USC, $0 \%$ indicated the offender threatened to harm them

- 2012 comparisons across years - no differences

- Class differences in 2012 - no differences 


\section{Offender Used Some Degree of Physical Force}

Percent of Women Who Experienced Unwanted Sexual Contact

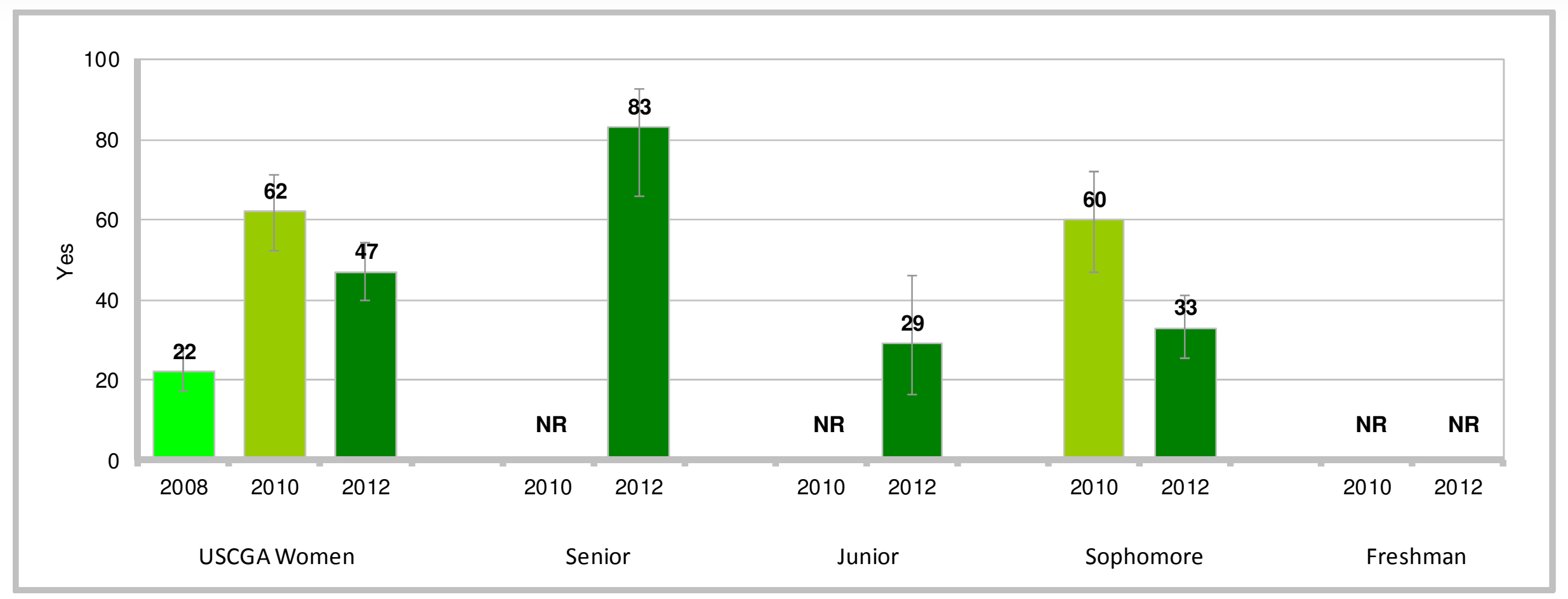

- Of the $9.8 \%$ of women who experienced USC, $47 \%$ indicated the offender threatened to use some degree of physical force

- 2012 comparisons across years - lower than 2010; higher than 2008

- Sophomores in 2012 lower than 2010

- Class differences in 2012 - higher response led by seniors; lower response led by juniors and sophomores 


\section{Contents}

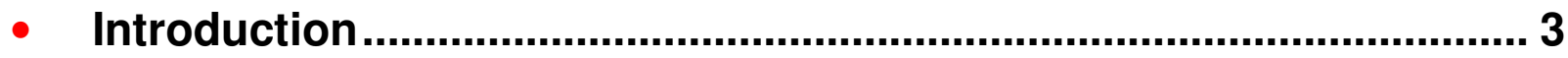

- Unwanted Sexual Contact ............................................................... 7

- Most Serious Behaviors Experienced....................................... 11

- Location of Incident ..................................................................... 13

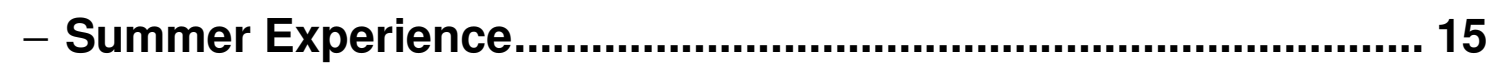

- Characteristics of Offender......................................................... 17

- Alcohol/Drug Involvement......................................................... 22

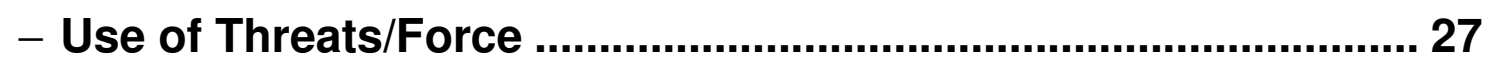

$\checkmark$ Experience of Sexual Harassment/Stalking .............................. 32

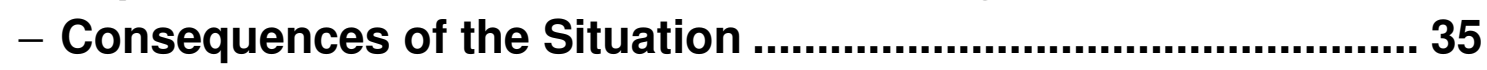

- Reporting the Situation ........................................................... 39

- Experience of Unwanted Sexual Contact Prior to Entering

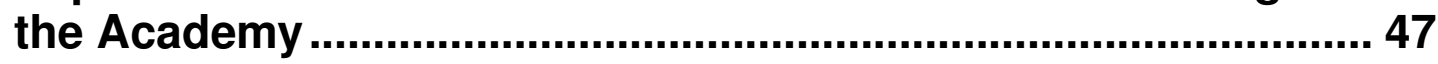

- Unwanted Gender-Related Behaviors ................................................ 49

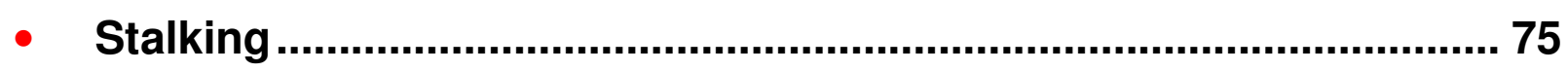

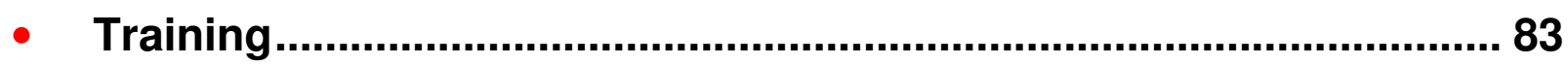

- Student Perceptions.................................................................... 93 


\section{Offender Sexually Harassed and/or Stalked You Before or After the Incident} Percent of Women Who Experienced Unwanted Sexual Contact

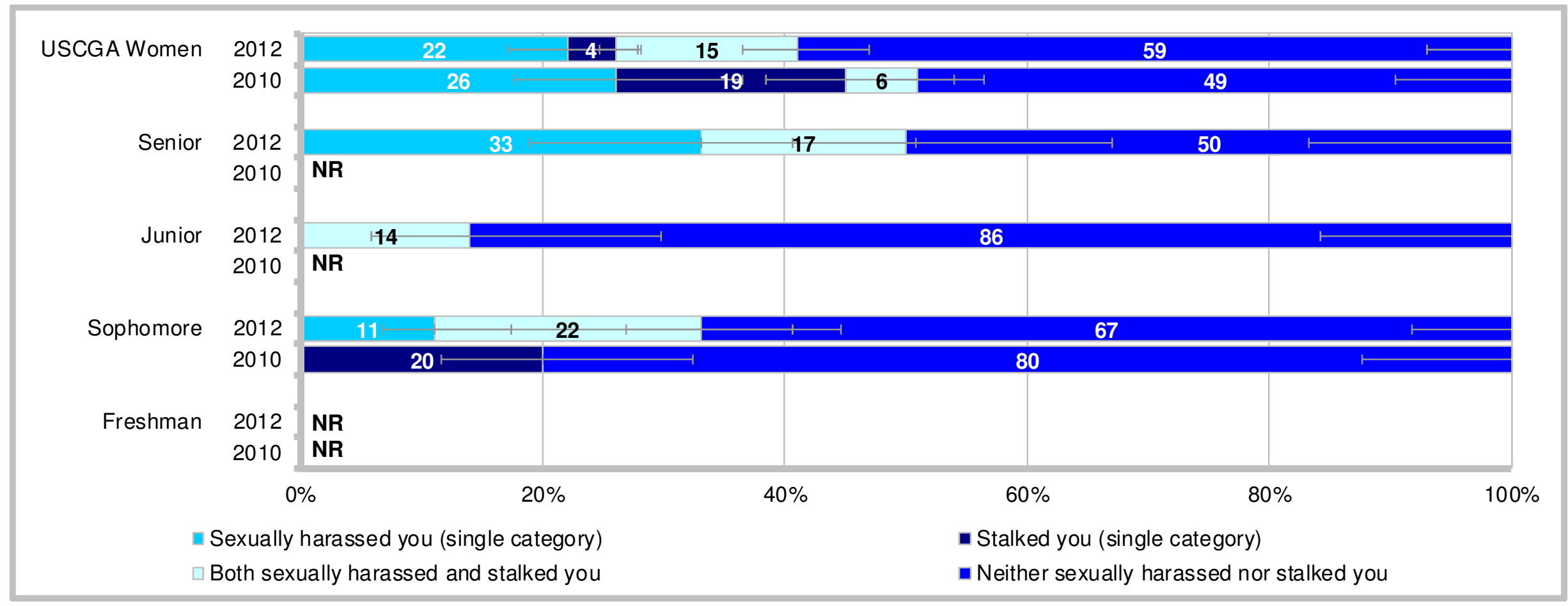

- Of the $9.8 \%$ of women who experienced USC, $22 \%$ indicated the offender sexually harassed them; $4 \%$ indicated the offender stalked them; $15 \%$ indicated the offender both sexually harassed and stalked them; and $59 \%$ indicated the offender neither sexually harassed nor stalked them

- 2012 comparisons across years - stalked lower than 2010; both higher than 2010

- Sophomores indicating stalked in 2012 lower than 2010; sophomores indicating neither in 2012 lower than 2010

- Class differences in 2012 - neither led by juniors 


\section{When Offender Sexually Harassed and/or Stalked You Percent of Women Who Experienced Unwanted Sexual Contact}

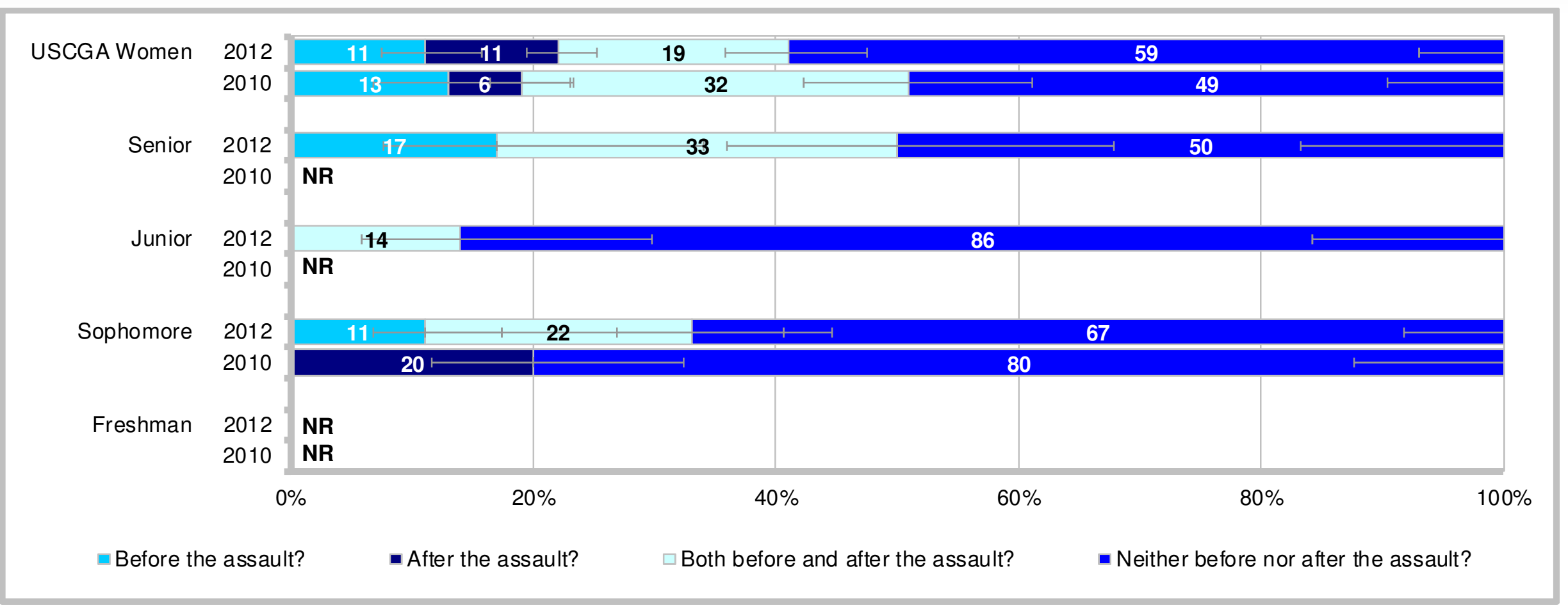

- Of the $9.8 \%$ of women who experienced USC, $11 \%$ indicated the offender sexually harassed or stalked them before the assault; $11 \%$ indicated the offender sexually harassed or stalked them after the assault; $19 \%$ indicated the offender sexually harassed or stalked them both before and after the assault; and $59 \%$ indicated neither before nor after the assault

- 2012 comparisons across years - after higher than 2010; both lower than 2010

- Sophomores indicating after in 2012 lower than 2010; sophomores indicating neither in 2012 lower than 2010

- Class differences in 2012 - neither led by juniors 


\section{Contents}

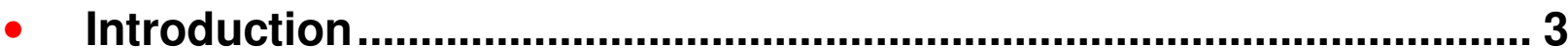

- Unwanted Sexual Contact ................................................................... 7

- Most Serious Behaviors Experienced............................................ 11

- Location of Incident ....................................................................... 13

- Summer Experience..................................................................... 15

- Characteristics of Offender....................................................... 17

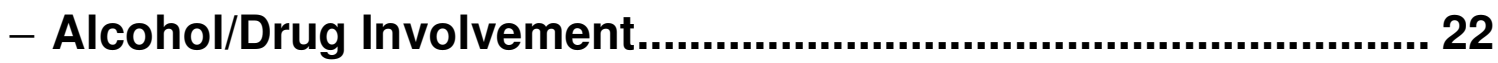

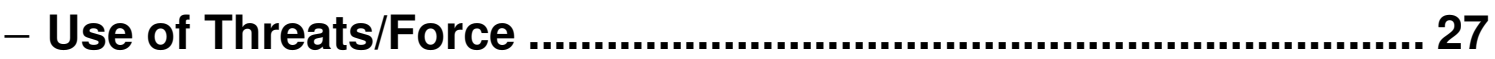

- Experience of Sexual Harassment/Stalking ............................... 32

$\checkmark$ Consequences of the Situation .................................................... 35

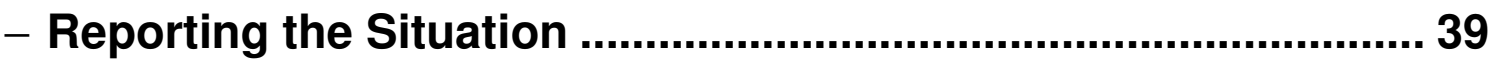

- Experience of Unwanted Sexual Contact Prior to Entering the Academy ..................................................................................... 47

- Unwanted Gender-Related Behaviors .............................................. 49

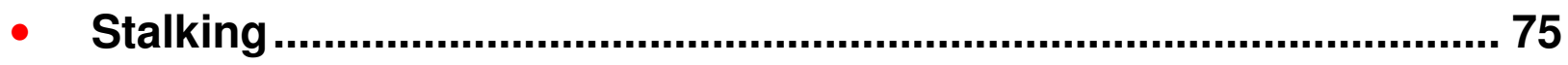

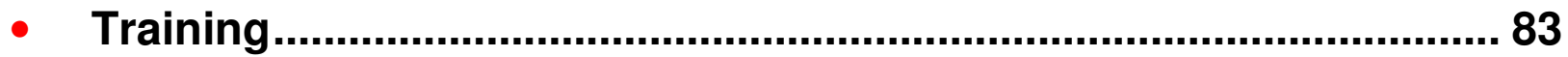

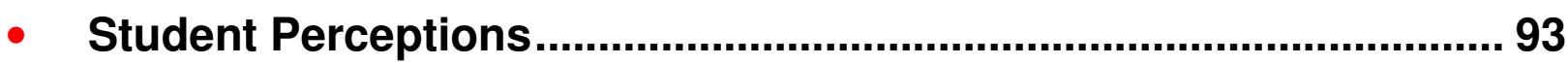




\section{Considered Requesting a Transfer to Another Company/Squadron}

Percent of Women Who Experienced Unwanted Sexual Contact

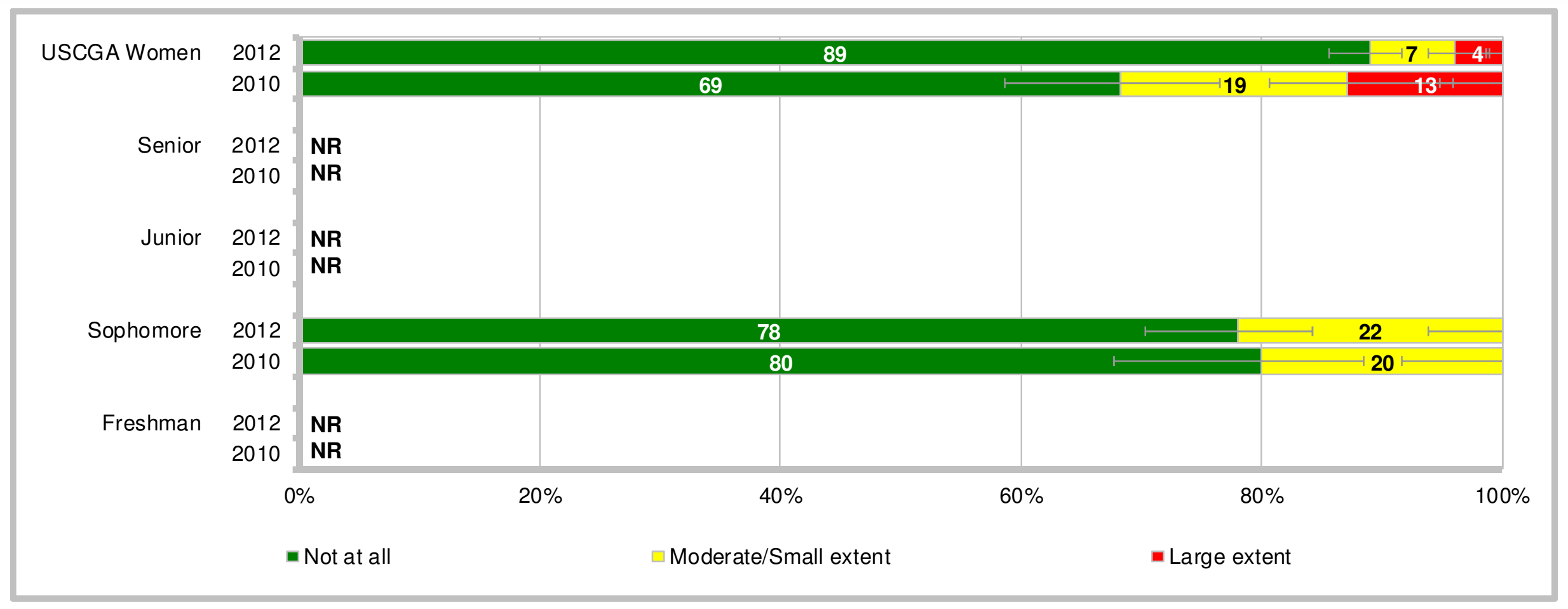

- Of the $9.8 \%$ of women who experienced USC, $11 \%$ indicated they considered a transfer to some extent; $89 \%$ indicated not at all

- 2012 comparisons across years - not at all higher than 2010; 2012 moderate/small extent lower than 2010; large extent lower than 2010

- Class differences in 2012 - no differences 


\section{Thought About Leaving Your Academy} Percent of Women Who Experienced Unwanted Sexual Contact

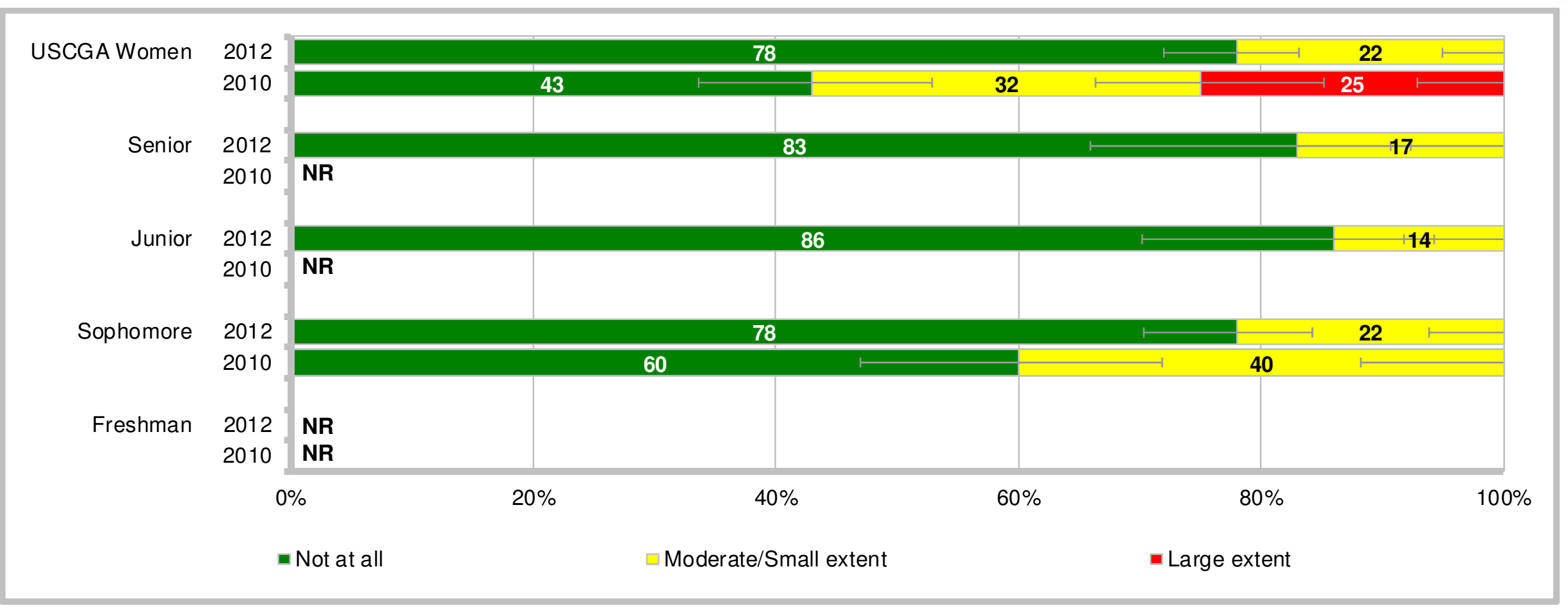

- Of the $9.8 \%$ of women who experienced USC, $22 \%$ indicated they thought about leaving the Academy to some extent; $78 \%$ indicated not at all

- 2012 comparisons across years - not at all higher than 2010; large extent lower than 2010

- Sophomores indicating not at all in 2012 higher than 2010; sophomores indicating moderate/small extent in 2012 lower than 2010

- Class differences in 2012 - no differences 


\section{Your Academic Performance Suffered}

\section{Percent of Women Who Experienced Unwanted Sexual Contact}

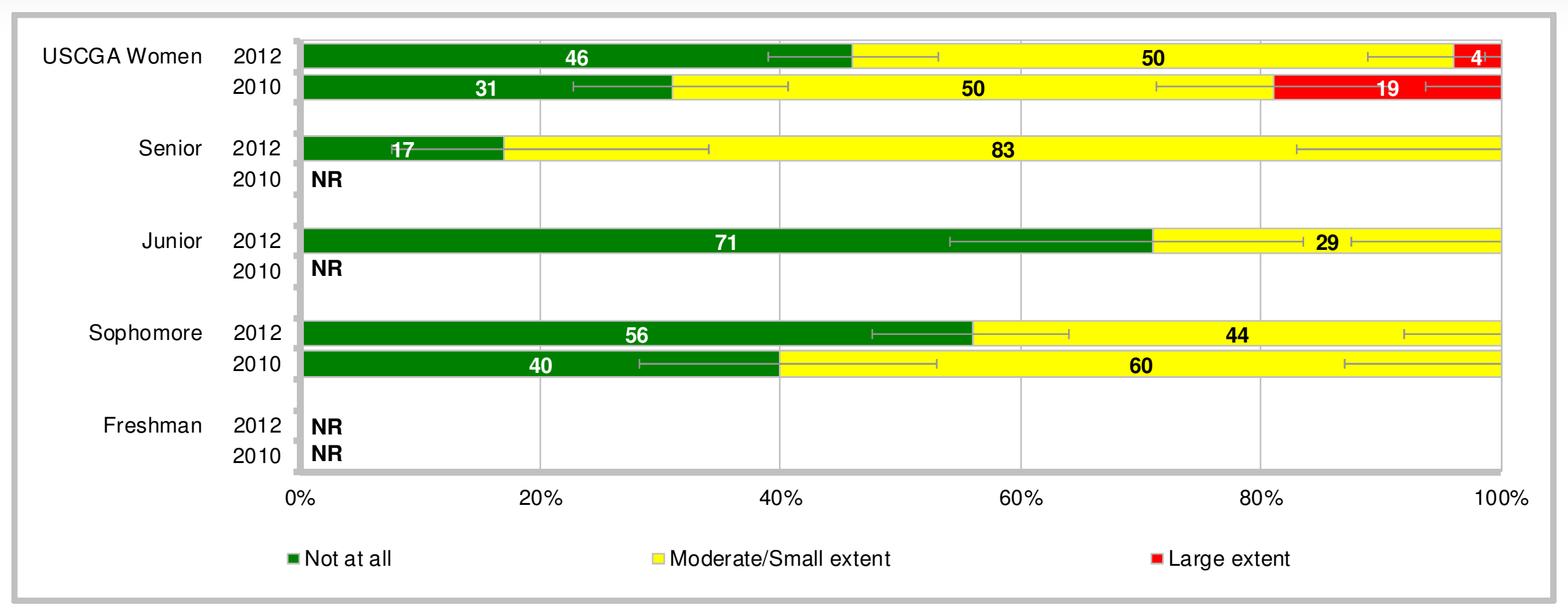

- Of the $9.8 \%$ of women who experienced USC, $54 \%$ indicated their academic performance suffered to some extent; $46 \%$ indicated not at all

- 2012 comparisons across years - not at all higher than 2010; large extent lower than 2010

- Sophomores indicating not at all in 2012 higher than 2010; sophomores indicating moderate/small extent in 2012 lower than 2010

- Class differences in 2012 - not at all led by juniors; moderate/small extent led by seniors 


\section{Contents}

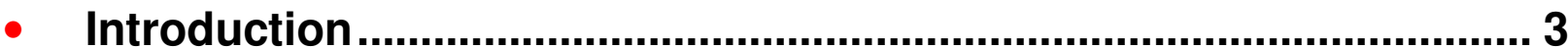

- Unwanted Sexual Contact ................................................................... 7

- Most Serious Behaviors Experienced.......................................... 11

- Location of Incident ....................................................................... 13

- Summer Experience..................................................................... 15

- Characteristics of Offender....................................................... 17

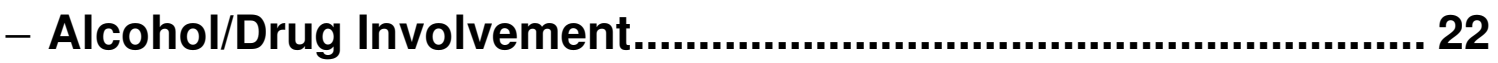

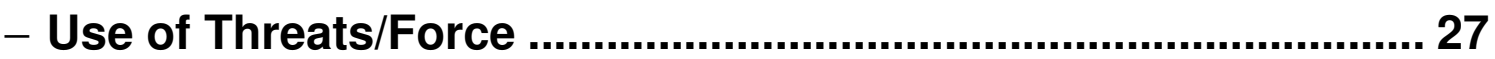

- Experience of Sexual Harassment/Stalking ............................... 32

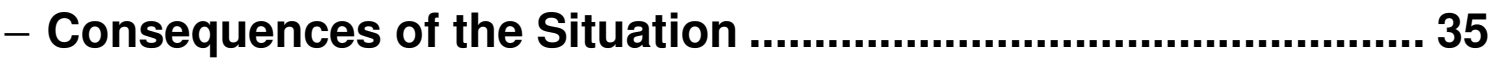

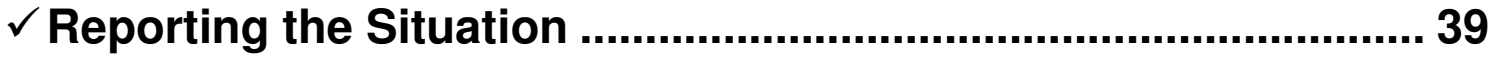

- Experience of Unwanted Sexual Contact Prior to Entering the Academy ..................................................................................... 47

- Unwanted Gender-Related Behaviors .............................................. 49

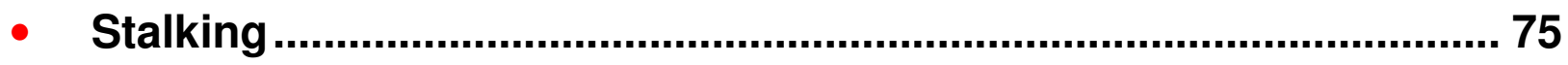

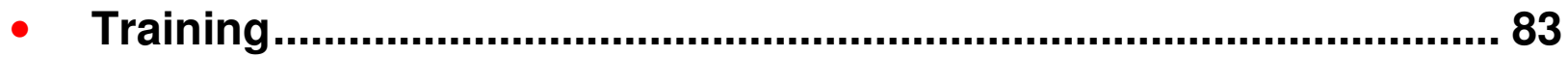

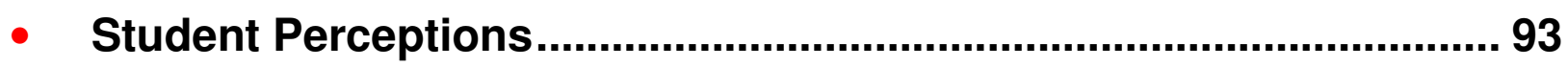




\section{Reported This Situation to a Military Authority or Organization Percent of Women Who Experienced Unwanted Sexual Contact}

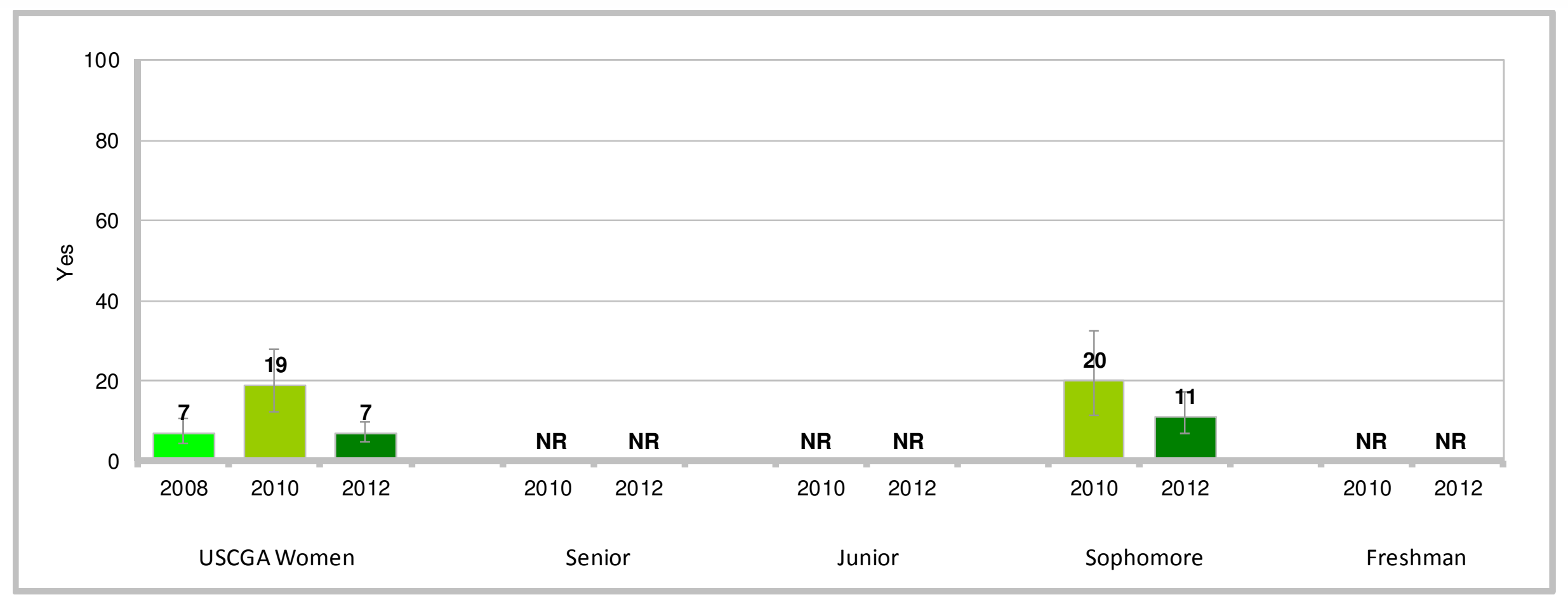

- Of the $9.8 \%$ of women who experienced USC, $7 \%$ indicated they reported the situation

- 2012 comparisons across years - lower than 2010

- Class differences in 2012 - no differences 


\section{Most Frequently Selected Reasons for Not Reporting Unwanted Sexual Contact Situation Percent of Women Who Experienced Unwanted Sexual Contact and Did Not Report It}

\begin{tabular}{|c|c|c|c|c|c|c|}
\hline Reasons for Not Reporting & $\begin{array}{c}\text { Survey } \\
\text { Year }\end{array}$ & Total & Senior & Junior & Sophomore & Freshman \\
\hline \multirow{2}{*}{ Took care of it myself } & 2012 & $86 \uparrow$ & 80 & 71 & 100 & NR \\
\hline & 2010 & 61 & NR & NR & NR & NR \\
\hline \multirow{2}{*}{$\begin{array}{l}\text { Thought it was not important enough to } \\
\text { report }\end{array}$} & 2012 & 73 & 60 & 71 & 88 & NR \\
\hline & 2010 & 60 & NR & NR & NR & $\mathrm{NR}$ \\
\hline \multirow{2}{*}{ Did not want people gossiping } & 2012 & 69 & 80 & 57 & 63 & NR \\
\hline & 2010 & 78 & NR & NR & NR & NR \\
\hline \multirow{2}{*}{ Did not want anyone to know } & 2012 & 65 & 80 & 57 & 63 & NR \\
\hline & 2010 & 56 & NR & NR & NR & NR \\
\hline \multirow{2}{*}{ Felt uncomfortable making a report } & 2012 & $61 \downarrow$ & 80 & 57 & 63 & NR \\
\hline & 2010 & 85 & NR & NR & NR & NR \\
\hline \multirow{2}{*}{ Felt shame/embarrassment } & 2012 & 61 & 80 & 57 & 38 & NR \\
\hline & 2010 & 62 & NR & NR & NR & NR \\
\hline \multirow{2}{*}{$\begin{array}{l}\text { Feared some form of retaliation from } \\
\text { offender or his/her friends }\end{array}$} & 2012 & 53 & 60 & 71 & 25 & NR \\
\hline & 2010 & 57 & NR & NR & NR & NR \\
\hline Margins of Error & & $\pm 8-12 \%$ & $\pm 20 \%$ & $\pm 17 \%$ & $\pm 8-9 \%$ & -- \\
\hline
\end{tabular}

Higher Response of Yes

Lower Response of Yes

Higher in 2012 than 2010

Lower in 2012 than 2010 


\section{Would Make the Same Decision About Reporting or Not Reporting if Could Do It Over}

Percent of Women Who Experienced Unwanted Sexual Contact and Reported or Did Not Report to a Military Authority

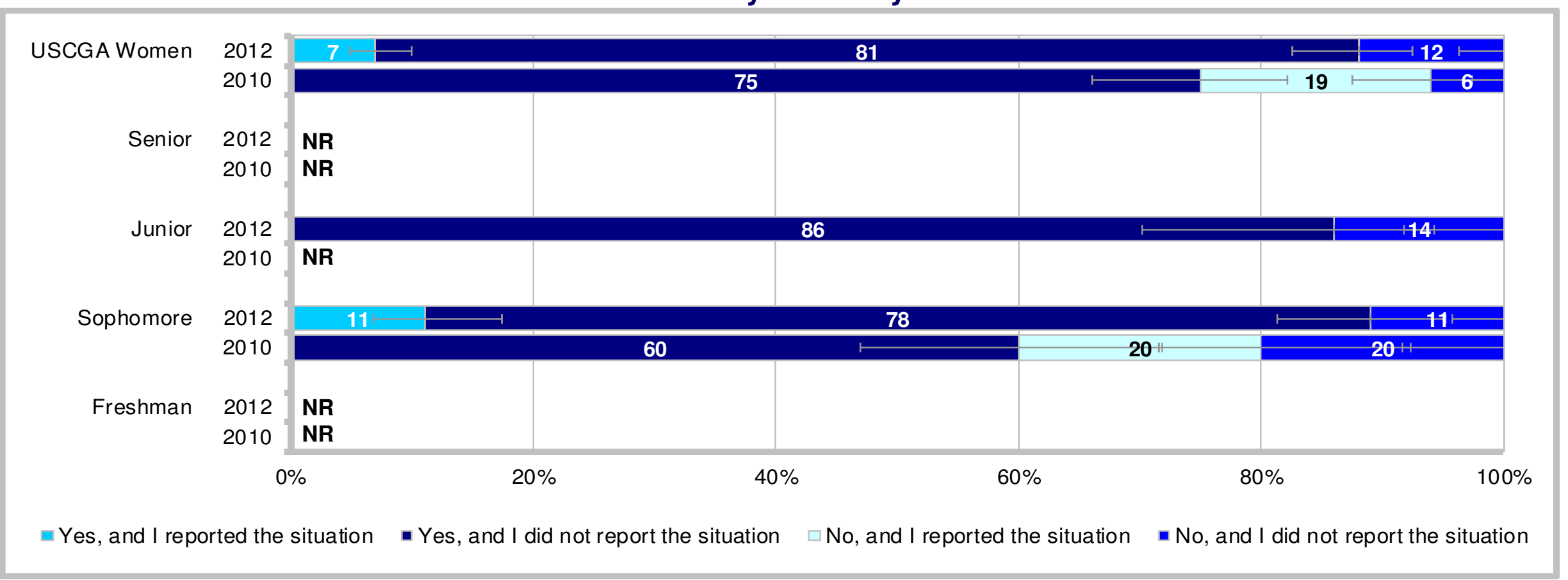

- Of the $9.8 \%$ of women who experienced USC, $7 \%$ indicated they reported the situation and would make the same decision again; $81 \%$ indicated they did not report the situation and would make the same decision again; $0 \%$ indicated they reported but would not make the same decision again; and $12 \%$ indicated they did not report the situation but would not make the same decision again

- 2012 comparisons across years - reported the situation and would make the same decision again higher than 2010; reported the situation but would not make the same decision again lower than 2010; did not report the situation but would not make the same decision again higher than 2010

- Sophomores indicating reported and would make the same decision in 2012 higher than 2010; sophomores indicating did not report and would make the same decision in 2012 higher than 2010; sophomores indicating reported but would not make the same decision in 2012 lower than 2010; sophomores indicating did not report and would not make the same decision in 2012 lower than 2010

- Class differences in 2012 - no differences 


\section{Contents}

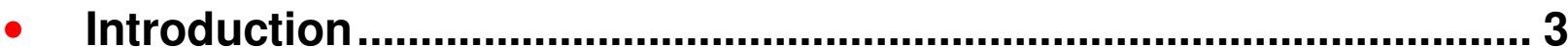

- Unwanted Sexual Contact ................................................................... 7

- Most Serious Behaviors Experienced........................................... 11

- Location of Incident ....................................................................... 13

- Summer Experience..................................................................... 15

- Characteristics of Offender....................................................... 17

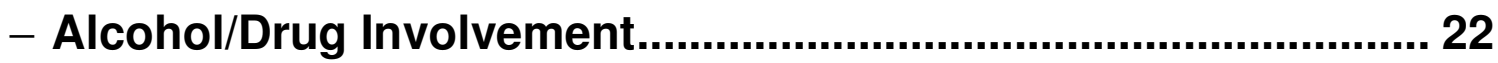

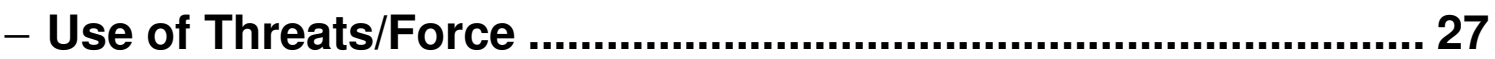

- Experience of Sexual Harassment/Stalking ............................... 32

- Consequences of the Situation ...................................................... 35

- Reporting the Situation .................................................................. 39

$\checkmark$ Experience of Unwanted Sexual Contact Prior to Entering the Academy .................................................................................... 43

- Unwanted Gender-Related Behaviors .............................................. 49

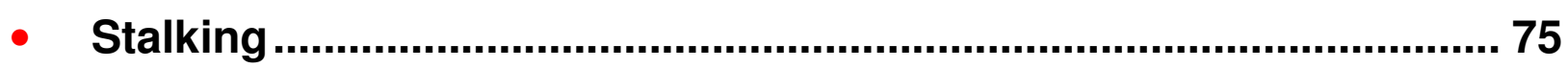

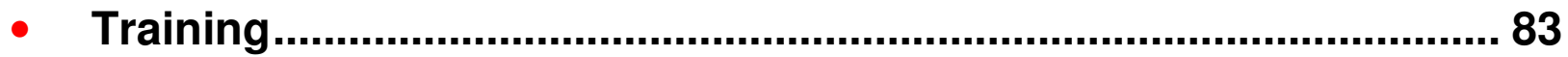

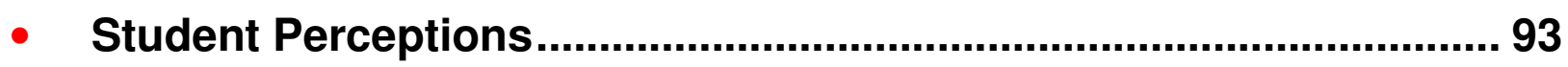




\section{Unwanted Sexual Contact Incident Rate Prior to Entering the Academy} Percent of All Students

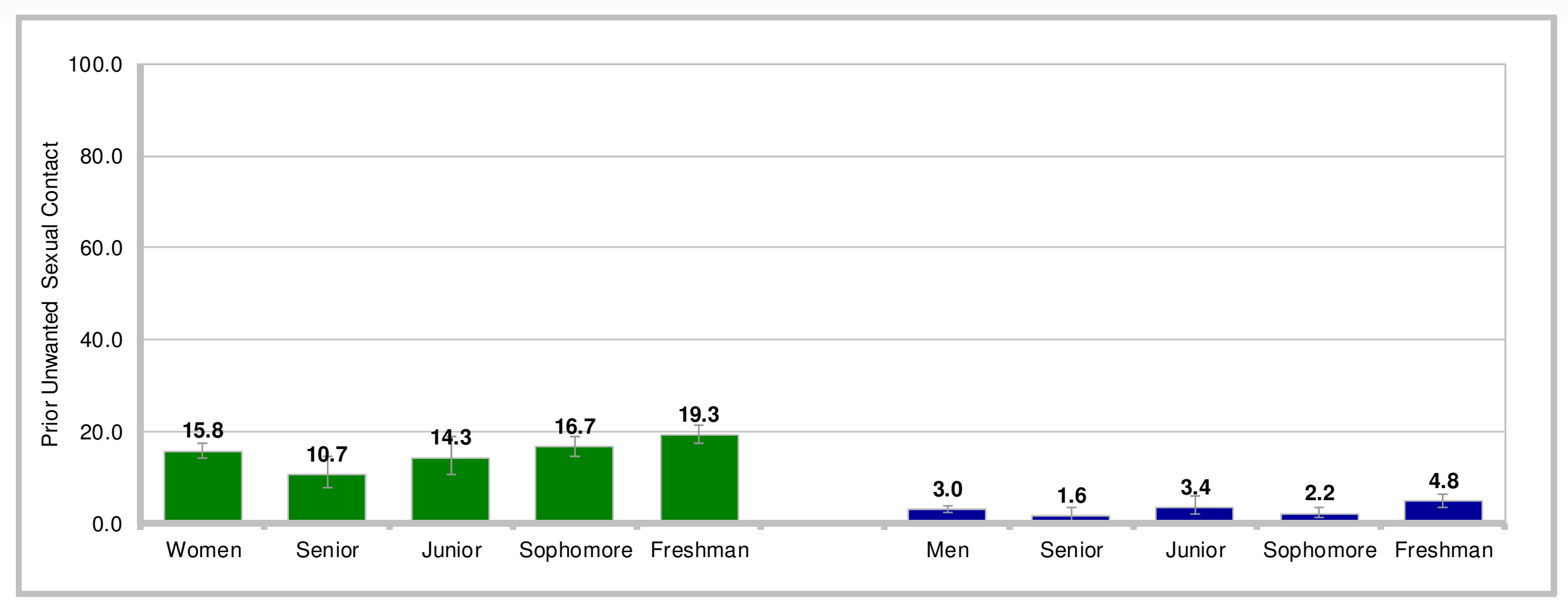

- $15.8 \%$ of women and $3.0 \%$ of men indicated experiencing unwanted sexual contact prior to entering the Academy

- 2012 comparisons across years - new question in 2012; no trend data available

- Class differences in $\mathbf{2 0 1 2 ~ - ~ f o r ~ w o m e n , ~ h i g h e r ~ r e s p o n s e ~ l e d ~ b y ~ f r e s h m e n , ~ l o w e r ~ r e s p o n s e ~ l e d ~ b y ~ s e n i o r s ; ~ f o r ~ m e n , ~}$ higher response led by freshmen 


\section{Contents}

Slide

• Introduction ...................................................................................... 3

- Unwanted Sexual Contact .................................................................. 7

$\checkmark \quad$ Unwanted Gender-Related Behaviors .......................................... 45

- Sexual Harassment ................................................................. 47

- Categories of Behaviors Associated with Sexual Harassment.................................................................................... 50

- Sexist Behavior ........................................................................... 57

- Organizational Affiliation of Offender ...................................... 60

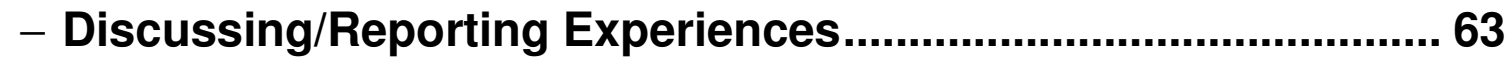

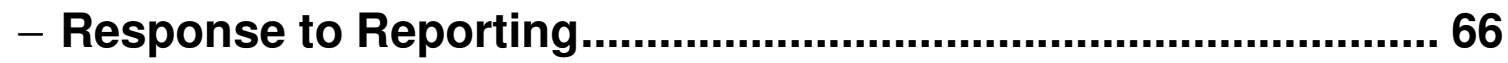

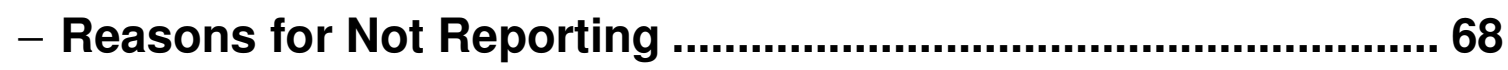

- Stalking ........................................................................................... 71

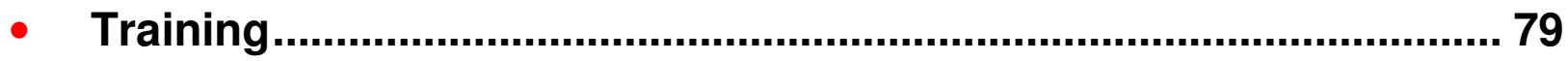

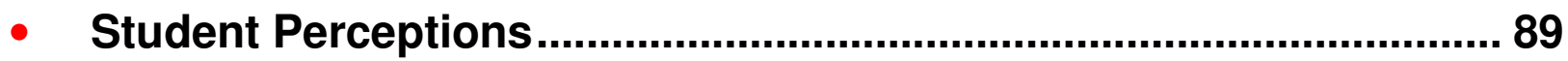




\section{Unwanted Gender-Related Behaviors:}

\section{Sexual Harassment and Sexist Behavior Incident Rates}

\section{- Definition and measure of sexual harassment and sexist behavior:}

- DoD defines sexual harassment as "a form of sex discrimination that involves unwelcome sexual advances, requests for sexual favors, and other verbal or physical conduct of a sexual nature when:

- Submission to such conduct is made either explicitly or implicitly a term or condition of a person's job, pay, or career, or

- Submission to or rejection of such conduct by a person is used as a basis for career or employment decisions affecting that person, or

- Such conduct has the purpose or effect of unreasonably interfering with an individual's work performance or creates an intimidating, hostile, or offensive working environment" (Department of Defense, 1995).

- The core measure of unwanted gender-related behaviors on the 2012 SAGR consists of 17 items

- Sexual harassment measure has 12 items in a three-factor structure and a single "labeling" item

- Crude/Offensive Behavior - four items regarding offensive or embarrassing verbal/nonverbal behaviors of a sexual nature

- Unwanted Sexual Attention - four items regarding unwanted attempts to establish a sexual relationship

- Sexual Coercion - four items regarding classic quid pro quo instances of special treatment or favoritism conditioned on sexual cooperation

- One "labeling" item that asks if the student considers any of the selected behaviors to be sexual harassment

- Sexist behavior measure has four items that include verbal/nonverbal behaviors that convey insulting, offensive, and/or condescending attitudes based on gender of student

- Write-in comments analyzed separately
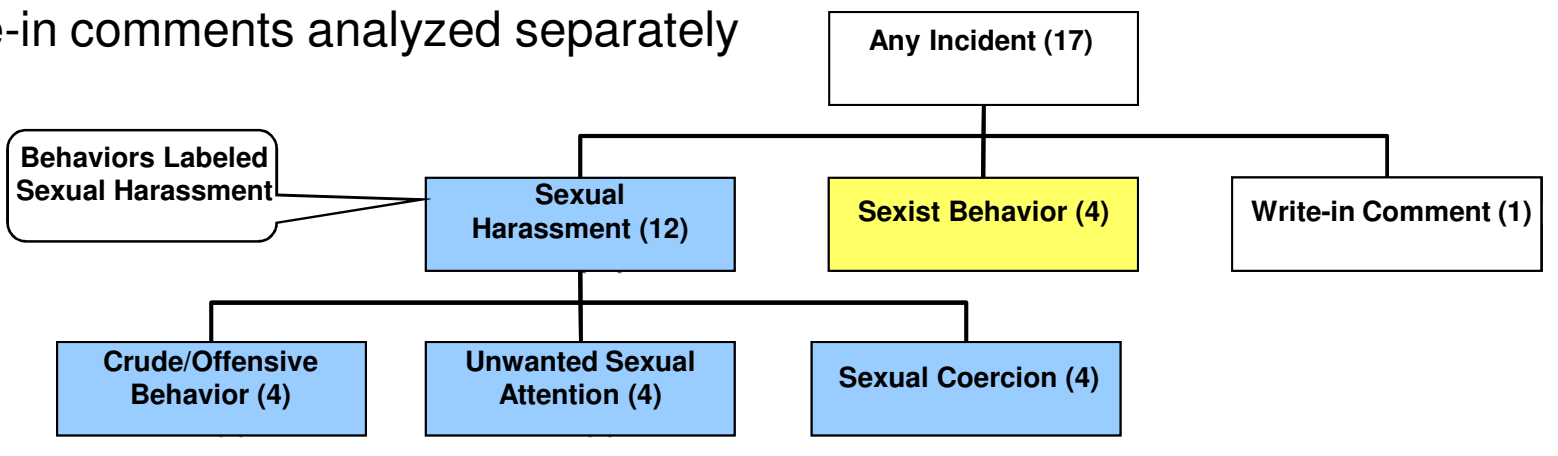


\section{Contents}

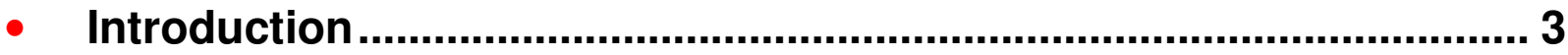

- Unwanted Sexual Contact ................................................................. 7

- Unwanted Gender-Related Behaviors ............................................. 45

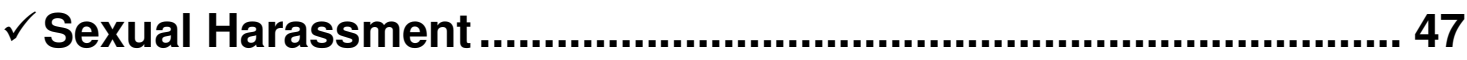

- Categories of Behaviors Associated with Sexual Harassment.................................................................................. 50

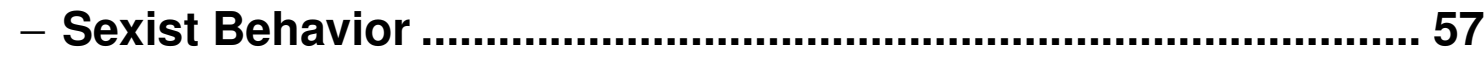

- Organizational Affiliation of Offender ...........................................6 60

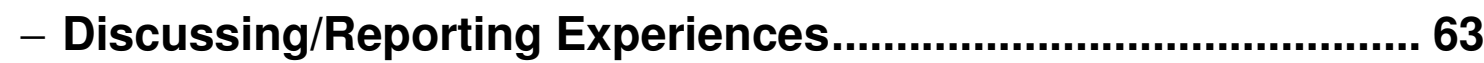

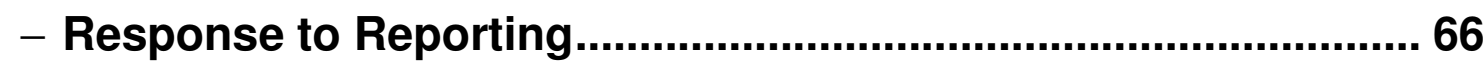

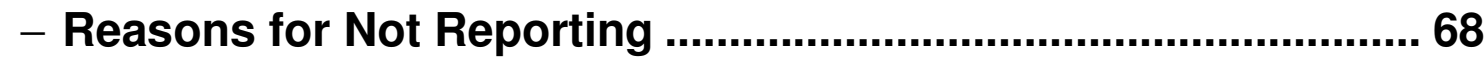

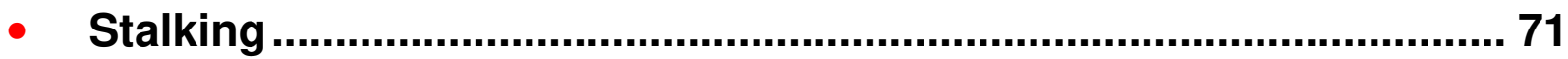

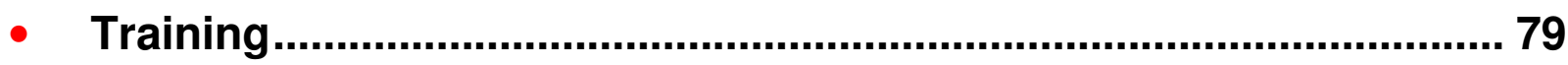

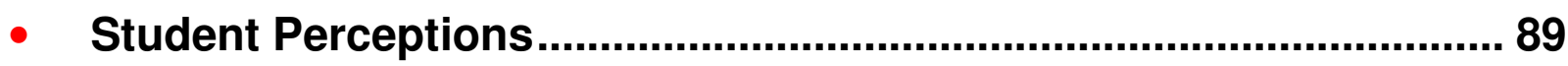




\section{Sexual Harassment Incident Rate Percent of Women}

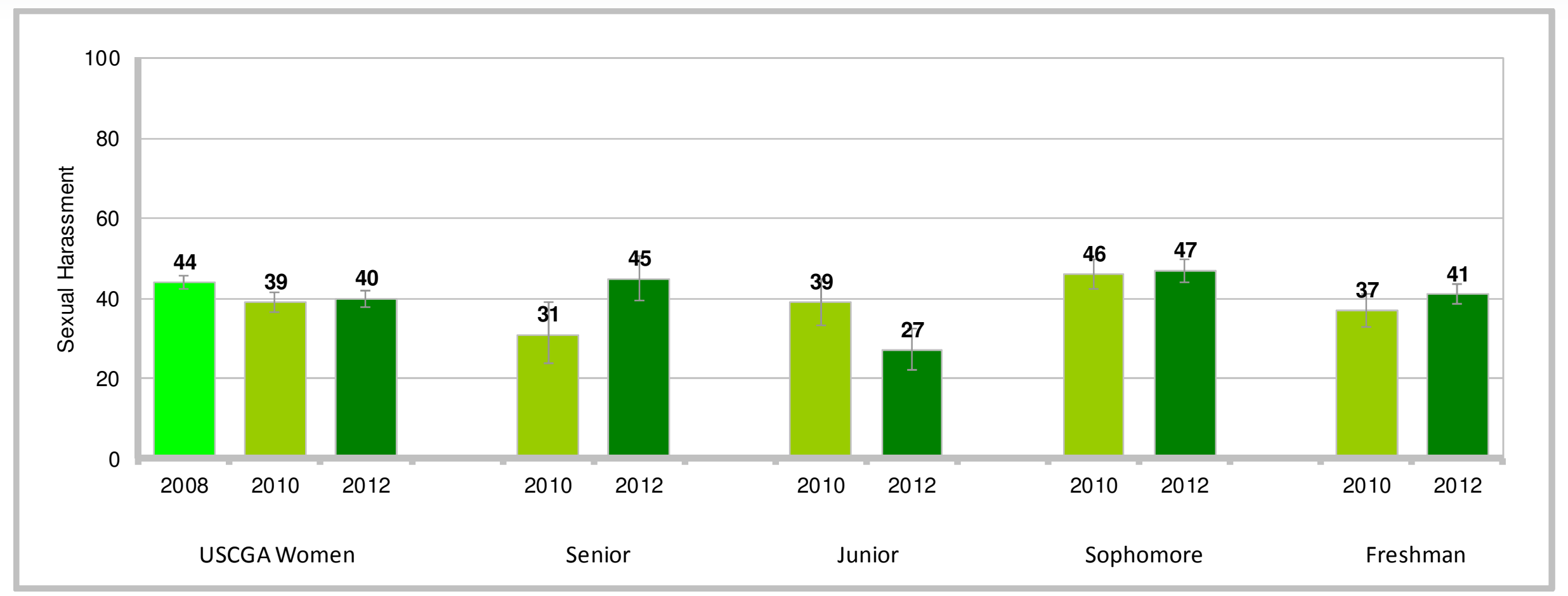

- $40 \%$ of women indicated experiencing sexual harassment in 2012

- 2012 comparisons across years - lower than 2008

- Seniors in 2012 higher than 2010, whereas juniors lower

- Class differences in $\mathbf{2 0 1 2}$ - higher response led by sophomores; lower response led by juniors 


\section{Sexual Harassment Incident Rate}

Percent of Men

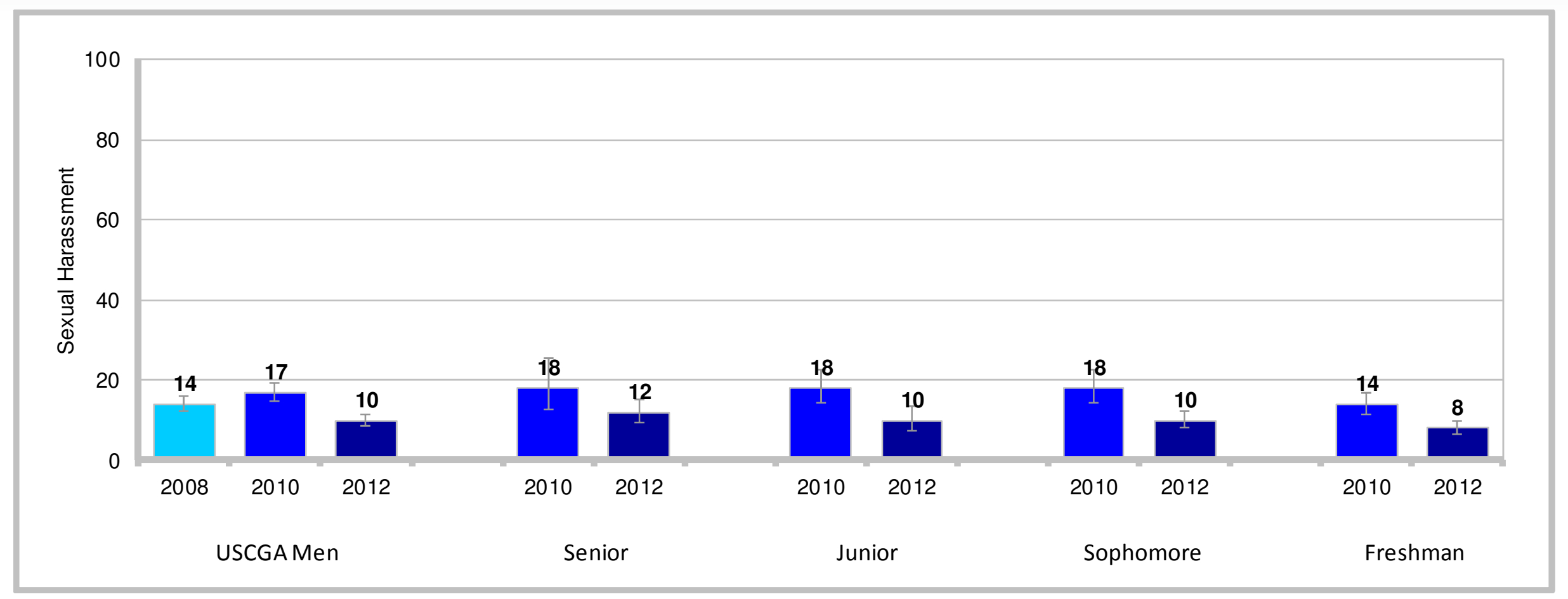

- $10 \%$ of men indicated experiencing sexual harassment in 2012

- 2012 comparisons across years - lower than 2010, 2008

- Juniors, sophomores, and freshmen in 2012 lower than 2010

- Class differences in 2012 - no differences 


\section{Contents}

Slide

- Introduction ............................................................................... 3

- Unwanted Sexual Contact ................................................................... 7

- Unwanted Gender-Related Behaviors ........................................ 45

- Sexual Harassment ..................................................................... 47

$\checkmark$ Categories of Behaviors Associated with Sexual Harassment................................................................................ 50

- Sexist Behavior ........................................................................... 57

- Organizational Affiliation of Offender ..................................... 60

- Discussing/Reporting Experiences......................................... 63

- Response to Reporting............................................................ 66

- Reasons for Not Reporting .................................................... 68

- Stalking ........................................................................................... 71

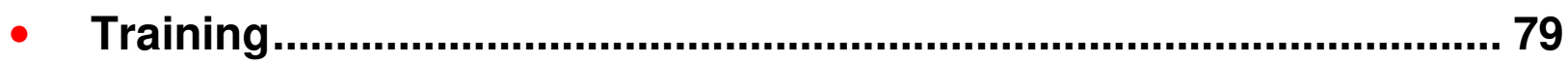

• Student Perceptions...................................................................... 89 


\section{Crude/Offensive Behavior Incident Rate Percent of Women}

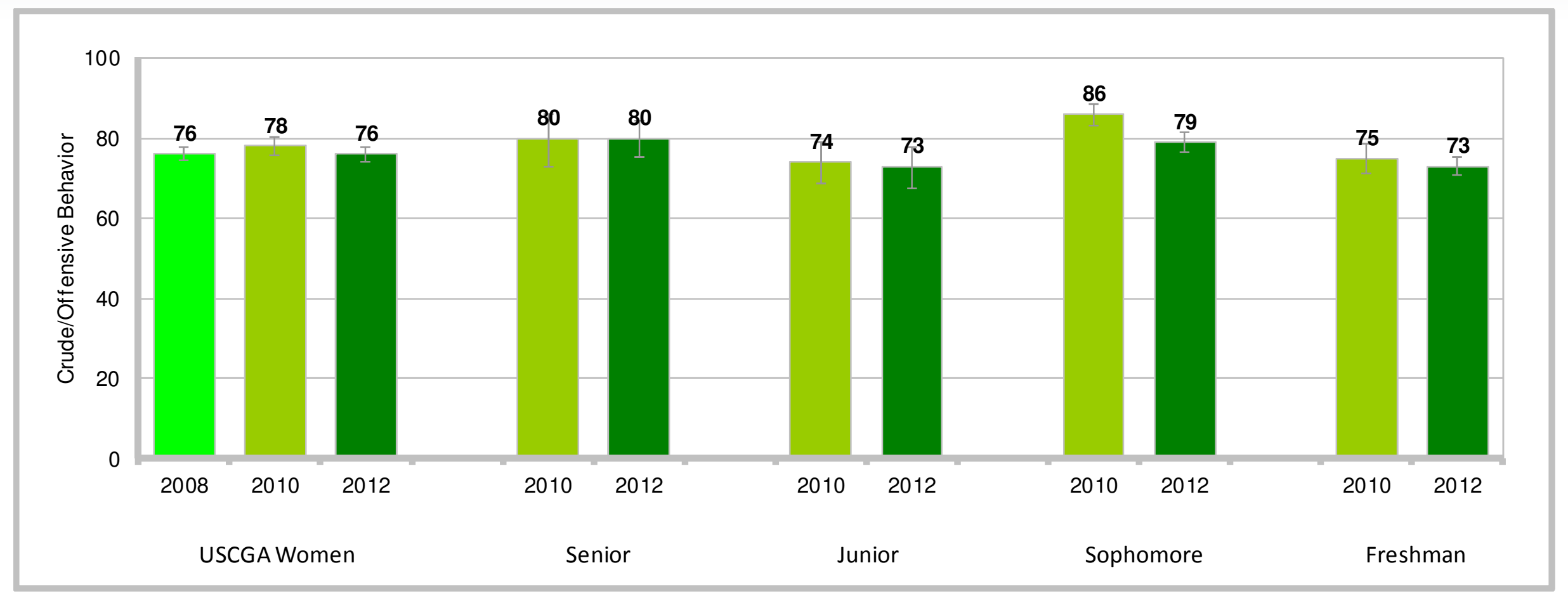

- $76 \%$ of women indicated experiencing crude/offensive behavior in 2012

- 2012 comparisons across years - no differences

- Sophomores in 2012 lower than 2010

- Class differences in 2012 - no differences 


\section{Crude/Offensive Behavior Incident Rate} Percent of Men

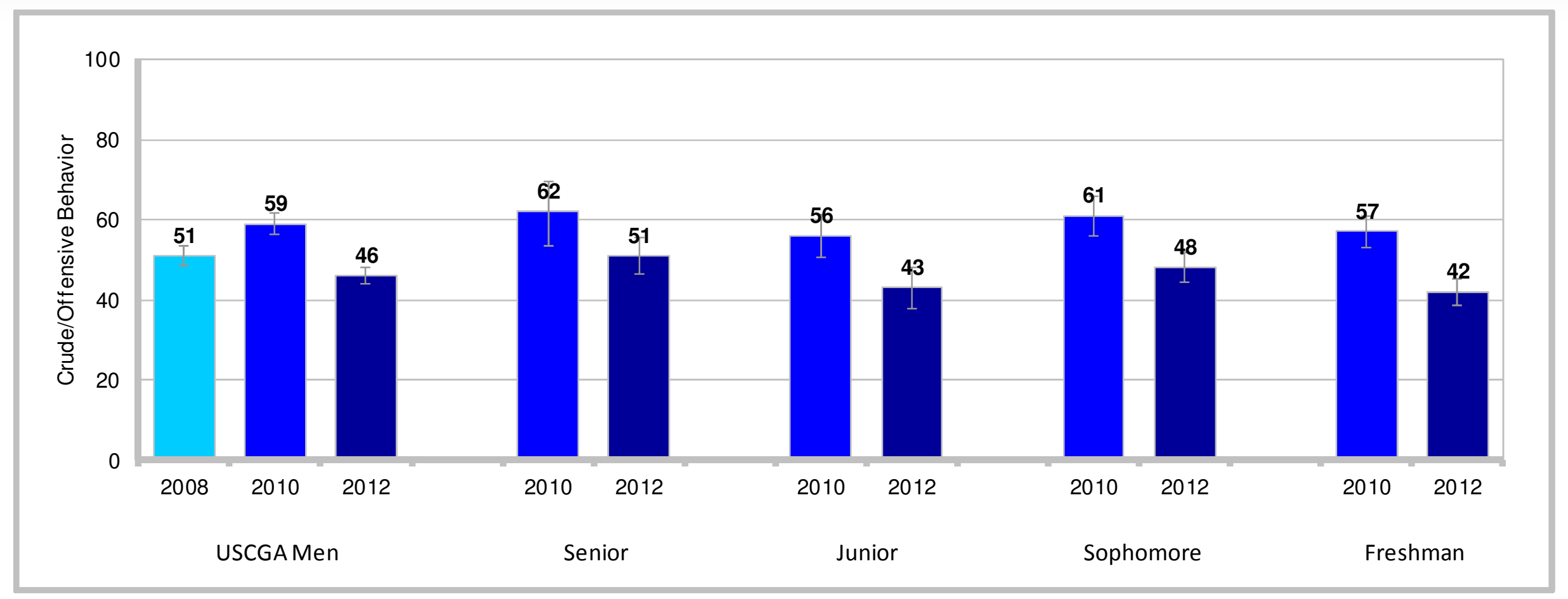

- $46 \%$ of men indicated experiencing crude/offensive behavior in 2012

- 2012 comparisons across years - lower than 2010, 2008

- All class years in 2012 lower than 2010

- Class differences in 2012 - higher response led by seniors; lower response led by freshmen 


\section{Unwanted Sexual Attention Incident Rate Percent of Women}

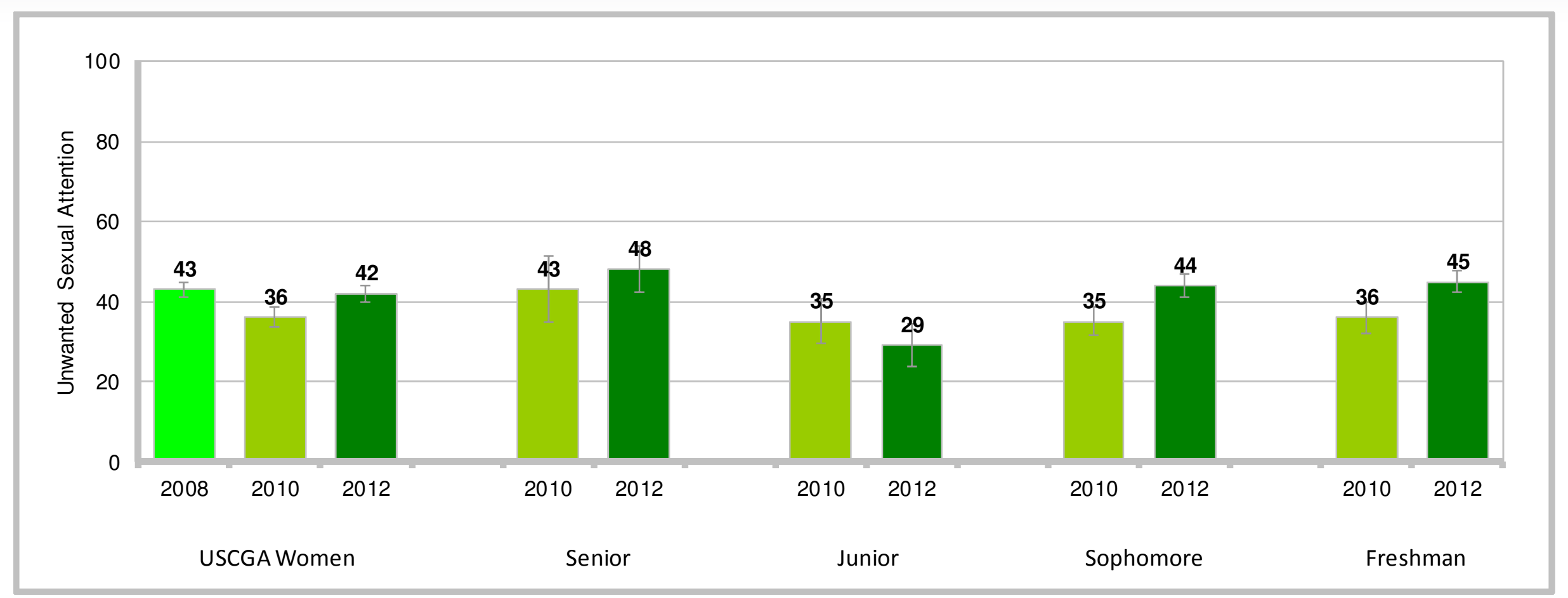

- $42 \%$ of women indicated experiencing unwanted sexual attention in 2012

- 2012 comparisons across years - higher than 2010

- Sophomores and freshmen in 2012 higher than 2010

- Class differences in 2012 - higher response led by seniors; lower response led by juniors 


\section{Unwanted Sexual Attention Incident Rate}

Percent of Men

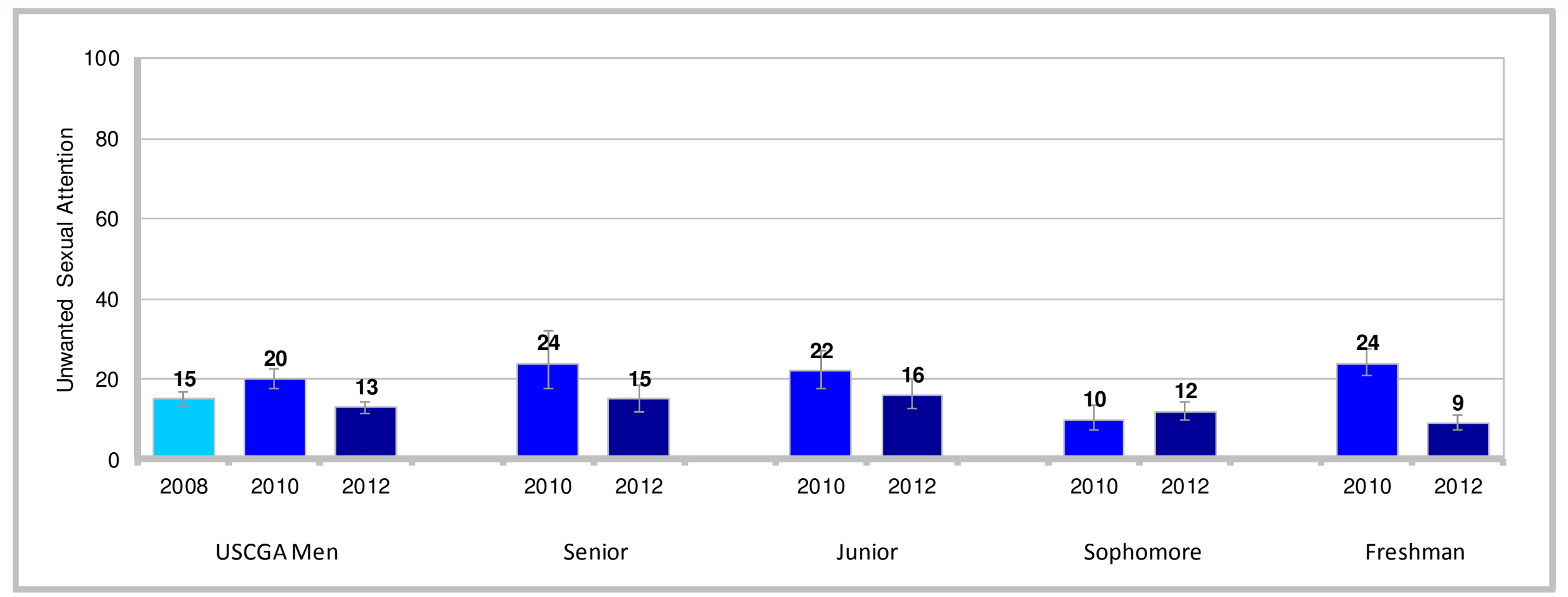

- $13 \%$ of men indicated experiencing unwanted sexual attention in 2012

- 2012 comparisons across years - lower than 2010

- Seniors, juniors, and freshmen in 2012 lower than 2010

- Class differences in 2012 - lower response led by freshmen 


\section{Sexual Coercion Incident Rate}

Percent of Women

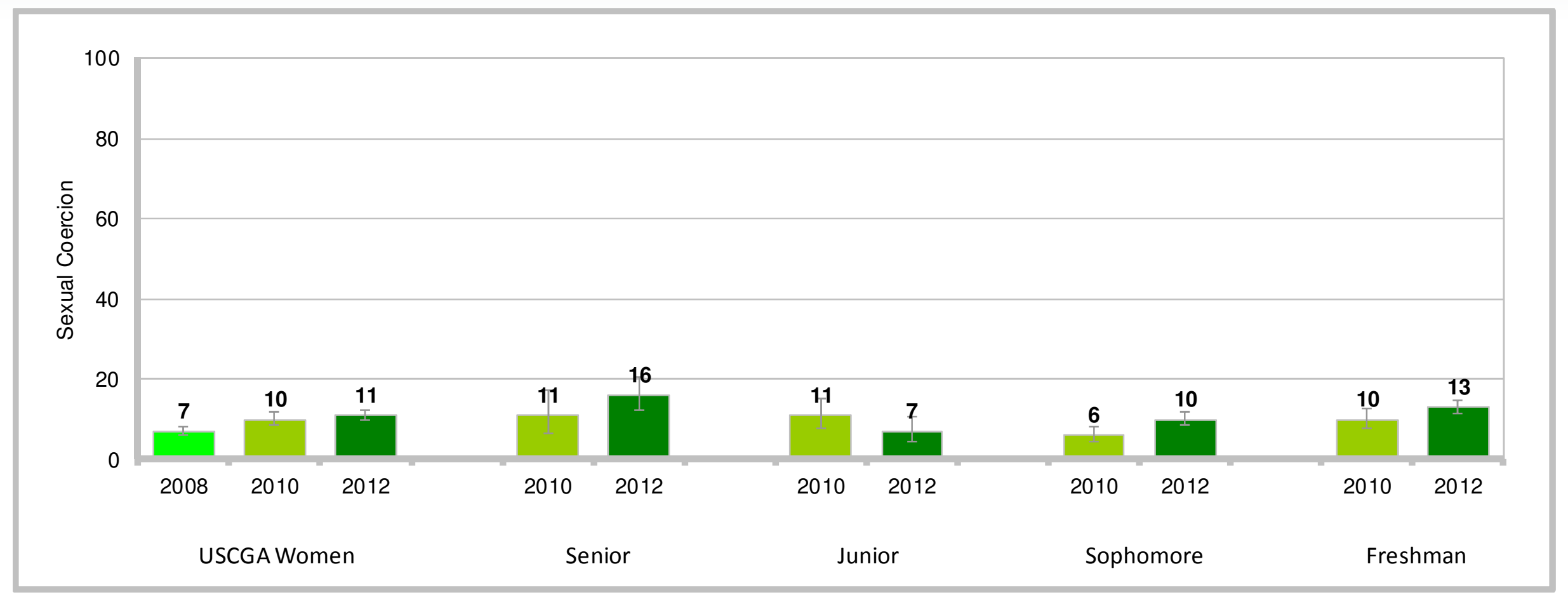

- $11 \%$ of women indicated experiencing sexual coercion in 2012

- 2012 comparisons across years - higher than 2008

- Sophomores in 2012 higher than 2010

- Class differences in 2012 - higher response led by seniors; lower response led by juniors 


\section{Sexual Coercion Incident Rate}

Percent of Men

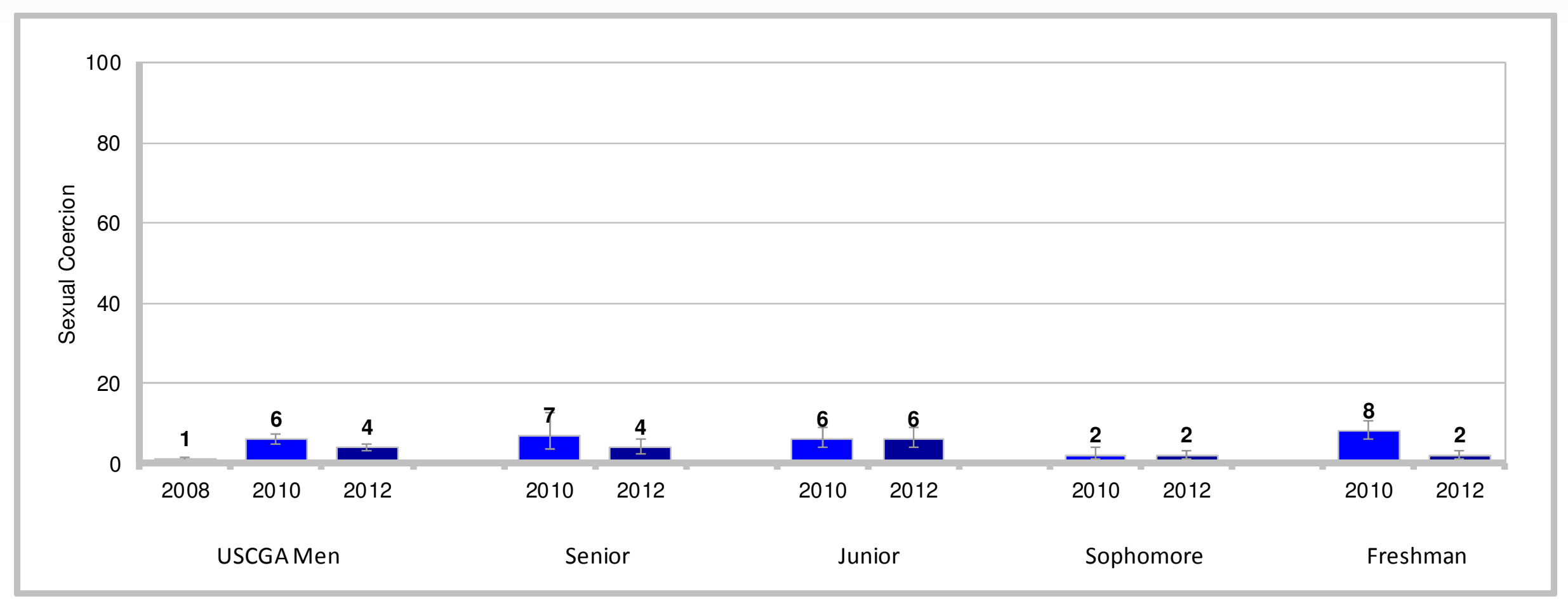

- $4 \%$ of men indicated experiencing sexual coercion in 2012

- 2012 comparisons across years - lower than 2010; higher than 2008

- Freshmen in 2012 lower than 2010

- Class differences in 2012 - lower response led by freshmen and sophomores 


\section{Contents}

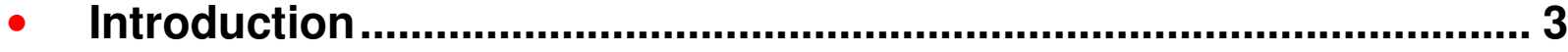

- Unwanted Sexual Contact ................................................................. 7

- Unwanted Gender-Related Behaviors ............................................. 45

- Sexual Harassment ..................................................................... 47

- Categories of Behaviors Associated with Sexual

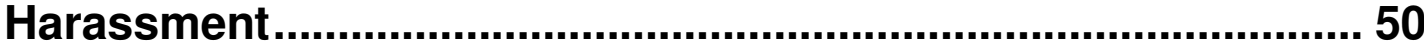

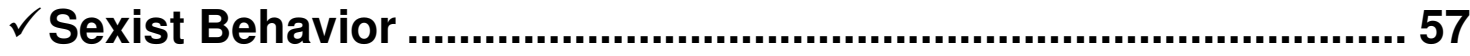

- Organizational Affiliation of Offender .......................................... 60

- Discussing/Reporting Experiences..............................................63 63

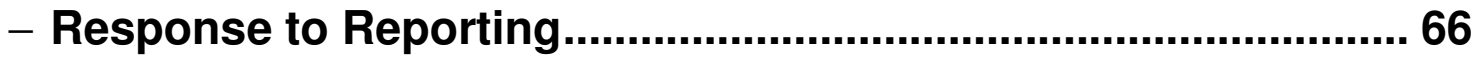

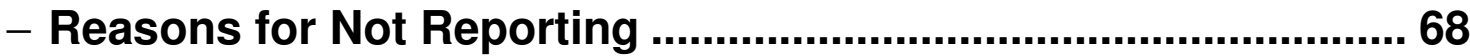

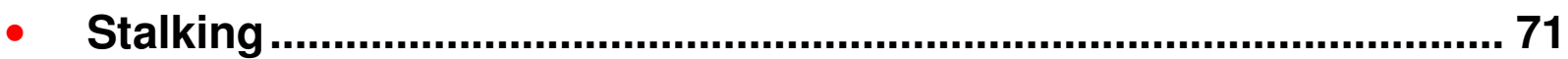

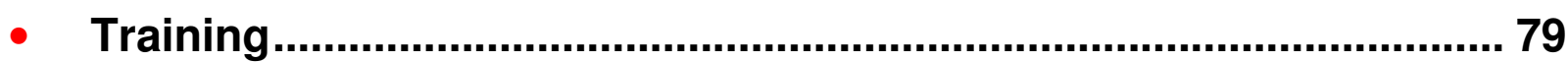

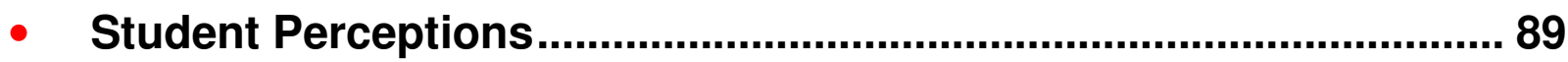




\section{Sexist Behavior Incident Rate Percent of Women}

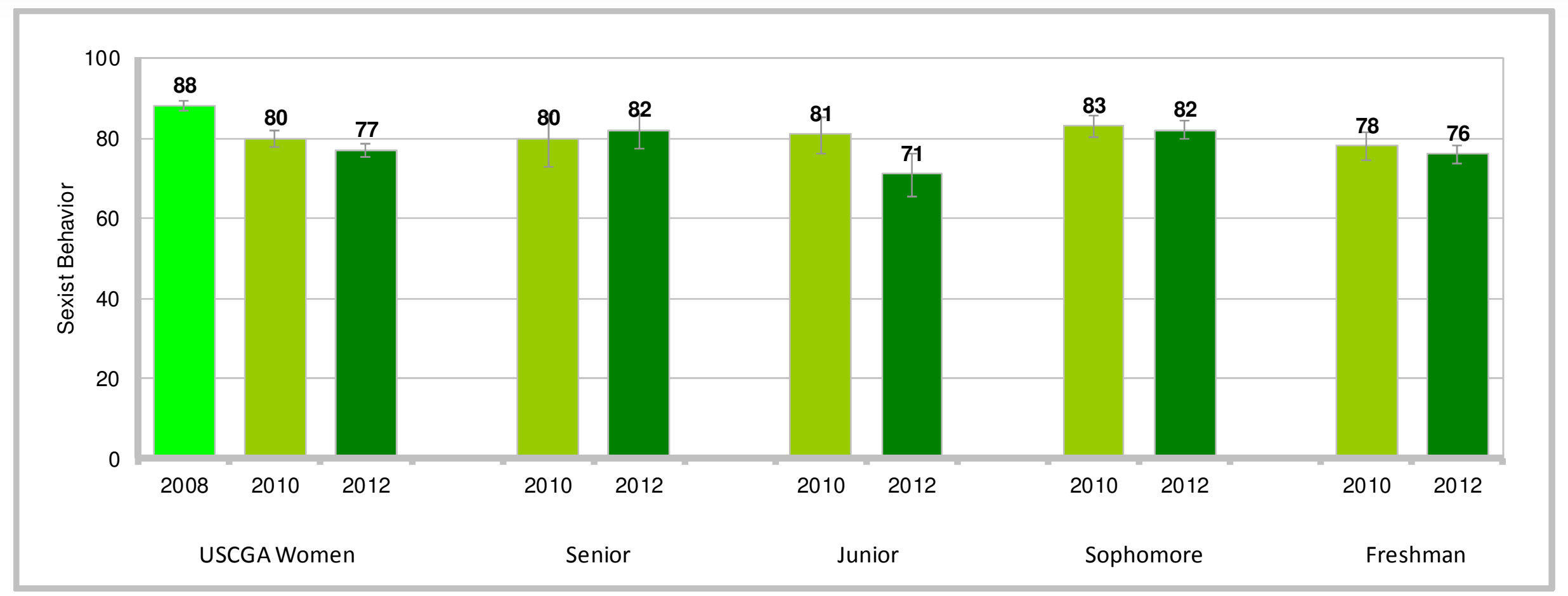

- 77\% of women indicated experiencing sexist behavior in 2012

- 2012 comparisons across years - lower than 2010, 2008

- Juniors in 2012 lower than 2010

- Class differences in 2012 - higher response led by seniors and sophomores; lower response led by juniors 


\section{Sexist Behavior Incident Rate}

Percent of Men

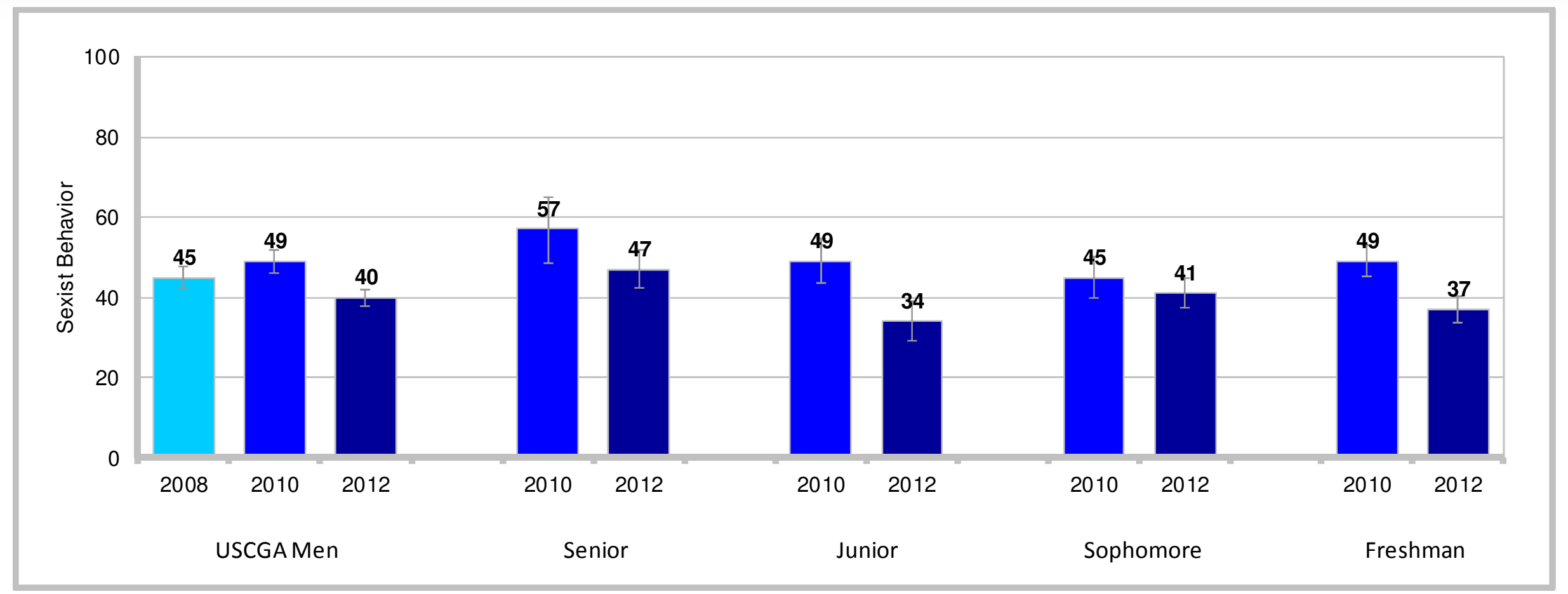

- $40 \%$ of men indicated experiencing sexist behavior in 2012

- 2012 comparisons across years - lower than 2010, 2008

- Seniors, juniors, and freshmen in 2012 lower than 2010

- Class differences in 2012 - higher response led by seniors 


\section{Contents}

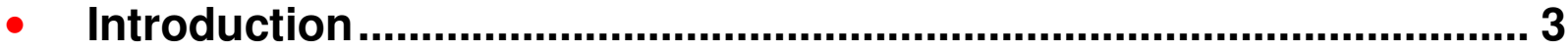

- Unwanted Sexual Contact ................................................................... 7

- Unwanted Gender-Related Behaviors ............................................. 45

- Sexual Harassment ...................................................................... 47

- Categories of Behaviors Associated with Sexual

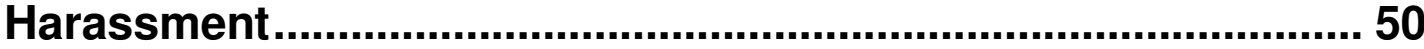

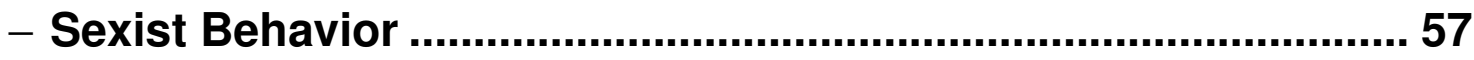

$\checkmark$ Organizational Affiliation of Offender .........................................6 60

- Discussing/Reporting Experiences...............................................63 63

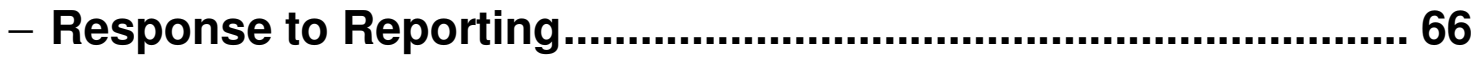

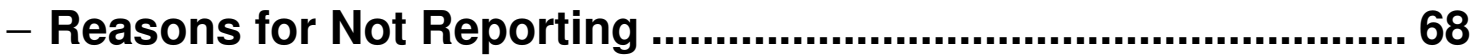

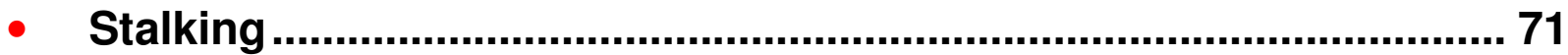

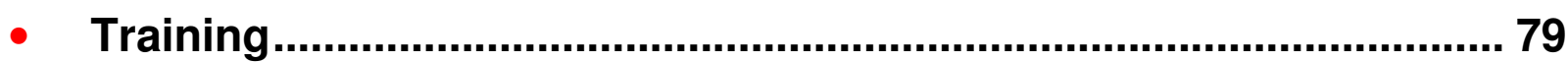

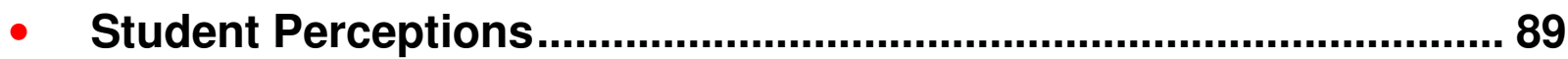




\section{Combinations of Offender Affiliations}

\section{Percent of Women Who Experienced Unwanted Gender-Related Behavior}

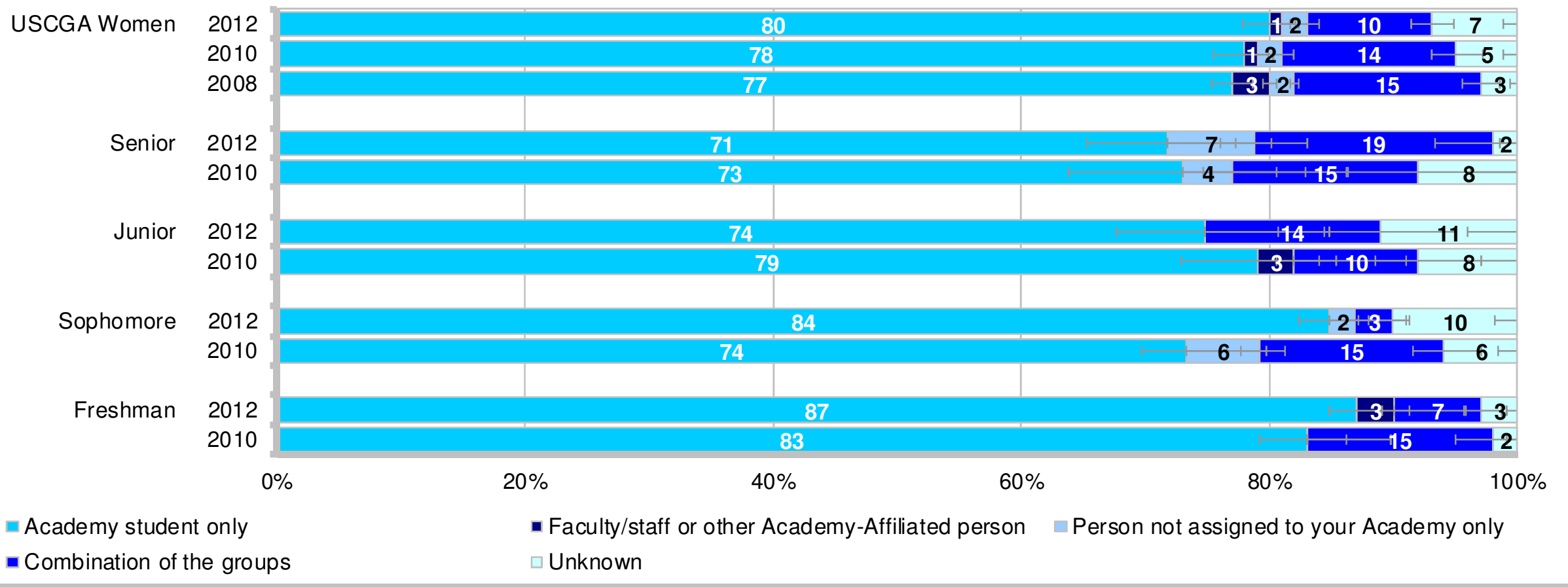

- $84 \%$ of women indicated experiencing one or more of the unwanted gender-related behaviors (i.e., crude/offensive behavior, unwanted sexual attention, sexual coercion, sexist behavior). Of those, $80 \%$ indicated the offender was an Academy student only; $1 \%$ indicated faculty/staff; $2 \%$ indicated person not assigned to the Academy; $10 \%$ indicated combination of groups; and $7 \%$ indicated unknown.

- 2012 comparisons across years - Academy student only higher than 2008; faculty/staff lower than 2008; combination of groups lower than 2010, 2008; unknown higher than 2010, 2008

- Sophomores indicating Academy student only in 2012 higher than 2010; juniors indicating faculty/staff in 2012 lower than 2010 , whereas freshmen higher; sophomores indicating person not assigned to the Academy in 2012 lower than 2010; sophomores and freshmen indicating combination of groups in 2012 lower than 2010; sophomores indicating unknown in 2012 higher than 2010, whereas seniors lower

- Class differences in 2012 - Academy student only led by freshmen and sophomores; faculty/staff led by freshmen; person not assigned to the Academy led by seniors; combination of groups led by seniors; unknown led by sophomores*

${ }^{*}$ Note that $11 \%$ of junior women indicated unknown. This percentage is not significantly different from the average of the other class years due to a higher margin of error for junior women responding to this question. 


\section{Combinations of Offender Affiliations}

\section{Percent of Men Who Experienced Unwanted Gender-Related Behavior}

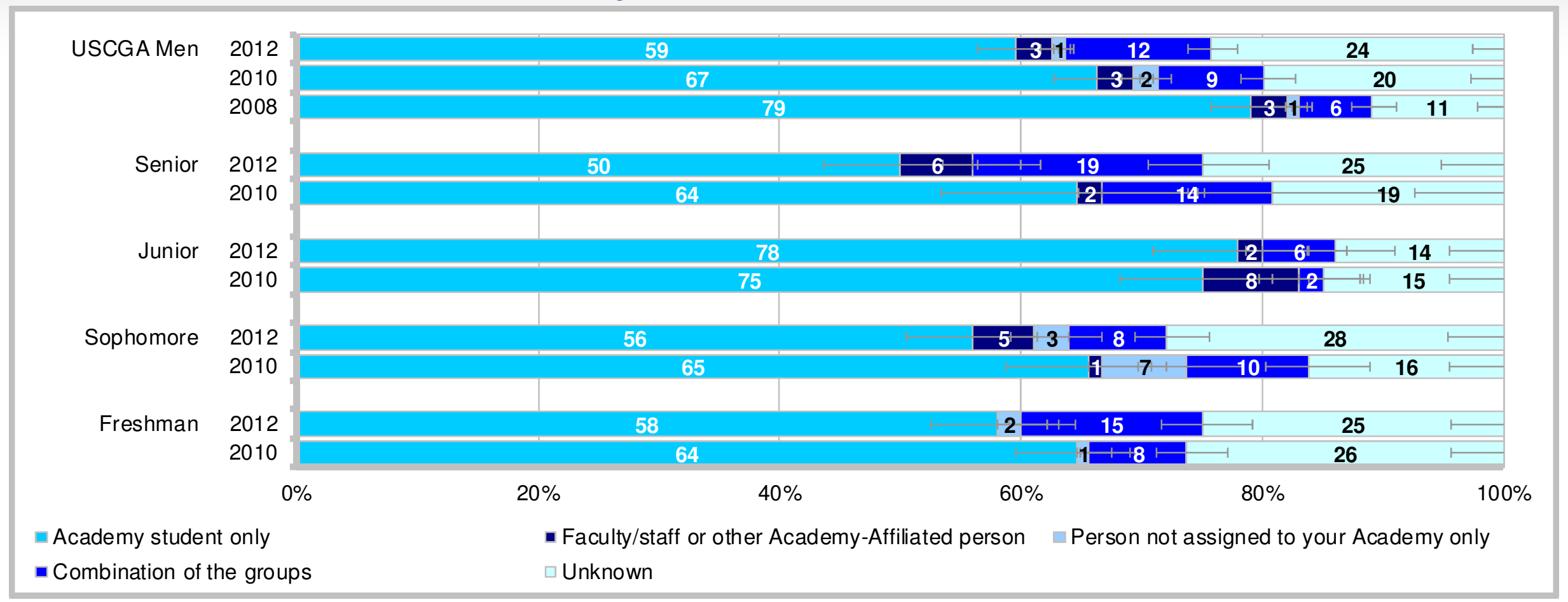

- $55 \%$ of men indicated experiencing one or more of the unwanted gender-related behaviors (i.e., crude/offensive behavior, unwanted sexual attention, sexual coercion, sexist behavior). Of those, $59 \%$ indicated the offender was Academy student only; $3 \%$ indicated faculty/staff; $1 \%$ indicated person not assigned to the Academy; $12 \%$ indicated combination of groups; and $24 \%$ indicated unknown.

- 2012 comparisons across years - Academy student only lower than 2010, 2008; combination of groups higher than 2008; unknown higher than 2010, 2008

- Seniors and sophomores indicating Academy student only in 2012 lower than 2010; sophomores indicating faculty/staff in 2012 higher than 2010, whereas juniors lower; freshmen indicating combination of groups in 2012 higher than 2010; sophomores indicating unknown in 2012 higher than 2010

- Class differences in 2012 - Academy student only led by juniors; person not assigned to the Academy led by sophomores; combination of groups led by seniors 


\section{Contents}

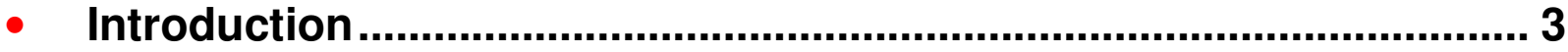

- Unwanted Sexual Contact ................................................................... 7

- Unwanted Gender-Related Behaviors ............................................. 45

- Sexual Harassment ...................................................................... 47

- Categories of Behaviors Associated with Sexual

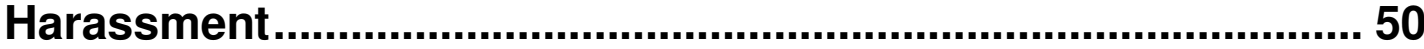

- Sexist Behavior ................................................................................ 57

- Organizational Affiliation of Offender ........................................6 60

$\checkmark$ Discussing/Reporting Experiences.............................................. 63

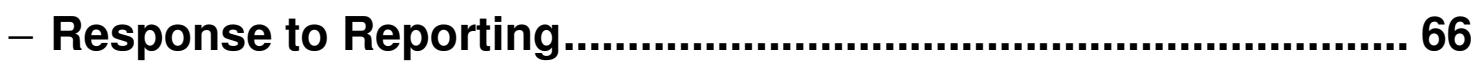

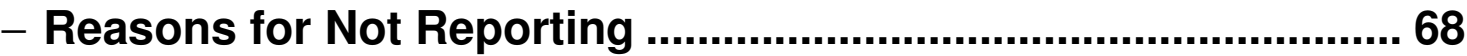

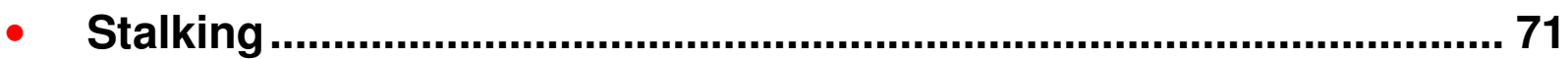

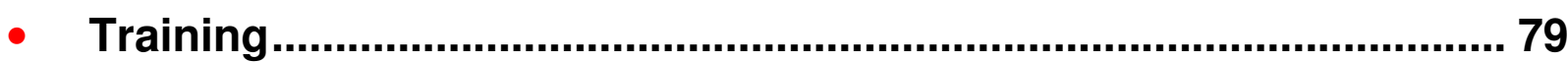

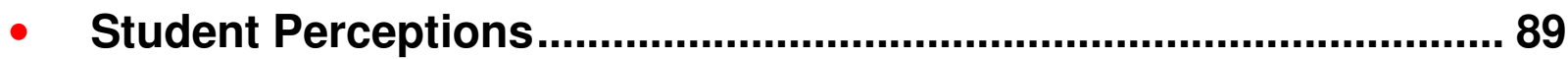




\section{Discuss/Report This Situation to an Authority or Organization} Percent of Women Who Experienced Unwanted Gender-Related Behavior

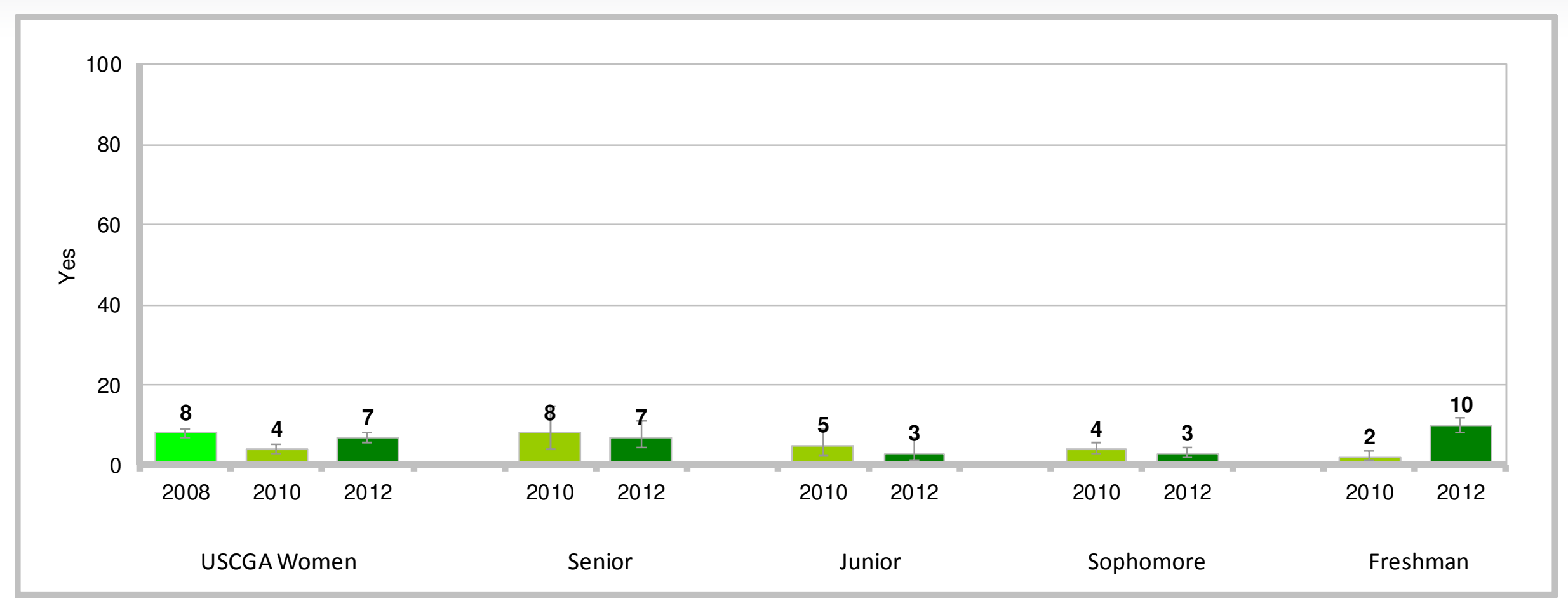

- $84 \%$ of women indicated experiencing one or more of the unwanted gender-related behaviors (i.e., crude/offensive behavior, unwanted sexual attention, sexual coercion, sexist behavior). Of those, $7 \%$ indicated they reported the situation to an authority or organization.

- 2012 comparisons across years - higher than 2010

- Freshmen in 2012 higher than 2010

- Class differences in 2012 - higher response led by freshmen; lower response led by sophomores*

*Note that both $3 \%$ of junior and sophomore women indicated they reported the situation in 2012.

The percentage for junior women is not significantly different from the average of the other class 


\section{Discuss/Report This Situation to an Authority or Organization Percent of Men Who Experienced Unwanted Gender-Related Behavior}

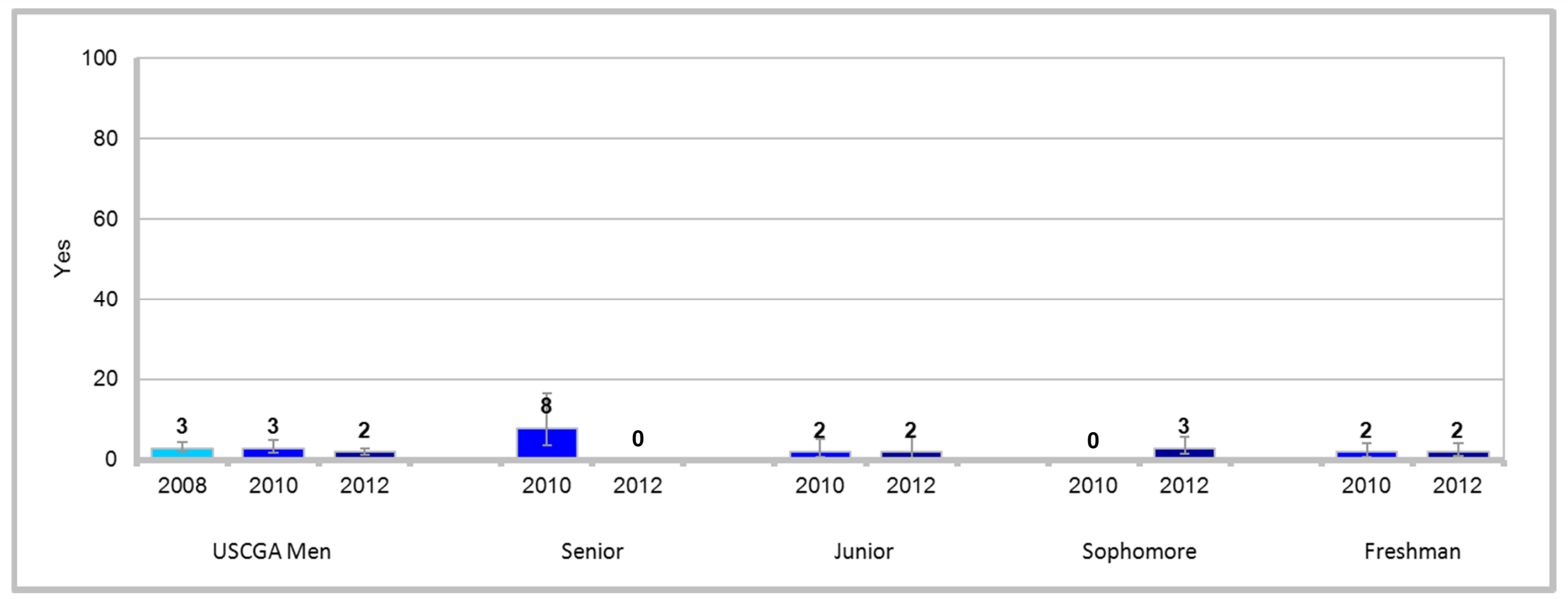

- $55 \%$ of men indicated experiencing one or more of the unwanted gender-related behaviors (i.e., crude/offensive behavior, unwanted sexual attention, sexual coercion, sexist behavior). Of those, $2 \%$ indicated they reported the situation to an authority or organization.

- 2012 comparisons across years - no differences

- Sophomores in 2012 higher than 2010, whereas seniors lower

- Class differences in 2012 - lower response led by seniors 


\section{Contents}

- Introduction ............................................................................................. 3

- Unwanted Sexual Contact ................................................................. 7

- Unwanted Gender-Related Behaviors .............................................. 45

- Sexual Harassment ..................................................................... 47

- Categories of Behaviors Associated with Sexual Harassment................................................................................ 50

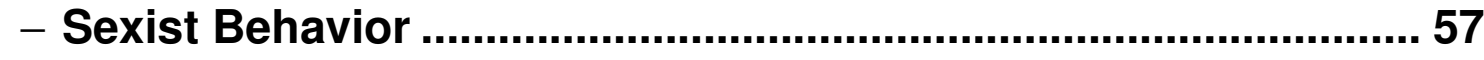

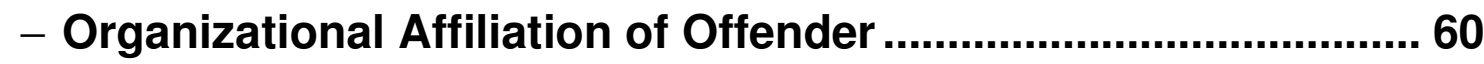

- Discussing/Reporting Experiences............................................... 63

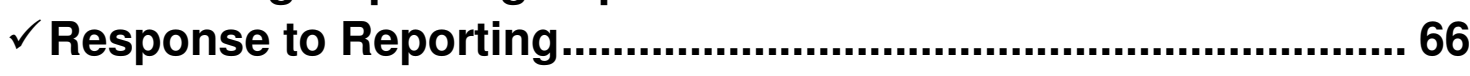

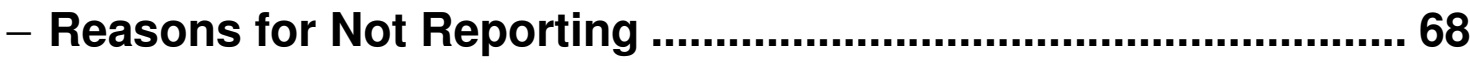

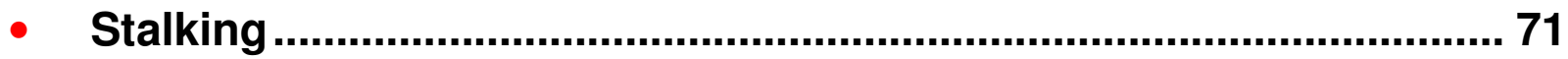

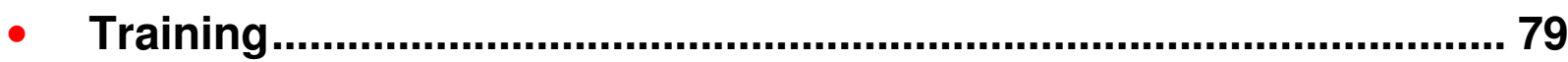

- Student Perceptions.................................................................... 89 


\section{Actions Taken in Response to Discussing/Reporting}

\section{Percent of Women Who Experienced Unwanted Gender-Related Behaviors and Reported It}

\begin{tabular}{|c|c|c|c|c|c|c|}
\hline Response to Reporting & $\begin{array}{l}\text { Survey } \\
\text { Year }\end{array}$ & Total & Senior & Junior & Sophomore & Freshman \\
\hline \multirow{2}{*}{ The situation was corrected } & 2012 & $68 \uparrow$ & NR & NR & NR & 83 \\
\hline & 2010 & 41 & NR & NR & NR & NR \\
\hline \multirow{2}{*}{ My situation was/is being investigated } & 2012 & $45 \uparrow$ & NR & NR & NR & 50 \\
\hline & 2010 & 28 & NR & NR & NR & NR \\
\hline \multirow{2}{*}{$\begin{array}{l}\text { I was kept informed of what actions were } \\
\text { being taken }\end{array}$} & 2012 & 46 & NR & NR & NR & 50 \\
\hline & 2010 & 56 & NR & NR & NR & NR \\
\hline \multirow{2}{*}{ I was encouraged to "tough it out" } & 2012 & $24 \downarrow$ & NR & NR & NR & 17 \\
\hline & 2010 & 43 & NR & NR & NR & NR \\
\hline \multirow{2}{*}{$\begin{array}{l}\text { My situation was discounted or not taken } \\
\text { seriously }\end{array}$} & 2012 & 8 & NR & NR & NR & 0 \\
\hline & 2010 & 16 & NR & NR & NR & NR \\
\hline \multirow{2}{*}{ Action was taken against me } & 2012 & $7 \uparrow$ & NR & NR & NR & \\
\hline & 2010 & 0 & NR & NR & NR & NR \\
\hline \multirow{2}{*}{$\begin{array}{l}\text { I was ridiculed or scorned by others for } \\
\text { discussing/reporting the situation }\end{array}$} & 2012 & $23 \uparrow$ & NR & NR & NR & 17 \\
\hline & 2010 & 0 & NR & NR & NR & NR \\
\hline \multirow{2}{*}{ Some other action was taken } & 2012 & 24 & NR & NR & NR & 17 \\
\hline & 2010 & 29 & NR & NR & NR & $\mathrm{NR}$ \\
\hline Margins of Error & & $\pm 0-19 \%$ & -- & -- & -- & $\pm 0-10 \%$ \\
\hline \multirow{4}{*}{ - Results for men not reportable } & & \multicolumn{3}{|c|}{ Higher Response of Yes } & \multirow{4}{*}{\multicolumn{2}{|c|}{ Margins of error range from $\pm 0 \%$ to $\pm 19 \%$}} \\
\hline & & \multicolumn{3}{|c|}{ Lower Response of Yes } & & \\
\hline & & \multicolumn{3}{|c|}{ Higher in 2012 than 2010} & & \\
\hline & 2 Q17a-g & \multicolumn{3}{|c|}{ Lower in 2012 than 2010} & & \\
\hline
\end{tabular}




\section{Contents}

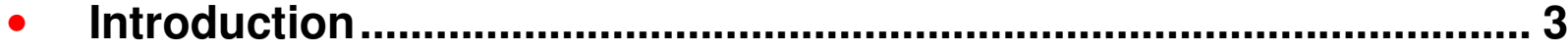

- Unwanted Sexual Contact ................................................................. 7

- Unwanted Gender-Related Behaviors ............................................. 45

- Sexual Harassment ..................................................................... 47

- Categories of Behaviors Associated with Sexual

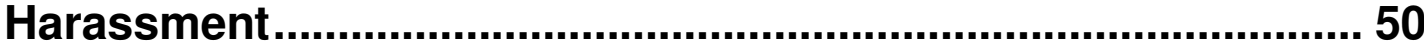

- Sexist Behavior .............................................................................. 57

- Organizational Affiliation of Offender ........................................6 60

- Discussing/Reporting Experiences...............................................63 63

- Response to Reporting .............................................................. 66

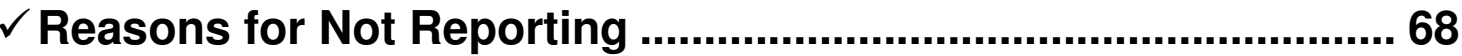

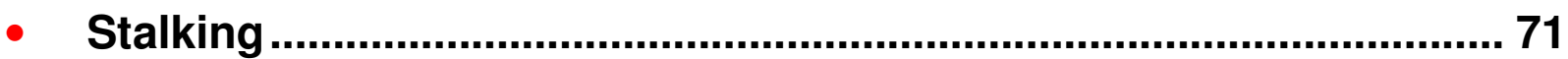

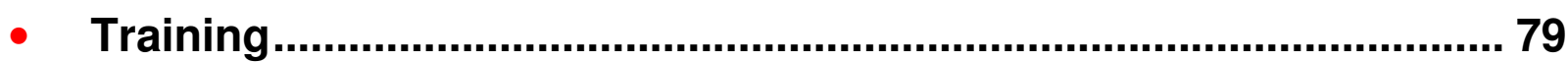

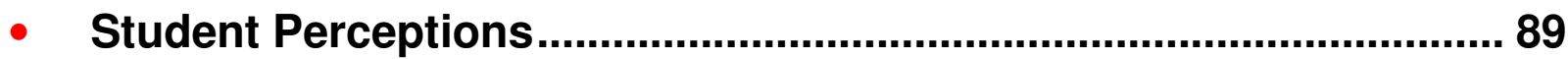




\section{Most Frequently Selected Reasons for}

\section{Not Reporting Unwanted Gender-Related Situation}

Percent of Women Who Experienced Unwanted Gender-Related Behaviors and Did Not Report It

\begin{tabular}{|c|c|c|c|c|c|c|}
\hline Reasons for Not Reporting & $\begin{array}{l}\text { Survey } \\
\text { Year }\end{array}$ & Total & Senior & Junior & Sophomore & Freshman \\
\hline \multirow{2}{*}{$\begin{array}{l}\text { Thought it was not important enough to } \\
\text { report }\end{array}$} & 2012 & 83 & 77 & 81 & $92 \uparrow$ & $80 \downarrow$ \\
\hline & 2010 & 82 & 77 & 83 & 78 & 88 \\
\hline \multirow{2}{*}{ Took care of the problem myself } & 2012 & 72 & 70 & 72 & $75 \uparrow$ & $70 \uparrow$ \\
\hline & 2010 & 70 & 77 & 74 & 69 & 63 \\
\hline \multirow{2}{*}{ Did not want people gossiping about me } & 2012 & 35 & 40 & 28 & 41 & 30 \\
\hline & 2010 & 36 & 50 & 26 & 37 & 35 \\
\hline \multirow{2}{*}{$\begin{array}{l}\text { Thought reporting would take too much } \\
\text { time and effort }\end{array}$} & 2012 & $28 \uparrow$ & 27 & 28 & $27 \uparrow$ & $30 \uparrow$ \\
\hline & 2010 & 24 & 32 & 23 & 22 & 22 \\
\hline \multirow{2}{*}{ Did not want to hurt the offender's career } & 2012 & $26 \uparrow$ & $35 \uparrow$ & 19 & 25 & $26 \uparrow$ \\
\hline & 2010 & 20 & 9 & 26 & 24 & 18 \\
\hline Margins of Error & & $\pm 3 \%$ & $\pm 7-11 \%$ & $\pm 7-8 \%$ & $\pm 3-4 \%$ & $\pm 3-5 \%$ \\
\hline
\end{tabular}

\begin{tabular}{|l|c|}
\hline Higher Response of Yes & \\
\hline Lower Response of Yes & \\
\hline Higher in 2012 than 2010 & $\uparrow$ \\
\hline Lower in 2012 than 2010 & $\downarrow$ \\
\hline
\end{tabular}




\section{Most Frequently Selected Reasons for Not Reporting Unwanted Gender-Related Situation} Percent of Men Who Experienced Unwanted Gender-Related Behaviors and Did Not Report It

\begin{tabular}{|c|c|c|c|c|c|c|}
\hline Reasons for Not Reporting & $\begin{array}{l}\text { Survey } \\
\text { Year }\end{array}$ & Total & Senior & Junior & Sophomore & Freshman \\
\hline \multirow{2}{*}{$\begin{array}{l}\text { Thought it was not important enough to } \\
\text { report }\end{array}$} & 2012 & $73 \downarrow$ & 73 & 73 & $73 \downarrow$ & 72 \\
\hline & 2010 & 79 & 81 & 82 & 84 & 70 \\
\hline \multirow{2}{*}{ Took care of the problem myself } & 2012 & $55 \downarrow$ & 59 & 67 & $47 \downarrow$ & $45 \downarrow$ \\
\hline & 2010 & 62 & 61 & 73 & 57 & 55 \\
\hline \multirow{2}{*}{$\begin{array}{l}\text { Thought reporting would take too much } \\
\text { time and effort }\end{array}$} & 2012 & 17 & 22 & 20 & $\overline{10 \downarrow}$ & 15 \\
\hline & 2010 & 21 & 19 & 21 & 23 & 19 \\
\hline \multirow{2}{*}{ Did not think anything would be done } & 2012 & $16 \uparrow$ & 24 & $16 \uparrow$ & 12 & $13 \uparrow$ \\
\hline & 2010 & 9 & 14 & 7 & 11 & 4 \\
\hline \multirow{2}{*}{ Thought I would be labeled a troublemaker } & 2012 & 11 & $15 \uparrow$ & 13 & 10 & $4 \downarrow$ \\
\hline & 2010 & 9 & 6 & 9 & 11 & 8 \\
\hline \multirow{2}{*}{$\begin{array}{l}\text { Thought it would hurt my reputation and } \\
\text { standing }\end{array}$} & 2012 & 11 & 8 & 16 & $8 \downarrow$ & 13 \\
\hline & 2010 & 10 & 3 & 13 & 15 & 9 \\
\hline Margins of Error & & $\pm 3-4 \%$ & $\pm 5-13 \%$ & $\pm 6-9 \%$ & $\pm 4-8 \%$ & $\pm 3-6 \%$ \\
\hline
\end{tabular}

\begin{tabular}{|l|c|}
\hline Higher Response of Yes & \\
\hline Lower Response of Yes & \\
\hline Higher in 2012 than 2010 & $\uparrow$ \\
\hline Lower in 2012 than 2010 & $\downarrow$ \\
\hline
\end{tabular}




\section{Contents}

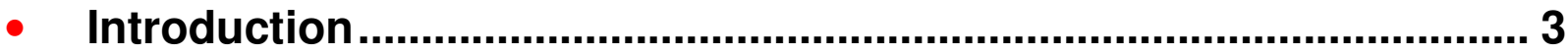

- Unwanted Sexual Contact ................................................................. 7

- Unwanted Gender-Related Behaviors .............................................. 45

$\checkmark$ Stalking ........................................................................................ 71

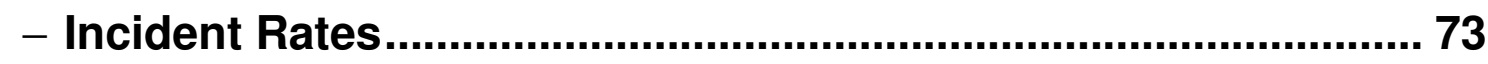

- Stalking and Sexual Harassment Related to Unwanted

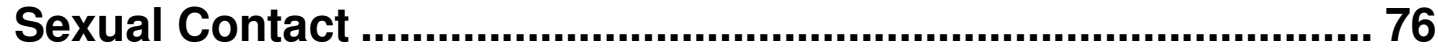

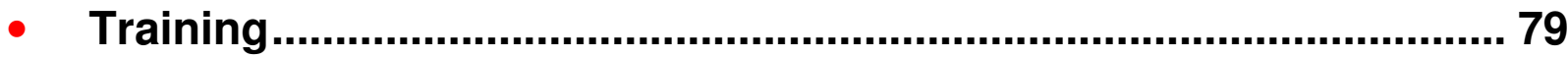

- Student Perceptions........................................................................... 89 


\section{Stalking Incident Rate}

\section{- Definition and measure of stalking:}

- Under Article 120a of the UCMJ, stalking is a crime. The UCMJ definition of stalking is "a course of conduct directed at a specific person that would cause a reasonable person to fear death or bodily harm, including sexual assault, to himself or herself or a member of his or her immediate family."

- Thus, to be punishable under the provision, stalking must be intentional, repeated (two or more occasions of such conduct), and cause unreasonable fear of physical injury. Note that this definition does not limit stalking to association with sexual harassment or sexual assault.

- The measure of stalking on the 2012 SAGR is consistent with the definition in Article 120a of the UCMJ. Students were asked (Q9) whether they had experienced any stalking behaviors (e.g., followed or spied on them in public areas; spied on them in private areas; showed up at places where they were even though he/she had no reason to be there; left unwanted items for them to find; stood outside or hung around their dorm room or classroom even though he/she had no reason to be there; and vandalized or tampered with their belongings) by someone assigned to their Academy, including students and military/civilian personnel, and whether they felt in danger of physical harm or sexual assault as a result of the experience (Q11). 


\section{Contents}

Slide

• Introduction .......................................................................................... 3

- Unwanted Sexual Contact ............................................................... 7

- Unwanted Gender-Related Behaviors ........................................... 45

- Stalking ........................................................................................... 71

$\checkmark$ Incident Rates............................................................................ 73

- Stalking and Sexual Harassment Related to Unwanted Sexual Contact ....................................................................... 76

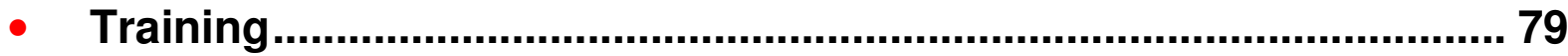

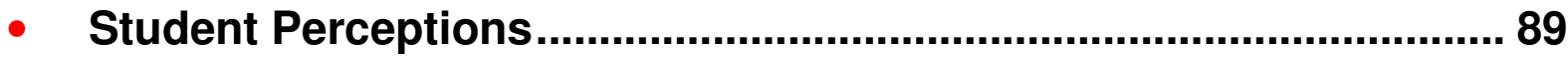




\section{Stalking Incident Rate}

Percent of Women Who Experienced Stalking Behavior and Felt in Danger

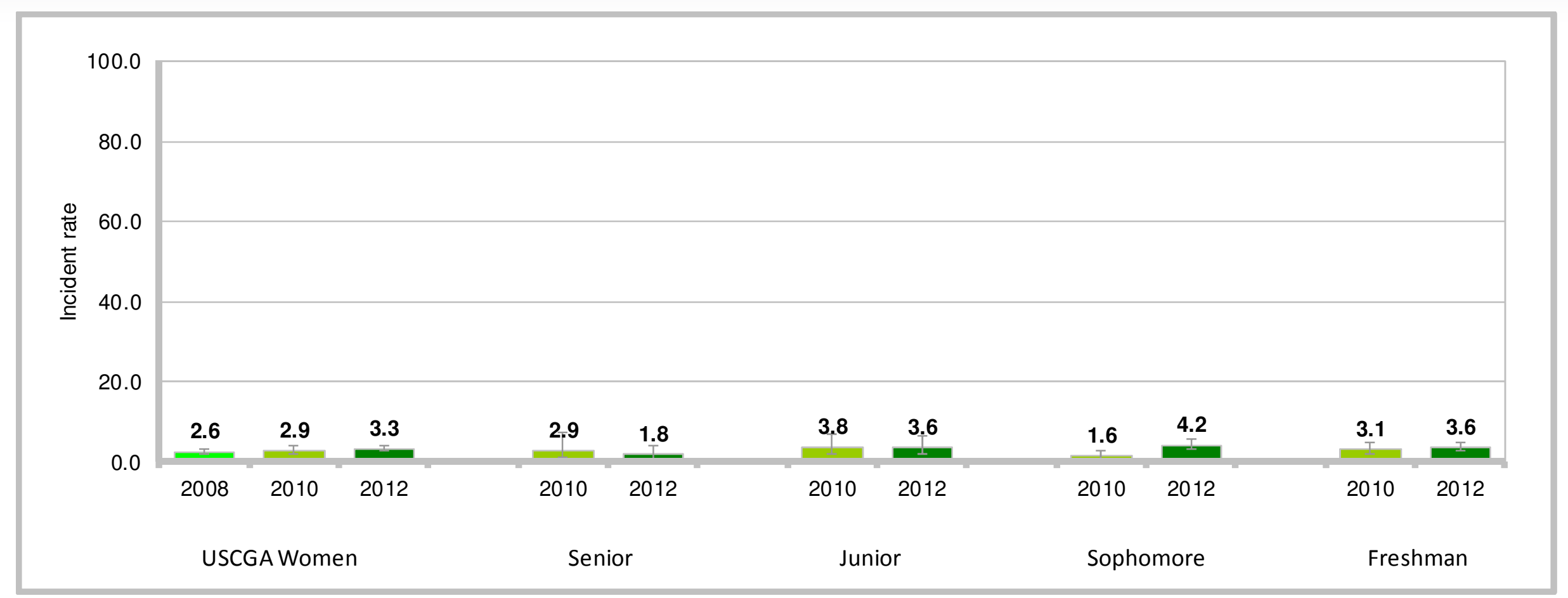

- $3.3 \%$ of women indicated experiencing stalking behavior in 2012

- 2012 comparisons across years - no differences

- Sophomores in 2012 higher than 2010

- Class differences in 2012 - no differences 


\section{Stalking Incident Rate}

Percent of Men Who Experienced Stalking Behavior and Felt in Danger

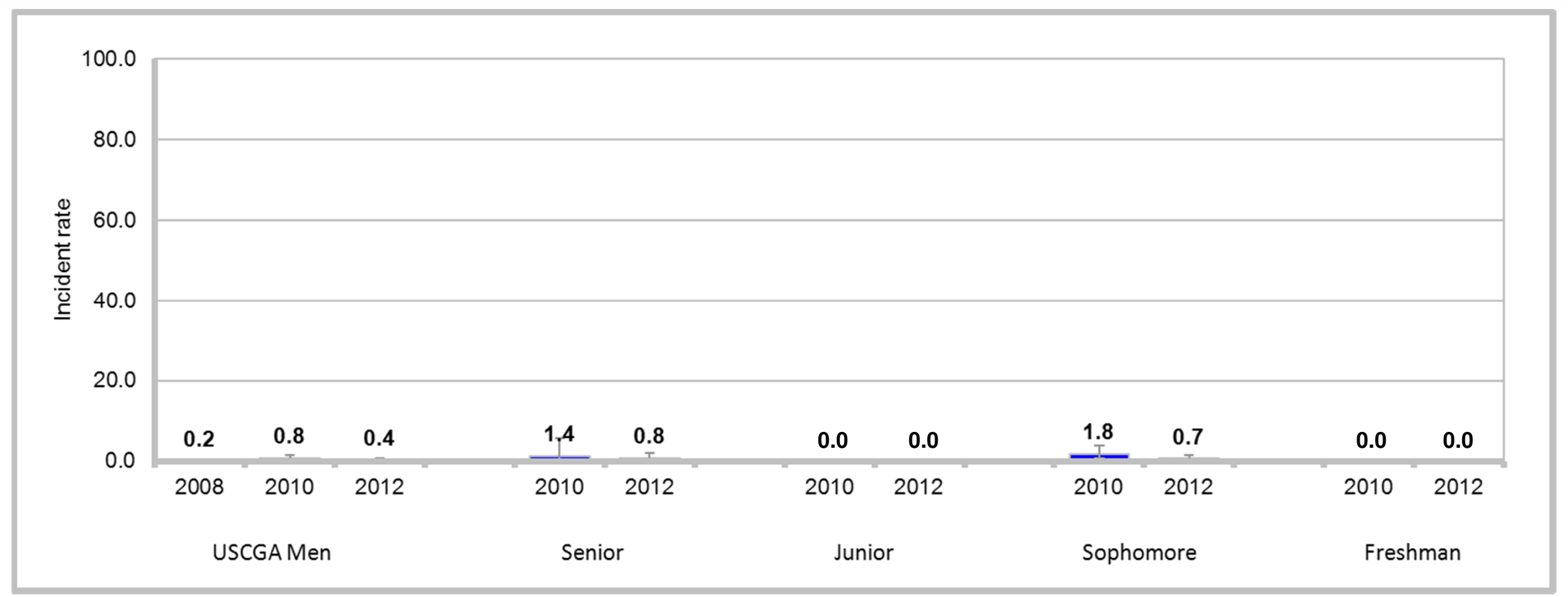

- $0.4 \%$ of men indicated experiencing stalking behavior in 2012

- 2012 comparisons across years - no differences

- Class differences in 2012 - lower response led by juniors and freshmen 


\section{Contents}

• Introduction .................................................................................. 3

- Unwanted Sexual Contact ................................................................ 7

- Unwanted Gender-Related Behaviors ........................................... 45

• Stalking ............................................................................................ 71

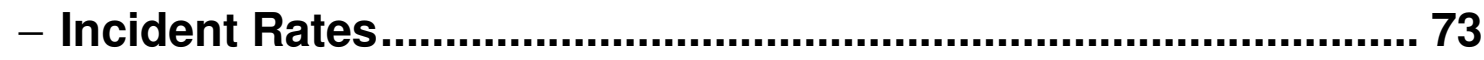

$\checkmark$ Stalking and Sexual Harassment Related to Unwanted

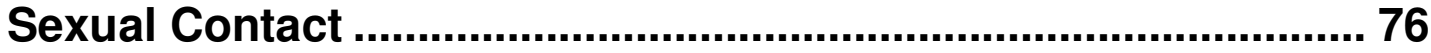

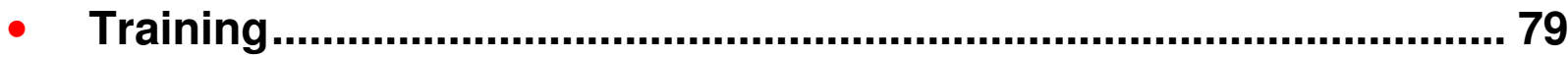

- Student Perceptions...................................................................... 89 


\section{Stalking and Sexual Harassment Possibly Lead to Sexual Assault}

\section{Percent of Women}

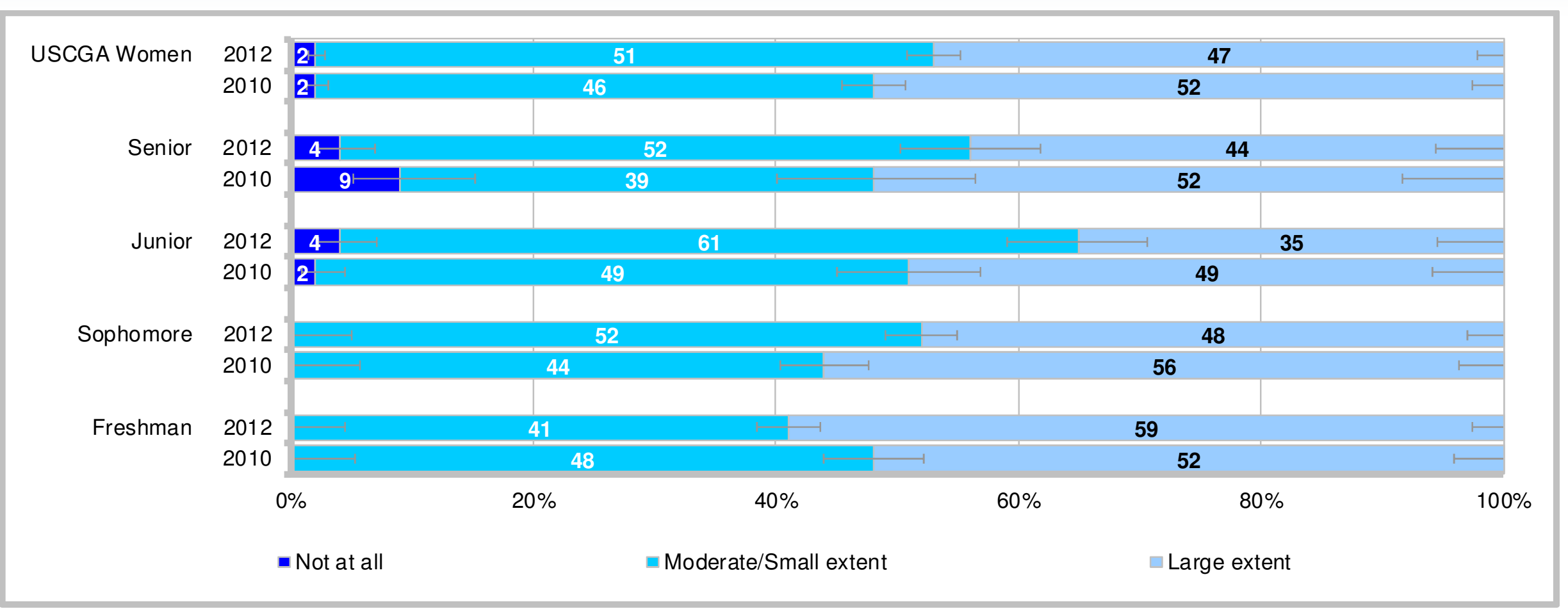

- $98 \%$ of women indicated stalking and sexual harassment possibly lead to sexual assault to at least some extent; $2 \%$ indicated stalking and sexual harassment do not lead to sexual assault

- 2012 comparisons across years - large extent lower than 2010; moderate/small extent higher than 2010

- Freshmen indicating large extent in 2012 higher than 2010, whereas juniors and sophomores lower; seniors, juniors, and sophomores indicating moderate/small extent in 2012 higher than 2010, whereas freshmen lower

- Class differences in 2012 - large extent led by freshmen; moderate/small extent led by juniors 


\section{Stalking and Sexual Harassment Possibly Lead to Sexual Assault} Percent of Men

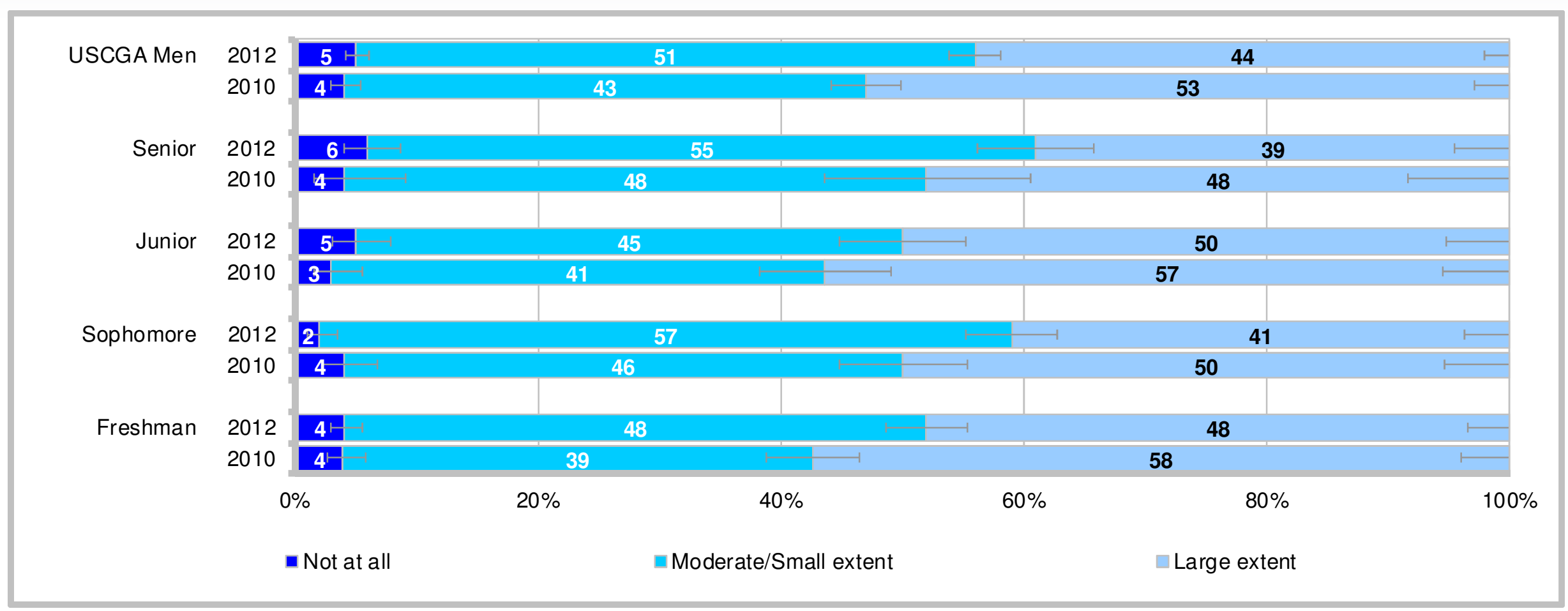

- $95 \%$ of men indicated stalking and sexual harassment possibly lead to sexual assault to at least some extent; $5 \%$ indicated stalking and sexual harassment do not lead to sexual assault

- 2012 comparisons across years - large extent lower than 2010; moderate/small extent higher than 2010

- Sophomores and freshmen indicating large extent in 2012 lower than 2010; sophomores and freshmen indicating moderate/small extent in 2012 higher than 2010

- Class differences in 2012 - moderate/small extent led by sophomores 


\section{Contents}

Slide

- Introduction ............................................................................................. 3

- Unwanted Sexual Contact ................................................................ 7

- Unwanted Gender-Related Behaviors ........................................... 45

• Stalking ........................................................................................ 71

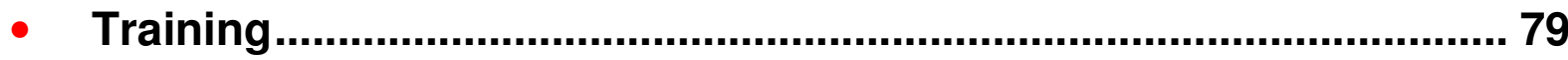

$\checkmark$ Sexual Assault Training Availability and Effectiveness ........... 79

- Sexual Harassment Training Availability and Effectiveness..... 84

- Student Perceptions....................................................................... 89 


\section{Received Training Since June 2011 on Topics Related to Sexual Assault Percent of Women}

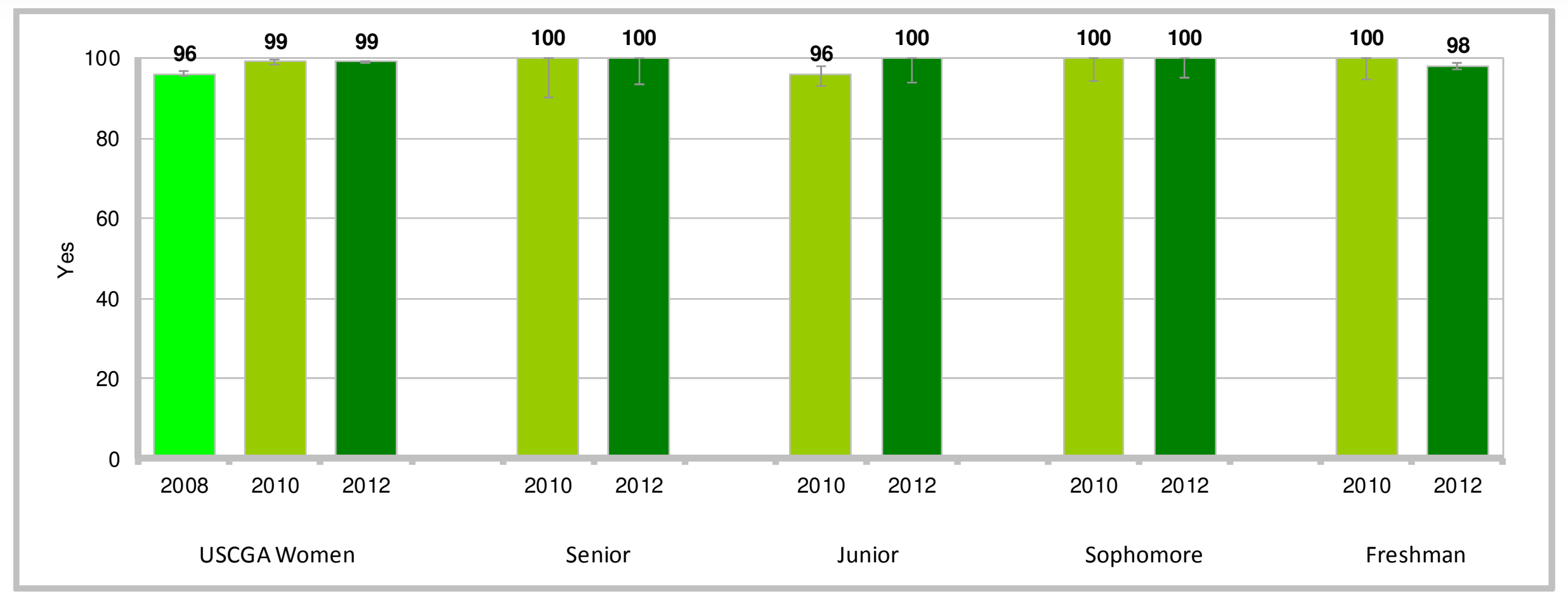

- $99 \%$ of women indicated receiving training on topics related to sexual assault in 2012

- 2012 comparisons across years - higher than 2008

- Juniors in 2012 higher than 2010; freshmen in 2012 lower than 2010

- Class differences in 2012 - higher response led by seniors, juniors, and sophomores; lower response led by freshmen 


\section{Received Training Since June 2011 on Topics Related to Sexual Assault} Percent of Men

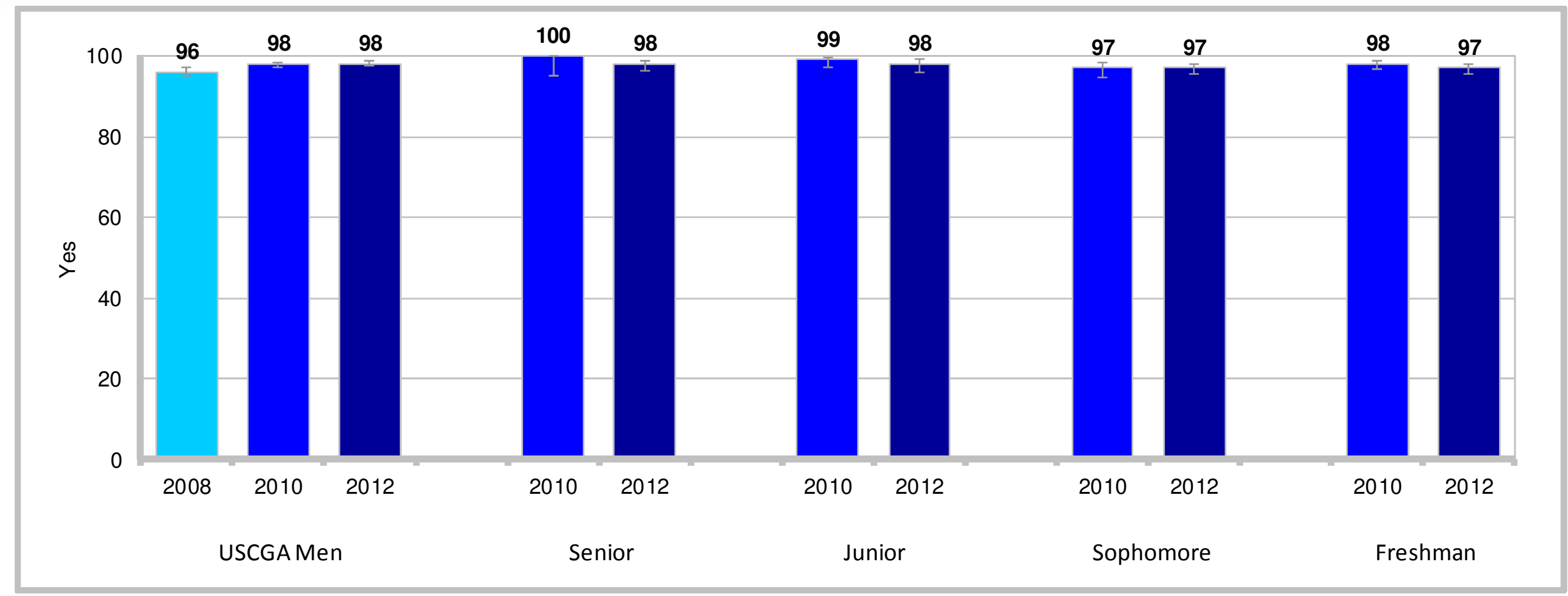

- $98 \%$ of men indicated receiving training on topics related to sexual assault in 2012

- 2012 comparisons across years - higher than 2008

- Seniors in 2012 lower than 2010

- Class differences in 2012 - no differences 


\section{Effectiveness of Training in Actually Reducing/Preventing Sexual Assault Percent of Women Who Had Training on Sexual Assault}

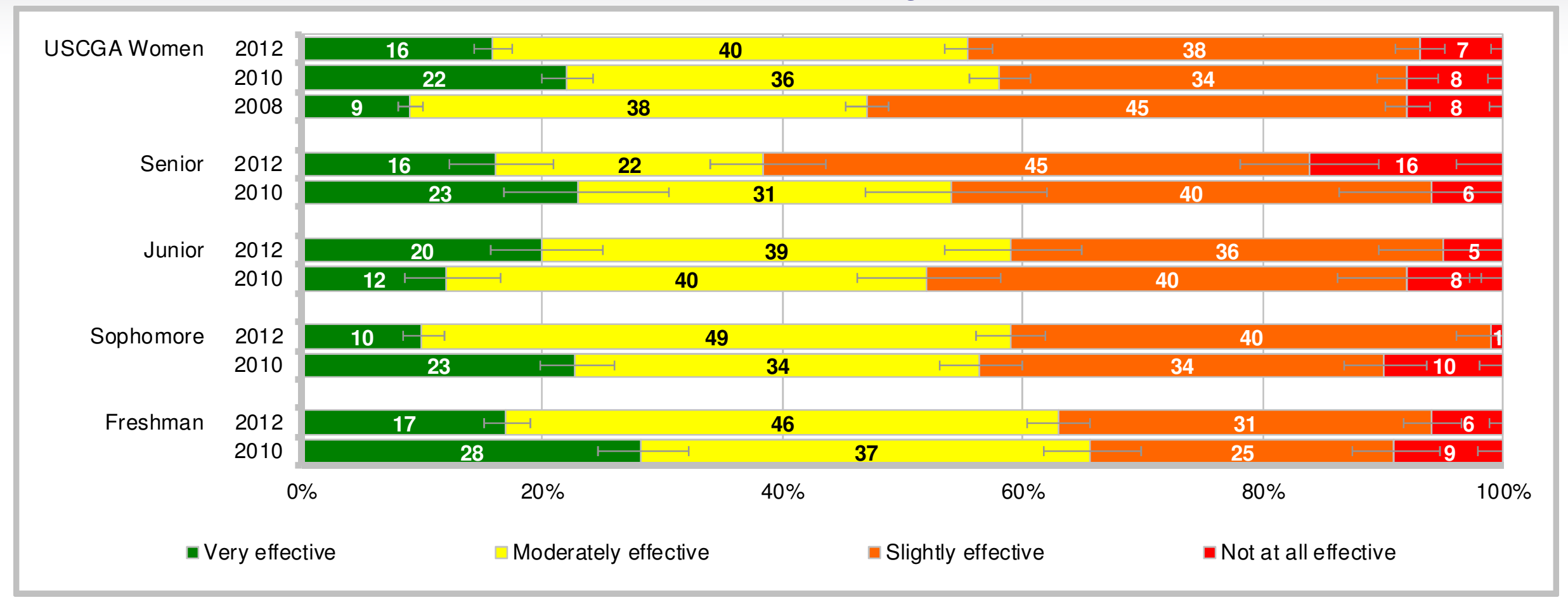

- $16 \%$ of women indicated their training was very effective in actually reducing/preventing sexual assault; $40 \%$ indicated moderately effective; $38 \%$ indicated slightly effective; and $7 \%$ indicated not at all effective

- 2012 comparisons across years - very effective lower than 2010, higher than 2008; moderately effective higher than 2010; slightly effective higher than 2010, lower than 2008

- Juniors indicating very effective in 2012 higher than 2010, whereas sophomores and freshmen lower; sophomores and freshmen indicating moderately effective in 2012 higher than 2010, whereas seniors lower; sophomores and freshmen indicating slightly effective in 2012 higher than 2010; seniors indicating not at all effective in 2012 higher than 2010, whereas sophomores and freshmen lower

- Class differences in 2012 - moderately effective led by sophomores and freshmen; slightly effective led by seniors; not at all effective led by seniors 


\section{Effectiveness of Training in Actually Reducing/Preventing Sexual Assault} Percent of Men Who Had Training on Sexual Assault

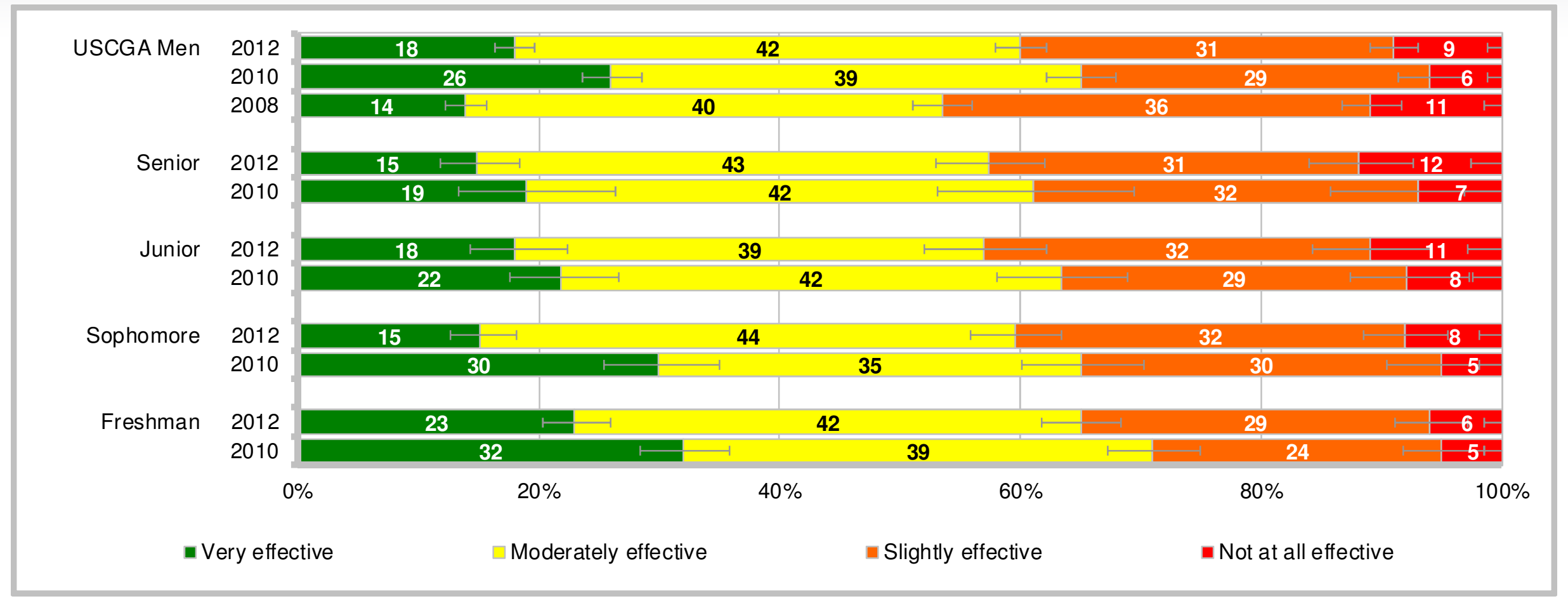

- $18 \%$ of men indicated their training was very effective in actually reducing/preventing sexual assault; $42 \%$ indicated moderately effective; $31 \%$ indicated slightly effective; and $9 \%$ indicated not at all effective

- 2012 comparisons across years - very effective lower than 2010, higher than 2008; slightly effective lower than 2008; not at all effective higher than 2010

- Sophomores and freshmen indicating very effective in 2012 lower than 2010; sophomores indicating moderately effective in 2012 higher than 2010; freshmen indicating slightly effective in 2012 higher than 2010

- Class differences in 2012 - very effective led by freshmen 


\section{Contents}

Slide

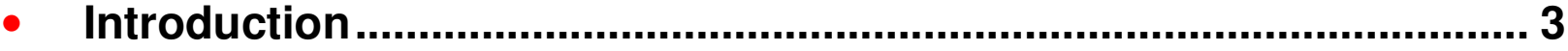

- Unwanted Sexual Contact .................................................................. 7

- Unwanted Gender-Related Behaviors .......................................... 45

• Stalking ......................................................................................... 71

• Training................................................................................................. 79

- Sexual Assault Training Availability and Effectiveness ............ 79

$\checkmark$ Sexual Harassment Training Availability and Effectiveness..... 84

- Student Perceptions....................................................................... 89 


\section{Received Training Since June 2011 on Topics Related to Sexual Harassment \\ Percent of Women}

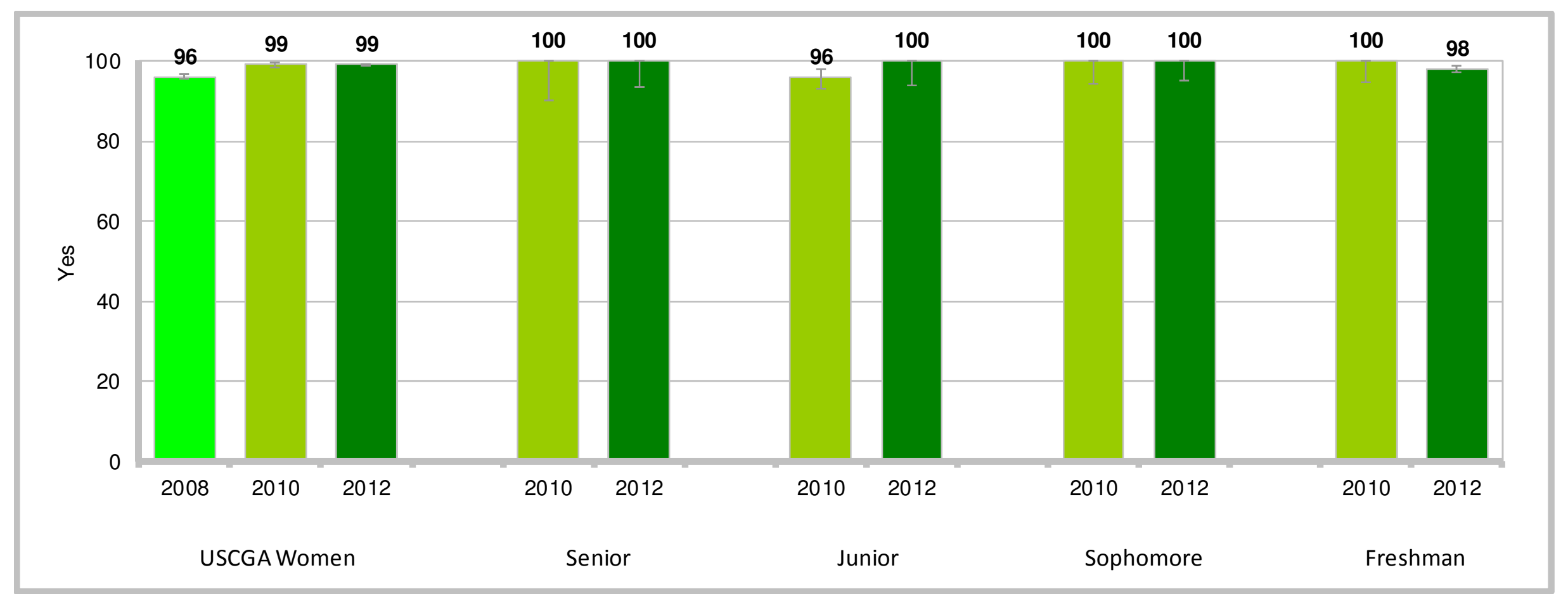

- $99 \%$ of women indicated receiving training on topics related to sexual harassment in 2012

- 2012 comparisons across years - higher than 2008

- Juniors in 2012 higher than 2010, whereas freshmen lower

- Class differences in $\mathbf{2 0 1 2}$ - higher response led by seniors, juniors, and sophomores; lower response led by freshmen 


\section{Received Training Since June 2011 on Topics Related to Sexual Harassment \\ Percent of Men}

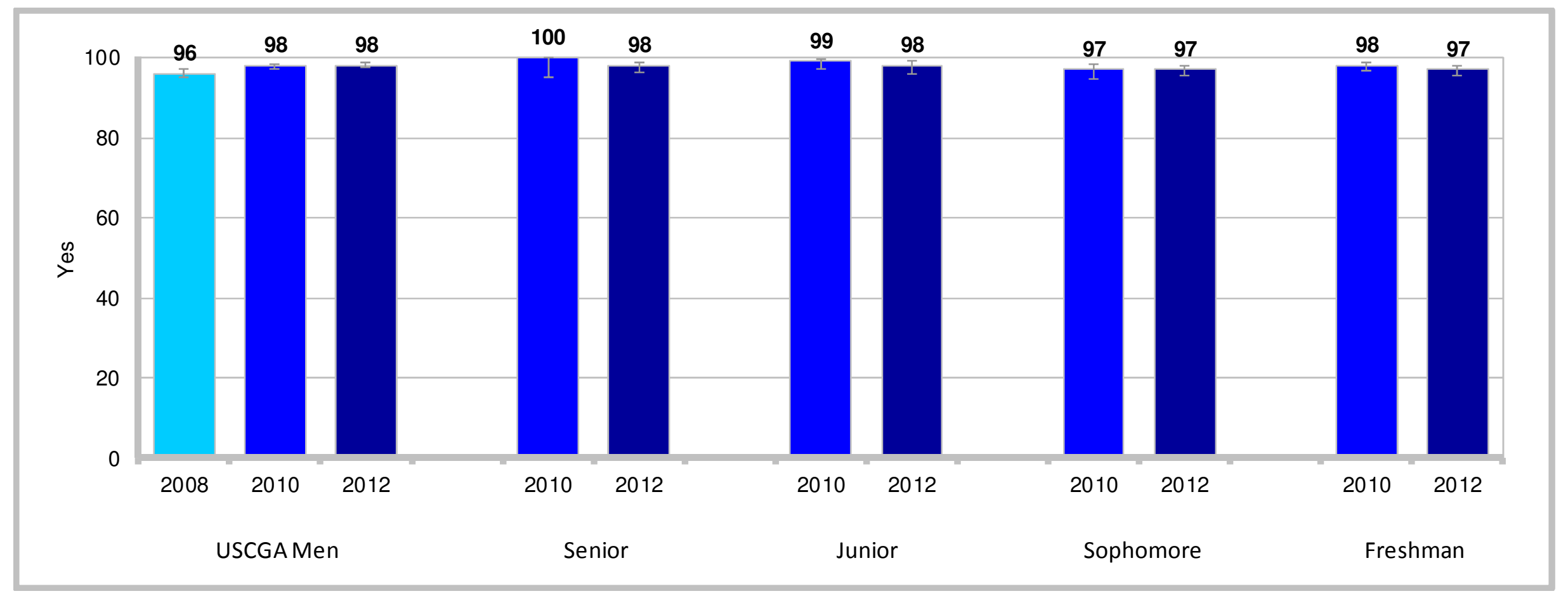

- $98 \%$ of men indicated receiving training on topics related to sexual harassment in 2012

- 2012 comparisons across years - higher than 2008

- Seniors in 2012 lower than 2010

- Class differences in 2012 - no differences 


\section{Effectiveness of Training in Actually Reducing/Preventing Sexual Harassment \\ Percent of Women Who Had Training on Sexual Harassment}

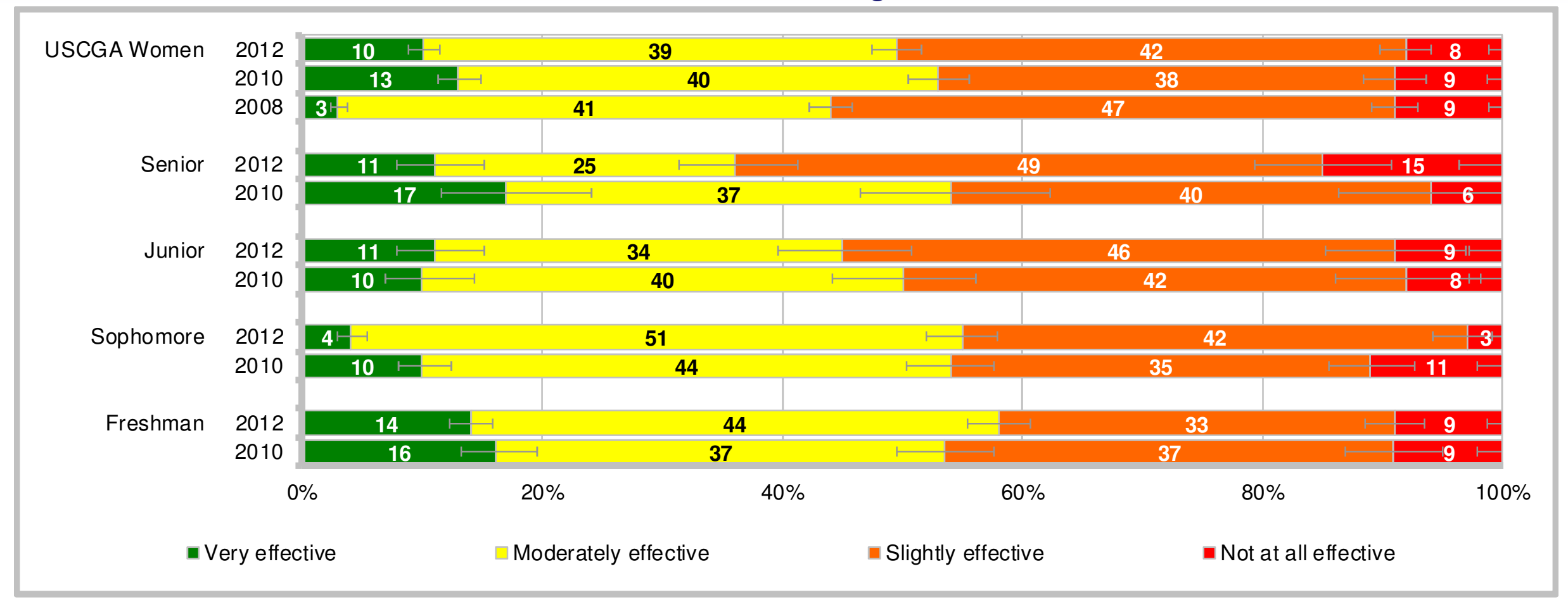

- $10 \%$ of women indicated their training was very effective in actually reducing/preventing sexual harassment; $39 \%$ indicated moderately effective; $42 \%$ indicated slightly effective; and $8 \%$ indicated not at all effective

- 2012 comparisons across years - very effective lower than 2010, higher than 2008; slightly effective higher than 2010 , lower than 2008

- Sophomores indicating very effective in 2012 lower than 2010; sophomores and freshmen indicating moderately effective in 2012 higher than 2010, whereas seniors lower; sophomores indicating slightly effective in 2012 higher than 2010; seniors indicating not at all effective in 2012 higher than 2010, whereas sophomores lower

- Class differences in 2012 - very effective led by freshmen; moderately effective led by sophomores and freshmen; slightly effective led by seniors; not at all effective led by seniors 


\section{Effectiveness of Training in Actually Reducing/Preventing Sexual Harassment \\ Percent of Men Who Had Training on Sexual Harassment}

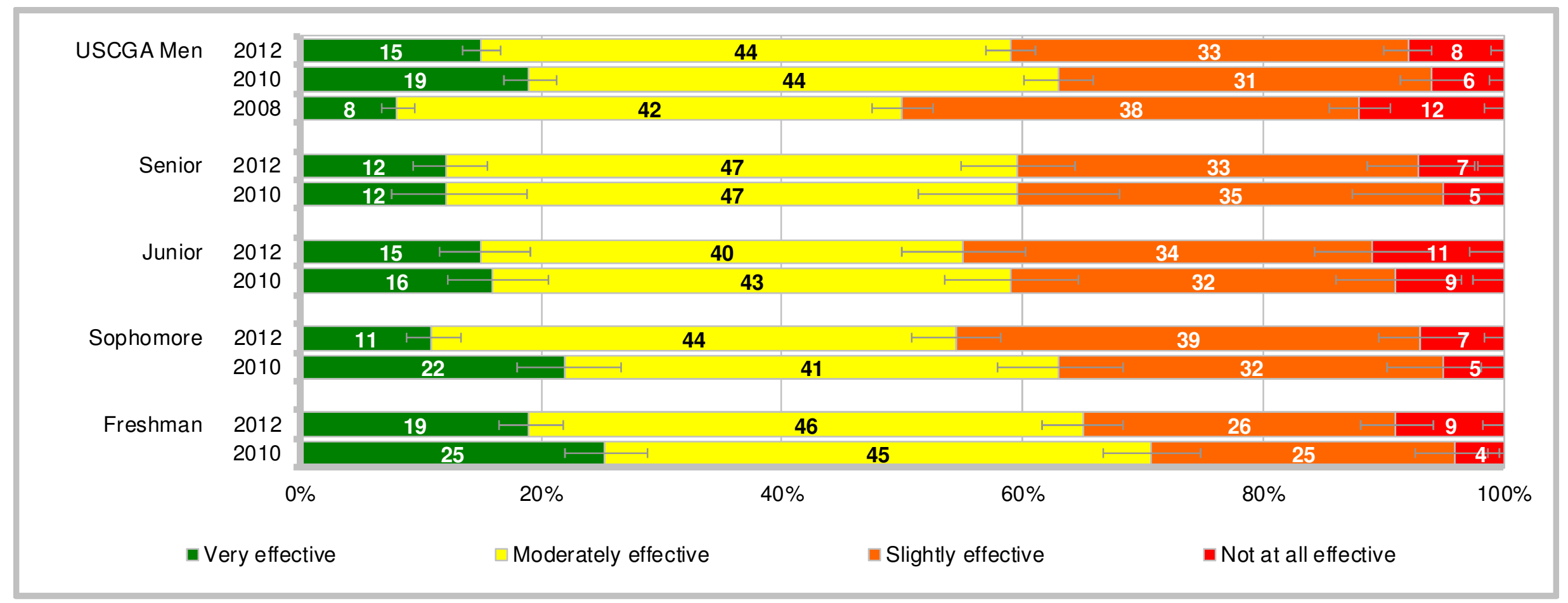

- $15 \%$ of men indicated their training was very effective in actually reducing/preventing sexual harassment; $44 \%$ indicated moderately effective; $33 \%$ indicated slightly effective; and $8 \%$ indicated not at all effective

- 2012 comparisons across years - very effective lower than 2010, higher than 2008; slightly effective lower than 2008; not at all effective higher than 2010, lower than 2008

- Sophomores and freshmen indicating very effective in 2012 lower than 2010; sophomores indicating slightly effective in 2012 higher than 2010; freshmen indicating not at all effective in 2012 higher than 2010

- Class differences in 2012 - very effective led by freshmen; slightly effective led by sophomores 


\section{Contents}

Slide

- Introduction ............................................................................................. 3

- Unwanted Sexual Contact ............................................................... 7

- Unwanted Gender-Related Behaviors ........................................... 45

• Stalking ............................................................................................ 71

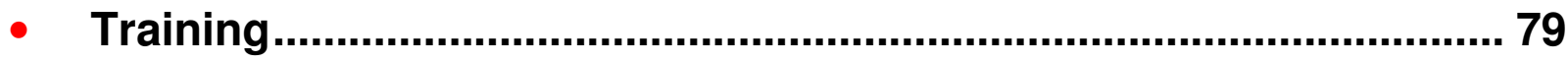

$\checkmark \quad$ Student Perceptions...................................................................... 89 


\section{Extent You Think Incidents of Sexual Assault Are Not Reported Percent of Women}

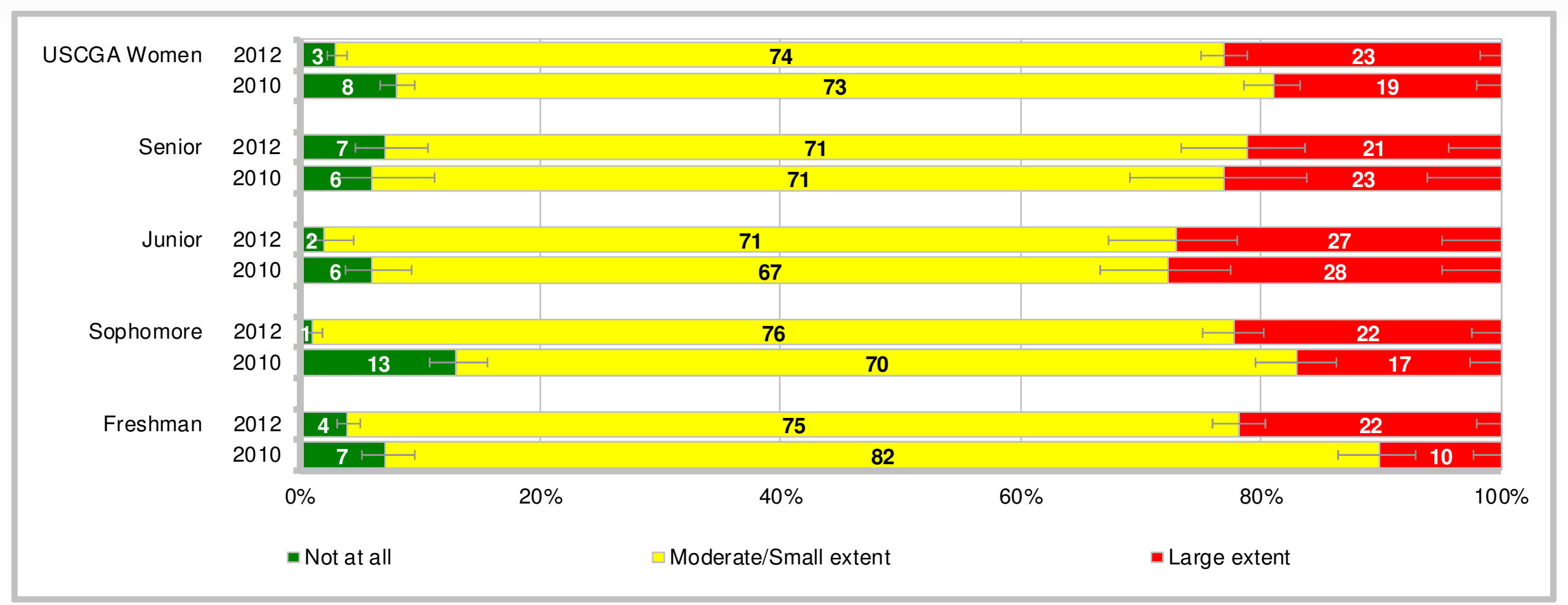

- $23 \%$ of women indicated to a large extent that incidents of sexual assault are not reported; $74 \%$ indicated moderate/small extent; and $3 \%$ indicated not at all

- 2012 comparisons across years - not at all lower than 2010; large extent higher than 2010

- Juniors, sophomores, and freshmen indicating not at all in 2012 lower than 2010; sophomores indicating moderate/small extent in 2012 higher than 2010, whereas freshmen lower; sophomores and freshmen indicating large extent in 2012 higher than 2010

- Class differences in 2012 - not at all led by seniors 


\section{Extent You Think Incidents of Sexual Assault Are Not Reported Percent of Men}

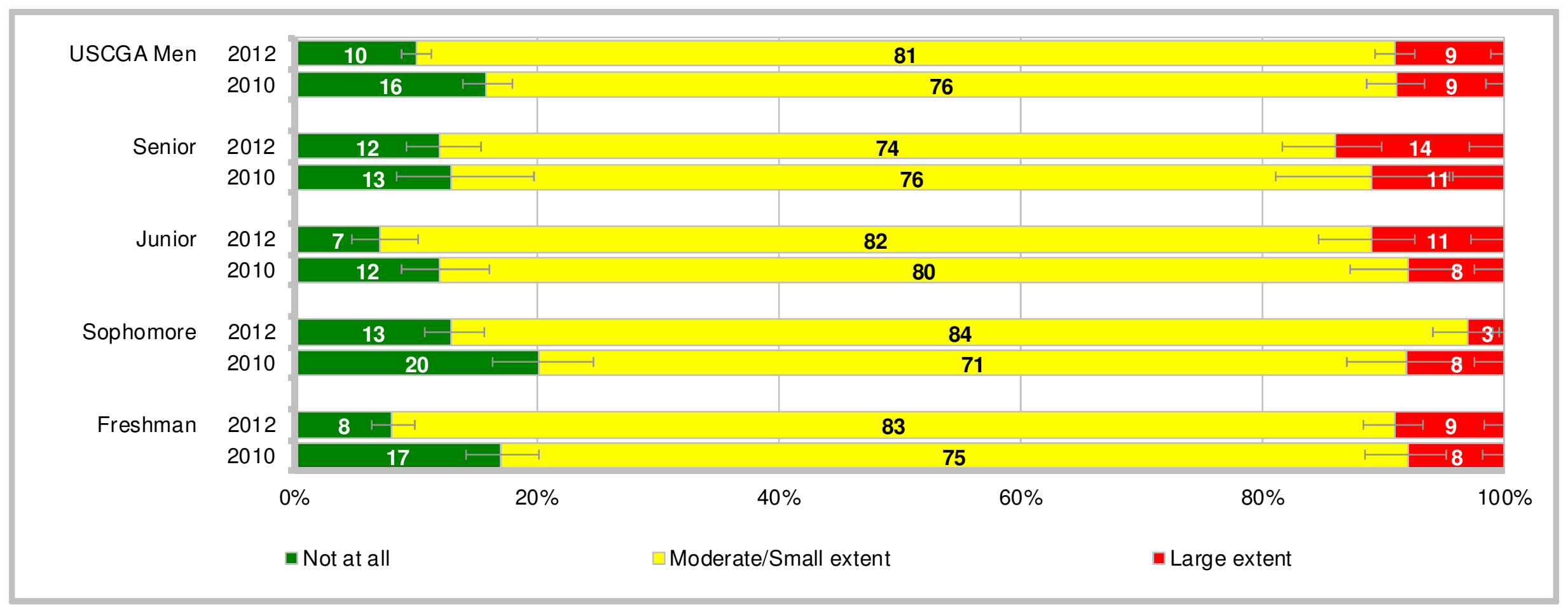

- $9 \%$ of men indicated to a large extent that incidents of sexual assault are not reported; $81 \%$ indicated moderate/small extent; and $10 \%$ indicated not at all

- 2012 comparisons across years - not at all lower than 2010; moderate/small extent higher than 2010

- Juniors, sophomores, and freshmen indicating not at all in 2012 lower than 2010; sophomores and freshmen indicating moderate/small extent in 2012 higher than 2010; sophomores indicating large extent in 2012 lower than 2010

- Class differences in 2012 - large extent led by seniors; not at all led by sophomores SAGR 2012 Q7a 


\section{Extent You Would Report a Sexual Assault Percent of Women}

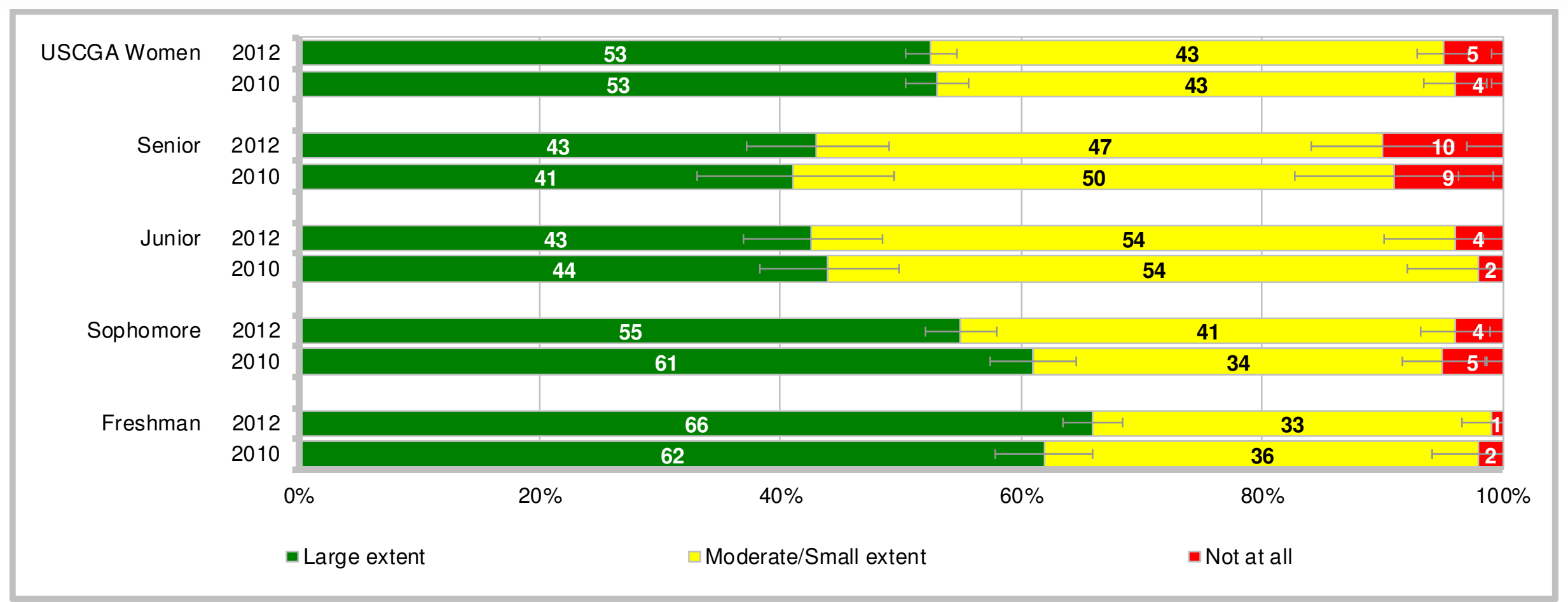

- $53 \%$ of women indicated to a large extent they would be willing to report a sexual assault; $43 \%$ indicated moderate/small extent; and $5 \%$ indicated not at all

- 2012 comparisons across years - no differences

- Sophomores indicating large extent in 2012 lower than 2010; sophomores indicating moderate/small extent in 2012 higher than 2010

- Class differences in 2012 - large extent led by freshmen; moderate/small extent led by juniors; not at all led by seniors 


\section{Extent You Would Report a Sexual Assault Percent of Men}

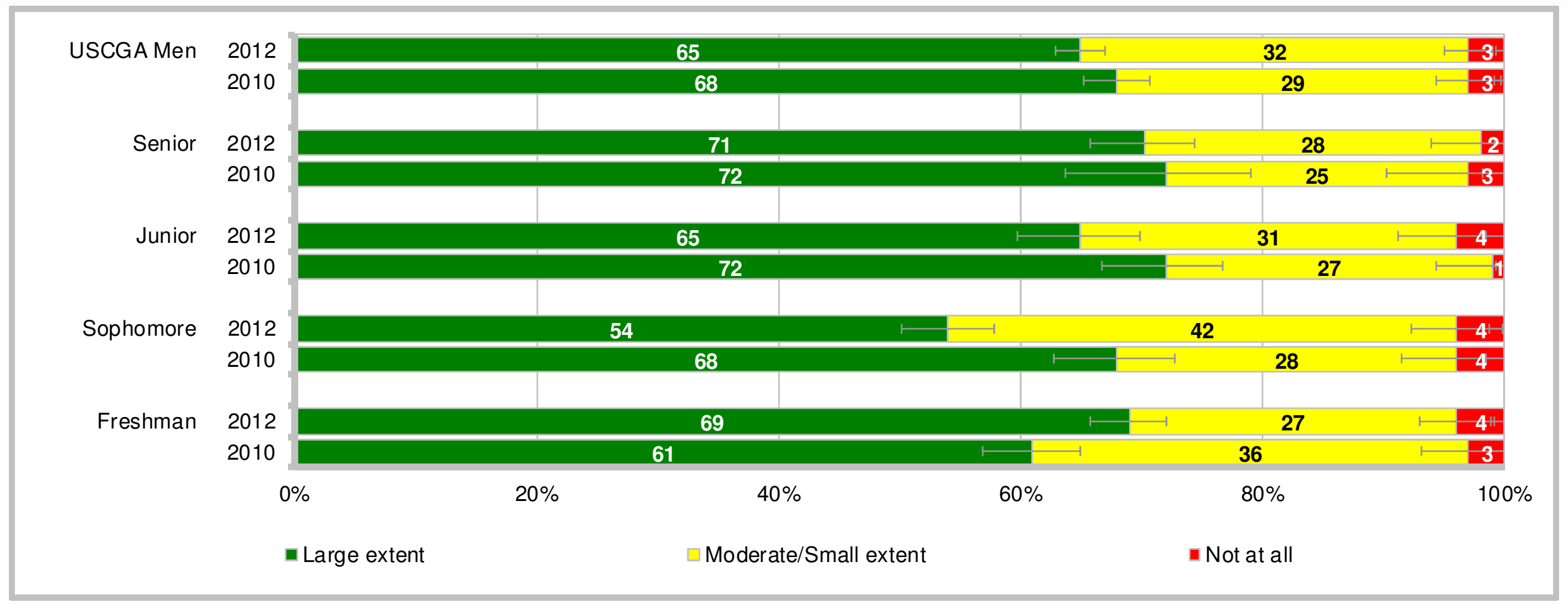

- $65 \%$ of men indicated to a large extent they would be willing to report a sexual assault; $32 \%$ indicated to a moderate/small extent; and $3 \%$ indicated not at all

- 2012 comparisons across years - no differences

- Sophomores indicating large extent in 2012 lower than 2010, whereas freshmen higher; sophomores indicating moderate/small extent in 2012 higher than 2010, whereas freshmen lower; juniors not at all in 2012 higher than 2010

- Class differences in 2012 - large extent led by seniors and freshmen; moderate/small extent led by sophomores 


\section{Extent You Think High-Profile Cases Deter Reporting of Sexual Assault Percent of Women}

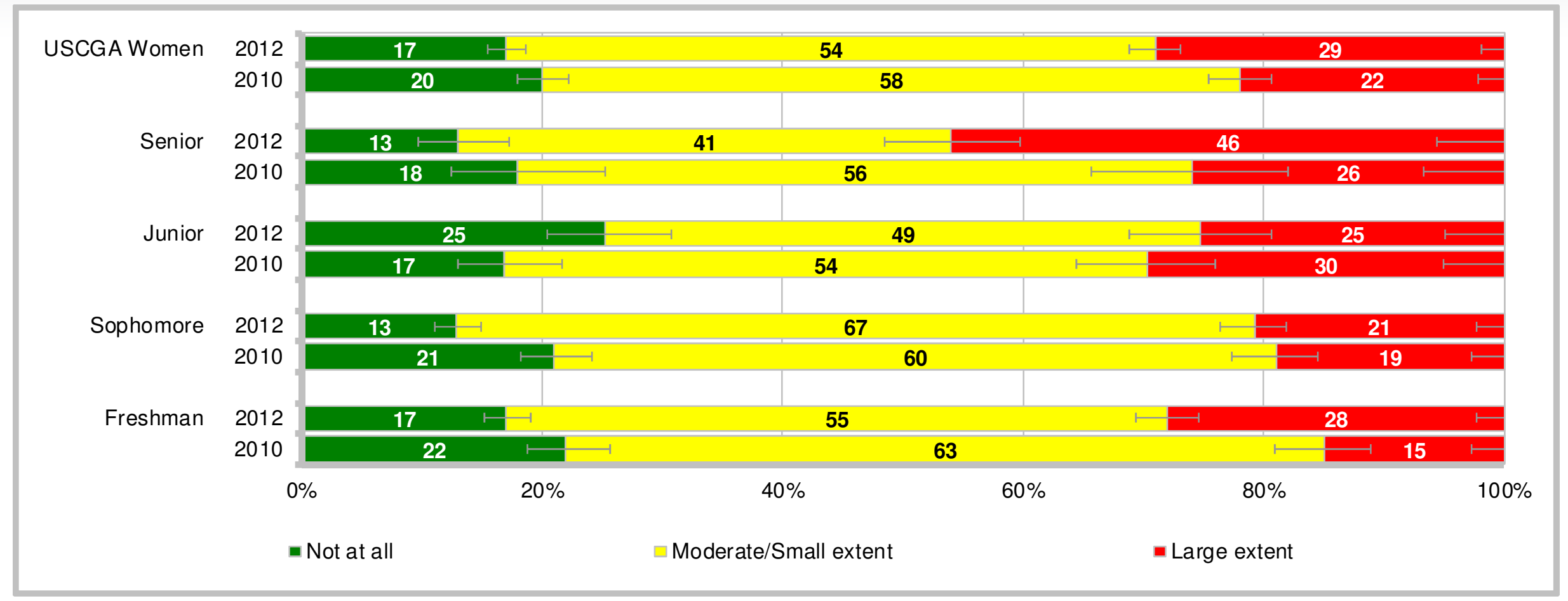

- $29 \%$ of women indicated to a large extent high-profile cases deter reporting of sexual assault; $54 \%$ indicated to a moderate/small extent; and $17 \%$ indicated not at all

- 2012 comparisons across years - not at all lower than 2010; moderate/small extent lower than 2010; large extent higher than 2010

- Juniors indicating not at all in 2012 higher than 2010, whereas sophomores and freshmen lower; sophomores indicating moderate/small extent in 2012 higher than 2010, whereas seniors and freshmen lower; seniors and freshmen indicating large extent in 2012 higher than 2010

- Class differences in 2012 - large extent led by seniors; moderate/small extent led by sophomores; not at all led by juniors 


\section{Extent You Think High-Profile Cases Deter Reporting of Sexual Assault} Percent of Men

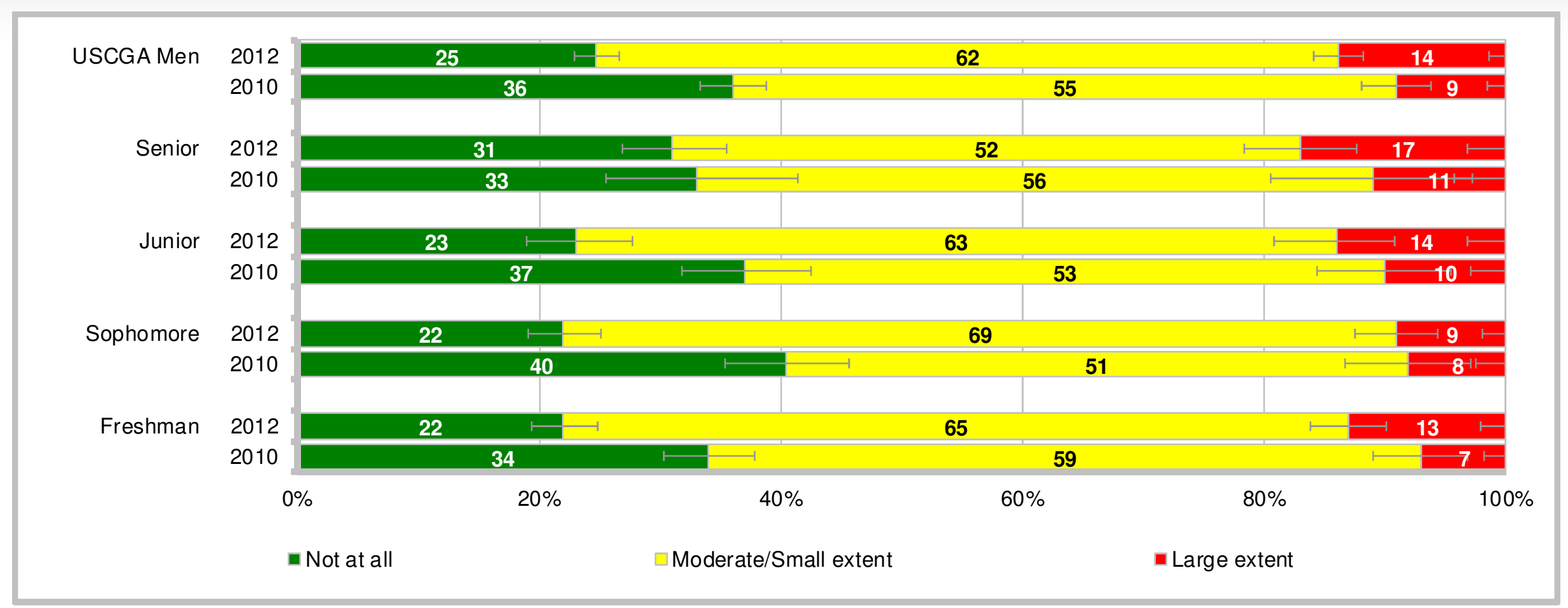

- $14 \%$ of men indicated to a large extent high-profile cases deter reporting of sexual assault; $62 \%$ indicated to a moderate/small extent; and $25 \%$ indicated not at all

- 2012 comparisons across years - not at all lower than 2010; moderate/small extent higher than 2010; large extent higher than 2010

- Juniors, sophomores, and freshmen indicating not at all in 2012 lower than 2010; juniors, sophomores, and freshmen indicating moderate/small extent in 2012 higher than 2010; freshmen indicating large extent in 2012 higher than 2010

- Class differences in 2012 - moderate/small extent led by sophomores; not at all led by seniors 


\section{You Would Discuss an Incident With a SARC if You Were To Experience} Sexual Assault in the Future

\section{Percent of Women Who Had Not Experienced Unwanted Sexual Contact in Past 12 Months}

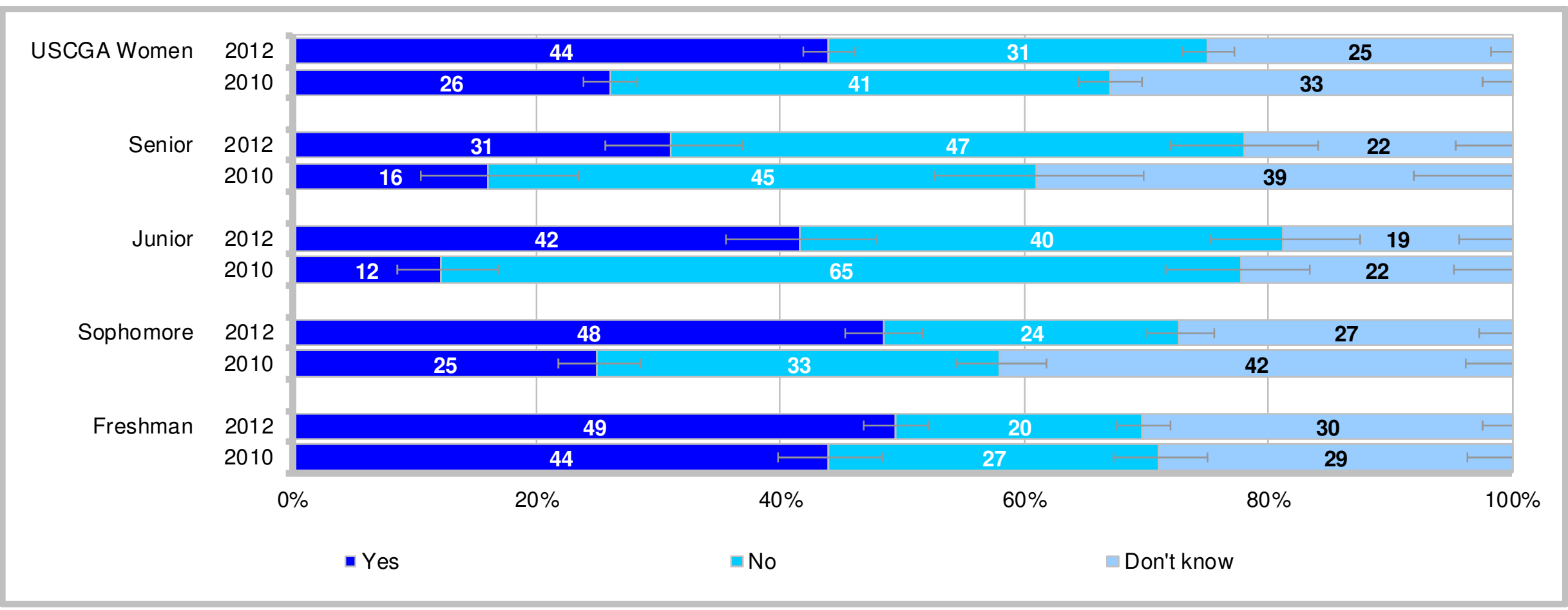

- $44 \%$ of women indicated yes, they would discuss an incident of sexual assault with a SARC; $31 \%$ indicated no; and $25 \%$ indicated don't know

- 2012 comparisons across years - yes higher than 2010; no lower than 2010; don't know lower than 2010

- Seniors, juniors, and sophomores indicating yes in 2012 higher than 2010; juniors, sophomores, and freshman indicating no in 2012 lower than 2010; seniors and sophomores indicating don't know in 2012 lower than 2010

- Class differences in 2012 - yes led by freshmen and sophomores; no led by seniors and juniors; don't know led by freshmen 


\section{You Would Discuss an Incident With a SARC if You Were To Experience} Sexual Assault in the Future

\section{Percent of Men Who Had Not Experienced Unwanted Sexual Contact in Past 12 Months}

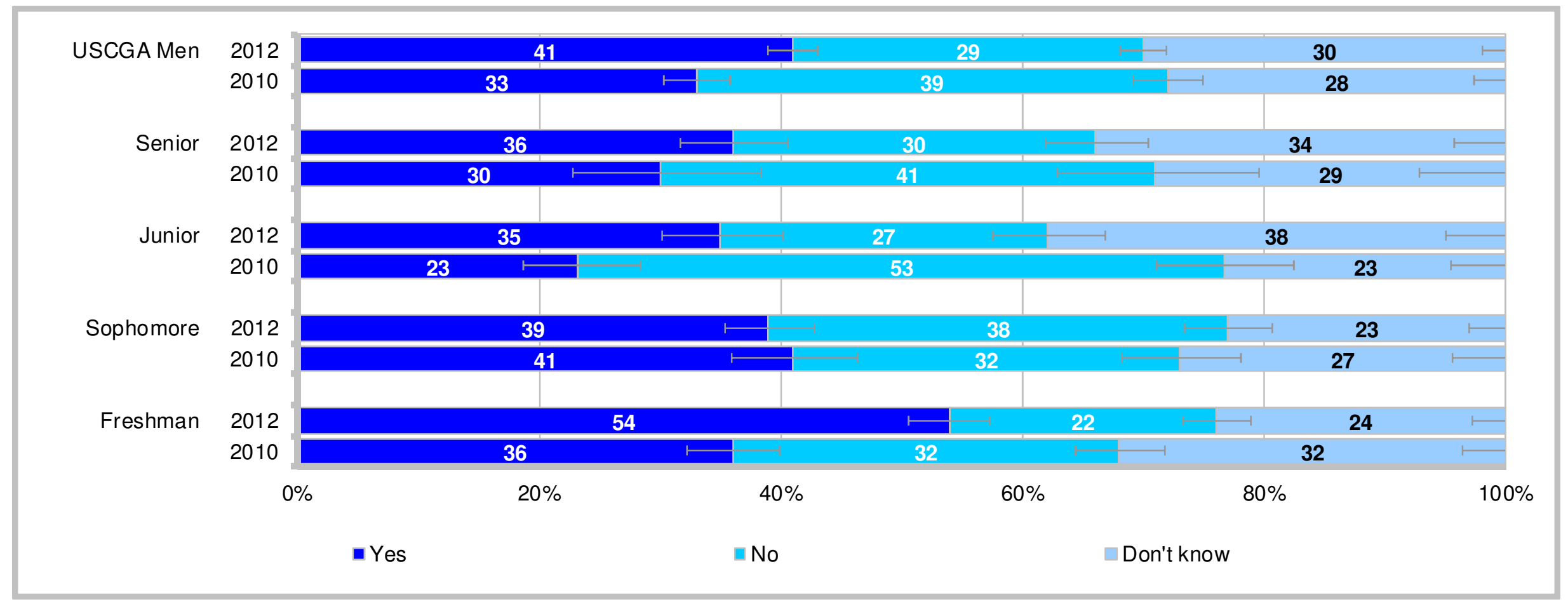

- $41 \%$ of men indicated yes, they would discuss an incident of sexual assault with a SARC; $29 \%$ indicated no; and $30 \%$ indicated don't know

- 2012 comparisons across years - yes higher than 2010; no lower than 2010

- Juniors and freshmen indicating yes in 2012 higher than 2010; seniors, juniors, and freshman indicating no in 2012 lower than 2010; freshmen indicating don't know in 2012 lower than 2010, whereas juniors higher

- Class differences in 2012 - yes led by freshmen; no led by sophomores; don't know led by juniors 


\section{You Would Trust the System To Protect Your Privacy if You Were To Experience Sexual Assault in the Future \\ Percent of Women Who Had Not Experienced Unwanted Sexual Contact in the Past 12 Months}

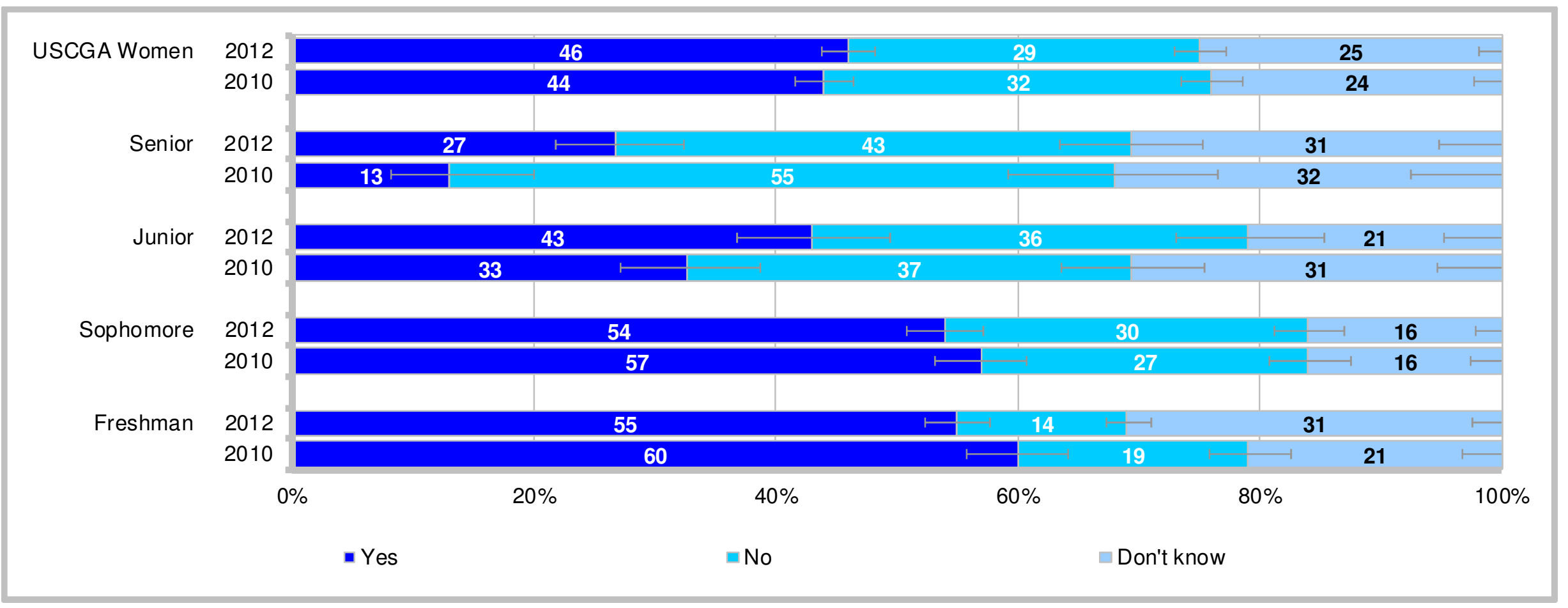

- $46 \%$ of women indicated yes, they would trust the system to protect their privacy if they experienced sexual assault; $29 \%$ indicated no; and $25 \%$ indicated don't know

- 2012 comparisons across years - no differences

- Seniors and juniors indicating yes in 2012 higher than 2010, whereas freshmen lower; seniors and freshmen indicating no in 2012 lower than 2010; juniors indicating don't know in 2012 lower than 2010, whereas freshmen higher

- Class differences in 2012 - yes led by freshmen and sophomores; no led by seniors and juniors; don't know led by freshmen 


\section{You Would Trust the System To Protect Your Privacy if You Were To Experience Sexual Assault in the Future \\ Percent of Men Who Had Not Experienced Unwanted Sexual Contact in the Past 12 Months}

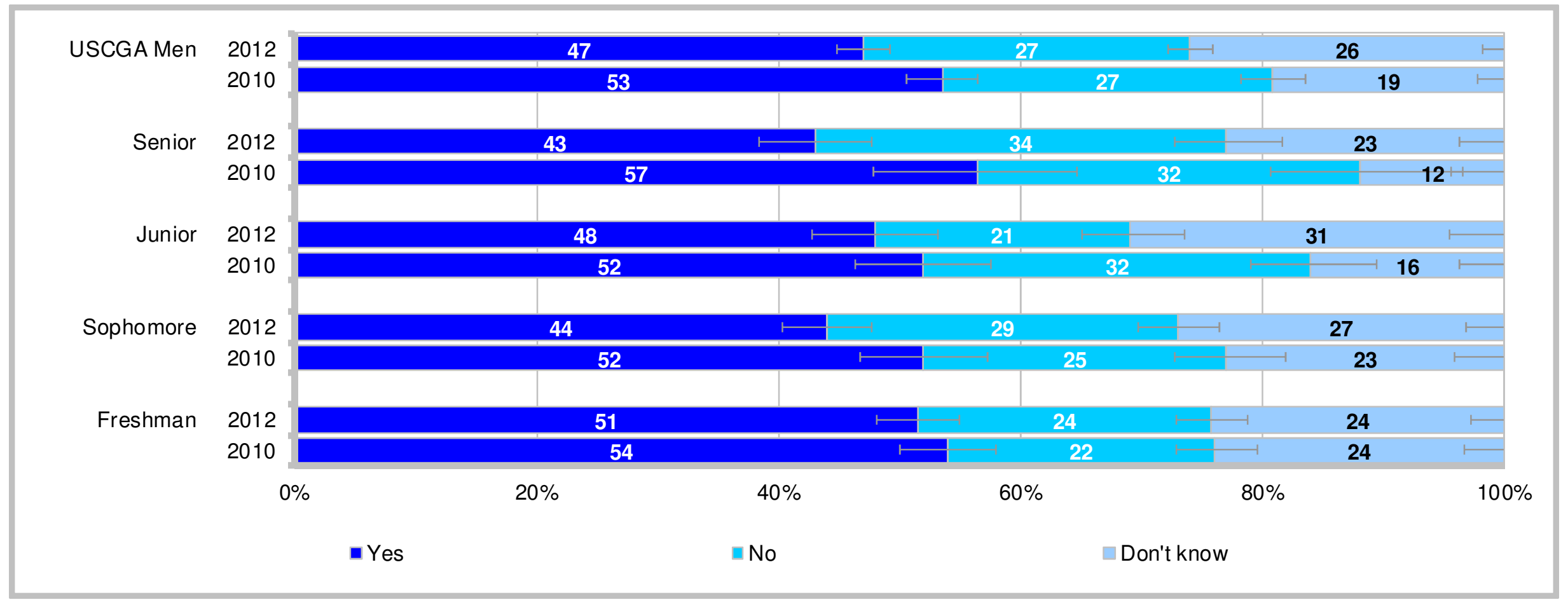

- $47 \%$ of men indicated yes, they would trust the system to protect their privacy if they experienced sexual assault; $27 \%$ indicated no; and $26 \%$ indicated don't know

- 2012 comparisons across years - yes lower than 2010; don't know higher than 2010

- Seniors and sophomores indicating yes in 2012 lower than 2010; juniors indicating no in 2012 lower than 2010; seniors and juniors indicating don't know in 2012 higher than 2010

- Class differences in 2012 - yes led by freshmen; no led by seniors 


\section{You Would Be Willing To Encourage Someone Who Has Experienced Sexual Assault To Report It Percent of Women}

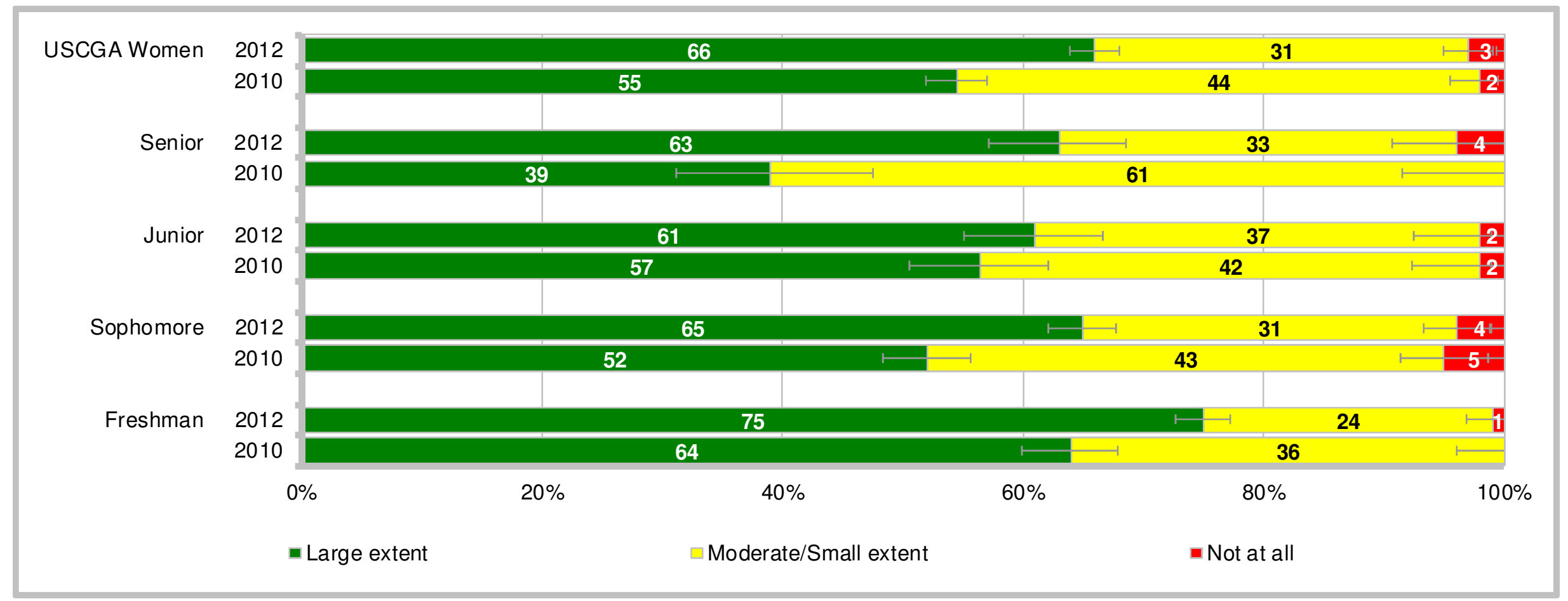

- $97 \%$ of women indicated they would encourage someone to report sexual assault to at least some extent; $3 \%$ indicated not at all

- 2012 comparisons across years - large extent higher than 2010; moderate/small extent lower than 2010

- Seniors, sophomores, and freshmen indicating large extent in 2012 higher than 2010; seniors, sophomores, and freshmen indicating moderate/small extent in 2012 lower than 2010; seniors indicating not at all in 2012 higher than 2010

- Class differences in 2012 - large extent led by freshmen; moderate/small extent led by juniors; not at all led by sophomores*

*Note that both $4 \%$ of senior and sophomore women indicated not at all in 2012 . The percentage for senior women is not significantly different from the average of the other class years due to a higher margin of error for senior women responding to this question. 


\section{You Would Be Willing To Encourage Someone Who Has Experienced Sexual Assault To Report It \\ Percent of Men}

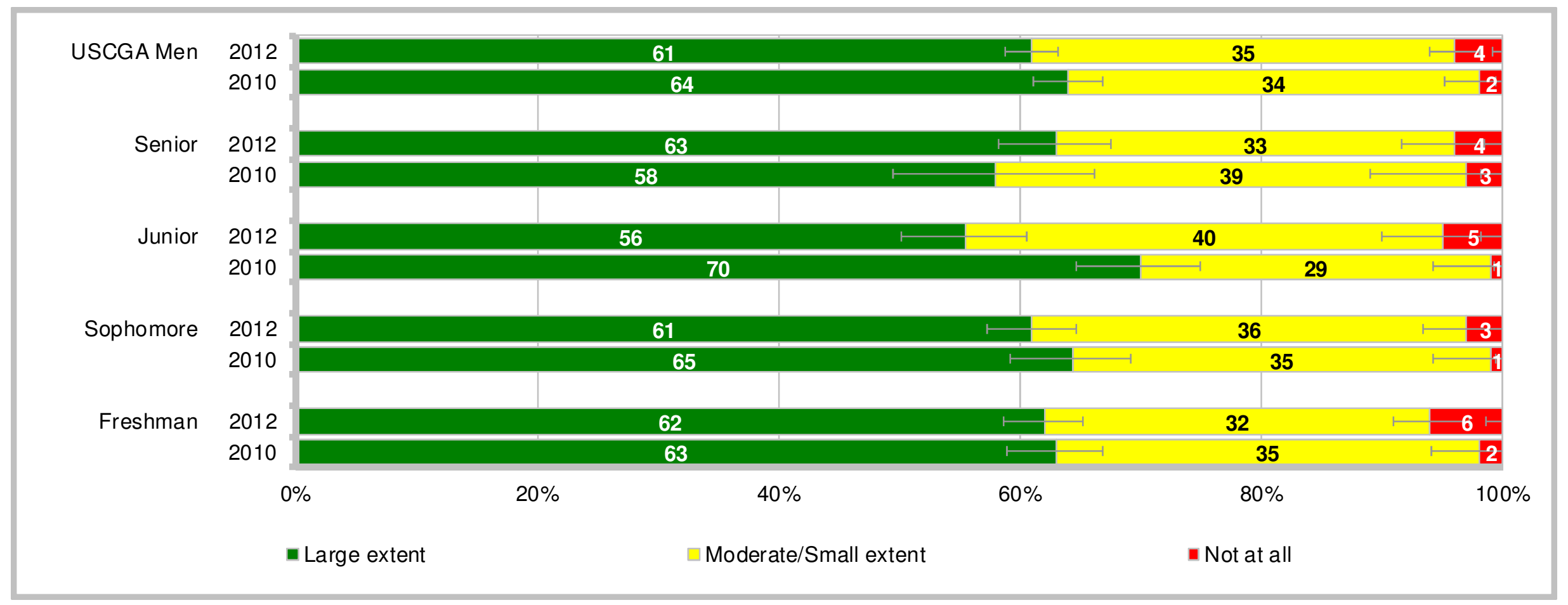

- $96 \%$ of men indicated they would encourage someone to report sexual assault to at least some extent; $4 \%$ indicated not at all

- 2012 comparisons across years - not at all higher than 2010

- Juniors indicating large extent in 2012 lower than 2010; juniors indicating moderate/small extent in 2012 higher than 2010; juniors, sophomores, and freshmen indicating not at all in 2012 higher than 2010

- Class differences in 2012 - no differences 


\section{You Would Step In and Stop a Situation That Might Lead to Sexual Assault} Percent of Women

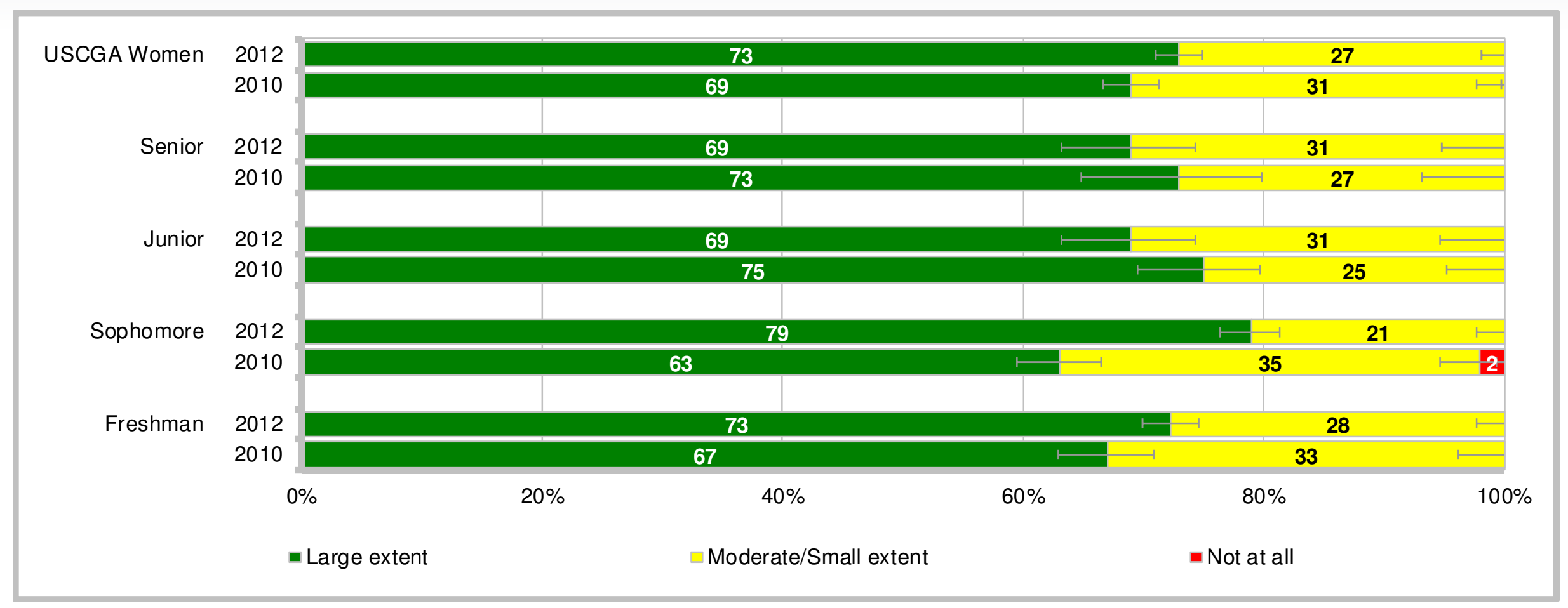

- $100 \%$ of women indicated they would step in and stop a situation that might lead to sexual assault to at least some extent; $0 \%$ indicated not at all

- 2012 comparisons across years - large extent higher than 2010; moderate/small extent lower than 2010

- Sophomores and freshmen indicating large extent in 2012 higher than 2010; sophomores and freshmen indicating moderate/small extent in 2012 lower than 2010; sophomores indicating not at all in 2012 lower 2010

- Class differences in $\mathbf{2 0 1 2}$ - large extent led by sophomores 


\section{You Would Step In and Stop a Situation That Might Lead to Sexual Assault}

Percent of Men

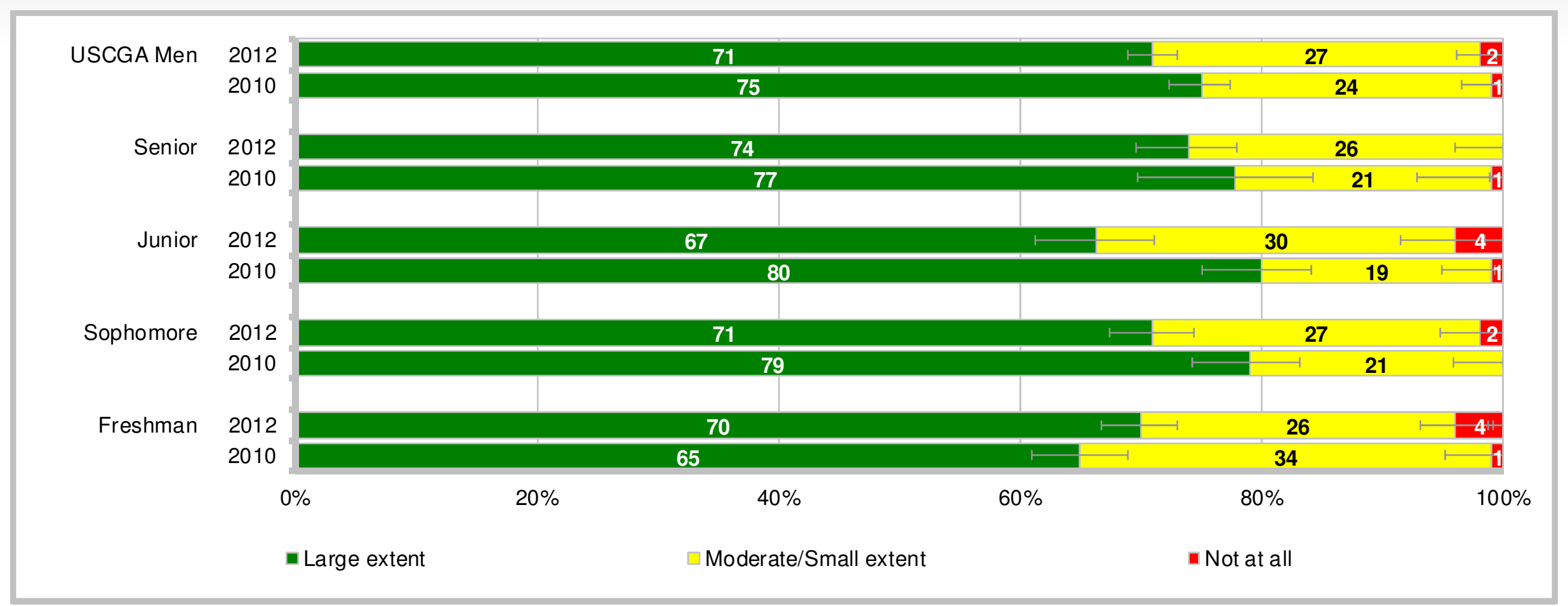

- $98 \%$ of men indicated they would step in and stop a situation that might lead to sexual assault to at least some extent; $2 \%$ indicated not at all

- 2012 comparisons across years - large extent lower than 2010

- Juniors and sophomores indicating large extent in 2012 lower than 2010; juniors and sophomores indicating moderate/small extent in 2012 higher than 2010, whereas freshmen lower; juniors, sophomores, and freshmen indicating not at all in 2012 higher 2010

- Class differences in 2012 - not at all led by freshmen*

*Note that both $4 \%$ of junior and freshman men indicated not at all in 2012 . The percentage for junior men is not significantly different from the average of the other class years due to a higher margin of error for junior men responding to this question. 


\section{You Would Point Out to Someone That They "Crossed the Line" With Comments or Jokes \\ Percent of Women}

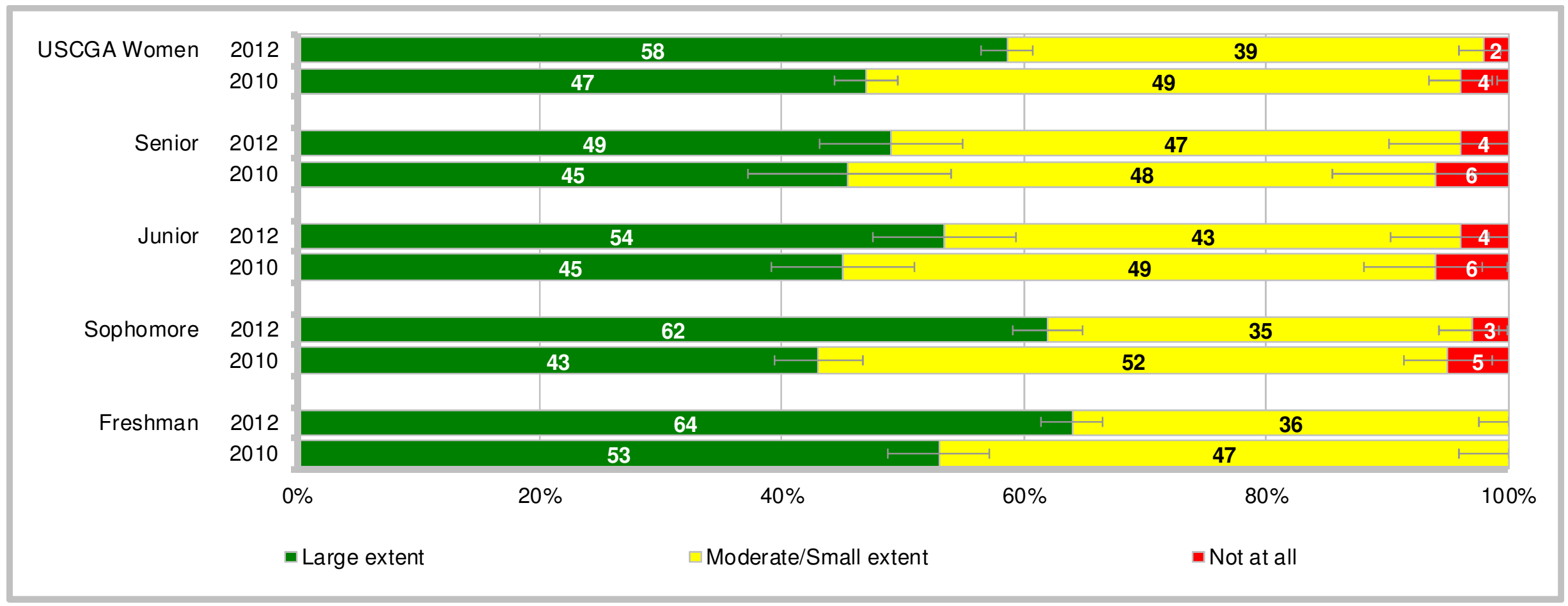

- $97 \%$ of women indicated they would point out to someone that they "crossed the line" with comments or jokes to at least some extent; $2 \%$ indicated not at all

- 2012 comparisons across years - large extent higher than 2010; moderate/small extent lower than 2010; not at all lower than 2010

- Juniors, sophomores, and freshmen indicating large extent in 2012 higher than 2010; sophomores and freshmen indicating moderate/small extent in 2012 lower than 2010; sophomores indicating not at all in 2012 lower than 2010

- Class differences in 2012 - large extent led by freshmen and sophomores; moderate/small extent led by seniors 


\section{You Would Point Out to Someone That They "Crossed the Line" With Comments or Jokes \\ Percent of Men}

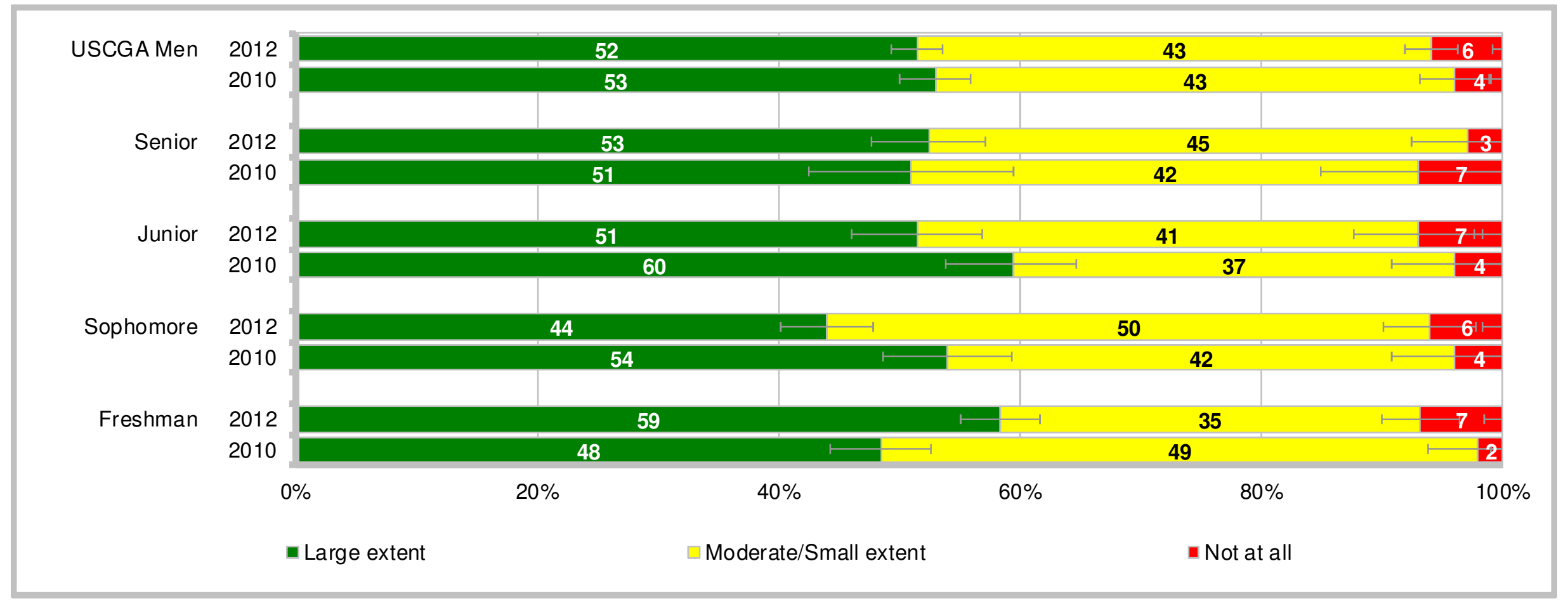

- $95 \%$ of men indicated they would point out to someone that they "crossed the line" with comments or jokes to at least some extent; $6 \%$ indicated not at all

- 2012 comparisons across years - not at all higher than 2010

- Juniors and sophomores indicating large extent in 2012 lower than 2010, whereas freshmen higher; sophomores indicating moderate/small extent in 2012 higher than 2010, whereas freshmen lower; freshmen indicating not at all in 2012 higher than 2010

- Class differences in 2012 - large extent led by freshmen; moderate/small extent led by sophomores 


\section{You Would Seek Help From Chain of Command in Stopping Other Students Who Continue To Engage in Sexual Harassment}

\section{Percent of Women}

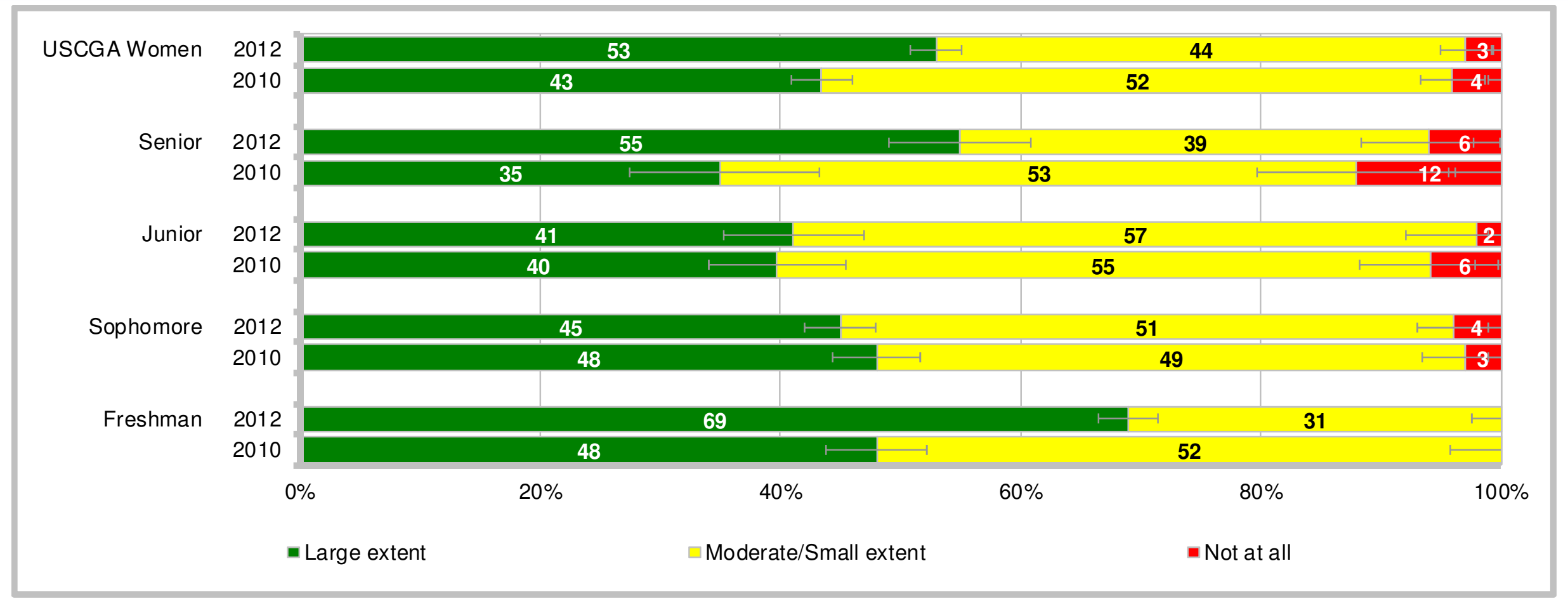

- $97 \%$ of women indicated they would seek help from the chain of command in stopping other students who continue to engage in sexual harassment to at least some extent; $3 \%$ indicated not at all

- 2012 comparisons across years - large extent higher than 2010; moderate/small extent lower than 2010

- Seniors and freshmen indicating large extent in 2012 higher than 2010; seniors and freshmen indicating moderate/small extent in 2012 lower than 2010; juniors indicating not at all in 2012 lower than 2010

- Class differences in 2012 - large extent led by freshmen; moderate/small extent led by juniors and sophomores; not at all led by seniors 


\section{You Would Seek Help From Chain of Command in Stopping Other Students Who Continue To Engage in Sexual Harassment}

Percent of Men

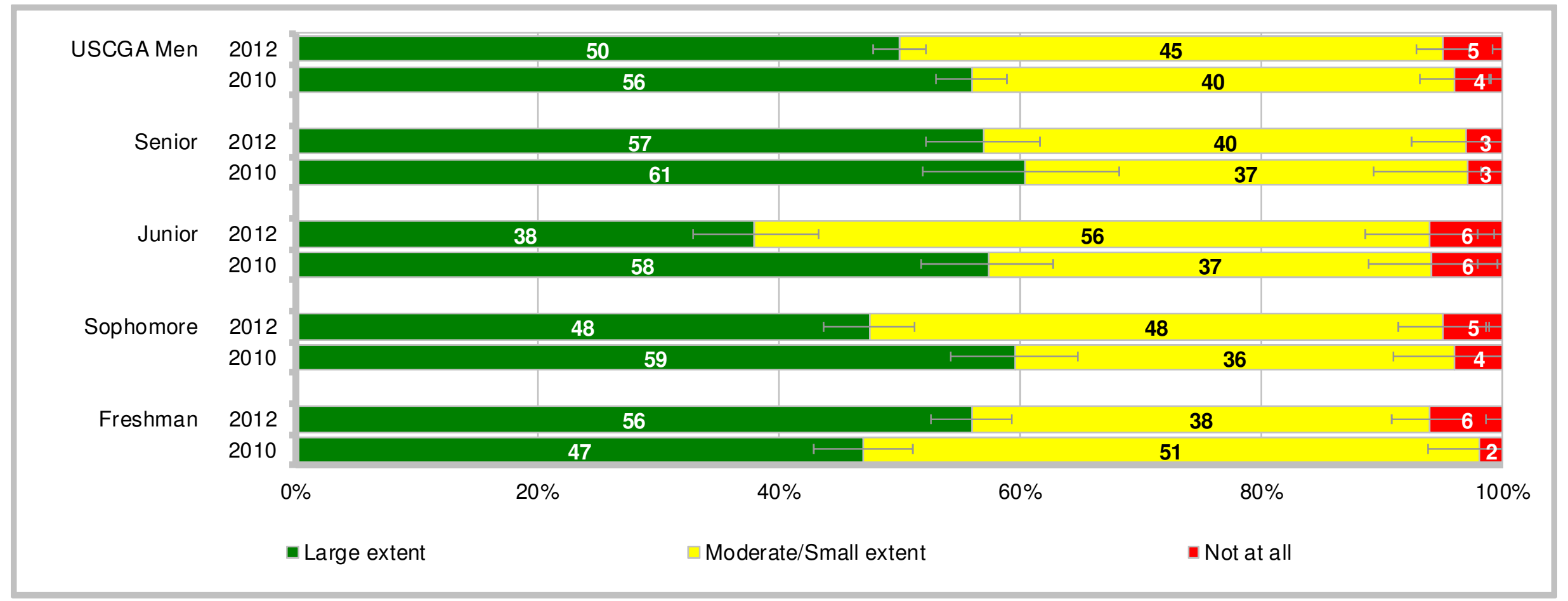

- $95 \%$ of men indicated they would seek help from the chain of command in stopping other students who continue to engage in sexual harassment to at least some extent; and $5 \%$ indicated not at all

- 2012 comparisons across years - large extent lower than 2010; moderate/small extent higher than 2010

- Juniors and sophomores indicating large extent in 2012 lower than 2010, whereas freshmen higher; juniors and sophomores indicating moderate/small extent in 2012 higher than 2010, whereas freshmen lower; freshmen indicating not at all in 2012 higher than 2010

- Class differences in 2012 - large extent led by seniors and freshmen; moderate/small extent led by juniors 